\title{
Sichere Knotenpunkte für schwächere Verkehrsteilnehmer
}

Jürgen Gerlach

Sebastian Seipel

Sebastian Poschadel

Dirk Boenke

Unfallforschung der Versicherer 


\section{Sichere Knotenpunkte für schwächere Verkehrsteilnehmer}

Univ.-Prof. Dr.-Ing. Jürgen Gerlach

Dipl.-Ing. Sebastian Seipel

Dr.-phil. Sebastian Poschadel

Dr.-Ing. Dirk Boenke 


\section{Impressum}

\section{Gesamtverband der Deutschen Versicherungswirtschaft e. V.}

\section{Unfallforschung der Versicherer}

Wilhelmstraße 43/43G, 10117 Berlin

Postfach 0802 64, 10002 Berlin

E-Mail: unfallforschung@gdv.de

Internet: www.udv.de

Facebook: www.facebook.com/unfallforschung

Twitter: @unfallforschung

YouTube: www.youtube.com/unfallforschung

ISBN-Nr.: 978-3-939163-52-7

Bildnachweis: UDV und siehe Quellenangaben

Erschienen: 2014 


\section{Sichere Knotenpunkte für schwächere Verkehrsteilnehmer}

bearbeitet durch:

Lehr- und Forschungsgebiet "Straßenverkehrsplanung und Straßenverkehrstechnik" am Fachzentrum Verkehr der Bergischen Universität Wuppertal Univ.-Prof. Dr.-Ing. Jüren Gerlach Dipl.-Ing. Sebastian Seipel

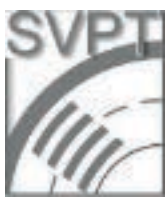

Leibniz-Institut für Arbeitsforschung an der Technischen Universität Dortmund

Dr.- phil. Sebastian Poschadel

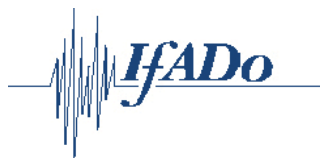

und Studiengesellschaft für unterirdische Verkehrsanlagen e. V. Dr.-Ing. Dirk Boenke

\section{STUV/A}

Bei der UDV betreut von: Dr.-Ing. Jean Emmanuel Bakaba

Dipl.-Ing. Jörg Ortlepp

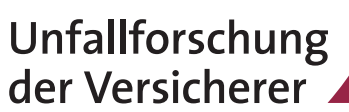


Abbildungen sind teilweise Videoausschnitte.

Die Bildauflösung wurde aus datenschutzrechtlichen Gründen reduziert.

Die Gesichter der Verkehrsteilnehmer sind im Einzelfall zusätzlich unkenntnlich gemacht worden. 


\section{Kurzfassung / Abstract}

\section{Sichere Knotenpunkte für schwächere Verkehrsteilnehmer}

Kinder, ältere Menschen und Menschen mit besonderen Mobilitätseinschränkungen oder Mobilitätsbehinderungen sind im Verkehrsraum aufgrund ihrer körperlichen und kognitiven Fähigkeiten häufig benachteiligt. Sie zählen zu den so genannten „schwächeren Verkehrsteilnehmern". Mit dem Ziel einen effizienten Ansatz zur Erhöhung der Verkehrssicherheit „schwächerer Verkehrsteilnehmer“ zu liefern, wurde das Forschungsvorhaben "Sichere Knotenpunkte für schwächere Verkehrsteilnehmer" durchgeführt.

Zunächst wurden die Anforderungen von Kindern (bis einschließlich 14 Jahre), älteren Menschen (65 Jahre und älter) und Menschen mit besonderen Mobilitäteinschränkungen oder Mobilitätsbehinderungen hinsichtlich der aktiven Teilnahme am Straßenverkehr, die sich vor allem aus den physiologischen und psychologischen Fähigkeiten ergeben, erarbeitet.

Auf Basis einer makroskopischen Unfallanalyse von rd. 350.000 Unfällen an Knotenpunkten aus fünf Bundesländern (rd. 280.000 Unfälle innerorts und rd. 65.000 Unfälle außerorts, ohne Autobahnen) wurden im Weiteren typische Unfallsituationen von Kindern und älteren Menschen ermittelt. Im Hinblick auf Unfälle innerorts lässt sich zusammenfassend festhalten, dass

- Kinder als Hauptverursacher oft als Radfahrer bei Einbiegen/Kreuzen-Unfällen, insbesondere Typ 342 und 321, und überproportional oft als Fußgänger bei Überschreiten-Unfällen erfasst sind,

- $\quad$ Kinder innerhalb ihrer Altersgruppe zusätzlich häufig bei Abbiege-Unfällen als weitere beteiligte Radfahrer und Fußgänger verunglücken,

- $\quad$ Ältere bei Abbiege-Unfällen, insbesondere im Typ 211, und Einbiegen/KreuzenUnfällen als Kraftfahrer am häufigsten vertreten sind (was auch der Verteilung aller Unfälle an Knotenpunkten entspricht) und

- $\quad$ Kinder und ältere Menschen in Relation zu den übrigen Verkehrsteilnehmern häufiger in Überschreiten-Unfälle verwickelt sind und bei diesen auch verunglücken.

Das Unfallgeschehen mobilitätsbehinderter Menschen wurde anhand von Fallbeispielen untersucht. Aus Unfallbeschreibungen, vorwiegend Abbiege- und Einbiegen/KreuzenUnfälle, lassen sich tendenziell die folgenden Ursachen ausmachen:

- fehlende Sichtbeziehungen (Kraftfahrer gaben z. B. an, Rollstuhlnutzer beim Abbiegen übersehen zu haben) und

- falsche Nutzung von Verkehrsflächen (Rollstuhlnutzer befuhren Radverkehrsanlagen, da im Bereich des Gehweges eine Nullabsenkung fehlte).

Aus einem Kollektiv von 291 Knotenpunkten, die unter anderem auf Basis des Unfallgeschehens identifiziert wurden, wurden für die weiteren Untersuchungen zunächst 50 und schließlich 15 Knotenpunkte exemplarisch für Detailuntersuchungen ausgewählt. Die Detailuntersuchungen beinhalteten 
- Unfallanalysen,

- Planaudits,

- Sicherheitsinspektionen vor Ort und

- Verhaltensbeobachtungen im Realverkehr.

Im Ergebnis konnten sicherheitstechnische Defizite an den Verkehrsanlagen festgestellt und prototypische Merkmale bzw. Situationen beobachtet und dokumentiert werden, die insbesondere aus Sicht der „schwächeren Verkehrsteilnehmer" sicherheitsrelevant sind, aber auch aus Sicht aller Verkehrsteilnehmer Sicherheitsrisiken bergen.

An den 15 Knotenpunkten wurden Defizite in den folgenden Kategorien festgestellt:

- Barrierefreie Gestaltung (Defizite an 13 von 15 Knotenpunkten festgestellt)

- Radverkehrsanlagen (Defizite an 9 von 15 Knotenpunkten festgestellt)

- Verkehrsregelung (Defizite an 8 von 15 Knotenpunkten festgestellt)

- Sichtbeziehungen (Defizite an 8 von 15 Knotenpunkten festgestellt)

- Verkehrsführung (Defizite an 6 von 15 Knotenpunkten festgestellt)

- Verkehrszeichen (Defizite an 5 von 15 Knotenpunkten festgestellt)

- Straßenentwurf (Defizite an 4 von 15 Knotenpunkten festgestellt)

- Fußgängerverkehrsanlagen (Defizite an 4 von 15 Knotenpunkten festgestellt)

- Signalsteuerung (Defizite an 1 von 15 Knotenpunkten festgestellt)

Die Verhaltensbeobachtungen der Verkehrsteilnehmer erfolgten neben der Beobachtung durch Personen mit Hilfe eines Funk-Kamera-Systems, mit dessen Hilfe Knotenpunkte aus bis zu vier verschiedenen Perspektiven zeitsynchron erfasst werden konnten. Insgesamt wurde das Verhalten von 24.598 Einzelpersonen als Fußgänger, Radfahrer oder Pkw-Fahrer unter Berücksichtigung der Altersgruppe oder einer Mobilitätseinschränkung/-behinderung analysiert.

Generell konnte bei allen Verkehrsteilnehmern eine hohe Regelkonformität beobachtet werden. In der Gruppe der Fußgänger und Radfahrer machten Kinder relativ gesehen mehr Fehler als Personen anderer Altersgruppen. Ältere wie jüngere Kraftfahrer begingen qualitativ dieselben Fehler. Allerdings machten älterer Kraftfahrer beim Linksabbiegen relativ mehr Fehler als jüngere Kraftfahrer.

Prototypische Merkmale/Situationen, die an den untersuchten Knotenpunkten festgestellt wurden und im Zusammenhang mit Konflikten und auch Unfällen standen, betrafen vor allem:

- die signaltechnisch nicht gesicherte Führung von Linksabbiegern,

- fehlende oder nicht mehr erkennbare Orientierungshilfen (Leitlinien, Wartelinien) für Linksabbieger im Kreuzungsbereich,

- die gemeinsame Führung von Linksabbiegern und Geradeausfahrern auf dem linken Fahrstreifen der Zufahrt, insbesondere wenn von den Geradeausfahrern auch die mittleren bzw. rechten Fahrstreifen genutzt werden dürfen (Geradeausfahrer scheren nach rechts aus, um wartepflichtige Linksabbieger zu umfahren; Verflechtungsvorgänge im Kreuzungsbereich),

- nicht gegebene oder eingeschränkte Sichtbeziehungen sowie 
- die nicht regelwerkskonforme Gestaltung von Fuß- und Radverkehrsanlagen bzw. fehlende Überquerungsanlagen.

Im Rahmen des Projektes konnten einige bereits bekannte Einflussfaktoren, die zu einer Gefährdung auch von schwächeren Verkehrsteilnehmern führen, auf Basis umfangreicher Unfallanalysen und Verkehrsbeobachtungen sicher verifiziert werden. Zudem konnten neue Erkenntnisse, insbesondere zum Verhalten älterer Pkw-Fahrer, gewonnen werden.

Grundlegend ist festzuhalten, dass ein Großteil der festgestellten Konflikte sehr wahrscheinlich nicht aufgetreten wäre, wenn die untersuchten Knotenpunkte im Bestand nach aktuellen Entwurfsregelwerken gestaltet gewesen wären. Empfehlungen zur Gestaltung von Knotenpunkten müssen daher im Wesentlichen auf die Ausführungen aktueller Entwurfsregelwerke innerstädtischer Straßen und Knotenpunkte sowie von Fuß-, Rad- und barrierefreier Verkehrsanlagen verweisen. Dabei ist immer die Gesamtanlage unter Berücksichtigung der Belange aller Verkehrsteilnehmer zu betrachten.

Neben der regelwerkskonformen Einrichtung und Ausführung der Verkehrsanlagen sind

- die Einrichtung eines Linksabbiegeschutzes für Kraftfahrer,

- die Einhaltung und Gewährleistung aller Sichtbeziehungen und

- die Gestaltung von Fußgänger-Überquerungsstellen mit differenzierten Bordhöhen

besonders hervorzuheben.

Diese Empfehlungen beziehen die aufgetretenen Konfliktsituationen von Kindern, älteren Menschen und Menschen mit besonderen Mobilitätseinschränkungen/-behinderungen mit ein. Viele Anforderungen an die Gestaltung im Hinblick auf diese Gruppen gelten auch für alle übrigen Verkehrsteilnehmer. So tragen u. a. die Einhaltung der Sichtbeziehungen und die Schaffung sicherer Überquerungsanlagen nicht nur, aber im Besonderen zur Erhöhung der Verkehrssicherheit für Kinder oder Rollstuhlfahrer bei. Klare Regelungen im Kreuzungsbereich (gesicherte Führung von Linksabbiegern, Führungslinien, Trennung des Links- und Geradeausverkehrs) unterstützen besonders ältere Kraftfahrer, bieten aber zugleich auch mehr Sicherheit für Kraftfahrer aller Altersklassen.

„Schwächere Verkehrsteilnehmer“ benötigen einen besonderen Schutz im Straßenverkehr. Allerdings bedarf es keiner grundlegend neuen alters- oder subgruppenspezifischen Entwurfsregelwerke. Die bestehenden aktuellen Regelwerke sind bei konsequenter Anwendung dazu geeignet, „schwächeren Verkehrsteilnehmern“ einen hohen Schutz zu gewährleisten.

Eine Verschärfung im Hinblick auf die Regelungen zum signaltechnisch geführten Linksabbieger würde allerdings besonders älteren Kraftfahrern helfen und darüber hinaus zur Erhöhung der Verkehrssicherheit an Knotenpunkten im Allgemeinen beitragen. Analog gilt dies für die Gewährleistung ausreichender Sichtbeziehungen, was einen Sicherheitsgewinn für alle Verkehrsteilnehmer bedeutet, insbesondere aber Kindern hilft.

Im Wesentlichen ergeben sich aus den Untersuchungen die folgenden Forderungen:

1. Die Verpflichtung zur Einrichtung eines Linksabbiegeschutzes für Kraftfahrer sollte bei bestimmten Rahmenbedingungen, wie z. B. eingeschränkte Sichtverhältnisse oder hohe Verkehrsmengen, verbindlich eingeführt werden. Eine entsprechende, 
einheitliche sprachliche Regelung sollte in den einschlägigen Entwurfsregelwerken Einzug finden.

2. Die Einhaltung der Sichtbeziehungen ist im Rahmen der Planung von Straßenverkehrsanlagen verbindlich zu gewährleisten und bereits in der (Vor)Entwurfsplanung, z. B. durch die Verpflichtung Sichtdreiecke in Planunterlagen einzuzeichnen, zu berücksichtigen.

3. Bei Neu- und Umbaumaßnahmen von Verkehrsanlagen sind die Gestaltungshinweise aktueller Entwurfsregelwerke verpflichtend einzuhalten. Verkehrsanlagen sind dabei in ihrer Gesamtheit, unter Berücksichtigung der sicherheitsrelevanten Belange aller Verkehrsteilnehmer, zu betrachten. Zudem sollte das Sicherheitsaudit von Straßen in allen Entwurfsphasen verbindlich eingeführt werden.

4. Das Bestandsaudit sollte anlassbezogen (Unfallhäufungsstelle), als systematischer Bestandteil der örtlichen Unfalluntersuchung verbindlich eingeführt werden. Maßnahmen zur Verbesserung der Verkehrssicherheit, die aus dem Bestandsaudit hervorgehen, sollten verbindlich umzusetzen sein.

\section{Design of safe intersections for weaker road users}

Children, elderly people and people with special mobility restrictions or disabilities are often disadvantaged in traffic on account of their physical and cognitive abilities. They count to the so-called "weaker road users". With the aim to deliver an efficient approach to improve the road safety of "weaker road users", the research project "Design of safe intersections for weaker road users“ was carried out.

First the requirements were compiled, which children (till 14 years including), elderly people (65 years and older) and people with special mobility restrictions or disabilities have from the point of view of their active participation in traffic. These requirements depend on the physiological and psychological abilities of these people.

On the basis of a macroscopic accident analysis of about 350000 accidents at intersections from five federal states (about 280000 accidents in urban areas and about 65000 accidents in rural areas, without motorways/highways) typical accident situations of children and elderly people were determined. In view of accidents in urban areas it can be found that

- children as bicyclists often cause accidents with vehicles that turn right or cross the sidewalk/cycle track (especially when children come from the right side of the driver's view),

- elderly people are mostly involved in left-turning accidents (one vehicle oncoming) and right-angle accidents as drivers (this corresponds to the distribution of all accidents at intersections) and that

- in relation to all road users children and elderly people are disproportionately often involved in accidents with vehicles as pedestrians (where they also often get injured).

Accidents of handicapped persons were examined on the basis of case studies. From accident descriptions the following causes, amongst others, can be put out: 
- insufficient fields of vision between road users (e.g., wheelchair users were not seen by turning drivers) and

- wrong use of traffic areas (e.g., wheelchair users on cycle tracks).

From a group of 291 intersections, identified on the basis of the accident analyses, first 50 and finally 15 intersections were be put out for further investigations. These investigations contained

- detailed accident analyses,

- safety audits,

- on-the-spot inspections and

- behavioral studies in real traffic.

In the result deficits that are relevant for road safety could be ascertained and prototype characteristics or situations were found, which are relevant for safety in particular from the point of view of the "weaker road users" but also from the point of view al all road users.

At the 15 intersections deficits were ascertained in the following categories:

- barrier-free designing (deficits were found at 13 of 15 intersections)

- traffic infrastructure for bicyclists (deficits were found at 9 of 15 intersections)

- traffic regulation (deficits were found at 8 of 15 intersections)

- fields of vision (deficits were found at 8 of 15 intersections)

- traffic routing (deficits were found at 6 of 15 intersections)

- traffic signs (deficits were found at 5 of 15 intersections)

- road design (deficits were found at 4 of 15 intersections)

- traffic infrastructure for pedestrians (deficits were found at 4 of 15 intersections)

- signal control (deficits were found at 1 of 15 intersections)

In addition to observations made by people, the observations of the behavior of road users were made by a wireless camera system that allows the view on intersections from up to four different perspectives. Overall, the behavior of 24598 individual pedestrians, bicyclists and drivers taking into account the age group or the mobility restriction or disability were analyzed.

Generally, a high regulatory compliance of all road users could be noticed. In the group of the pedestrians and bicyclists children relatively made more mistakes than people of other age groups. Elderly drivers committed the same mistakes than younger drivers. However, elderly drivers made relatively more mistakes than younger drivers when turning left.

Prototype characteristics or situations which were ascertained at the examined intersections and which stood in connection with conflicts and also accidents concerned above all:

- left turning traffic is not protected by traffic lights,

- missing or not more recognizable guidance (guidelines, waiting lines) for vehicles turning left

- vehicles turning left and vehicles going straight use the left lane together (especially when vehicles that are going straight could use the middle or the right lanes, too)

- insufficient fields of vision,

- design of pedestrian and bicycle facilities is not compliant to the guidelines and 
- lack of crossing facilities for pedestrians and bicyclists.

Within the scope of the project some already known factors which lead to a danger also from weaker road users could be verified on the basis of extensive accident analyses and traffic observations. Besides, new knowledge could be won, in particular to the behavior of older drivers when turning left.

Basically, it should be noted that most of the identified conflicts would most likely not have occurred if the examined intersections had been designed according to the current guidelines and directives. Therefore, recommendations for the design of intersections must relate mainly to the current guidelines and directives for the design of urban roads and intersections. Besides, the whole road infrastructure is always to be considered, taking into account the needs of all road users.

Besides the design of intersections according to the current guidelines

- the protected left turn by traffic signals,

- the observance of sufficient fields of vision and

- the design of crossing facilities for pedestrian with differentiated hights (zero drawdown for wheelchair users, tactile elements for people using a white cane)

are particularly emphasized.

These recommendations comprise the requirements of children, elderly people and people with special mobility restrictions or disabilities. Many requirements for the design of intersections in terms of these groups also apply to all other road users. The observance of sufficient fields of vision and the design of safe crossing facilities are particularly important for the safety of children and wheel chair users but they are also important for all road users. Unequivocal regulations in the intersection area (e.g., secure guidance of vehicles that turn left) are particularly important for elderly drivers; however, offer also more security for drivers of all age groups.

"Weaker road users" need a special protection in traffic. However, it requires no fundamentally new design guidelines for these people. The existing current regulations are capable to ensure a high level of protection for "weaker road users", when they are used consistently.

However, an accentuation in terms of the rules for the protection of vehicles that turn left, would particularly help elderly drivers and beyond that also contribute to the improvement of traffic safety at intersections in general. Analogously this is valid for the observance of sufficient fields of vision. This means a greater safety for all road users and especially helps children.

Above all, the following demands arise from the investigations:

1. The duty to establish a fully protected left turn for motorists should be made obligatory in certain circumstances, e.g., insufficient fields of vision or high traffic volume. A corresponding uniform linguistic regulation should be included in the relevant guidelines and directives.

2. The observance of sufficient fields of vision is obligingly to guarantee in planning of road traffic facilities and already to consider during the preliminary design, e.g., through the duty to draw vision triangles in plans. 
3. The design informations of current design guidelines should be obligingly for new construction and reconstruction measures of traffic facilities. Traffic facilities are in their entirety to consider taking into account the security concerns of all road users. In addition, the safety audit should be made mandatory in all stages of design of roads.

4. The road safety inspection should be obligingly in case of black spots. Measures to improve traffic safety which arise from the inspection audit, should be mandatory to implement. 


\section{Inhaltsverzeichnis}

Einleitung. 1

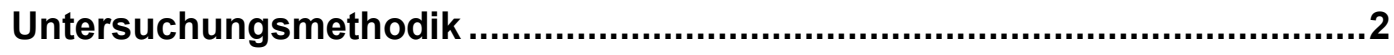
Grundalgenermittlung / Literaturanalyse .5

Psychologische Aspekte der Teilnahme von Kindern und älteren Menschen am Straßenverkehr .5

Vorbemerkung: Kognitive Entwicklung über die Lebensspanne .5 Entwicklungspsychologische Aspekte von Kindern als aktive Teilnehmer am Straßenverkehr

Psychologische Aspekte von älteren Menschen als aktive Teilnehmer am Straßenverkehr .26

Knotenpunkte: Anforderungen und Unfälle mobilitätsbehinderter Menschen ......38

Behinderung und Einschränkung - Definition und Bedeutung ..... .38

Anforderungen mobilitätsbehinderter Menschen an Überquerungsstellen ..... 42

Kompensationsmöglichkeiten durch barrierefreie Gestaltung ..... 46 Unfälle mobilitätsbehinderter Menschen an Knotenpunkten 46 Quellen für die Unfallaufnahme bei Unfällen mit Beteiligung behinderter Menschen

Zusammenfassung: Unfalluntersuchungen mobilitätsbehinderter Menschen .....56

Makroskopische Unfallanalyse 57

Datengrundlage. .57

Unfalltypen von Unfällen an Knotenpunkten .58

Typische Unfallsituationen von Kindern und Älteren. .64

Zusammenfassung: Typische Unfallsituationen. .68

Auswahl von Knotenpunkten für die Sicherheitsanalysen und Verhaltensbeobachtungen .69

Methodik zur Auswahl relevanter Knotenpunkte. 69 Auswahl relevanter Knotenpunkte. .70

Zusammenfassung: Auswahl von Knotenpunkten .71

Sicherheitsanalysen und Verhaltensbeobachtungen 72

Vorgehen .72

Unfallanalysen. .72

Sicherheitsanalysen der Verkehrsinfrastruktur .72

Verhaltensbeobachtungen der Verkehrsteilnehmer im Realverkehr .72 
6.2 Überblick über das Unfallgeschehen an den ausgewählten Knotenpunkten .......77

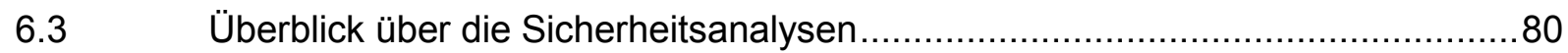

6.4 Überblick über die Verhaltensbeobachtungen ................................................82

6.5 Sicherheitsanalysen und Verhaltensbeobachtungen nach Gruppe und

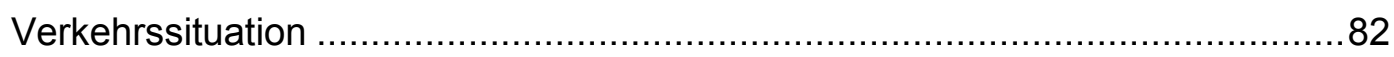

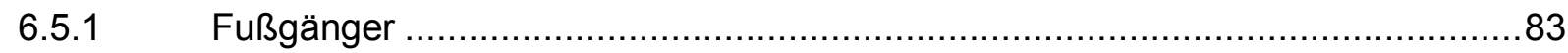

6.5.2 Menschen mit besonderen Mobilitätseinschränkungen oder

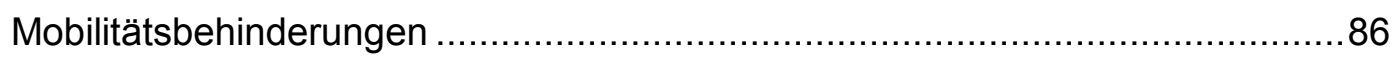

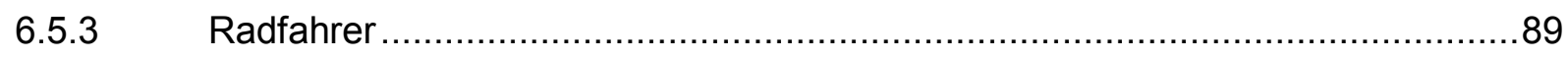

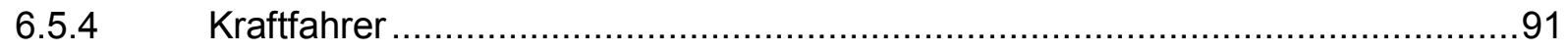

6.6 Sicherheitsanalysen und Verhaltensbeobachtungen - wesentliche Aspekte je

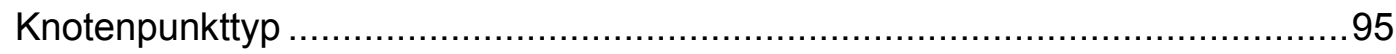

7 Prototypische Merkmale/Situationen nach Art der Verkehrsbeteiligung .....96

$8 \quad$ Ergebnisdiskussion im Rahmen eines Expertenrunde ..............................101

$9 \quad$ Ansatz zur Beschreibung der Komplexität von Kontenpunkten................105

10 Empfehlungen zur Gestaltung von Knotenpunkten ................................113

10.1 Empfehlungen aus Sicht der Fußgänger (insbesondere für Kinder, ältere Menschen und Menschen mit Mobilitätseinschränkungen/-behinderungen) .....113

10.2 Empfehlungen aus Sicht der Radfahrer (insbesondere für Kinder und ältere Menschen) ....................................................................................... 115

10.3 Empfehlungen aus Sicht der Kraftfahrer (insbesondere für ältere Menschen) ..117

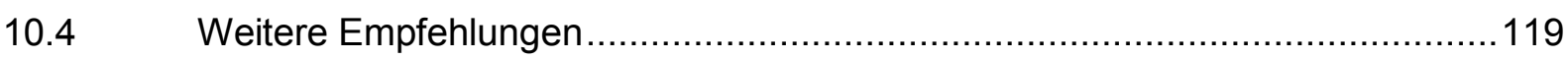

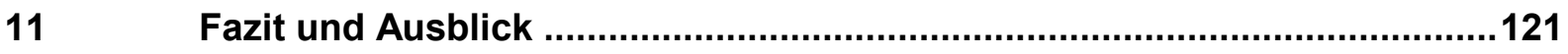

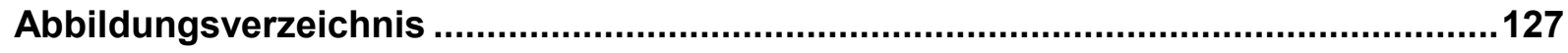

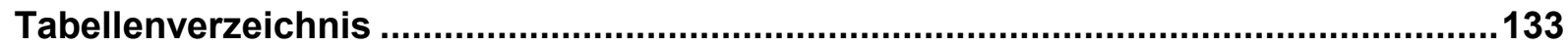

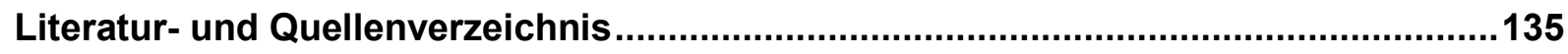

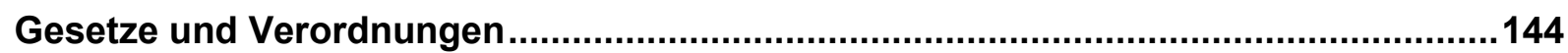

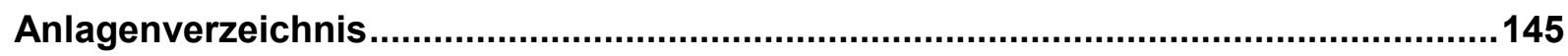




\section{Einleitung}

Kinder, ältere Menschen und Mobilitätseingeschränkte sind im Verkehrsraum aufgrund ihrer körperlichen und kognitiven Einschränkungen häufig benachteiligt - sie zählen zu den so genannten „schwächeren Verkehrsteilnehmern“. Doppelt schwer wiegt die Tatsache, dass diese Personengruppen bei Verkehrsunfällen zu Fuß oder mit dem Fahrrad besonders häufig (Kinder) oder besonders schwerwiegende Verletzungen bis hin zur Todesfolge (ältere Verkehrsteilnehmer) erleiden. Bei den älteren Menschen kommt das erhöhte Mortalitätsrisiko bei gleicher Unfallschwere als zusätzlicher negativer Einflussfaktor bei den Unfallfolgen hinzu. So war z. B. 2006 jeder zweite getötete Fußgänger oder Radfahrer mindestens 65 Jahre alt. Bei den Kindern verunglückte etwa ein Drittel mit dem Fahrrad, ein Viertel als Fußgänger (DESTATIS 2007). Knotenpunkte haben dabei ein besonders hohes Risikopotenzial. Mehr als jeder zweite Unfall in Ortschaften geschieht an einem Knotenpunkt. Ein Drittel dieser Unfälle ereignet sich an lichtsignalgeregelten, die Hälfte an vorfahrtgeregelten und die restlichen an rechts-vor-links-geregelten Knotenpunkten (DVR 2008). Nicht alle Defizite schlagen sich dabei in der Unfallstatistik nieder. Als Beispiel sei eine fehlende Absenkung für Rollstuhlbenutzer an Überquerungsstellen genannt. Diese kann dazu führen, dass der Rollstuhlfahrer die Fahrbahn nicht schnell genug verlassen kann, dennoch wird ein Unfall zwischen einem Kraftfahrzeug und einem querenden Rollstuhlfahrer ein äußerst seltenes Ereignis sein. Neben der Annahme, dass die Beteiligung der genannten Gruppen an Unfällen auf physiologische und kognitive Schwächen zurückzuführen ist, setzt sich immer häufiger die Ansicht durch, dass die Verkehrsräume den Anforderungen dieser Menschen nicht weit genug entsprechen.

Das hohe Gefährdungsrisiko für die genannten Gruppen kann sich dabei aus mehreren Gründen ergeben. Möglicherweise werden die Anforderungen von Kindern, älteren Menschen und Mobilitätseingeschränkten bei der Gestaltung von Verkehrsräumen in den Regelwerken nicht ausreichend berücksichtigt. Ein weiterer Aspekt könnte sein, dass ein potenziell vorhandener Spielraum bei den Vorgaben aus den Regelwerken nicht immer im Sinne schwächerer Verkehrsteilnehmer umgesetzt wird, da z. B. Nachteile für die Verkehrsabwicklung (Leistungsfähigkeit) befürchtet werden. Viele Sicherheitsdefizite entstehen zudem erst während der Planung oder Umsetzung, was Erfahrungen aus der Auswertung von Pilotaudits bei der Qualifizierung von Sicherheitsauditoren zeigen (Kesting \& Gerlach 2006).

Im Rahmen dieser Studie werden für Kinder und Jugendliche (14 Jahre und jünger) die Themenbereiche Fußgänger und Radfahrer als aktive Verkehrsteilnehmer besonders hervorgehoben (die motorisierte Teilnahme am Verkehr, z. B. als Mitfahrer, wird in dieser Studie ausgeklammert). Für die älteren Verkehrsteilnehmer (65 Jahre und älter) werden ebenfalls die Themenbereiche Fußgänger und Radfahrer hervorgehoben, daneben wird der ältere Mensch vor allem als Kraftfahrer dargestellt. Die Teilnahme am öffentlichen Verkehr oder als Mitfahrer (Bus und Bahn) wird für die bestehende Fragestellung ausgeklammert. Die Darstellung für die Gruppe der mobilitätsbehinderten Menschen richtet sich zusätzlich nach den Erfordernissen, die sich aus der Anfrage bei relevanten Dachorganisationen ergeben. 


\section{Untersuchungsmethodik}

Die vorliegende Untersuchung stützt sich auf die im Folgenden aufgelisteten und im Weiteren beschriebenen Arbeitspakete:

AP1 Grundlagenermittlung

AP1.1 Internet- und Literaturrecherche

AP1.2 Abfrage Dachorganisationen und Interessenverbände

AP1.3 Erhebung von Unfalldaten

AP2 Makroskopische Unfalldatenanalyse

AP2.1 Unfalldatenanalyse

AP2.2 Ableitung Gesetzmäßigkeiten

AP2.2 Ermittlung Typischer Unfallsituationen

AP3 Sicherheitsanalyse Knotenpunkte

AP3.1 Auswahl prototypischer Knotenpunkte

AP3.2 Beschaffung von Planunterlagen

AP3.3 Sicherheitsanalyse prototypischer Knotenpunkte

AP3.4 Vor-Ort-Untersuchungen

AP4 Verkehrsbeobachtungen

AP4.1 Beobachtung Realverkehr

AP4.2 Auswertung Videobeobachtung

AP5 Prototypische Verbesserungsvorschläge

AP5.1 Ergebnisbündelung, Vorbereitung Expertenrunden

AP5.2 Durchführung und Auswertung Expertenrunden

AP5.3 Zusammenführung der Ergebnisse (Defizite, Positives)

AP5.4 Prototypische Verbesserungsvorschläge

\section{P6 Regelwerke}

AP6.1 Anwendungsanalyse Regelwerke

AP6.2 Handlungsempfehlungen Regelwerke

\section{AP7 Berichterstellung}

\section{AP1 - Grundlagenermittlung}

Im ersten Schritt (AP1) wurden Daten und Informationen als Grundlage für die folgende Erstellung eines Anforderungskatalogs (AP2) sowie die weiteren Analysen erhoben. Dafür wurde zuerst die bereits bekannte Literatur ausgewertet, anschließend eine ergänzende Literaturrecherche in wissenschaftlichen Datenbanken sowie dem Internet durchgeführt. Hiermit wurden die bisher bekannten Schwierigkeiten, Hemmnisse und Einschränkungen hinsichtlich der Nutzung von Verkehrsräumen bei Kindern, mobilitätseingeschränkten Menschen und insbesondere bei älteren Menschen ermittelt und zusammenfasst.

In Kapitel 3 wird ein Überblick über die entwicklungspsychologischen Eckpunkte beim Heranwachsen von Kindern in Bezug auf die Teilnahme am Straßenverkehr gegeben. Eine Übersicht zeigt, über welche Fähigkeiten Kinder etwa in welchem Alter verfügen und auf welche Besonderheiten der Wahrnehmung von Kindern bei der Gestaltung der Verkehrsumwelt besonders geachtet werden muss. Für die älteren Verkehrsteilnehmer wird ebenfalls ein Überblick gezeigt, wie sich Wahrnehmung und Kognition über die Lebensspanne entwickelt und mit welchen Einschränkungen bei der Gruppe der älteren Verkehrsteilnehmer zu rechnen ist. Die Übersicht zeigt, welche kognitiven Besonderheiten 
bei der Gestaltung der verkehrlichen Umwelt in Bezug auf ältere Menschen besonders beachtet werden sollten.

Ergänzt wurde diese Recherche durch Abfrage bei Dachorganisationen von Interessenverbänden (u. a. DBSV, VdK), auch um zusätzliche spezifische Anforderungen für spezielle Nutzergruppen zu ermitteln, z. B. der Anforderungen hörbehinderter Menschen. Kapitel 3.2 führt die Anforderungen mobilitätsbehinderter und -eingeschränkter Menschen an Knotenpunkte auf.

Zur Ermittlung des Anforderungskataloges bzw. zur Bestimmung häufiger Unfalltypen von Kindern und älteren Menschen, wurden elektronische Unfalldaten aus fünf Bundesländern erhoben, aufbereitet und analysiert. Zudem bilden diese Daten die Grundlage zur Auswahl von Knotenpunkten für die Sicherheitsanalysen und Verhaltensbeobachtungen im Realverkehr.

\section{AP2 - Makroskopische Unfalldatenanalyse}

Im zweiten Arbeitspaket wurde zunächst die Unfalldatenbank analysiert, indem nach Auffälligkeiten bei den Unfalltypen geschaut wurde. Somit konnten für jede Subgruppe typische Unfälle identifiziert und in einer Rangliste dargestellt werden.

\section{AP3 - Sicherheitsanalyse von ausgewählten Knotenpunkten}

Im dritten Arbeitspaket wurden detaillierte Untersuchungen an ausgesuchten Knotenpunkten durchgeführt. Die ausgewählten Knotenpunkte wurden einzeln einer Sicherheitsanalyse unterzogen, sowie das reale Verhalten der Verkehrsteilnehmer im Realverkehr mit verdeckter Kamera beobachtet (AP4). Mit dem Ziel, verkehrstechnische Gestaltungselemente an Knotenpunkten zu identifizieren, die für die jeweiligen Subgruppen ein Risikopotenzial hinsichtlich der Verkehrssicherheit bergen.

\section{AP4 - Verkehrsbeobachtungen im Realverkehr an den ausgewählten Knotenpunkten}

Ergänzt wurden die Analysen aus AP3 in einem parallelen vierten Schritt (AP4) durch Verhaltensbeobachtungen im Realverkehr (mit verdeckter Kamera), wodurch typische Verhaltensweisen jeder Subgruppe und Konfliktsituationen analysiert werden konnten. Dabei wurde auf ein standardisiertes Beobachtungsverfahren zurückgegriffen, das für die Analyse von Kinderunfällen entwickelt wurde. Hierbei wird das Verhalten im Realverkehr zunächst mit verdeckter Kamera beobachtet, um zu verhindern, dass allein die Verkehrsbeobachtung schon das reale Verhalten verändert und anschließend ausgewertet.

\section{AP5 - Prototypische Verbesserungsvorschläge / Expertenrunden}

Im Rahmen des Arbeitspaketes 5 wurden prototypischer Verbesserungsvorschläge im Hinblick auf die Gestaltung von Knotenpunkten auf Basis der gewonnenen Erkenntnisse erarbeitet. Die festgestellten prototypischen Merkmale wurden im Rahmen einer Expertenrunde unter Beteiligung verschiedener Fachdisziplinen (Verkehrsplanung und technik, Verkehrspsychologie, barrierefreie Verkehrsplanung) vorgestellt und diskutiert.

Auf Grundlage der Zusammenführung der Erkenntnisse aus Unfallanalyse, Sicherheitsanalyse, Videobeobachtung und den Ergebnissen aus der Expertenrunde wurden prototypische Verbesserungsvorschläge zur Erhöhung der Verkehrssicherheit, insbesondere für die Zielgruppen, an Knotenpunkten erarbeitet. 


\section{AP6 - Regelwerksanalyse}

In einem sechsten Schritt (AP6) wurde überprüft, ob sich trotz Beachtung gültiger Regelwerke Sicherheitsdefizite ergeben oder ob die Defizite aus Mängeln bei der Planung oder Umsetzung resultieren.

\section{AP7 - Berichterstellung}

Die Ergebnisse der Untersuchungen wurden in dem vorliegenden Bericht, einschließlich des Anlagenbandes dokumentiert und aufbereitet. 


\section{Grundalgenermittlung / Literaturanalyse}

\subsection{Psychologische Aspekte der Teilnahme von Kindern und älteren Menschen am Straßenverkehr}

Im Folgenden wird ein Überblick aus psychologischer Perspektive gegeben, bei dem die wichtigsten kognitiven und körperlichen Rahmenbedingungen dargestellt werden, unter denen sich Kinder und ältere Menschen im Straßenverkehr bewegen.

Insbesondere wird aufgezeigt, wie sich für die Teilnahme am Straßenverkehr wichtige Fähigkeiten nach der Geburt erst mit zunehmendem Alter entwickeln, im Laufe des Lebens einen Zenit erreichen und manche dieser Fähigkeiten im höheren Alter wieder abnehmen. Dieser Überblick kann wegen der zahlreichen Literatur nicht erschöpfend sein.

\subsubsection{Vorbemerkung: Kognitive Entwicklung über die Lebensspanne}

Über die Lebensspanne verändern sich die kognitive und körperliche Leistungsfähigkeit in vielen Bereichen und damit auch die kognitive Leistungsfähigkeit im Altagsleben. Die Fähigkeit der Wahrnehmung und Informationsverarbeitung (für den Straßenverkehr vor allem "Sehen“, „Hören“ und „Entscheidungsfindung“) ist deshalb sicherlich eine der wichtigsten Ressourcen des Menschen. Die Wahrnehmung von Umweltinformationen steht am Anfang eines Prozesses, der in einer möglichst angemessenen, kontextadäquaten Handlung mündet. Zwischen diesen beiden Zeitpunkten liegt der Prozess der Informationsverarbeitung. Beobachtbar ist, dass sich trotz gleicher äußerlicher Reize das Verhalten von Kindern, Erwachsenen und älteren Menschen in vielen Situationen grundsätzlich voneinander unterscheiden können, obwohl sie sich in einer Umgebung mit genau denselben äußerlichen Informationen und Reizen befinden. Das gilt auch für Situationen im Straßenverkehr. Die Veränderungen der kognitiven und körperlichen Leistungsfähigkeit spiegeln sich auch im unterschiedlichen Unfallgeschehen von Kindern, Erwachsenen und älteren Menschen im Straßenverkehr wieder. Das „Altern“ ist ein Prozess, der sich auf die gesamte Lebensspanne bezieht.

Was „Altern“ im Detail ist und wie es sich wissenschaftlich exakt definieren lässt, ist derzeit in der Wissenschaft noch nicht eindeutig definiert.

Als gesichert kann gelten, dass mit zunehmendem Alter während des normalen Lebens- und Alternsprozesses kognitive, perzeptive und körperliche Fähigkeiten über die Lebensspanne gesehen zunächst in allen Bereichen zunehmen, im Erwachsenenalter ihren Zenit erreichen und mit zunehmendem Alter wieder nachlassen (Churchill et al. 2002; Craik \& Bialystok 2006).

Unterschiede über die Lebensspanne sind schon dadurch begründet, dass das Gehirn bei Kindern bei der Geburt noch nicht voll entwickelt ist. Der Großteil der Synapsen (einzelne Gehirnzellen) vernetzt sich erst nach der Geburt und auch die Gehirnmasse insgesamt vergrößert sich nach der Geburt noch etwa um das Vier- bis Fünffache bis zu einem Alter von etwa 20 Jahren. Im Alter nimmt die reine Gehirnmasse wieder ab (vgl. Craik \& Bialystok 2006). 
In der Wissenschaft wird inzwischen davon ausgegangen, dass das Gehirn die vollständige Funktionsreife sogar erst im frühen Erwachsenenalter besitzt (vgl. Luna, Thulborn, Munoz, Merriam, Garver et al. 2001) und als letztes die Vernetzung des Frontallappens des Gehirns vollständig abgeschlossen ist (vgl. Span, Ridderinkhof \& van der Molen 2004).

Erstaunlich ist, dass der Alterungsprozess schon ab einem Alter von etwa 40 Jahren einsetzt, bei der Bewegungskoordination teilweise sogar noch früher. Der Präfrontallappen reift zwar als letztes, aber dort zeigt sich auch der erste Abbau. Das spiegelt sich in Untersuchungen über den Leistungsabbau von für das Autofahren wichtigen Funktionen wider: Leistungsabbau zeigt sich nicht erst im höheren Lebensalter, sondern teilweise schon sehr viel früher (TÜV 1998): „Zumeist wird mit zunehmendem Alter ein Anstieg der Variation deutlich, d. h. die Extremwerte der Gruppenleistungen rücken mit zunehmendem Alter weiter auseinander. Dieser Prozess beginnt teils schon früh (31-40 Jahre, Bewegungskoordination), teils ganz spät (71-80 Jahre, Reaktion), er ist auch bei manchen Leistungen gering ausgeprägt (Wahrnehmung) oder schwankend (Vigilanz).“

Früher ging man beim Altern von einem übergreifenden Leistungsabbau aus, der alle entscheidenden kognitiven Funktionen betrifft (z. B. Salthouse 1996). Inzwischen ist jedoch gut belegt, dass im Alter nicht alle, sondern nur bestimmte kognitive Funktionen spezifisch beeinträchtigt oder verlangsamt sind (z. B. Yordanova et al. 2004).

Die sprachliche oder soziale Kompetenz z. B. bleibt im Alter anscheinend unverändert bestehen oder wird teilweise sogar besser (z. B. Persson et al. 2004). Auch sind Reizverarbeitungs- und Entscheidungszeiten bei einfachen Aufgaben nicht unbedingt altersbedingt verlangsamt (z. B. TÜV Kraftfahrt GmbH 1998).

Bei Tempoprozessen allerdings oder bei Zeitdruck kann man beobachten, dass Ältere oft langsamer sind als Jüngere und auch mehr Fehler machen (z. B. Churchill et al. 2002). Gleichzeitig ist zu beobachten, dass die interindividuelle Variation des Alternsprozesses sehr groß ist und sich teilweise schon ab dem Alter von 30 Jahren beobachten lässt (s. o., TÜV Kraftfahrt $\mathrm{GmbH}$ 1998). Es ist also nicht möglich, auf Basis des kalendarischen Alters eines Menschen auf seine kognitiven und körperlichen Fähigkeiten zu schließen. Manche Menschen laufen z. B. mit 70 Jahren noch Marathon oder geben Computerkurse, während andere Menschen in demselben Alter bereits bettlägerig und auf Pflegehilfe angewiesen sind.

Je älter Menschen dem kalendarischen Alter nach im Durchschnitt sind, desto größer ist die Variation ihres individuellen Leistungsvermögens.

\subsubsection{Die Unterscheidung von kristallinen und fluiden Fähigkeiten nach Cattell}

Um die verschiedenen Dimensionen des Denkens und Wahrnehmens bzw. der Kognition zu erfassen, wird in den Neurowissenschaften allgemein noch immer von Cattells grundsätzlicher Unterscheidung zwischen der fluiden und kristallinen Intelligenz (1963) ausgegangen.

Mit kristallinen kognitiven Leistungen werden in erster Linie Kulturtechniken assoziiert wie z. B. Lesen und Schreiben; aber auch das Langzeitgedächtnis wird hierzu gezählt, ebenso wie Erfahrung, Wissen, Sozialkompetenz und Urteilsvermögen. Diese Leistungen werden im 
Alter eher besser oder nehmen wenigstens nicht oder kaum ab („gesundes“ Altern vorausgesetzt).

Zu den fluiden kognitiven Fähigkeiten werden vor allem sog. „Kontrollprozesse“ gezählt, das ist z. B. der Umgang mit Mehrfachanforderungen oder die Bewältigung von Tempoprozessen (für Kinder nicht bewältigbar, für ältere Menschen oft schon mit erheblichen Einbußen verbunden).

Dass gerade Kontrollfunktionen im höheren Lebensalter wieder abnehmen, wird damit erklärt, dass an ihrer Realisierung der präfrontale Kortex beteiligt ist. Dieser reift in der Kindheit am spätesten aus, und zeigt im Alter als erster einen Abbau (s. o., vgl. auch Span et al. 2004).

Craik \& Bialystok (2006) präzisieren die Begriffe Cattells noch ein wenig in [Kristalline] Repräsentation \& [Fluide] Kontrolle des Verhaltens.

Wenn die Entwicklung von Denken bzw. Kognitionen über die Lebensspanne betrachtet wird, kann man auf Basis des gegenwärtigen wissenschaftlichen Standes in etwa von folgendem Bild ausgehen:

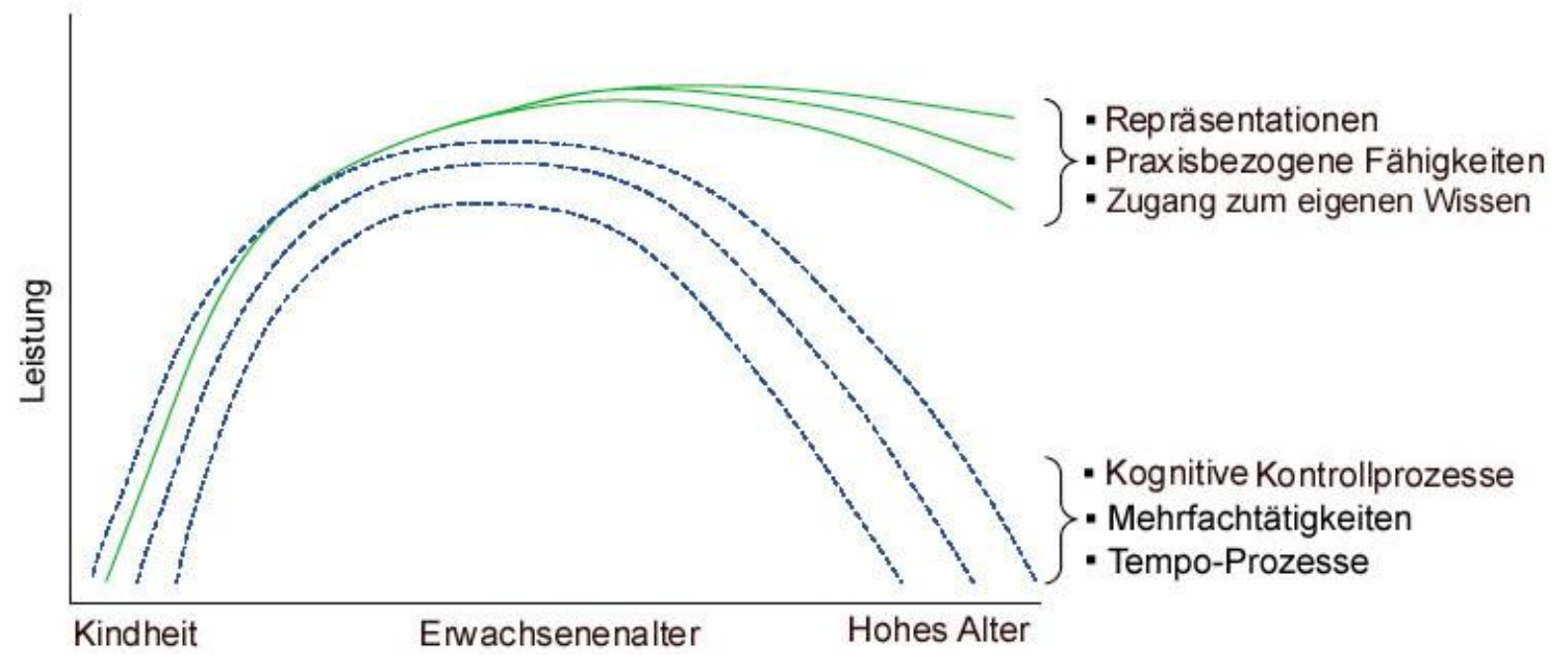

Abbildung 1: Kognitive Entwicklung über die Lebensspanne [Poschadel \& Sommer, 2007: übersetzt aus: Craik \& Bialystok, 2006]

Abbildung 1 macht deutlich, dass sich viele kognitive Fähigkeiten (also auch die, die eine sichere Teilnahme am Straßenverkehr ermöglichen), erst mit zunehmendem Alter in der Kindheit ausbilden und im Erwachsenenalter einen Zenit erreichen. Die verschiedenen Linien bei den Kontrollprozessen im Bild repräsentieren die Variabilität einzelner Prozesse. Während bestimmte Fähigkeiten auch im höheren Lebensalter nicht so sehr nachlassen (Repräsentationen; kristalline Fähigkeiten, die oberen Linien in der Abbildung), baut die Leistungsfähigkeit von Kontrollprozessen (fluide Fähigkeiten, die unteren Linien in der Abbildung) mit zunehmendem Lebensalter doch teils erheblich ab. Das sind vor allem Fähigkeiten, die Multitaskingprozesse, parallele Informationsverarbeitung und Tempoprozesse repräsentieren. 
Es kann hier durchaus ein Zusammenhanghang zwischen dem Nachlassen bestimmter kognitiver Fähigkeiten und Verkehrsunfällen von älteren Menschen als Fahrer in komplexen Situationen (große Kreuzungen, Linksabbiegen) hergestellt werden.

Nach dieser kurzen Einführung über die kognitive Entwicklung über die Lebensspanne wird im Folgenden dargestellt, wie sich die für die Teilnahme am Straßenverkehr wichtigen Fähigkeiten bei Kindern erst mit zunehmendem Alter entwickeln und welche Rolle sie für die aktive Teilnahem am Straßenverkehr spielen.

In einem weiteren Abschnitt werden die speziellen Anforderungen an ältere Verkehrsteilnehmer aus psychologischer Sicht beschrieben. 


\subsubsection{Entwicklungspsychologische Aspekte von Kindern als aktive Teilnehmer am Straßenverkehr}

In diesem Abschnitt wird neben einem aktuellen Überblick über das Unfallgeschehen ein Überblick über die Entwicklung von Kindern in Bezug auf den Straßenverkehr gegeben. Nach einer kurzen allgemeinen Einführung wird getrennt auf die Entwicklung als Fußgänger und als Radfahrer eingegangen.

\subsubsection{Vorbemerkung: Unfallgeschehen von Kindern als Verkehrsteilnehmer (Statistisches Bundesamt 2009)}

„Unfälle mit Kindern gehören nach wie vor zum traurigsten Kapitel unseres Verkehrsalltages. Im Durchschnitt des letzten Jahres kam alle 17 Minuten ein Kind im Straßenverkehr zu Schaden, jeden vierten Tag wurde ein Kind getötet. Im Jahr 2008 verunglückten 31.648 Kinder auf Deutschlands Straßen, das waren 6,4\% weniger als 2007. Die Zahl der im Straßenverkehr getöteten Kinder ist um 8,1\% auf 102 gesunken. Insgesamt betrug der Anteil der Kinder an allen Verunglückten im Straßenverkehr 7,7 \%, ihr Bevölkerungsanteil lag bei $14 \%$ (11,2 Millionen). Bezogen auf die Einwohner ihrer Altersgruppe verunglückten 281 Kinder unter 15 Jahren je 100.000 Einwohner.

Trotz der noch immer hohen Zahlen ist das Unfallrisiko für Kinder in den vergangenen 30 Jahren deutlich geringer geworden: Im Jahr 1978 verunglückten in Deutschland 72.129 Kinder, mehr als doppelt so viele wie im Jahr 2008. Das Risiko, als Kind im Straßenverkehr getötet zu werden, war 1978 mit 1.449 getöteten Kindern vierzehn mal so hoch wie im Jahr 2008 mit 102. Besonders hat sich die Situation bei den Fußgängern verbessert: 2008 kamen 23 Kinder als Fußgänger ums Leben, 1978 waren es mit 701 noch das Dreißigfache.

Überdurchschnittlich häufig verunglücken Kinder als Fußgänger oder Radfahrer. $24 \%$ aller verunglückten Fußgänger und $14 \%$ aller verunglückten Fahrer und Mitfahrer von Fahrrädern hatten das 15. Lebensjahr noch nicht vollendet.

36 \% der im vergangenen Jahr im Straßenverkehr verunglückten Mädchen und Jungen im Alter unter 15 Jahren waren mit dem Fahrrad unterwegs. Jedes dritte verunglückte Kind saß als Mitfahrer in einem Personenkraftwagen (33\%) und jedes vierte verunglückte als Fußgänger (25\%).

Dabei ergibt sich in den einzelnen Altersgruppen ein unterschiedliches Bild:

Es verunglückten

- Kinder unter 6 Jahren zu 58 \% im Pkw, zu $26 \%$ als Fußgänger und zu 9,9 \% als Fahrradbenutzer.

- Kinder im Alter von 6- bis unter 10 Jahren zu $35 \%$ als Insasse im Pkw, zu 33 \% als Fußgänger und zu $27 \%$ als Fahrradbenutzer.

- Kinder über 10 Jahren zu $50 \%$ als Fahrradbenutzer, zu $23 \%$ als Pkw-Insasse und zu $20 \%$ als Fußgänger.

Von den im Jahr 2008 getöteten Kindern saß jedes zweite Kind in einem Pkw (51 Kinder), jeweils knapp ein Viertel (23\%) nahm als Fußgänger oder Fahrradfahrer am Straßenverkehr teil, als der Unfall passierte“. 
Um die unterschiedliche Gefährdung von Kindern in verschiedenen Altersgruppen zu verdeutlichen, werden die absoluten Unfallzahlen je Altersgruppe von 2008 in folgender Abbildung zusammengefasst (Daten aus Statistisches Bundesamt, 2009):

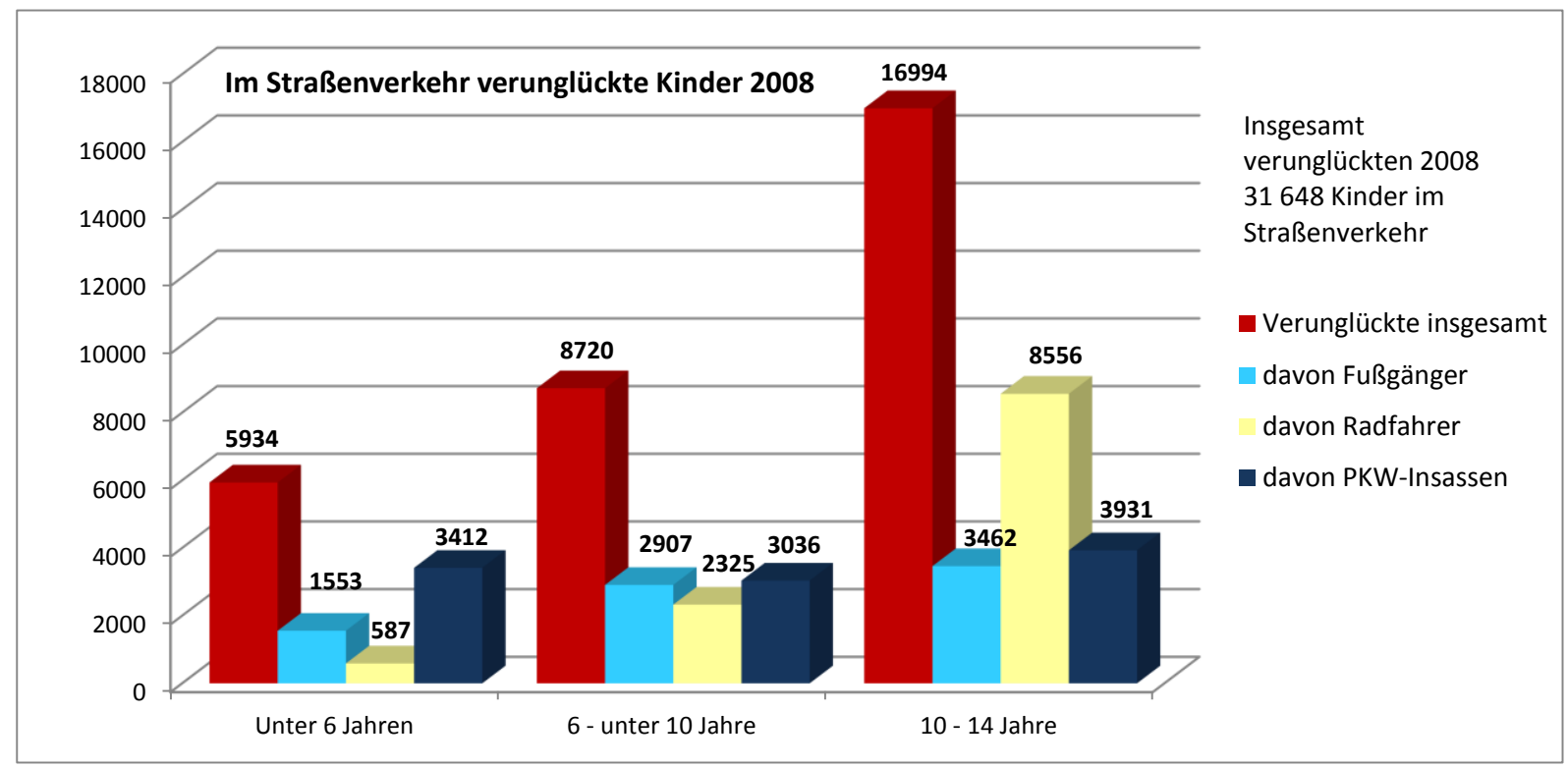

Abbildung 2: Im Straßenverkehr verunglückte Kinder 2008 in absoluten Zahlen [Quelle: Statistisches Bundesamt 2009]

Wie die Abbildung 2 zeigt, verunglückten die meisten Kinder in der Altersgruppe von 10 bis 14 Jahren. Etwa die Hälfte von innen als Radfahrer. Überhaupt steigt die Zahl der verunglückten Radfahrer über die drei Altersgruppen hinweg in der letzten Altersgruppe dramatisch an. Das ist die Altersgruppe der Kinder, die eine weitergehende Schule besuchen (oder bald besuchen werden). Es kann vermutet werden, dass sich mit dem Besuch der weitergehenden Schule auch das Verkehrsmittelwahlverhalten verändert und (nach dem „Fahrradführerschein“) das Rad signifikant häufiger für die täglichen Wege genutzt wird.

Die Unfälle als Pkw-Insassen verteilen sich vergleichbar auf die drei Altersgruppen.

Unfälle zu Fuß nehmen über die Altersgruppen hinweg betrachtet ebenfalls zu und kommen in der letzten Altersgruppe am häufigsten vor.

Bei der Betrachtung der relativen Verunglücktenzahlen - bezogen auf die jeweilige Altersgruppe - wird deutlich, welche Kinder in welcher Altersgruppe relativ am meisten gefährdet sind: 


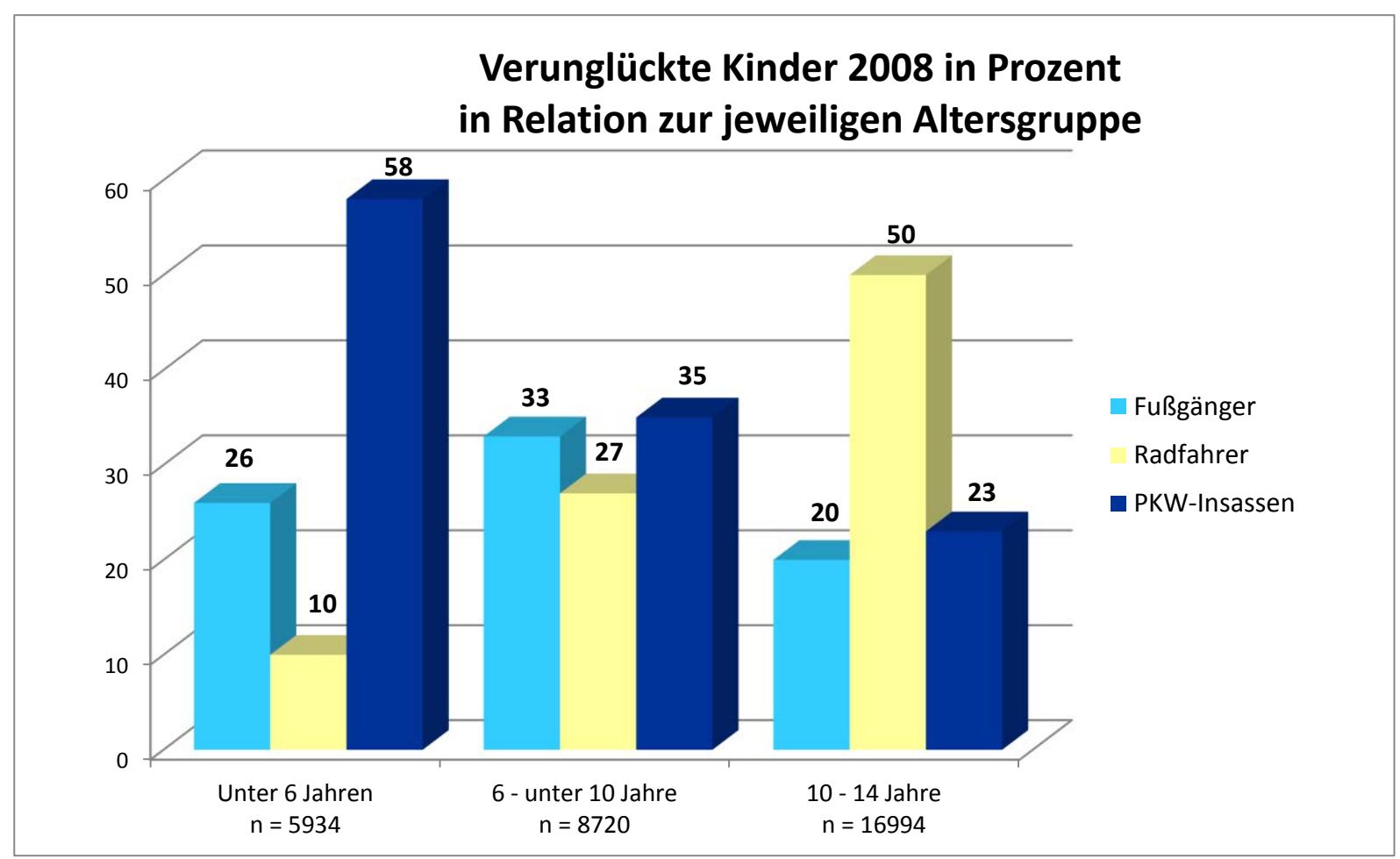

Abbildung 3: Verunglückte Kinder 2008 in Prozent in Relation zur jeweiligen Altersgruppe [Quelle: Statistisches Bundesamt 2009]

Abbildung 3 zeigt, bei welcher Verkehrsbeteiligungsart die Kinder der einzelnen Altersgruppen relativ gefährdet sind: Während die jüngeren Kinder in ihrer Altersgruppe besonders als Pkw-Insassen verunglücken, ist es bei den Kindern der mittleren Altersgruppe eher ausgeglichen.

Ältere Kinder sind besonders als Radfahrer gefährdet. In Verbindung mit den absoluten Zahlen ist diese Teilgruppe überhaupt die, die am häufigsten im Straßenverkehr verunglückt.

Dieser Überblick soll dazu dienen, die folgenden entwicklungspsychologischen Aspekte besser zum Gesamtunfallgeschehen in Beziehung setzen zu können.

\subsubsection{Entwicklung der Informationsverarbeitungsprozesse bei Kindern}

Mit Hilfe moderner, nicht invasiver Methoden der kognitiven Neurowissenschaft ist es in den letzten Jahrzehnten möglich geworden, Informationsverarbeitungsprozesse im menschlichen Gehirn immer präziser zu analysieren. Beispielsweise ist das möglich durch die funktionelle Magnetresonanztomografie (fMRT), die Ableitung Ereignis Korrelierter Potenziale (EKP) und andere Verfahren.

Das Verfahren der „Ereignis Korrelierten Potenziale“ ist eine Untersuchungsmethode, die am IfADo (Institut für Arbeitsforschung an der TU Dortmund) seit vielen Jahren praktiziert wird.

Das „Ereignis“ ist bei EKP-Untersuchungen in der Regel ein visueller oder auditiver Reiz, der mittels Computer dargeboten wird. Dabei werden die Hirnströme mittels EEG abgeleitet und zu den dargebotenen Reizen in Beziehung gesetzt. Dadurch ist es möglich, Rückschlüsse über kognitive Verarbeitungsprozesse zu ziehen. 
Während früher davon ausgegangen wurde, dass die Informationsverarbeitung bei Kindern nicht so schnell wie bei Erwachsenen funktioniert, wird in den letzten Jahren immer deutlicher, dass auch Kinder dargebotene Reize sehr schnell wahrnehmen. Im Vergleich mit Erwachsenen unterscheiden sich aber die Gehirnareale, in denen die Reizverarbeitung stattfindet. Es sind also an der Informationsverarbeitung andere Hirnareale beteiligt.

Für die Teilnahme am Straßenverkehr ist die Entwicklung der selektiven Wahrnehmung von besonderer Bedeutung. Schließlich sollten Kinder sicher unterscheiden können zwischen Wahrnehmungen, die für sie gefährlich sein können (z. B. ein herannahendes Auto) und Wahrnehmungen, die nicht unbedingt gefährlich sind (z. B. laute Geräusche von einer Baustelle in der Nähe).

Perchet \& Garcia-Larrea (2005) konnten z. B. zeigen, dass sich die selektive Wahrnehmung von Kindern im Alter zwischen 6 und 9 Jahren (das sind Grundschulkinder) und Erwachsenen deutlich unterscheidet. In dem Experiment ging es darum, nur bei bestimmten Reizen (einem roten Stern) zu reagieren und andere Reize (rote Kreise) nicht zu beachten. Während Erwachsene zwischen relevanten uns irrelevanten Reizen gut unterscheiden können, ist für Kinder zunächst einmal jeder Reiz ein relevanter Reiz. Die Unterschiede in der Informationsverarbeitung zwischen Erwachsenen und Kindern werden darauf zurückgeführt, dass Kinder noch nicht zwischen relevanten und irrelevanten Hinweisreizen in einem Experiment unterscheiden können, während diese Fähigkeit bei Erwachsenen sehr wohl vorhanden ist.

Ähnliche Effekte zur selektiven Aufmerksamkeit zeigen sich auch im auditiven Bereich.

In Experimenten von Määttä, Pääkkönen, Saavalainen \& Partanen (2005) ging es darum, dass die Versuchspersonen nur bei bestimmten Hinweistönen reagieren sollten, bei anderen Tonhöhen nicht. Während für die Erwachsenen gezeigt werden konnte, dass sie bei entsprechenden akustischen Hinweisreizen auf den folgenden Ton vorbereitet waren, zeigten bestimmte Komponenten der EKPs bei Kindern Muster, die darauf hindeuten, dass Kinder auf einer frühen Stufe der Informationsverarbeitung nicht zwischen relevanten und irrelevanten Reizen unterscheiden können.

Die beiden exemplarischen Studien zur selektiven Aufmerksamkeit von Kindern sollen vor allem eines zeigen:

Während die Kinder im Labor immer auf einen bestimmten Reiz oder insgesamt nur sehr wenige Reize reagieren sollten, müssen sie im Straßenverkehr selbständig aus der Vielzahl der Sinneseindrücke diejenigen herausfiltern, die für die Situation, in der sie sich befinden, relevant sind. Vor allem müssen sie auch zwischen „gefährlichem Reiz“ und „ungefährlichem Reiz" unterscheiden können. Das gelingt ihnen aber noch nicht (immer). Wie die beiden beispielhaften Experimente zur Entwicklung der geteilten bzw. selektiven Aufmerksamkeit zeigen, ist aber gerade diese Auswahl für Kinder schwierig, weil verschiedene Hinweisreize für Kinder gleichberechtigt nebeneinander stehen und jeder Hinweisreiz zunächst eine Relevanz besitzen kann. Sie können noch nicht sicher zwischen „relevant“ und „irrelevant“ für die eigene Situation unterscheiden. 


\subsubsection{Entwicklung des Gefahrenbewusstseins von Kindern}

Mit dem Begriff "Gefahrenbewusstsein“ soll in erster Linie die Kompetenz bezeichnet werden, das (unmittelbare) Risiko der Umwelt für die eigene Gesundheit in konkreten Situationen zu antizipieren und durch entsprechende kontextadäquate Handlungen zu vermeiden bzw. zu minimieren. Vielleicht sollte der Begriff "Gefahrenbewusstsein“ auch umschrieben werden mit "Gewahrwerden“ der eigenen Gefährdung bzw. der Gefährdung der eigenen Gesundheit. Die Entwicklung dieser Kompetenzen ist mit der Entwicklung der inhaltlichen Bedeutung des Begriffes "Gefahr“ im Kindesalter verbunden.

Für den deutschsprachigen Raum (es gibt kulturelle Unterscheide, je nach der Umwelt, in der ein Kind aufwächst) wurde eine Alters- und Stufeneinteilung von Limbourg (1997) erarbeitet:

\section{„1. Stufe:}

Auf der ersten Stufe lernen die Kinder, gefährliche Situationen im Straßenverkehr zu erkennen, aber erst dann, wenn sie schon akut gefährdet sind. Wenn z. B. ein Kind, das einen steilen Abhang mit dem Fahrrad herunterfährt und immer schneller wird, Angst bekommt, hat es schon ein akutes Gefahrenbewusstsein. In diesem Stadium lässt sich der Unfall kaum mehr vermeiden, die Wahrnehmung der Gefahr kommt in der Regel zu spät. Diese erste Stufe wird mit ca. 5 bis 6 Jahren erreicht.

2. Stufe:

Auf der zweiten Stufe lernen die Kinder, Gefahren vorauszusehen, d. h. sie lernen zu erkennen, durch welche Verhaltensweisen sie in Gefahr geraten könnten (vorausschauendes Gefahrenbewusstsein). Erkennt das Kind z. B., dass Radfahren auf abschüssigen Wegen gefährlich ist, hat es schon ein vorausschauendes Gefahrenbewusstsein. Diese Stufe wird mit ca. 8 Jahren erreicht.

\section{Stufe:}

Auf der dritten Stufe lernen Kinder, vorbeugende Verhaltensweisen bewusst einzusetzen, um Gefahren zu reduzieren (Präventionsbewusstsein). Ein Kind, das einen Umweg in Kauf nimmt, um eine Straße sicher zu überqueren, hat schon ein Bewusstsein für vorbeugende Maßnahmen entwickelt. Diese Stufe wird erst mit ca. 9 bis 10 Jahren erreicht.

Die einzelnen Altersangaben für das Erreichen bestimmter Stufen können nur als Durchschnittswerte betrachtet werden mit großen interindividuellen Schwankungen. Aus diesem Grund muss man immer mit Kindern rechnen, die sich langsamer entwickeln und die einzelnen Stufen wesentlich später erreichen.

Eine einigermaßen „sichere“ Teilnahme am Straßenverkehr kann erst dann erwartet werden, wenn die Kinder die dritte Stufe erreicht haben (Bewusstsein für vorbeugende Maßnahmen), aber auch das Erreichen der zweiten Stufe (vorausschauendes Gefahrenbewusstsein) bringt schon eine deutliche Sicherheitserhöhung für die Kinder“.

Bei dieser Einteilung wird deutlich, dass Kinder eigentlich erst mit dem Besuch einer weiterführenden Schule in der Lage sind, Gefahren für die eigene Gesundheit zu erkennen. 


\subsubsection{Entwicklung Sehen und Hören bei Kindern (in Bezug auf den Straßenverkehr)}

Da gerade jüngere Kinder häufig als Fußgänger verunfallen, wurde angenommen, dass sich besonders das periphere Sehen, das die Erkennung von Reizen im äußeren Gesichtsfeld ermöglicht, bei Kindern unterentwickelt ist. Dazu machte besonders die Arbeitsgruppe um David, Foot \& Chapman Anfang der 1990er Jahre mehrere Experimente. Dabei zeigte sich jedoch, dass das periphere Sehen von Kindern im Vergleich zu Erwachsenen nur minimal schlechter ausgebildet ist. Es gibt zwar minimale statistische Unterschiede bei der Entdeckung von Reizen im peripheren Gesichtsfeld, die jedoch so gering sind, dass sie nach Meinung der Autoren nicht Ursache der hohen Zahl von Fußgängerunfällen von Kindern sein können (David, Foot, Chapman, \& Sheehy 1986).

In einem Nachfolgeexperiment (David, Foot \& Chapman 1990) wurde die Untersuchungsmethode verfeinert und untersucht, ob sich die erhöhte Unfallgefahr für Kinder gegenüber Erwachsenen aus einer langsameren kognitiven Verarbeitung der optischen Reize im peripheren Sehfeld ergeben könnte.

An der Untersuchung nahmen vier verschiedene Altersgruppen teil: Erwachsene (Durchschnittsalter 21 Jahre), 11-jährige Kinder, 9-jährige Kinder und 7-jährige Kinder. In dieser Versuchsreihe sollten Kinder jetzt nicht nur einen optischen Reiz entdecken, sondern auch entscheiden, ob ein bestimmter optischer Reiz im peripheren Gesichtsfeld eine Gefahr für sie selbst darstellt. Hierfür wurden "gefährliche Situationen“ (ein sich näherndes Auto) und „ungefährliche Situationen“ (ein sich entfernendes Auto) im Realverkehr gefilmt. Hier konnten die Autoren zeigen, dass es signifikante Zeitunterschiede bei der Entdeckung und gleichzeitigen Bewertung eines sich nähernden Autos gibt. Je jünger Kinder sind, desto länger brauchen sie zur Entdeckung. Allerdings entdeckten 9-jährige Kinder die Reize am schnellsten. Doch trotz der wiederum gemessenen marginalen Mittelwertunterscheide zwischen den Gruppen sind die Autoren, wie schon beim ersten Experiment, der Meinung, dass sie nicht Ursache für die hohe Zahl von Fußgängerunfällen von Kindern sein können.

Die Ergebnisse legen die Vermutung nahe, dass das „Falschverhalten“ in bestimmten Verkehrssituationen nicht durch die physiologischen Sehunterschiede zwischen Kindern und Erwachsenen erklärt werden kann, sondern vor allem durch den entwicklungspsychologischen Unterscheid insgesamt, da die kognitiven Systeme von Kindern, die eine adäquate Einschätzung einer Situation erst ermöglichen, noch nicht voll ausgebildet sind.

Beim Hören ließen sich allerdings wesentliche Unterschiede zwischen Erwachsenen und Kindern finden:

Pfeffer \& Barnecutt (1996) gingen der Frage nach, warum sich nach ihren Erkenntnissen die meisten Unfälle von Kindern in eher ruhigen Straßen mit Sichtbehinderungen ereignen. Im Experiment wurde eine solche Situation simuliert. Die Kinder sollten auf Basis realer Motorengeräuschen von Autos (die Geräusche wurden im „echten“ Straßenverkehr aufgenommen), eine Richtungslokalisation dieser Geräusche vornehmen.

Die Ergebnisse sind eindeutig: Sie zeigen, dass selbst 11-jährige Kinder nur etwa $60 \%$ der dargebotenen Stimuli richtig identifizieren können, bei den jüngeren Kindern waren es noch weniger. 
Werden auch noch visueller und optischer Reiz kombiniert (Januschke-Stämpfli, 1978), zeigt sich das Dilemma, in dem sich Kinder befinden, wenn sie sich in komplexen Situationen mit Autos und Sichthindernissen im Straßenverkehr befinden. Die Ergebnisse der Versuche zeigten, dass insbesondere jüngere Kinder ein akustisches Signal jeweils mit einem visuellen Signal assoziierten, obwohl sich beide Objekte mit unterschiedlicher Geschwindigkeit bewegten. „Angenommen, ein Kind steht am Straßenrand und will die Straße überqueren. In einer geringen Entfernung sieht es ein Fahrzeug herankommen. Weil die Straße eine Biegung macht, nimmt das Kind das Auto lediglich von der Seite wahr. Dadurch kann es ein zweites Fahrzeug, welches gerade dabei ist das andere zu überholen, nicht sehen [...] geht das Kind in unserem Beispiel über die Fahrbahn in der Meinung, das langsame Fahrzeug könne ihm nichts anhaben, kann es eine böse Überraschung erleben. Hinter dem langsamen kann plötzlich das schnellere hervorkommen. So kann das Kind sich unmittelbar in Gefahr begeben“ (Januschke-Stämpfli, 1978). Januschke-Stämpfli zeigt, dass es besonders für jüngere Kinder schwer ist, visuelle und auditive Reize zu kombinieren, vor allem dann, wenn sich die beiden Reize nicht gleich schnell bewegen. Dann kommt es zu einer Fehllokalisation des auditiven Reizes. Je jünger die Kinder sind, desto stärker ist die Fehllokalisation.

Insgesamt kann aus den Ergebnissen der Untersuchungen zur Entwicklung der visuellen und auditiven Wahrnehmung von Kindern der Schluss gezogen werden, dass jüngere Kinder über wichtige Eigenschaften der menschlichen Wahrnehmung noch nicht, bzw. anders verfügen als Erwachsene. Optische und akustische Reize werden anders - und auf der Ebene der Verhaltensbeobachtung oft auch langsamer - verarbeitet als bei Erwachsenen. Vor allem jüngere Kinder als Fußgänger können nicht alle Fähigkeiten, die eigentlich zur Teilnahme am Straßenverkehr nötig wären, zu einem beliebigen Zeitpunkt erlernen (z. B. kombinierte Aufmerksamkeitsprozesse, Übertragung von Regeln auf verschiedene Kontexte, Geschwindigkeitsschätzung, auditive Richtungslokalisation usw.). Dementsprechend muss inre Verkehrssicherheit durch externe (das heißt vor allem bauliche) Faktoren im Straßenraum erhöht werden, um diese entwicklungsbedingten Nachteile der Wahrnehmung zu kompensieren.

\subsubsection{Entwicklungsaspekte von Kindern als Fußgänger aus psychologischer Sicht}

In diesem Abschnitt soll ebenfalls wieder an zwei exemplarischen Studien gezeigt werden, wie sich die Fähigkeiten von Kindern bei Straßenquerungen erst mit zunehmendem Alter ausbilden.

Welche Fähigkeiten 5-, 7-, 9- und 11-jährige Kinder haben, um sichere Routen für eine Straßenquerung zu finden, untersuchten Ampofo-Boateng \& Thomson (1991). Bei den Experimenten mussten die Kinder die sichersten Routen entweder in einer nachgestellten Situation (auf einem Tisch) oder anhand von Fotografien oder im realen Verkehr suchen. Bei allen Experimenten zeigte sich (vgl. Ampofo-Boateng \& Thomson 1991), dass 5- und 7 jährige Kinder nur über sehr rudimentäre Fähigkeiten verfügen, sichere Stellen für die Straßenquerung zu finden. Ihre Entscheidungen richteten sich nur danach, ob sie direkt Autos sehen konnten. Andere Faktoren, wie etwa Sichthindernisse oder komplexe Kreuzungen, flossen nicht in ihre Entscheidungen ein und wurden vor allem nicht als potenziell gefährlich erkannt. 
Im Alter von 9 Jahren zeigten die Kinder schon ganz gute Einschätzungen der Routen für eine sichere Straßenquerung, bei 11-jährigen Kindern konnte eine gute Einschätzung der Routenwahl gezeigt werden. In Deutschland entspricht dieses Alter etwa der 5. oder 6. Klasse der weiterführenden Schulen.

In einer nachfolgenden Untersuchung konnte Thompson (1997) diese Ergebnisse bestätigen:

Es zeigte sich auch dort ein klarer Entwicklungstrend. Je älter Kinder werden, desto sicherer sind die Straßenüberquerungsrouten, die sie wählen. Bei 11-jährigen konnte beobachtet werden, dass etwa $75 \%$ der gewählten Routen als sicher gelten können. Bei den jüngeren Kindern war es ganz anders. Nur etwa $10 \%$ der Routen, die von 5-jährigen Kindern gewählt wurden, konnten als sichere Routen gelten.

Bei den jüngeren Kindern ( 5 und 7 Jahre) zeigten sich mehrere typische und fundamentale Fehler:

Zum einen konnten sie bei gefährlichen Stellen nicht sagen, warum sie für eine Straßenquerung gefährlich sind und glaubten zudem auch nicht, dass diese gefährlichen Stellen überhaupt gefährlich sind.

Jüngere Kinder zeigten die Tendenz, die kürzesten Wege als die sichersten anzusehen, wie auch schon in den Vorläuferexperimenten.

Jüngere Kinder schätzen vor allem solche Stellen als gefährlich ein, bei denen Autos zu sehen sind, wie sich auch schon im ersten Experiment zeigte. Sind keine Autos (direkt) zu sehen, schätzen sie die Stellen weniger gefährlich ein. Vor allem Sichthindernisse werden nicht als solche wahrgenommen. Konnten die Kinder kein Auto sehen (auch, wenn es nur durch Sichthindernisse verdeckt war), schien die Stelle für sie sicher.

Fasst man die Ergebnisse in Bezug auf Straßenquerungen von Kindern zusammen ergibt sich folgendes Bild:

- Wenn jüngere Kinder eine Straße queren möchten, wählen sie häufig die kürzeste Route, weil sie innen als sicherste erscheint, auch wenn sie tatsächlich gefährlich ist. Dabei werden Sichthindernisse oder komplexe Verkehrssituationen nicht als Gefahr wahrgenommen.

- Ab einem Alter von etwa 11 Jahren können Kinder selbständig einigermaßen sichere Routen für die Straßenquerung wählen.

- Abseits von Kreuzungen (also auf "freier Strecke“) wird die Entscheidung einer Straßenquerung hauptsächlich beeinflusst von der räumlichen Lücke im Verkehrsfluss unabhängig von der Geschwindigkeit der Fahrzeuge.

- Bis zu einer Geschwindigkeit von etwa $55 \mathrm{~km} / \mathrm{h}$ treffen die Kinder in den allermeisten Fällen unabhängig von Alter und Geschlecht „sichere“ Entscheidungen, allerdings mit „Ausreißern“. Bei Fahrzeuggeschwindigkeiten darüber nehmen „unsichere“ Entscheidungen bei Kindern unter 10 Jahren progressiv zu. Je höher die Geschwindigkeit der Autos, desto häufiger treffen Kinder Fehlentscheidungen. 
- Besonders die jüngeren Kinder zeigen ein inkonsistentes Verhalten und sind sehr unsicher bei Straßenquerungen.

Werden diese Aspekte auf die Verkehrsplanung übertragen, ergibt sich ein besonderes Schutzbedürfnis für Kinder als Fußgänger. Ihre passive Sicherheit muss vor allem durch gute Baumaßnahmen im Straßenverkehr gesichert werden. Dabei müssen zwei zentrale Aspekte als Grundlage dienen:

- Je höher die tatsächlichen Fahrzeuggeschwindigkeiten sind, desto schwieriger ist es für Kinder, bei Straßenquerungen die Geschwindigkeit von Fahrzeugen einzuschätzen, desto gefährlicher sind die Situationen für Kinder als Fußgänger.

- Je mehr Sichthindernisse, desto schwieriger ist es für Kinder, die Verkehrssituation zu überblicken, desto höher die Gefährdung für Kinder als Fußgänger.

\subsubsection{Die Körpergröße von Kindern als wichtiger Einflussfaktor bei der Entwicklung}

Kinder sind schon wegen ihrer geringeren Körpergröße im Straßenverkehr benachteiligt.

Aufgrund ihrer geringen Körpergröße fällt es ihnen schwer, sich einen Überblick über Straßenverkehrssituationen zu verschaffen (ganz abgesehen von den entwicklungspsychologischen Einschränkungen des Sehens und Hörens, s. o.). Die nachfolgende Abbildung soll diesen Nachteil verdeutlichen. In der Abbildung 4 wird dargestellt, wie groß Kinder im Alter von 4, 6, 8 und 10 Jahren in Relation zur Höhe eines geparkten Pkw sind. Die Augenhöhe der Kinder liegt noch 10 cm (4 Jahre) bzw. 20 cm (10 Jahre) darunter.

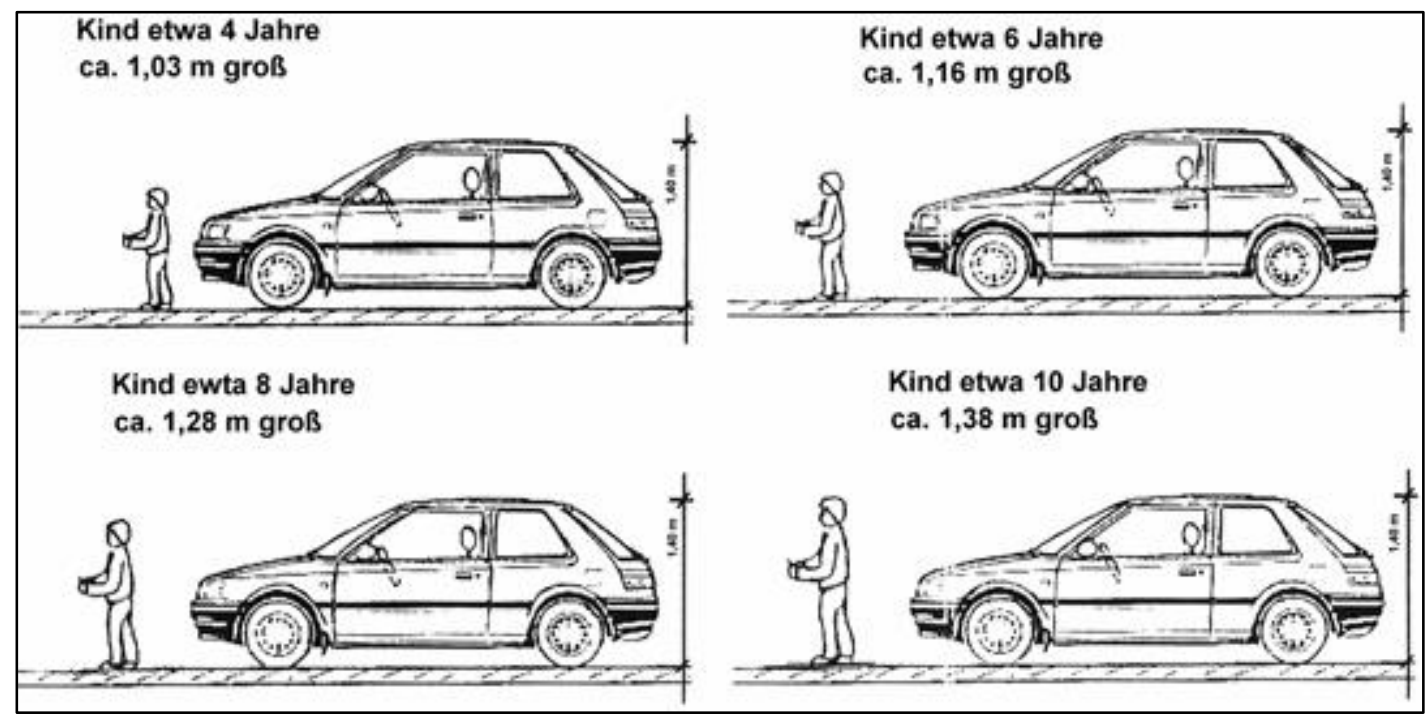

Abbildung 4: Größe eines Kindes abhängig vom Alter in Relation zu einem Auto [Poschadel 2006]

Erst im Alter von ca. 10 Jahren ist ein Kind so groß, wie es der Höhe eines „Mittelklasse$\mathrm{Pkw}^{\prime \prime}$ entspricht (mit einer Höhe von etwa 1,40 m). Bevor ein Kind diese Körpergröße 
erreicht, hat es keine Möglichkeiten zu sehen, was sich auf einer Straße hinter einem Auto verbirgt.

Aus der Perspektive eines Pkw-Fahrers stellt sich die Situation ähnlich dar. Auch er kann nicht sehen, was sich hinter einem geparkten Auto verbirgt. Die nachfolgende Abbildung zeigt diese Situation schematisch:

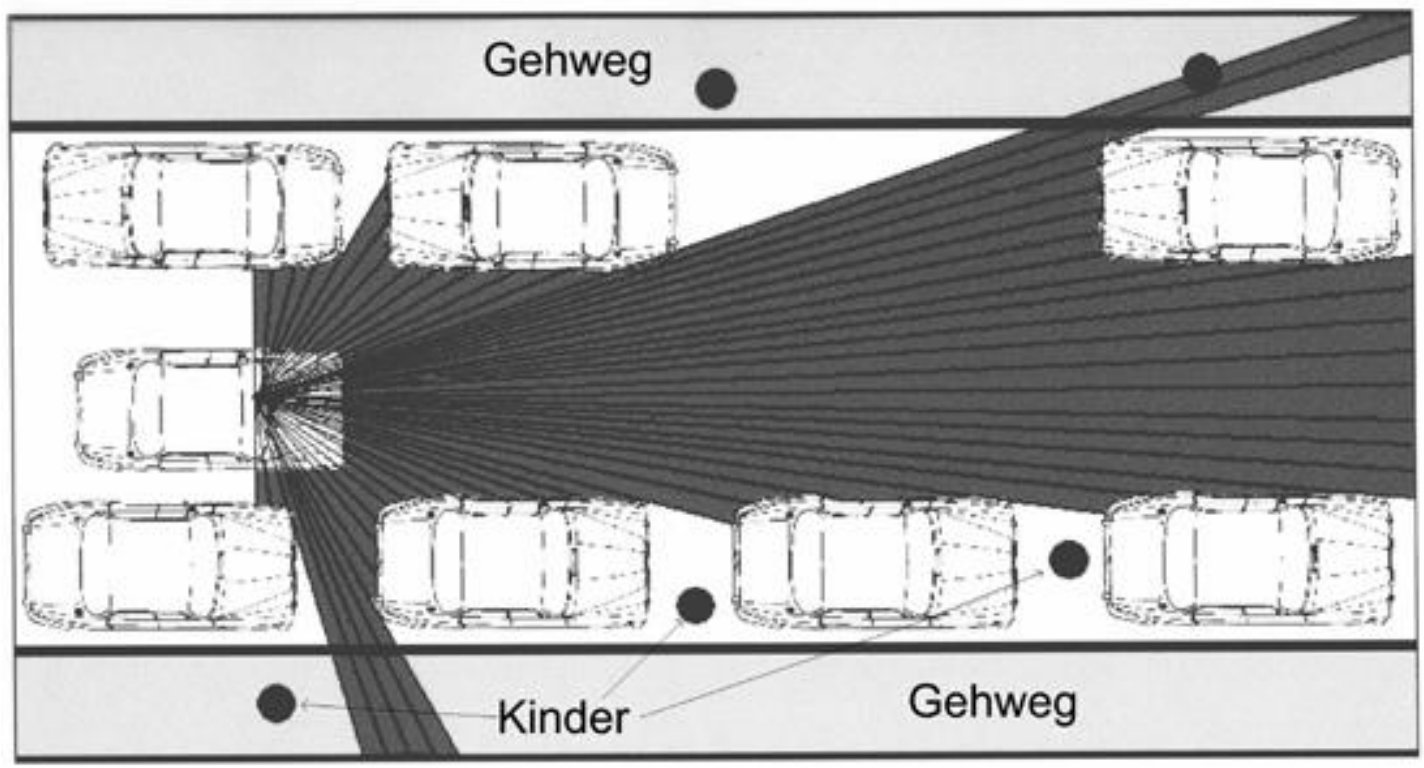

Abbildung 5: Schematisiertes Blickfeld eines Pkw-Fahrers in einer beidseitig beparkten Straße [Poschadel 2006]

Abbildung 5 zeigt die Situation für einen Pkw-Fahrer, der durch eine Straße fährt, in der beidseitig Autos geparkt sind. In der Abbildung wird davon ausgegangen, dass das Kind etwa 6 Jahre alt ist und die Kopfhöhe somit in etwa der Höhe einer Motorhaube entspricht. Die "Strahlen" entsprechen dem Blickfeld eines Pkw-Fahrers. Obwohl sich in dieser schematisierten Darstellung 5 Kinder in unmittelbarer Nähe des Pkw befinden, kann nur ein einziges (das hinter der Parklücke im oberen Bildrand) vom Pkw-Fahrer gesehen werden. Eine solch schematisch dargestellte Situation ist vor allem problematisch vor Knotenpunkten, da die geparkten Autos die Sichtbeziehungen erheblich einschränken und hierdurch Konfliktsituationen „vorprogrammiert“ sind.

\subsubsection{Entwicklungsaspekte von Kindern als Radfahrer aus psychologischer Sicht}

Limbourg (1997) hat sehr kurz zusammengefasst, in welchem Alter welche VerkehrsKompetenzen von Kindern zu erwarten sind: „Zur Entwicklung von ,sicheren' Verhaltensweisen im Verkehr benötigen Kinder eine Reihe von Fähigkeiten, die erst Schritt für Schritt im Laufe der Kindheit ausgebildet werden. Kinder werden erst mit ca. 8 - 10 Jahren zu Fußgängern und mit ca. 13 bis 15 Jahren zu Radfahrern, deren Verhalten im Straßenverkehr berechenbar ist. Vor diesen Altersstufen führen diese Defizite zu gefährlichen Konflikten mit anderen Verkehrsteilnehmern, Unfälle können dann die Folge sein. [...] Die motorischen Fähigkeiten für die sichere Beherrschung des Fahrrads sind erst 
mit ca. 9 bis 10 Jahren ausreichend ausgebildet, und auch mit dem Rad können die Kinder ihre Handlungen nur schlecht abbrechen - sie fahren deshalb plötzlich vom Gehweg auf die Fahrbahn. [...] Das Verhalten von Kindern als Radfahrer ist bis zum Alter von ca. 8 Jahren so defizitär, dass eine Teilnahme am Straßenverkehr nicht zu empfehlen ist. Zwischen 8 und 14 Jahren entwickeln sich die erforderlichen Fertigkeiten und es kommt zu einer deutlichen Verbesserung des Fehlverhaltens. Mit ca. 14 Jahren sind die Fähigkeiten zum sicheren Radfahren vollständig entwickelt“" (Limbourg 1997b).

Diese grundsätzlichen Aussagen lassen sich durch mehrere Untersuchungen sehr gut belegen. Pafferott etwa hat die kindliche Entwicklung der Fähigkeit Fahrrad zu fahren, wie folgt beschrieben:

„Von den psychomotorischen Reifungsvorgängen aus betrachtet, sind Kinder ab etwa 3 Jahren in der Lage, einfache Manöver mit dem Fahrrad auszuführen (Auf- und Absteigen, Bremsen, Geradeausfahren, Kurvenfahren). Komplexere psychomotorische Anforderungen wie Stabilisieren des Rades bei langsamer Fahrt, Slalomfahren oder Einhändigfahren können Kinder kaum vor Vollendung des 8. Lebensjahres erfüllen. Erst ab diesem Alter bringen sie die Voraussetzungen mit, die zur Lösung komplexer Fahraufgaben mindestens erforderlich sind. [...] Die Möglichkeiten zur visuellen Orientierung sind mindestens bis zu diesem Alter noch deutlich begrenzt. So fällt es den Kindern z. B. schwer, in eine von der Fahrtrichtung abweichende Richtung zu blicken. Versuchen sie es doch, kommen sie aus dem Gleichgewicht. Von daher ist es verständlich, daß die visuelle Orientierung junger Radfahrer einem ,Minimalprogramm' folgt. Je jünger ein Kind ist, desto starrer ist sein gesamter Wahrnehmungsprozeß auf den Freiraum unmittelbar vor inm gerichtet“ (Pfafferott 1994).

In experimentellen Studien der Ruhr-Universität Bochum wurden diese Aussagen in Experimenten aus den Jahren 1993 (Basner \& de Marées) und 1997 (Bongert \& Henke) sehr gut belegt.

In der ersten Studie (1993) wurden speziell Grundschüler untersucht. Die Ergebnisse der Untersuchungen lassen sich wie folgt zusammenfassen: „Mit sechs Jahren werden bereits 90-95\% der Kopfhöhe des Erwachsenen erreicht, d. h. in dieser Phase ist der Kopf überproportional groß. Für die Massenverhältnisse, z. B. auf dem Fahrrad, bedeutet das, dass eine relativ große Teilmasse des Körpers in relativ großem Abstand von der durch die Reifenauflagepunkte verlaufende Drehachse positioniert ist, was sich auf die Gleichgewichtssituation des Kindes auf dem Fahrrad destabilisierend auswirkt" (Basner \& de Marées 1993, S. 16). Standuntersuchungen zur Balancierfähigkeit haben gezeigt, dass die Haltung von Grundschulkindern im Verhältnis zu Erwachsenen drei- bis sechsmal instabiler ist, d. h. 6- bis 7-jährige Kinder erreichen erst $15 \%$ der Leistungsfähigkeit von 25-jährigen Erwachsenen (vgl. ebd.). Aber auch die Feinmotorik ist beim Radfahren aufgrund von Reifungsprozessen noch nicht voll ausgeprägt: „Im Grundschulalter ist die Muskelmasse und davon abhängig die Muskelkraft der Kinder noch relativ gering. Die Beugemuskulatur ist im Allgemeinen besser ausgeprägt. Ein geringer ausgeprägter Muskeltonus mit höherer Flexibilität im Gelenkbereich ermöglicht zwar einen größeren Bewegungsradius, ist aber für feinkoordinierte, präzise Bewegungen eher von Nachteil“ (ebd., S. 18f.). 
Neben der motorischen Kontrolle der Muskulatur und des Gleichgewichtes beim Radfahren, müssen im Straßenverkehr aber auch zusätzliche Reize bewältigt werden und auch eine adäquate Reaktion muss gewährleistet sein: „Ein weiterer Parameter der periphere Sinnesreize optischer, akustischer, vestibulärer, kinästhetischer und taktiler Art mit der zentralen Wahrnehmungsleistung verknüpft, ist die Reaktionszeit, die bei 5-jährigen Kindern gegenüber Erwachsenen noch auf das Doppelte verlängert, aber bereits ca. $40 \%$ kürzer ist als beim 2- bis 3jährigen. [...] Darüber hinaus ist zu beachten, dass Kinder bei Reizüberflutung eher überfordert sind. Dadurch verlängert sich die Entscheidungszeit zum motorischen Handeln. Diese starke Reizgebundenheit belegt, dass Kinder in diesem Alter noch nicht dazu in der Lage sind, ihre Aufmerksamkeit problemorientiert zu zentrieren. Erst allmählich entwickelt sich zunächst die fluktuierende zu einer willentlich, auswählenden, stark reizabhängigen, fixierenden Aufmerksamkeit. Aber noch das ältere Schulkind hat oft Schwierigkeiten, seine Aufmerksamkeit zu teilen und sich willentlich auf ein bestimmtes Objekt zu konzentrieren“ (ebd.).

„Kinder lernen frühzeitig und schnell [gemeint ist das Lebensalter zwischen 4 und 6 Jahren, Anm. d. Verf.], auch in der ständig labilen Gleichgewichtssituation, rein motorisch ihr Fahrrad zu beherrschen, wenn ihre volle Konzentration auf diese Tätigkeit gerichtet ist. Werden Kinder aber, wie es die Verkehrswirklichkeit ständig erfordert [...], mit unvorhergesehenen, ungeübten oder auch unbekannten Situationen und Aufgaben konfrontiert, so zeigen sich bei Kindern im Alter unter acht Jahren rasch Überforderungen, insbesondere in der Koordination und zentralen Verarbeitung verschiedener Außenreize mit der spezifischen Konsequenz einer erhöhten Sturzgefahr im Radfahrbereich“ (ebd.).

Der Gleichgewichtssinn (quasi-statisches Gleichgewichtsvermögen) ist so stark gestreut, dass eine Kollektivtrennung nicht signifikant ist. „Die große Streubreite deutet auf ein stark interindividuell unterschiedlich ausgeprägtes Regulationsvermögen in der labilen Gleichgewichtssituation hin" (ebd.).

In Bezug auf das „Können“ als solches sind vor allem Übungseffekte beobachtbar. Kinder, die regelmäßig Rad fahren, zeigen eine sehr viel höhere Radfahrkompetenz als Kinder, die nicht geübt sind. Die Experimente belegen deutlich, dass geübte Radfahrer und eher weniger geübte Radfahrer sich in wesentlichen Kompetenzen unterschieden: „Aus diesen Befunden ist ableitbar, dass 7/8-jährige als Ergebnis einer signifikant häufigeren Radnutzung deutliche Übungseffekte im Sinne einer verbesserten spezifischen Gleichgewichtsregulation in den getesteten Standardsituationen auf dem Rad zeigen. Die im Allgemeinen als Habituation bezeichnete spezifische Gleichgewichtsanpassung ist bei den jüngeren Kindern des ländlichen Bereichs bereits so ausgeprägt, dass diese in einer Reihe von Prüfsituationen das Niveau der älteren Kinder aus der Großstadt nicht nur erreichen, sondern sogar übertreffen“ (ebd.).

Aus den Befunden kann in jedem Fall abgeleitet werden, dass es sinnvoll ist, intensiv und häufig mit Kindern das Rad fahren zu üben, wenn sie damit regelmäßig am Straßenverkehr teilnehmen sollen. Es zeigte sich, dass ein aus 14 Übungseinheiten bestehendes Fahrtraining besonders für die gleichgewichtsregulatorisch defizitären jüngeren Großstadtkinder signifikante Verbesserungen zeigte. 
In dem Folgeprojekt (1997) wurden viele Aspekte der Verkehrswirklichkeit von Schülern betrachtet, die das Fahrrad häufig schon regelmäßig (z. B. auf dem Weg zur Schule) einsetzen (Bongert \& Henke 1997).

„Besondere Schwierigkeiten macht radfahrenden Kindern die Bewältigung von Mehrfachaufgaben, wie sie im Straßenverkehr abverlangt werden. Hierunter versteht man die simultane und koordinierte Realisierung von Teilaufgaben, wie sie z. B. bei der Steuerung und Stabilisierung des Fahrrades und dem gleichzeitigen Erkennen der Verkehrssituation erforderlich ist" (ebd.).

Auch in dieser Studie plädieren die Autoren für ein umfangreiches Einüben der Radfahrkompetenzen, um eine möglichst sichere Teilnahme am Straßenverkehr zu ermöglichen. Das Training ist der wichtigste Aspekt für eine sichere Teilnahme am Straßenverkehr. Je weniger Training, desto unsicherer fahren auch ältere Kinder (weiterführende Schule) im Straßenverkehr: „Zusammenfassend ist der motorische Lernprozess durch einen Anstieg der koordinativen Leistungsfähigkeit und eine Abnahme der dafür erforderlichen Aufmerksamkeit gekennzeichnet. [...] Die Bewegungsautomatisierung wird hierbei als Effekt extensiven Übens angesehen, in Analogie zu motorischen Lernprozessen auf höchstem Lernniveau. [...] Damit reduzieren sich die aufmerksamkeitspflichtigen Anteile und die Bewegung läuft weitgehend automatisch ab. Neben der automatisierten Bewegung können zusätzliche (kognitive) Anforderungen bewältigt werden, wie die Hinwendung zu übergeordneten taktischen und strategischen Aspekten“ (ebd.).

Aus psychologischer Perspektive kann der Kompetenzgewinn der Radfahrer durch Fahrtraining so interpretiert werden, dass durch die Verbesserung der Bewegungsautomatisation die Möglichkeit geschaffen wird, durch die freigewordenen kognitiven Ressourcen besser auf die Verkehrsumwelt achten zu können. Durch die Automatisation der Bewegungsabläufe sind erheblich mehr Ressourcen für die Teilnahme am Straßenverkehr frei und Kinder können hierdurch sehr viel besser auf plötzliche Situationen im Straßenverkehr reagieren.

Ein optimales Training würde demnach darin bestehen, nicht nur das Rad fahren an sich zu trainieren, sondern es sollte nach und nach auch explizit trainiert werden - mit der Basisfähigkeit der Bewegungsautomatisation als Voraussetzung - gleichzeitig die Aufmerksamkeit beim Rad fahren teilen zu können, indem gleichzeitig verkehrsrelevante Aufgaben gelöst werden müssen.

\subsubsection{Zusammenfassung: Psychologische Aspekte der Entwicklung von Fähigkeiten für die aktive Verkehrsteilnahme von Kindern (vgl. auch Poschadel, 2006)}

\section{Entwicklung kognitiver Funktionen im Allgemeinen}

Bei der Geburt ist das menschliche Gehirn noch längst nicht vollständig ausgewachsen und entwickelt .Die Gehirnmasse von Kindern vergrößert sich noch um das Vier- bis Fünffache bis zu einem Lebensalter von etwa 20 Jahren. Während früher angenommen wurde, dass die Informationsverarbeitung von Kindern vor allem wesentlich langsamer ist als die von Erwachsenen, zeigt sich durch neue Untersuchungsmethoden immer deutlicher, dass 
Informationsverarbeitungsprozesse bei Kindern vor allem auf andere Weise verlaufen. Informationsverarbeitungsprozesse finden bei Kindern - im Vergleich zu Erwachsenen zum Teil auch in anderen Gehirnarealen statt. Die für die Teilnahme am Straßenverkehr wichtigen kognitiven Funktionen, wie etwa die Fähigkeit zur selektiven Aufmerksamkeit, entwickeln sich erst mit zunehmendem Alter. Heute wird sogar davon ausgegangen, dass das Gehirn die vollständige Funktionsreife erst im frühen Erwachsenenalter besitzt. Je jünger Kinder sind, desto geringer ist der funktionale Reifegrad des Gehirns, desto unterschiedlicher sind Informationsverarbeitungsprozesse im Vergleich zu Erwachsenen, desto schlechter finden sie sich im Straßenverkehr zurecht.

Entwicklung der visuellen Wahrnehmung im Hinblick auf die Teilnahme am Straßenverkehr

Die Entwicklung des „Sehens“ im Zusammenhang mit der Anteilnahme am Straßenverkehr sollte unter Berücksichtigung von Informationsverarbeitungsprozessen erfolgen. Auch wenn es beispielsweise bei der Entdeckung von visuellen Stimuli im peripheren Sehfeld nur geringe Mittelwertunterschiede zwischen Erwachsenen und Kindern gibt, muss doch angenommen werden, dass Kinder visuelle Reize anders verarbeiten und beispielsweise nicht die „richtigen“ Entscheidungen treffen. Sie gefährden sich eventuell selbst, weil sie Reize nicht richtig interpretieren (können).

Entwicklung der auditiven Wahrnehmung im Hinblick auf die Teilnahme am Straßenverkehr

Auch die auditive Entwicklung sollte unter dem Aspekt betrachtet werden, dass noch nicht alle kognitiven Fähigkeiten von Kindern voll entwickelt sind. Bei Untersuchungen zur Entwicklung des Hörens zeigt sich vor allem, dass gerade jüngere Kinder bei der Richtungslokalisation sich bewegende Reize nur unzulänglich richtig identifizieren können. Selbst 11-jährige Kinder konnten nur etwa $60 \%$ der dargebotenen Reize eines sich bewegenden Autos richtig einordnen und die Richtung des Geräusches erkennen. Die Autoren kommen zum Schluss, dass diese Fehllokalisation - neben den in anderen Untersuchungen nachgewiesenen Problemen bei der visuellen Informationsverarbeitung entscheidend zum Unfallrisiko beitragen kann und auch einfache Anweisungen an die Kinder nicht helfen, das Problem zu lösen. Hier wird ebenfalls deutlich, dass sich entwicklungspsychologische Reifeprozesse nicht einfach durch klare Anweisungen kompensieren lassen.

Insbesondere die entwicklungspsychologisch bedingten Nachteile der Wahrnehmung (Sehen und Hören), bedingt durch im Gegensatz zu Erwachsenen andere Informationsverarbeitungsprozesse, führen dazu, dass komplexe Situationen im alltäglichen Verkehrsgeschehen von Kindern oft nicht adäquat erfasst werden können; für die Teilnahme am Straßenverkehr wichtige Reize können im Gegensatz zu Erwachsenen nicht so schnell von unwichtigen Reizen unterschieden werden. Dabei zeigt sich insgesamt: Je jünger Kinder sind, desto weniger entspricht die Wahrnehmung und die Verarbeitung der Umweltstimuli denen eines erwachsenen Menschen.

\section{Entwicklung des Gefahrenbewusstseins}

Wird "Gefahrenbewusstsein" vor allem als die Kompetenz betrachtet, das (unmittelbare) Risiko für die eigene Gesundheit in konkreten Situationen zu antizipieren und durch entsprechende kontextadäquate Handlungen zu vermeiden bzw. zu minimieren, entwickelt 
sich bei Kindern ein "Gefahrenbewusstsein“ erst mit zunehmendem Alter. Die wenigen Forschungsarbeiten deuten auf ein Bewusstsein von Gefahr und Sicherheit, das sich nach Limbourg in drei Stufen unterteilen lässt: Auf der ersten Stufe können Kinder gefährliche Situationen im Straßenverkehr erkennen, aber erst dann, wenn sie schon akut gefährdet sind (mit 5-6 Jahren erreicht). Auf der zweiten Stufe können Kinder Gefahren voraussehen, d. h. sie lernen zu erkennen, durch welche Verhaltensweisen sie in Gefahr geraten könnten (erreicht mit etwa 8 Jahren). Auf der dritten Stufe können Kinder vorbeugende Verhaltensweisen bewusst einsetzen, um Gefahren zu reduzieren (erreicht mit etwa 10 Jahren). Erst mit zunehmendem Alter lernen sie also, in entsprechenden Situationen gesundheitsschädigendes Verhalten zu antizipieren und durch Handlungsalternativen ihre Gesundheit aktiv zu schützen.

\section{Entwicklung des Verhaltens von Kindern als Fußgänger}

Auch bei der Entwicklung des Verhaltens als Fußgänger gilt: Je jünger Kinder sind, desto schlechter sind unter dem Aspekt der Verkehrssicherheit die entwicklungspsychologischen Voraussetzungen zur Teilnahme am Straßenverkehr. Intensiv wurde das Verhalten bei der Straßenquerung untersucht. Folgende Erkenntnisse, die sich aus verschiedenen Forschungsarbeiten ableiten lassen, sollten bei der Verkehrssicherheitsarbeit unbedingt berücksichtigt werden:

- Jüngere Kinder wählen gerne die kürzeste Route, weil sie innen als sicherste erscheint, auch wenn sie tatsächlich gefährlicher ist.

- Sichthindernisse oder komplexe Verkehrssituationen werden besonders von jüngeren Kindern nicht als Gefahr erkannt.

- Erst 11-jährige Kinder können sichere Routen für die Straßenquerung wählen.

- Die Entscheidung von Kindern, eine Straße zu queren oder nicht, wird hauptsächlich beeinflusst von der räumlichen Lücke im Verkehrsfluss, unabhängig von der Geschwindigkeit der Fahrzeuge.

- Bis zu einer Geschwindigkeit von etwa $55 \mathrm{~km} / \mathrm{h}$ treffen die Kinder im Durchschnitt unabhängig von Alter und Geschlecht „sichere“ Entscheidungen für eine Straßenquerung, allerdings sind die Entscheidungen individuell oft inkonsistent und es gibt „Ausreißer“.

- Bei Fahrzeuggeschwindigkeiten über $55 \mathrm{~km} / \mathrm{h}$ nehmen „unsichere“ Entscheidungen zur Straßenquerung bei Kindern unter 10 Jahren progressiv zu. Je höher die Geschwindigkeit der Autos, desto häufiger treffen Kinder Fehlentscheidungen.

- Besonders die jüngeren Kinder zeigen ein inkonsistentes Verhalten.

- Im Realverkehr werden von Kindern durch Spontanreaktionen oder Ablenkung wahrscheinlich häufiger Fehlentscheidungen getroffen, als bei begleitenden Beobachtungen wissenschaftlicher Erhebungen.

\section{Entwicklung der Körpergröße von Kindern}

Kinder sind schon aufgrund ihrer im Gegensatz zu Erwachsenen geringeren Körpergröße im Straßenverkehr benachteiligt. Erst im Alter von etwa 10 Jahren erreichen sie die 
Körpergröße, die der Höhe eines Mittelklasse-Pkw entspricht. Sie werden durch geparkte Autos verdeckt und können sich (rein visuell) ihrerseits keinen Überblick über das Verkehrsgeschehen verschaffen. Besonders problematisch ist dieser Zusammenhang vor Knotenpunkten, da dort die Sichtbeziehungen durch geparkte Pkw erheblich eingeschränkt werden können.

\section{Entwicklung des Verhaltens von Kindern als Radfahrer}

Auch bei den älteren Kindern, für die das Risiko als Radfahrer im Straßenverkehr zu verunfallen besonders hoch ist, ist die Kompetenz zum Rad fahren noch nicht voll entwickelt. Wichtige Fähigkeiten zur sicheren Teilnahme am Straßenverkehr entwickeln Kinder Schritt für Schritt im Laufe der Kindheit. Erst mit etwa 13 bis 15 Jahren verfügen Kinder als Radfahrer über die Fähigkeiten, die sie für andere Verkehrsteilnehmer im Verkehr berechenbar machen.

Kinder können zwar schon sehr früh (im Alter von 3-6 Jahren) lernen, ihr Fahrrad motorisch zu beherrschen, wenn sie sich hierauf voll konzentrieren. Werden sie aber mit unbekannten Aufgaben konfrontiert oder müssen auf unvorhergesehene Situationen schnell reagieren, sind sie bei der Koordination und der gleichzeitigen Verarbeitung verschiedener Außenreize schnell überfordert mit der spezifischen Gefahr eines Sturzes.

Folgende Befunde sind besonders wichtig im Hinblick auf die Verkehrssicherheit von Kindern im Grundschulalter als Radfahrer:

- Bei Grundschulkindern ist der Gleichgewichtssinn (quasi-statisches Gleichgewichtsvermögen) stark gestreut. Es gibt große interindividuelle Unterschiede.

- 7- bis 8-jährige Kinder, die ihr Fahrrad signifikant häufiger nutzen als eine Vergleichsgruppe, zeigen deutliche Übungseffekte im Sinne einer verbesserten spezifischen Gleichgewichtsregulation in Standardsituationen auf dem Rad.

- Ein aus praktischen Übungen bestehendes Fahrradtraining zeigt bei der Gleichgewichtsregulation von weniger geübten Kindern signifikante Verbesserungen.

- In realitätsnahen Situationen (z. B. Zusatzlast, verschiedene Geschwindigkeiten, Einhändigfahren und Kopfwendung mit Symbolerkennung) schwanken Kinder viel mehr mit dem Rad, als es in einer einfachen Fahrsituation der Fall ist. Es kann vermutet werden, dass hierdurch das Fahrkönnen der Kinder insgesamt (beispielsweise durch die Eltern) fälschlich zu hoch eingeschätzt wird.

Folgende Befunde sind besonders wichtig im Hinblick auf die Verkehrssicherheit von Kindern weiterführender Schulen (Sek. 1) als Radfahrer:

- Es gibt Belege, dass das tatsächliche Verhalten mit dem Rad im Straßenverkehr an Einmündungen und Kreuzungen in keinem Zusammenhang zum theoretischen Wissen steht. Es kann deshalb vermutet werden, dass mangelnde Regelkenntnisse kaum eine Ursache für Radunfälle darstellen. 
- Besonders schwierig ist die Bewältigung von Mehrfachaufgaben, wie sie im Straßenverkehr abverlangt werden (z. B. bei der Steuerung und Stabilisierung des Fahrrades und dem gleichzeitigen Erkennen der Verkehrssituation).

- Es existiert kein flächendeckendes, psychomotorisches Training an weiterführenden Schulen. Wegen der nachweisbaren Trainingseffekte ist dies als eine ungenutzte Ressource zur Unfallprävention anzusehen.

- Aus sportwissenschaftlicher Sicht wird deutlich, dass je mehr die Bewegungsabläufe beim Rad fahren geübt werden, desto besser wird die Bewegungsautomatisation, desto mehr Kapazitäten der Aufmerksamkeit stehen für weitere (unvorhergesehene) Reize zur Verfügung, desto besser wird die Verkehrssicherheit.

- Je mehr das Rad fahren realitätsnah trainiert wird - also auch mit Zusatzaufgaben, bei denen geübt wird, die Aufmerksamkeit zu teilen - desto besser wird die motorische Radfahrkompetenz.

\subsubsection{Was kann aus den Befunden in Bezug auf Knotenpunkte abgeleitet werden?}

- Knotenpunkte müssen im Nahbereich von Überquerungsstellen frei von parkenden Autos sein, da besonders Kinder durch diese verdeckt werden können.

- Hohe Geschwindigkeiten an Überquerungsstellen müssen vermieden werden.

- Radverkehrsanlagen auf denen insbesondere Kinder fahren, sollten eine Wegeführung haben, die ausreichende Überquerungsmöglichkeiten und gute Sichtbeziehungen bietet. 


\subsubsection{Psychologische Aspekte von älteren Menschen als aktive Teilnehmer am Straßenverkehr}

In den folgenden Abschnitten sollen die psychologischen Aspekte der Verkehrsteilnahme Älterer betrachtet werden, mit einem besonderen Schwerpunkt bei der Entwicklung der kognitiven Ressourcen.

Als „Vorbemerkung" ist ein Abschnitt aufgeführt, der das aktuelle Unfallgeschehen älterer Menschen von 2008 in Deutschland aufzeigt (Statistisches Bundesamt 2010). In dieser Vorbemerkung wird deutlich, in welchen Situationen ältere Menschen besonders häufig verunfallen, gleichzeitig aber auch, dass ihr Unfallrisiko in vielen Bereichen deutlich geringer ist als im Vergleich zur Gesamtbevölkerung und dass dies auch mit der kognitiven und körperlichen Leitungsfähigkeit eng in Verbindung steht.

\subsubsection{Vorbemerkung: Das Unfallgeschehen Älterer in Deutschland (Statistisches Bundesamt, 2010)}

„Im Jahr 2008 lebten 16,6 Mill. Personen im Alter von mindestens 65 Jahren in Deutschland; inr Anteil an der Gesamtbevölkerung hat aufgrund der demographischen Entwicklung in Deutschland zugenommen und liegt zurzeit bei $20 \%$. Als Beteiligte an Unfällen mit Personenschaden hatten die über 65-Jährigen in 2008 aber „nur“ einen Anteil von 11 \%. Aus dieser unterproportionalen Unfallbeteiligung kann jedoch nicht geschlossen werden, dass ältere Fahrer die sichereren Fahrer sind, sondern sie spiegelt in weiten Teilen deren geringere Verkehrsteilnahme als Fahrzeugführer wider. Ihre durchschnittliche Fahrleistung pro Pkw und Jahr ist deutlich niedriger als bei den jüngeren Altersgruppen. Zwar nehmen immer mehr Senioren mittels Pkw am Straßenverkehr teil und sichern sich so eine große Mobilität bis ins hohe Alter, dennoch ist die Pkw-Verfügbarkeit der älteren Menschen, insbesondere älterer Frauen, erheblich geringer als die der übrigen Erwachsenen. Die Gruppe der Senioren ist eine sehr inhomogene Altersgruppe, was ihre Wahrnehmungs- und Leistungsfähigkeit sowie ihren Gesundheitszustand anbelangt. Hier seien insbesondere die Verschlechterung des Sehvermögens und die Verringerung der Reaktionsgeschwindigkeit bei vielen älteren Menschen genannt. Art, Dauer und Häufigkeit der Verkehrsbeteiligung weisen bei den älteren Menschen deutliche Unterschiede zu den jüngeren Altersgruppen auf und haben damit auch Auswirkungen auf das Unfallgeschehen der Senioren.

\section{Überblick über die Entwicklung im Jahr 2008}

Im Jahr 2008 verunglückten insgesamt 44.527 ältere Menschen im Alter von 65 oder mehr Jahren im Straßenverkehr, das waren 1,2\% weniger als im Vorjahr. Davon wurden 32.147 Senioren leicht $(-1,4 \%)$ und 11.314 schwer verletzt $(+0,1 \%)$. Die Zahl der getöteten Senioren ist um 7,5\% auf 1.066 zurückgegangen. Senioren stellten damit $11 \%$ aller Verunglückten dar, ihr Anteil an den Todesopfern betrug allerdings $24 \%$. Je 100.000 Einwohner im Alter von über 65 Jahren sind 268 Personen im Straßenverkehr verunglückt, damit ist das Unfallrisiko der älteren Menschen im Vergleich zum Durchschnitt der Gesamtbevölkerung mit 503 Verunglückten je 100.000 Einwohnern nur etwa halb so hoch. Weitaus größer als bei jüngeren Menschen ist aber die Gefahr bei einem Unfall schwer verletzt zu werden oder gar ums Leben zu kommen. So wurden $25 \%$ der verunglückten älteren Menschen schwer verletzt. Bei den unter 65-Jährigen trugen mit $16 \%$ deutlich 
weniger der Verunglückten schwerwiegende Verletzungen davon. Darüber hinaus haben die über 65-Jährigen eine geringere Chance, einen Verkehrsunfall zu überleben. Während der Anteil der Getöteten an den Verunglückten der jeweiligen Altersgruppe für die Kinder und Jugendlichen bis 18 Jahre bei $0,5 \%$ und für die Altersgruppe der 18- bis 64-Jährigen bei $1,0 \%$ liegt, steigt er bei den Senioren auf 2,4\%. Bezogen auf je 100.000 Einwohner der Altersgruppe wurden 6 Senioren im Straßenverkehr getötet. Hierin spiegelt sich zum einen die mit zunehmenden Alter nachlassende physische Widerstandskraft wider, zum anderen ist das höhere Unfallrisiko durch die Art der Verkehrsteilnahme bedingt: Ältere Menschen nehmen häufiger als - ungeschützte - Fußgänger am Verkehr teil und sind daher einem größeren Risiko ausgesetzt, schwerwiegende Verletzungen zu erleiden. [...]

\section{Verunglückte nach Art der Verkehrsbeteiligung}

Weniger als die Hälfte der verunglückten Senioren kam 2008 als Pkw-Insassen zu Schaden, nämlich 20.130 bzw. 45 \% (zum Vergleich alle Altersgruppen: $55 \%$ ). 12.546 bzw. $28 \%$ verunglückten als Fahrradfahrer, 7.136 bzw. $16 \%$ als Fußgänger, 1.275 bzw. 2,9 \% als Businsassen und 3.440 bzw. $7,7 \%$ in den übrigen Verkehrsbeteiligungsarten. Die meisten der getöteten Senioren (437 Personen oder 41 \%) starben als Pkw-Insassen. 325 bzw. 30 \% der getöteten Senioren waren Fußgänger und 218 bzw. $20 \%$ waren Fahrradfahrer. Die Verletzungsschwere bei den älteren Fußgängern ist jedoch im Vergleich zu anderen Verkehrsbeteiligungsarten besonders hoch: Während im Durchschnitt 1,2\% der verunglückten unter 65- jährigen Fußgänger an den Unfallfolgen verstarben, waren es bei den Senioren 4,6\%. Ungefähr jeder zweite getötete Fußgänger und Fahrradfahrer war mindestens 65 Jahre alt. Bei den getöteten Pkw-Insassen gehörte etwa jeder 5. zur Altersgruppe der Senioren. [...]

Überwiegend waren Senioren als Pkw-Fahrer an einem Unfall mit Personenschaden beteiligt (62 \%), $20 \%$ waren als Radfahrer und $11 \%$ als Fußgänger in einen Unfall verwickelt.

$11 \%$ aller Beteiligten an Unfällen mit Personenschaden waren mindestens 65 Jahre alt. An allen beteiligten Fußgängern stellten sie einen Anteil von $20 \%$, an allen Radfahrern von $15 \%$ und an allen Pkw-Fahrern von $10 \%$.

Sofern über 65-jährige Pkw-Fahrer in einen Unfall verwickelt waren, trugen sie sehr häufig $(66 \%)$ die Hauptschuld. Bei den mindestens 75-Jährigen wurde sogar drei von vier unfallbeteiligten Pkw-Fahrern die Hauptschuld am Unfall zugewiesen (76 \%).

Eine differenzierte Betrachtung des Anteils der Hauptverursacher an allen Unfallbeteiligten nach Geschlecht zeigt folgendes Bild: In den Altersklassen bis 64 Jahren war im Jahr 2008 der Anteil der Männer als Hauptverursacher höher als der der Frauen. Bei den Unfallbeteiligten der Altersgruppen 65 Jahre oder älter sind dagegen Frauen häufiger Hauptverursacher der Unfälle, in die sie verwickelt sind. [...] 


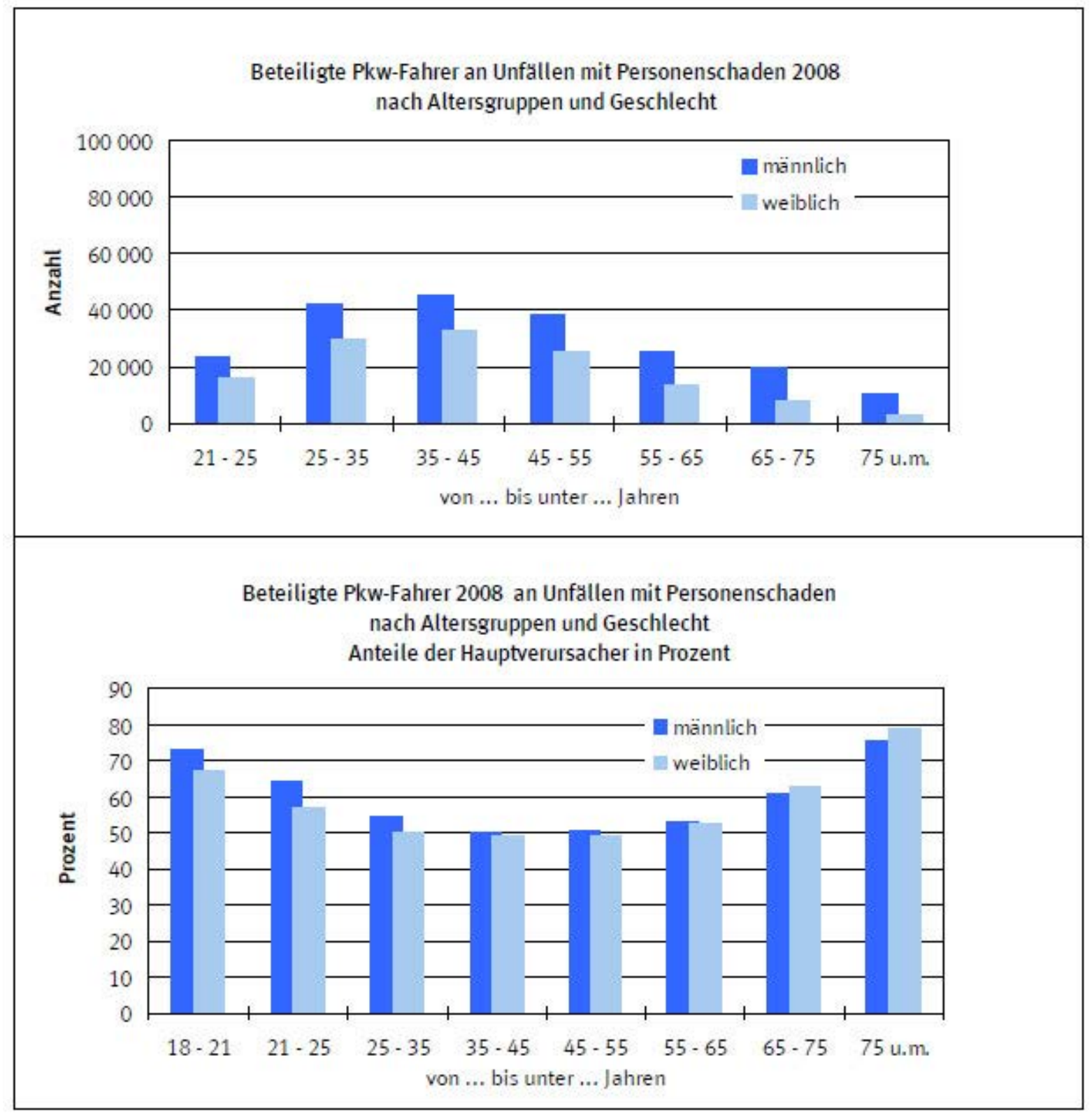

Abbildung 6: Beteiligte Pkw-Fahrer an Unfällen mit Personenschaden nach Alter und Geschlecht, absolut und in Prozent [Quelle: Statistisches Bundesamt 2010]

\section{Unfallursachen}

Ältere Menschen verlieren in komplexen Situationen schneller den Überblick als Verkehrsteilnehmer der jüngeren Altersgruppen. So waren bei den Senioren über 65 Jahren „Vorfahrtsfehler" die häufigste Unfallursache der 40.373 beteiligten Pkw-Fahrer an Personenschadensunfällen. Diese Ursache wurde fast jedem 5. Unfallbeteiligten dieser Altersklasse (19\%) vorgeworfen. Es folgten „Abbiegefehler“ mit 9,7\%. Diese beiden Unfallursachen wurden Senioren wesentlich häufiger angelastet als im Durchschnitt den Pkw-Fahrern aller Altersgruppen. Dagegen spielten „Abstandsfehler“ (8,0\%), „nicht angepasste Geschwindigkeit“ (4,9\%), „falsche Straßenbenutzung“ (3,1\%), „Fehler beim Überholen“ (2,4 \%) sowie „Alkoholeinfluss“ (1,0\%) relativ zu den anderen Altersklassen eine 
geringere Rolle. Die Unfallursachen deuten somit eher auf altersbedingte Einschränkungen der Wahrnehmungsfähigkeit als auf leichtsinniges Verhalten hin“.

\subsubsection{Kognitiver und körperlicher Leistungswandel im Alter mit Auswirkungen auf die Teilnahme am Straßenverkehr.}

Im Zuge der demografischen Entwicklung wird es in Zukunft zu einem immer größeren Teil an älteren Menschen im Straßenverkehr kommen, vor allem als Pkw-Fahrer. Während Anfang der 1990er Jahre in Deutschland nur etwa $10 \%$ der 80-jährigen Frauen einen Führerschein besaßen, werden es im Jahr 2025 etwa 80 \% Führerscheinbesitzerinnen bei den 80-jährigen Frauen sein. Für die 80-jährigen Männer wird dieser Anteil in diesem Zeitraum sogar auf fast 100 \% geschätzt (vgl. Kaiser, H. J. \& Oswald, W. D. 2000).

Während für junge Menschen der Erwerb eines Führerscheins heute als Regel angenommen werden kann (diese werden den Führerschein im Jahre 2025 dann voraussichtlich noch immer besitzen), war dies bei den heute 80-Jährigen nicht der Fall. Viele Menschen haben erst im Laufe des Erwachsenenlebens ihre Fahrerlaubnis erworben, bei Frauen war es sogar eher die Ausnahme. Vor allem durch die hohe Zahl der Menschen, die in den vergangenen Jahren schon zu Anfang ihres Erwachsenenlebens den Führerschein erwerben, ist diese prognostizierte Steigerung zu erklären.

\section{Bevölkerungsentwicklung bis 2060}

Die Gruppe der Älteren wird bis zum Jahre 2025 absolut und relativ im Vergleich zu den jüngeren Altersgruppen ebenfalls anwachsen. Das Statistische Bundesamt macht bis zum Jahr 2060 folgende Vorausberechnung. Der Altenquotient berechnet sich dabei wie folgt: Altenquotient ist das Verhältnis der 65-Jährigen und Älteren im Vergleich zu je 100 Personen im Alter von 20 bis 64 Jahren.

„Der Altenquotient wird [bis 2060, Anm. d. Verf.] stark zunehmen. Die Verschiebungen in der Altersstruktur bewirken, dass der Bevölkerung im Erwerbsalter künftig immer mehr Seniorinnen und Senioren gegenüberstehen werden. Im Jahr 2008 entfielen auf 100 Personen im Erwerbsalter von 20 bis unter 65 Jahren 34 Personen, die 65 Jahre oder älter waren“ (Statistisches Bundesamt 2009).

Zusammenfassend kommt das statistische Bundesamt in seiner Prognose für $2060 \mathrm{zu}$ folgenden Schlüssen:

„Das Altern der heute stark besetzten mittleren Jahrgänge führt zu gravierenden Verschiebungen in der Altersstruktur. Im Ausgangsjahr 2008 bestand die Bevölkerung zu $19 \%$ aus Kindern und jungen Menschen unter 20 Jahren, zu $61 \%$ aus 20- bis unter 65Jährigen und zu $20 \%$ aus 65-Jährigen und Älteren. Im Jahr 2060 wird bereits jeder Dritte (34\%) mindestens 65 Lebensjahre durchlebt haben und es werden doppelt so viele 70Jährige leben, wie Kinder geboren werden.

Die Alterung schlägt sich insbesondere in den Zahlen der Hochbetagten nieder. Im Jahr 2008 lebten etwa 4 Millionen 80-Jährige und Ältere in Deutschland, dies entsprach $5 \%$ der Bevölkerung. Ihre Zahl wird kontinuierlich steigen und mit über 10 Millionen im Jahr 2050 den bis dahin höchsten Wert erreichen. Zwischen 2050 und 2060 sinkt dann die Zahl der Hochbetagten auf 9 Millionen. Es ist also damit zu rechnen, dass in fünfzig Jahren etwa 
$14 \%$ der Bevölkerung - das ist jeder Siebente - 80 Jahre oder älter sein wird“ (Statistisches Bundesamt 2009).

Es kann davon ausgegangen werden, dass viele dieser Menschen zumindest theoretisch noch den Führerschein besitzen können, da die meisten von ihnen eine Fahrerlaubnis im Laufe ihres Lebens erworben haben.

\subsubsection{Veränderungen des allgemeinen Leistungsvermögens mit zunehmendem Lebensalter}

Ältere Menschen unterliegen, genau wie Kinder, in besonderem Maße dem Wandel ihres kognitiven und körperlichen Leistungsvermögens, wie in der „Vorbemerkung“, Kapitel 3.1.2 , bereits beschrieben.

Mit zunehmendem Alter verändern sich allerdings nicht alle, sondern vor allem die sogenannten Kontrollfunktionen (oder auch exekutive Funktionen), die vorwiegend für die Steuerung von Multitasking- und Tempoprozessen verantwortlich sind. Gerade diese Funktionen sind neben den körperlich-physischen Leistungsparametern aus verkehrspsychologischer Sicht besonders bedeutsam für die aktive Teilnahme am Straßenverkehr (Falkenstein und Poschadel 2008).

Für bestimmte Unfallsituationen kann ein Zusammenhang zu bestimmten Altersgruppen hergestellt werden (siehe Vorbemerkung zu diesem Kapitel). Speziell bei der Altersgruppe der über 70-jährigen Fahrer gibt es typische Unfallmuster, insbesondere an Knotenpunkten (z. B. Schlag 1990, 2007). Leider lässt sich aus Unfallstatistiken nicht ermitteln, inwieweit einzelne Unfälle kausal tatsächlich auf altersbegleitende Leistungsveränderungen zurückzuführen sind. Es kann lediglich ein statistischer Zusammenhang zwischen typischen Unfallsituationen und bestimmten Altersklassen in Verbindung gebracht werden (Poschadel \& Sommer 2007). Nachfolgend werden ausgewählte altersbegleitende kognitive Beeinträchtigungen mit Bezug zum Autofahren dargestellt.

\section{Hohe Variabilität in allen verkehrssicherheitsbezogenen Leistungsbereichen von Älteren}

Es ist nach dem gegenwärtigen Stand der Wissenschaft nicht möglich, aufmerksamkeitsbasierte, neurologische oder motorische Defizite allein auf Grund des Alters eines Menschen vorherzusagen.

Zu groß sind interindividuelle Unterschiede in allen drei zu beschreibenden Teilbereichen, die sich mit zunehmendem Lebensalter in der Amplitude noch weiter erhöhen.

Deshalb lassen sich auch Kohortenvorhersagen zu verkehrsrelevanten Leistungsdefiziten nach derzeitigem Stand der Forschung nicht treffen. Altersbedingte Veränderungen im verkehrsrelevanten Leistungspotenzial zeigen sich mit hoher interindividueller Variabilität bei gleichem kalendarischen Alter (z. B. Kaiser \& Oswald 2000; Becker \& Albrecht 2003). Weiterhin zeigen sich fahrrelevante interindividuelle Leistungsunterschiede nicht erst im höheren Lebensalter, sondern teilweise schon sehr viel früher (TÜV Kraftfahrt GmbH, Institut für Verkehrssicherheit, Herberg, K.-W., 1998). 


\subsection{Prävalenz altersbedingter Krankheiten}

Für Deutschland liegen insgesamt nur sehr wenige Daten vor, aus denen sich abschätzen lässt, in welchem Alter wie viel Prozent der Bevölkerung von einer bestimmten altersbegleitenden Einschränkung oder Krankheit betroffen sind (Prävalenz). „In Deutschland existieren bevölkerungsbezogene Krankheitsregister lediglich für einige wenige Krankheitsbilder. Vielfach stehen die rechtlichen Rahmenbedingungen, insbesondere die im internationalen Vergleich sehr restriktiven Datenschutzbestimmungen einer für die Erfüllung der Registerfunktion erforderlichen Vollständigkeit der Registrierung entgegen“ (Bundesministerium für Familie, Senioren, Frauen und Jugend 2002).

Deshalb kann gegenwärtig nicht abgeschätzt werden, welche altersbegleitenden bzw. krankhaften Einschränkungen bei der Planung von verkehrssicherheitserhöhenden Maßnahmen genau getroffen werden müssen. Allerdings lassen sich einige Fakten nennen:

Einen guten und komprimierten Überblick über Veränderungen der Leistungsfähigkeit (auch auf Grund von Krankheiten) in Bezug auf den Straßenverkehr gab 2004 die Schweizerische Beratungsstelle für Unfallverhütung (BFU) in ihrem Handbuch der Sicherheitsarbeit "Sicherheit für alle“ (2004) im Kapitel „Senioren im Straßenverkehr“:

\section{„Die Augen}

80 bis 90 Prozent der für das Fahren benötigten Informationen werden durch das Auge aufgenommen. Altersbedingte Veränderungen zeigen sich insbesondere

- als Beeinträchtigung des Sehvermögens bei Dämmerung und Dunkelheit. Ein 60jähriger Pkw-Fahrer braucht achtmal soviel Licht wie ein 20-jähriger, um bei Dunkelheit noch richtig sehen zu können;

- als Zunahme der Blendempfindlichkeit;

- als Verschlechterung des Sehens von Bewegungsvorgängen in der Randzone des Gesichtsfeldes und als Verlängerung der Zeit, die das Auge braucht, bis es ein fixiertes Objekt scharf abbildet. Die Blickfolge 'Strasse - Tachometer - Strasse' dauert im Alter deutlich länger.

\section{Das Gehör}

Etwa 30 Prozent aller 65-Jährigen sind hörbehindert. [...]

\section{Der Bewegungsapparat}

Arthrose, Rheuma oder ungenügende Blutversorgung - insbesondere der Beine und Füße können das Lenken eines Fahrzeuges erschweren. Wenn eine Versteifung des Halses auftritt, kann es Schmerzen bereiten, den Kopf zu drehen, weshalb ältere Leute dies oft unterlassen. [...]

\section{Das Reaktionsvermögen}

Im Alter erhöht sich einerseits die Reaktionszeit bis zum Beginn einer Bewegung; andererseits nimmt die Zeit zu, die für die Ausführung der Bewegung erforderlich ist. Ältere Menschen reagieren oft langsamer als jüngere [allerdings trifft das nicht auf Einfachwahlreaktionsaufgaben zu, Anm.d.Verf.]. Bei begrenzter Entscheidungszeit unterlaufen innen mehr Fehleinschätzungen. 


\section{Krankheit und Medikamente}

Außer den erwähnten Veränderungen können sich auch verschiedene Krankheiten negativ auf das sichere Lenken eines Fahrzeugs auswirken, wie z. B. Herz- oder Kreislaufleiden, Bluthochdruck oder Zuckerkrankheit. Das Problem der Behandlung solcher Krankheiten besteht darin, dass entsprechende Medikamente oft das Fahrerverhalten in sicherheitsgefährdendem Maß beeinträchtigen.“

\section{Demenzen}

Neben den von der BFU genannten Einschränkungen zeigen sich in höherem Lebensalter oft vor allem kognitive Beeinträchtigungen wie Demenzen, die die Teilnahme am Straßenverkehr zunächst erschweren und schließlich gänzlich unmöglich machen können. Hier liegen für die Bundesrepublik Zahlen vor, die jedoch teilweise auf alten oder sehr alten Datenbeständen beruhen.

Im „Vierten Bericht zur Lage der Älteren Generation“ wird auf das Thema Prävalenz der Demenz in einem eigenen Abschnitt eingegangen (Bundesministerium für Familie, Senioren, Frauen und Jugend 2002): „Vereinfacht kann zwischen leichten, mittelschweren und schweren Demenzen unterschieden werden. Von leichten Demenzen spricht man, wenn zwar kognitive Störungen vorliegen, die die Bewältigung schwieriger Aufgaben kaum erlauben oder zumindest erheblich einschränken, die aber noch nicht so ausgeprägt sind, dass die Betroffenen im Altag von anderen Personen abhängig sind“.

Es kann davon ausgegangen werden, dass Menschen im Frühstadium oder mit ganz leichten Demenzen noch in der Lage sind, Auto zu fahren. Nur, an welchem Punkt genau die Grenze gezogen werden muss, ist bisher ungeklärt.

„Derzeit liegen keine Angaben über die Zahl der leicht bis mittelschwer demenzkranken Autofahrerinnen und Autofahrer in Deutschland, sowie Häufung und Art der Vergehen bzw. Unfälle im Straßenverkehr vor" (Bundesministerium für Familie, Senioren, Frauen und Jugend 2002). Trotzdem lassen sich vorsichtige Aussagen zur Prävalenz der Demenz machen. Für Deutschland wird gegenwärtig von folgender Prävalenz bei leichten Demenzen ausgegangen: „Die Prävalenz leichter Demenzen liegt nach deutschen Untersuchungen in ähnlicher Höhe wie die der schweren Formen. Es werden Raten von etwa $5 \%$ berichtet, mit sehr geringen regionalen Unterschieden. [...] In internationalen Studien schwanken die Schätzungen jedoch sehr stark. Dies hängt auch damit zusammen, dass es noch an klaren Kriterien mangelt, anhand derer man in Bevölkerungsstudien frühe Stadien von gutartigen kognitiven Einbußen von lebenslang bestehenden Leistungsminderungen und von vorübergehenden Beeinträchtigungen abgrenzen kann. Subklinische kognitive Defizite, die nicht die Demenzkriterien erfüllen, treten bei älteren Menschen relativ häufig auf. Auf Testleistungen und klinischen Beobachtungen beruhende Schätzungen beziffern die Prävalenz auf 16 bis $34 \%$, wobei ebenfalls eine starke Altersabhängigkeit festzustellen ist" (Bundesministerium für Familie, Senioren, Frauen und Jugend 2002).

Wegen der insgesamt eher schlechten Datenlage in Deutschland und weil individuell verlässliche Aussagen zu altersbegleitenden Leistungsveränderungen nicht auf Basis des kalendarischen Alters getroffen werden können, existieren derzeit kaum Konzepte, um die individuelle „Verkehrsteilnahmefähigkeit“ älterer Menschen durch eine Verbesserung der 
individuellen physiologisch / kognitiven / körperlichen Leistungsmerkmale kohortenweise gezielt zu verlängern.

\subsubsection{Altersbegleitende Veränderungen der kognitiven Leistungsfähigkeit mit zentraler Bedeutung für die Verkehrsteilnahme}

Das sogenannte „Useful Field of View”

Bei der Verkehrsteilnahme spielt die visuelle Wahrnehmung und Aufmerksamkeit eine zentrale Rolle. Die Menge an visueller Information, die bei einer einzelnen Fixierung aus der Umgebung extrahiert werden kann, wird durch die Größe des visuellen Aufmerksamkeitsbereichs bestimmt. Informationen außerhalb dieses Bereichs werden nur rudimentär oder gar nicht verarbeitet. Im Bereich von Fahraufgaben wird der effektive Aufmerksamkeitsbereich durch das sogenannte "Useful Field of View" (UFOV) beschrieben (Ball et al. 1993). Viele Untersuchungen hierzu legen nahe, dass Einschränkungen im UFOV mit einem erhöhten Unfallrisiko eng in Verbindung stehen (Ball et al. 1993, 2006; Rubin et al. 2007) und der UFOV somit als wichtiger Indikator für die Fahreignung angesehen werden kann. Viele Untersuchungen zeigen, dass sich das UFOV mit zunehmendem Alter verringert.

\section{Visuelle Suche}

„Visuelle Suche" bedeutet das Finden von einem oder mehreren Zielreizen in einem Umfeld von Nicht-Zielreizen (Treisman und Gelade 1980). Sie kostet mit steigender Anzahl der zu durchsuchenden Zeichen zunehmend mehr Zeit, aber nur, falls sich der Zielreiz aus einer Kombination von Eigenschaften zusammensetzt (z. B. rote Ampel rechts).

Eine weitere Beeinträchtigung im Alter dieser für die Teilnahme am Straßenverkehr relevanten Funktion ist durch eine Vielzahl von Studien belegt (z. B. Madden et al. 1999). In Laboraufgaben konnte die Arbeitsgruppe um Falkenstein mit neurophysiologischen Maßen zeigen, dass die visuelle Suche von Zielreizen, die durch mehrere Eigenschaften definiert sind („conjunction search“), unter Zeitdruckbedingungen für Ältere (55 bis 65-jährige) mit erhöhter Anstrengung verbunden ist und dennoch schlechter bewältigt wird als von einer jungen Vergleichsgruppe (Wild-Wall et al. 2007).

Diese Probleme beeinträchtigen nicht nur die Suche selbst, sondern auch die gleichzeitig ausgeführte Tätigkeiten. In verkehrsähnlichen Laboraufgaben mit visueller Suche waren Ältere beeinträchtigt, vor allem bei stark strukturiertem visuellem Hintergrund und gleichzeitiger Ausführung einer weiteren Aufgabe (McPhee et al. 2004). Ältere zeigen im Vergleich zu Jüngeren schwächere Leistungen bei der visuellen Suche von Informationen, insbesondere in einem stark strukturierten visuellen Feld, z. B. komplexe Hinweisschilder. Es besteht daher das Risiko, dass z. B. eine nötige Reaktion im Straßenverkehr verspätet ausgeführt wird, weil Ältere durch den visuellen Suchprozess abgelenkt oder zu stark beansprucht werden.

\section{Orientierung}

Orientierung ist die unwillkürliche Hinwendung der Aufmerksamkeit auf neue und deswegen möglicherweise relevante oder sogar gefährliche Reize in der Umwelt. Sie spiegelt sich in bestimmten EKP-Komponenten, P3a (Orientierung) und RON (Reorientierung) (Mager et al. 2005). Zusammenfassend lässt sich sagen, dass Ältere ihre Aufmerksamkeit länger auf 
irrelevante Reize (z. B. neue Werbeplakate) richten und länger brauchen, um die Aufmerksamkeit wieder zurück auf die Hauptaufgabe (z. B. fahrrelevante Informationen) zu richten. Der unmittelbare Zusammenhang zur Verkehrssicherheit wird hier besonders deutlich: Solange Ältere durch irrelevante Reize abgelenkt sind, können sie auf die aktuell relevanten Reize nicht reagieren. Im Extremfall kann dies zum gänzlichen Ausfall einer Reaktion führen, indem z. B. wichtige Verkehrsschilder wegen Ablenkung einfach übersehen werden (z. B. rote Ampel).

\section{$\underline{\text { Inhibition }}$}

Eine weitere bei Älteren vermutlich insgesamt eingeschränkte Funktion ist die Inhibition. Dies betrifft zum einen die Hemmung irrelevanter Reizaspekte, als auch die Hemmung falscher (Re-)aktionen (z. B. Falkenstein et al. 1995, 1999a, 1999b, 2002, 2007). Ein immer wiederkehrender Befund in der kognitiven Altersforschung ist die stärkere Interferenz in der Stroop-Aufgabe (Spieler, Balota und Faust 1996). Bei der Benennung der Druckfarbe eines Farbwortes (z. B. Rot) sind Ältere langsamer und machen mehr Fehler als Jüngere, wenn Farbe und Wort nicht übereinstimmen. Dies deutet auf eine schlechtere Hemmung der automatisierten Reaktion "Lesen der Worte“ hin.

Ein solches Inhibitionsdefizit scheint insbesondere bei der Verarbeitung irrelevanter Reize zu bestehen. Hasher et al. (1991) postulieren in ihrer Theorie, dass viele altersbedingte kognitive Defizite durch eine verringerte Fähigkeit zur Unterdrückung von irrelevanten Informationen im Arbeitsgedächtnis zustande kommen.

Vor allem ist die Inhibition bei Älteren dann gestört, wenn dabei ein bestimmter Kontext beachtet werden muss. Eine Fehlbewertung kann bei Älteren z. B. beim Linksabbiegen zustande kommen, wo unter Zeitdruck und in Abhängigkeit vom Kontext (z. B. wer hat Vorfahrt?) die Entscheidung zu fahren oder zu stoppen gefällt werden muss. Im Einklang mit dieser Beobachtung postulieren Braver et al. (2001), dass ein Kernproblem Älterer eine dysfunktionale Kontextverarbeitung ist. In realen Lebens- und Arbeitssituationen findet Kontrolle (wie z. B. Inhibition) immer unter Beachtung eines Kontextes statt (z. B. Bremsen, wenn die Ampel der eigenen Spur, jedoch nicht die der benachbarten Spur rot zeigt).

Zusammenfassend scheint die Inhibition irrelevanter Reize oder Reaktionen bei älteren Personen beeinträchtigt zu sein und damit eine zentrale Rolle bei den Fähigkeiten einzunehmen, die für die Teilnahme am Straßenverkehr von entscheidender Bedeutung sind. Ältere Verkehrsteilnehmer könnten daher eher als junge auf irrelevante Reize (z. B. grüne Rechtsabbiegerampel als Geradeausfahrer) fälschlich reagieren.

\section{Mehrfachtätigkeit}

Die Durchführung von zwei oder mehr Aufgaben ist im Alltag häufig erforderlich. Die Teilnahme am Straßenverkehr ist eine typische derartige Anforderung, da neben der Steuerung des eigenen Verhaltens (sei es als Pkw-Fahrer, Radfahrer oder Fußgänger) verschiedene Aufgaben gleichzeitig durchgeführt (z. B. Beobachtung der Umwelt). Dadurch erhöht sich bei Doppelaufgaben die Reaktionszeit und z. T. die Fehlerrate verglichen mit der Einzelaufgabe. Defizite bei Mehrfachtätigkeit bzw. bei Doppelaufgaben bei älteren Personen werden immer wieder gefunden (Verhaeghen \& Cerella 2002) und gehören zu den am besten belegten Altersdefiziten. Li \& Lindenberger (2002) konnten vor allem zeigen, dass 
Ältere bei gleichzeitiger Durchführung sensomotorischer und kognitiver Aufgaben Probleme haben. Genau diese Situation ist der Teilnahme am Straßenverkehr gegeben: Neben dem eigentlichen Steuern des eigenen Verhaltens (also einer „Tracking“-Aufgabe) müssen häufig weitere Aufgaben ausgeführt werden, insbesondere das Beachten und Bewerten von Verkehrssituationen und -zeichen.

Zusammenfassend besteht ein deutliches Doppelaufgaben-Defizit bei Älteren, v. a. wenn Reiz und motorische Aspekte der Teilaufgaben ähnlich sind, vor allem aber, wenn sie in komplexen Situationen ausgeführt werden müssen.

\section{Aufgabenwechsel}

Eine ähnliche kognitiv-funktionelle Bedeutung wie die Mehrfachtätigkeit hat der Wechsel zwischen unterschiedlichen Aufgaben, die Teilaufgaben folgen hier aufeinander und sind somit zeitlich getrennt. Man kann sowohl unterscheiden zwischen regelmäßigen und daher vorhersagbaren Wechseln zweier Teilaufgaben (A und B) als auch einer Wechselaufgabe, in der durch Hinweisreize die aktuelle Teilaufgabe „angesagt“ wird. Im letzten Fall ist der Wechsel unvorhersagbar und durch die Hinweisreize extern gesteuert (Rogers und Monsell 1995; Schuch und Koch 2003). Beide Versionen kommen auch in Verkehrssituationen vor. Kray und Lindenberger (2000) fanden bei vorhersagbaren Wechseln (AA, BB...) bei älteren Probanden deutliche Defizite, die interpretiert wurden als Schwierigkeit Älterer, zwei Aufgaben-Sets im Arbeitsgedächtnis zu halten.

\section{Handlungsüberwachung}

Damit ist die Fähigkeit gemeint, die eigene Tätigkeit zu überwachen und auf Fehler angemessen zu reagieren. Nach Fehlern bei einer Reaktionsaufgabe zeigt sich im EKP ein negatives ( $\mathrm{Ne}$ oder $\mathrm{ERN}$ ) und ein nachfolgendes positives Potential $(\mathrm{Pe})$ (Gehring et al. 1993). Vor allem bei schwierigen Aufgaben zeigten Ältere eine kleinere Fehlerkorrekturrate als Jüngere (Band und Kok 2000). Dies weist darauf hin, dass die Fehlererkennung sowie die Reaktionsüberwachung bei älteren gegenüber jüngeren Personen verändert sind. Für die Fahrzeugführung im Straßenverkehr bedeutet es, dass Ältere weniger in der Lage sind, falsches Fahrverhalten unmittelbar zu bemerken bzw. zu korrigieren, da sie Fahrfehler ungenauer registrieren.

$\underline{\text { Randbedingungen, die kognitive Defizite bei der Teilnahme am Straßenverkehr verstärken }}$ können

Altersbedingte Veränderungen zeigen sich mit hoher interindividueller Variabilität (s. o.). Es hängt offenbar sehr von den Randbedingungen $a b$, ob altersbedingte Funktionsveränderungen auftreten, wie stark diese Veränderungen sind und ob sie sich überhaupt auf das offene Verhalten auswirken. Eine wichtige Randbedingung ist „Zeitdruck“. Eine für Ältere kritische Situation ist z. B. an Knotenpunkten beim Abbiegen gegeben, wo unter Zeitdruck die Entscheidung zu fahren oder zu stoppen in Abhängigkeit vom Kontext (z. B. wer hat Vorfahrt?) gefällt werden muss. Eine weitere Randbedingung ist „Mehrfachtätigkeit“. Altersbegleitende Defizite sind oft dann besonders groß, wenn mehrere Aufgaben gleichzeitig zu bearbeiten sind und daher Verarbeitungskapazität flexibel und variabel verteilt werden muss. Eine für ältere Kraftfahrer schwierige Situation und typische Mehrfachtätigkeit ist deshalb oft das Befahren komplexer Kreuzungen, bei der mehrere 
Aufgaben gleichzeitig erledigt werden müssen (visuelles Tracking, Inhibition irrelevanter Informationen, Bewerten der Verkehrssituation, Orientierung usw.) und bei der häufig noch zusätzlich Zeitdruck besteht.

\subsubsection{Möglichkeiten zum Erhalt kognitiver Leistungen (vgl. Poschadel \& Sommer 2007)}

Es ist schon seit langer Zeit bekannt, dass auch ältere Menschen in Bezug auf ihre kognitiven Fähigkeiten noch über ein erhebliches Entwicklungspotenzial verfügen: „Insbesondere im Hinblick auf die Förderung kognitiver Leistungen dokumentieren eine große Zahl experimenteller Trainingsstudien übereinstimmend das erhebliche Entwicklungspotenzial und die Plastizität auch physiologischer Alterungsprozesse" (Oswald et al. 2002). Ähnliches wird auch von den Wissenschaftlern der Langzeitstudie ACTIVE berichtet: „Recent research from both human and animal studies indicates that neural plasticity endures across the lifespan, and that cognitive stimulation in the environment is an important predictor of enhancement and maintenance of cognitive functioning, even in old age" (Ball et al. 2002). Ebenso stellt Simoes fest: „Numerous studies have found significant improvements in the performance of adults over 60 years old as a result of behavioural interventions, and also that cognitive training is able to resolve the decline in healthy older people's cognitive performance" (Simoes 2003).

„Frühere" Ergebnisse der Forschung zur Trainierbarkeit fluider Fähigkeiten wurden gerne in der Wendung „Use it or lose it“ zusammengefasst (vgl. Oswald, Hagen, Rupprecht \& Gunzelmann 2002). Damit wurde zum Ausdruck gebracht, dass das bloße Ausführen selbst „alltäglicher“ Tätigkeiten schon ein Training bedeutet, dass Abbau entgegenwirken kann.

Churchill et al. (vgl. 2002) konnten für Fähigkeiten, die für das Autofahren wichtig sind (Mutiple-Task-Aufgaben), bei einem entsprechenden Training positive Effekte über einen Zeitraum von mehreren Monaten nachweisen.

\subsubsection{Zusammenfassung: Leistungswandel Älterer in Bezug auf den Straßenverkehr}

Kognitive Fähigkeiten, die mit Erfahrungswissen im Zusammenhang stehen, bleiben auch im hohen Erwachsenenalter eher stabil. Andererseits nehmen kognitive Fähigkeiten, die mit Tempoprozessen und Mehrfachanforderungen im Zusammenhang stehen, im hohen Erwachsenenalter eher ab. Dieser Leistungswandel macht die typischen Schwierigkeiten älterer Verkehrsteilnehmer im Straßenverkehr plausibel, da vor allem solche kognitiven Fähigkeiten nachlassen, die mit Aufmerksamkeitsprozessen im engen Zusammenhang stehen. Das ist vor allem in komplexen Situationen der Fall, wie beispielsweise dem „LinksAbbiegen" oder dem Befahren von komplexen Kreuzungen.

Die kognitive Laborforschung zeigt jedoch eindeutig, dass sich derartige Veränderungen durch gezieltes Training (auch langfristig) positiv beeinflussen lassen. 
3.1.3.7 Was kann aus den Befunden in Bezug auf Knotenpunkte für ältere Verkehrsteilnehmer (insbesondere Pkw-Fahrer) abgeleitet werden?

- Knotenpunkte sollten einfach strukturiert sein.

- Im Bereich von Knotenpunkten sollten ablenkende Reize vermieden werden (z. B. bunte Reklame, zu viele Schilder auf einmal, leuchtende Werbung).

- Wichtige Informationen sollten rechtzeitig, vielleicht auch mehrfach, vor Knotenpunkten angekündigt werden.

- Die Verkehrsführung sollte einfach, eindeutig und kontrastreich sein. 


\subsection{Knotenpunkte: Anforderungen und Unfälle mobilitätsbehinderter Menschen}

\subsubsection{Behinderung und Einschränkung - Definition und Bedeutung}

Menschen haben unterschiedliche Fähigkeiten und daher unterschiedliche Anforderungen und Bedürfnisse an die Gestaltung der gebauten Umwelt. Die Anforderungen an die Gestaltung öffentlicher Verkehrsanlagen ergeben sich z. B. aufgrund von Mobilitätseinschränkungen, die dauerhaft oder auch temporär vorliegen können. Demnach ist zu unterscheiden zwischen mobilitätseingeschränkten Menschen im engeren Sinne sowie mobilitätseingeschränkten Menschen im weiteren Sinne (vgl. Abbildung 7).

\section{Mobilitätseingeschränkte Menschen}

\section{mobilitätseingeschränkt im engeren Sinne}

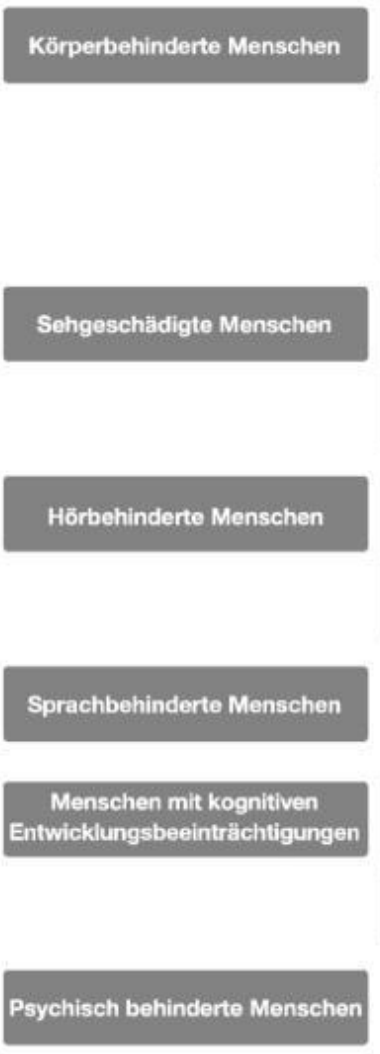

Gehbehinderte Menschen

Rollstuhinutzende Menschen

Arm- und handbehinderte Menschen

Sehbehinderte Menschen

Blinde Menschen

Schwerhörige Menschen

Gehőrlose Menschen

Lernbehinderte Menschen

Geistig behinderte Menschen

mobilitätseingeschränkt im weiteren Sinne

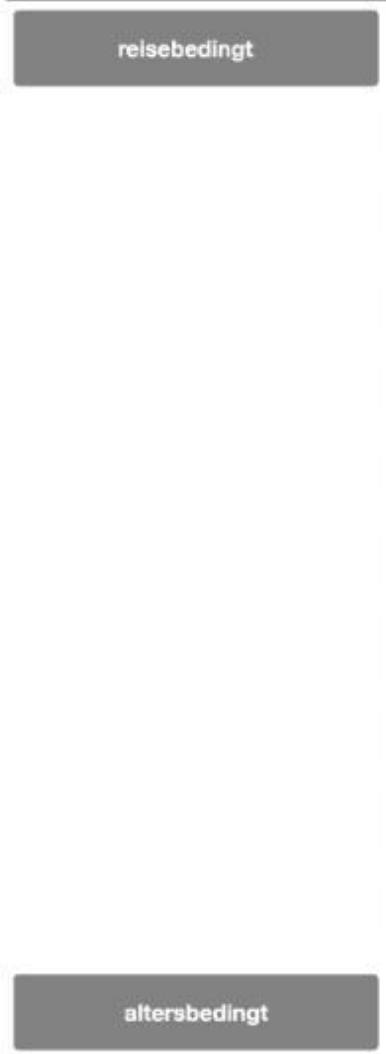

Fahrgäste mit Gepăck

Fahrgäste mit Kinderwagen

Fahrgäste mit Fahrrädern

Fahrgäste mit Einkaufs- $/$ Gepăckwagen

Fahrgåste mit Hunden

Werdende Mūtter

Übergewichtige Menschen

Ortsunkundige Menschen

Menschen mit temporären

Einschrănkungen

Menschen mit Allergien

Sprachunkundige Menschen

Ältere Menschen

Kleinkinder

Abbildung 7: Mobilitätseingeschränkte Menschen - Übersicht [Quelle: (FGSV 2011), korrigierte Fassung]

\subsubsection{Mobilitätseinschränkung im engeren Sinne - Mobilitätsbehinderung}

Als mobilitätseingeschränkt im engeren Sinne gelten Menschen, die aufgrund dauernder Beeinträchtigung oder akuter Erkrankung in ihrer Mobilität stark eingeschränkt sind. Dazu zählen Menschen mit verschiedenen Formen einer körperlichen, sensorischen oder geistigen 
Behinderung (vgl. Abbildung 7). Für den Personenkreis mit Mobilitätseinschränkungen im engeren Sinne wird häufig auch der Begriff "Mobilitätsbehinderte Menschen“ verwendet. Behinderung wird hier im Sinne des Gesetzes verstanden. Das Gesetz zur Gleichstellung behinderter Menschen (BGG) definiert dazu in § 3: „Menschen sind behindert, wenn ihre körperliche Funktion, geistige Fähigkeit oder seelische Gesundheit mit hoher Wahrscheinlichkeit länger als sechs Monate von dem für das Lebensalter typischen Zustand abweichen und daher ihre Teilhabe am Leben in der Gesellschaft beeinträchtigt ist“ (Deutscher Bundestag 2002). ${ }^{1}$

Die Auswirkungen auf die Teilhabe am Leben in der Gesellschaft, die sich aus einer Behinderung ergeben, werden als Grad der Behinderung (GdB) nach Zehnergraden (20 100) abgestuft festgestellt. Als schwerbehinderte Menschen gelten Personen, denen von den Versorgungsämtern ein Grad der Behinderung $(\mathrm{GdB})$ von 50 oder mehr zuerkannt worden ist. Auf Antrag wird für diese Personen ein Ausweis über die Eigenschaft als schwerbehinderter Mensch ausgestellt [Quelle: SGB IX].

\subsubsection{Mobilitätseinschränkung im weiteren Sinne}

Zu den Personen mit Mobilitätseinschränkungen im weiteren Sinne zählen Menschen, deren Mobilität zeitweise oder in bestimmten Situationen erschwert ist (vgl. Abbildung 7), z. B.

- ältere und hochbetagte Menschen,

- Kinder,

- werdende Mütter,

- vorübergehend mobilitätseingeschränkte Personen mit zeitlich begrenzten Unfall-, Krankheitsfolgen oder postoperativen Beeinträchtigungen,

- Analphabeten oder fremdsprachige Personen sowie

- Personen mit Kinderwagen oder schwerem bzw. sperrigem Gepäck.

\subsubsection{Bedeutung der Gruppe der mobilitätseingeschränkten Menschen für die Verkehrsplanung}

Ältere Menschen und Kinder zählen demnach zunächst zu den mobilitätseingeschränkten Menschen im weiteren Sinne (altersbedingte Mobilitätseinschränkungen). Gerade im Alter können allerdings mehrere Einschränkungen auftreten, welche die Mobilität beeinträchtigen. Diese erreichen jeweils für sich genommen nicht die Schwere einer Behinderung. In der Summe können die Einschränkungen aber die Auswirkung einer schweren Behinderung erreichen (Boenke 2010). Daraus ergibt sich allerdings nicht zwangsläufig ein Anspruch auf einen Schwerbehindertenausweis gemäß SGB IX. Daraus folgt, dass die Gruppe der Menschen mit Behinderung größer ist, als die statistischen Daten auf Basis der in Deutschland ausgegebenen Schwerbehindertenausweise aufzeigen. Die Übergänge zwischen Einschränkung und Behinderung sind teils fließend, Anforderungen deckungsgleich - je nach Art der Einschränkung bzw. Behinderung. Der Fokus der

\footnotetext{
${ }^{1}$ Gleiche Definition im neunten Sozialgesetzbuch (SGB IX).
} 
Untersuchung in diesem Abschnitt liegt zunächst auf der Gruppe der Menschen mit einer Behinderung gemäß der gesetzlichen Definition. Wegen der Überschneidungen sind immer wieder Querverweise möglich (beispielsweise muss ein Rollatornutzer nicht zwangsläufig behindert gemäß der gesetzlichen Definition sein, kann aber erhebliche Schwierigkeiten bei der Mobilität im Verkehrsraum haben).

Zum Stichtag 31. Dezember 2011 nennt das Bundesministerium für Arbeit und Soziales ca. 7,336 Mio. gültige Schwerbehindertenausweise für Menschen mit Behinderung in Deutschland. Das entsprach 8,9\% der Gesamtbevölkerung zu diesem Zeitpunkt (Bundesministerium für Arbeit und Soziales (BMAS) 2012). Ein nicht unerheblicher Teil dieser Bevölkerungsgruppe ist in seiner Mobilität deutlich eingeschränkt (Merkzeichen „aG“, „B“, „H“ und „BL“; „RF“ und „GL“ vgl. Tabelle 1). Diese Menschen benötigen für ihre Fortbewegung zwingend eine barrierefrei gestaltete Umwelt.

Tabelle 1: Aufteilung der Merkzeichen der amtlich registrierten schwerbehinderten Menschen in Deutschland zum 31.12.2011 [Quelle: (Bundesministerium für Arbeit und Soziales (BMAS) Referat V2, 2012)]

\begin{tabular}{|l|c|}
\hline Merkzeichen & in \% der Ausweisinhaber \\
\hline „G“ (gehbehindert) & 48,2 \\
\hline „aG“ (außergewöhnlich gehbehindert) & 9,5 \\
\hline „H“ (hilflos) & 12,5 \\
\hline „BL“ (blind) & 1,4 \\
\hline „RF“ (Befreiung von der Rundfunkgebührenpflicht) & 14,0 \\
\hline „GL“ (gehörlos) & 0,5 \\
\hline „1.KL“ (1. Klasse Nutzung erlaubt) & 0,1 \\
\hline „B“ (Begleitperson benötigt) & 25,7 \\
\hline „Kriegsbeschädigt“ & 0,5 \\
\hline „VB“ (weniger als 50 \% Erwerbsfähigkeit) & $>0,1$ \\
\hline „EB“ (weniger als 50 \% Erwerbsfähigkeit) & $>0,1$ \\
\hline
\end{tabular}

Hinzu kommen ca. 2,27 Mio. Menschen mit einem GdB von mindestens 30 aber unter 50, die mit schwerbehinderten Personen gleichgestellt sind, aber keinen Ausweis erhalten. ${ }^{4}$ Damit ergibt sich derzeit eine Zahl von 9,6 Mio. behinderter Menschen in Deutschland (Bundesministerium für Arbeit und Soziales (BMAS) 2011), das entspricht einem Bevölkerungsanteil von $11,7 \%$. Weiterhin besteht eine Dunkelziffer all derjenigen, die keinen Schwerbehindertenausweis beantragt haben und keine sozialen Leistungen beziehen (vgl.

${ }^{2}$ Es besteht die Möglichkeit des Eintrags mehrerer Merkzeichen für eine Person.

3 Die gesundheitlichen Voraussetzungen für die Befreiung von der Rundfunkgebührenpflicht sind erfüllt bei blinden oder nicht nur vorübergehend wesentlich sehbehinderten Menschen mit einem GdB von wenigstens 60 allein wegen der Sehbehinderung, hörgeschädigten Menschen sowie behinderten Menschen mit einem GdB von wenigstens 80, die wegen ihres Leidens an öffentlichen Veranstaltungen ständig nicht teilnehmen können.

${ }^{4}$ Vgl. SGB IX § 2 Abs. 3 i. d. F. v. 19.6.2001. 
Neumann 2006). ${ }^{5}$ Etwa die Hälfte der schwerbehinderten Menschen lässt sich der Gruppe der älteren Menschen (ab 65 Jahre) zurechnen (Statistisches Bundesamt 2009).

Der Anteil der im weiteren Sinne mobilitätseingeschränkten Menschen an der deutschen Gesamtbevölkerung kann derzeit mit mindestens $30 \%$ angenommen werden; alleine der Anteil der Kinder und älteren Menschen beträgt fast $26 \%$ (Statistisches Bundesamt 2012). Aufgrund der prognostizierten starken Veränderungen der Altersstruktur in den kommenden Jahrzehnten ist zu erwarten, dass der Anteil der mobilitätseingeschränkten Menschen (und auch der mobilitätsbehinderten Menschen) weiter ansteigen wird (vgl. Abbildung 8) (Statistisches Bundesamt 2009).

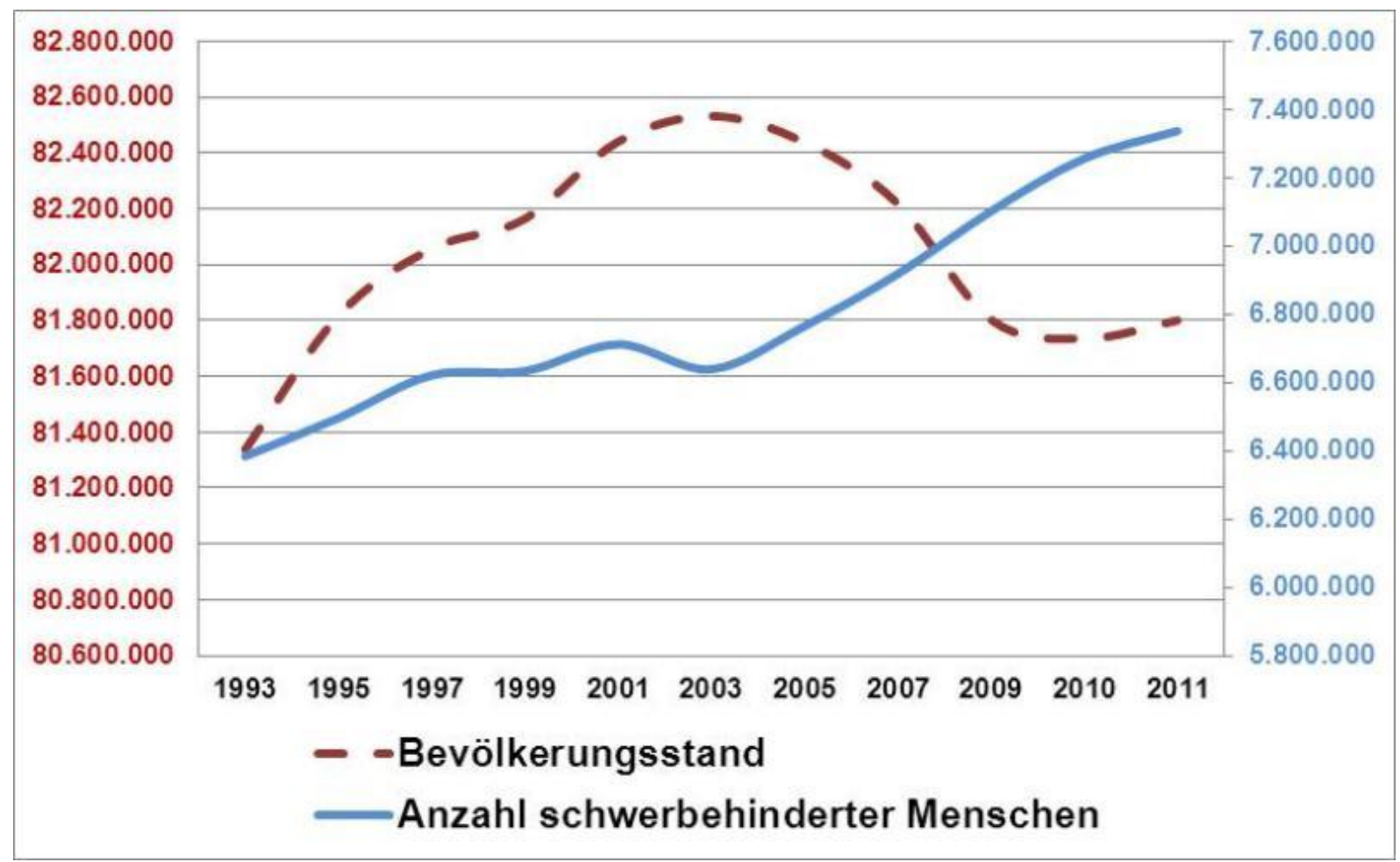

Abbildung 8: Anzahl schwerbehinderter Menschen und Bevölkerungsentwicklung in Deutschland im Vergleich [Zahlen: Statistisches Bundesamt und BMAS (Bevölkerung 2011 vorläufig)]

Insbesondere durch den Zuwachs bei älteren und damit auch hochbetagten Menschen mit alterstypischen Einschränkungen ist zu erwarten, dass gerade die Zahl der Menschen mit Sehbehinderungen und Gehbehinderungen deutlich zunehmen wird (vgl. z. B. (Pro Retina Deutschland e. V. 2008) und (Abbildung 9)). Damit erhöht sich auch der dringende Bedarf, Verkehrsräume an die Anforderungen mobilitätsbehinderter Menschen anzupassen.

${ }^{5}$ Bei einer Behinderung besteht in Deutschland keine Meldepflicht und der Antrag auf Ausstellung eines Schwerbehindertenausweises ist freiwillig (vgl. SGB IX § 69 Abs. 5 i. d. F. v. 19.6.2001). 


\section{Gehbehinderungen, Sehbeeinträchtigungen und Medikamenteneinnahme nehmen im Alter zu}

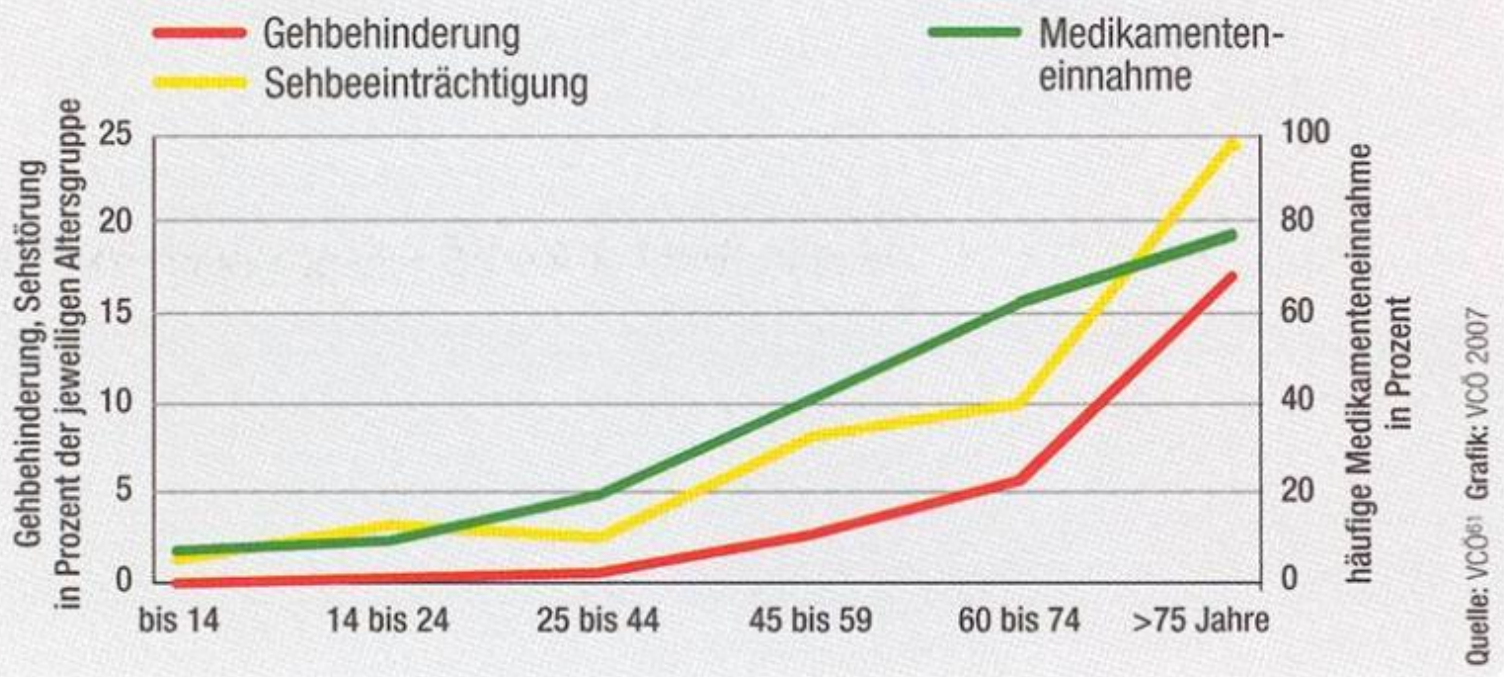

Abbildung 9: Zunahme von Mobilitätseinschränkungen und Medikamenteneinnahme im Alter [Dangschat et. al. 2007]

Durch die Analyse der Straßenverkehrsunfälle älterer Menschen und Kinder als Hauptaufgabe des Forschungsvorhabens erfolgte bereits eine Verkehrssicherheitsbetrachtung eines großen Teils schwächerer Verkehrsteilnehmer. In diesem Arbeitspaket lag der Fokus speziell auf der Betrachtung der Menschen mit einer Mobilitätsbehinderung und hier insbesondere auf den Gruppen der Personen mit einer Sehschädigung oder einer Gehbehinderung einschl. Rollstuhlnutzern. Für diese Gruppen sind aufgrund der körperlichen und sensorischen Einschränkungen bei der Überquerung von Straßen besondere Schutzziele zu formulieren.

\subsubsection{Anforderungen mobilitätsbehinderter Menschen an Überquerungsstellen}

Im Folgenden werden die Anforderungen ausgesuchter Gruppen mobilitätsbehinderter Menschen an die Gestaltung von Knotenpunkten erläutert. Dabei beschränkt sich die Zusammenfassung auf die Gruppen von Menschen mit Gehbehinderung und Menschen mit Sehbehinderung, welche auch zahlenmäßig die größte Bedeutung haben.

\subsubsection{Rollstuhlnutzer}

Grundsätzlich ist zwischen mit Muskelkraft betriebenen Rollstühlen (Greifreifen- oder Schieberollstühle) sowie Elektro-Rollstühle zu unterscheiden. Erste gelten als Fußgänger im Sinne der StVO. Letztere gelten als Fahrzeuge im Sinne der StVZO. Die Spannbreite der Leistungsfähigkeit und der Fortbewegungsgeschwindigkeit ist daher bei den Rollstuhlnutzern individuell sehr verschieden und nicht zuletzt abhängig von der Antriebsart. Die Fortbewegungsgeschwindigkeit hat z. B. Bedeutung bei der Überquerung von Fahrbahnen. Sie kann bei Rollstühlen mit Muskelkraftantrieb (Greifreifen) durchschnittlich mit etwa 1,0 m/s 
angenommen werden. Bei Elektro-Rollstühlen, die für die Fahrt auf Fußgänger- oder Radverkehrsflächen zugelassen sind, gibt es vier verschiedene Geschwindigkeitsklassen: $6 \mathrm{~km} / \mathrm{h}, 10 \mathrm{~km} / \mathrm{h}, 12 \mathrm{~km} / \mathrm{h}$ und $15 \mathrm{~km} / \mathrm{h}$ (MEYRA-ORTOPEDIA Vertriebsgesellschaft $\mathrm{mbH}$ 2005). $)^{6}$. Aufgrund der geringen Körperhöhe (Sitzposition) besteht die Gefahr, dass Rollstuhlbenutzer, die die Fahrbahn überqueren möchten, für herannahende Kraftfahrer nicht gut erkennbar sind (vgl. wie bei Kindern).

Rollstuhlnutzer können Schwierigkeiten beim Überwinden niedriger Kanten und Schwellen haben (z. B. niedrige Bordsteinkante), wodurch das zügige Verlassen der Fahrbahn nach dem Überqueren erschwert werden kann. Insbesondere bei manuell bedienten Rollstühlen besteht durch die ungünstige Verlagerung der Schwerlinie die Gefahr des Kippens nach hinten. Das Überwinden von Bordkanten o. Ä. erfordert daher einige Übung bezüglich der Gewichtsverlagerung und des Kraftaufwands.

\subsubsection{Rollatornutzer und gehbehinderte Menschen}

Die Anforderungen gehbehinderter Menschen ergeben sich aus Schwierigkeiten beim Gehen und Unsicherheiten beim Stehen. Ein großer Teil der gehbehinderten Menschen nutzt daher Hilfsmittel. Dazu zählen neben den bereits genannten Rollstühlen insbesondere Rollatoren, Gehstöcke, sowie weniger verbreitet Unterarm- und Achselstützen und Vierfußgehhilfen.

Gerade Rollatoren finden seit einigen Jahren als Hilfsmittel immer mehr Verbreitung. Allein die Krankenkassen übernehmen die Versorgung von jährlich 500 Tsd. Versicherten mit diesen Gehhilfen (Brust 2011). Dazu kommen zahlreiche Verkäufe über weitere Quellen, z. B. Internetverkäufe und auch Apotheken und Discounter usw., die nicht statistisch erfasst werden. Viele Personen, die ständig auf Rollatoren angewiesen sind, haben Gleichgewichtsstörungen, eingeschränkte Balancefähigkeit und/oder verminderte physische Kräfte. Viele Rollatornutzer haben bereits große Schwierigkeiten, Bordsteinkanten von lediglich $3 \mathrm{~cm}$ zu überwinden (Bundesarbeitsgemeinschaft SELBSTHILFE von Menschen mit Behinderung und chronischer Erkrankung und ihren Angehörigen e. V 2006). Es gibt inzwischen auch Rollatoren am Markt, die kleinere Kanten mit geringem Kraftaufwand überwinden können. Diese Hilfsmittel kosten jedoch ein Vielfaches des von den Krankenkassen erstatteten Kostenanteils. Sie sind daher für eine Vielzahl von potenziellen Nutzern nicht erschwinglich. Daher hat die Qualität der Mobilität von Rollatornutzern auch eine gesellschaftlich-soziale Komponente.

Gehbehinderte Menschen haben gegenüber Menschen ohne diese Einschränkungen häufig eine stark verminderte Gehgeschwindigkeit. Diese kann auf bis zu 0,6 m/s absinken (vgl. z. B. König 2007). Aus diesen beiden Handicaps (Stufenüberwindung und Gehgeschwindigkeit) ergeben sich besondere Probleme bei der Überquerung von Fahrbahnen:

- An einer signalisierten Fußgängerüberquerung können die Räumzeiten für die Anforderungen dieser Gruppe zu kurz sein. An unsignalisierten Überquerungsstellen

\footnotetext{
${ }^{6}$ Elektro-Rollstühle können sogar eine Höchstgeschwindigkeit von $45 \mathrm{~km} / \mathrm{h}$ erreichen. E-Rollstühle dieser Bauart müssen allerdings die Fahrbahn benutzen. Bei E-Rollstühlen, die als Fußgänger im Sinne der StVO zu verstehen sind, ist die Höchstgeschwindigkeit auf $15 \mathrm{~km} / \mathrm{h}$ begrenzt.
} 
besteht die Gefahr, dass Menschen mit Gehbehinderungen die Fahrbahn nicht schnell genug verlassen können bzw. den Zeitbedarf zum Überschreiten der Fahrbahn unterschätzen und somit in Konfliktsituationen mit dem Fahrverkehr in Längsrichtung geraten.

Gleichfalls ergeben sich für viele Rollatornutzer Schwierigkeiten bei der Überwindung von Bordsteinkanten. Daher bevorzugen insbesondere Rollatornutzer niveaugleiche Verkehrsflächen. Mit diesem Punkt stehen ihre Anforderungen vor allem im Gegensatz zu den Anforderungen von blinden und sehbehinderten Menschen, die eine eindeutig erkennbare (ertastbare oder sichtbare) Trennung der Geh- und Fahrbahnbereiche fordert. Teilweise lassen sich Strategien für die einfachere Bewältigung von Bordsteinkanten o. Ä. trainieren. Derartige Sicherheits- und Mobilitätstrainings werden z. B. von sozialen Diensten oder Verkehrsunternehmen durchgeführt (Eickler 2012 und VDV 2012). Eine Strategie kann z. B. sein, dass sich die Person mit dem Rollator auf der Fahrbahn dreht, um dann rückwärts auf den Gehweg zu gelangen. Dadurch ergeben sich allerdings u. U. Sicherheitsrisiken, weil die Fahrbahn nicht schnell genug verlassen werden kann.

\subsubsection{Sehgeschädigte Menschen}

Eine Sehschädigung gehört zu den sensorischen Behinderungen. Sie kann erhebliche Einschränkungen für die Qualität der Mobilität nach sich ziehen und hat einen hohen Einfluss auf die Sicherheit bei der Fortbewegung im Straßenverkehrsraum. Sehschädigung umfasst alle Sehbehinderungen in unterschiedlicher Ausprägung bis zur Blindheit mit dem vollständigen Verlust der Sehfähigkeit. Der Mensch nimmt den überwiegenden Teil der Informationen aus seiner Umwelt über die Augen auf. Menschen mit Sehschädigungen müssen diese Informationsaufnahme teilweise oder ganz kompensieren. Da mit steigendem Alter in der Regel die Sehkraft nachlässt und die Wahrscheinlichkeit für bestimmte Augenerkrankungen zunimmt, sind insbesondere ältere Menschen von Sehschädigungen betroffen.

\subsection{Blindheit}

Unter Blindheit versteht man in Deutschland nach der gesetzlichen Definition eine Sehschärfe (einen Visus) von höchstens 0,02 oder eine Einschränkung des Gesichtsfeldes auf $5 \mathrm{Grad}$ und weniger, jeweils bezogen auf das bessere und voll korrigierte Auge. Das bedeutet, dass die Sehleistung eines der Definition nach blinden Menschen $2 \%$ oder weniger der Sehkraft eines normal Sehenden beträgt. Ein Sehrest von $2 \%$ kann bedeuten, dass ein Mensch einen Gegenstand, den ein normal Sehender bereits in $100 \mathrm{~m}$ Entfernung erkennen würde, erst in $2 \mathrm{~m}$ Entfernung erkennt. Es kann aber auch bedeuten, dass sich ein „Tunnelblick“ mit lediglich $2 \%$ des Gesichtsfeldes ergibt. Nach der gesetzlichen Definition können blinde Menschen theoretisch eine Restsehkraft besitzen. Diese beschränkt sich allerdings maximal auf eine Hell-/ Dunkelwahrnehmung.

Blinde Menschen nutzen zur Kompensation des fehlenden Sehsinns den Geruchs- und Tastsinn sowie das Gehör. Blinde Menschen nutzen bei der Fortbewegung im öffentlichen Raum einen Langstock oder auch einen Führhund, der sie bei der Orientierung unterstützt. Der Langstock dient dabei überwiegend der frühzeitigen Erkennung von Hindernissen, kann aber auch beim Ertasten von Leit- und Orientierungselementen. In den letzten Jahren gab es 
einen Trend zu größeren Stockspitzen (Rollspitzen aus Kunststoff). Diese erfordern insbesondere bei taktilen Strukturen eine stärkere Rückmeldung, als das bei den früher verwendeten Metallspitzen der Fall war. Allerdings tragen die neuen Rollspitzen zu einer einfacheren und weniger ermüdenden Handhabung bei und werden daher bevorzugt. Führhunde helfen ebenfalls dabei, die selbstständige Mobilität zu verbessern. Sie sind z. B. auf das Erkennen von Bordsteinkanten trainiert, so dass sie den blinden Menschen vor dem Betreten der Fahrbahn warnen können.

\subsection{Sehbehinderung}

Per Definition ist zwischen „sehbehinderten“ und „hochgradig sehbehinderten“ Menschen zu unterscheiden. Ein Mensch gilt nach deutschem Recht als sehbehindert, wenn er auf dem besseren Auge selbst mit Brille oder Kontaktlinse nicht mehr als $30 \%$ von dem sieht, was ein Mensch mit voller Sehkraft sieht (s. o.). Hochgradig sehbehinderte Menschen besitzen der Definition nach eine Restsehkraft von höchstens 5 \% (Visus höchstens 0,05).

Sehbehinderte Menschen können niedrige Hindernisse oder Höhenunterschiede schlecht erkennen, wenn sie nicht optisch hervorgehoben sind (z. B. niedrige Bordsteinkanten). Zudem haben sie Schwierigkeiten, Informationen zu erfassen, die kleinflächig dargestellt sind sowie aufgrund ihres eingeschränkten Visus Schwierigkeiten bei der Beobachtung des fließenden Verkehrs, z. B. an Überquerungsstellen.

Sehbehinderte Menschen sind - auch wenn es zunächst überraschend erscheint - in besonderem Maße auf optische Informationen und Reize angewiesen, um ihre Restsehkraft nutzen und etwas von ihrer Umgebung erkennen zu können. Solche Reize werden z. B. durch Farbe und Leuchtdichte (Helligkeit) eines Objektes im Kontrast zum Hintergrund sowie durch seine Größe und Entfernung bewirkt. Damit sehbehinderte Menschen Objekte erkennen können, müssen sie demnach kontrastreich gestaltet und ausreichend groß sein. Mit steigendem Grad der Sehbehinderung spielt die akustische Orientierung eine steigende Rolle.

\subsection{Anforderungen blinder und sehbehinderter Menschen im Verkehrsraum}

Für blinde und stark sehbehinderte Menschen ist eine sicher ertastbare Bordsteinkante zur Abtrennung der Gehflächen von der Fahrbahn essentiell. Diese ist ein eindeutiges Erkennungssignal für die Grenze zwischen Gehfläche und Fahrbahn. Damit konkurrieren ihre Anforderungen mit denen der gehbehinderten Menschen, die niveaugleiche Flächen bevorzugen. Seit Jahren wird in vielen deutschen Kommunen der Bordstein daher auf eine Kompromisshöhe von $3 \mathrm{~cm}$ eingebaut. ${ }^{7}$ Der Zielkonflikt verschärft sich aufgrund der demografischen Entwicklung jedoch, da es immer mehr sehbehinderte und immer mehr gehbehinderte Menschen (vor allem Rollatornutzer) gibt.

Blinde und sehbehinderte Menschen orientieren sich bevorzugt in einem orthogonalen Wegesystem. Bietet die Umwelt keine Struktur zur Orientierung und wird von einem

\footnotetext{
7 Insbesondere in Norddeutschland werden auch geringere Bordsteinhöhen eingesetzt. Eine umfassende Evaluation über die Auswirkungen auf die Sicherheit der Mobilität blinder und sehbehinderter Menschen gibt es jedoch nicht.
} 
orthogonalen System abgewichen (z. B. im Kreisverkehr), dann sind Orientierungshilfen erforderlich.

Blinde und stark sehbehinderte Menschen können ohne taktile oder akustische Signale nur über kurze Strecken auf der gewünschten Gehlinie bleiben. Mit steigender Entfernung und ohne ausreichende Orientierungshilfen nimmt die Abweichung mit steigender Entfernung vom Ausgangspunkt immer weiter zu (vgl. dazu auch Böhringer 2007). An Überquerungsstellen sind daher Orientierungshilfen erforderlich. Dabei kann es sich um taktile Orientierungshilfen (z. B: eine Bordsteinkante parallel zur Fahrbahnachse der zu überquerenden Straße) oder um akustische Hilfen handeln.

Insbesondere für blinde und stark sehbehinderte Menschen spielt aus Gründen der objektiven und subjektiven Sicherheit vor allem die Zuverlässigkeit geschlossener Orientierungs- und Mobilitätsketten eine Rolle (Grossmann et. al. 2008). Eine Vereinheitlichung von Orientierungshilfen im öffentlichen Raum im Sinne einer Standardisierung fördert und verbessert die Mobilität dabei wesentlich.

\subsubsection{Kompensationsmöglichkeiten durch barrierefreie Gestaltung}

Ausgehend vom Benachteiligungsverbot des Grundgesetzes wurde mit Erlass des Behindertengleichstellungsgesetzes (BGG) des Bundes das Ziel verfolgt, behinderten Menschen eine selbstbestimmte und gleichberechtigte Teilhabe am Leben in der Gesellschaft zu ermöglichen. Dies soll in den Bereichen von Bau und Verkehr durch die Herstellung einer möglichst weitreichenden Barrierefreiheit erfolgen. Der öffentliche Raum soll gemäß der Zielvorgabe des BGG für behinderte Menschen „in der allgemein üblichen Weise ohne besondere Erschwernisse und grundsätzlich ohne fremde Hilfe zugänglich und nutzbar" werden (§ 4 BGG) (Deutscher Bundestag 2002). Diese Forderung schließt die Verkehrssicherheit natürlich mit ein. Die Konkretisierung dieser Zielvorgabe erfolgt in Fachgesetzen, technischen Regelwerken, Programmen und Zielvereinbarungen. Im Zuständigkeitsbereich der Bundesländer sind inzwischen überwiegend gleichlautende oder ähnliche Zielvorgaben durch Behindertengleichstellungsgesetze auf Länderebene getroffen worden.

Mobilitätseingeschränkte und vor allem behinderte Menschen sind dabei mehr als jede andere Gruppe auf lückenlose Mobilitätsketten angewiesen. Durch eine geeignete Gestaltung der Infrastruktur im Sinne der gesetzlich beschriebenen, weitreichenden Barrierefreiheit können die Schwierigkeiten von Menschen mit Mobilitätseinschränkungen in Teilen kompensiert und kann die Verkehrssicherheit für alle Menschen damit erhöht werden.

\subsubsection{Unfälle mobilitätsbehinderter Menschen an Knotenpunkten}

Über Unfälle behinderter Menschen im Straßenverkehr ist bisher sehr wenig bekannt. Unfälle dieser Personengruppen werden z. B. in der veröffentlichten Unfallstatistik nicht ausgewiesen. Das über Straßenverkehrsunfälle von Menschen mit Behinderung wenig bekannt ist, dafür können folgende Thesen eine Erklärung liefern:

- Die Größe einzelner Gruppen mobilitätsbehinderter Menschen ist zahlenmäßig gering, so dass es sich im Vergleich zur Summe aller Straßenverkehrsunfälle bei Unfällen mit mobilitätsbehinderten Personen um eher seltene Ereignisse handelt. 
Dadurch wird seitens der Verkehrssicherheitsarbeit kein Handlungsbedarf gesehen, Unfälle mit dem Merkmal „Behinderung“ zu erfassen (vgl. z. B. Sonderauswertung „Kinderunfälle“).

- Mobilitätsbehinderte Menschen sind deutlich weniger mobil, als Menschen ohne Mobilitätseinschränkungen. Sie nehmen daher seltener als aktive Verkehrsteilnehmer am Straßenverkehr teil (das gilt z. B. vor allem für Menschen mit geistiger Behinderung, die oftmals von speziellen Fahrdiensten transportiert werden). Daraus resultiert eine überdurchschnittlich geringe Beteiligung an Unfällen.

- Mobilitätsbehinderte Personen vermeiden Wege, die von innen subjektiv als gefährlich eingeschätzt werden und nehmen dafür Umwege in Kauf. Die eigene, vorbeugende Unfallprävention durch das Verkehrsverhalten kann bei mobilitätsbehinderten Menschen demnach als sehr hoch ausgeprägt bezeichnet werden. Die Häufigkeit von Unfällen wird dadurch stark reduziert.

- Die Erfassung des Merkmals „Behinderung“ gilt als diskriminierend gegenüber den Betroffenen und verstößt gegen gesetzlich verbriefte Grundsätze der Gleichbehandlung (z. B. Grundgesetz, UN-Konvention über die Rechte von Menschen mit Behinderungen).

Die unterschiedlichen Thesen werden nachfolgend detaillierter analysiert:

\subsubsection{These 1: Zahlenmäßige Bedeutung der Gruppen mobilitätseingeschränkter Menschen}

Die Schätzungen über die Anzahl von Menschen in Deutschland, die dauerhaft oder temporär einen Rollstuhl zur Fortbewegung nutzen, schwankt zwischen 900 Tsd. (Berufsgenossenschaft für Gesundheitsdienst und Wohlfahrtspflege - BGW 2009) und 1,56 Mio. (VdK Medien- und Anzeigenverwaltung GmbH 2011).

Genaue Zahlen über die Anzahl der Personen mit Gehbehinderungen liegen nicht vor. Die Anzahl lässt sich anhand der Menschen mit gültigen Schwerbehindertenausweisen, welche mit einem anerkannten Grad der Behinderung von mindestens 50 über das Kennzeichen "gehbehindert“ oder „außergewöhnlich gehbehindert“ verfügen, auf etwa 4,25 Mio. Menschen schätzen (BMAS 2012, Statistisches Bundesamt 2009) und vgl. Tabelle 1.

Die Anzahl der Menschen in Deutschland, die blind sind oder von einer Sehbehinderung betroffen sind, kann ebenfalls nicht genau beziffert werden. Das Bundesministerium für Arbeit und Soziales gibt die Anzahl der blinden Menschen in Deutschland zum Stichtag 31.12.2011 mit 100.384 an (BMAS 2012). Die Zahl der Menschen mit einer hochgradigen Sehbehinderung liegt nach Angaben des Statistischen Bundesamtes bei ca. 51.000, etwa 220.000 Personen in Deutschland haben eine sonstige Sehbehinderung (Statistisches Bundesamt 2009). Damit sind etwa 370.000 Menschen in Deutschland statistisch erfasst, die blind oder sehbehindert sind (Personen mit einem amtlichen Schwerbehindertenausweis der einen Grad der Behinderung von mindestens 50 aufführt). In der Statistik sind allerdings nicht alle Personen mit einer Sehschädigung erfasst, da der Antrag auf einen Schwerbehindertenausweis vom Betroffenen freiwillig erfolgen kann und nicht obligatorisch ist. 
Nach Angaben des DBSV leben nach Schätzwerten auf Basis alter Zahlen aus der ehemaligen DDR sowie der World Health Organization (WHO) in Deutschland sogar 1,2 Mio. Menschen mit einer Sehschädigung, etwa mindestens 150 Tsd. davon sind blind (DBSV 2011). Viele ältere Menschen erreichen mit ihrer Sehbehinderung nicht die gesetzlich definierten Grenzwerte, haben aber dennoch Schwierigkeiten bei der Wahrnehmung optischer Informationen. Bei vielen Augenkrankheiten gilt die Dunkelziffer der Erkrankten aufgrund des schleichenden Krankheitsverlaufes als besonders hoch. Pro Retina nennt sogar eine Zahl von 3,5 Millionen Menschen in Deutschland, die an der Altersabhängigen Makuladegeneration (AMD) leiden (Pro Retina Deutschland e. V. 2008). AMD ist bereits heute die häufigste Ursache für schwere Seheinschränkungen und tritt aufgrund der steigenden Lebenserwartung immer häufiger auf.

Insgesamt sind demnach bereits diese drei Gruppen mobilitätsbehinderter Menschen (Rollstuhlnutzer, gehbehinderte und sehgeschädigte Menschen) zahlenmäßig durchaus relevant. In der Summe sind etwa 5,5 Mio. Menschen in Deutschland statistisch gesichert einer dieser drei Gruppen zuzuordnen (6,7 \%). Dennoch bestehen keinerlei Hinweise, dass eine dieser Gruppen auffällig oft an Straßenverkehrsunfällen beteiligt ist.

\subsubsection{These 2: Mobilität von Menschen mit Mobilitätseinschränkungen}

Bei der Erhebung „Mobilität in Deutschland“ im Jahr 2008 wurde erstmals auch gezielt nach gesundheitlichen Einschränkungen und ihren Auswirkungen auf die Mobilität gefragt. Die Erhebung beschränkte sich auf die Unterscheidung nach "Gehbehinderung“, "Sehbehinderung“ und „Anderer gesundheitlicher Einschränkung“ (Infas Institut für angewandte Sozialwissenschaften $\mathrm{GmbH}$ 2010). Im Ergebnis zeigte sich, dass Menschen mit gesundheitlichen Einschränkungen (am Stichtag der Befragung) häufiger nicht mobil waren als Menschen ohne Einschränkungen (Tabelle 2). Durchschnittlich legen gesundheitlich beeinträchtigte Menschen auch weniger Wege zurück und verbringen weniger Zeit im Verkehr. Vor allem die täglich zurückgelegte Wegstrecke bleibt mit einer Differenz von $38 \%$ unter der Tagesstrecke gesunder Menschen. 
Tabelle 2: Mobilitätskenngrößen von Personen ab 60 Jahren mit und ohne gesundheitlicher Einschränkungen (MiD 2008)

\begin{tabular}{|c|c|c|c|}
\hline & \multicolumn{2}{|c|}{ Gesundheitliche Einschränkungen } \\
\hline & & mit & ohne \\
\hline Anteil mobiler Personen & $\%$ & 78 & 87 \\
\hline Wege pro Person und Tag & Anzahl & 2,7 & 3,4 \\
\hline Tagesstrecke & $\mathrm{km}$ & 18 & 29 \\
\hline Unterwegszeit (ohne rbW) ${ }^{8}$ & $\min$ & 65 & 82 \\
\hline \multicolumn{4}{|l|}{ Modal Split } \\
\hline zu Fuß & \multirow{5}{*}{$\%$} & 33 & 33 \\
\hline Fahrrad & & 8 & 11 \\
\hline MIV-Mitfahrer & & 12 & 10 \\
\hline MIV-Fahrer & & 39 & 40 \\
\hline Öffentlicher Verkehr & & 9 & 7 \\
\hline
\end{tabular}

Personen ab 60 Jahren;

ohne Stellvertreterinterviews

Demnach kann es für Gruppen mobilitätseingeschränkter Menschen sein, dass sie aufgrund ihrer im Allgemeinen geringeren Verkehrsleistung auch seltener an Verkehrsunfällen beteiligt sind. Diese These kann allerdings nicht weiter verifiziert werden, da keine genauen Zahlen über die Unfallbeteiligung behinderter Menschen vorliegen.

\subsubsection{These 3: Verringerung des Risikopotenzials durch Wegewahl}

Mobilitätseingeschränkte Menschen bevorzugen - je nach Schwere der Auswirkungen der Einschränkung auf die Mobilität - häufig oder immer Wege, die sie als subjektiv sicher empfinden. Dafür nehmen sie auch gewisse Umwege in Kauf. Z. B. bevorzugen blinde Menschen im Zuge der Überquerung von Hauptverkehrsstraßen eine Nutzung von lichtsignalgeregelten Übergängen, statt an Fußgängerüberwegen oder gar unsignalisierten Stellen die Fahrbahn zu überqueren. Letztendlich entscheiden die als individuelle Barrieren empfundenen Hindernisse die Wegewahl, Diese Feststellungen basieren allerdings auf persönlichen Mitteilungen Betroffener. Es fehlen allerdings bisher differenzierte Verkehrsdaten z. B. über die Wegewahl für unterschiedliche Behinderungsgruppen (Step by Step 2009). Kompensationsstrategien sind aber z. B. auch bei der Wegewahl älterer PkwFahrer bekannt.

\subsubsection{These 4: Vermeidung der Diskriminierung}

Durch den Paradigmenwechsel der letzten Jahre steht nicht mehr die Behinderung im Fokus, sondern der Mensch. Dies drückt sich u. a. darin aus, dass z. B. nicht mehr von

\footnotetext{
${ }^{8} \mathrm{rbW}=$ regelmäßig berufliche Wege.
} 
„Sehbehinderten“ gesprochen wird, sondern von „Menschen mit Sehbehinderung“. Eine Funktionseinschränkung rückt in den Hintergrund.

Die Unterscheidung und Kategorisierung von Beteiligten an einem Straßenverkehrsunfall aufgrund des Merkmals einer Behinderung ist gesellschaftspolitisch im Sinne einer Gleichbehandlung nicht gewünscht und würde gegen Gleichstellungsgesetze verstoßen (vergleichbar dem Sachverhalt, wenn die Polizei bei der Unfallaufnahme nach der ethnischen Herkunft fragen würde).

Die Erfassung eines Merkmals „Behinderung“ ist folgerichtig auch nicht nach dem Gesetz über die Statistik der Straßenverkehrsunfälle (Deutscher Bundestag 1990) vorgesehen und dieses Merkmal wird daher nicht in der Verkehrsunfallanzeige erfasst.

\subsubsection{Quellen für die Unfallaufnahme bei Unfällen mit Beteiligung behinderter Menschen}

Als Ansatzpunkt für die Ermittlung von Unfallzahlen mobilitätsbehinderter Menschen kommen unterschiedliche Quellen in Betracht:

- Unfallaufnahme durch die Polizei und amtliche Statistik,

- Statistiken der Behindertenverbände,

- Berufsgenossenschaften als Träger von Einrichtungen für behinderte Menschen,

- Gesetzliche Unfallversicherung,

- Krankenkassen bzw. Krankenhäuser,

- $\quad$ weitere Quellen, z. B. Untersuchungen zur Thematik.

Im Folgenden werden die einzelnen potenziellen Quellen analysiert.

\subsubsection{Polizeiliche Unfallaufnahme}

Die Sicherheit eines Verkehrsraums kann sich u. a. in der Anzahl und Schwere der Unfälle an einem Knotenpunkt oder auf einem Streckenabschnitt widerspiegeln. Unfälle im Straßenverkehr in Deutschland werden - sofern die Polizei Kenntnis von einem Unfall erlangt - von den örtlichen Polizeidienststellen aufgenommen. Von dort werden sie an die Statistischen Landesämter und dann an das Statistische Bundesamt gemeldet. Zumindest bei den Unfällen der Kategorien 1 bis 2 (Unfall mit mindestens einem getöteten oder schwerverletzten Verkehrsteilnehmer) sowie Unfällen mit schwerem Sachschaden kann von einer nahezu vollständigen Erfassung der Verkehrsunfälle ausgegangen werden. Bei Unfällen der Kategorie 3 (Unfall mit mindestens einem leichtverletzten Verkehrsteilnehmer) oder leichten Sachschäden kann davon nicht mehr ausgegangen werden. ${ }^{9}$ Gerade bei Unfällen mit Fußgängern, zu denen gemäß StVO z. B. auch Rollstuhlnutzer zählen, und Radfahrern, wird eine hohe Dunkelziffer vermutet (Hautzinger 1993).

9 Vgl. in den Jahresberichten zur Unfallentwicklung des Statistischen Bundesamts, Abschnitt „Allgemeine und methodische Erläuterungen zur Straßenverkehrsunfallstatistik“. 
Versucht man, Straßenverkehrsunfälle mit Beteiligung mobilitätsbehinderter Personen aus der polizeilichen Statistik zu extrahieren, scheitert man somit an der unzureichenden Kennzeichnung relevanter Datensätze. Über die Häufigkeit von Unfällen mobilitätsbehinderter Menschen im Straßenverkehr oder speziell an Knotenpunkten liegen daher in der amtlichen Statistik (Bundes- und Landesstatistik) keine auswertbaren Daten vor.

Üblicherweise erfasst die Polizei bei der Unfallaufnahme über die Verkehrsunfallanzeige eine Reihe von Merkmalen, die eine detaillierte Unfallanalyse im Hinblick auf bestimmte Personen- oder Altersgruppen, Unfalltyp und Unfallursachen oder auch zum Unfallort ermöglichen. Diese Merkmale werden auf Landes- oder Bundesebene weiter verarbeitet. Das derzeitige Gesetz über die Statistik der Straßenverkehrsunfälle (StVUnfStatG) sieht die Erfassung eines Merkmals „Behinderung“ nicht vor. Behinderte Menschen werden zunächst als Fußgänger (oder ggf. Rad- oder Kraftfahrer) erfasst. Ist eine Behinderung eines der Unfallbeteiligten augenscheinlich erkennbar, besteht die übliche Praxis darin, das Merkmal einer Behinderung als Freitext unter „sonstige geistige oder körperliche Mängel“ aufzunehmen. Eine gezielte Abfrage eines Merkmals „Behinderung“ wird nicht vorgenommen (vgl. auch Abschnitt 3.2.4.4).

In seltenen Fällen (z. B. Berlin) werden Hinweise über ein Merkmal einer Behinderung der Unfallbeteiligten notiert (z. B. Rollstuhlbenutzer). Der Vermerk erfolgt in einem Sonderfeld der Unfalldatenbank (z. B. „Beteiligtenbemerkung“ in Berlin). Es erfolgt allerdings keine systematische Erfassung solcher Merkmale, da dafür auch keine Pflicht besteht. Somit ist keine umfassende Auswertung über die polizeilichen Datenbanken der Verkehrsunfälle möglich (Zimmermann 2008).

Als einziges Merkmal in der amtlichen Unfallstatistik, das auf eine Beteiligung eines Menschen mit Behinderung an einem Straßenverkehrsunfall hinweist, werden unter der Schlüsselnummer 59 Krankenfahrstühle erfasst. Ein motorisierter Krankenfahrstuhl ist ein nach der Bauart zum Gebrauch durch behinderte Personen bestimmtes Kraftfahrzeug (GNP Arbeitskreis Fahreignung 2007). Es handelt sich um einsitzige, elektrobetriebene Fahrzeuge mit einer zulässigen Höchstgeschwindigkeit von $15 \mathrm{~km} / \mathrm{h}$ Für das Führen eines Krankenfahrstuhls besteht keine Fahrerlaubnispflicht. Ein motorisierter Krankenfahrstuhl darf im Straßenverkehr auch dort gefahren werden, wo Fußgänger gehen dürfen, jedoch nur mit Schrittgeschwindigkeit (§24 Abs. 2 StVO).

Die Schlüsselzuweisung in der amtlichen Unfallstatistik geschieht zusammengefasst mit einer Reihe weiterer Fahrzeuge (z. B. Feuerwehr, Straßenreinigung) in der Kategorie „Übriges Kraftfahrzeug“. Somit lässt sich bei einer Auswertung dieser Schlüsselnummer der reine Anteil der Krankenfahrstühle an der Unfallbeteiligung nur über die Schlüsselnummer nicht filtern. Da im Rahmen dieser Untersuchungen die Unfälle zu Fuß gehender, mobilitätseingeschränkter Personen an Knotenpunkten untersucht werden, werden Krankenfahrstühle im weiteren nicht weiter betrachtet.

\subsubsection{Untersuchungen der Gesetzlichen Unfallversicherung}

In einer Untersuchung, die sich dem Thema „Schulwegunfälle“ widmete, konnte die Relevanz der Datenbanken der gesetzlichen Unfallversicherung neben der amtlichen Statistik für die Unfallanalyse bei Straßenverkehrsunfällen nachgewiesen werden 
(Langescheid 2005). Die von der Polizei erfassten Unfälle stellen demnach nicht zwangsläufig die Gesamtheit aller Unfälle im Straßenverkehr für bestimmte Gruppen dar. Der Verband Deutsche Gesetzliche Unfallversicherung (DGUV) ist der Spitzenverband der gewerblichen Berufsgenossenschaften und der öffentlichen Hand. Beim DGUV bzw. seinen Mitgliedsverbänden werden alle Unfälle der Versicherten, die im Zusammenhang mit dem Weg zur Arbeit stehen, erfasst. Eine zentrale Datenbankabfrage mit dem Merkmal "Behinderung" ist beim DGUV allerdings nicht möglich, da auch in der Statistik der Unfallversicherungsträger das Merkmal „Behinderung“ nicht explizit erfasst wird (Wilhelm 2009) (vgl. auch Abschnitt 3.2.4.4).

Mit der Lebenssituation behinderter Menschen befasst sich insbesondere die Berufsgenossenschaft für Gesundheitsdienst und Wohlfahrtspflege (BGW). Üblicherweise finden keine Analysen der Unfälle von Menschen mit Behinderungen statt. Im Rahmen einer Studie zur Verbesserung der Präventionsarbeit wurden die Unfälle behinderter Menschen im Zusammenhang mit ihrer Beschäftigung (Arbeitsunfälle und Unfälle auf dem Weg zur Arbeit) detailliert untersucht (Wilhelm 2009). Anlass für die Untersuchung war der Umstand, dass die „BGW in Branchen, in denen Menschen mit Behinderungen stark vertreten sind, [...] ein hohes Unfallgeschehen“ verzeichnet. Dies gilt einerseits für Arbeitsunfälle, aber auch für Unfälle auf arbeitsbedingten Wegen. Im Rahmen der Untersuchung konnten in mühevoller Handarbeit (Akteneinsicht) etwa 4.500 registrierte Unfälle behinderter Menschen ausgewertet werden. 1.884 davon standen im unmittelbaren Zusammenhang mit Mobilität. Da es sich um eine Untersuchung im Schwerpunktbereich der Rehabilitationsmaßnahmen für Menschen mit Behinderung handelte, spielten allerdings auch Unfälle beim Transport durch die Fahrdienste eine große Rolle (also Unfälle, bei denen behinderte Menschen nicht aktiv am Verkehrsgeschehen teilnahmen).

Für die Präventionsarbeit zur Vermeidung von Unfällen behinderter Menschen stehen bisher Fragestellungen nach dem Unfallhergang und der Unfallschwere im Vordergrund. Weitere Details zu den Unfällen, z. B. zum Unfallort, spielen keine Rolle. Die Untersuchung stellt fest, dass Unfälle mobilitätsbehinderter Menschen im öffentlichen Raum nicht detailliert hinsichtlich des Unfallortes erfasst werden und somit nicht im Detail zu analysieren sind. Eine Ableitung von Empfehlungen für die Straßenraumgestaltung kann daher aus den Ergebnissen dieser Untersuchung nicht getroffen werden.

\subsubsection{Pressemitteilungen der Polizei}

Aus den Vorgaben aus den Landespressegesetzen sind die Polizeibehörden dazu verpflichtet, den Medien und damit der Öffentlichkeit Informationen aus ihrer Tätigkeit zur Verfügung zu stellen, soweit keine Sicherheitsinteressen berührt werden (z. B. § 4 Abs. 1 Landespressegesetz NRW).

Die jeweiligen Stellen für Presse- und Öffentlichkeitsarbeit der Polizeibehörden geben laufend aktuelle Pressemeldungen zu verschiedenen Themenbereichen heraus, die über die jeweiligen Presseportale verfügbar sind. Über besondere Nachrichtennetzwerke für Presseinformationen werden diese zusätzlich den Medienvertretern und der Öffentlichkeit zugänglich gemacht. 
Die Pressemitteilungen werden bei den jeweiligen Polizeidienststellen archiviert, es besteht jedoch keine Möglichkeit, eine Schlagwortsuche durchzuführen. ${ }^{10}$ Allerdings besteht bei den Nachrichtennetzwerken die Möglichkeit, in den Online-Datenbanken gezielt nach Schlagwörtern in den Pressemitteilungen zu suchen (z. B. „Rollstuhl“, „Unfall“).

Die Pressemeldungen spiegeln jedoch nicht das vollständige Unfallaufkommen wider, da nicht jeder Unfall per Pressemeldung veröffentlicht wird. Eine auf Basis dieser Quellen erhobene Statistik wird daher eher lückenhaft sein. Zudem kann diese Vorgehensweise zunächst nur dazu dienen, Unfälle mit Beteiligung von Menschen mit Behinderung zu identifizieren. Eine detaillierte Auswertung, um Unfallursachen zu ermitteln und daraus Empfehlungen für die Gestaltung des Straßenraums abzuleiten, erfordert wiederum eine Akteneinsicht bei der zuständigen Polizeidienststelle.

\subsubsection{Unfälle mobilitätsbehinderter Menschen - eine Stichprobenauswertung}

Aus der Unfallanalyse für Berlin war bekannt, dass Polizeidienststellen $u$. U. eine hausinterne Statistik über die Unfälle mit Beteiligung von Menschen mit Mobilitätsbehinderungen führen. Aus diesem Wissen wurde der Versuch einer Stichprobenerhebung bei ausgewählten Polizeibehörden durchgeführt. Diese diente dem Zweck, weitere für eine Analyse verwertbare Unfall-Datensätze mit Beteiligten mit dem Merkmal „Behinderung“ ausfindig zu machen.

Die Befragung zielte dabei auf zwei Stadttypen:

- Großstädte, da hier bei einer hohen Unfallzahl auch die Wahrscheinlichkeit von Unfällen von Menschen mit einer Mobilitätsbehinderung größer wäre,

- Städte mit Rehabilitations- oder Bildungseinrichtungen für Menschen mit Behinderungen; es galt die Hypothese: je höher die Zahl mobiler mobilitätsbehinderter Menschen, desto höher die Anzahl der Unfällen mit deren Beteiligung. ${ }^{11}$

Trotz des deutlich höheren Anteils von Menschen mit Behinderungen in den ausgewählten Städten war den zuständigen Polizeidienststellen kein besonderes Unfallaufkommen mit Beteiligung dieser Personengruppen bekannt. Daher wurde bei den Dienststellen keine Erhebung mit dem Merkmal „Behinderung“ durchgeführt (Tabelle 3).

\footnotetext{
${ }^{10}$ Exemplarisch erhoben für Nordrhein-Westfalen.

${ }^{11}$ Die Auswahl fokussierte sich entsprechend der Aufgabenstellung auf Einrichtungen für blinde, sehbehinderte und körperbehinderte Menschen.
} 
Tabelle 3: Städte mit Einrichtungen für Menschen mit Behinderungen und polizeiliche Unfalldatenerfassung mit dem Merkmal „Behinderung“

\begin{tabular}{|l|l|l|}
\hline Stadt & Einrichtung & $\begin{array}{l}\text { Unfalldaten mit } \\
\text { Merkmal ,Behinderung“ }\end{array}$ \\
\hline $\begin{array}{l}\text { Marburg an der } \\
\text { Lahn }\end{array}$ & $\begin{array}{l}\text { Blindenstudienanstalt (Blinde und } \\
\text { sehbehinderte Menschen) }\end{array}$ & Keine Erfassung \\
\hline $\begin{array}{l}\text { Wetter an der } \\
\text { Ruhr }\end{array}$ & $\begin{array}{l}\text { Evangelische Stiftung Volmarstein } \\
\text { (körperbehinderte Menschen) }\end{array}$ & Keine Erfassung \\
\hline Düren & $\begin{array}{l}\text { Berufsförderungswerk Düren } \\
\text { (Rehabilitationseinrichtung für blinde und } \\
\text { sehbehinderte Menschen) }\end{array}$ & Keine Erfassung \\
\hline
\end{tabular}

Auch stichprobenhafte Interviews mit Bediensteten der Träger der Einrichtungen für die Menschen mit Behinderungen zeigten auf Nachfrage keine Kenntnis von Besonderheiten bei Unfällen mit Beteiligung dieser Personengruppen. Ein Orientierungs- und Mobilitätstrainer der Deutschen Blindenstudienanstalt äußerte jedoch, häufiger kritische Situationen bei blinden oder sehbehinderten Menschen zu beobachten, wenn diese z. B. eine Straße überqueren müssten. Dass sich diese Konflikte nicht in der Unfallstatistik niederschlagen, wurde u. a. damit erklärt, dass aufgrund des hohen Anteils von blinden und sehbehinderten Menschen im Verkehrsraum die Kraftfahrer und anderen Verkehrsteilnehmer sensibilisiert sind und mehr Rücksicht auf schwächere Verkehrsteilnehmer nehmen.

\subsubsection{Auswertung von Unfällen mit Beteiligung behinderter Menschen - Beispiel Berlin}

Eine statistische Erfassung der Unfälle mit Beteiligung von Menschen mit Behinderung durch die Polizei ist somit nur aus Berlin bekannt. Dort werden die Unfälle mit Beteiligung mobilitätsbehinderter Menschen als Sondermerkmal in der Rubrik "Beteiligten-Bemerkung“ (Zusatzfeld) erfasst. Eine Weiterleitung dieser Bemerkung für die elektronische Unfallauswertung (Euska) findet nicht statt, da dieses Merkmal keinen nativen Bestandteil der Verkehrsunfallanzeige darstellt und daher ein entsprechendes Feld in Euska ebenfalls nicht vorhanden ist. Somit kann die Unfallauswertung auf Basis der Excel-Tabellen nur „zu Fuß" erfolgen.

Aus Berlin wurden für die Jahre 2004 bis 2010 (7 Jahre) insgesamt 63 Unfälle übermittelt, an denen mindestens ein Mensch mit einer Mobilitätsbehinderung beteiligt war. Davon ließen sich 46 Verkehrsunfälle dem Unfallort „Knotenpunkt“ zuordnen. Der überwiegende Teil (44 Unfälle) fand jeweils mit Beteiligung eines Rollstuhlnutzers statt, davon in 14 Fällen eines elektrisch angetriebenen Rollstuhls. Jeweils lediglich ein Unfall war jeweils mit Beteiligung eines sehbehinderten Menschen bzw. eines Rollatornutzers.

Bei zwölf Unfällen (26 \%) wurde von der Polizei der mobilitätseingeschränkte Unfallbeteiligte als Verursacher des Unfalls festgelegt. In der überwiegenden Zahl der Fälle (74 \%) waren die mobilitätseingeschränkten Verkehrsteilnehmer allerdings Unfallopfer. 
Fast Dreiviertel der Unfälle fanden im Zusammenhang mit Abbiegen oder Einbiegen/Kreuzen statt (Abbildung 10).

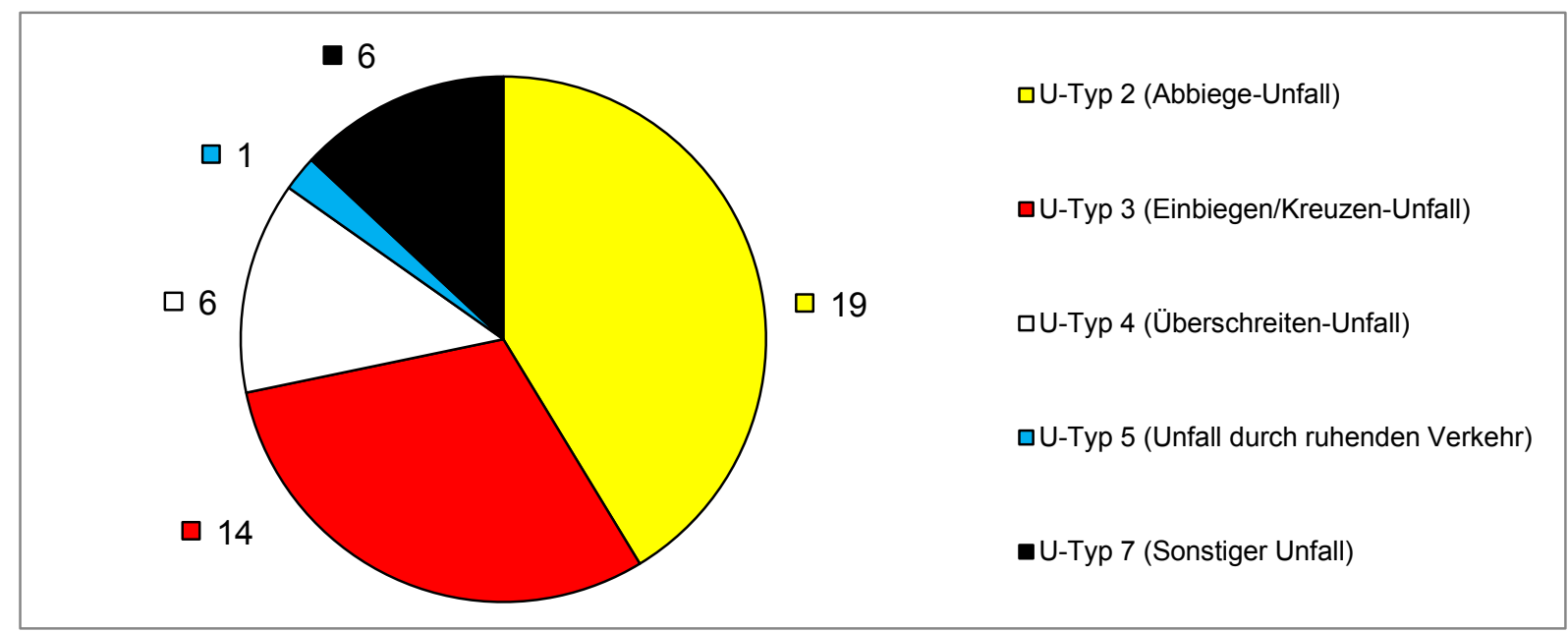

Abbildung 10: Unfälle mit Beteiligung mobilitätsbehinderter Menschen - Unfalltypen (Auswertung Berlin, 2004 bis 2010)

In der Unfallbeschreibung (lag nicht für alle Unfälle vor) wurde in insgesamt 10 Fällen bei Unfällen mit Beteiligung von Rollstuhlnutzern erwähnt, dass der Kraftfahrer den Rollstuhlnutzer beim Abbiegevorgang übersehen hätte. Die Sichtverhältnisse spielen demnach eine zentrale Rolle bei Unfällen mit Beteiligung von Rollstuhlnutzern.

Weiterhin lassen sich aus den vorhandenen Beschreibungen zum Unfallhergang Hinweise auf eine dringende Notwendigkeit einer an die Anforderungen mobilitätseingeschränkter bzw. behinderter Menschen angepassten Infrastruktur ableiten. In fünf Fällen nutzten Rollstuhlnutzer eine Verkehrsfläche nicht in der vorgeschriebenen Weise (Tabelle 4). Fünfmal davon wurde eine Radverkehrsfläche befahren, weil im Zuge der Radwegverbindung der Bordstein abgesenkt ist. Es wird deutlich, dass ein abgesenkter Bordstein für Rollstuhlnutzer (und auch für Rollatornutzer) eine große Bedeutung bei der sicheren Fortbewegung im Verkehrsraum hat. Allerdings stehen dem Wunsch nach abgesenkten Bordbereichen auch die Sicherheitsinteressen blinder und sehbehinderter Menschen entgegen (s. Abschnitt 3.2.2.3). 
Tabelle 4: Unfälle im Zusammenhang mit der nicht bestimmungsgemäßen Nutzung von Verkehrsflächen durch Rollstuhlnutzer

\begin{tabular}{|c|c|c|}
\hline Nr. & $\begin{array}{l}\text { Genutzte } \\
\text { Verkehrsfläche }\end{array}$ & Unfallhergang (Auszug) \\
\hline 1 & Radweg & $\begin{array}{l}\text { "[...]. Ord.-Nr. } 02 \text { nutzte den auf der südlichen Fahrbahnseite befindlichen } \\
\text { Radweg, da dort der Bordstein abgesenkt ist. [...]" }\end{array}$ \\
\hline 2 & Schutzstreifen & $\begin{array}{l}\text { „[...] übersah dabei den von links kommenden Rollstuhl-fahrer, (02), } \\
\text { welcher auf dem Radschutzstreifen }[\ldots] \text { fuhr. [...]" }\end{array}$ \\
\hline 3 & Radweg & $\begin{array}{l}\text { „[...] nutzte nur an der o. a. Örtlichkeit den Fahrradweg um die }[\ldots] \text { zu } \\
\text { überqueren. }[\ldots] \text { ". }\end{array}$ \\
\hline 4 & Radweg & $\begin{array}{l}\text { „[...]übersah er die aus nordwestlicher Richtung, den Fahrradweg } \\
\text { befahrende Rollstuhlfahrerin 02. [...]" }\end{array}$ \\
\hline 6 & Fahrbahn & $\begin{array}{l}\text { „[...] am rechten Fahrbahnrand stehenden Rollstuhlfahrerin (02) [...] } 02 \text { gab } \\
\text { an, dass sie mit ihrem Rollstuhl die [...] in falscher Richtung befuhr, um im } \\
\text { dortigen Kreuzungsbereich den abgesenkten Bordstein zu benutzen, um } \\
\text { auf den Gehweg zu gelangen. }[\ldots] \text {. }\end{array}$ \\
\hline
\end{tabular}

\subsubsection{Zusammenfassung: Unfalluntersuchungen mobilitätsbehinderter Menschen}

Bisher gibt es in Deutschland aber auch weltweit keinerlei Untersuchungen darüber, welche Defizite an Knotenpunkten zu Unfällen mobilitätsbehinderter Menschen führen könnten. Ansätze finden sich im Bereich der Präventionsarbeit bzw. Rehabilitation. Allerdings werden die Unfälle dort unter anderen Aspekten erfasst. Somit gibt es auch dort keine Datensätze, die sich einfach nach dem Merkmal „Behinderung“ auswerten lassen bzw. eine genaue Zuordnung an Knotenpunkten scheitert an der Qualität der Datensätze (anderer Schwerpunkt bei der Erfassung).

Die Unfälle mobilitätsbehinderter Menschen in Deutschland werden derzeit (2012) von den Polizeidienststellen nicht systematisch erfasst und tauchen daher auch nicht in den Statistiken der Länder oder des Bundes auf. Eine seltene Ausnahme bildet die Stadt Berlin. Dort wird von der Polizei erfasst, ob an einem Unfall eine Person mit Rollstuhl, Rollator oder Langstock beteiligt war. Von einer vollständigen Erfassung kann allerdings nicht ausgegangen werden. Der Anteil dieser Unfälle an der gesamten Anzahl der Unfälle in Berlin ist zudem sehr gering. In Städten, in denen Einrichtungen für behinderte Menschen vorhanden sind, lässt sich kein besonders auffälliges Unfallbild erkennen.

Die Auswertung der Berliner Unfälle zeigt eine besonders hohe Beteiligung von Rollstuhlnutzern. Langstock oder Rollator spielen praktisch keine Rolle. Fehlt an einem Knotenpunkt eine Absenkung auf Fahrbahnniveau, kann es passieren, dass Rollstuhlnutzer auf Radverkehrsanlagen oder die Fahrbahn ausweichen. Dort kommt es immer wieder zu Konflikten mit abbiegendem Kfz-Verkehr. 


\section{Makroskopische Unfallanalyse}

Im Folgenden Kapitel werden häufige (typische) Unfallsituationen von Kindern und Älteren an Knotenpunkten auf der Grundlage elektronischer Unfalldatenbestände ermittelt. Für mobilitätsbehinderte Menschen ließen sich anhand der vorliegenden Unfalldaten keine weitreichenden Aussagen hinsichtlich des Unfallgeschehens ermitteln. Neben der geringen Anzahl von Menschen, die aufgrund ihrer Hilfsmittel bei der Unfallaufnahme für die Polizei vor Ort als Mensch mit Behinderung zu identifizieren sind, enthält die derzeitige Form der Verkehrsunfallanzeige kein Feld für das Merkmal „Behinderung“ für die weitergehende statistische Auswertung. Wird bei einem der Unfallbeteiligten eine Behinderung erkannt, wird diese i. d. R. als „geistiger oder körperlicher Mangel“ unter Bemerkungen als Freitextfeld erfasst.

\subsection{Datengrundlage}

Grundlage der makroskopischen Unfalluntersuchung sind die elektronischen Datenbestände zu Unfällen mit Personenschaden und zu schwerwiegenden Unfällen mit Sachschaden (Unfallkategorien 1 bis 4$)^{12}$ aus fünf Bundesländern an inner- und außerörtlichen Knotenpunkten. Unfälle an Knotenpunkten auf Bundesautobahnen sind nicht Bestandteil der Untersuchung. Die Tabelle 5 listet die fünf Bundesländer auf und gibt einen Überblick über die Zeiträume der zugrunde liegenden Unfalldaten.

Tabelle 5: Übersicht der Bundesländer und der Zeiträume zu den vorliegenden elektronischen Unfalldaten

\begin{tabular}{|l|l|c|}
\hline Bundesland & Betrachtungszeitraum & Jahre \\
\hline Berlin & $01.01 .2006-31.12 .2008$ & 3 \\
\hline Nordrhein-Westfalen & $01.01 .2004-31.12 .2008$ & 5 \\
\hline Rheinland-Pfalz & $01.01 .2004-31.07 .2009$ & 5,5 \\
\hline Sachsen & $01.01 .2004-31.12 .2007$ & 4 \\
\hline Sachsen-Anhalt & $01.01 .2006-31.12 .2008$ & 3 \\
\hline
\end{tabular}

Erfasst wurden in den aufgeführten Zeiträumen insgesamt 697.386 Unfälle außerhalb von Bundesautobahnen (Tabelle 6). Die darin enthaltenen 351.351 Unfälle an Knotenpunkten zeigt Tabelle 7. Die weiteren Analysen konzentrieren sich auf die Unfälle an Knotenpunkten.

12 Vgl. Merkblatt zur Örtlichen Unfalluntersuchung in Unfallkommissionen, FGSV (Hrsg.): Unfallkategorie 1: Unfälle mit Getöteten (U(GT): mindestens ein getöteter Verkehrsteilnehmer). Unfallkategorie 2: Unfälle mit Schwerverletzten (U(SV): mindestens ein schwerverletzter Verkehrsteilnehmer, aber keine Getöteten). Unfallkategorie 3: Unfälle mit Leichtverletzten (U(LV): mindestens ein leichtverletzter Verkehrsteilnehmer, aber keine Getöteten und keine Schwerverletzten). Unfallkategorie 4: Schwerwiegende Unfälle mit Sachschaden (U(SS): Unfälle mit Sachschaden und Straftatbestand oder Ordnungswidrigkeits-Anzeige (unfallursächlich), bei denen mindestens ein Kraftfahrzeug nicht mehr fahrbereit ist (abschleppen). S. 8, Ausgabe 2012 
Tabelle 6: Unfälle außerhalb von Bundesautobahnen

\begin{tabular}{|c|c|c|c|c|c|c|}
\hline Bundesland & Jahre & U-Kat 1 & U-Kat 2 & U-Kat 3 & U-Kat 4 & Summen \\
\hline Berlin & 3 & 182 & 5.308 & 38.240 & 3.602 & 47.332 \\
\hline Nordrhein-Westfalen & 5 & 3.191 & 60.980 & 249.013 & 92.664 & 405.848 \\
\hline Rheinland-Pfalz & 5,5 & 1.154 & 18.346 & 66.557 & 37.163 & 123.220 \\
\hline Sachsen & 4 & 962 & 15.783 & 43.971 & 23.215 & 83.931 \\
\hline Sachsen-Anhalt & 3 & 506 & 6.341 & 21.380 & 8.828 & 37.055 \\
\hline \multicolumn{2}{|c|}{ Summen } & 5.995 & 106.758 & 419.161 & 165.472 & 697.386 \\
\hline
\end{tabular}

Tabelle 7: Unfälle an Knotenpunkten außerhalb von Bundesautobahnen

\begin{tabular}{|l|c|r|r|r|r|r|}
\hline Bundesland & Jahre & \multicolumn{1}{|c|}{ U-Kat 1 } & U-Kat 2 & U-Kat 3 & U-Kat 4 & Summen \\
\hline Berlin & 3 & 93 & 3.068 & 23.555 & 2.175 & 28.891 \\
\hline Nordrhein-Westfalen & 5 & 1.134 & 27.531 & 129.003 & 59.928 & 217.596 \\
\hline Rheinland-Pfalz & 5,5 & 192 & 5.591 & 26.127 & 16.071 & 47.981 \\
\hline Sachsen & 4 & 236 & 5.829 & 21.218 & 13.177 & 40.460 \\
\hline Sachsen-Anhalt & 3 & 115 & 2.221 & 9.426 & 4.661 & 16.423 \\
\hline \multicolumn{2}{|c|}{ Summen } & 1.770 & 44.240 & 209.329 & 96.012 & 351.351 \\
\hline
\end{tabular}

\subsection{Unfalltypen von Unfällen an Knotenpunkten}

Die rund 350.000 Unfälle an Knotenpunkten verteilen sich zu etwa $81 \%$ auf Bereiche innerorts und zu etwa $19 \%$ auf Bereiche außerorts (ohne Autobahnen) (Abbildung 11). Dabei nehmen Abbiege-Unfälle (Unfalltyp 2) und Einbiegen/Kreuzen-Unfälle (Unfalltyp 3) erwartungsgemäß den größten Anteil bei der Betrachtung von Unfällen an Knotenpunkten, unter Berücksichtigung aller Arten der Verkehrsbeteiligung, ein (Abbildung 12 und Abbildung 13). Die drei häufigsten Unfälle an Knotenpunkten

1. Einbiegen/Kreuzen-Unfälle (44 bzw.48 \%),

2. Abbiege-Unfälle ( 25 bzw. $27 \%)$ und

3. Unfälle im Längsverkehr (13 bzw. $12 \%)$

umfassen innerorts und außerorts 82 bzw. 87 \% aller Unfälle an Knotenpunkten.

Die Verteilung der einzelnen Unfalltypen unterscheidet sich nur geringfügig zwischen dem außerörtlichen und dem innerörtlichen Bereich. Lediglich ist der Unfalltyp 4 (ÜberschreitenUnfall) in innerörtlichen Bereichen anteilig häufiger vertreten als in den außerörtlichen Bereichen. 


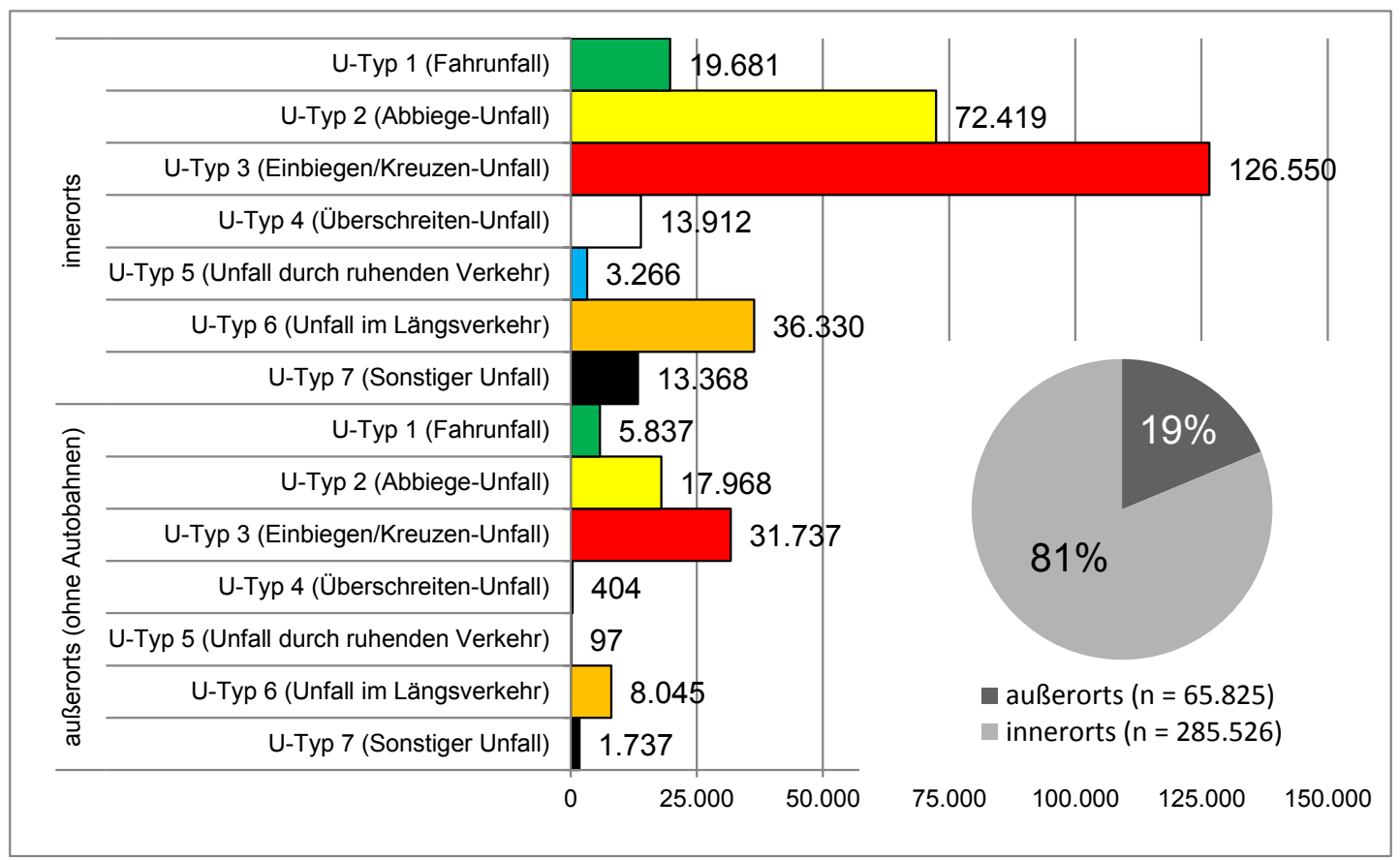

Abbildung 11: Häufigkeit von Unfällen an Knotenpunkten nach Ortslage und Unfalltyp ( $\mathrm{n}=$ 351.351 Unfälle)

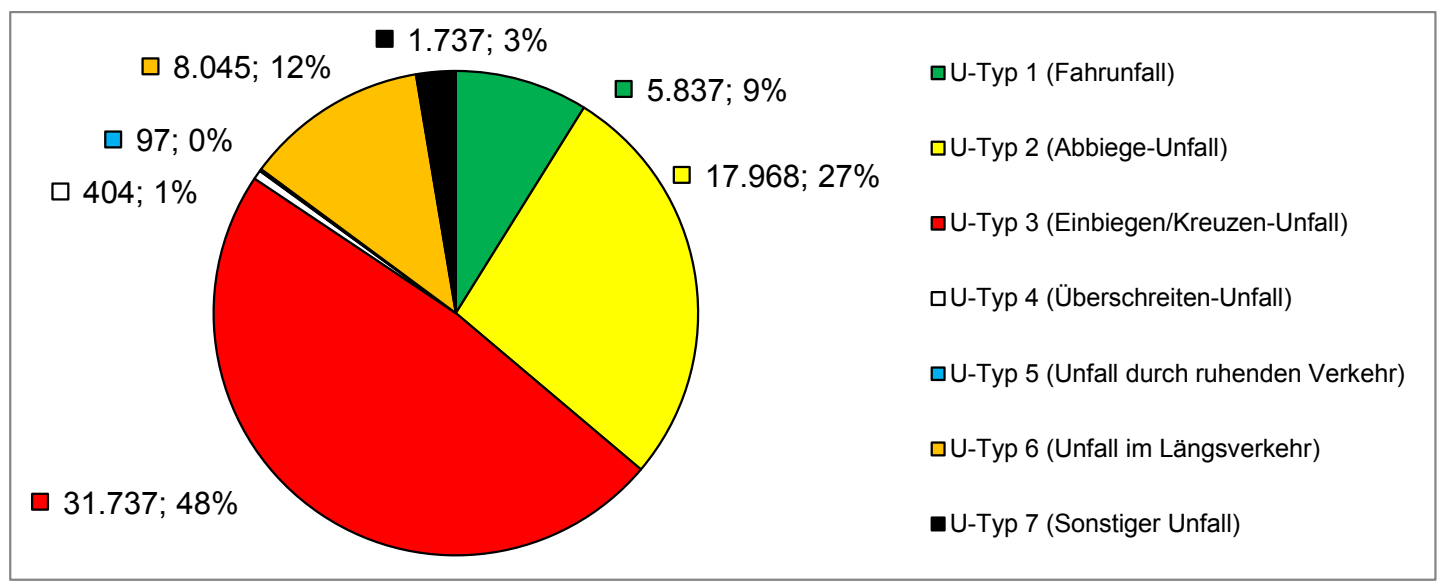

Abbildung 12: Verteilung der Unfalltypen von Unfällen an Knotenpunkten, außerorts, ohne Autobahnen ( $n=65.825$ Unfälle)

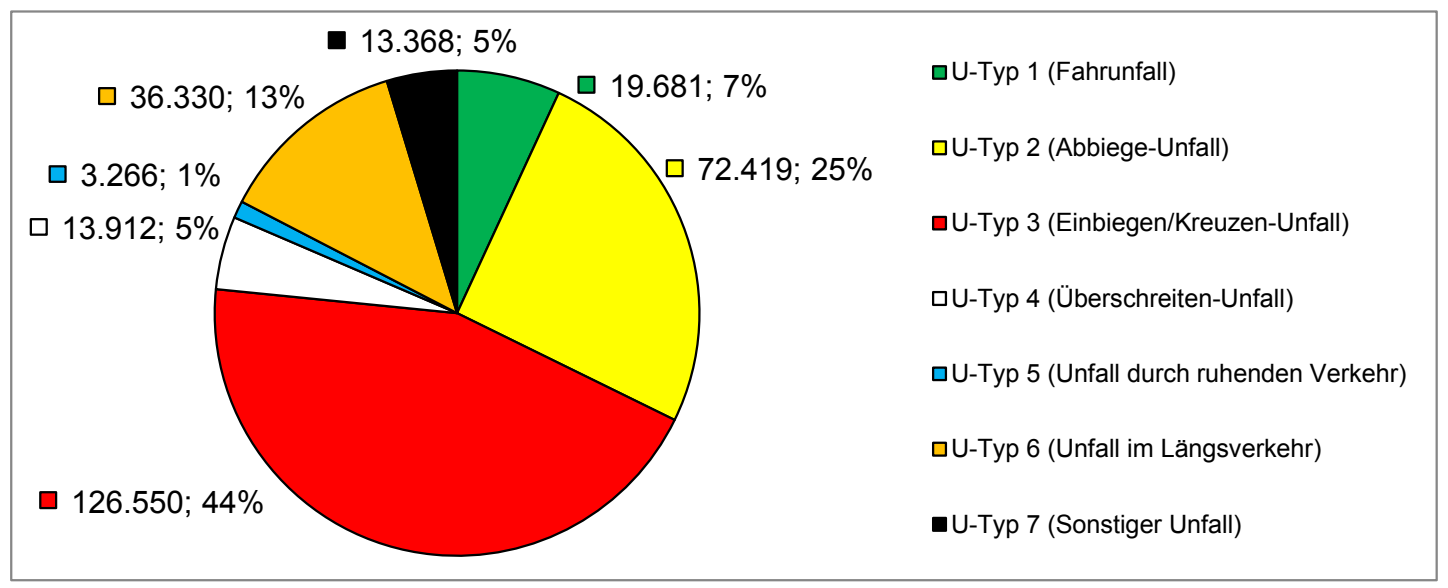

Abbildung 13: Verteilung der Unfalltypen von Unfällen an Knotenpunkten, innerorts ( $n=$ 285.526 Unfälle) 
Abbildung 14 führt im Weiteren die absoluten Zahlen für Unfälle mit Beteiligung von Kindern und Unfällen mit Beteiligung von Älteren hinsichtlich der Unfalltypen auf. Während die Unfälle mit Beteiligung von Älteren qualitativ im Wesentlichen der Verteilung aller Unfälle auf die Unfalltypen entsprechen, konzentrieren sich die Unfälle mit Beteiligung von Kindern vornehmlich auf Abbiege-Unfälle (Typ 2), Einbiegen/Kreuzen-Unfälle (Typ 3) und Überschreiten-Unfälle (Typ 4). Die anteilige Verteilung nach Altersgruppen verdeutlicht zudem, dass Kinder insbesondere an Überschreiten-Unfällen beteiligt sind (Abbildung 15).

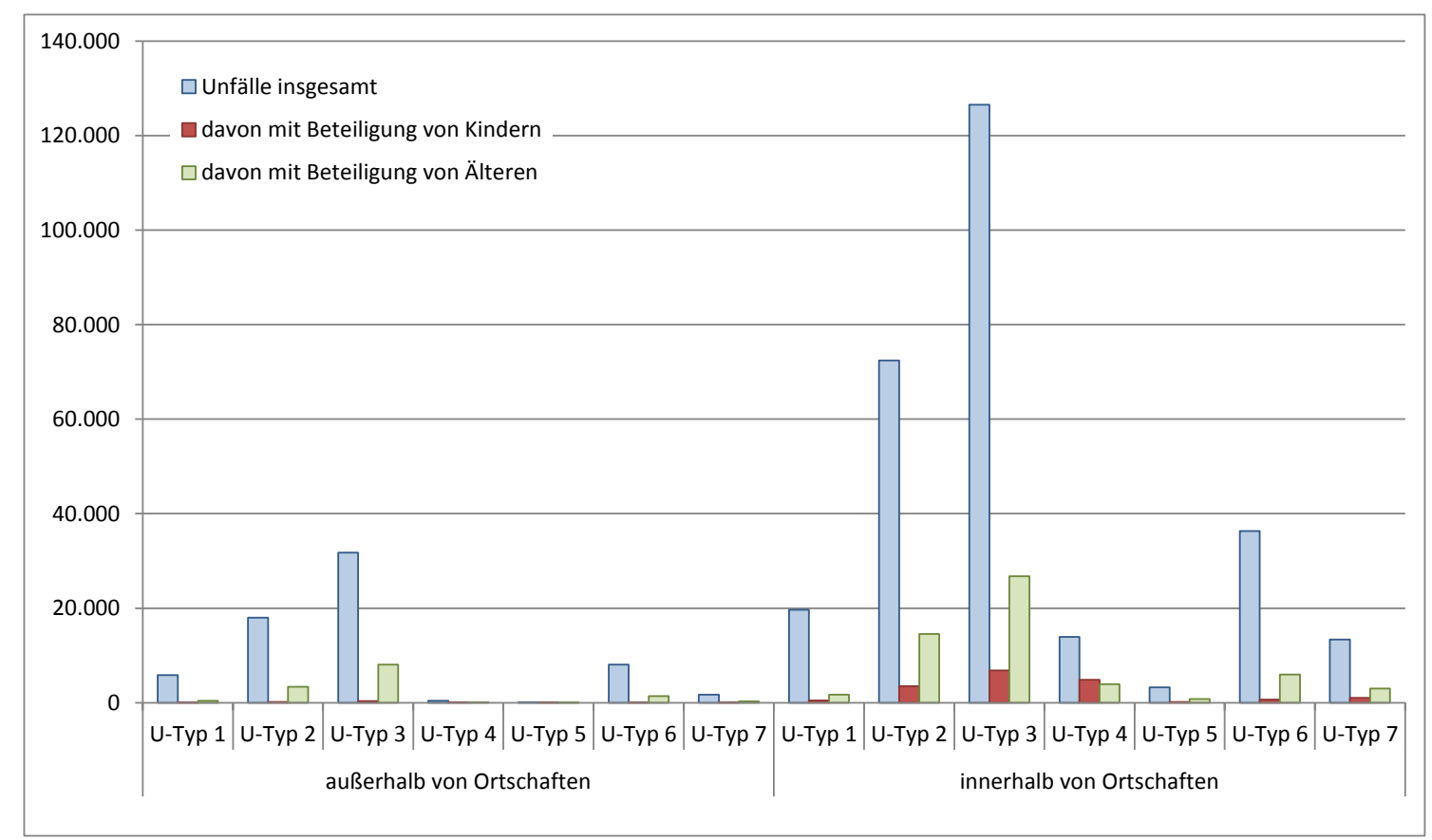

Abbildung 14: Unfällen mit Personenschaden und schwerwiegende Unfälle mit Sachschaden an Knotenpunkten innerorts $\left(n_{\text {ges }}=351.351\right)$, einschließlich der Unfälle mit Beteiligung von Kindern und Älteren

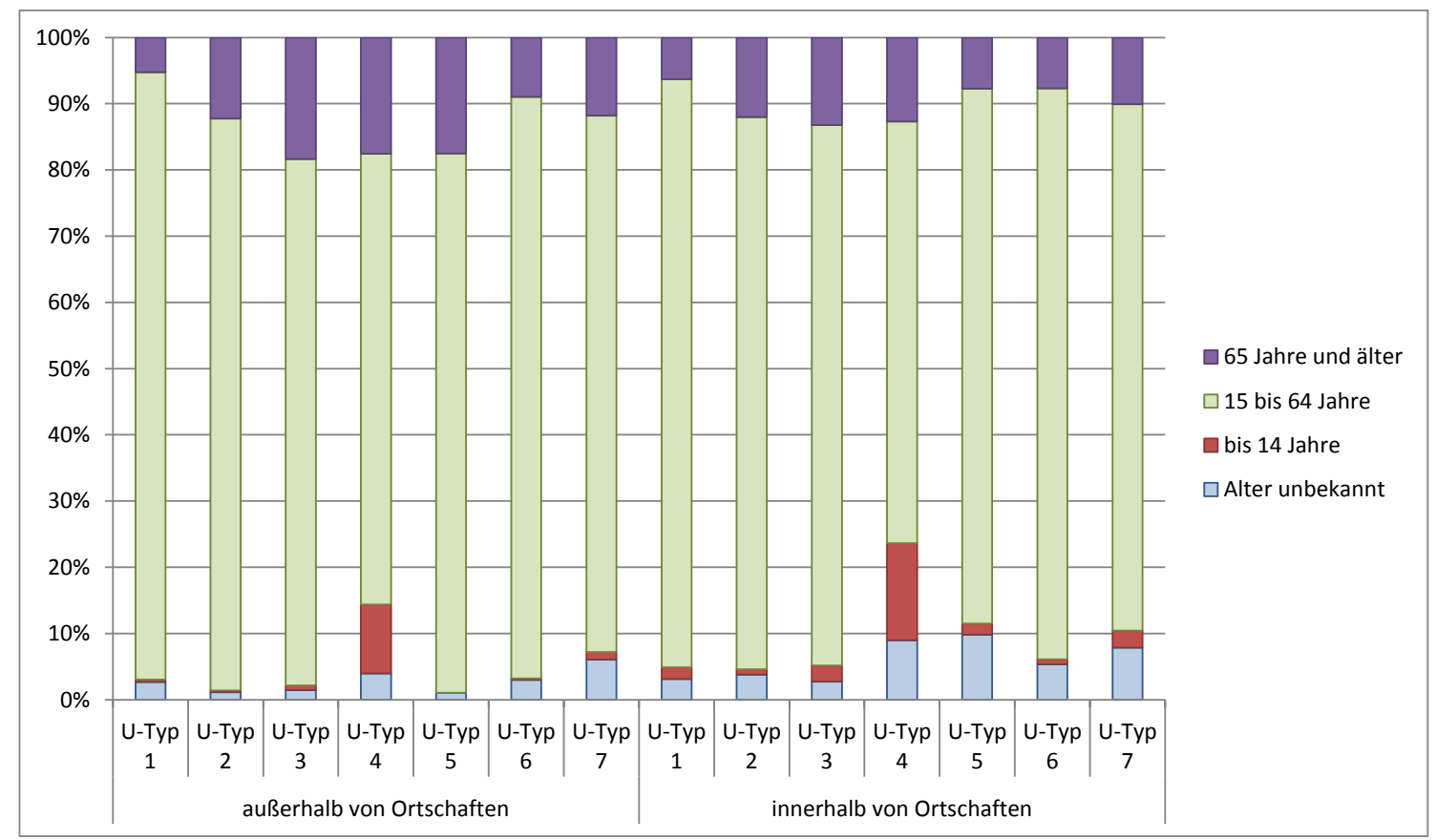

Abbildung 15: Unfällen mit Personenschaden und schwerwiegende Unfälle mit Sachschaden an Knotenpunkten innerorts $\left(n_{\text {ges }}=351.351\right.$ ), anteilig nach Altersgruppen 
Die weitere Betrachtung konzentriert sich auf die rund 285.000 Unfälle innerhalb geschlossener Ortschaften, da hier der Schwerpunkt der Untersuchung gesehen wird.

Abbildung 16 zeigt als Ausschnitt der Abbildung 14 die Verteilung der Unfälle auf die Unfalltypen, einschließlich der Unfälle mit Beteiligung von Kindern und Älteren.
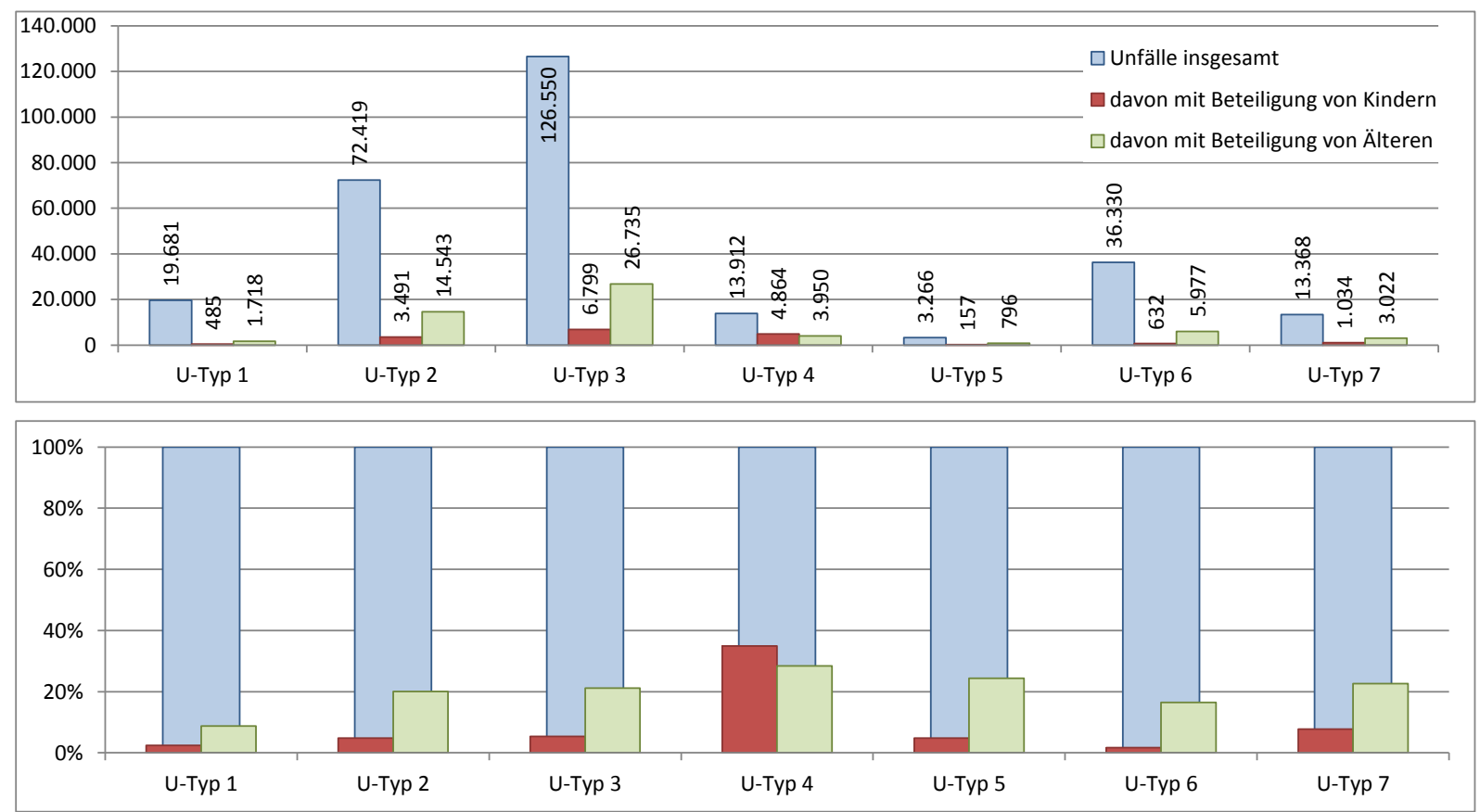

Abbildung 16: Unfälle mit Personenschaden und schwerwiegende Unfälle mit Sachschaden an Knotenpunkten innerorts $\left(\mathbf{n}_{\text {ges }}=\mathbf{2 8 5 . 5 2 6}\right)$

Auffällig ist, dass Kinder und Ältere relativ gesehen häufiger an Überschreiten-Unfällen beteiligt sind als andere Altersgruppen. Insgesamt nehmen Unfälle mit Beteiligung von Kindern rd. $35 \%$ aller Überschreiten-Unfälle an Knotenpunkten ein, Unfälle mit Beteiligung von Älteren in etwa $28 \%$ (Tabelle 8). Zwar ist der Unfalltyp 4 relativ betrachtet seltener erfasst, da hier nur Unfälle an Knotenpunkten betrachtet werden, dennoch ist es ersichtlich, dass trotz der insgesamt geringeren Anzahl von Überschreiten-Unfällen an Knotenpunkten ggü. Streckenabschnitten, ältere Menschen und Kinder beim Überschreiten der Fahrbahn grundsätzlich gefährdeter sind als übrige Altersgruppen. Kinder und Ältere sind, im Vergleich zu allen erfassten Unfällen an Knotenpunkten, bei Überschreiten-Unfällen überdurchschnittlich oft vertreten.

Tabelle 8: Unfälle an Knotenpunkten innerhalb geschlossener Ortschaften ( $\mathrm{n}=\mathbf{2 8 5 . 5 2 6}$ )

\begin{tabular}{|c|c|c|c|c|c|c|c|c|}
\hline \multirow{2}{*}{ Unfälle } & \multicolumn{7}{|c|}{ Unfalltyp } & \multirow{2}{*}{ Summe } \\
\hline & 1 & 2 & 3 & 4 & 5 & 6 & 7 & \\
\hline insgesamt & 19.681 & 72.419 & 126.550 & 13.912 & 3.266 & 36.330 & 13.368 & 285.526 \\
\hline $\begin{array}{l}\text { davon mit } \\
\text { Beteiligung von } \\
\text { Kindern } \\
\end{array}$ & 485 & 3.491 & 6.799 & 4.864 & 157 & 632 & 1.034 & 17.462 \\
\hline $\begin{array}{l}\text { davon mit } \\
\text { Beteiligung von } \\
\text { Älteren }\end{array}$ & 1.718 & 14.543 & 26.735 & 3.950 & 796 & 5.977 & 3.022 & 56.741 \\
\hline $\begin{array}{l}\text { Beteiligung Kinder } \\
\text { (anteilig in \%) }\end{array}$ & 2,5 & 4,8 & 5,4 & 35,0 & 4,8 & 1,7 & 7,7 & 6,1 \\
\hline $\begin{array}{l}\text { Beteiligung Ältere } \\
\text { (anteilig in \%) }\end{array}$ & 8,7 & 20,1 & 21,1 & 28,4 & 24,4 & 16,5 & 22,6 & 19,9 \\
\hline
\end{tabular}


Der Blick auf die Hauptverursacher, einschließlich der verunglückten Hauptverursacher, von Unfällen an Knotenpunkten und auf die weiteren Verunglückten (nur aktiv Beteiligte, keine Mitfahrer) bestätigt diesen Trend. Zudem zeigt sich, dass Kinder, i. d. R. Fußgänger und Radfahrer, im Vergleich zu den anderen Altersgruppen, häufiger als Hauptverursacher auch selber bei Unfällen verunglücken und insbesondere bei Überschreiten-Unfällen verunglücken. Ältere verunglücken im Vergleich $\mathrm{zu}$ den anderen Altersgruppen bei Überschreiten-Unfällen eher als weitere Beteiligte und nicht als Hauptverursacher (Abbildung 17 bis Abbildung 19).
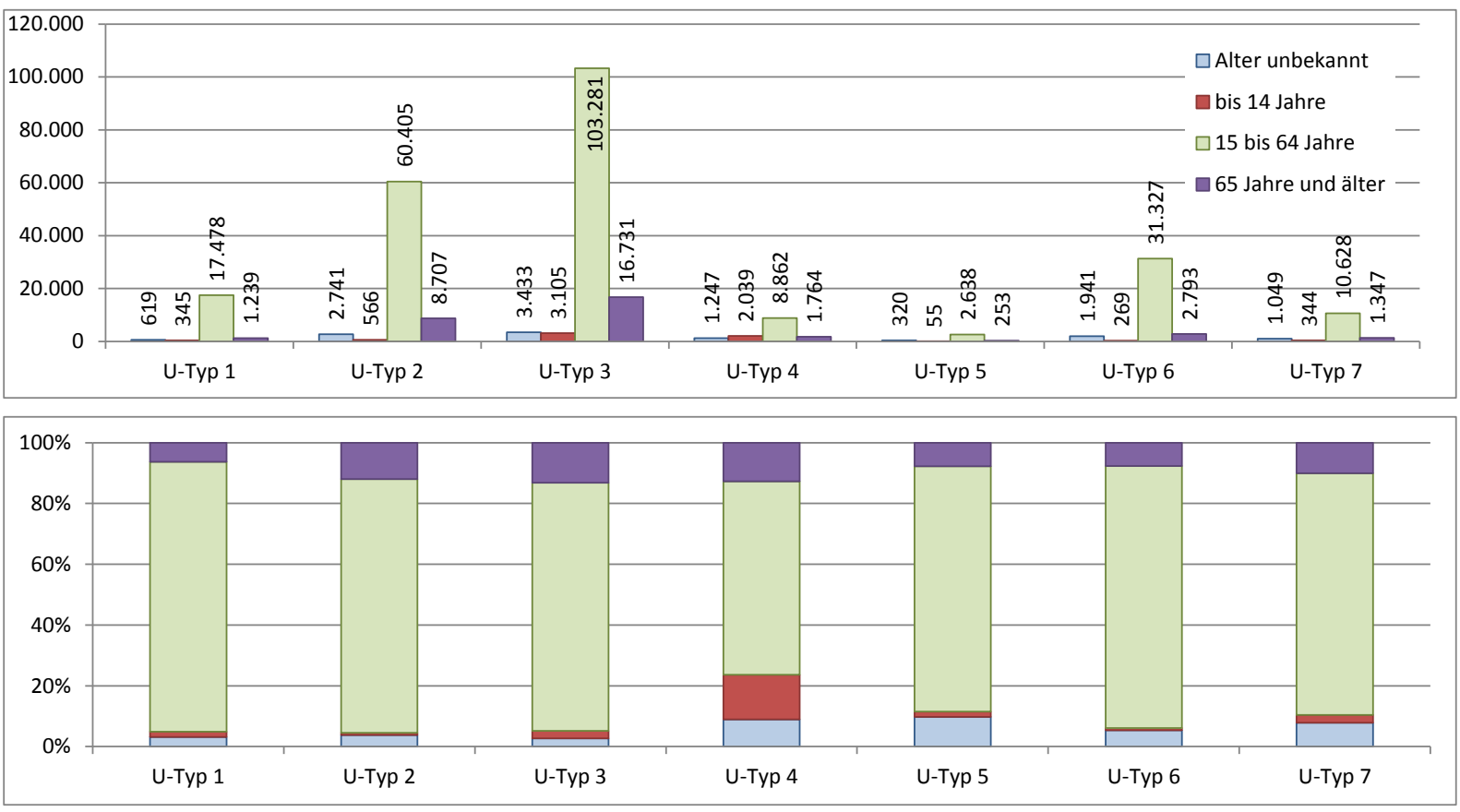

Abbildung 17: Hauptverursacher von Unfällen mit Personenschaden und schwerwiegende Unfälle mit Sachschaden an Knotenpunkten innerorts $\left(\mathrm{n}_{\text {ges }}=\mathbf{2 8 5 . 5 2 6}\right)$ 

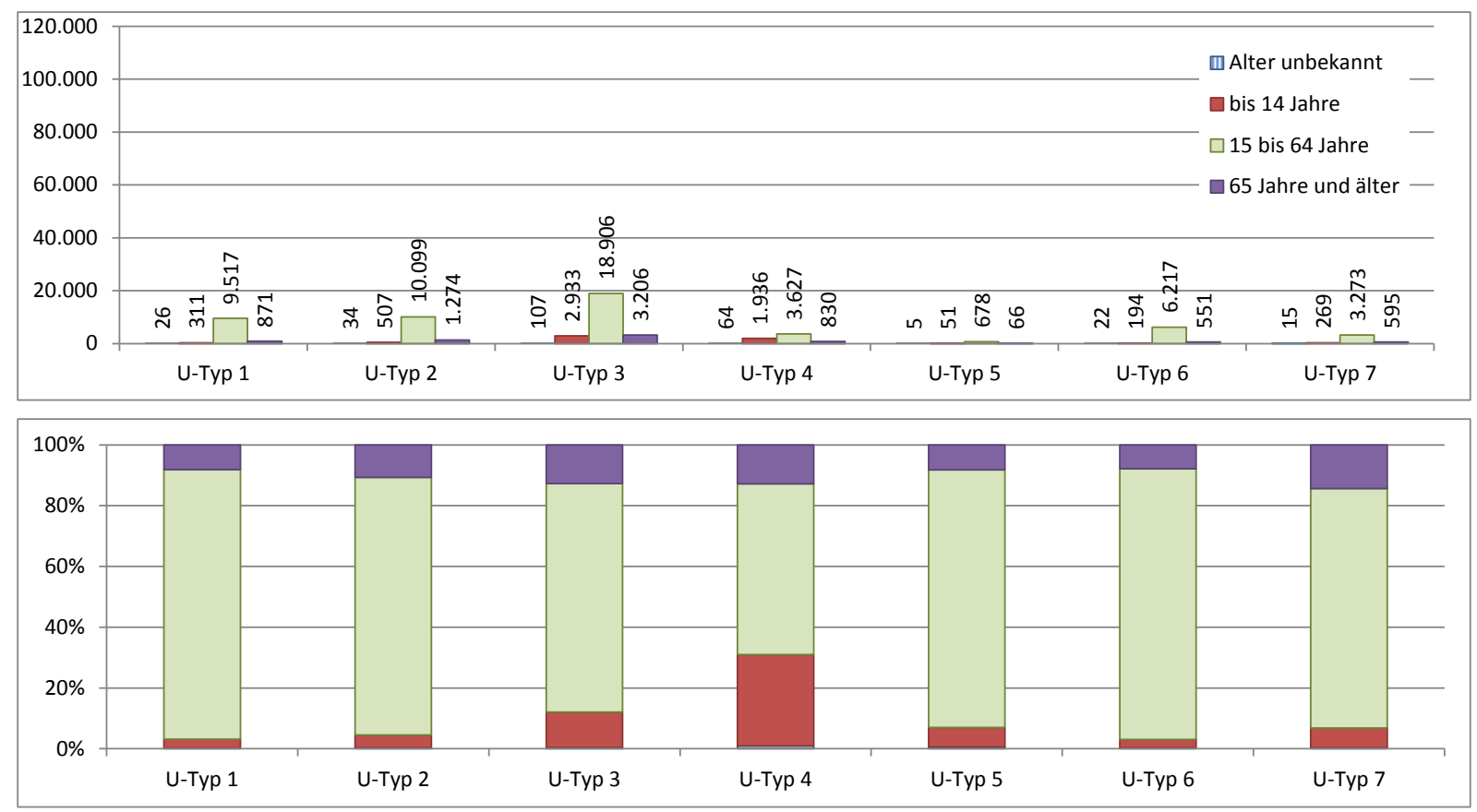

Abbildung 18: Verunglückte Hauptverursacher bei Unfällen mit Personenschaden an Knotenpunkten innerorts $\left(n_{\text {ges }}=66.172\right)$
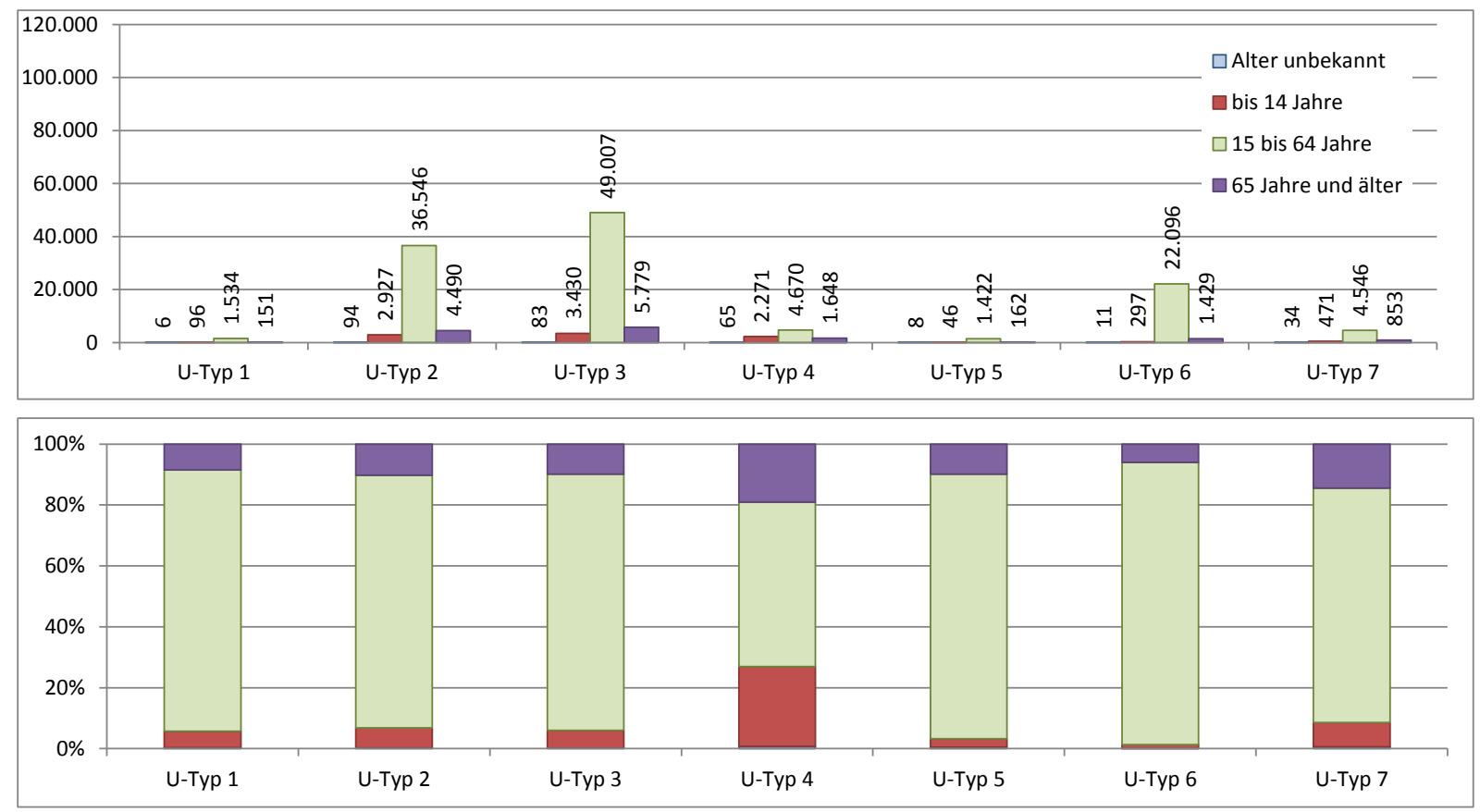

Abbildung 19: Verunglückte weitere Beteiligte (ohne Mitfahrer) bei Unfällen mit Personenschaden an Knotenpunkten innerorts $\left(n_{\text {ges }}=144.172\right)$ 


\subsection{Typische Unfallsituationen von Kindern und Älteren}

Kinder sind als Hauptverursacher (Fußgänger und Radfahrer) zumeist an Einbiegen/Kreuzen- und Überschreiten-Unfällen beteiligt, wobei sie auch selber häufig verunglücken ${ }^{13}$. (Abbildung 20). Zudem verunglücken sie als weitere Beteiligte (keine Mitfahrer), neben diesen beiden Unfalltypen, auch häufig bei Abbiege-Unfällen.

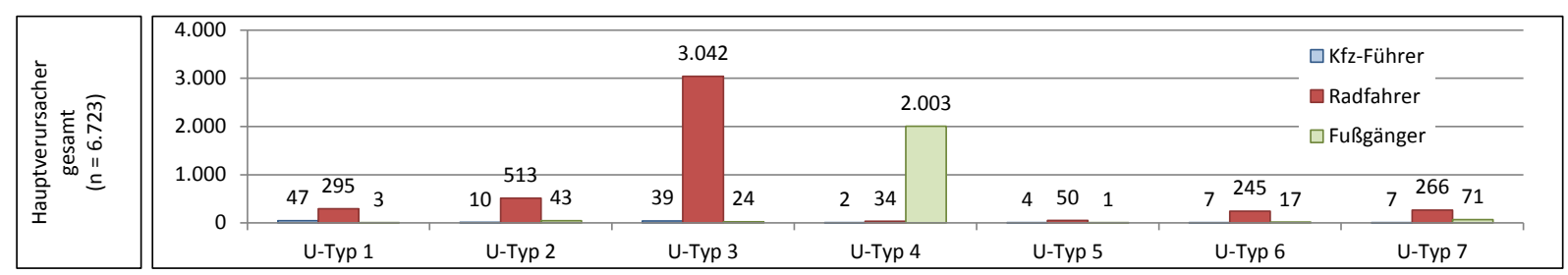

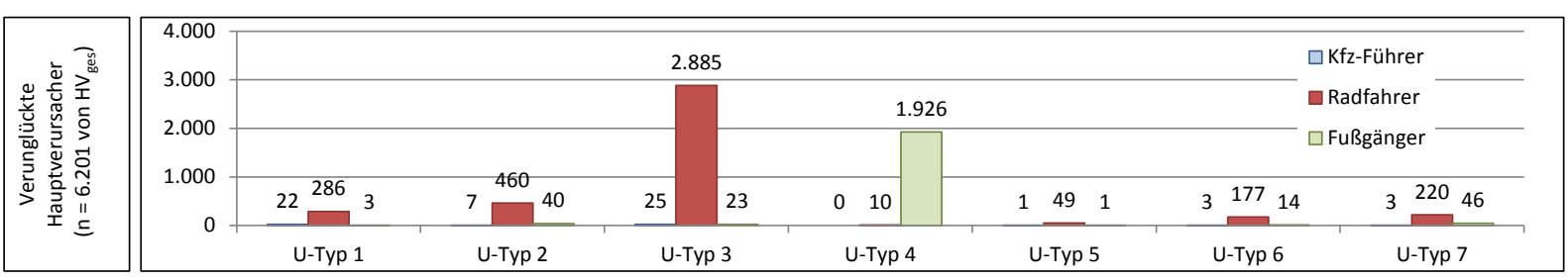

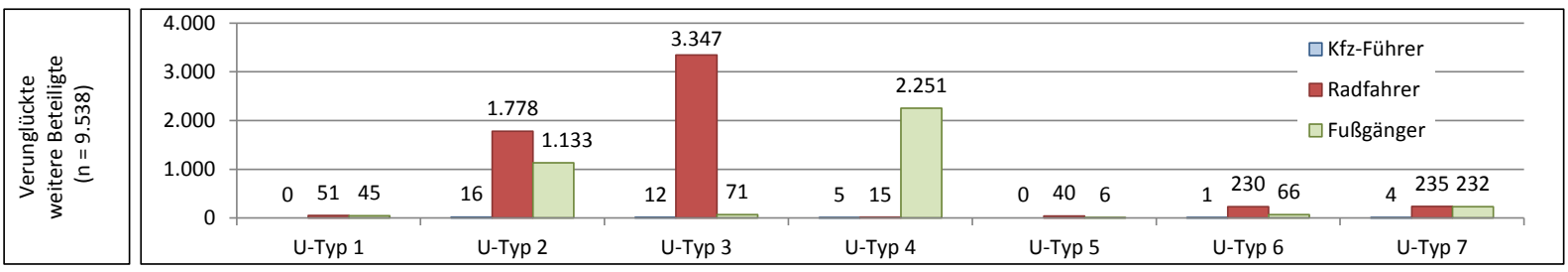

Abbildung 20: Kinder als Hauptverursacher und verunglückte weitere Beteiligte ${ }^{14}$

Ältere Menschen verursachen Unfälle vornehmlich als Kraftfahrer (Abbildung 21). Dabei kommen insbesondere Abbiege- und Einbiegen/Kreuzen-Unfälle vor. An dritter Position stehen mit einigem Abstand Unfälle im Längsverkehr. Im Vergleich zu unfallverursachenden Kindern (Fußgänger und Radfahrer), sind ältere Hauptverursacher als Kraftfahrer besser geschützt. Sie verunglücken als Hauptverursacher weitaus seltener als $\operatorname{Kinder}^{15}$.

Als weitere Unfallbeteiligte verunglücken Ältere, ebenso wie Kinder, häufig als Radfahrer und Fußgänger bei Abbiege-, Einbiegen/Kreuzen- und Überschreiten-Unfällen. Als Führer von Kraftfahrzeugen verunglücken Ältere neben Abbiege- und Einbiegen/Kreuzen-Unfällen zudem bei Unfällen im Längsverkehr.

\footnotetext{
${ }^{13}$ Kinder verunglücken bei Einbiegen/Kreuzen- und Überschreiten-Unfällen, die sie als Fußgänger oder Radfahrer verursachen, in etwa $95 \%$ der Fälle.

14 Anmerkung: Die Auswertung beruht auf den zur Verfügung gestellten elektronischen Unfalldatenbeständen. Kinder (14 Jahre und jünger), die als Kfz-Führer in den Datenbeständen aufgeführt sind, waren vornehmlich 13 bis 14 Jahre alt und nutzen ein kleinmotoriges Kraftrad.

${ }^{15}$ Ältere Menschen verunglücken bei Abbiege- und Einbiegen/Kreuzen-Unfällen, die sie als Kraftfahrer verursachen, in etwa $10 \%$ der Fälle.
} 

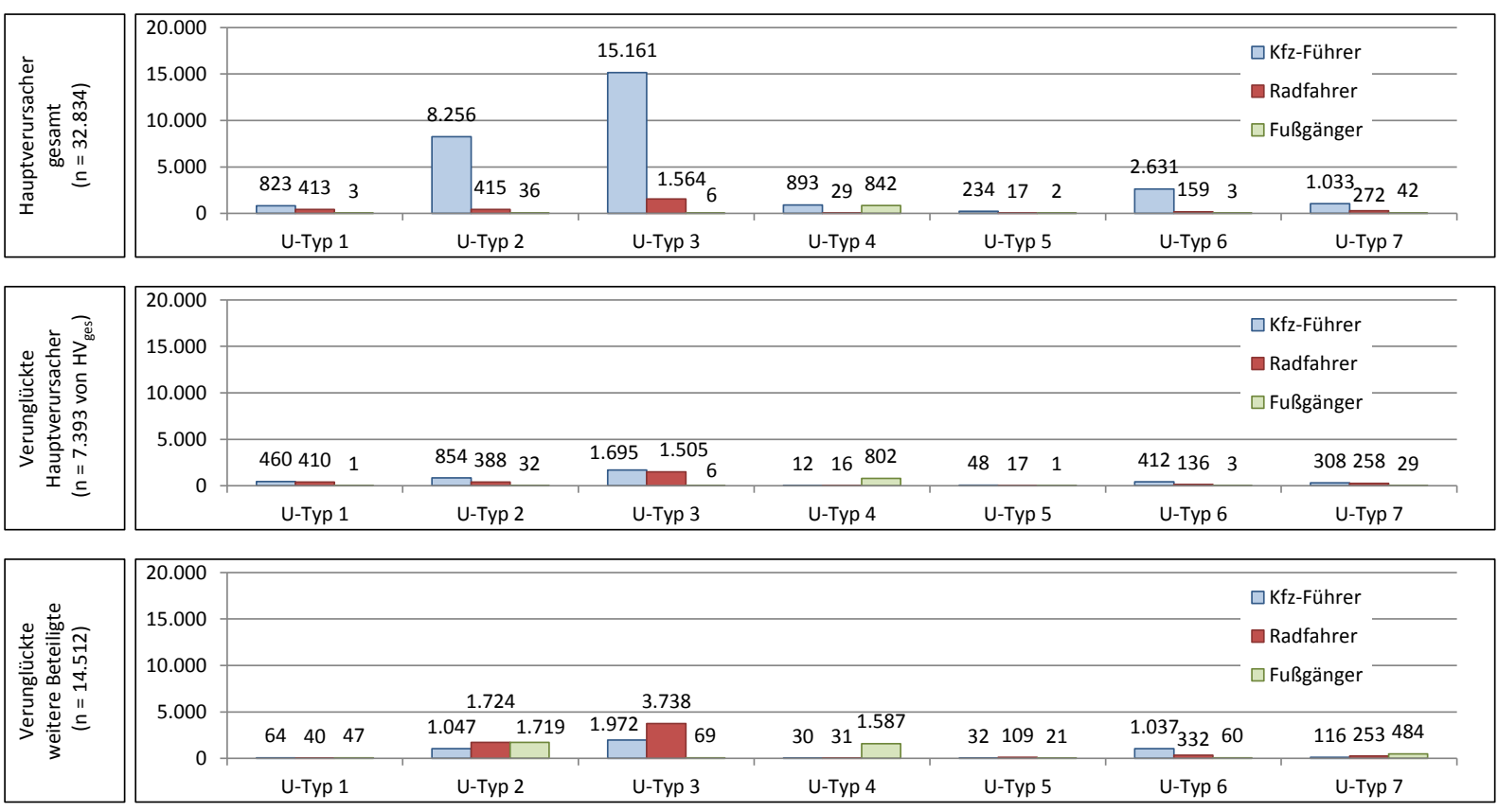

Abbildung 21: Ältere als Hauptverursacher und verunglückte weitere Beteiligte ${ }^{16}$

$\mathrm{Zu}$ weiteren Spezifizierung wurden die 3-stelligen Unfalltypen am Beispiel der Verkehrsunfalldaten aus NRW (2004 bis 2008) hinsichtlich ihres Aufkommens untersucht. Insgesamt fällt dabei auf, dass es eine hohe Konzentration bestimmter Unfalltypen gibt. So umfassen acht Unfalltypen bereits die Hälfte aller untersuchten Unfälle an Knotenpunkten (Abbildung 22). Bei Kinderunfällen stellen die vier häufigsten Unfalltypen ein Viertel aller in dieser Gruppe erfassten Unfälle dar (Abbildung 23). Bei Älteren umfassen die vier häufigsten Unfalltypen sogar rund $40 \%$ aller Unfälle (Abbildung 24).

Der am häufigsten vorkommende dreistellige Unfalltyp an Kreuzungen ist, bezogen auf alle Knotenpunktunfälle aus NRW, der Unfalltyp 211, bei dem ein nach links abbiegender Kraftfahrer (Wartepflicht) mit einem Fahrzeug des Gegenverkehrs zusammenstößt. In dieser Häufigkeitsbetrachtung folgen im Weiteren vornehmlich Einbiegen-Kreuzen-Unfälle (Abbildung 22).

Bei Knotenpunktunfällen mit Beteiligung von Kindern ist der Unfalltyp 342, bei dem ein einbiegendes bzw. kreuzendes Fahrzeug (Wartepflicht) mit einem von rechts kommenden, auf dem zur Einmündung angrenzenden Radweg fahrenden, Radfahrer zusammenstößt, am häufigsten vertreten. Gefolgt wird dieser vom Unfalltyp 321, bei dem ebenfalls, ein Konflikt zwischen einen kreuzenden und einem von rechts kommenden Verkehrsteilnehmer besteht. Diese beiden Unfalltypen nehmen in etwa $18 \%$ aller Unfälle mit Beteiligung von Kindern ein (Abbildung 23).

Die Verteilung der dreistelligen Unfalltypen bei Unfällen mit Beteiligung von Älteren entspricht in etwa der Unfalltypenverteilung aller Knotenpunktunfälle. Auch hier ist der Unfalltyp 211 am häufigsten vertreten, gefolgt von Einbiegen/Kreuzen-Unfällen (Abbildung 24).

${ }^{16}$ Vgl. Anmerkungen zu Abbildung 20. 


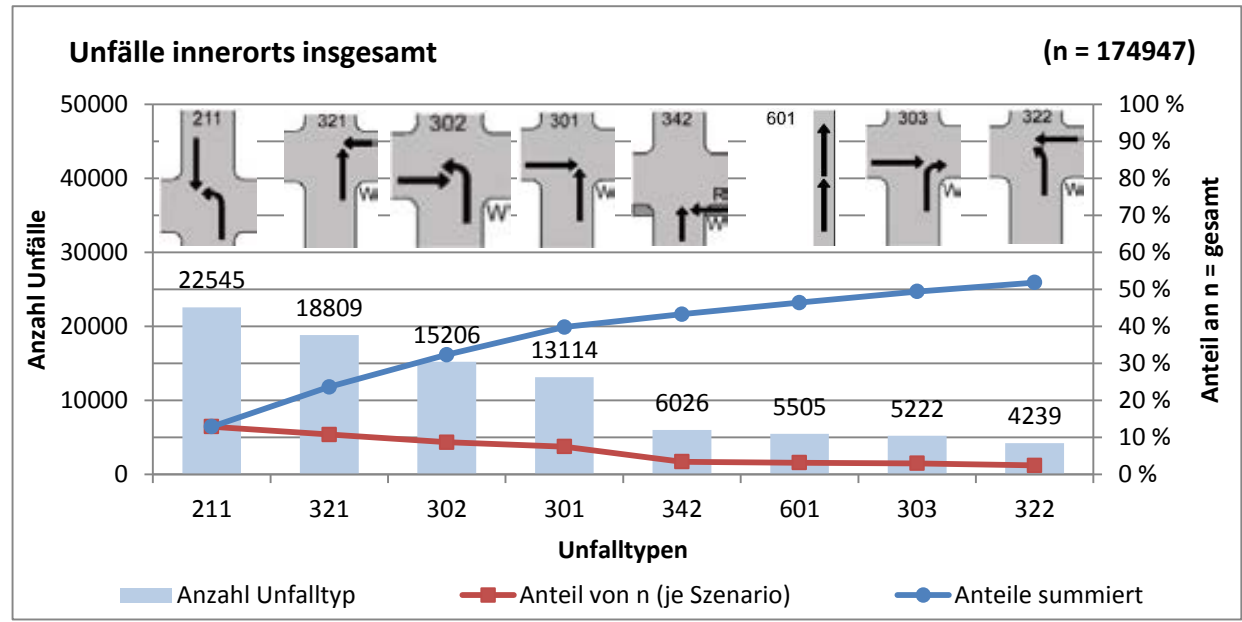

Abbildung 22: Häufigste dreistellige Unfalltypen von Unfällen mit Personenschaden und schwerwiegenden Unfällen mit Sachschaden an Knotenpunkten innerorts am Beispiel der Unfalldaten Nordrhein-Westfalen aus 2004 bis $2008\left(n_{\text {ges }}=174.947\right)$

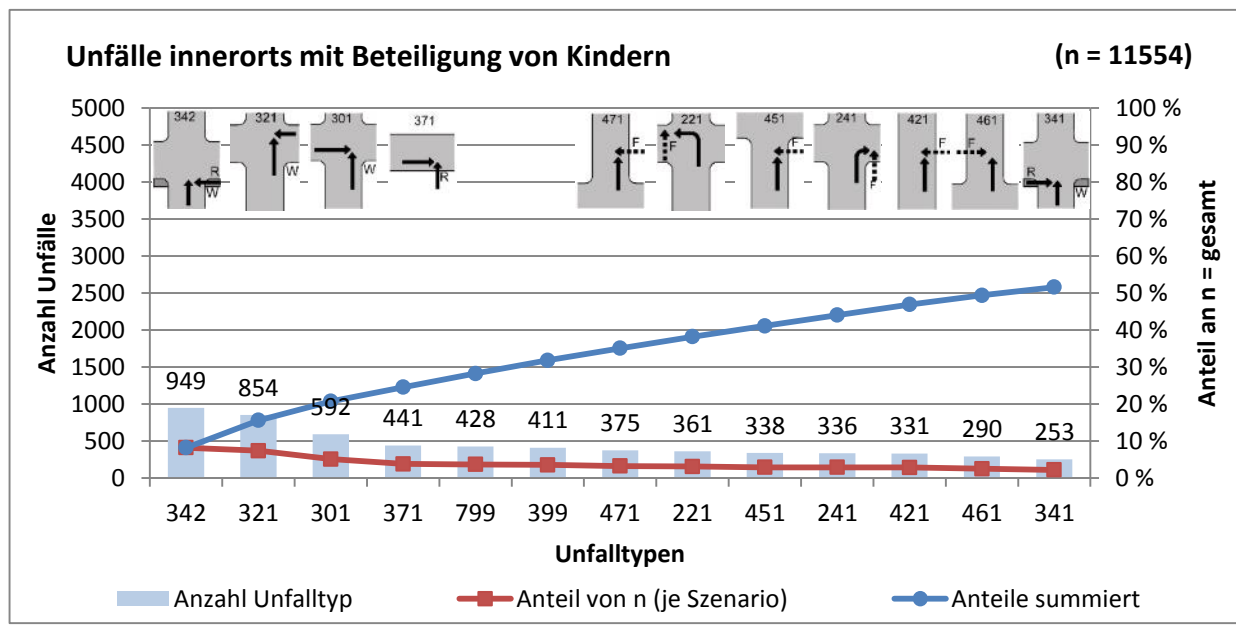

Abbildung 23: Häufigste dreistellige Unfalltypen von Unfällen mit Personenschaden und schwerwiegenden Unfällen mit Sachschaden mit Beteiligung von Kindern an Knotenpunkten innerorts am Beispiel der Unfalldaten Nordrhein-Westfalen aus 2004 bis $2008\left(n_{\text {ges }}=11.554\right)$

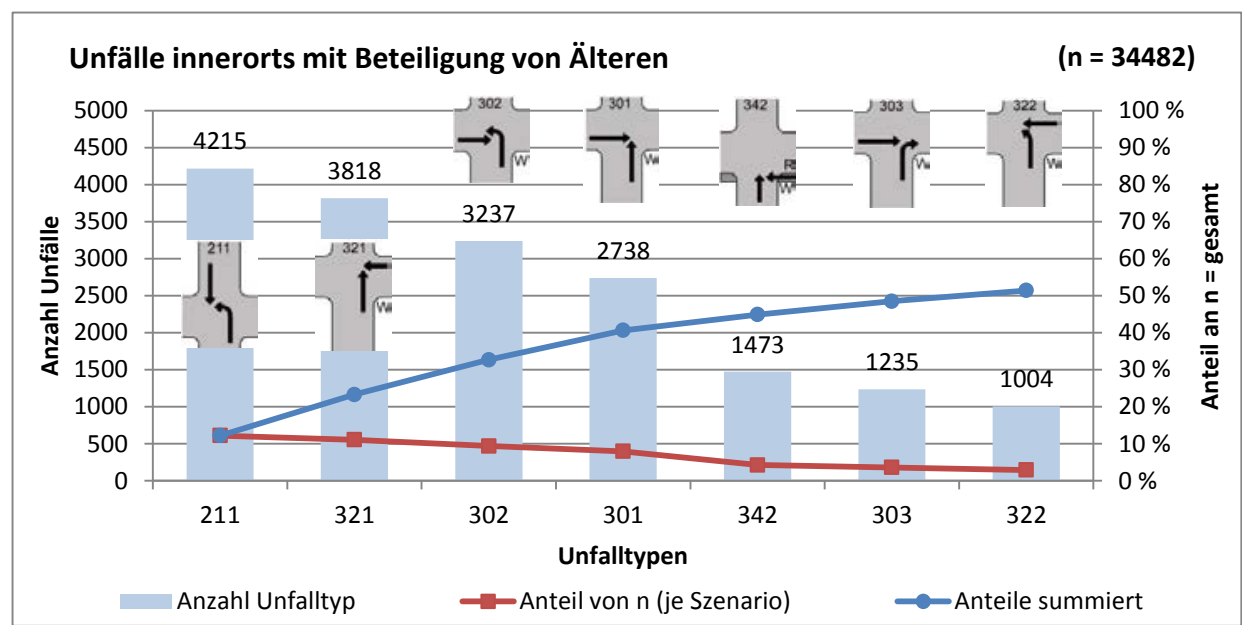

Abbildung 24: Häufigste dreistellige Unfalltypen von Unfällen mit Personenschaden und schwerwiegenden Unfällen mit Sachschaden mit Beteiligung von Älteren an Knotenpunkten innerorts am Beispiel der Unfalldaten Nordrhein-Westfalen aus 2004 bis $2008\left(n_{\text {ges }}=34.482\right)$ 
$\mathrm{Da}$ nicht für alle Unfalldaten dreistellige Unfalltypen vorlagen wurden auch häufige Kombination von einstelligem Unfalltyp und Unfallart betrachtet. Für Kinder wie für Ältere Hauptverursacher und Verunglückte ergeben sich die Kombinationen aus

- Unfalltyp 2 „Abbiege-Unfall“ und Unfallart 5 „Zusammenstoß mit einbiegenden/kreuzenden Fahrzeug" (mit 50 bis 60\% der Fälle die häufigste Unfallart bei Unfällen des Typs 2)

- Unfalltyp 3 „Einbiegen/Kreuzen-Unfall“ und Unfallart 5 „Zusammenstoß mit einbiegenden/kreuzenden Fahrzeug" (mit 90 bis 95\% der Fälle die häufigste Unfallart bei Unfällen des Typs 3)

- Unfalltyp 4 „Überschreiten-Unfall“ und Unfallart 6 „Zusammenstoß zwischen Fahrzeug und Fußgänger" (mit rund $95 \%$ die häufigste Unfallart bei Unfällen des Typs 4).

Im Hinblick auf Unfälle an Knotenpunkten innerhalb geschlossener Ortschaften lässt sich zusammenfassend festhalten, dass

- Kinder als Hauptverursacher oft bei Unfällen des Typs 3 (Einbiegen/Kreuzen-Unfälle) als Radfahrer, insbesondere Typ 342 und 321, und überproportional oft bei Unfällen des Typs 4 (Überschreiten-Unfälle) als Fußgänger erfasst sind,

- Kinder innerhalb ihrer Altersgruppe, zusätzlich zu den beiden Unfalltypen 3 und 4, zudem noch im Unfalltyp 2 (Abbiege-Unfälle) als weitere beteiligte Radfahrer und Fußgänger häufig verunglücken,

- Ältere in den Unfalltypen 2, insbesondere im Typ 211, und 3 als Kfz-Führer absolut gesehen am häufigsten vertreten sind (was auch der Verteilung aller Unfälle an Knotenpunkten entspricht) und

- im Weiteren Kinder und ältere Menschen in Relation zu den übrigen Verkehrsteilnehmern häufiger in Unfälle des Typs 4 verwickelt sind und bei diesen auch verunglücken.

Abbildung 25 stellt die für Kinder und Ältere typischen Unfallsituationen grafisch dar.

\begin{tabular}{|c|c|c|c|c|c|c|c|c|c|c|c|c|c|c|c|c|c|c|c|c|c|}
\hline \multirow[b]{2}{*}{ Art der Verkehrsbeteiligung } & \multicolumn{3}{|c|}{$\begin{array}{l}\text { Unfalltyp } 1 \\
\text { Fahrunfal (F) }\end{array}$} & \multicolumn{3}{|c|}{$\begin{array}{c}\text { Unfalltyp } 2 \\
\text { Abbiege-Unfall (AB) }\end{array}$} & \multicolumn{3}{|c|}{$\begin{array}{l}\text { Unfalltyp } 3 \\
\text { Enbiegent/Kreuzen- } \\
\text { Unfall (EK) }\end{array}$} & \multicolumn{3}{|c|}{$\begin{array}{c}\text { Unfalltyp } 4 \\
\text { Überschreiten-Unfall } \\
\text { (US) }\end{array}$} & \multicolumn{3}{|c|}{\begin{tabular}{|c|} 
Unfalltyp 5 \\
Unfal durth ruhenden \\
Verkehr (RV)
\end{tabular}} & \multicolumn{3}{|c|}{$\begin{array}{c}\text { Unfalltyp } 6 \\
\text { Unfall imLängsverkehr } \\
\text { (LV) }\end{array}$} & \multicolumn{3}{|c|}{$\begin{array}{c}\text { Unfalltyp } 7 \\
\text { Sonstiger Unfall (SO) }\end{array}$} \\
\hline & $\mathrm{Kfz}$ & $\mathrm{Rf}$ & $\mathrm{Fg}$ & Kfz & $\mathrm{Rf}$ & $\mathrm{Fg}$ & $\mathrm{Kfz}$ & Rf & $\mathrm{Fg}$ & Kfz & $\mathrm{Rf}$ & $\mathrm{Fg}$ & $\mathrm{Kfz}$ & $\mathrm{Rf}$ & $\mathrm{Fg}$ & \begin{tabular}{l|l}
$\mathrm{Kfz}$ \\
\end{tabular} & Rf & $\mathrm{Fg}$ & $\mathrm{Kfz}$ & $\mathrm{Rf}$ & $\mathrm{Fg}$ \\
\hline \multirow{2}{*}{ Als Hauptverursacher } & & & & & & & & $\mathbf{K}$ & & & & $\mathrm{K}$ & & & & & & & & & \\
\hline & & & & À & & & A & & & & & & & & & & & & & & \\
\hline \multirow{2}{*}{$\begin{array}{l}\text { Als Hauptverursacher } \\
\text { (selber verunglückt) }\end{array}$} & & & & & & & & $\mathbf{K}$ & & & & $\mathrm{K}$ & & & & & & & & & \\
\hline & & & & & & & Ä & $\dot{A}$ & & & & & & & & & & & & & \\
\hline \multirow{2}{*}{$\begin{array}{c}\text { Als weitere Beteiligte } \\
\text { (verunglückt) }\end{array}$} & & & & & $\mathbf{K}$ & $\mathbf{K}$ & & $\mathrm{K}$ & & & & K & & & & & & & & & \\
\hline & & & & A & $\bar{A}$ & $\bar{A}$ & $\bar{A}$ & $\bar{A}$ & & & & A & & & & A & & & & & \\
\hline \multirow[t]{4}{*}{ Legende/Erläuterungen: } & \multicolumn{9}{|c|}{ Relevante Unfalltypen für: } & \multicolumn{9}{|c|}{ Art der Verkehrsbeteiligung: } & & & \\
\hline & \multicolumn{2}{|l|}{ Kinder } & $\mathrm{K}$ & & & & & & & & KGz & \multicolumn{7}{|c|}{ Führer eines Krafffahrzeuges } & & & \\
\hline & Ältere & & $\bar{A}$ & & & & & & & & $\mathrm{Rf}$ & \multicolumn{7}{|c|}{ Radfahrer } & & & \\
\hline & & & & & & & & & & & $\mathrm{Fg}$ & \multicolumn{7}{|c|}{ Fußgänger } & & & \\
\hline
\end{tabular}

Abbildung 25: Relevante Unfalltypen für Unfälle mit Beteiligung von Kindern und Älteren an Knotenpunkten innerorts 


\subsection{Zusammenfassung: Typische Unfallsituationen}

Die makroskopische Unfallanalyse basierte auf elektronischen Unfalldaten aus fünf Bundesländern. Insgesamt wurden über 350.000 Unfälle an Knotenpunkten außerhalb von Bundesautobahnen innerhalb und außerhalb geschlossener Ortschaften erfasst. Davon entfielen rd. 280.00 Unfälle (80 \%) auf Bereiche innerorts und etwa 70.000 Unfälle (20 \%) auf Bereiche außerorts.

Die meisten Unfälle an Knotenpunkten sind Einbiegen/Kreuzen-Unfälle, gefolgt von AbbiegeUnfällen. Mit einigem Abstand kommen auch Unfälle im Längsverkehr vor. In der Detailbetrachtung der dreistelligen Unfalltypen liegt allerdings der Unfalltyp 211 (AbbiegeUnfall: Missachtung der Vorfahrt des Gegenverkehrs durch Linksabbieger) vor den dreistelligen Unfalltypen der Einbiegen/Kreuzen-Unfälle. Überschreiten-Unfälle kommen in der Gesamtschau aller Verkehrsbeteiligungsarten seltener vor.

Kinder sind als Hauptverursacher (Fußgänger und Radfahrer) zumeist an Einbiegen/kreuzen- und Überschreiten-Unfällen beteiligt, wobei sie auch selber häufig verunglücken. Zudem verunglücken sie als weitere Beteiligte häufig bei Abbiege-Unfällen. Unter den dreistelligen Unfalltypen stechen bei Kindern die Unfalltyp 321 und 342 (Radfahrer von rechts) hervor.

Ältere Menschen verursachen Unfälle vornehmlich als Kfz-Führer. Dabei kommen insbesondere Abbiege- und Einbiegen/Kreuzen-Unfälle vor.

Als Kfz-Führer verunglücken sie weitaus seltener wie Kinder als Hauptverursacher. Als weitere Unfallbeteiligte verunglücken Ältere, ebenso wie Kinder, häufig als Radfahrer und Fußgänger bei Abbiege-, Einbiegen/Kreuzen- und Überschreiten-Unfällen.

Auch in dieser Altersgruppe liegt der Unfalltyp 211 insgesamt noch vor den dreistelligen Unfalltypen der Einbiegen/Kreuzen-Unfälle. 


\section{Auswahl von Knotenpunkten für die Sicherheitsanalysen und Verhaltensbeobachtungen}

Mit dem Ziel, verkehrstechnische Gestaltungselemente an Knotenpunkten zu identifizieren, die insbesondere für die jeweiligen Untersuchungsgruppen (Kinder, Ältere, weitere mobilitätseingeschränkte und mobilitätsbehinderte Personen) ein Risikopotenzial hinsichtlich der Verkehrssicherheit bergen, wurden auf der Grundlage der elektronischen Unfalldatenbestände Knotenpunkte für weitere Detailanalysen bestimmt. Der Schwerpunkt wurde dabei auf Knotenpunkte innerhalb geschlossener Ortschaften gelegt. Die späteren Detailanalysen umfassen die Auswertung von Verkehrsunfallanzeigen, Sicherheitsanalysen der Verkehrsinfrastruktur und Verhaltensbeobachtungen der Verkehrsteilnehmer im Realverkehr.

\subsection{Methodik zur Auswahl relevanter Knotenpunkte}

Die zentrale Grundlage zur Auswahl der Knotenpunkte, stellten die im Vorfeld ermittelten häufigen (typischen) Unfallsituationen von Kindern und Älteren dar.

Im Rahmen der Untersuchung wurden drei Methoden angewandt, die voneinander unabhängig durchgeführt wurden und mit deren Hilfe relevante Knotenpunkte auf Basis der elektronischen Datenbestände aus den fünf Bundesländern identifiziert werden konnten:

Methode 1

1. Filterung von Unfällen nach Art der Knotenpunkte (Kreuzung, Einmündung)

2. Filterung der Unfälle mit Beteiligung von Kindern oder Älteren

3. Identifizierung von Knotenpunkten

\section{Methode 2}

1. Filterung der am häufigsten auftretenden Kombinationen von Unfalltyp und Unfallart (bzw. dreistelligen Unfalltypen)

2. Filterung relevanter Altersgruppen (Kinder, Ältere)

3. Identifizierung von Knotenpunkten

Methode 3

1. Filterung der relativ häufig auftretenden Kombinationen Unfalltypen/Unfallarten (bzw. dreistelligen Unfalltypen) von Kindern und Älteren im Vergleich zu den übrigen Verkehrsteilnehmern

2. Identifizierung von Knotenpunkten 


\subsection{Auswahl relevanter Knotenpunkte}

Insgesamt wurden 291 Knotenpunkte exemplarisch ausgewählt, die in einem oder mehreren der oben genannten Methoden (vgl. Abschnitt 5.1) auffällig waren. Davon lagen 262 Knotenpunkte (rd. $90 \%$ ) innerorts und 29 Knotenpunkte (rd. $10 \%$ ) außerorts (ohne Autobahnen). Von den innerörtlichen Knotenpunkte waren

- 181 lichtsignalgeregelte Kreuzungen (69,1\%),

- 30 vorzeichengeregelte Kreuzungen (11,5\%),

- 23 lichtsignalgeregelte Einmündungen $(8,8 \%)$ und

- 24 vorzeichengeregelte Einmündungen (9,2\%).

Auf alle 291 Knotenpunkte entfielen 5.365 Unfälle, davon 4.891 Unfälle (91 \%) auf Bereiche innerorts und 474 Unfälle ( $9 \%$ ) auf Bereiche außerorts. Von den Unfällen innerorts entfielen

- auf lichtsignalgeregelte Kreuzungen 4.048 Unfälle (82,8 \%),

- auf vorzeichengeregelte Kreuzungen 337 Unfälle (6,9\%),

- auf lichtsignalgeregelte Einmündungen 237 Unfälle (5,6 \%) und

- auf vorzeichengeregelte Einmündungen 225 Unfälle (4,6 \%).

Rechts-vor-links-geregelte Kreuzungen bzw. Einmündungen und Kreisverkehre kamen nur vereinzelt vor.

In weiteren Auswahlschritten, die u. a. den Anteil der Unfälle von Kindern und Älteren an allen Unfällen des jeweiligen Knotenpunktes sowie Knotenpunkte, die über mehrere oder alle der insgesamt drei Filtermethoden auffällig waren und die weitere Kriterien, wie die Art der Verkehrsregelung, der typischen Unfallsituationen berücksichtigen, wurden stellvertretend zunächst rd. 50 und daraus schließlich 15 Knotenpunkte innerhalb geschlossener Ortschaften für die Detailuntersuchungen ausgewählt (Tabelle 9).

Tabelle 9: Übersicht der 15 Knotenpunkte für die Detailuntersuchungen

\begin{tabular}{|c|l|l|l|}
\hline Knotenpunkt Nr. & \multicolumn{1}{|c|}{ Typ } & \multicolumn{1}{c|}{ Verkehrsregelung } & \multicolumn{1}{c|}{ Zielgruppe } \\
\hline 1 & Kreuzung & LSA & Kinder und Ältere \\
\hline 2 & Kreuzung & LSA & Ältere \\
\hline 3 & Kreuzung & LSA & Kinder und Ältere \\
\hline 4 & Kreuzung & LSA & Ältere \\
\hline 5 & Kreuzung & LSA & Kinder \\
\hline 6 & Kreuzung & LSA & Ältere \\
\hline 7 & Kreuzung & LSA & Kinder \\
\hline 8 & Kreuzung & LSA & Ältere \\
\hline 9 & Kreuzung & LSA & Ältere \\
\hline 10 & Kreuzung & VZ, FG-LSA & Kinder \\
\hline 11 & Kreuzung & VZ & Ältere \\
\hline 12 & Kreuzung & VZ Ältere \\
\hline 13 & Kreuzung & VZ & Kinder \\
\hline 14 & Einmündung & VZ & Kinder \\
\hline 15 & Einmündung & VZ & Kinder \\
\hline \multicolumn{2}{|l|}{} \\
\hline Erläuterungen: \\
\hline
\end{tabular}




\subsection{Zusammenfassung: Auswahl von Knotenpunkten}

Die Auswahl der Knotenpunkte für die Detailuntersuchungen basierte grundlegend auf den elektronischen Unfalldaten der fünf Bundesländer und berücksichtigte dabei typische Unfallsituationen von Kindern und älteren Menschen.

Zunächst wurden mithilfe dreier, voneinander unabhängig durchgeführten Methoden 291 Knotenpunkte identifiziert, an denen die (typischen) Unfälle von Kindern und älteren Menschen (relativ häufig) auftraten.

In weiteren Auswahlschritten, die u. a. den Knotenpunkttyp, die Verkehrsregelung und den Anteil der Unfälle mit Kindern und/oder Älteren am Gesamtunfallgeschehen berücksichtigten, wurden zunächst etwa 50 Knotenpunkte und aus diesem Kollektiv schließlich 15 Knotenpunkte für die Detailuntersuchungen ausgewählt. 


\section{Sicherheitsanalysen und Verhaltensbeobachtungen}

Im Folgenden wird das Vorgehen der Detailuntersuchungen (Abschnitt 6.1) sowie die wesentlichen Aspekte des Unfallgeschehens, der Sicherheitsanalysen und die grundlegenden Ergebnisse der Verhaltensbeobachtungen an den 15 untersuchten Knotenpunkten unterschieden nach Art der Verkehrsbeteiligung dargestellt (Abschnitt 6.2 und 6.3). Ausführliche Berichte zu den einzelnen Knotenpunkten sind im Anhang A 2 bis A 5, die detaillierte Auswertung des Verkehrsverhaltens der betrachteten Gruppen ist im Anhang A 6 aufgeführt.

Die Ergebnisse der Verhaltensbeobachtungen von Menschen mit besonderen Mobilitätseinschränkungen oder Mobilitätsbehinderungen werden aufgrund der geringen Fallzahlen im Rahmen der Beobachtungen durch Ergebnisse von Studien Dritter ergänzt.

\subsection{Vorgehen}

Für die 15 Knotenpunkte, die aufgrund des Typs (Kreuzung, Einmündung), der Verkehrsregelung (mit LSA, mit vorfahrtregelnden Verkehrszeichen) und des Unfallgeschehens stellvertretend aus den 262 Knotenpunkten innerhalb geschlossener Ortschaften ausgewählt wurden, wurde zunächst das Unfallgeschehen detailliert untersucht. Im Weiteren wurden Sicherheitsanalysen der Verkehrsinfrastruktur sowie Verhaltensbeobachtungen im Realverkehr durchgeführt.

\subsubsection{Unfallanalysen}

Im Rahmen der Detailuntersuchungen wurden anonymisierte Verkehrsunfallanzeigen zu Unfällen mit Personenschaden (Unfallkategorie 1 bis 3) und zu schwerwiegenden Unfällen mit Sachschaden (Kategorie 4) für die ausgewählten Knotenpunkte bei den zuständigen Polizeidienststellen möglichst der letzten fünf Jahre (2006 - 2010) angefragt und analysiert.

Einen Überblick über das Unfallgeschehen gibt Kapitel 6.2. Die zentralen Aussagen zum Unfallgeschehen für die jeweiligen Kontenpunkttypen sind in Kapitel 6.3 dargestellt, ausführliche Berichte enthalten die Ausführungen im Anhang (A 2 bis A 5).

\subsubsection{Sicherheitsanalysen der Verkehrsinfrastruktur}

Die Sicherheitsanalysen orientierten sich methodisch am Verfahren des Sicherheitsaudits von Straßen und erfolgten auf der Grundlage zur Verfügung gestellter Planunterlagen, z. B. Ausführungspläne, Signalzeitenpläne. Zudem wurde jeder Knotenpunkt im Rahmen von VorOrt-Begehungen begutachtet. Zu einigen Knotenpunkten konnten keine entsprechenden Planunterlagen seitens der Stadtverwaltungen zur Verfügung gestellt werden, hier wurden in erster Linie Vor-Ort-Untersuchungen durchgeführt.

Die wesentlichen Aspekte der einzelnen Feststellungen sind in Kapitel 6.3 aufgeführt. Detaillierte Berichte können dem Anhang (A 2 bis A 5) entnommen werden.

\subsubsection{Verhaltensbeobachtungen der Verkehrsteilnehmer im Realverkehr}

Die Verhaltensbeobachtungen der Verkehrsteilnehmer an Knotenpunkten erfolgten neben der Beobachtung durch Personen mit Hilfe eines Funk-Kamera-Systems, das für dieses Forschungsprojekt konzipiert und realisiert wurde. 
Das Kamerasystem besteht aus 4 Kameras (mit Sendeeinheiten), die jeweils auf einem Stativ befestigt werden (Abbildung 26) und einem zentralem Empfangsstativ, auf dem die Empfänger aller 4 Kameras montiert sind. Die Zentrale Aufzeichnungseinheit besteht aus einem Festplattenrekorder, der gleichzeitig die Signale der 4 Kameras aufzeichnen kann.

Die aufgezeichneten Bilder aller 4 Kameras können bei der späteren Auswertung zeitgleich und absolut synchron auf einem Monitor betrachtet werden (Abbildung 27). Bei Bedarf können die Bilder einer einzelnen Kamera bei der Auswertung jeweils zum Vollbild vergrößert werden.

Dieses Verfahren ermöglicht, dass Knotenpunkte aus vier verschiedenen Perspektiven erfasst werden können (Abbildung 28).

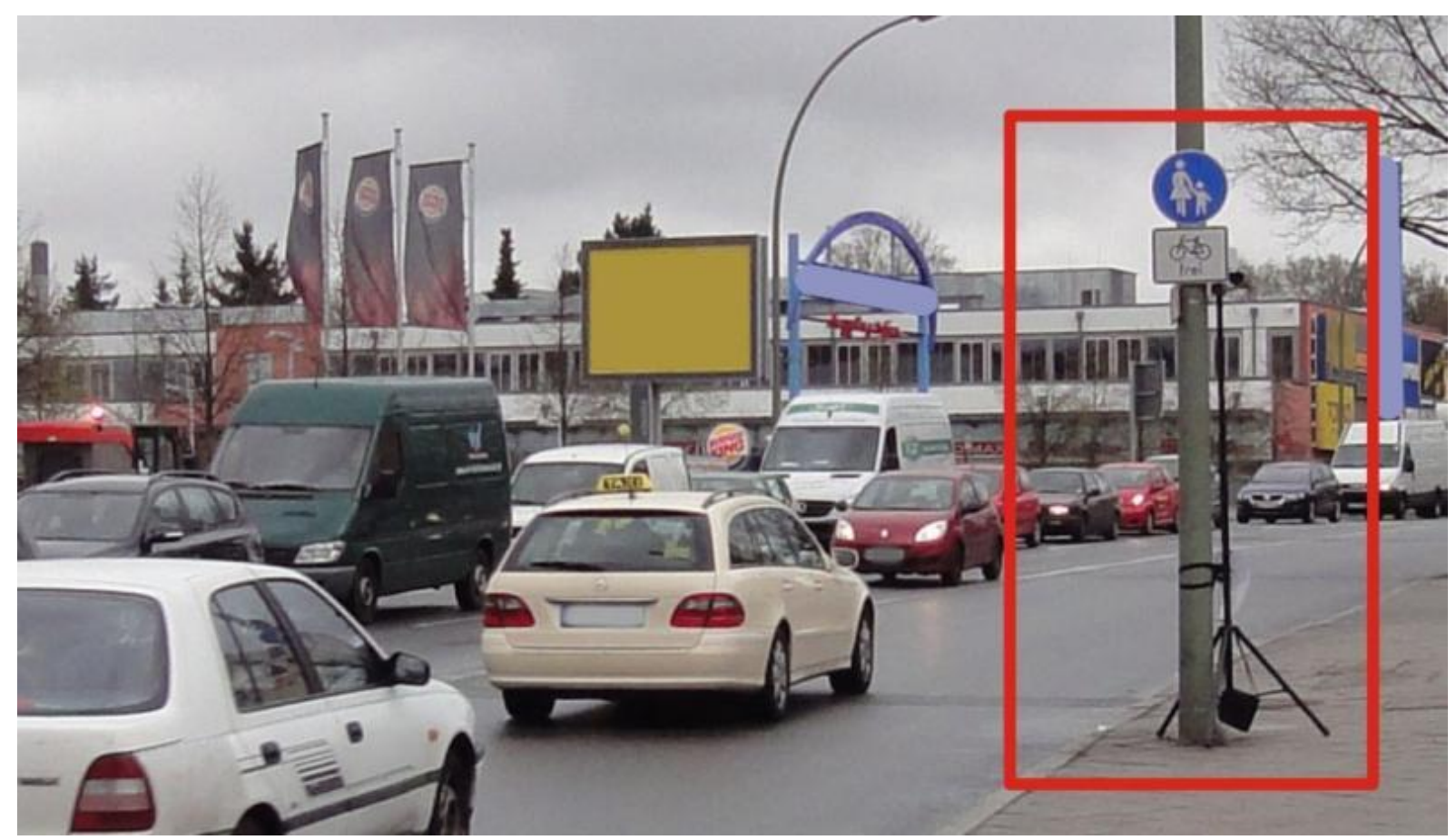

Abbildung 26: Exemplarische Darstellung eines Kamerastandortes 


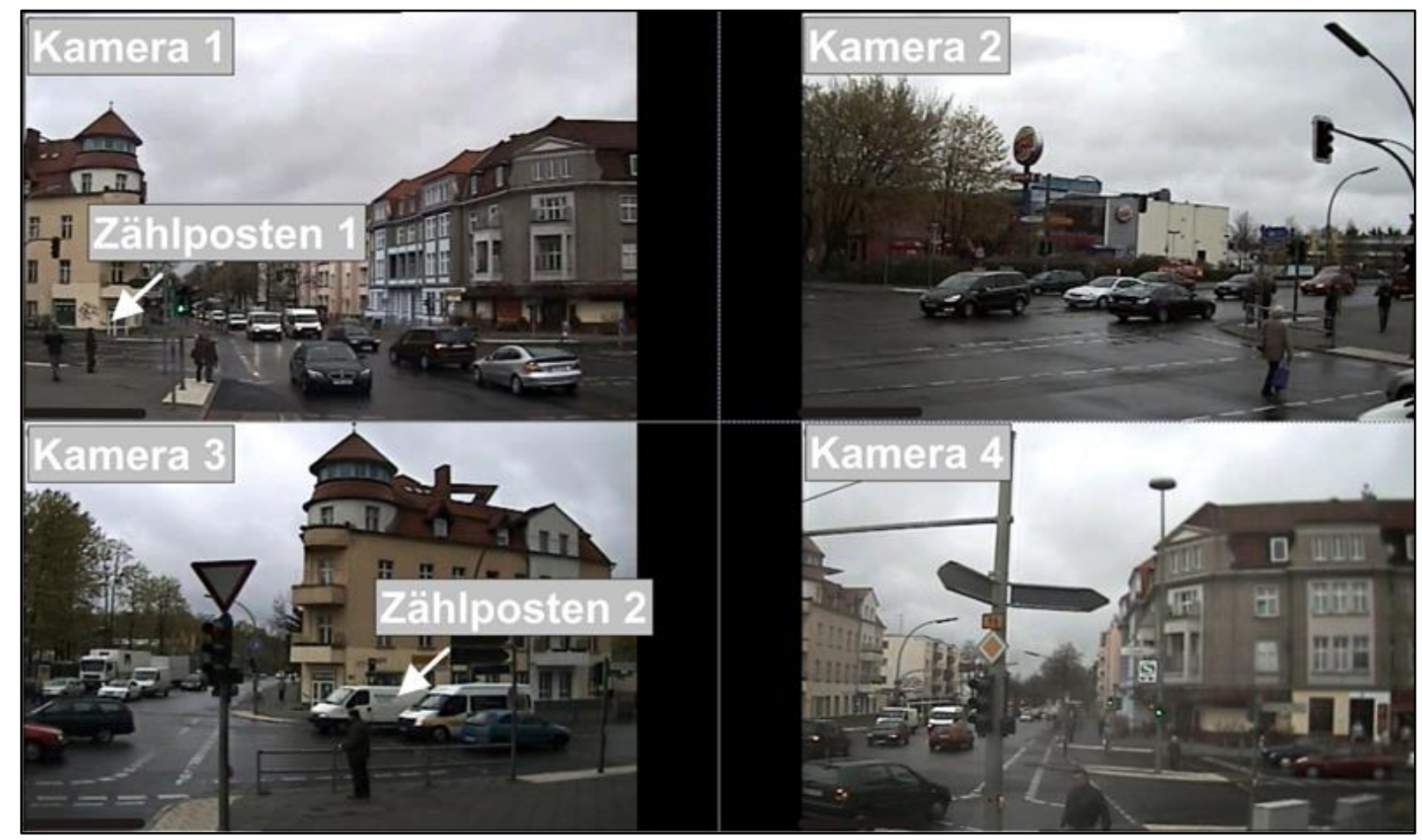

Abbildung 27: Exemplarische Darstellung der Kamerabilder

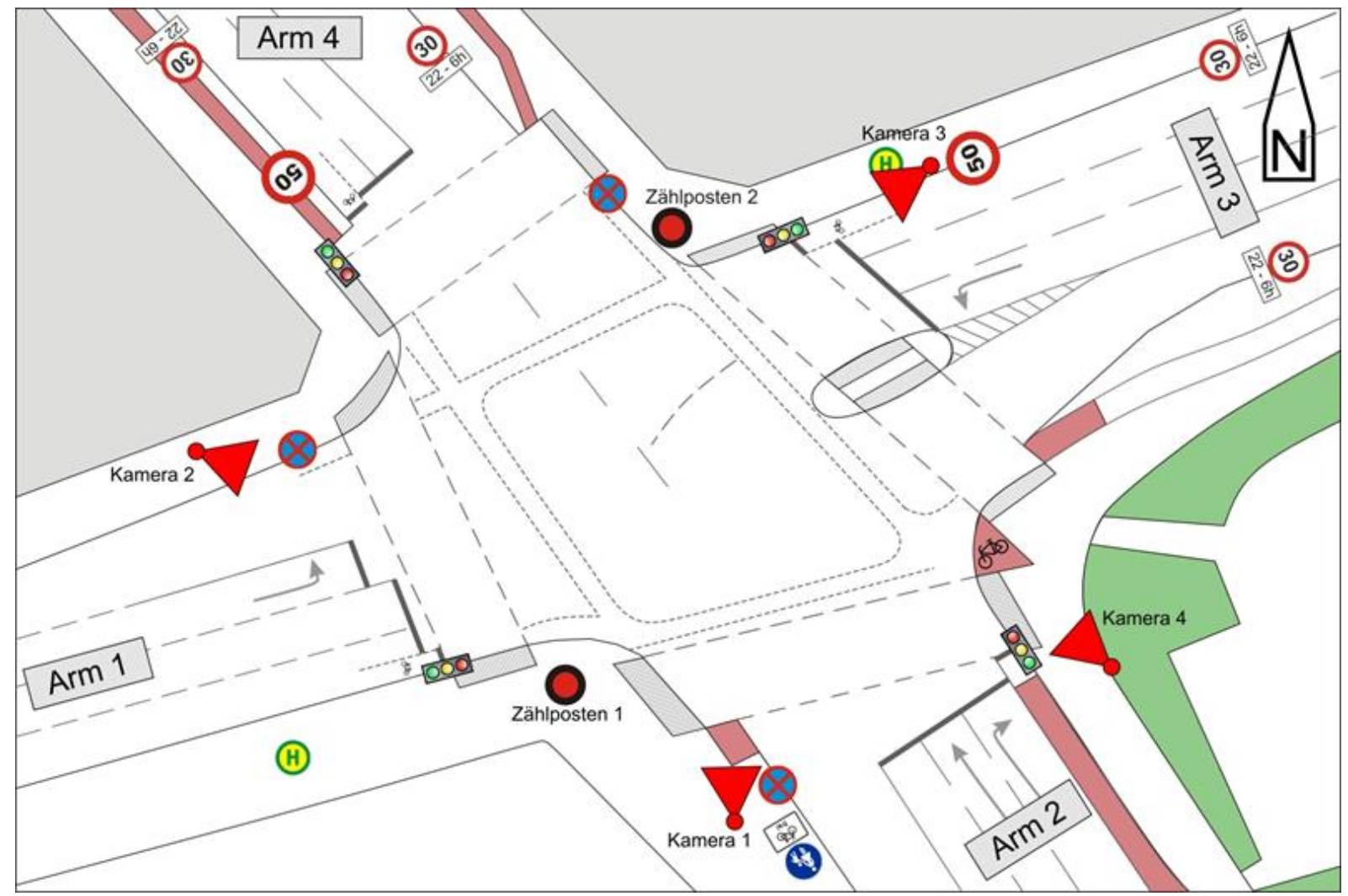

Abbildung 28: Exemplarische Darstellung der Kamerastandorte und Positionen der Zählposten für die Verhaltensbeobachtungen 
Die zahlenmäßige Auswertung des Verhaltens der Verkehrsteilnehmer erfolgte nachträglich anhand der Videobilder, einschließlich der Altersschätzung und der Einschätzung, ob eine Mobilitätsbehinderung bei einer beobachteten Person vorlag.

Die Alterseinschätzung ist im Fall von Fußgängern, unter Einschränkung der Bildqualität, in der Regel gut möglich, obwohl aus datenschutzrechtlichen Gründen die Kameraperspektiven so gewählt wurden, dass eine Identifizierung (und genaue Altersschätzung) einzelner Personen im Nachhinein nicht möglich ist. Es wurden lediglich „Übersichtaufnahmen“ durchgeführt. Das Alter von Kraftfahrern lässt sich anhand der Videobilder im Nachhinein nicht einschätzen. Aus diesem Grund positionierten sich während der Aufnahmen Zählposten für vorher bestimmte Verkehrsströme am Knotenpunkt und gaben Zeichen, wenn ein älterer Kraftfahrer / Radfahrer vorbeifuhr. Anhand dieser Zeichen konnten den im Videomaterial sichtbaren Fahrzeugen ältere Kraftfahrer zugeordnet werden. Bei den folgenden Auswertungen der Verhaltensbeobachtungen ist daher zu berücksichtigen, dass die Altersklassen nicht auf Basis des "echten“ kalendarischen Alters bestimmt werden konnten. Das Alter (bis einschließlich 14 Jahre und 65 Jahre und älter) wurde aufgrund des Aussehens vor Ort geschätzt. Aus diesem Grund wurde die Anzahl älterer Verkehrsteilnehmer tendenziell unterschätzt (wer in Wirklichkeit schon „alt“ war, aber jünger aussah, wurde nicht als „alt“ identifiziert). Ebenso sind Kinder tendenziell unterschätzt (besonders Mädchen sahen teilweise älter aus, als sie in Wirklichkeit vielleicht waren; gerade nach dem Einsetzen der Pubertät ist aufgrund der Bekleidungswahl eine genaue Schätzung schwer möglich).

Bei der späteren Auswertung der Videoaufnahmen wurde festgehalten, ob sich die beobachtete Person "StVO-konform“ verhalten hat. Dabei wurden die Maßstäbe der Straßenverkehrsordnung "streng“ angelegt. Hierdurch lassen sich auch die vielen Fehler von Fußgängern erklären, die z. B. teilweise nicht auf dem kürzesten Weg eine Fahrbahn überquert haben, wie es die StVO für Fußgänger vorsieht. Falls ein Fehler beobachtet wurde, wurde für jeden beobachteten Fehler auf einem Protokollbogen notiert, wie das Verhalten vom verkehrsgerechten Verhalten abweicht (z. B. Straßenüberquerung trotz rotem Signal). Auf dem Protokollbogen wurde natürlich ebenfalls notiert, wie viele Personen der intendierten Zielgruppen beobachtet wurden.

Außerdem wurde notiert, ob bestimmte Schwierigkeiten bei bestimmten Nutzergruppen auf dem Videomaterial erkennbar waren, die zwar nicht direkt der Straßenverkehrsordnung entgegenstehen, aber eben den subgruppenspezifischen Defiziten entsprechen.

Das Verfahren eignet sich generell auch dazu, subgruppenspezifische Schwierigkeiten und Hemmnisse zu dokumentieren, die durch die Gestaltung der Knotenpunkte ausgelöst werden können bzw. durch subgruppenspezifische Besonderheiten bedingt sein können (z. B. langes Verweilen als älterer Kraftfahrer im Linksabbiegebereich einer Kreuzung, obwohl der kreuzende Verkehr schon GRÜN signalisiert bekommt).

Insofern handelt es sich bei dem entwickelten Verfahren um eine Mischung aus einem quantitativen und qualitativen Beobachtungsverfahren. Für jede Zielgruppe können typische Schwierigkeiten / Hemmnisse ermittelt werden.

Die Videobeobachtungen fanden werktags in der Zeit zwischen 10 und 15 Uhr statt (an zwei Knotenpunkten zwischen 8 und $13 \mathrm{Uhr}$, bevor die genaue Standardisierung des Vorgehens 
bei der Beobachtung an den Knotenpunkten festgelegt wurde). Diese Zeiträume wurden aufgrund der zeitlichen Beteiligung von Kindern und Älteren an Unfällen auf Basis der Unfalldaten der 291 Knotenpunkte bestimmt (Abbildung 29 und Abbildung 30).

Zusätzlich zu den Verhaltensbeobachtungen wurden an jedem Knotenpunkt die Verkehrsmengen (alle Verkehrsteilnehmer) zwischen 10 und $11 \mathrm{Uhr}$ (vgl. Unfallzeiten von Älteren) und zwischen 13 und 14 Uhr (vgl. Unfallzeiten von Kindern) gezählt, auch um Vergleichskollektive für die spätere Auswertung bilden zu können. In diesen Zeitbereichen wurde das Verhalten aller Verkehrsteilnehmer beurteilt, das Verhalten von Kindern und Älteren wurde über den gesamten Beobachtungszeitraum beurteilt.

\begin{tabular}{|l|c|c|c|c|c|c|c|c|}
\hline beteiligte Kinder (igO) & Mo & Di & Mi & Do & Fr & Sa & So & Woche \\
\hline $0: 00$ - 0:59 & 1 & 0 & 0 & 0 & 0 & 0 & 0 & 1 \\
\hline $1: 00-1: 59$ & 0 & 0 & 0 & 0 & 0 & 0 & 0 & 0 \\
\hline $2: 00-2: 59$ & 0 & 0 & 0 & 0 & 0 & 0 & 0 & 0 \\
\hline $3: 00-3: 59$ & 0 & 0 & 0 & 0 & 0 & 0 & 0 & 0 \\
\hline $4: 00-4: 59$ & 0 & 0 & 0 & 0 & 0 & 0 & 0 & 0 \\
\hline $5: 00-5: 59$ & 0 & 0 & 0 & 0 & 0 & 0 & 0 & 0 \\
\hline $6: 00-6: 59$ & 3 & 2 & 4 & 1 & 1 & 0 & 0 & 11 \\
\hline $7: 00-7: 59$ & 10 & 10 & 7 & 15 & 11 & 0 & 0 & 53 \\
\hline $8: 00-8: 59$ & 5 & 0 & 3 & 4 & 1 & 0 & 0 & 13 \\
\hline $9: 00-9: 59$ & 1 & 0 & 1 & 1 & 1 & 1 & 1 & 6 \\
\hline $10: 00-10: 59$ & 2 & 0 & 0 & 4 & 0 & 1 & 0 & 7 \\
\hline $11: 00-11: 59$ & 5 & 1 & 0 & 4 & 1 & 0 & 2 & 13 \\
\hline $12: 00-12: 59$ & 5 & 3 & 4 & 4 & 5 & 4 & 1 & 26 \\
\hline $13: 00-13: 59$ & 10 & 10 & 12 & 3 & 9 & 2 & 1 & 47 \\
\hline $14: 00-14: 59$ & 8 & 8 & 11 & 5 & 6 & 2 & 1 & 41 \\
\hline $15: 00-15: 59$ & 9 & 9 & 7 & 10 & 12 & 2 & 0 & 49 \\
\hline $16: 00-16: 59$ & 6 & 7 & 10 & 12 & 11 & 0 & 2 & 48 \\
\hline $17: 00-17: 59$ & 5 & 7 & 5 & 8 & 1 & 6 & 1 & 33 \\
\hline $18: 00-18: 59$ & 4 & 7 & 2 & 4 & 4 & 4 & 1 & 26 \\
\hline $19: 00-19: 59$ & 2 & 3 & 2 & 2 & 4 & 2 & 1 & 16 \\
\hline $20: 00-20: 59$ & 0 & 1 & 0 & 1 & 1 & 0 & 0 & 3 \\
\hline $21: 00-21: 59$ & 0 & 1 & 0 & 0 & 2 & 0 & 1 & 4 \\
\hline $22: 00-22: 59$ & 0 & 0 & 0 & 0 & 1 & 0 & 0 & 1 \\
\hline $23: 00-23: 59$ & 0 & 0 & 0 & 0 & 0 & 2 & 0 & 2 \\
\hline
\end{tabular}

Abbildung 29: Beteiligte Kinder an Unfällen innerorts ( $n=400$ Beteiligte) 


\begin{tabular}{|c|c|c|c|c|c|c|c|c|}
\hline beteiligte Ältere (igO) & Mo & $\mathrm{Di}$ & $\mathrm{Mi}$ & Do & $\mathrm{Fr}$ & $\mathrm{Sa}$ & So & Woche \\
\hline $0: 00-0: 59$ & 0 & 3 & 1 & 0 & 0 & 1 & 4 & 9 \\
\hline $1: 00-1: 59$ & 0 & 0 & 0 & 1 & 1 & 1 & 0 & 3 \\
\hline $2: 00-2: 59$ & 0 & 0 & 0 & 0 & 0 & 0 & 0 & 0 \\
\hline 3:00 - 3:59 & 1 & 0 & 0 & 0 & 0 & 0 & 0 & 1 \\
\hline 4:00-4:59 & 0 & 1 & 0 & 0 & 0 & 0 & 1 & 2 \\
\hline 5:00- 5:59 & 0 & 0 & 0 & 1 & 1 & 0 & 0 & 2 \\
\hline 6:00- 6:59 & 1 & 0 & 3 & 2 & 3 & 2 & 0 & 11 \\
\hline 7:00 - 7:59 & 5 & 3 & 5 & 10 & 4 & 2 & 0 & 29 \\
\hline $8: 00-8: 59$ & 7 & 7 & 17 & 8 & 5 & 4 & 4 & 52 \\
\hline 9:00 - 9:59 & 20 & 14 & 27 & 18 & 19 & 17 & 3 & 118 \\
\hline $10: 00$ - 10:59 & 39 & 23 & 20 & 24 & 17 & 18 & 8 & 149 \\
\hline $11: 00$ - 11:59 & 31 & 32 & 23 & 22 & 30 & 13 & 9 & 160 \\
\hline $12: 00$ - $12: 59$ & 19 & 15 & 14 & 15 & 10 & 11 & 12 & 96 \\
\hline $13: 00-13: 59$ & 10 & 17 & 19 & 15 & 23 & 14 & 6 & 104 \\
\hline $14: 00$ - 14:59 & 14 & 15 & 17 & 15 & 12 & 9 & 15 & 97 \\
\hline $15: 00$ - 15:59 & 20 & 13 & 20 & 22 & 25 & 7 & 5 & 112 \\
\hline $16: 00$ - $16: 59$ & 8 & 10 & 12 & 17 & 14 & 11 & 7 & 79 \\
\hline $17: 00$ - 17:59 & 16 & 11 & 13 & 20 & 8 & 16 & 15 & 99 \\
\hline $18: 00$ - 18:59 & 17 & 18 & 8 & 7 & 9 & 6 & 6 & 71 \\
\hline $19: 00$ - 19:59 & 12 & 8 & 10 & 7 & 4 & 5 & 8 & 54 \\
\hline 20:00 - 20:59 & 3 & 5 & 3 & 1 & 3 & 4 & 2 & 21 \\
\hline $21: 00$ - 21:59 & 1 & 3 & 2 & 0 & 2 & 1 & 4 & 13 \\
\hline $22: 00$ - 22:59 & 1 & 2 & 2 & 4 & 2 & 2 & 1 & 14 \\
\hline $23: 00$ - 23:59 & 1 & 1 & 3 & 1 & 1 & 6 & 0 & 13 \\
\hline
\end{tabular}

Abbildung 30: Beteiligte Ältere an Unfällen innerorts ( $n=1.309$ Beteiligte)

\section{2 Überblick über das Unfallgeschehen an den ausgewählten Knotenpunkten}

Zu den 15 Knotenpunkten der Untersuchung lagen Unfälle für die in aufgeführten Zeitbereiche in Form anonymisierter Verkehrsunfallanzeigen (VUA) und aus Unfallisten vor ${ }^{17}$ (Tabelle 10). Insgesamt entfielen 259 Unfälle auf die 15 Knotenpunkte. Abbildung 31 zeigt die Verteilung auf die Unfalltypen, Tabelle 11 zeigt die anteilsmäßige Verteilung je Unfallkategorie.

Die prozentuale Verteilung der Unfalltypen weicht von der Verteilung der Unfalltypen der rd. 285.000 Innerortsunfälle der makroskopischen Untersuchung ab. Hier war der Unfalltyp 3 am häufigsten vertreten. Dies kann dadurch begründet sein, dass die Auswahl der zu untersuchenden Knotenpunkte und somit auch der Unfälle an diesen, unter besonderer Berücksichtigung von Unfällen mit Kindern und/oder Älteren erfolgte und somit z. B. Überschreiten-Unfälle in der Auswahl der 15 Knotenpunkte überrepräsentiert sind. Insgesamt nehmen die drei, für Kinder und ältere Menschen typischen Unfälle (Abbiege-, Einbiegen/Kreuzen- und Überschreiten-Unfälle), aber einen vergleichbaren Anteil von etwa $83 \%$, gegenüber rd. $75 \%$ bei der makroskopischen Betrachtung von Unfällen an Knotenpunkten, ein.

Auf die wesentlichen Aspekte des Unfallgeschehens der jeweiligen Knotenpunkttypen wird in Abschnitt 6.3 eingegangen, detaillierte Informationen enthalten die Berichte zu den einzelnen Knotenpunkten im Anhang (A 2 bis A 5)

${ }^{17}$ Ausnahme: Knotenpunkt 11. Hier konnten ausschließlich Unfalllisten zur Verfügung gestellt werden. 
Tabelle 10: Anzahl der Unfälle und Auswertungszeiträume

\begin{tabular}{|l|l|l|l|}
\hline Knotenpunkt Nr. & Zeitraum & Jahre & Anzahl Unfälle (nur Kategorie 1 bis 4) \\
\hline 1 & $2006-2010$ & 5 & 20 \\
\hline 2 & $2007-2007$ & 4 & 14 \\
\hline 3 & $2006-2010$ & 5 & 26 \\
\hline 4 & $2006-2009$ & 4 & 18 \\
\hline 5 & $2008-2010$ & 3 & 10 \\
\hline 6 & $2006-2010$ & 5 & 42 (für Teilbereich des Knotenpunktes) \\
\hline 7 & $2007-2010$ & 4 & 19 \\
\hline 8 & $2006-2010$ & 5 & 12 \\
\hline 9 & $2006-2010$ & 5 & 26 \\
\hline 10 & $2006-2009$ & 4 & 12 \\
\hline 11 & $2004-2009$ & 6 & 21 \\
\hline 12 & $2006-2010$ & 5 & 8 (keine Unfälle in 2009 und 2010) \\
\hline 13 & $2004-2010$ & 6 & 14 (VUA für 11 Unfälle aus 2006 - 2010, zudem 3 \\
\hline 14 & & & Unfälle aus Unfalllisten) \\
\hline 15 & $2006-2010$ & 5 & 12 (keine Unfälle in 2010) \\
\hline
\end{tabular}

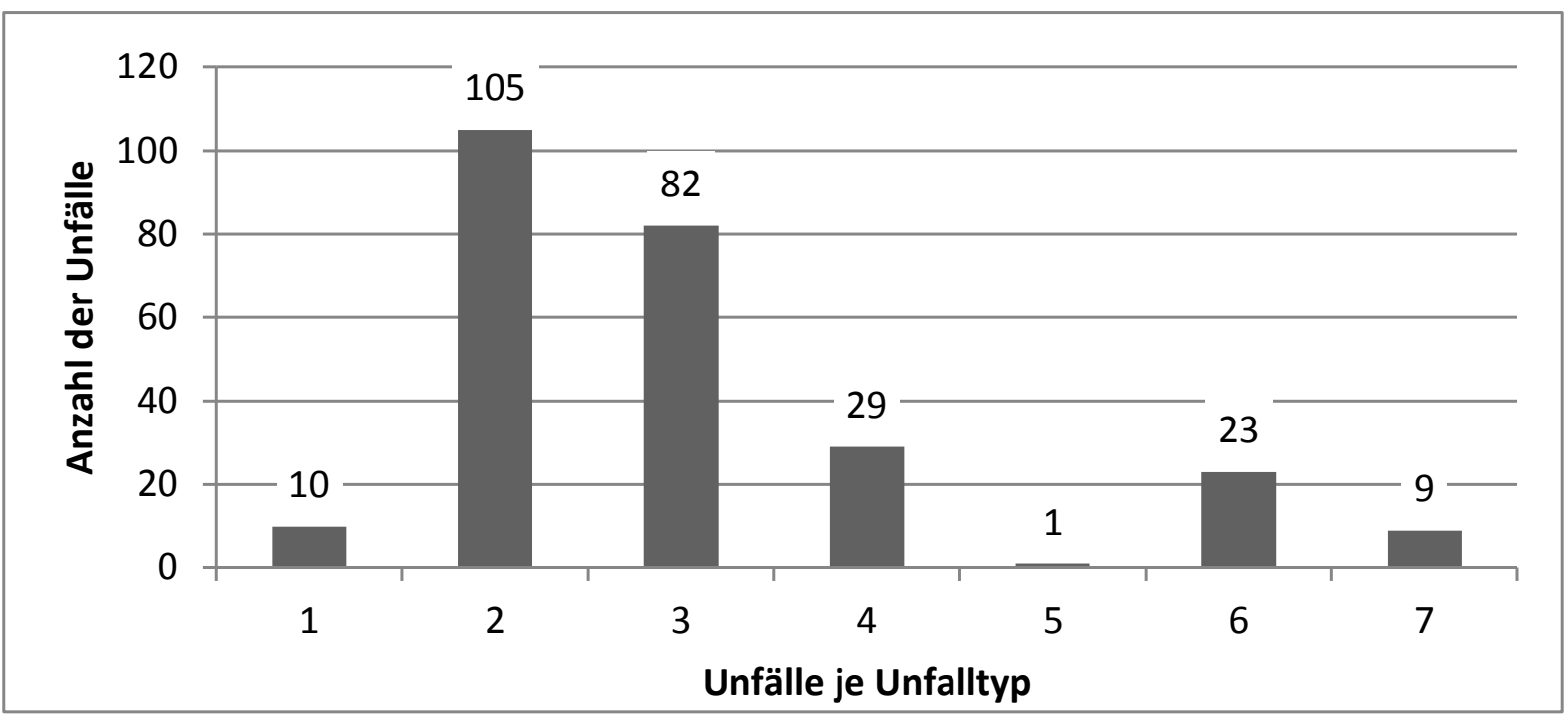

Abbildung 31: Unfälle je Unfalltyp der 15 ausgewählten Knotenpunkte $(n=259)$

Tabelle 11: Prozentuale Verteilung der Unfallkategorien je Unfalltyp für die Unfälle an den 15 ausgewählten Knotenpunkte $(n=259)$

\begin{tabular}{|c|r|r|r|r|r|r|r|r|}
\hline \multirow{2}{*}{ Unfallkategorie } & \multirow{2}{*}{$\begin{array}{c}\text { Unfälle } \\
\text { insgesamt }\end{array}$} & \multicolumn{8}{|c|}{ Unfalltypen (Anteile je Zeile in \%) } \\
\cline { 3 - 9 } & 1 & \multicolumn{1}{|c|}{2} & \multicolumn{1}{c|}{3} & 4 & 5 & \multicolumn{1}{c|}{6} & 7 \\
\hline 1 & 1 & 0,0 & 0,0 & 100,0 & 0,0 & 0,0 & 0,0 & 0,0 \\
\hline 2 & 23 & 13,0 & 34,8 & 8,7 & 26,1 & 4,4 & 13,0 & 0,0 \\
\hline 3 & 174 & 1,7 & 43,7 & 27,0 & 13,2 & 0,0 & 9,2 & 5,2 \\
\hline 4 & 61 & 6,6 & 34,4 & 52,5 & 0,0 & 0,0 & 6,6 & 0,0 \\
\hline Summe & 259 & 3,9 & 40,5 & 31,7 & 11,2 & 0,4 & 8,9 & 3,5 \\
\hline
\end{tabular}

Zum Vergleich die prozentuale Verteilung auf Basis der 285.526 Innerortsunfälle der makroskopischen

Betrachtung

Summe

285.526

\begin{tabular}{l|l}
6,9 & 25,4 \\
\hline
\end{tabular}

44,3

4,9

1,1

12,7 


\section{3 Überblick über die Sicherheitsanalysen}

Die Sicherheitsanalysen wurden an insgesamt 15 Knotenpunkten durchgeführt. Abbildung 32 zeigt die Anzahl der Knotenpunkte, bei denen Defizite in einer der folgenden Kategorien festgestellt wurden:

- Defizite/Sicherheitsrisiken im Bereich der Fußgängerverkehrsanlagen, z. B. fehlende Überquerungsanalgen, nicht regelwerkskonforme Ausführung von Teilen von Überquerungsanlagen

- Defizite/Sicherheitsrisiken im Bereich der Radverkehrsanlagen, z. B. nicht regelwerkskonforme Ausführung, fehlende Radverkehrsanlagen

- Defizite/Sicherheitsrisiken im Bereich der barrierefreien Gestaltung, z. B. fehlende Bodenindikatoren (Belange Menschen mit Sehbehinderungen), keine Nullabsenkung (Belange von Menschen mit besonderer Mobilitätseinschränkung).

- Defizite/Sicherheitsrisiken im Bereich der Sichtbeziehungen, z. B. eingeschränkte Anfahrsicht, Sichtbehinderungen durch Straßenbegleitgrün oder ruhenden Verkehr

- Defizite/Sicherheitsrisiken im Bereich der Verkehrsregelung, z.B. signaltechnisch nicht gesichert geführter Linksabbieger bei hohen Verkehrsmengen und/oder fehlenden Führungshilfen im Kreuzungsbereich, Grünpfeil (Z 720, vgl. Anlage A 1) bei weit abgesetzten Haltlinien

- Defizite/Sicherheitsrisiken im Bereich der Verkehrsführung, z. B. Linksabbieger und Geradeausfahrer auf einem gemeinsamen Fahrstreifen bei mehr als einem Fahrstreifen der Zufahrt, Fahrstreifensubtraktion unmittelbar nach dem Kreuzungsbereich, fehlende Leitmarkierungen und/oder Wartemarkierungen im Kreuzungsbereich für Linksabbieger

- Defizite/Sicherheitsrisiken im Bereich der Straßengeometrie und des Entwurfes, z. B. zu geringe/zu große Fahrstreifenbreite, spitze Kreuzungswinkel der Straßen (z. B. wenn daraus Sichtbehinderungen oder Konflikte durch missverstandene Vorfahrtregelung entstanden)

- Defizite im Bereich der Verkehrszeichen, z.B. schlechter Zustand von Markierungen, nicht regelwerkskonforme Ausführung von Markierungen

- Defizite im Bereich der Signalsteuerung (Einzelfall: geringe Zwischenzeit)

Die festgestellten Defizite wurden nachträglich in die aufgeführten Defizitkategorien eingeordnet. In dieser Darstellung sind nur Defizite berücksichtigt, die nach evtl. Umbaumaßnahmen der Verkehrsanlage noch festgestellt wurden. Sie beschreiben somit die Situation zum Zeitpunkt der Vor-Ort-Begehungen. 


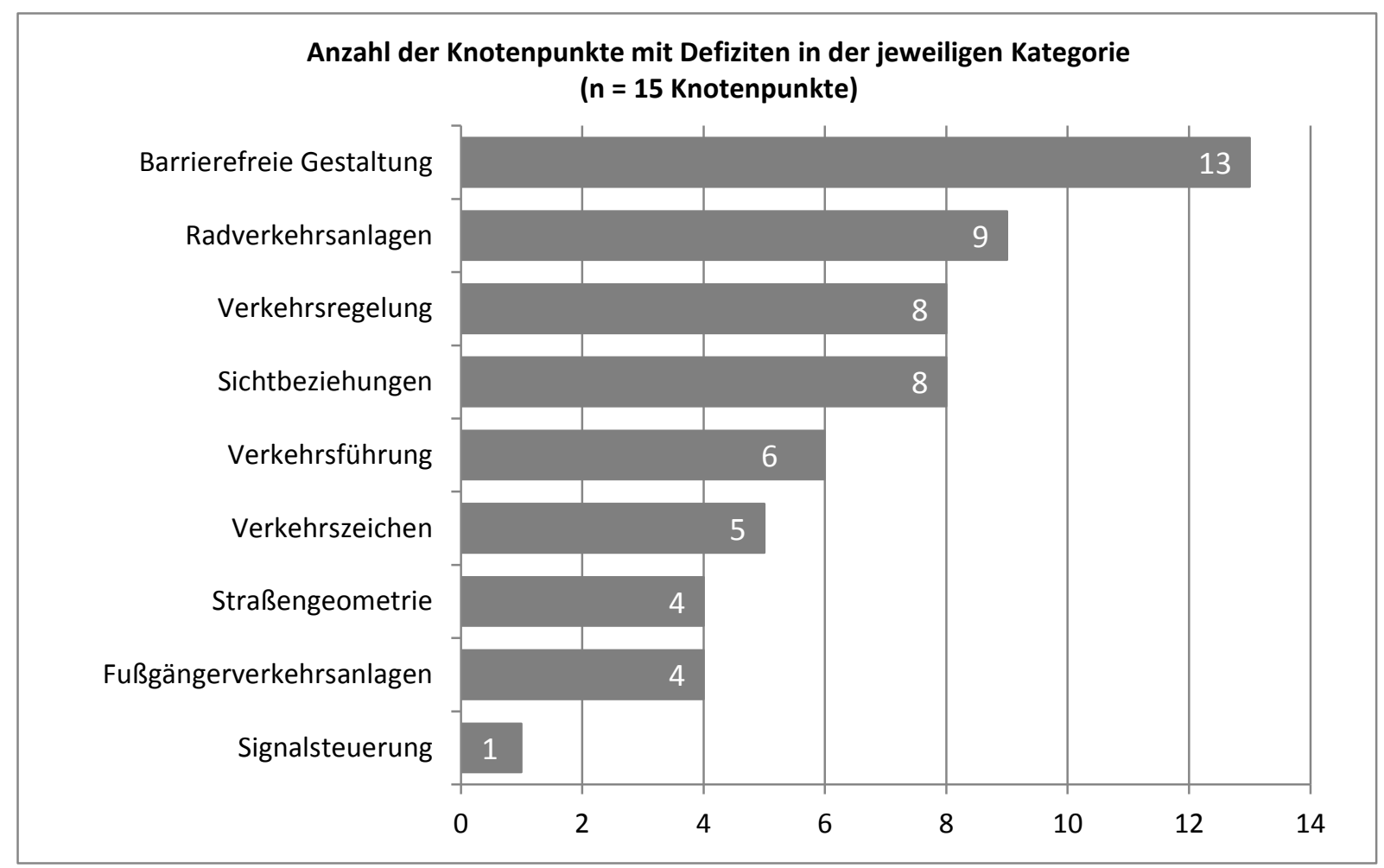

Abbildung 32: Anzahl der Knotenpunkte mit Defiziten je Kategorie ( $\mathrm{n}=15$ Knotenpunkte)

Nahezu an allen Knotenpunkten (13 von 15) waren die Belange von Menschen mit besonderer Mobilitätseinschränkung (z. B. Menschen mit Rollator oder Kinderwagen) oder Mobilitätsbehinderung (z. B. blinde Menschen oder Rollstuhlfahrer) nicht oder nicht ausreichend berücksichtigt.

Verkehrsanlagen für den Radverkehr waren oft ebenfalls in nicht regelwerkskonformer Ausführung oder fehlten vollständig ${ }^{18}$ An 9 der insgesamt 15 Knotenpunkte konnten diesbezüglich Defizite festgestellt werden.

Weitere häufig festgestellte Defizite betrafen Mängel in der Verkehrsregelung oder der Verkehrsführung des Kfz-Verkehrs.

An insgesamt 8 der 15 Knotenpunkte konnten Defizite im Bereich der Sichtbeziehungen festgestellt werden.

${ }^{18}$ Als Defizit erfasst, wenn die Empfehlungen für Radverkehrsanlagen (ERA 2010) bei bestimmten Randbedingungen Schutzstreifen, Radfahrstreifen oder Radwege empfehlen (vgl. ERA 2010, S. 18 f.). 


\section{4 Überblick über die Verhaltensbeobachtungen}

Insgesamt wurden etwa 300 Stunden Videomaterial an den 15 beobachteten Knotenpunkten aufgenommen und anschließend im Detail ausgewertet. Dabei wurde das Verhalten von 24.598 Einzelpersonen als Fußgänger, Radfahrer oder Pkw-Fahrer unter Berücksichtigung der Altersgruppe analysiert.

Generell konnte bei allen Verkehrsteilnehmern eine hohe Regelkonformität beobachtet werden. In der Gruppe der Fußgänger und Radfahrer machten Kinder relativ gesehen mehr Fehler als Personen anderer Altersgruppen (vgl. Abbildung 35 und Abbildung 38). Ältere wie jüngere Kraftfahrer begingen prinzipiell qualitativ dieselben Fehler. Allerdings machten älterer Kraftfahrer beim Linksabbiegen relativ mehr Fehler als jüngere Kraftfahrer (vgl. Abbildung 44).

\subsection{Sicherheitsanalysen und Verhaltensbeobachtungen nach Gruppe und Verkehrssituation}

Im Folgenden sind die Ergebnisse der Sicherheitsanalysen und der Verhaltensbeobachtungen der 15 Knotenpunkte für die Gruppen

- Fußgänger

- Radfahrer

- Menschen mit besonderen Mobilitätseinschränkungen (z. B. Menschen mit Rollatoren, Kinderwagen) oder Mobilitätsbehinderungen (z. B. Blinde, Rollstuhlfahrer) und

- Kraftfahrer

zusammenfassend dargestellt.

Die Kurzberichte der einzelnen Knotenpunkte sind in den Anlagen A 2 bis A 5 aufgeführt, die detaillierte Auswertung der Verhaltensbeobachtungen nach Art der Verkehrsbeteiligung und Knotenpunkttyp enthält die Anlage A 6.

Zudem ist in Kapitel 6.6 eine zusammenfassende Darstellung der wesentlichen Aspekte je Knotenpunkttyp beschrieben. 


\subsubsection{Fußgänger}

Überschreiten-Unfälle und Abbiege-Unfälle sind die häufigsten Unfalltypen bei denen Fußgänger an Knotenpunkten gefährdet werden bzw. verunglücken.

Sicherheitsrisiken ergeben sich für Fußgänger insbesondere an Knotenpunkten ohne Lichtsignalregelung. Vor allem dann, wenn Überquerungsanlagen (auch Teile davon) nicht dem Stand der Technik entsprechen oder fehlen.

Ebenso sind Fußgänger - speziell Kinder - gefährdet, wenn Sichtbeziehungen nicht gegeben oder z. B. durch parkende Fahrzeuge am Fahrbahnrand eingeschränkt sind. Dies gilt besonders auch für Rollstuhlfahrer.

An Knotenpunkten ohne Lichtsignalregelung, mit einem deutlichen Hauptstrom, ergeben sich weitere Schwierigkeiten für Fußgänger. Die Zeitlücken im Hauptstrom müssen zeitgleich von einbiegenden, ggf. auch wendenden, Fahrzeugen und überquerenden Fußgängern genutzt werden. Den Fußgängern wird dabei ihr Vorrangsrecht gegenüber den einbiegenden $\mathrm{Kfz}$ oft nicht eingeräumt (Abbildung 33).

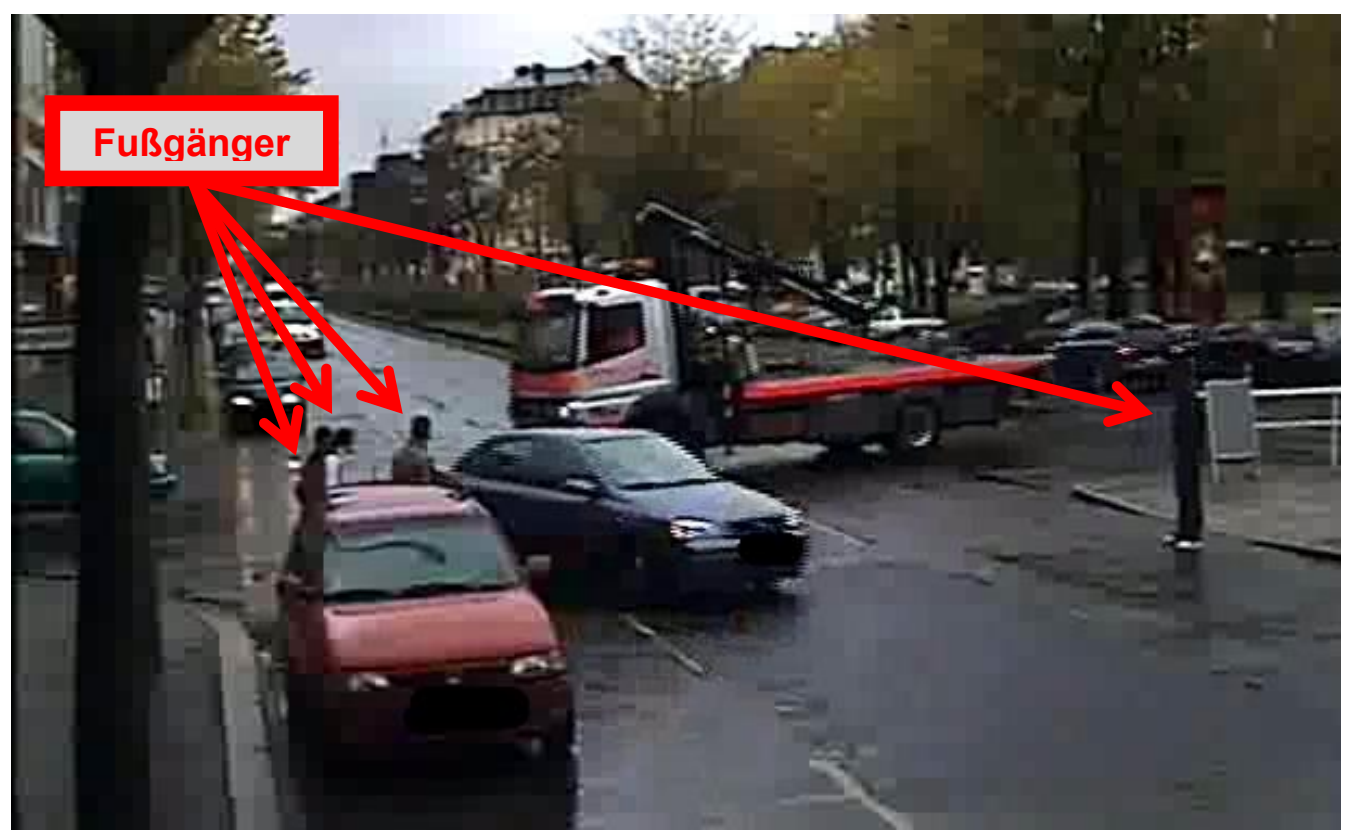

Abbildung 33: Einbiegende (und wendende) Kraftfahrer missachten Vorrangsrecht überquerender Fußgänger

Zur Hervorhebung der Bevorrechtigung und zur Sicherheit von Fußgängern gegenüber einbiegenden und ggf. wendenden Fahrzeugen sollten an Knotenpunkten mit Lichtsignalanlage die Hilfssignalgeber (gelbes Blicklicht an Fußgängerfurt, Abbildung 34 und Abbildung 62, Seite 154) einen Durchmesser von 300 statt 200 mm aufweisen. 


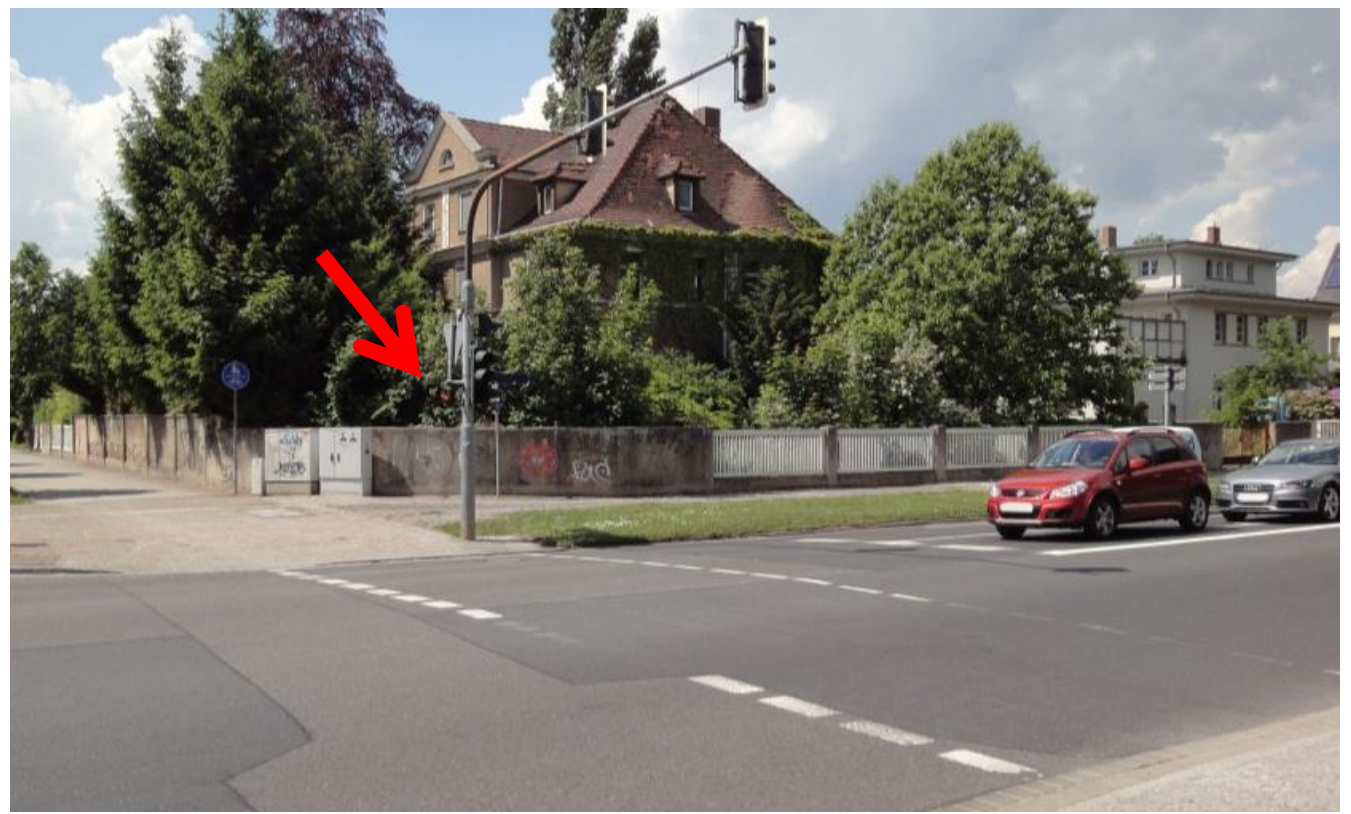

Abbildung 34: Hilfssignalgeber (gelbes Blinklicht) mit 200 mm Durchmesser an Fußgänger Furt schlecht zu erkennen

Im Weiteren sollten an Knotenpunkten mit Lichtsignalanlagen geringe Wartezeiten für Fußgänger eingehalten werden. Insbesondere dann, wenn die Überquerung der Straße auch zum Erreichen von Haltestellen des ÖV dient und/oder Schulwege über den Knotenpunkt führen. Auch wenn keine Konflikte zwischen „Rotläufern“ und kreuzenden Kfz an den 15 Knotenpunkten beobachtet werden konnten, stieg die Zahl der „Rotläufer“ bei längeren Wartezeiten, insbesondere an Knotenpunkten in der Nähe von ÖV-Haltestellen.

Im Rahmen der Verhaltensbeobachtungen wurden insgesamt 9.474 Überquerungen von Fußgängern ausgewertet. Am regelkonformsten verhielten sich ältere Menschen, gefolgt von den 15 bis 64-Jährigen. Kinder machten relativ gesehen, die meisten Fehler beim Überqueren der Straße.

Schwere Verstöße wurden sehr selten beobachtet. Das meiste nicht StVO-konforme Verhalten bezog sich darauf, die Fahrbahn nicht auf dem allerkürzesten Weg überquert zu haben oder nicht immer auf der Furt gegangen zu sein. Acht Fälle, in denen nicht beurteilt werden konnte, ob das Verhalten korrekt war, sind in der folgenden Grafik (Abbildung 35) nicht gesondert aufgeführt, sondern unter „korrekt“ berücksichtig. 


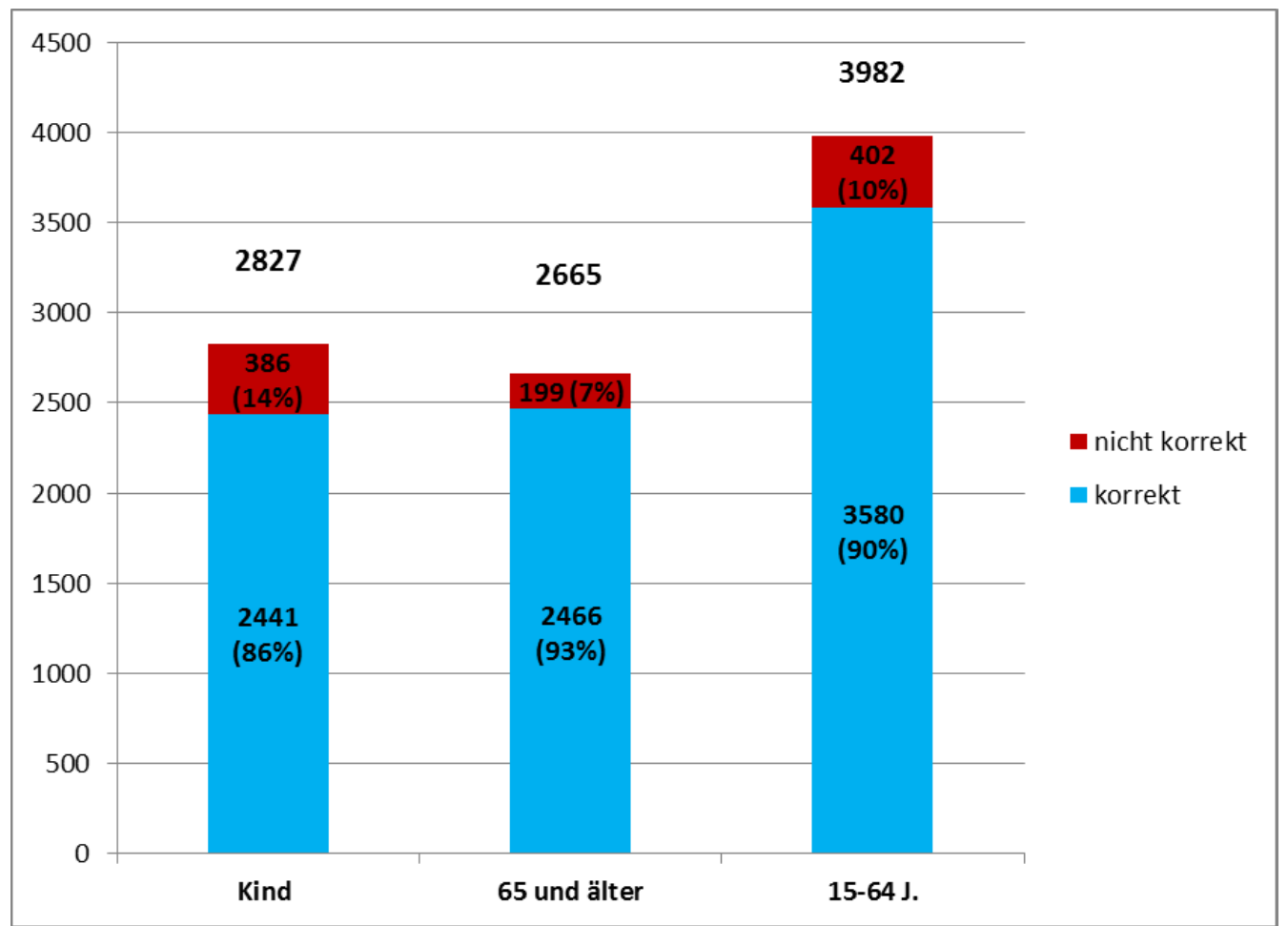

Abbildung 35: Gesamtüberblick Fußgängerverhalten an 15 Knotenpunkten 


\subsubsection{Menschen mit besonderen Mobilitätseinschränkungen oder Mobilitätsbehinderungen}

Im Rahmen der durchgeführten Videobeobachtungen konnte nur eine kleine Zahl von Menschen mit Behinderungen (bzw. Menschen mit Mobilitätseinschränkungen) beobachtet werden. Die Beobachtungen stimmen aber mit Beobachtungen Dritter überein (s. Abschnitt 6.5.2.2), so dass bisherige Thesen zur barrierefreien Gestaltung weiter gefestigt werden können.

\subsubsection{Auswertung der Videobeobachtungen}

Bei der Auswertung der Videos ergaben sich für die Gestaltung von Knotenpunkten zusammenfassend folgende Hinweise aus Sicht der Barrierefreiheit:

- Rollatornutzer betreten/verlassen den Gehweg bevorzugt an Stellen, an denen der Bord auf Fahrbahnniveau abgesenkt wurde. Besteht keine eigene Absenkung im Fußgängerbereich, aber eine Absenkung an einem parallelen Radweg, dann wird diese genutzt. Dieses Verhalten lässt sich auch bei Kinderwagen beobachten. Es wird auch für Rollstuhlnutzer bestätigt (vgl. Abschnitt 3.2.5.5, Tabelle 4). Dadurch können Konflikte mit dem Radverkehr entstehen und ebenfalls mit abbiegenden Kfz, wenn die Kfz-Lenker nicht mit Rollstuhlnutzern o. Ä. in diesen Bereichen rechnen.

- Bei Abbiegevorgängen kürzen Rollatornutzer (oder andere „Rollende“) über die Fahrbahn im Bereich des Knotenpunktes ab, wenn keine Absenkungen der Bordkante vorhanden sind. Hierdurch können Konflikte mit dem Kfz-Verkehr entstehen (vgl. Tabelle 4, Nr. 5).

- Je kleiner der Raddurchmesser „rollender“ Fußgänger (Kinderwagen, Rollatoren, Rollstuhl) ist, desto größer die Probleme an Bordkanten bei der Überquerung. Das ist insoweit von Interesse, da es keine Standardisierung bei Raddurchmessern für Rollatoren oder Rollstühlen gibt. Eine große Gruppe von Rollatornutzern hat Schwierigkeiten, ihr technisches Hilfsmittel anzukippen, um derartige Kanten einfacher überwinden zu können.

- „Rollende“ Fußgänger bevorzugen ebene Oberflächen. Es wurde beobachtet, dass bei unbefestigten Oberflächen ein Ausweichen auf die Fahrbahn stattfindet.

Vor allem eine Absenkung von Bordsteinkanten im Bereich von Überquerungsstellen ist für eine stetig wachsende Zahl von Fußgängern mit „rollenden Hilfsmitteln“ relevant. Aus Sicht von blinden und sehbehinderten Menschen besteht dann allerdings die Gefahr, dass bei Absenkung des Bords auf Fahrbahnniveau die Sicherheit an Knotenpunkten nicht mehr gewährleistet ist (fehlende Bordsteinkante als eindeutige Grenze zwischen Fahrbahn und Gehweg). Die Oberflächengestaltung spielt bei der Wahl der Verkehrsfläche ebenfalls eine Rolle. „Rollende“ nutzen bevorzugt glatte und ebene Oberflächen, da sie sich auf diesen einfacher fortbewegen können.

\subsubsection{Verhaltensbeobachtungen und Untersuchungen Dritter}

Im Rahmen von verschiedenen Vorhaben Dritter wurden Videobeobachtungen von Überquerungsvorgängen behinderter Menschen und Tests mit behinderten Probanden zur Funktionalität von Bodenindikatoren durchgeführt, die Hinweise auf eine verkehrssichere Gestaltung von Knotenpunkten liefern. 


\subsection{Hessische Straßen- und Verkehrsverwaltung}

Die Hessische Straßen- und Verkehrsverwaltung hat im Jahre 2006 den Leitfaden „Unbehinderte Mobilität“ herausgebracht. Dort wurden u. a. Hinweise zur Gestaltung von Überquerungsstellen unter Berücksichtigung der Anforderungen insbesondere gehbehinderter und sehgeschädigter Menschen gegeben. Im Jahr 2010 wurden im Rahmen einer kleinen Evaluationsstudie ergänzend Videobeobachtungen an Überquerungsstellen, die nach den Hinweisen des Leitfadens umgebaut worden waren, durchgeführt. Die Erfahrungen wurden in einem Ergänzungsband zum Leitfaden veröffentlicht (Hessische Straßen- und Verkehrsverwaltung - HSVV 2010). Zusammenfassend wurde Folgendes beobachtet (Auszug):

- Gehbehinderte Menschen nutzen die Nullabsenkung;

- Bodenindikatoren können eine wichtige Hilfe bei der Orientierung sein; die Unterscheidung der Strukturen (Noppe, Rippe) scheint für einige Nutzer schwierig zu sein;

- Lichtsignalanlagen sind für blinde und stark sehbehinderte Menschen eine Hilfe bei der Orientierung;

- Der Lichtsignalmast steht idealerwiese in der Mitte der Furt, um von allen Nutzern sicher aufgefunden und bedient (Anforderungstaster) werden zu können;

- Die Auffindbarkeit des Lichtsignalmastes lässt sich durch Auffindestreifen deutlich verbessern.

\subsection{Tests zur Erkennbarkeit und Berollbarkeit von Bodenindikatoren beim Hamburger Verkehrsverbund}

Der HVV hat eine kleine Testreihe (36 Personen) zur Erkennbarkeit und Berollbarkeit von Bodenindikatoren durchgeführt (Hamburger Verkehrsverbund $\mathrm{GmbH}$ 2011). Hintergrund war einerseits die Entwicklung bei den Langstöcken von den feinen Metallspitzen zu den größeren Kugelrollspitzen, welche gröbere Strukturen erforderlich machen. Andererseits häufen sich Rückmeldungen von Rollstuhl- und Rollatornutzern, dass die teils großflächig verlegten Bodenindikatoren ein Hindernis bei der Überrollung darstellen können. Die Ergebnisse der Tests lassen sich wie folgt zusammenfassen:

- Noppenplatten mit markanterer und dicht besetzter Struktur sind für Nutzer eines Langstocks einfacher zu ertasten (z. B Kegelstümpfe, 50 Noppen); Noppen mit weniger markanter Struktur mit geringer Dichte (z. B. Kugelschalen, 32 Noppen) geben nur eine schwache Rückmeldung;

- Für Rippenplatten mit einem Achsabstand von 40 mm wurden von Langstocknutzern die besten Noten hinsichtlich Erkennbarkeit und Handling vergeben;

- Für Rollstuhl- und Rollatornutzer sind Rippenstrukturen mit wachsendem Rippenabstand (im Rahmen der vorgegebenen DIN) schwieriger zu befahren; beim maximalen Achsabstand gemäß technischem Regelwerk rütteln Rollstuhl und Rollator stark; dies kann u. U. Spastiken auslösen;

- Bei dicht besetzten Noppenstrukturen spielt die Art der Noppen (Kugelschalen, Kegelstümpfe) für Nutzer von Rollatoren oder Rollstühlen keine Rolle; beide werden als „unangenehm zu befahren“ bewertet; 
- Dichter besetzte Noppenplatten und größere Rippenabstände lassen sich mit hochhackigem Schuhwerk schlechter begehen (1 Testperson).

\subsection{Untersuchung von "getrennten Überquerungsstellen“}

Aus Sicht blinder und sehbehinderter Verkehrsteilnehmer sollte ein taktil ertastbarer Bordstein im Bereich von Überquerungsstellen den Gehweg von der Fahrbahn trennen. Demgegenüber stehen die Wünsche „rollender“ Mobilitätseingeschränkter, Nullabsenkungen einzubauen. In den letzten Jahren wurde versucht, diesen Kompromiss durch Überquerungsanlagen mit differenzierten Bordhöhen zu lösen. Dabei wird immer auch wieder die notwendige bzw. aus Gründen der Verkehrssicherheit maximale Breite der Nullabsenkung diskutiert. In technischen Regelwerken zum barrierefreien Bauen wird die Breite derzeit auf maximal $1 \mathrm{~m}$ beschränkt (FGSV 2011) (DIN 2011). Der Beauftragte für barrierefreies Gestalten des Verbandes der Blinden- und Sehbehindertenpädagogen und pädagoginnen e. V. (VBS) hat an einer Überquerungsstelle mit differenzierten Bordhöhen in Kassel Beobachtungen durchgeführt. Die Ergebnisse hat er in einem beim DBSV veröffentlichten Papier zusammengefasst (Böhringer 2010).

Demnach überquerten rund drei Viertel der beobachteten Verkehrsteilnehmer die Fahrbahn direkt am 3,5 cm hohen Bordauftritt und $12 \%$ über am Hochbord von $12 \mathrm{~cm}$. Nur wenige Passanten nutzten konsequent alle auf Fahrbahnniveau abgesenkten Bereiche im Zuge der Überquerung. Dies erfolgte z. B. durch einen von sechs beobachteten Personen mit Kinderwagen sowie zwei von drei Rollatornutzern. Aus Sicht des Beobachters war „in keinem Fall [...] erkennbar, dass die Bordsteinkanten von ca. $3 \mathrm{~cm}$ bzw. $12 \mathrm{~cm}$ Höhe die nicht behinderten Passanten störten, bremsten oder gar gefährdeten.“

Aus den Beobachtungen wird abgeleitet, dass keine Notwendigkeit besteht, die abgesenkten Bereiche breiter als mit $1 \mathrm{~m}$ Breite auszuführen. Einen „Rückstau“ an der Nullabsenkung durch eine hohe Nutzung wäre zu keinem Zeitpunkt zu erkennen gewesen. Der Beobachter räumt allerdings ein, dass die abgesenkten Bereiche für Rollatornutzer und auch für Rollstuhlnutzer eine wertvolle Hilfe darstellen können. 


\subsubsection{Radfahrer}

Die häufigsten Unfalltypen bei denen Radfahrer an Knotenpunkten verunglücken sind Abbiege-Unfälle und Einbiegen/Kreuzen-Unfälle.

Wesentliche Defizite ergaben sich im Rahmen der Sicherheitsanalysen durch die nicht regelwerkskonforme Ausführung von Radverkehrsanlagen. Hierbei wurden zum Teil die Mindestbreiten der entsprechenden Führungsformen (Radweg, Radfahrstreifen oder Schutzstreifen) unterschritten. Es fehlten Überquerungsanlagen oder an einigen Knotenpunkten, für die aufgrund der verkehrlichen Belastung Radverkehrsanlagen gemäß aktuellen Entwurfsregelwerken empfohlen werden, waren gar keine Radverkehrsanlagen vorhanden.

Dies hatte zur Folge, dass in vielen Fällen die Radfahrer auf die (vermeintlich sicheren) Gehwege auswichen und hier auch „entgegen“ der Fahrrichtung fuhren (Unfalltyp 342) bzw. in Konflikt zu Fußgängern gerieten.

Wie auch bei den Fußgängern konnten die wesentlichen Sicherheitsrisiken für Radfahrer an Knotenpunkten ohne Lichtsignalanale festgestellt werden. Insbesondere an den einmündenden Straßen, wenn die Anfahrsicht für die Kraftfahrer eingeschränkt (Abbildung 36) oder die Radverkehrsfurten nicht zu erkennen war (Abbildung 37).
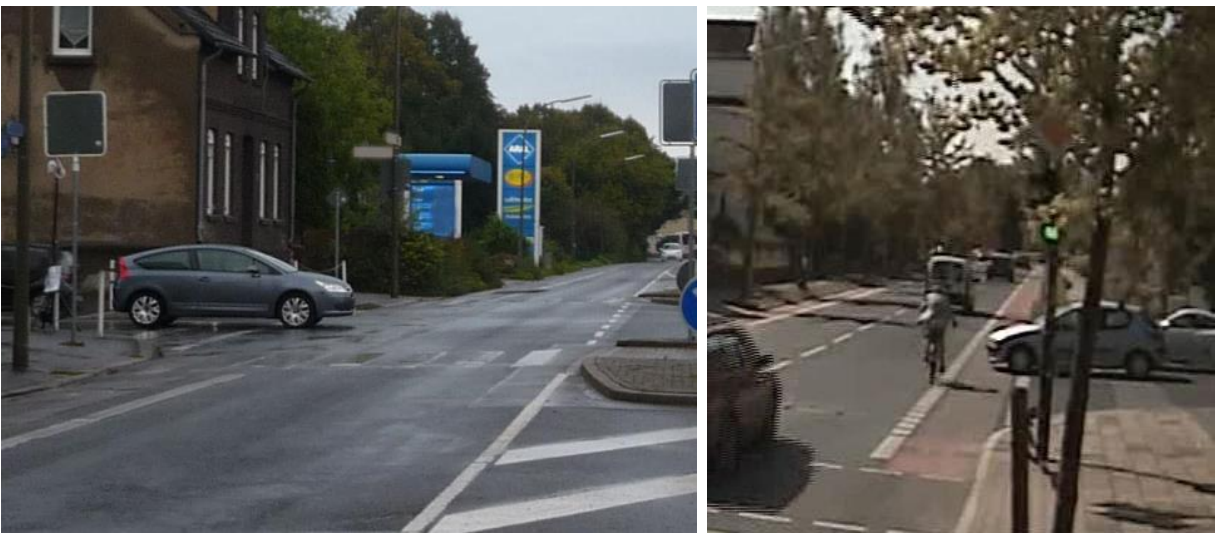

Abbildung 36: Einbiegende/kreuzende Fahrzeuge warten auf Radverkehrsfurt bei eingeschränkter Anfahrsicht

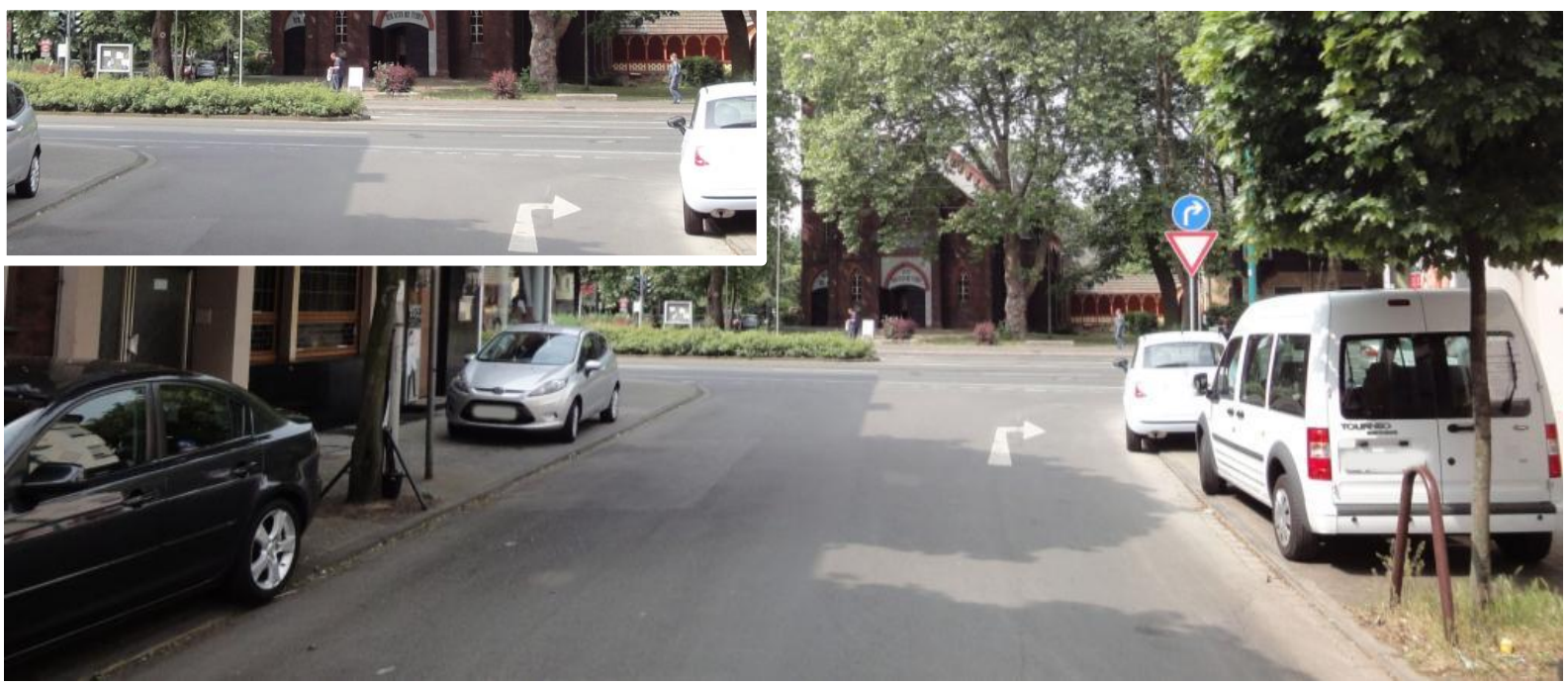

Abbildung 37: Radverkehrsfurt aus Sicht der einbiegenden Kraftfahrer nicht zu erkennen 
Einen Überblick des Verhaltens der Radfahrer zeigt Abbildung 38. Insgesamt wurde das Verhalten von 2.709 Radfahrern ausgewertet.

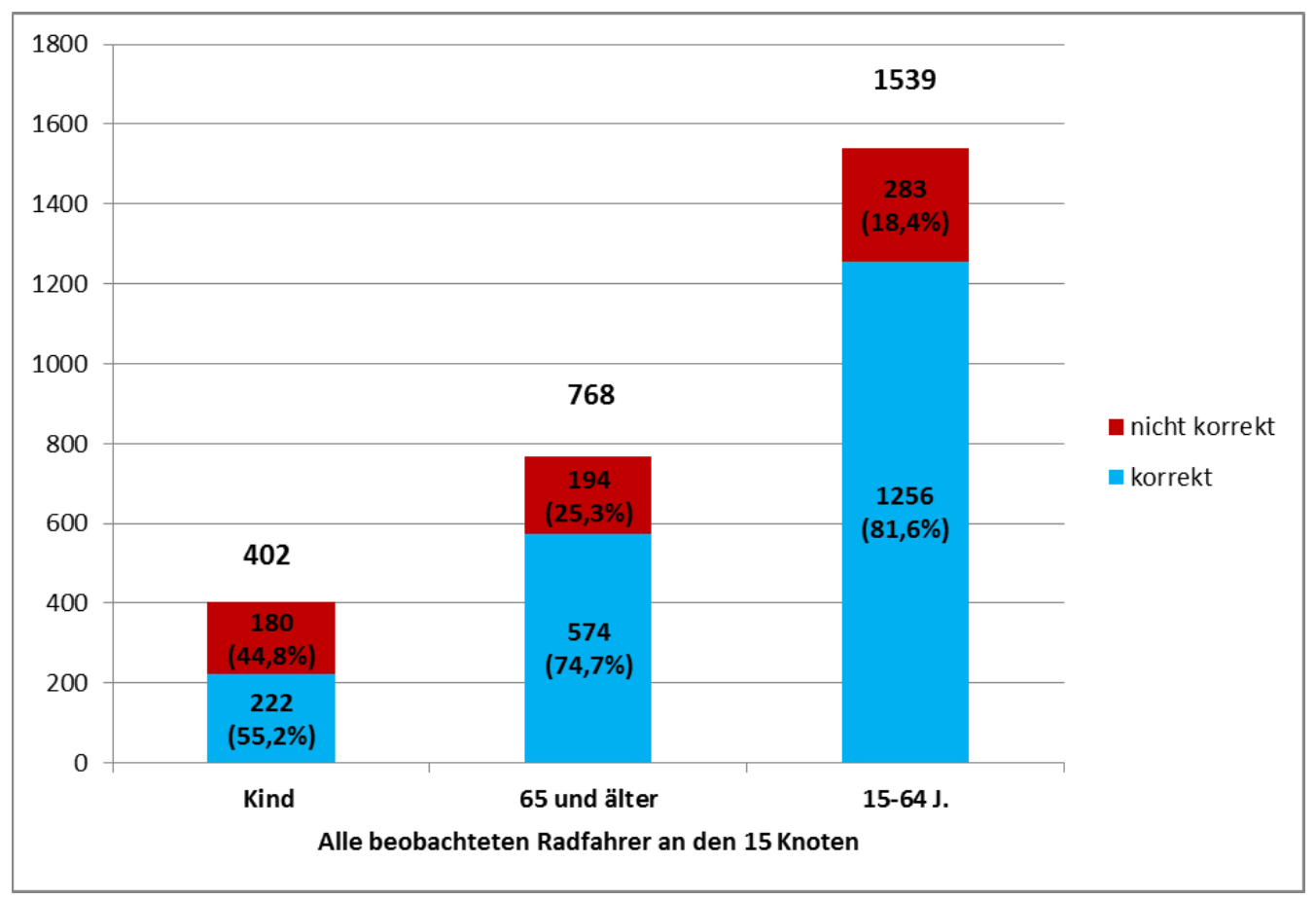

Abbildung 38: Gesamtüberblick Radfahrerverhalten an 15 Knotenpunkten

Wie die Abbildung zeigt, machten Kinder relativ gesehen die meisten Fehler beim Radfahren, gefolgt von den älteren Radfahrern und den Radfahrern zwischen 15 und 64 Jahren.

Die häufigsten Fehler bestanden bei Kindern und älteren Menschen in der Nutzung von Fußgängerverkehrsanlagen: Teilweise vielleicht auch aus Bequemlichkeit, aber wohl vor allem, weil teilweise selbst an sehr großen und hochfrequentierten Knotenpunkten keine Radverkehrsanlagen entsprechend den Empfehlungen von Entwurfsregelwerken vorhanden waren. Selbst wenn Radverkehrsanlagen für den Längsverkehr an solchen Knotenpunkten vorhanden waren, wurden trotzdem häufig die Fußgängeranlagen zur Überquerung der Straße vorgezogen.

Fehlten Überquerungsanlagen für Radfahrer auf längeren Streckenabschnitten wurde auch der Radweg in Gegenrichtung genutzt. In Ausnahmefällen wurde das allerdings auch an allen anderen Radwegen beobachtet.

Vorhandene Radverkehrsanlagen wurden nicht immer vorschriftsmäßig genutzt, sofern die vorschriftsmäßige Nutzung einen erheblichen Umweg oder erhebliche Wartezeiten bedeutete.

Insgesamt wurden sehr wenige schwerwiegende Verstöße beobachtet, Rotlichtverstöße waren die Ausnahme in allen Altersgruppen.

Beim Abbiegen fehlte häufig das Handzeichen. 


\subsubsection{Kraftfahrer}

Die meisten Unfälle an Knotenpunkten im Kfz-Verkehr sind Einbiegen/Kreuzen-Unfälle, gefolgt von Abbiege-Unfällen. Auch Unfälle im Längsverkehr, z. B. bedingt durch Verflechtungsvorgänge im Kreuzungsbereich oder durch plötzliches Abbremsen vor Überquerungsanlagen kommen vor.

In der Detailbetrachtung der dreistelligen Unfalltypen sticht allerdings der Unfalltyp 211 (Abbiege-Unfall, vgl. Abbildung 39) bei Kraftfahrern aller Altersklassen hervor und steht noch vor den dreistelligen Unfalltypen der Einbiegen/Kreuzen-Unfälle ${ }^{19}$. Bei diesem Unfalltyp wird das Vorrangrecht geradeausfahrender Kraftfahrer von wartepflichtigen Linksabbiegern der Gegenrichtung missachtet.

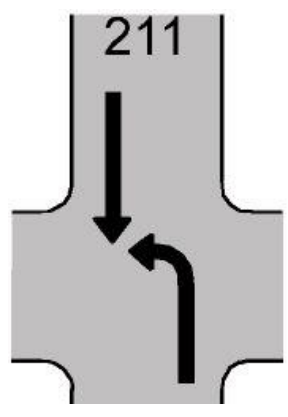

Abbildung 39: Unfalltyp 211

Die wesentlichen Defizite bzw. Sicherheitsrisiken ergaben sich für Kraftfahrer bei Knotenpunkten mit Lichtsignalanlage vor allem dann, wenn der Linksabbieger signaltechnisch nicht gesichert geführt wurde. Insbesondere wenn zudem Orientierungshilfen im Kreuzungsbereich (Leitlinien, Wartelinien) fehlten oder nicht mehr zu erkennen waren.

Konflikte ergaben sich im Weiteren, wenn Verflechtungsvorgänge im Kreuzungsbereich durchgeführt werden mussten, weil z. B.

- Linksabbieger und Geradeausfahrer auf einem Fahrstreifen fuhren und die Geradeausfahrer hinter den wartenden Linksabbieger auf den rechten Fahrstreifen z. T. plötzlich - ausscherten oder

- direkt hinter dem Kreuzungsbereich ein Fahrstreifen des Geradeausverkehrs - bei zwei Fahrstreifen der Zufahrt - eingezogen wurde.

Weitere Konflikte für Kraftfahrer - auch in Verbindung mit nicht motorisierten Verkehrsteilnehmern - konnten bei Kreuzungen mit Lichtsignalanlage im Zusammenhang mit weit abgesetzten Haltlinien der Zufahrten beobachtet werden, wenn

- ein Grünpfeil (Zeichen 720, vgl. Anlage A 1) angebracht war und Rechtsabbieger bei ROT losfuhren, das Signal aber vor Erreichen der einmündenden Straße auf GRÜN (dann auch für die dort wartenden Fußgänger und Radfahrer) umschlug oder wenn

\footnotetext{
${ }^{19}$ Auf Grundlage der Auswertung der dreistelligen Unfalltypen am Beispiel elektronischer Unfalldaten aus Nordrhein-Westfalen (2004 bis 2008).
} 
- Linksabbieger eine Zugabezeit erhielten und Geradeausfahrer, die einen gemeinsamen Fahrstreifen mit den Linksabbiegern nutzen durften, während ihrer eigenen Freigabezeit keine ausreichend großen Zeitlücken fanden, um sich in den Geradausverkehr des rechten Fahrstreifen einordnen zu können aber bereits hinter der LSA warteten und den Kreuzungsbereich erst dann räumten, als die Linksabbieger der Gegenrichtung per grünem Pfeil die Zugabezeit angezeigt bekamen (vgl. Abbildung 40).

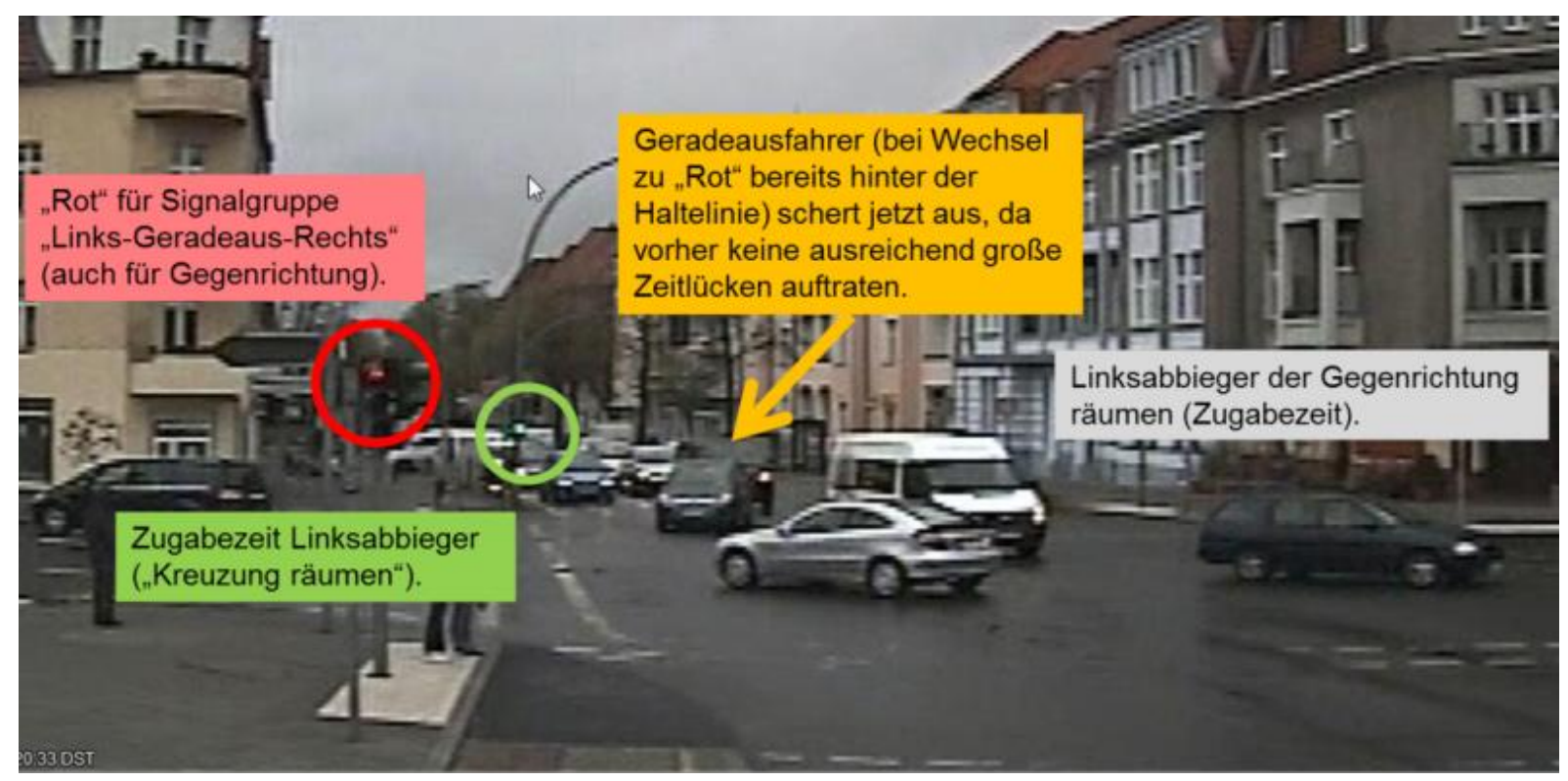

Abbildung 40: Konflikte bei Zugabezeit für Linksabbieger und weit abgesetzter Haltlinie

An Knotenpunkten mit vorfahrtregelnden Verkehrszeichen konnten insbesondere Defizite im Bereich der Sichtbeziehungen festgestellt werden. Hier ist vor allem die durch Bäume, Masten, Randbebauung, Grundstücksmauern oder auch Haltestellen eingeschränkte Anfahrsicht zu nennen.

An den 15 Knotenpunkten wurde das Verhalten von 9.337 jüngeren und 2.338 älteren PkwFahrern beobachtet (zusätzlich von 740 Fahrern am Knotenpunkt 5, bei denen nicht zwischen Jung und Alt unterschieden wurde). Zusammen sind das 12.415 Verhaltensbeobachtungen von Pkw-Fahrern.

Den Gesamtüberblick der Auswertung der Verhaltensbeobachtungen zeigt (Abbildung 41). Wie das Diagramm zeigt, machten ältere Fahrer in der Gesamtschau relativ gesehen nur wenig mehr Fehler als die Fahrer jüngerer Altersgruppen. Die Differenz beträgt etwa 2 \%. 


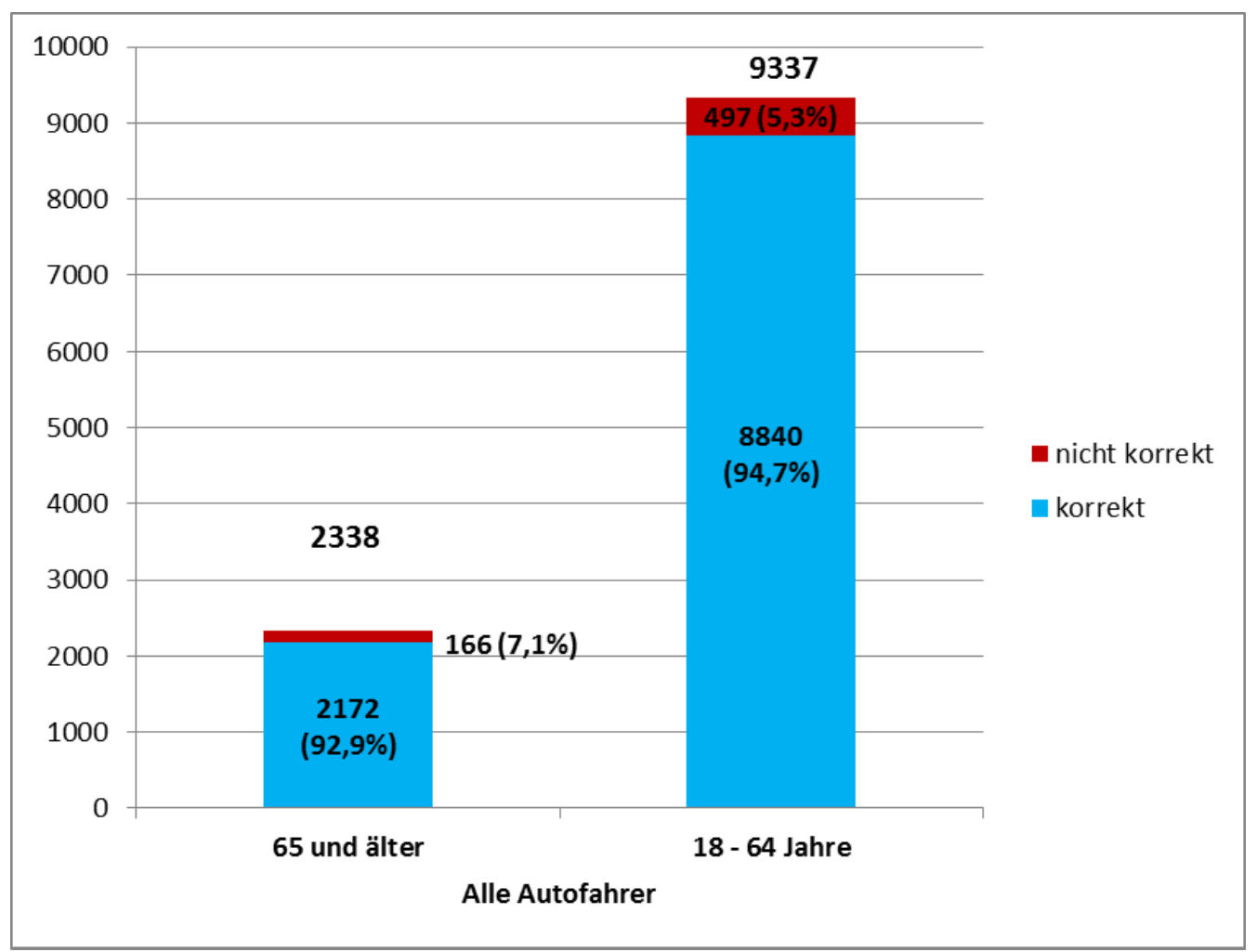

Abbildung 41: Gesamtüberblick Verhalten von Pkw-Fahrern an 15 Knotenpunkten

Bei den Geradeausfahrern an komplexen Knotenpunkten lagen die Fehler in beiden Altersgruppen deutlich unter den Gesamtzahlen (Abbildung 42). Der relative Fehlerunterschied beträgt $0,2 \%$ zwischen älteren und jüngeren Fahrer. Hier sind die Gruppen in der Leistung sehr vergleichbar.

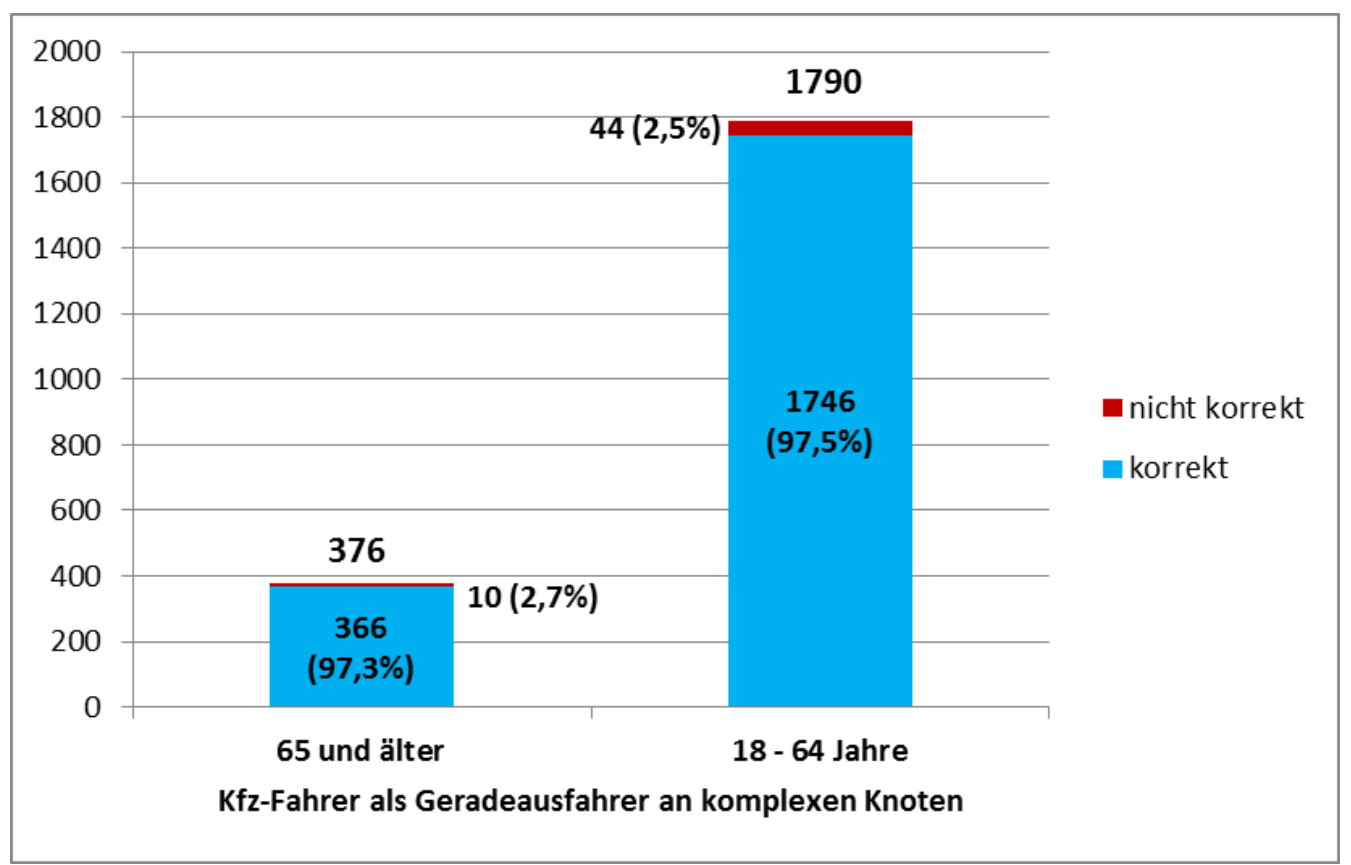

Abbildung 42: Pkw-Fahrer als Geradeausfahrer an den 15 Knotenpunkten 
Betrachtet man nur die Rechtsabbieger, ist das relative Verhältnis im Vergleich zum Gesamtergebnis genau umgekehrt. Die Differenz beträgt knapp $2 \%$ zwischen älteren und jüngeren Fahrern, wobei sich bei dieser Betrachtung die älteren Fahrer regelkonformer verhielten (Abbildung 43).

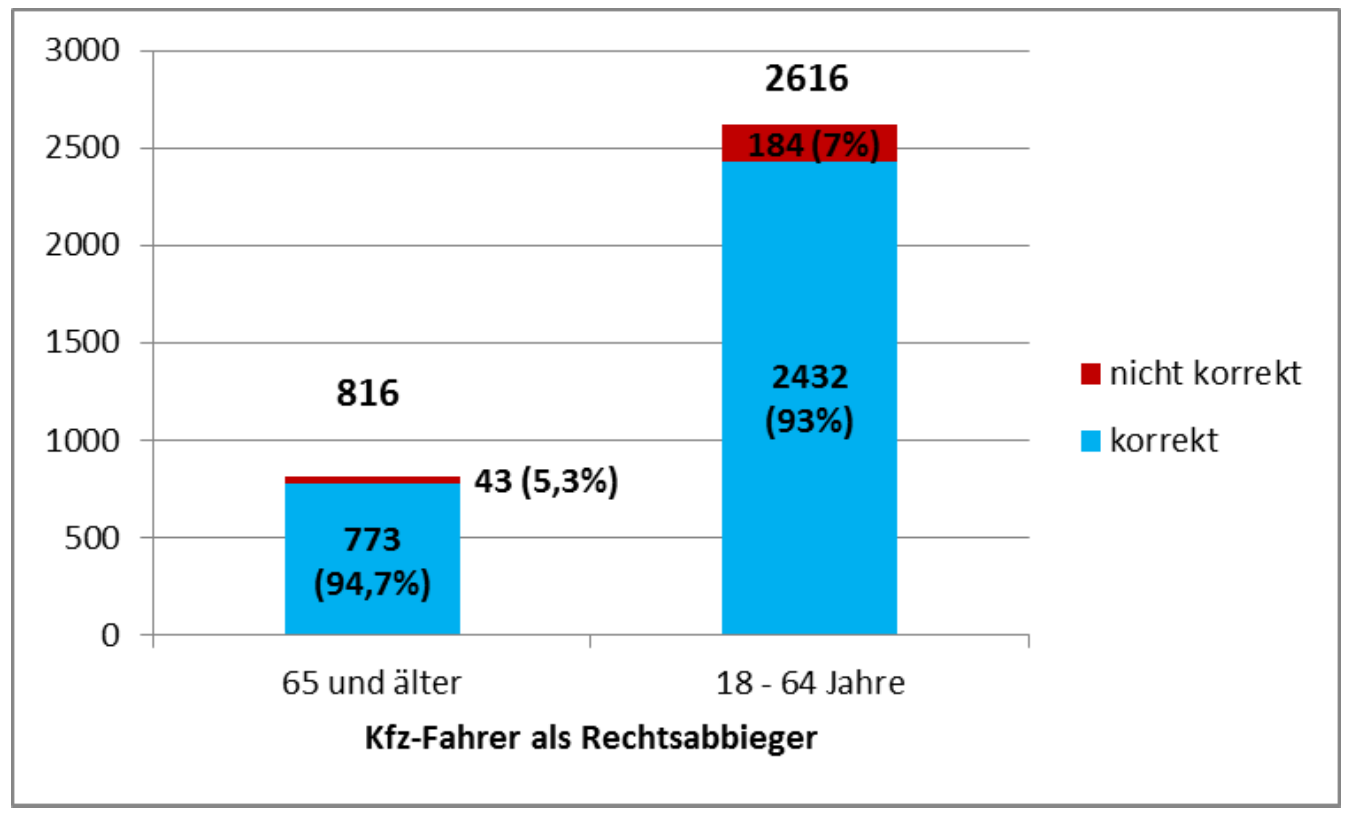

Abbildung 43: Pkw-Fahrer als Rechtsabbieger an den 15 Knotenpunkten

Bei den Linksabbiegern besteht jedoch ein erheblicher Unterschied zwischen älteren und jüngeren Fahrern. Abbildung 44 zeigt deutlich, dass bei gleichen Situationen ältere Linksabbieger mehr Fehler machten als Jüngere. Es wird vermutet, dass dies mit der nachlassenden Multitasking-Fähigkeit im Alter zusammenhängt. Wenn viele Eindrücke gleichzeitig in einer komplexen Situation verarbeitet werden müssen und teilweise an den beobachteten Knotenpunkten Führungs- und Wartelinien gefehlt haben, ist es für ältere Fahrer schwieriger regelkonform links abzubiegen.

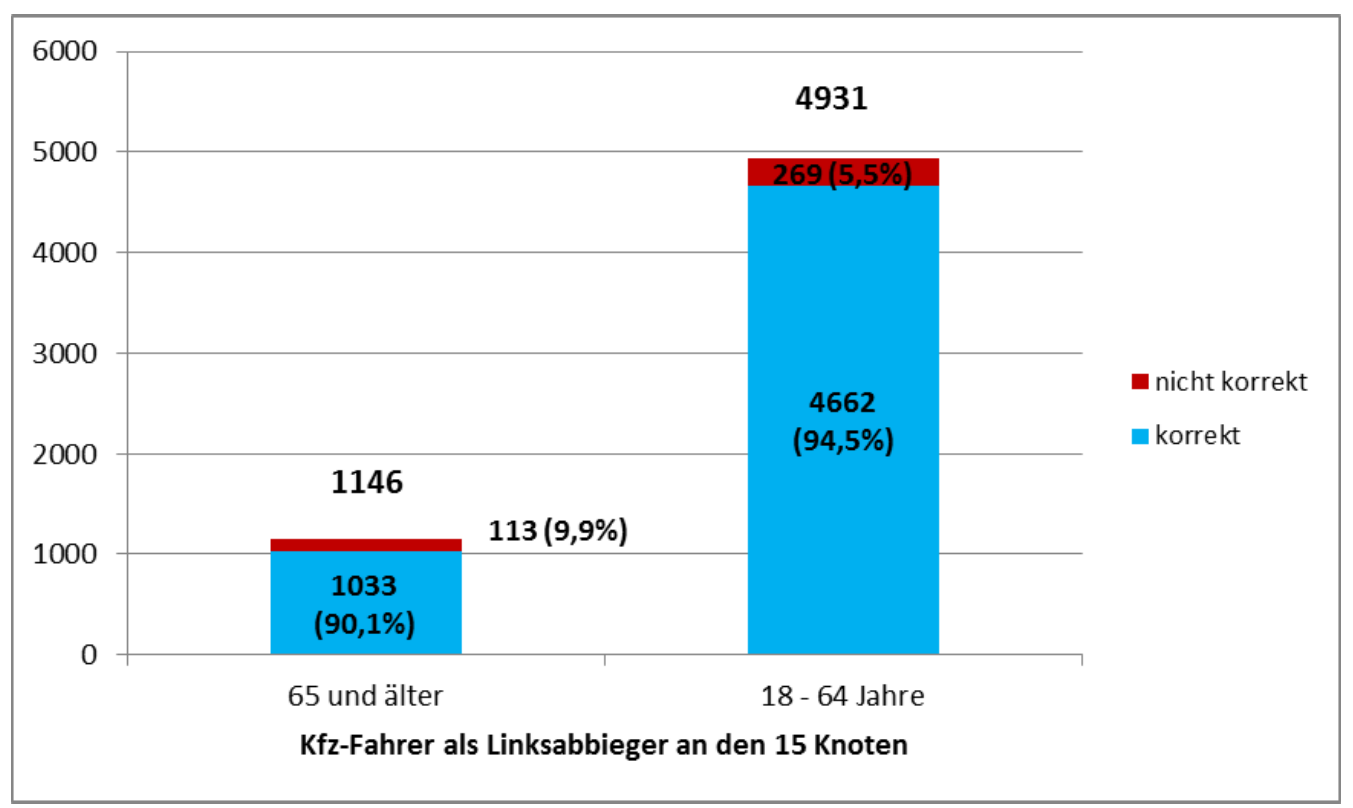

Abbildung 44: Pkw-Fahrer als Linksabbieger an den 15 Knotenpunkten 


\subsection{Sicherheitsanalysen und Verhaltensbeobachtungen - wesentliche Aspekte je Knotenpunkttyp}

Im Folgenden werden die wesentlichen Defizite und Sicherheitsrisiken tabellarisch je Knotenpunkttyp überblicksgebend dargestellt. Darüber hinaus enthält die Tabelle Defizite, die unabhängig vom Knotenpunkttyp festgestellt wurden bzw. für weitgehend alle betrachteten Knotenpunkten gelten.

Tabelle 12: Sicherheitsaspekte je Knotenpunkttyp

\begin{tabular}{|c|c|}
\hline Knotenpunkttyp & Defizit bzw. Sicherheitsrisiko \\
\hline \multirow{5}{*}{$\begin{array}{l}\text { Kreuzung mit } \\
\text { Lichtsignalanlage }\end{array}$} & Signaltechnisch nicht gesichert geführte Linksabbieger \\
\hline & $\begin{array}{l}\text { Fehlende oder nicht mehr erkennbare Orientierungshilfen (Leitlinien, } \\
\text { Wartelinien) für Linksabbieger im Kreuzungsbereich }\end{array}$ \\
\hline & $\begin{array}{l}\text { Verflechtungsvorgänge im Kreuzungsbereich aufgrund der Führung von } \\
\text { Linksabbiegern und Geradeausfahrern auf einem Fahrstreifen (bei } \\
\text { mehr als einem Fahrstreifen der Zufahrt) oder einer } \\
\text { Fahrstreifensubtraktion hinter dem Kreuzungsbereich }\end{array}$ \\
\hline & $\begin{array}{l}\text { Grünpfeil (Zeichen 720) oder Zugabezeit für Linksabbieger (wenn } \\
\text { Linksabbieger und Geradeausfahrer auf einem gemeinsamen } \\
\text { Fahrstreifen zugelassen sind) bei weit abgesetzten Haltlinien der } \\
\text { Zufahrten }\end{array}$ \\
\hline & $\begin{array}{l}\text { Lange Wartezeiten für Fußgänger und Radfahrer (insbesondere in der } \\
\text { Nähe von Schulen oder Haltestellen des ÖV) }\end{array}$ \\
\hline \multirow{4}{*}{$\begin{array}{l}\text { Knotenpunkte } \\
\text { (Kreuzung und } \\
\text { Einmündungen) } \\
\text { mit } \\
\text { vorfahrtregelnden } \\
\text { Verkehrszeichen }\end{array}$} & Eingeschränkte Sichtbeziehungen, auch eingeschränkte Anfahrsicht \\
\hline & $\begin{array}{l}\text { Fehlende oder nicht regelwerkskonforme Fußgängerverkehrsanlagen } \\
\text { (insbesondere Überquerungsanlagen) }\end{array}$ \\
\hline & $\begin{array}{l}\text { Nicht erkennbare Radverkehrsfurt im Bereich der einmündenden } \\
\text { Straße }\end{array}$ \\
\hline & $\begin{array}{l}\text { Fehlende Überquerungsanalgen für den Radverkehr (Radfahrer nutzen } \\
\text { Überquerungsanlagen des Fußgängerverkehrs oder Rad-/Gehwege } \\
\text { entgegen der Fahrtrichtung) }\end{array}$ \\
\hline \multirow{4}{*}{$\begin{array}{l}\text { Knotenpunkte } \\
\text { allgemein }\end{array}$} & Eingeschränkte Sichtbeziehungen, auch eingeschränkte Anfahrsicht \\
\hline & $\begin{array}{l}\text { Fehlende oder nicht regelwerkskonform ausgeführte } \\
\text { Radverkehrsanlagen }\end{array}$ \\
\hline & $\begin{array}{l}\text { Fehlende oder nicht regelwerkskonform ausgeführte Analgen für } \\
\text { Menschen mit besonderer Mobilitätseinschränkung (z. B. Rollator, } \\
\text { Kinderwagen) oder Mobilitätsbehinderung (z. B. Menschen mit } \\
\text { schwerer Seh- oder Gehbehinderung) }\end{array}$ \\
\hline & $\begin{array}{l}\text { Die Vorfahrtsregelung (rechtsabbiegender Kraftfahrer ggü. kreuzenden } \\
\text { Fußgängern oder Radfahrern) an freien Rechtsabbiegestreifen } \\
\text { (mit/ohne FGÜ, Radverkehrsfurt) scheint häufig nicht bekannt zu sein. }\end{array}$ \\
\hline
\end{tabular}




\section{Prototypische Merkmale/Situationen nach Art der Verkehrs- beteiligung}

Auf Grundlage der durchgeführten Sicherheitsanalysen und Verhaltensbeobachtungen, werden im Folgenden die wesentlichen, prototypischen Merkmale/Situationen hinsichtlich der Knotenpunktart, der Verkehrsführung und der verkehrstechnischen und baulichen Einrichtungen sowie die dazu in Verbindung stehenden Verhaltensweisen und/oder Konfliktsituationen wiedergegeben.

Dies erfolgt getrennt für Fußgänger (Tabelle 13), für Radfahrer (Tabelle 14), für Menschen mit besonderen Mobilitätseinschränkungen oder Mobilitätsbehinderungen (Tabelle 15) und für Kraftfahrer (Tabelle 16). Wobei auch Wechselwirkungen bestehen und beispielsweise Defizite im Kfz-Verkehr zu Konflikten mit anderen Verkehrsteilnehmern führen können.

Die prototypischen Merkmale werden in Tabellenform aufgeführt. Dabei wird den Merkmalen sowohl das beobachtete Verhalten der Verkehrsteilnehmer als auch die Knotenpunktform, an der dieses vornehmlich zutraf gegenübergestellt. Wenn auf Grundlage der zur Verfügung stehenden Unfalldaten, ein Bezug zu bestimmten Unfalltypen festgestellt werden konnte, ist dies ebenfalls kenntlich gemacht.

Im Anhang A 7 sind im Bezug zu den prototypischen Merkmalen/Situationen zusätzlich mögliche Maßnahmen aufgeführt, die helfen können, die beobachteten Konfliktsituationen und festgestellten Unfälle zu vermeiden. 
Tabelle 13: Prototypische Merkmale/Situationen für Fußgänger

\begin{tabular}{|c|c|c|c|c|}
\hline $\begin{array}{l}\text { Lfd.- } \\
\mathrm{Nr} .\end{array}$ & Merkmale / Situationen & Verhalten / Konflikte & $\begin{array}{l}\text { Knotenpunktart / } \\
\text { Verkehrsregelung }\end{array}$ & Bezug zu Unfällen \\
\hline 1 & $\begin{array}{l}\text { Keine Überquerungsanlage } \\
\text { an mehrstreifiger Fahrbahn } \\
\text { bei hohen Verkehrsmengen }\end{array}$ & $\begin{array}{l}\text { - Unsicheres } \\
\text { Überquerungsverhalt } \\
\text { en } \\
\text { - „Vor- und } \\
\text { Zurückgehen“ } \\
\text { - Bei längerer } \\
\text { Wartezeit werden } \\
\text { auch kurze } \\
\text { Zeitlücken genutzt, } \\
\text { riskante } \\
\text { Überquerungsversuc } \\
\text { he }\end{array}$ & $\begin{array}{l}\text { Einmündungen (VZ) } \\
\text { und Kreuzungen } \\
(\mathrm{VZ})\end{array}$ & $\begin{array}{l}\text { Überschreiten- } \\
\text { Unfälle }\end{array}$ \\
\hline 2 & $\begin{array}{l}\text { Sichtbeziehungen zwischen } \\
\text { Fußgängern und Kfz-Verkehr } \\
\text { durch ruhenden Verkehr } \\
\text { eingeschränkt }\end{array}$ & $\begin{array}{l}\text { - Unsicheres } \\
\text { Überquerungsverhalt } \\
\text { en } \\
\text { - Gefahr insbesondere } \\
\text { für Kinder und } \\
\text { Rollstuhlfahrer }\end{array}$ & $\begin{array}{l}\text { Einmündungen (VZ) } \\
\text { und Kreuzungen } \\
(\mathrm{VZ})\end{array}$ & $\begin{array}{l}\text { Überschreiten- } \\
\text { Unfälle }\end{array}$ \\
\hline 3 & $\begin{array}{l}\text { Erkennbarkeit der } \\
\text { Überquerungsstelle ist für den } \\
\text { Kfz-Verkehr nicht gegeben, } \\
\text { wartende Fußgänger werden } \\
\text { spät gesehen } \\
\text { (Straßenführung, Parken am } \\
\text { Fahrbahnrand, keine } \\
\text { Beschilderung, Bepflanzung) }\end{array}$ & $\begin{array}{l}\text { - Hohe } \\
\text { Geschwindigkeiten } \\
\text { im Kfz-Verkehr } \\
\text { - Z. T. abruptes } \\
\text { Abbremsen der Kfz } \\
\text { zu beobachten } \\
\text { - Unsicheres } \\
\text { Überquerungsverhalt } \\
\text { en der Fußgänger }\end{array}$ & $\begin{array}{l}\text { Einmündungen (VZ) } \\
\text { und Kreuzungen } \\
(\mathrm{VZ})\end{array}$ & $\begin{array}{l}\text { Überschreiten- } \\
\text { Unfälle und bedingt } \\
\text { Unfälle im } \\
\text { Längsverkehr }\end{array}$ \\
\hline 4 & $\begin{array}{l}\text { Keine oder nicht ausreichend } \\
\text { gesicherte Aufstellfläche für } \\
\text { Fußgänger an Überquerung }\end{array}$ & $\begin{array}{l}\text { - Ausweichen auf } \\
\text { Fahrbahn oder } \\
\text { Radverkehrsanlagen } \\
\text { - Unkontrolliertes, } \\
\text { unvorhersehbares } \\
\text { und unsicheres } \\
\text { Überquerungsverhalt } \\
\text { en von Fußgängern }\end{array}$ & $\begin{array}{l}\text { Einmündungen (VZ) } \\
\text { und Kreuzung (VZ } \\
\text { und LSA) }\end{array}$ & $\begin{array}{l}\text { Überschreiten- } \\
\text { Unfälle }\end{array}$ \\
\hline 5 & $\begin{array}{l}\text { Durchmesser Warnsignal } \\
\text { (gelbes Blinklicht) an } \\
\text { Fußgängerfurten beträgt } 200 \\
\text { mm }\end{array}$ & $\begin{array}{l}\text { - Schlechte } \\
\text { Wahrnehmbarkeit für } \\
\text { Kfz-Verkehr }\end{array}$ & $\begin{array}{l}\text { Knotenpunkte mit } \\
\text { LSA }\end{array}$ & $\begin{array}{l}\text { Kein Bezug auf } \\
\text { Basis der } \\
\text { Datengrundlage } \\
\text { herstellbar. }\end{array}$ \\
\hline 6 & $\begin{array}{l}\text { Fußgängersignalanlagen } \\
\text { (Anforderungssignalanlagen) }\end{array}$ & $\begin{array}{l}\text { - Werden gut } \\
\text { angenommen, wenn } \\
\text { Anforderungsdauer } \\
\text { kurz ist, trotz } \\
\text { einzelner „Rotläufer“. }\end{array}$ & $\begin{array}{l}\text { Knotenpunkte mit } \\
\text { VZ }\end{array}$ & $\begin{array}{l}\text { Unabhängig vom } \\
\text { Unfallgeschehen. }\end{array}$ \\
\hline
\end{tabular}


Tabelle 14:Prototypische Merkmale/Situationen für Radfahrer

\begin{tabular}{|c|c|c|c|c|}
\hline Lfd.- & Merkmale / Situationen & Verhalten / Konflikte & $\begin{array}{l}\text { Knotenpunktart / } \\
\text { Verkehrsregelung }\end{array}$ & Bezug zu Unfällen \\
\hline 1 & $\begin{array}{l}\text { Erkennbarkeit der } \\
\text { Radverkehrsfurt an } \\
\text { einmündenden Straßen ist } \\
\text { nicht gegeben }\end{array}$ & $\begin{array}{l}\text { Einbiegende und } \\
\text { kreuzende Kraftfahrer } \\
\text { fahren zügig bis an } \\
\text { den Fahrbahnrand } \\
\text { und warten auf den } \\
\text { Bereichen der } \\
\text { Radverkehrsfurt }\end{array}$ & $\begin{array}{l}\text { Einmündungen } \\
(V Z) \text { und } \\
\text { Kreuzungen (VZ) }\end{array}$ & $\begin{array}{l}\text { Einbiegen/Kreuzen- } \\
\text { Unfälle }\end{array}$ \\
\hline 2 & $\begin{array}{l}\text { Nicht regelkonform gestaltete } \\
\text { und/oder dimensionierte } \\
\text { Radverkehrsanlagen (z. B. } \\
\text { Aufstellflächen für indirekte } \\
\text { Linksabbieger fehlen, zu } \\
\text { geringe Breiten bei } \\
\text { Radfahrstreifen, überlagernde } \\
\text { Wegebeziehungen) }\end{array}$ & $\begin{array}{l}\text { - Gefährdung von } \\
\text { Radfahrern durch } \\
\text { überholende und } \\
\text { abbiegende Kfz } \\
\text { - Anlagen werden von } \\
\text { Radfahrern zum Teil } \\
\text { nicht genutzt } \\
\text { - Radfahrer weichen } \\
\text { auf die (sichereren) } \\
\text { Anlagen für } \\
\text { Fußgänger aus } \\
\text { - Behinderung/Konflikte } \\
\text { mit Fußgängern }\end{array}$ & Alle & $\begin{array}{l}\text { Bedingter Bezug zu } \\
\text { Abbiege- und } \\
\text { Einbiegen/Kreuzen- } \\
\text { Unfällen, vor allem, } \\
\text { wenn Radfahrer auf } \\
\text { Gehwege } \\
\text { ausweichen und } \\
\text { Kraftfahrer hier nicht } \\
\text { mit Radfahrern } \\
\text { rechnen. }\end{array}$ \\
\hline 3 & $\begin{array}{l}\text { Nicht ausreichende und/oder } \\
\text { sich überlagernde } \\
\text { Aufstellflächen für Radfahrer } \\
\text { und Fußgänger }\end{array}$ & $\begin{array}{l}\text { - Gegenseitige } \\
\text { Behinderung. } \\
\text { Allerdings keine } \\
\text { Konflikte beobachtet, } \\
\text { da die Radfahrer, } \\
\text { insbesondere bei } \\
\text { hoher } \\
\text { Passantendichte sich } \\
\text { zumeist defensiv } \\
\text { verhalten }\end{array}$ & Alle & $\begin{array}{l}\text { Kein Bezug auf } \\
\text { Basis der } \\
\text { Datengrundlage } \\
\text { herstellbar. }\end{array}$ \\
\hline 4 & $\begin{array}{l}\text { Radwegenetz (Straßenzug) } \\
\text { ohne ausreichende } \\
\text { Überquerungsmöglichkeiten } \\
\text { für Radfahrer }\end{array}$ & $\begin{array}{l}\text { - Radfahrer auf } \\
\text { Einrichtungsradwege } \\
\mathrm{n} \text { entgegen der } \\
\text { vorgeschriebenen } \\
\text { Fahrtrichtung } \\
\text { - Radfahrer nutzen } \\
\text { Gehwege (in beiden } \\
\text { Fahrtrichtungen) }\end{array}$ & Alle & $\begin{array}{l}\text { Einbiegen/Kreuzen- } \\
\text { Unfälle } \\
\text { (insbesondere Typ } \\
342 \text { ) }\end{array}$ \\
\hline
\end{tabular}


Tabelle 15: Prototypische Merkmale/Situationen für Menschen mit besonderen Mobilitätseinschränkungen oder Mobilitätsbehinderungen

\begin{tabular}{|c|c|c|c|c|}
\hline $\begin{array}{l}\text { Lfd.- } \\
\mathrm{Nr} \text {. }\end{array}$ & Merkmale / Situationen & Verhalten / Konflikte & $\begin{array}{l}\text { Knotenpunktart } \\
\text { Verkehrsregelung }\end{array}$ & Bezug zu Unfällen \\
\hline 1 & $\begin{array}{l}\text { Keine } \\
\text { Überquerungsbereiche } \\
\text { mit Nullabsenkung } \\
\text { vorhanden }\end{array}$ & $\begin{array}{l}\text { - Menschen mit Rollatoren } \\
\text { und Rollstühlen weichen auf } \\
\text { Radverkehrsanlagen (z. T. } \\
\text { auch auf die Fahrbahn) aus }\end{array}$ & Alle & $\begin{array}{l}\text { Abbiege-Unfälle unc } \\
\text { Einbiegen/Kreuzen- } \\
\text { Unfälle }\end{array}$ \\
\hline 2 & Bord mit $3 \mathrm{~cm}$ Auftritt & $\begin{array}{l}\text { - Menschen mit Rollatoren } \\
\text { und Rollstuhlfahrer haben } \\
\text { auch bei } 3 \mathrm{~cm} \text { Bordhöhe } z \text {. } \\
\text { T. erhebliche Probleme, } \\
\text { dieses Hindernis zu } \\
\text { überwinden }\end{array}$ & Alle & $\begin{array}{l}\text { Kein Bezug auf Basis } \\
\text { der Datengrundlage } \\
\text { herstellbar. }\end{array}$ \\
\hline 3 & $\begin{array}{lr}\text { Keine } & \text { oder } \\
\text { unzureichende } & \text { Anlagen } \\
\text { für blinde } & \text { und } \\
\text { sehbehinderte } & \\
\text { Menschen } & \end{array}$ & $\begin{array}{l}\text { Wurde an nahezu allen } \\
\text { Knotenpunkten beobachtet, } \\
\text { zumeist keine Neubauten, } \\
\text { festgestellt. Konflikte } \\
\text { konnten allerdings nicht } \\
\text { beobachtet werden (kaum } \\
\text { blinde oder sehbehinderte } \\
\text { Menschen beobachtet) }\end{array}$ & Alle & $\begin{array}{l}\text { Kein Bezug auf Basis } \\
\text { der Datengrundlage } \\
\text { herstellbar. }\end{array}$ \\
\hline
\end{tabular}


Tabelle 16: Prototypische Merkmale/Situationen für Kraftfahrer

\begin{tabular}{|c|c|c|c|c|}
\hline $\begin{array}{l}\text { Lfd.- } \\
\mathrm{Nr} .\end{array}$ & Merkmale / Situationen & Verhalten / Konflikte & $\begin{array}{l}\text { Knotenpunktart / } \\
\text { Verkehrsregelung }\end{array}$ & Bezug zu Unfällen \\
\hline 1 & $\begin{array}{l}\text { Nicht gesichert geführte } \\
\text { Linksabbieger }\end{array}$ & $\begin{array}{l}\text { - Schwierigkeiten für Ältere } \\
\text { und Jüngere gleichermaßen }\end{array}$ & $\begin{array}{l}\text { Kreuzung (LSA } \\
\text { und VZ) }\end{array}$ & $\begin{array}{l}\text { Abbiege-Unfälle } \\
\text { (Typ 211) }\end{array}$ \\
\hline 2 & $\begin{array}{l}\text { Keine } \\
\text { Orientierungshilfen für } \\
\text { Linksabbieger (Leitlinie, } \\
\text { Wartelinie) im } \\
\text { Kreuzungsbereich }\end{array}$ & $\begin{array}{l}\text { - Ungeordnete } \\
\text { Aufstellpositionen (auch für } \\
\text { nMIV problematisch) } \\
\text { - Nebeneinanderaufstellung } \\
\text { (,Doppelabbieger“) } \\
\text { - "Unsicheres Vorziehen“ } \\
\text { - „Vortaster“ }\end{array}$ & $\begin{array}{l}\text { Kreuzung (VZ und } \\
\text { LSA bei } \\
\text { signaltechnisch } \\
\text { nicht gesichert } \\
\text { geführten } \\
\text { Linksabbieger) }\end{array}$ & Abbiege-Unfälle \\
\hline 3 & $\begin{array}{l}\text { Sichtbehinderung (für } \\
\text { Linksabbieger) durch } \\
\text { Begrünung auf } \\
\text { Mittelstreifen (oder } \\
\text { Verkehr der } \\
\text { Gegenrichtung) }\end{array}$ & $\begin{array}{l}\text { - Schwierigkeiten für Jüngere } \\
\text { wie Ältere gleichermaßen }\end{array}$ & $\begin{array}{l}\text { Kreuzung (VZ und } \\
\text { LSA bei } \\
\text { signaltechnisch } \\
\text { nicht gesichert } \\
\text { geführten } \\
\text { Linksabbieger) }\end{array}$ & Abbiege-Unfälle \\
\hline 4 & $\begin{array}{l}\text { Eingeschränkte } \\
\text { Anfahrsicht, z. B. durch } \\
\text { Baumreihe, Hecken } \\
\text { oder ruhenden Verkehr }\end{array}$ & $\begin{array}{l}\text { - Vorziehen in den } \\
\text { Kreuzungsbereich, z. T. auf } \\
\text { Radfahrstreifen } \\
\text { - Unsicheres Anfahren }\end{array}$ & $\begin{array}{l}\text { Einmündung (VZ) } \\
\text { und Kreuzung (VZ) }\end{array}$ & $\begin{array}{l}\text { Einbiegen/Kreuzen } \\
\text {-Unfälle }\end{array}$ \\
\hline 5 & $\begin{array}{l}\text { Links abbiegender und } \\
\text { geradeaus fahrender } \\
\text { Verkehr auf einem } \\
\text { Fahrstreifen bei mehr } \\
\text { als einem Fahrstreifen in } \\
\text { der Zufahrt }\end{array}$ & $\begin{array}{l}\text { - Nachfolgende } \\
\text { Verkehrsteilnehmer scheren } \\
\text { nach rechts (auch auf } \\
\text { Radfahrstreifen/Schutzstreife } \\
\text { n) aus } \\
\text { - Besondere Gefährdung bei } \\
\text { Zugabezeit für Linksabbieger } \\
\text { wenn Geradeausverkehr } \\
\text { (der Gegenrichtung) noch } \\
\text { räumen muss } \\
\text { - Schwierigkeiten für Ältere } \\
\text { - und Jüngere gleichermaßen } \\
\text { Benötigt viel Aufmerksamkeit }\end{array}$ & $\begin{array}{l}\text { Kreuzung (LSA } \\
\text { und VZ) }\end{array}$ & $\begin{array}{l}\text { Unfälle im } \\
\text { Längsverkehr und } \\
\text { bedingt Abbiege- } \\
\text { Unfälle }\end{array}$ \\
\hline 6 & $\begin{array}{l}\text { Fahrstreifenreduktion } \\
\text { hinter Kreuzungsbereich } \\
\text { bei } 2 \text { oder mehr } \\
\text { Fahrstreifen des } \\
\text { zufahrenden } \\
\text { Geradeausverkehrs }\end{array}$ & $\begin{array}{l}\text { - Riskante } \\
\text { Verflechtungsvorgänge im } \\
\text { Kreuzungsbereich } \\
\text { - Plötzliches Ausscheren der } \\
\text { Geradeausfahrer nach } \\
\text { rechts }\end{array}$ & Kreuzung (LSA) & $\begin{array}{l}\text { Unfälle im } \\
\text { Längsverkehr }\end{array}$ \\
\hline 7 & $\begin{array}{l}\text { Grünpfeil (StVO-Zeichen } \\
720, \text { vgl. Anlage A 1), } \\
\text { insbesondere bei weit } \\
\text { abgesetzter Haltlinie }\end{array}$ & $\begin{array}{l}\text { - Verhalten der } \\
\text { Verkehrsteilnehmer oft nicht } \\
\text { StVO-konform } \\
\text { - Wird von Jüngeren und } \\
\text { Älteren gleichermaßen } \\
\text { missachtet } \\
\text { - Gefährdung von Radfahrern } \\
\text { und Fußgängern an } \\
\text { „paralleler“ Furt }\end{array}$ & Kreuzung (LSA) & Abbiege-Unfälle \\
\hline 8 & $\begin{array}{l}\text { Lange, gerade } \\
\text { Straßenflucht der } \\
\text { Hauptachse }\end{array}$ & $\begin{array}{l}\text { - Subjektive Einschätzung: zu } \\
\text { hohe Geschwindigkeiten } \\
\text { - Spätes, z. T. abruptes } \\
\text { Abbremsen an Fußgänger- } \\
\text { Überquerungsanlage }\end{array}$ & Kreuzung (VZ) & $\begin{array}{l}\text { Überschreiten- } \\
\text { Unfälle und Unfälle } \\
\text { im Längsverkehr }\end{array}$ \\
\hline
\end{tabular}




\section{Ergebnisdiskussion im Rahmen eines Expertenrunde}

Die Ergebnisse der Untersuchung zeigten, dass maßgebende bzw. häufige Defizite (Sicherheitsrisiken) an den 15 betrachteten Knotenpunkten im Bereich

- der signaltechnisch nicht gesichert geführten Linksabbieger,

- der Markierung (Leitorientierung für Kfz-Verkehr, insbesondere für Linksabbieger),

- der Sichtbeziehungen (auch im Hinblick auf Defizite aus dem Entwurf, der Gestaltung heraus)

- der Fußgängerverkehrsanlagen (insbesondere der Überquerungsanlagen)

- der Radverkehrsanlagen und auch

- der barrierefreien Gestaltung

lagen. Zudem schien die Komplexität eines Knotenpunktes Einfluss auf das Verhalten der Verkehrsteilnehmer zu haben. Zum Beispiel wurden Unsicherheiten beim Linksabbiegen vermehrt an Knotenpunkten beobachtet, die u. a. aufgrund ihrer Gestaltung, des Zustandes ihrer Markierung und/oder ihrer Umfeldnutzung als komplex bzw. „unübersichtlich“ eingeschätzt wurden.

Zur Diskussion und zur Validierung der Ergebnisse sowie zur Einbeziehung weitergehender Aspekte, die insbesondere die Belange der intendierten Untersuchungsgruppen (Kinder, ältere Menschen und Menschen mit besonderen Mobilitätseinschränkungen/-behinderungen) betreffen, wurde ein Expertenworkshop durchgeführt. $\mathrm{Zu}$ den Bereichen der Verkehrsplanung und -technik, der barrierefreien Verkehrsplanung und der Verkehrspsychologie nahmen fünf externe Experten teil, namentlich:

- Herr Prof. Friedrich (TU Braunschweig), insbesondere zu Themen der Verkehrstechnik,

- Herr Prof. Maier (TU Dresden), insbesondere zu Themen der Verkehrsplanung,

- Herr Mattner (Straßen NRW), insbesondere zu Themen des Straßenentwurfes und der Markierungen,

- Herr Prof. Topp (TU Kaiserslautern), insbesondere zu Themen der barrierefreien Verkehrsplanung und

- Herr Prof. Vollrath (TU Braunschweig), insbesondere zu Themen der Verkehrspsychologie.

Neben der Ergebnisvorstellung und -diskussion wurden dabei auch folgende Leitfragen gestellt:

1. Welcher Handlungsbedarf kann aus den Ergebnissen für die Planung von Knotenpunkten abgeleitet werden?

2. Welcher Handlungsbedarf ergibt sich auf Basis der Untersuchungsergebnisse sich für die Praxis?

3. Geben die bestehenden Regelwerke genug Spielraum, um Schwächere Verkehrsteilnehmer (einschließlich älterer Kraftfahrer) ausreichend zu schützen?

4. Könnte „schlechte Planung“ an fehlender Fortbildung der Planer in den Städten liegen?

5. Welche Verbesserungsmöglichkeiten sehen die Experten für ihre jeweils eigene Disziplin? 
Einleitend erkannte das Expertengremium die geleistete Arbeit an und stimmte der verwendeten Methodik grundsätzlich zu. Bekannte Einflussfaktoren, die zu einer Gefährdung schwächerer Verkehrsteilnehmer führen, konnten durch die umfangreichen Unfallanalysen und Verkehrsbeobachtungen sicher verifiziert werden.

Die technischen Regelwerke zur Gestaltung von Verkehrsinfrastruktur wurden insgesamt als sehr umfassend und hochwertig (mit Blick auf die Verkehrssicherheit) eingeschätzt. Als Ursache für die Entwicklung von Defiziten wurden z. B. ein nicht mehr aktueller Kenntnisstand, zu geringe Ressourcen (Personal), nicht ausreichende Fortbildung und nicht ausreichende Mittel für umfassende Maßnahmen nicht ausgeschlossen.

Vergleicht man die bauliche Gestaltung der beobachteten (komplexen) Knotenpunkte mit den aktuellen Entwurfsregelwerken, würde deutlich, dass Knotenpunkte im Bestand nicht dem Stand der Technik entsprechen. Dies mag daran liegen, dass sich die Regeln im Laufe der Zeit verändert haben - wahrscheinlich wurde in der Vergangenheit aber häufig nicht regelkonform gebaut. Auch die externen Experten kamen zu dem Schluss, dass bei konsequenter Anwendung der bestehenden Regelwerke viele der beobachteten Verkehrssituationen gar nicht vorkommen dürften.

Dennoch ließen auch die aktuellen Regelwerke große Spielräume zu und seien in ihren Ausführungen teilweise uneinheitlich. Als Beispiel wurden seitens des Auftragnehmers die verschiedenen Ausführungen zum „Linksabbiegeschutz“ genannt:

Richtlinien für die Anlage von Landstraßen (RAL-Entwurf):

„Linksabbieger sollen aus Sicherheitsgründen durch eine eigene Phase geschützt werden“-vgl. RAL 200x, Ziffer 6.3.3.4.

Richtlinien für die Anlage von Stadtstraßen (RASt 2006)

„Einmündungen oder Kreuzungen mit Lichtsignalanlage (in der Regel mit Linksabbiegeschutz) sind in der Regel geeignet" (...es folgt eine Aufzählung) - vgl. RASt 2006, Ziffer 5.3.2.

Richtlinien für Lichtsignalanlagen (RiLSA 2010):

„Die nicht gesicherte Führung der Linksabbieger sollte nur bei geringer Stärke mindestens eines der beiden Fahrzeugströme angewendet werden“ - vgl. RiLSA 2010, Ziffer 2.3.1.2.

„Aus Gründen der Verkehrssicherheit sind Steuerungen mit mehr als zwei Phasen im Allgemeinen günstiger zu beurteilen“-vgl. RiLSA 2010, Ziffer 2.3.2.

Im Sinne der Verkehrssicherheit seien hier eine einheitliche sprachliche Regelung und z. B. die Verpflichtung zur Einrichtung des Linksabbiegeschutzes zumindest bei zu definierenden Rahmenbedingungen wie eingeschränkte Sichtverhältnisse oder hohen Verkehrsmengen wünschenswert.

Im Weiteren unterschieden die Regelwerke bei den Lösungen nicht nach den Kompetenzen verschiedener Gruppen von Verkehrsteilnehmern. Beispielsweise schätzen ältere Menschen ihre Leistungsfähigkeit selbst ein, während Kinder eher ein Verhalten nachahmen, da sie 
über keine oder geringe Erfahrungen verfügen. Es ist zu überlegen, ob diese Unterschiede nicht von den Regelwerken stärker berücksichtigt werden müssten.

Knotenpunkte würden nach gültigen technischen Regelwerken geplant und gebaut. Im Laufe der Zeit würden zum Teil Änderungen an der Geometrie oder Infrastruktur durchgeführt. Dies kann Auswirkungen auf die Verkehrssicherheit haben, würde aber i. d. R. nicht ausreichend berücksichtigt. Bei nachträglichen Veränderungen müsste der gesamte Knotenpunkt hinsichtlich der Auswirkungen auf die Verkehrssicherheit betrachtet werden. Ein geeignetes Instrument hierfür könnte z. B. das Bestandsaudit sein.

Für die Anwendung eines Bestandsaudits wurde ein Katalog für Mindestanforderungen an Knotenpunkte, der sich u. a. aus den im Rahmen der Untersuchung festgestellten Hauptdefiziten ergibt, als hilfreich erachtet (Vgl. Kap. 9).

Auch ein Qualitätsmanagement für die Straßenraumgestaltung wäre anzudenken, um einen kontinuierlichen Prozess im Sinne der Verkehrssicherheit zu erreichen. So ließe sich das Auftreten von Sicherheitsdefiziten durch Änderungen an der Infrastruktur ohne ein geprüftes Gesamtkonzept vermeiden. Dem Aspekt Qualitätsmanagement wird mehr Potenzial eingeräumt, als der weiteren Verschärfung der technischen Regelwerke. Allerdings wird ergänzend eingebracht, dass ein Qualitätsmanagement eigentlich bereits durch „Verkehrssicherungspflicht" und „Verkehrsschau“ geregelt ist. Das Problem wird eher in der Umsetzung gesehen.

Neben den bisherigen Instrumenten zur Verbesserung der Verkehrssicherheit, wurde ein "systematisches Qualitätsmanagement speziell für Knotenpunkte" empfohlen, das die Verkehrsanlage als Ganzes betrachtet und sich auf alle Phasen der Lebensdauer der Verkehrsanlage bezieht. Dieses Qualitätsmanagement kann in ein allgemeines Qualitätsmanagement für Straßenräume integriert werden. Im Weiteren sollte überprüft werden, in welcher Weise ein solches Qualitätsmanagement die derzeit in der Erarbeitung befindlichen Empfehlungen für die Durchführung eines Bestandaudits von Straßen einbezieht und in welcher Weise neuere Sicherheitsanforderungen an Straßen auf bestehende Straßennetze und Verkehrsanlagen übertragen werden können. Das Qualitätsmanagement für Knotenpunkte sollte, ähnlich dem Qualitätsmanagement der Richtlinien für Lichtsignalanlagen, in die relevanten Regelwerke zum Straßenentwurf als zusätzliches Kapitel mit aufgenommen werden.

Weiterer Forschungsbedarf wurde im Hinblick auf die Komplexität von Knotenpunkten gesehen. Die derzeitige Praxis bei der Beseitigung von Unfallhäufungsstellen sei ein wichtiger Punkt zur Erhöhung der Verkehrssicherheit. Eine Definition der Komplexität könnte es zudem ermöglichen, auch speziell für Kinder und ältere Menschen, präventiv zu arbeiten und ggf. die Entstehung von Unfallschwerpunkten zu vermeiden (vgl. Kap. 9).

Zudem würde sich die Art und die Zusammensetzung der städtischen Mobilität, z. B. aufgrund geänderter Rahmenbedingungen (Gesetzesänderungen, Verkehrszunahmen $u$. w.) verändern. Beispielsweise in der Form starker Zuwächse beim Fußgänger- und Radverkehr bei derzeit relativ geringen Verkehrsflächen oder durch einen höheren Anteil des Radverkehrs auf der Fahrbahn. Auch ergäben sich durch die zunehmende Anzahl von Rollatornutzern zusätzliche Anforderungen an die Infrastruktur. Eine Überprüfung des 
bestehenden Verkehrsnetzes auf die Benutzbarkeit durch derartige „Verkehrsmittel“ wurde empfohlen. 


\section{Ansatz zur Beschreibung der Komplexität von Kontenpunkten}

Im Folgenden wird ein grundlegender Ansatz zur Einschätzung der Komplexität von Knotenpunkten beschrieben. Mögliche Folgen der Komplexität bzw. Zusammenhänge zwischen Komplexität und Verkehrssicherheit bzw. Gefährdung der Verkehrsteilnehmer werden thesenhaft aufgeführt.

Der Begriff der Komplexität ist allgemeingültig nicht definiert und wird von verschiedenen Wissenschaftsgebieten und Autoren teilweise unterschiedlich erfasst. Ein Ansatz aus der Systemtheorie sagt, dass „die Komplexität eines Systems mit der Anzahl an Elementen, der Anzahl an Verknüpfungen zwischen diesen Elementen sowie der Funktionalität dieser Verknüpfungen steigt (zum Beispiel Nicht-Linearität).“ (Milling 1981).

In den Wirtschaftswissenschaften wird die Komplexität auch als die Vielfalt der Beziehungen der Elemente eines Systems bezeichnet:

„1. Begriff: Gesamtheit aller voneinander abhängigen Merkmale und Elemente, die in einem vielfältigen aber ganzheitlichen Beziehungsgefüge (System) stehen. Unter Komplexität wird die Vielfalt der Verhaltensmöglichkeiten der Elemente und die Veränderlichkeit der Wirkungsverläufe verstanden.

2. Merkmale: Komplexität ist durch Anzahl und Art der Elemente und deren Beziehungen untereinander bestimmbar. Komplexe Prozesse weisen eine Eigendynamik auf und sind meist irreversibel, so dass Handlungen nicht rückgängig gemacht werden können. Wichtigstes Merkmal komplexer Situationen ist die Intransparenz für den Entscheider: Er hat keine Möglichkeit, das Netzwerk zirkulärer Kausalität intuitiv zu erfassen, keine Möglichkeit exakter Modellierung und exakter Prognosen, er muss mit Überraschungen und Nebenwirkungen rechnen. Der Umgang mit komplexen Systemen erfordert ein hohes Maß an Wissen über die kausalen Zusammenhänge der Systemelemente (Art der Vernetzung) und die Fähigkeit, Komplexität auf wenige Merkmale und Muster zu reduzieren (Komplexitätsreduktion)." (Gabler Wirtschaftslexikon 2012)

Für Knotenpunkte gibt es derzeit keine geeigneten Kenngrößen, mit denen die Komplexität eines Knotenpunktes umfassend bezeichnet, eingestuft und/oder bewertet werden kann. Hagen Schüller formuliert allerdings eine Methodik zur Beschreibung von Komplexität von innerstädtischen Straßen, die auf der Umfeldnutzung basiert:

„Ausgangspunkt der Überlegungen ist die Abschätzung, wie stark eine Randnutzung in Verbindung mit dem Aufkommen an nichtmotorisierten Verkehrsteilnehmern - vor allem Fußgänger - steht. Die gewerbliche Nutzung (GB) erhält die niedrigste Gewichtung. Arbeitsstätten generieren maßgeblich früh und am Nachmittag starke Fußgängerströme und in den restlichen Zeiten nur ein geringes Aufkommen an nichtmotorisierten Verkehrsteilnehmern. Sonstige gewerbliche Bebauung findet sich häufig an mehrstreifigen Zufahrtsstraßen (Gewerbegebiete), welche eine geringe Knotenpunktdichte, wenige Haltestellen und ein geringes Aufkommen an nichtmotorisierten Verkehrsteilnehmern aufweisen. Solche Straßen können als wenig komplex im Sinne der Umfeldnutzung bezeichnet werden, $d$. $h$., sie besitzen eine maßgebliche 
Verbindungsfunktion und nur eine geringe Erschließungsfunktion. Reine Wohnbebauung $(W)$ findet sich an nahezu allen Arten von Straßen im Netz. Aufgrund eines höheren Aufkommens an ruhendem Verkehr sowie einer vermehrten Nutzung durch Fußgänger und Radfahrer ist diese Art der Umfeldnutzung als komplexer gegenüber der gewerblichen Nutzung einzustufen. Einzelhandel wie z. B. allein stehende Supermärkte mit zugehörigem Parkplatz ergänzen häufig punktuell die reine Wohnbebauung in Wohngebieten. Eingeschossige Bebauung mit ausschließlich Geschäftsbesatz (EH) wird aufgrund des hohen Platzverbrauchs in Relation zum Verkehrsaufkommen, aber auch der häufigen Verwendung von Pkw, um diese Örtlichkeiten zu erreichen (große Parkplätze an Supermärkten), ähnlich der reinen Wohnbebauung gewichtet. Geschäftsstraßen weisen die komplexesten Straßenräume auf. Hier ist aufgrund häufiger Ein- und Ausparkvorgänge, eines hohen Fußgänger- und Radverkehrsaufkommens, einer häufigen Nutzung des öffentlichen Nahverkehrs (wartende Fahrgäste) und des Auftretens von Liefer- und Ladeverkehr ein hoher Erschließungsgrad gegeben. Solche Straßenräume sind häufig geprägt von einer geschlossenen Wohnbebauung mit Geschäftsbesatz in den unteren Geschossen (WEH). Die Gewichtung der Randnutzung erfolgt spezifisch für das Dresdner Hauptverkehrsstraßennetz, für die Anwendung in anderen Städten sind gegebenenfalls Anpassungen vorzunehmen."(Schüller 2011).

Diese Ausführungen decken sich grundsätzlich mit den Verkehrsbeobachtungen an den 15 Knotenpunkten. Je höher der Erschließungsrad war, z. B. in Kernstadtbereichen gegenüber Stadtrandbereichen, desto mehr Reize mussten von den Verkehrsteilnehmern, insbesondere von den Kraftfahrern, erfasst und - zum Teil unter Zeitdruck - verarbeitet werden und umso mehr Konfliktsituationen konnten beobachtet werden. Dabei war die Verkehrsbelastung ein beeinflussender, aber nicht entscheidender Faktor. Hochfrequentierte Knotenpunkte in Stadtrandlage bargen zum Teil weniger Schwierigkeiten für die Verkehrsteilnehmer als Knotenpunkte in zentraler Stadtlage. Dementsprechend könnte bei der Risikominimierung auch für die schwächeren, nichtmotorisierten Verkehrsteilnehmer vor allem auf solche Straßen ein Schwerpunkt gelegt werden, die sich neben einer bestimmten Verkehrsbelastung, insbesondere durch einen hohen Erschließungsgrad auszeichnen.

Hinsichtlich der Komplexität an Knotenpunkten werden die folgenden Thesen aufgestellt, die zunächst vornehmlich den Blick der Kraftfahrer aufnehmen:

- Je höher die Anzahl der möglichen Konflikte je Zeiteinheit (z. B. bei sich kreuzenden/entgegenkommenden Verkehrsteilnehmern) und je höher die Geschwindigkeit, desto höher die Komplexität und desto größer das Gefahrenpotenzial.

- Je weniger Hilfe durch die Verkehrsinfrastruktur gegeben ist (z. B. bei fehlenden Führungs- und Haltlinien für Linksabbieger oder nicht eindeutigen Vorfahrtsregelungen), desto höher die Komplexität.

- Je mehr ablenkende Reize (z. B. Geschäftsbesatz, Straßenbahn, Passanten) bestehen, desto höher die Komplexität, insbesondere für den $\mathrm{Kfz}-V e r k e h r$. 
Zur ersten allgemeinen Einschätzung der Komplexität der 15 beobachteten Knotenpunkte können im Wesentlichen einzelne Indikatoren bzw. Randbedingungen genannt werden, die die Komplexität beeinflussen können. Im Rahmen der Untersuchung wurden zunächst die folgenden Indikatoren herangezogen:

1. Hohes Kfz-Aufkommen (hier: DTV-Knotenpunkt $\geq 15.000 \mathrm{Kfz} / 24 \mathrm{~h}$ bei Einmündungen bzw. $\geq 25.000 \mathrm{Kfz} / 24 \mathrm{~h}$ bei Kreuzungen)

2. Zufahrende Verkehrsströme haben in Summe mindestens 5 Fahrstreifen (an Einmündungen) bzw. 6 Fahrstreifen (an Kreuzungen)

3. nicht gesichert geführter Linksabbieger der Hauptachse

4. nicht gesichert geführter Linksabbieger der Nebenachse

5. Linksabbieger und Geradeausfahrer auf einem Fahrstreifen bei mehr als einem Fahrstreifen in der Zufahrt zum Knotenpunkt

6. Fehlende oder unklare Führung (Leit- und Wartelinien) für Linksabbieger

7. Grünpfeil (Z 720), insbesondere bei weit abgesetzter Haltlinie

8. Straßenbahnführung über Knotenpunkt

9. ÖPNV-Haltestelle in unmittelbarer Nähe zum Knotenpunkt

10. hohes Fußgängeraufkommen

11. Überquerungsanlagen für Fußgänger sind nicht oder in nicht regelkonformer Ausführung vorhanden

12. hohes Radverkehrsaufkommen

13. Radverkehrsanlagen sind nicht bzw. in nicht regelkonformer Ausführung vorhanden

14. Sichtbehinderung

15. Geschäfts-/Bürobesatz

Zur Bestimmung der Komplexitätsgrade (Komplexitäts-Index) der jeweiligen Knotenpunkte, wurden im Folgenden für jeden Knotenpunkt überprüft, wie viele der 15 Indikatoren zutreffend sind. Alle Indikatoren wurden mit dem Wert 1 berücksichtigt, einfach gewichtet und je Knotenpunkt aufsummiert (vgl. Tabelle 18). So ist einem Knotenpunkt der geringsten Komplexitätsstufe theoretisch der Wert 0 zugewiesen, der höchst mögliche KomplexitätsIndex hat den Wert 15. Im Fall von baulichen oder verkehrstechnischen Änderungen an den Knotenpunkten wurden die Situationen vor der Umsetzung der Maßnahmen betrachtet und zur Bestimmung der Komplexität herangezogen.

Die so ermittelte Rangfolge, bezogen auf die Komplexität der Knotenpunkte, stimmt mit den (subjektiven) Einschätzungen auf Basis der Vor-Ort-Beobachtungen und Plananalysen überein.

Im Weiteren wurden Unfallkenngrößen zur Einschätzung der Zusammenhänge zwischen Komplexität und Verkehrssicherheit für die Knotenpunkte gebildet. Berücksichtigt wurden dabei ebenfalls nur die Unfälle, die vor der Umsetzung baulicher oder verkehrstechnischer Maßnahmen am Knotenpunkt geschahen.

Ermittelt wurde die durchschnittlich jährliche Unfalldichte (UD) und Unfallrate (UR) sowie die durchschnittlich jährliche Unfallkostendichte (UKD) und Unfallkostenrate (UKR) auf Basis der Unfälle zeitlich vor evtl. Umsetzungen baulicher und/oder verkehrstechnischer Maßnahmen 
(Tabelle 17) und auf Grundlage der Kostensätze zum Preisstand $2005^{20}$. Für die einzelnen Knotenpunkte lagen die Unfalldaten aus unterschiedlichen Betrachtungszeiträumen vor. Die Durchschnittliche Tägliche Verkehrsstärke (DTV) wurde auf Basis der zwei 1Stundenzählungen während der Vor-Ort-Untersuchungen nach dem Verfahren Schmidt/Thomas ${ }^{21}$ hochgerechnet (Tabelle 17).

Tabelle 17: Unfälle an den 15 untersuchten Knotenpunkten vor der Umsetzung baulicher oder verkehrstechnischer Maßnahmen zur Berechnung der Unfallkenngrößen ( $n=238)$

\begin{tabular}{|c|c|c|c|c|c|c|c|c|c|c|c|}
\hline 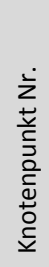 & 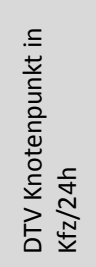 & 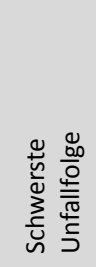 & 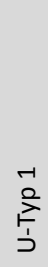 & 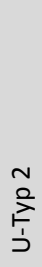 & 吕 & 竝 & 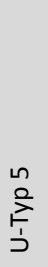 & $\begin{array}{l}0 \\
\sum_{2}^{0} \\
j \\
j\end{array}$ & 帝 & 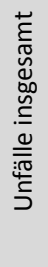 & 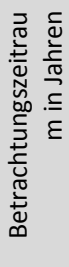 \\
\hline \multirow{3}{*}{1} & \multirow{3}{*}{42.699} & $U(S P)$ & 0 & 0 & 0 & 0 & 0 & 0 & 0 & \multirow{3}{*}{20} & \multirow{3}{*}{5} \\
\hline & & $\mathrm{U}(\mathrm{LV})$ & 0 & 13 & 0 & 7 & 0 & 0 & 0 & & \\
\hline & & $\mathrm{U}(\mathrm{SS})$ & \multicolumn{7}{|c|}{0} & & \\
\hline \multirow{3}{*}{2} & \multirow{3}{*}{27.947} & $U(S P)$ & 0 & 0 & 1 & 0 & 0 & 0 & 0 & \multirow{3}{*}{14} & \multirow{3}{*}{4} \\
\hline & & $\mathrm{U}(\mathrm{LV})$ & 0 & 6 & 1 & 0 & 0 & 0 & 0 & & \\
\hline & & $\mathrm{U}(\mathrm{SS})$ & \multicolumn{7}{|c|}{6} & & \\
\hline \multirow{3}{*}{3} & \multirow{3}{*}{34.048} & $\mathrm{U}(\mathrm{SP})$ & 0 & 1 & 0 & 0 & 0 & 1 & 0 & \multirow{3}{*}{26} & \multirow{3}{*}{5} \\
\hline & & $\mathrm{U}(\mathrm{LV})$ & 1 & 14 & 2 & 0 & 0 & 2 & 0 & & \\
\hline & & $\mathrm{U}(\mathrm{SS})$ & \multicolumn{7}{|c|}{5} & & \\
\hline \multirow{3}{*}{4} & \multirow{3}{*}{26.577} & $U(S P)$ & 1 & 0 & 0 & 1 & 0 & 0 & 0 & \multirow{3}{*}{18} & \multirow{3}{*}{4} \\
\hline & & $\mathrm{U}(\mathrm{LV})$ & 0 & 2 & 6 & 0 & 0 & 4 & 0 & & \\
\hline & & $\mathrm{U}(\mathrm{SS})$ & \multicolumn{7}{|c|}{4} & & \\
\hline \multirow{3}{*}{5} & \multirow{3}{*}{33.328} & $U(S P)$ & 0 & 0 & 0 & 0 & 0 & 0 & 0 & \multirow{3}{*}{10} & \multirow{3}{*}{3} \\
\hline & & $\mathrm{U}(\mathrm{LV})$ & 0 & 0 & 4 & 2 & 0 & 1 & 2 & & \\
\hline & & $\mathrm{U}(\mathrm{SS})$ & \multicolumn{7}{|c|}{1} & & \\
\hline \multirow{3}{*}{6} & \multirow{3}{*}{24.366} & $U(S P)$ & 1 & 0 & 0 & 0 & 0 & 0 & 0 & \multirow{3}{*}{37} & \\
\hline & & $\mathrm{U}(\mathrm{LV})$ & 0 & 3 & 9 & 0 & 0 & 4 & 0 & & 4 \\
\hline & & $\mathrm{U}(\mathrm{SS})$ & & & & 20 & & & & & \\
\hline & & $\mathrm{U}(\mathrm{SP})$ & 0 & 0 & 0 & 3 & 0 & 0 & 0 & & \\
\hline 7 & 23.222 & $\mathrm{U}(\mathrm{LV})$ & 0 & 3 & 0 & 5 & 0 & 1 & 1 & 16 & 3 \\
\hline & & $\mathrm{U}(\mathrm{SS})$ & & & & 3 & & & & & \\
\hline & & U(SP) & 0 & 0 & 0 & 0 & 0 & 0 & 0 & & \\
\hline 8 & 19.286 & $\mathrm{U}(\mathrm{LV})$ & 0 & 5 & 1 & 0 & 0 & 0 & 0 & 9 & 2 \\
\hline & & $\mathrm{U}(\mathrm{SS})$ & & & & 3 & & & & & \\
\hline & & $U(S P)$ & 0 & 0 & 0 & 0 & 0 & 0 & 0 & & \\
\hline 9 & 28.677 & $\mathrm{U}(\mathrm{LV})$ & 0 & 9 & 1 & 1 & 0 & 0 & 0 & 16 & 2 \\
\hline & & $U(S S)$ & & & & 5 & & & & & \\
\hline & & $U(S P)$ & 0 & 1 & 0 & 0 & 0 & 1 & 0 & & \\
\hline 10 & 13.126 & $\mathrm{U}(\mathrm{LV})$ & 1 & 3 & 4 & 1 & 0 & 0 & 0 & 12 & 4 \\
\hline & & $U(S S)$ & & & & 1 & & & & & \\
\hline
\end{tabular}

${ }^{20}$ Kostensätze für Unfälle nach Unfalltypen in Abhängigkeit von Unfallkategorie und Straßenkategorie (Unfallstruktur 2005) für Unfälle mit Getöteten und Verletzten und Pauschale Unfallkostensätze in Abhängigkeit von Unfallkategorie und Straßenkategorie für schwerwiegende Unfälle mit Sachschaden.

${ }^{21} \mathrm{Vgl}$. Bundesministerium für Verkehr (Hrsg.), Hochrechnungsfaktoren für manuelle und automatische Kurzzeitzählungen im Innerortsbereich, aus Forschung Straßenbau und Straßenverkehrstechnik, Heft 732, Ausgabe 1996 


\begin{tabular}{|c|c|c|c|c|c|c|c|c|c|c|c|}
\hline 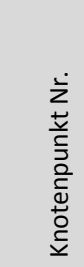 & 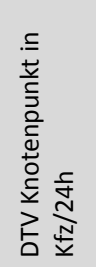 & 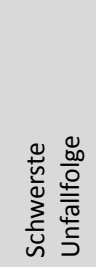 & 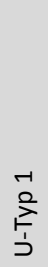 & 芳 & $\sum_{j}^{n}$ & $\begin{array}{l}+ \\
\sum_{\vec{z}}^{2} \\
j\end{array}$ & 足 & $\begin{array}{l}0 \\
\sum_{2}^{0} \\
\end{array}$ & $\sum_{j}^{\circ}$ & 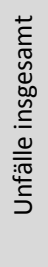 & 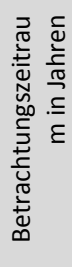 \\
\hline \multirow{3}{*}{11} & \multirow{3}{*}{14.002} & $\mathrm{U}(\mathrm{SP})$ & 0 & 2 & 1 & 0 & 0 & 0 & 0 & \multirow{3}{*}{21} & \multirow{3}{*}{6} \\
\hline & & $\mathrm{U}(\mathrm{LV})$ & 0 & 4 & 7 & 0 & 0 & 1 & 0 & & \\
\hline & & $\mathrm{U}(\mathrm{SS})$ & \multicolumn{7}{|c|}{6} & & \\
\hline \multirow{3}{*}{12} & \multirow{3}{*}{18.537} & $\mathrm{U}(\mathrm{SP})$ & 0 & 0 & 0 & 0 & 0 & 0 & 0 & \multirow{3}{*}{8} & \multirow{3}{*}{5} \\
\hline & & $\mathrm{U}(\mathrm{LV})$ & 0 & 1 & 5 & 0 & 0 & 0 & 0 & & \\
\hline & & $U(S S)$ & \multicolumn{7}{|c|}{2} & & \\
\hline \multirow{3}{*}{13} & \multirow{3}{*}{10.249} & $\mathrm{U}(\mathrm{SP})$ & 0 & 0 & 0 & 0 & 0 & 0 & 0 & \multirow{3}{*}{14} & \multirow{3}{*}{6} \\
\hline & & $\mathrm{U}(\mathrm{LV})$ & 0 & 1 & 2 & 1 & 0 & 4 & 2 & & \\
\hline & & $\mathrm{U}(\mathrm{SS})$ & \multicolumn{7}{|c|}{4} & & \\
\hline \multirow{3}{*}{14} & \multirow{3}{*}{28.722} & $\mathrm{U}(\mathrm{SP})$ & 1 & 1 & 0 & 2 & 1 & 0 & 0 & \multirow{3}{*}{12} & \multirow{3}{*}{5} \\
\hline & & $\mathrm{U}(\mathrm{LV})$ & 0 & 0 & 0 & 3 & 0 & 0 & 4 & & \\
\hline & & $\mathrm{U}(\mathrm{SS})$ & \multicolumn{7}{|c|}{0} & & \\
\hline \multirow{3}{*}{15} & \multirow{3}{*}{16.061} & $\mathrm{U}(\mathrm{SP})$ & 0 & 0 & 0 & 0 & 0 & 0 & 0 & \multirow{3}{*}{5} & \multirow{3}{*}{4} \\
\hline & & $\mathrm{U}(\mathrm{LV})$ & 1 & 1 & 2 & 0 & 0 & 1 & 0 & & \\
\hline & & $\mathrm{U}(\mathrm{SS})$ & \multicolumn{7}{|c|}{0} & & \\
\hline \multicolumn{12}{|c|}{$\begin{array}{l}\text { Erläuterungen: } \\
\text { DTV = Durchschnittlich Tägliche Verkehrsstärke, U-Typ = Unfalltyp, U(SP) = } \\
\text { Unfall mit Getöteten oder Schwerverletzten, U (LV) = Unfall mit Leichtverletzten, } \\
\text { U(SS) = Schwerwiegender Unfall mit Sachschaden }\end{array}$} \\
\hline
\end{tabular}

Tabelle 18: Unfallkenngrößen und Komplexitätsstufen (K-Index) an den Knotenpunkten der Untersuchung

\begin{tabular}{|c|c|c|c|c|c|c|c|c|c|c|}
\hline$\grave{z}$ & 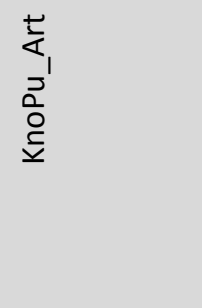 & 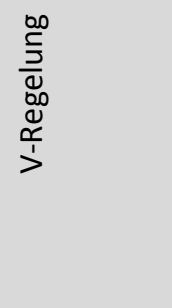 & 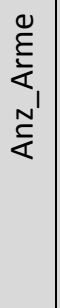 & $\begin{array}{l}\vec{J} \\
N_{1} \\
\stackrel{+}{<}\end{array}$ & $\begin{array}{l}\frac{n}{Z} \\
\frac{1}{4} \\
\frac{N}{4}\end{array}$ & $\begin{array}{l}\frac{\pi}{\supset} \\
\stackrel{D}{\supset}\end{array}$ & 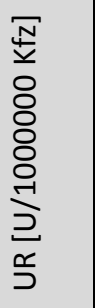 & 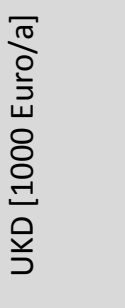 & 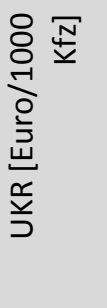 & 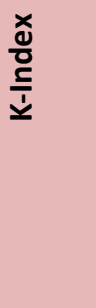 \\
\hline 1 & Kreuzung & LSA & 4 & 4 & 4 & 2,67 & 0,39 & 43,30 & 2,78 & 10,00 \\
\hline 2 & Kreuzung & LSA & 4 & 3 & 4 & 3,00 & 0,29 & 65,75 & 6,45 & 9,00 \\
\hline 3 & Kreuzung & LSA & 4 & 4 & 4 & 4,00 & 0,26 & 103,00 & 8,29 & 12,00 \\
\hline 4 & Kreuzung & LSA & 4 & 4 & 4 & 2,33 & 0,62 & 123,25 & 12,71 & 11,00 \\
\hline 5 & Kreuzung & LSA & 4 & 4 & 4 & 3,50 & 0,68 & 36,17 & 2,97 & 7,00 \\
\hline 6 & Kreuzung & LSA & 4 & 2 & 2 & 9,25 & 1,04 & 139,50 & 15,69 & 5,00 \\
\hline 7 & Kreuzung & LSA & 5 & 4 & 5 & 5,33 & 0,63 & 201,83 & 23,81 & 12,00 \\
\hline 8 & Kreuzung & LSA & 4 & 4 & 4 & 4,50 & 0,64 & 49,50 & 7,03 & 4,00 \\
\hline 9 & Kreuzung & LSA & 4 & 4 & 4 & 8,00 & 0,76 & 87,75 & 8,38 & 8,00 \\
\hline 10 & Kreuzung & VZ, FG-LSA & 4 & 4 & 4 & 3,50 & 0,34 & 89,88 & 18,76 & 5,00 \\
\hline 11 & Kreuzung & VZ & 4 & 4 & 4 & 5,33 & 0,63 & 93,08 & 18,21 & 5,00 \\
\hline 12 & Kreuzung & VZ & 4 & 4 & 4 & 8,00 & 0,76 & 29,33 & 4,34 & 2,00 \\
\hline 13 & Kreuzung & VZ & 4 & 4 & 4 & 5,20 & 0,42 & 25,75 & 6,88 & 4,00 \\
\hline 14 & Einmündung & VZ & 3 & 3 & 3 & 3,33 & 0,27 & 195,88 & 18,68 & 13,00 \\
\hline 15 & Einmündung & VZ & 3 & 3 & 3 & 3,00 & 0,63 & 13,88 & 2,37 & 9,00 \\
\hline
\end{tabular}


Abbildung 45 und Abbildung 46 stellen den Komplexitätsindex (Abszissenachse) den Unfallkenngrößen (Ordinatenachse) gegenüber. Bedingt zeigt sich, dass die als komplex eingestuften Knotenpunkte nicht zwingend mit vielen Unfällen (hohe UD oder UR) einhergehen bzw. diese hervorrufen (Abbildung 45). Wobei ein Anstieg der Unfalldichten im Zusammenhang mit einer zunehmenden Komplexität eher festzustellen ist (Abbildung 45, links), als ein Zusammenhang zwischen der Komplexität und den Unfallraten (Abbildung 45, rechts). Dies gilt analog auch für die Unfallkostendichten bzw. Unfallkostenraten (Abbildung 46).

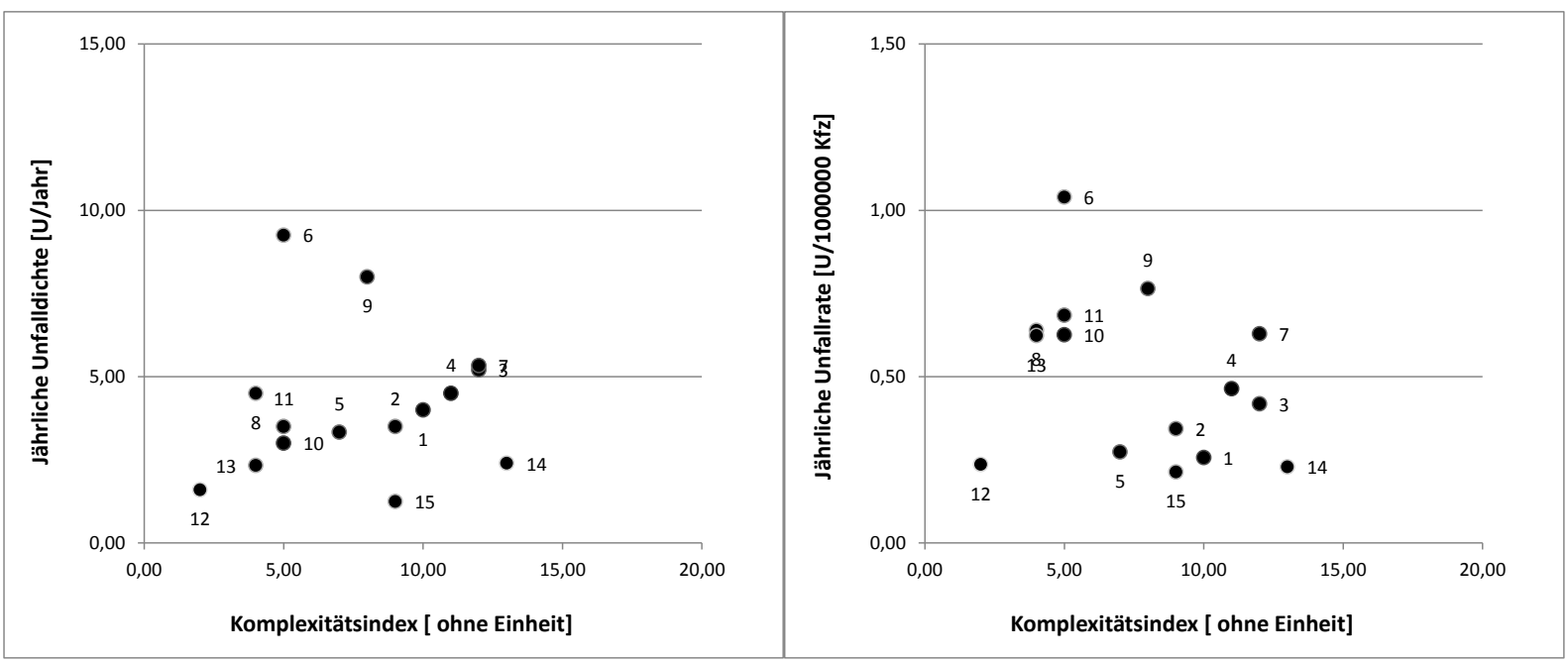

Abbildung 45: Komplexitätsindex zu Unfalldichte (Bild links) und Unfallrate (Bild rechts) für die Knotenpunkte der Untersuchung (1 bis 15)

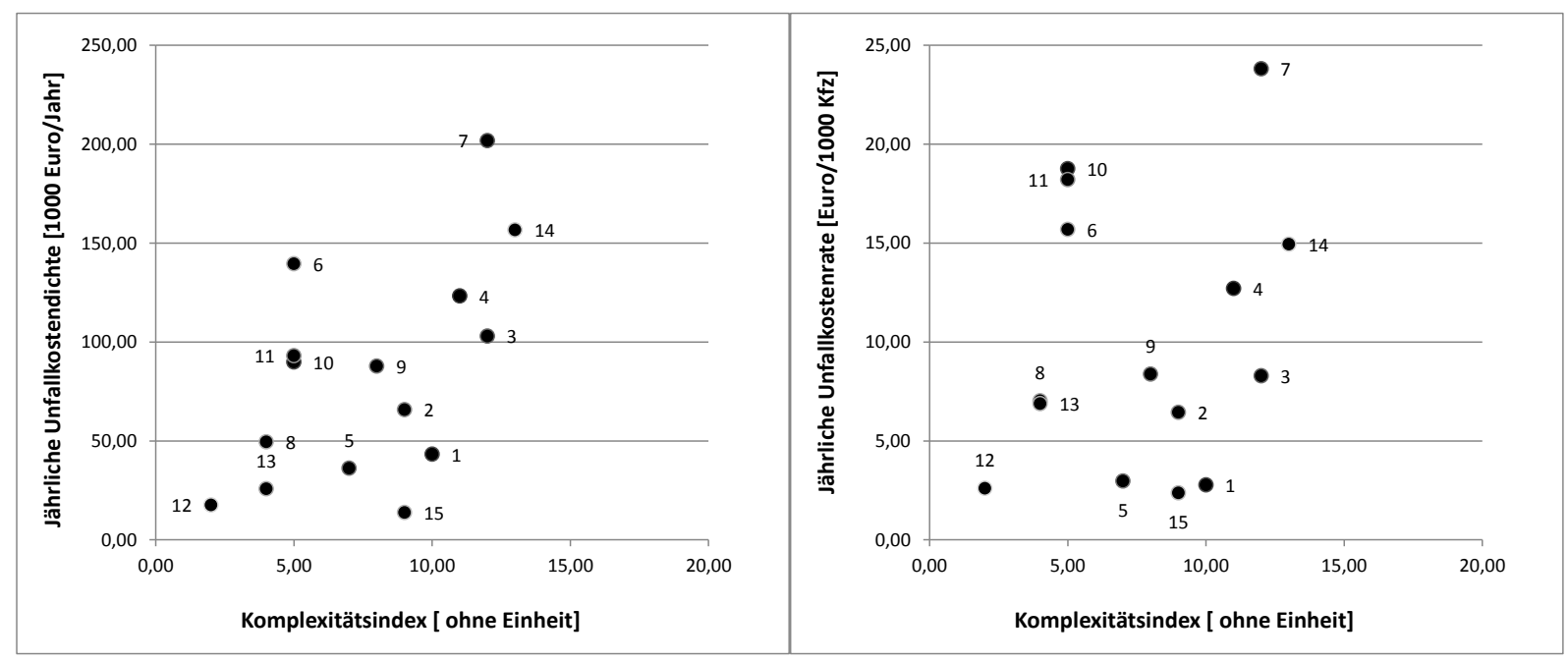

Abbildung 46: Komplexitätsindex zu Unfallkostendichte (Bild links) und Unfallkostenrate (Bild rechts) für die Knotenpunkte der Untersuchung (1 bis 15)

Aufgrund der kleinen Fallzahlen können im Folgenden keine belastbaren Aussagen getroffen werden. Es zeigt sich allerdings auf Grundlage der Untersuchungsdaten die Tendenz, dass mit zunehmender Komplexität die Unfalldichte und Unfallkostendichte ansteigt, während ein 
Zusammenhang zwischen der Komplexität und der Unfallrate bzw. Unfallkostenrate weitgehend nicht hergestellt werden kann.

Exemplarisch sind die linearen Regressionskurven und das Bestimmtheitsmaß der Verteilungen dargestellt (Abbildung 47 und Abbildung 48). Dabei wurde der Knotenpunkt 6 in dieser Darstellung nicht weiter berücksichtigt, da er einen Sonderfall darstellt. Diese Kreuzung ist auf Grundlage der o.g. Indikatoren als weniger komplex einzustufen, insbesondere aus Sicht der Pkw-Fahrer. Allerdings weist sie viele Unfälle auf, die aber aus einer sehr speziellen, sich wiederholenden Situation geschehen sind (hauptsächlich Rotlichtverstöße, vgl. Anlage A 3). Einen vollständigen Überblick der Regressionsanalysen unter Berücksichtigung aller Knotenpunkte enthält die Anlage A 9.

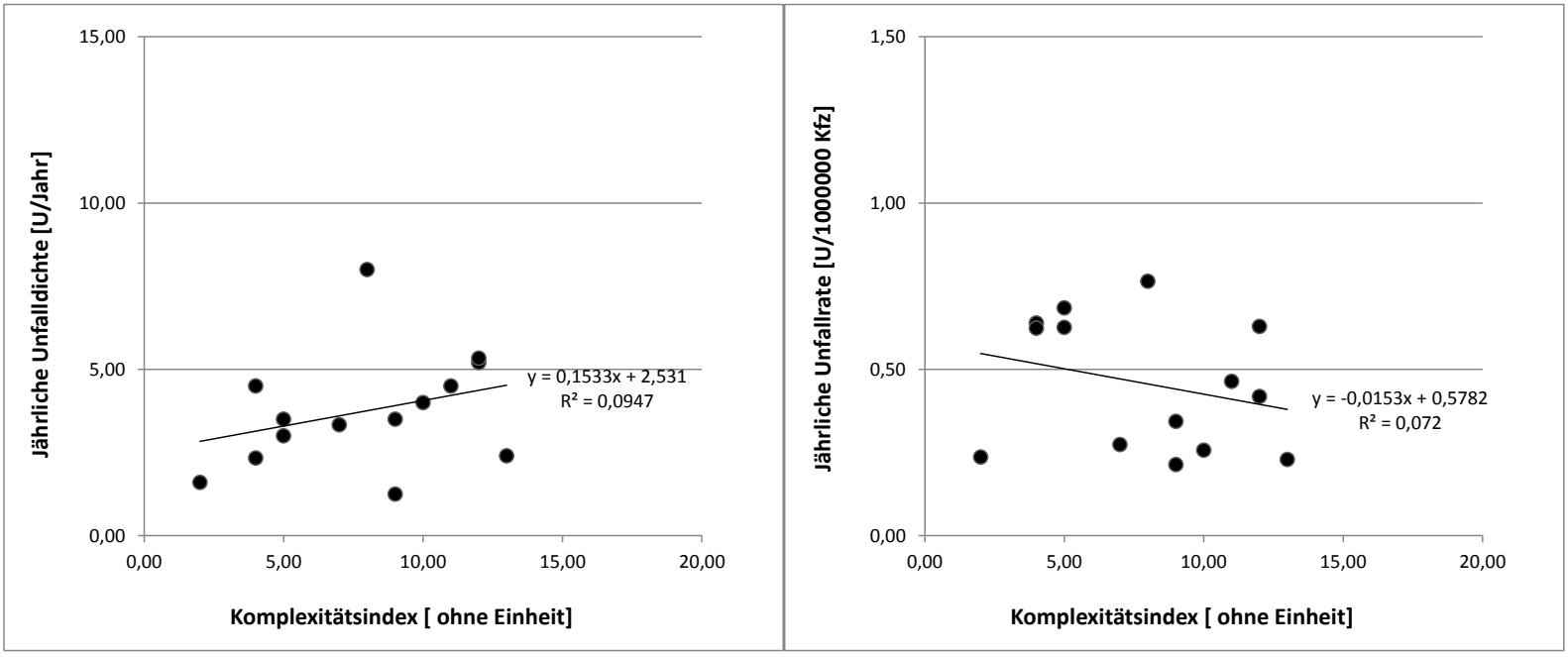

Abbildung 47: Lineare Regressionskurven und Bestimmtheitsmaß für den Zusammenhang zwischen Komplexitätsgrad und Unfalldichte (Bild links) bzw. Unfallrate (Bild rechts)
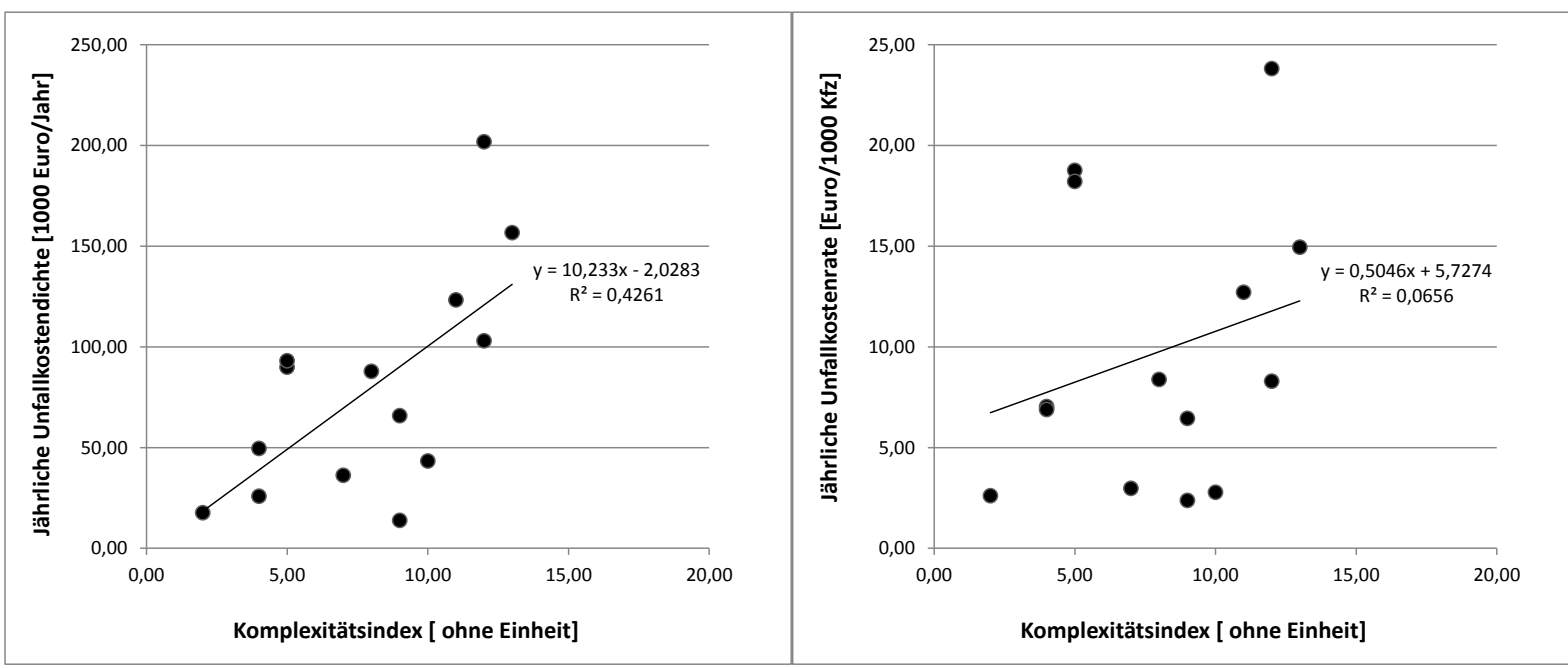

Abbildung 48: Lineare Regressionskurven und Bestimmtheitsmaß für den Zusammenhang zwischen Komplexitätsgrad und Unfallkostendichte (Bild links) bzw. Unfallkostenrate (Bild rechts) 
Unter den vorgenannten Einschränkungen ist zu erkennen, dass die Regressionskurven der Merkmale Komplexität und Unfalldichte bzw. Unfallkostendichte ein höheres Bestimmtheitsmaß (ca. 10 bzw. $43 \%$ ) erreichen, als dies zwischen den Merkmalen Komplexität und Unfallrate bzw. Unfallkostenrate erreicht wird (ca. $7 \%$ ). Dies führt zu den Thesen, dass mit zunehmender Komplexität eines Knotenpunktes die Anzahl der Unfälle und auch die Schwere der Unfälle ansteigen. Zudem sind nicht motorisierte Verkehrsteilnehmer an diesen Knotenpunkten besonders gefährdet.

Zur Bestätigung dieser Thesen sind weitergehende Untersuchungen notwendig. Die oben aufgeführten Aussagen stellen einen ersten Versuch dar, die Komplexität eines Knotenpunktes zu bestimmen und mögliche Zusammenhänge zwischen der Komplexität und der Verkehrssicherheit aufzudecken. Dies geschah unter Zuhilfenahme hypothetischer Indikatoren, die die Komplexität eines Knotenpunktes in der Gesamtbetrachtung beschrieben und vornehmlich den Blickwinkel der Pkw-Fahrer einbeziehen.

Insofern ist dies als Vorschlag zu verstehen, die Einstufung eines Knotenpunktes in bestimmte Komplexitätsgrade mit Hilfe geeigneter Indikatoren vorzunehmen. Dazu könnten die genannten Indikatoren als Grundlage für weitere Betrachtungen herangezogen und verifiziert bzw. falsifiziert werden. Zusätzlich Indikatoren wie z. B.

- die Art der Überquerungsanlage (LSA, FGÜ, mit/ohne Mittelinsel),

- die Art der Radverkehrsführung (Radfahrstreifen, Radweg auf Gehwegniveau),

- die Anzahl der zu überquerenden Fahrstreifen (ggf. zzgl. Radweg) beim Linksabbiegen,

- zusätzlich zu überquerende Gleiskörper von Straßenbahn/Stadtbahn,

- der Kreuzungswinkel (Kfz) bzw. der Winkel der Überquerung (FG/Rad) oder

- die Art der Signalisierung bzw. die Synchronität der Freigabe mit angrenzenden LSA für Fahrbahnüberquerungen

könnten aufgenommen werden.

Weitere Betrachtungen sollten zudem zwischen „allgemeinen“ und „speziellen“ Indikatoren differenzieren. So könnten sowohl grundlegende Aspekte, wie der Knotenpunkttyp, als auch verschiedenste Belange berücksichtigt werden. Es wird davon ausgegangen, dass die Komplexität eines Knotenpunktes für Fußgänger, Radfahrer oder Kraftfahrer sowie für Kinder, ältere Menschen oder Menschen mit besonderen Mobilitätseinschränkungen/behinderungen sehr verschieden sein kann. Ebenso stellt z. B. die Fahraufgabe des Linksabbiegens Pkw-Fahrer vor andere Herausforderungen wie die des Rechtsabbiegens.

Auch scheint eine Gewichtung möglicher Indikatoren sinnvoll, um z. B. die Verkehrsmenge oder die Bedeutung einzelner Indikatoren für verschiedene Verkehrsteilnehmer differenziert darstellen zu können. 


\section{Empfehlungen zur Gestaltung von Knotenpunkten}

Bei den folgenden Empfehlungen zur Gestaltung von Knotenpunkten wird der Schwerpunkt auf Maßnahmen zur Einschränkung von Konfliktsituationen gelegt, die während der Untersuchung besonders ins Gewicht gefallen sind (vgl. Abschnitt 6.3 und 7). Im Wesentlichen beziehen sich die Empfehlungen auf die Ausführungen aktueller Entwurfsregelwerke zur Gestaltung innerstädtischer Straßen und Knotenpunkte sowie von Fuß-, Rad- und barrierefreier Verkehrsanlagen. Grundlegend kann davon ausgegangen werden, dass an Knotenpunkten, die nach aktuellen Entwurfsregelwerken gestaltet sind, ein Großteil der festgestellten Konflikte nicht auftreten würde.

Generell ist im Hinblick auf die Verkehrssicherheit bei Neu- und Umbauten von Knotenpunkten - auch bei Änderungen von Teilbereichen - immer die Gesamtanlage unter Berücksichtigung der Belange aller Verkehrsteilnehmer zu betrachten.

Die folgenden Empfehlungen aus Sicht der Verkehrsbeteiligungsarten beziehen aufgetretene Konfliktsituationen von Kindern, älteren Menschen und Menschen mit besonderen Mobilitätseinschränkungen oder -behinderungen mit ein und gelten ausdrücklich auch für diese Personengruppen. Viele Anforderungen an die Gestaltung im Hinblick auf diese Gruppen, gelten aber auch für alle übrigen Verkehrsteilnehmer. So tragen u. a. die Einhaltung der Sichtbeziehungen und die Schaffung sicherer Überquerungsanlagen nicht nur, aber im Besonderen zur Erhöhung der Verkehrssicherheit für Kinder und auch für Rollstuhlfahrer bei. Klare Regelungen im Kreuzungsbereich (gesicherte Führung von Linksabbiegern, Führungslinien, Trennung des Links- und Geradeausverkehrs) unterstützen besonders ältere Kraftfahrer, bieten aber zugleich auch mehr Sicherheit für Kraftfahrer aller Altersklassen.

\subsection{Empfehlungen aus Sicht der Fußgänger (insbesondere für Kinder, ältere Menschen und Menschen mit Mobilitätseinschränkungen/- behinderungen)}

Das besondere Augenmerk gilt der Überquerung der Straße. An Überquerungsanlagen (Überquerungsstellen) wurden im Rahmen der Untersuchung wesentliche Defizite und Sicherheitsrisiken (auch in Verbindung mit Überschreiten-Unfällen) für Fußgänger festgestellt, wenn die diese ganz oder in Teilen, z. B. im Bereich der Aufstellflächen, fehlten bzw. nicht regelwerkskonform ausgeführt waren oder auch die Erkennbarkeit für die Kraftfahrer nicht gegeben war (vgl. u. a. prototypische Merkmale / Konfliktsituationen der Anlage A 7, Tabelle 30, Zeilen 1, 3 und 4).

Stellen, an denen ein Überquerungsbedarf durch Fußgänger besteht, z. B. zum Erreichen von Haltestellen, auf Schulwegen u. w., sollten mit geeigneten, dem Verkehrsaufkommen und den zu überquerenden Straßenbreiten angepassten Überquerungsanlagen ausgestattet sein. Bei Neu- und Umbaumaßnahmen sind die Überquerungsanlagen nach aktuellen Entwurfsregelwerken zu gestalten.

Ein weiterer wesentlicher Sicherheitsaspekt ist die Einhaltung der Sichtbeziehungen (vgl. u. a. prototypische Merkmale / Konfliktsituationen der Anlage A 7, Tabelle 30, Zeile 2), insbesondere im Bereich von Überquerungen. 
Das Parken am Fahrbahnrand im unmittelbaren Bereich von Überquerungsstellen ist wirksam zu unterbinden. Vorgezogene Seitenräume können auch in Bereichen mit hohem Parkdruck dafür Sorge tragen und die Sichtbeziehungen verbessern. Insbesondere zum Schutz von Kindern, die aufgrund ihrer Körpergröße hinter parkenden Fahrzeugen spät oder gar nicht zu sehen sind, ist dies ein grundlegender Faktor. Zudem kommt, dass Kinder z. T. auch unvermittelt auf die Straße laufen. Wenn zusätzlich die Sichtbeziehungen durch parkende Fahrzeuge am Fahrbahnrand eingeschränkt sind, können herannahende Kraftfahrer oft nicht rechtzeitig bremsen. Beispielsweise belegen dies auch die Unfallanzeigen der Unfälle am Knotenpunkt 14 (vgl. Anlage A 5).

Überquerungsanlagen (z. B. Fußgängerüberwege) sollten zudem deutlich gekennzeichnet werden. Besonders an Straßen, die einer langen, geraden Flucht folgen, damit Kfz-Führer frühzeitig auf mögliche Überquerungen von Fußgängern aufmerksam gemacht werden. Ggf. mit innen beleuchteten Verkehrszechen (StVO-Zeichen 350 „Fußgängerüberweg“, Abbildung 49). Auch ungesicherte Überquerungsanlagen können mit Hilfe baulicher Elemente, z. B. Mittelinsel, vorgezogene Seitenräume gekennzeichnet werden.

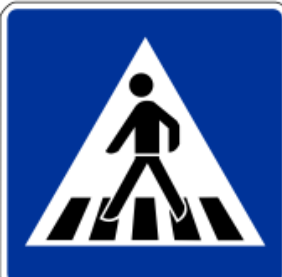

\section{Abbildung 49: StVO Zeichen 350 "Fußgänger Überweg"}

An lichtsignalgeregelten Knotenpunkten sollten die Wartezeiten für Fußgänger allgemein möglichst gering gehalten werden, um die Akzeptanz der Sperrzeit zu erhöhen und das Überqueren bei ROT einzuschränken. Fußgänger-Signalanlagen (Anforderungsanlagen) sollten möglichst kurz nach der Anforderung GRÜN zeigen damit sie von Fußgängern genutzt werden.

Auch für Menschen mit Mobilitätseinschränkung oder -behinderung stellt die Überquerung der Straße eine besondere Herausforderung dar. Die in Abschnitt 3.2 genannten Ausführungen zu mobilitätsbehinderten Menschen zeigen jedoch auch, dass eine entsprechend ihren Anforderungen angepasste Gestaltung an Knotenpunkten die Verkehrssicherheit erhöhen kann. Viele der Anforderungen sind dabei Deckungsgleich mit den Anforderungen anderer Gruppen. Daher kommen viele der Maßnahmen, die insbesondere Menschen mit Mobilitätseinschränkungen/-behinderungen helfen, auch allen Menschen zugute.

Folgende Elemente können für mobilitätsbehinderte Menschen eine Unterstützung bei der Überquerung von Straßen an Knotenpunkten sein und erhöhen die Sicherheit (vgl. u. a. prototypische Merkmale / Konfliktsituationen der Anlage A 7, Tabelle 32, Zeilen 1 bis 3):

- Ausreichende Sichtbeziehungen zwischen Fahrverkehr und überquerenden Fußgängern bzw. Rollstuhlnutzern;

- Mittelinseln;

- taktile Bodeninformationen (Bodenindikatoren);

- Überquerungsstellen mit differenzierten Bordhöhen: 
- visuell kontrastreich gestaltete Elemente im Bereich von Überquerungsstellen;

- akustische und taktile Signalgeber;

- längere Grünphasen bzw. geringere Räumgeschwindigkeit.

Eine detaillierte Beschreibung der vorgenannten Maßnahmen und Elemente kann der Anlage A 8 entnommen werden.

\subsection{Empfehlungen aus Sicht der Radfahrer (insbesondere für Kinder und ältere Menschen)}

Neben der Einhaltung der Sichtbeziehungen sollte dem Radverkehr ein dem Bedarf angepasstes Verkehrsinfrastrukturangebot zur Verfügung stehen. Dabei ist bei Neu- und Umbaumaßnahmen auf die regelwerkskonforme Ausführung der Radverkehrsanlagen besonders zu achten.

Die Untersuchungen zeigten insbesondere dort Mängel und Sicherheitsrisiken für Radfahrer, wo Radverkehrsanlagen fehlten ${ }^{22}$ oder nicht regelwerkskonform ausgebaut waren. Wesentliche Punkte waren, dass

- die Erkennbarkeit der Radverkehrsfurt an einmündenden Straßen nicht gegeben war (vgl. u. a. prototypische Merkmale / Konfliktsituationen der Anlage A 7, Tabelle 31, Zeile 1),

- die Radverkehrsanlage nicht regelwerkskonform gestaltet war, z. B. Unterschreitung der Mindestmaße (vgl. u. a. prototypische Merkmale / Konfliktsituationen der Anlage A 7, Tabelle 31, Zeile 2) und/oder

- Aufstellflächen für indirekt linksabbiegende Radfahrer oder Überquerungsanlagen fehlten (vgl. u. a. prototypische Merkmale / Konfliktsituationen der Anlage A 7, Tabelle 31, Zeilen 3 und 4).

Um die Erkennbarkeit von Radverkehrsfurten zu erhöhen, sind rote Einfärbungen der Furt zu empfehlen (Abbildung 50). An einigen Knotenpunkten war zwar der Radfahrtreifen/Radweg längs der Straße rot eingefärbt, aber gerade im Bereich der Einmündung, eine für Radfahrer oft gefährliche Stelle (vgl. Kap. 4, Einbiegen/Kreuzen-Unfälle), war die rote Einfärbung unterbrochen. Das StVO-Zeichen 138 „Radfahrer kreuzen“ kann zusätzlich an Einmündungen angebracht (Abbildung 51) oder als Piktogramm auf die Fahrbahn aufgebracht werden. Besonders für Kinder, die häufig bei Unfällen des Typs 342 verunglücken (Typ 342 „Radfahrer von rechts“, vgl. Abschnitt 4.3), ist dies eine Maßnahme zur Erhöhung der Verkehrssicherheit.

In diesem Zusammenhang ist auch die Einhaltung der Anfahrsichtweiten für einbiegende und kreuzende Kfz sicherzustellen, um das Vorziehen der Fahrzeuge auf die Radverkehrsfurt zu verhindern.

\footnotetext{
${ }^{22}$ Als Defizit erfasst, wenn die Empfehlungen für Radverkehrsanlagen (ERA 2010) bei bestimmten Randbedingungen Schutzstreifen, Radfahrstreifen oder Radwege empfehlen (vgl. ERA 2010, S. 18 f.).
} 

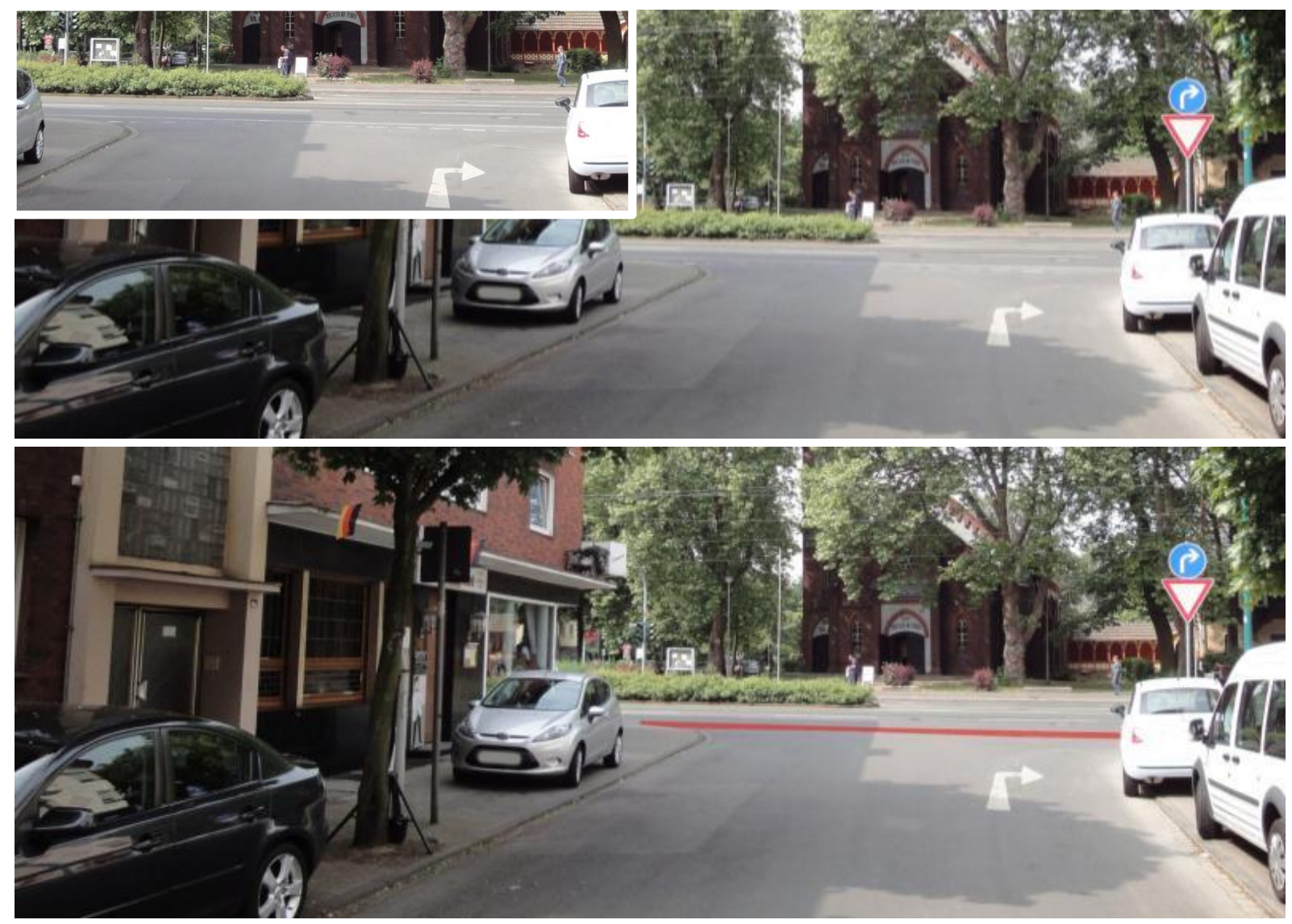

Abbildung 50: Radverkehrsfurt an einmündender Straße ohne Einfärbung (Bild oben) und mit Einfärbung (Bild unten, Retusche)
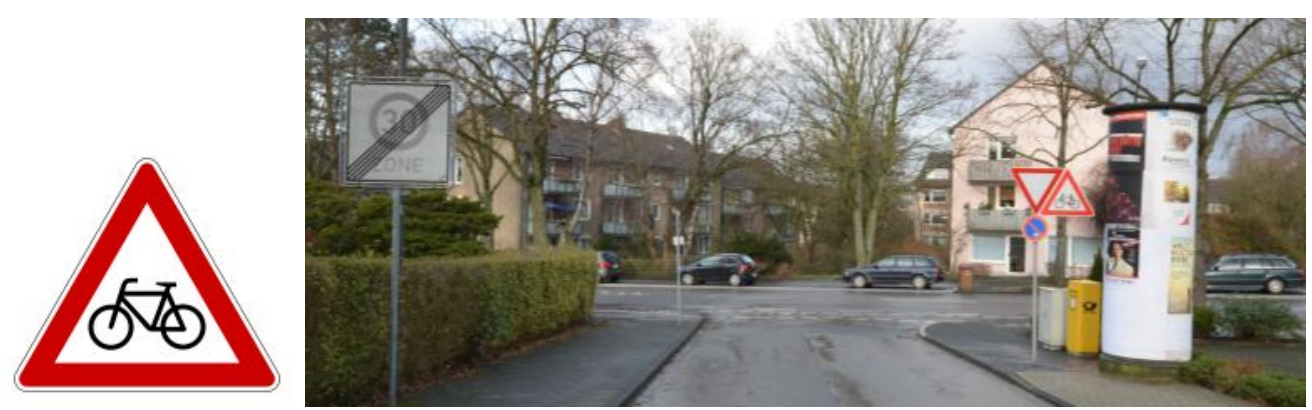

Abbildung 51: Sinnbild StVO-Zeichen 138 „Radfahrer kreuzen“ (Bild links) und an einmündender Straße (Bild rechts)

Im Weiteren sollten ausreichende Überquerungsmöglichkeiten im Hauptnetz der Radwege geschaffen werden, um ein Fahren auf Gehwegen und/oder entgegen der Fahrtrichtung auf Radwegen zu reduzieren. Damit sind auch Zugangsmöglichkeiten zu den Radverkehrsanlagen gemeint, z. B. das Erreichen eines gegenüberliegenden Radwegs bei der Ausfahrt aus einer untergeordneten Straße. 
Auf den Grünpfeil (StVO-Zeichen 720, vgl. Anhang A 1) sollte bei hohem Radverkehrsaufkommen generell verzichtet werden, insbesondere aber dann, wenn

„der freigegebene Fahrradverkehr auf dem zu kreuzenden Radweg für beide Richtungen zugelassen ist oder der Fahrradverkehr trotz Verbotes in der Gegenrichtung in erheblichem Umfang stattfindet und durch geeignete Maßnahmen nicht ausreichend eingeschränkt werden kann“ (VWV-StVO, § 37, XI Grünpfeil, Ziffer 1e).

\subsection{Empfehlungen aus Sicht der Kraftfahrer (insbesondere für ältere Menschen)}

Im Allgemeinen sind Einbiegen/Kreuzen-Unfälle, gefolgt von Abbiege-Unfällen die häufigsten Unfalltypen im Kfz-Verkehr an Knotenpunkten (vgl. Kap. 4). Insbesondere fällt allerdings der Unfalltyp 211 auf, der in der Detailbetrachtung der dreistelligen Unfalltypen noch vor den dreistelligen Unfalltypen der Einbiegen/Kreuzen-Unfälle rangiert ${ }^{23}$. Neben diesem Unfalltyp kamen sowohl bei älteren Kraftfahrern, wie auch bei allen Kraftfahrern die Unfalltypen 301, 302 und 321 häufig vor (vgl. Abschnitt 0 allgemein und Auswertung der 3-stelligen Unfalltypen am Beispiel der Unfalldaten aus NRW).
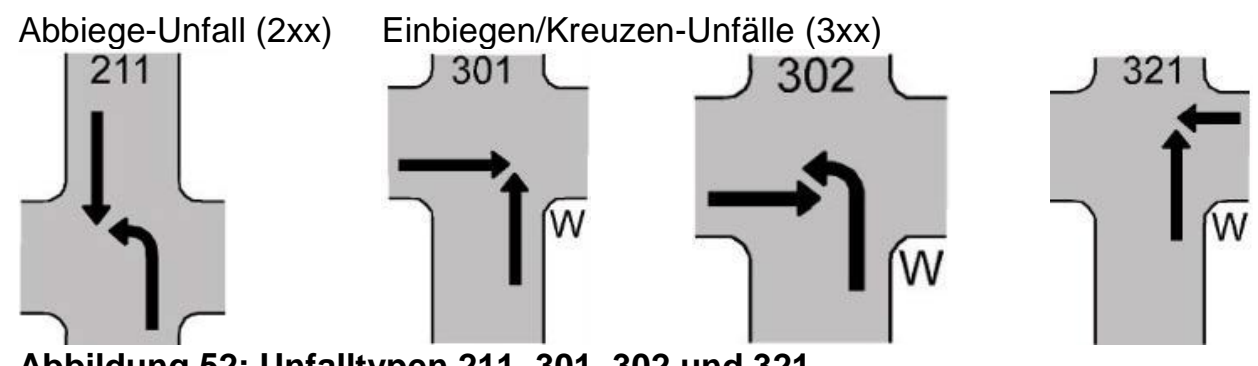

Abbildung 52: Unfalltypen 211, 301, 302 und 321

Im Zuge der Verhaltensbeobachtungen konnten gerade bei den Linksabbiegevorgängen die meisten Konflikte und Schwierigkeiten für die Verkehrsteilnehmer beobachtet werden, insbesondere dann, wenn Führungshilfen im Kreuzungsbereich (Leitlinien, Wartelinien) nicht gegeben waren (vgl. u. a. prototypische Merkmale / Konfliktsituationen der Anlage A 7, Tabelle 33, Zeilen 1, 2, 3 und 5).

Die für Kraftfahrer aller Altersklassen sicherste Führung bei Linksabbiegevorgängen an Kreuzungen mit Lichtsignalanlagen, ist die signaltechnisch gesicherte Führung. Diese sollte zur Erhöhung der Verkehrssicherheit anderen Lösungen vorgezogen werden. Im Sinne der Verkehrssicherheit ist in diesem Zusammenhang eine einheitliche sprachliche Regelung in den einschlägigen Richtlinien und die Verpflichtung zur Einrichtung des Linksabbiegeschutzes - zumindest bei zu definierenden Rahmenbedingungen wie eingeschränkte Sichtverhältnisse oder hohen Verkehrsmengen - wünschenswert.

\footnotetext{
${ }^{23}$ Auf Grundlage der Auswertung der dreistelligen Unfalltypen am Beispiel elektronischer Unfalldaten aus Nordrhein-Westfalen (2004 bis 2008).
} 
Im Weiteren bieten klare Führungshilfen, wie äußere Leitlinien und Wartelinien für Linksabbieger im Kreuzungsbereich, insbesondere für ältere Kraftfahrer, eine gute Unterstützung sich im Knotenpunkt zurechtzufinden.

Linksabbieger und Geradausfahrer sollten getrennt geführt werden. „Vor-Kopf“ angebrachte Linksabbiegestreifen der beiden Gegenrichtungen bieten zusätzliche Sicherheit (Abbildung 53 und Anhang A 3, Knotenpunkt 8). Eine Lösung, die auch an Kreuzungen ohne Lichtsignalanlagen angebracht werden kann.

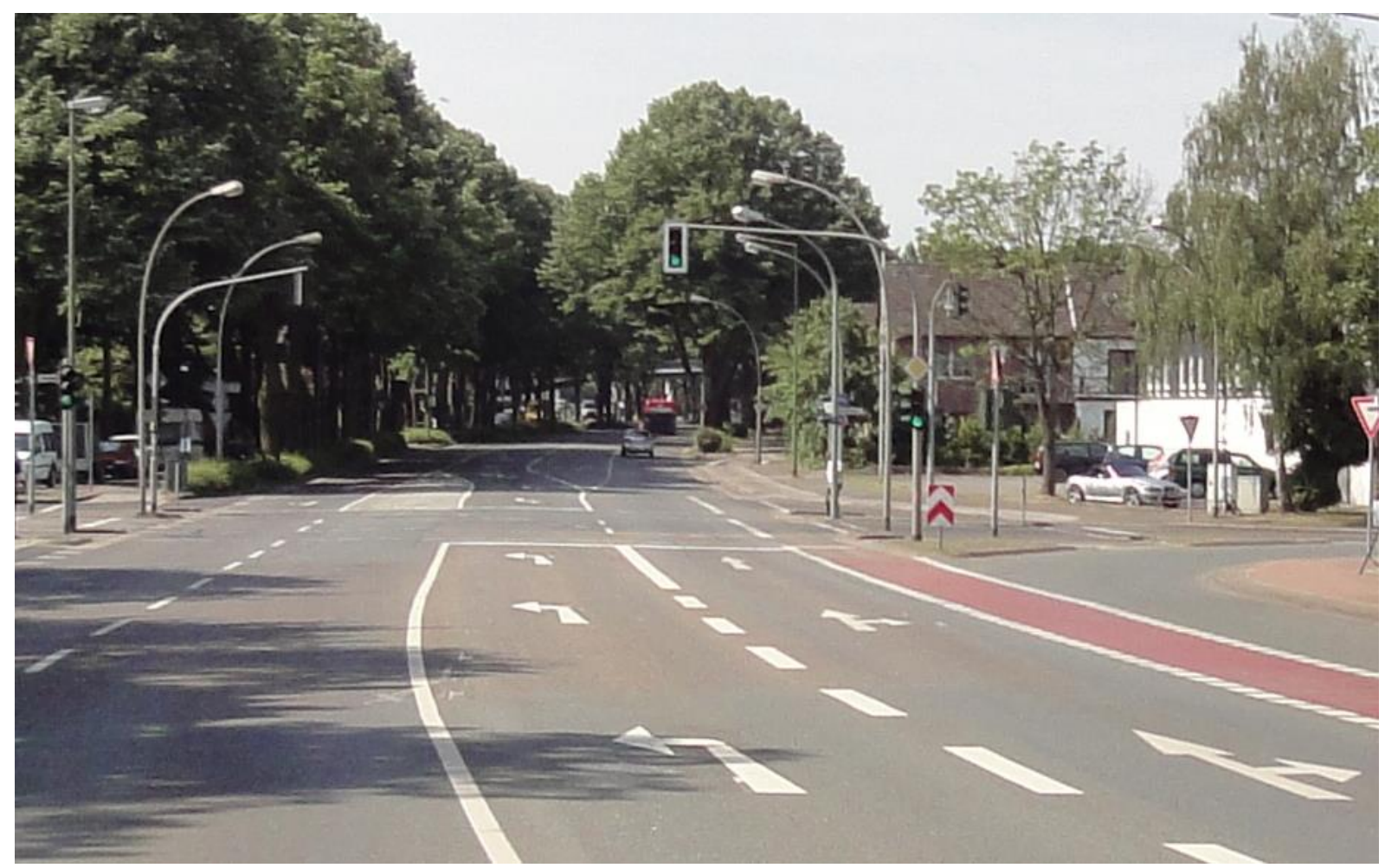

Abbildung 53: „Vor-Kopf“ gesetzte Linksabbiegestreifen

Zudem unterbindet eine Trennung dieser beiden Verkehrsströme in den Zufahrten von Knotenpunkten auch Verflechtungsvorgänge im Kreuzungsbereich, wenn hinter der Kreuzung eine Fahrstreifensubtraktion erfolgt (vgl. Abbildung 54). Diese Verflechtungsvorgänge führten im Rahmen der Vor-Ort-Beobachtungen zum Teil zu sehr unübersichtlichen Situationen. Auch werden die Sichtbeziehung durch weitere, im Kreuzungsbereich festgehaltene Fahrzeuge des Geradeausverkehrs, die keine ausreichend großen Zeitlücken zur Einordnung finden, weiter eingeschränkt. 


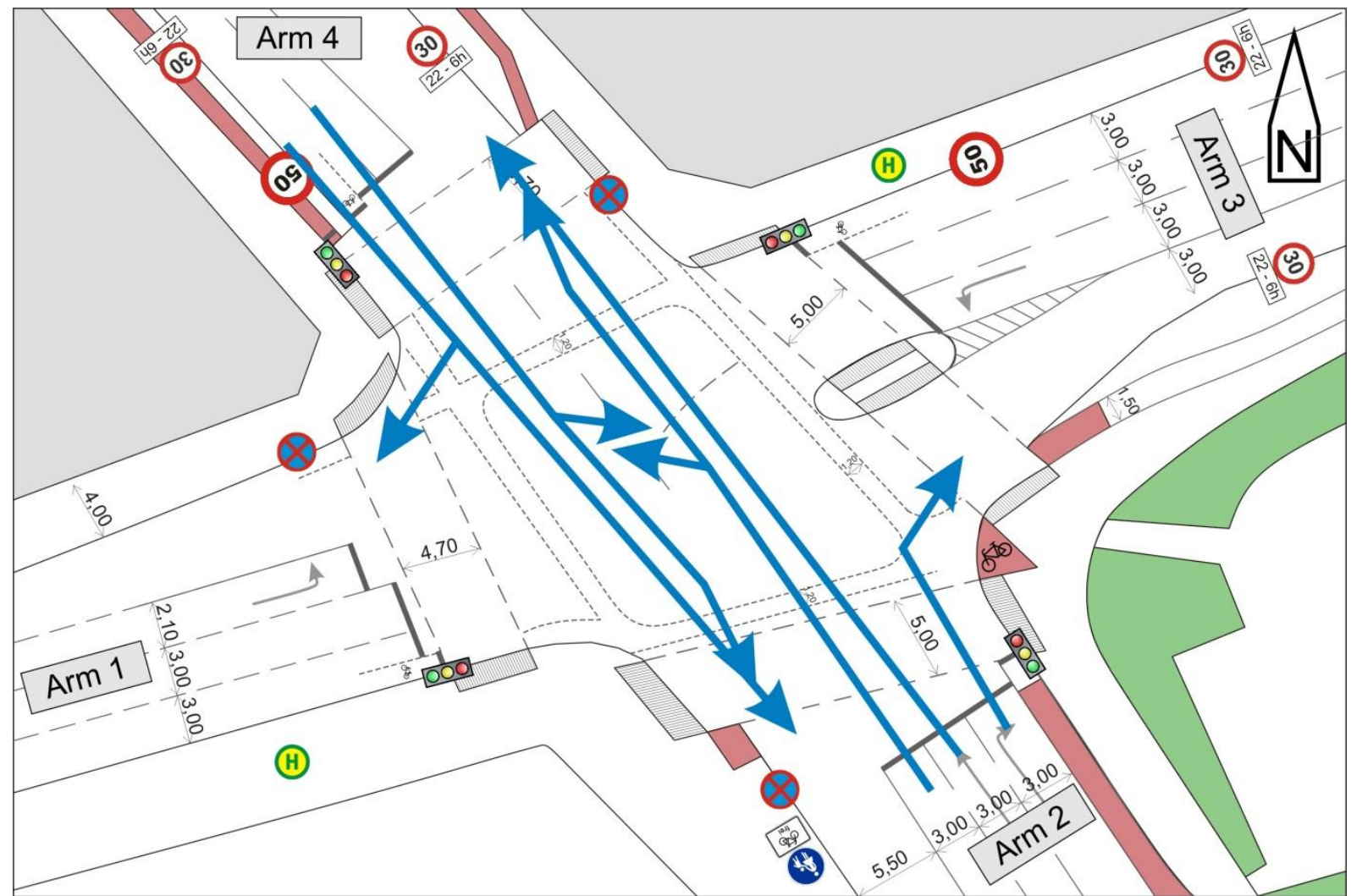

Abbildung 54: Verflechtungsvorgänge im Kreuzungsbereich am Beispiel des Knotenpunktes 1

Auch für den Kfz-Verkehr gilt, dass die Sichtbeziehungen zu anderen Verkehrsteilnehmer von entscheidender Bedeutung sind. Einschränkungen der Sicht, z. B. durch den Bewuchs auf dem Mittelstreifen oder durch im Kreuzungsbereich wartende andere Verkehrsteilnehmer, können mit Hilfe einfacher Maßnahmen (Rückschnitt der Begrünung, Führung der Linksabbieger, Trennung von Geradeausverkehr und Linksabbiegern) vermieden werden.

Anfahrsichtweiten sind in jedem Fall einzuhalten. Gestaltungen von Straßenräumen, z. B. mittels Baumreihen, haben dem gegenüber keine Priorität. Wenn die Anfahrsichtweiten, z. B. aufgrund einer geschlossenen Bebauung nicht eingehalten werden können, sollten die Zufahrten aus den untergeordneten Straßen mit Hilfe einer Lichtsignalanlage geregelt werden.

\subsection{Weitere Empfehlungen}

Im Rahmen dieser Untersuchung wurden wesentliche Defizite und Sicherheitsrisiken an innerstädtischen Knotenpunkten für alle, insbesondere aber für die nicht motorisierten Verkehrsteilnehmer (vor allem auch für Kinder und für Rollstuhlfahrer) identifiziert, die im Zusammenhang mit schlechten oder nicht gegebenen Sichtbeziehungen standen.

Neben der Einhaltung von Gestaltungshinweisen aktueller Entwurfsregelwerke, sollen im Sinn der Richtlinien für die Anlage von Straßen (RASt 2006) bereits in der Vorentwurfsplanung (entsprechend Entwurfsplanung nach HOAI), die Sichtdreiecke 
dargestellt werden ${ }^{24}$. Empfohlenen wird eine verbindliche Regelung zum Einzeichnen der Sichtdreiecke in die Entwurfspläne. Dies sollte in die „Richtlinien für die Gestaltung von einheitlichen Entwurfsunterlagen im Straßenbau“ übernommen werden.

Im Weiteren wäre ein Qualitätsmanagement für die Straßenraumgestaltung wünschenswert, um einen kontinuierlichen Prozess im Sinne der Verkehrssicherheit zu erreichen. Neben den bisherigen Instrumenten zur Verbesserung der Verkehrssicherheit, ist ein „systematisches Qualitätsmanagement speziell für Knotenpunkte" zu empfehlen, das die Verkehrsanlage als Ganzes betrachtet und sich auf alle Phasen der Lebensdauer der Verkehrsanlage bezieht. Dieses Qualitätsmanagement kann in ein allgemeines Qualitätsmanagement für Straßenräume integriert werden.

Im Weiteren sollte überprüft werden, in welcher Weise ein solches Qualitätsmanagement die derzeit in der Erarbeitung befindlichen Empfehlungen für die Durchführung eines Bestandaudits von Straßen einbezieht und in welcher Weise neuere Sicherheitsanforderungen an Straßen auf bestehende Straßennetze und Verkehrsanlagen übertragen werden können.

Ein Bestandsrege/werk für Straßen wäre wünschenswert, ist aber u. a. aufgrund begrenzter Ressourcen nicht einzuhalten. Allerdings sollte das Bestandsaudit in den systematischen Vorgang im Rahmen der örtlichen Unfalluntersuchung verbindlich eingeführt werden, um so die Unfallkommissionen unterstützen und auch Defizite im Straßenraum dokumentieren zu können. Die Ergebnisse der Untersuchung sollten dabei Berücksichtigung finden (vgl. u. a. prototypische Merkmale / Konfliktsituationen der Anlage A 7). Maßnahmen zur Verbesserung der Verkehrssicherheit, die aus dem Bestandsaudit hervorgehen, sollten verbindlich umzusetzen sein.

${ }^{24}$ Vgl. RASt 2006, FGSV[Hrsg.]: Richtlinie für die Anlage von Stadtstraßen, Ausgabe 2006, S. 23 


\section{Fazit und Ausblick}

Kinder, ältere Menschen und Menschen mit besonderen Mobilitätseinschränkungen oder Mobilitätsbehinderungen sind im Verkehrsraum aufgrund ihrer körperlichen und kognitiven Fähigkeiten häufig benachteiligt. Sie zählen zu den so genannten „schwächeren Verkehrsteilnehmern". Doppelt schwer wiegt die Tatsache, dass diese Personengruppen bei Verkehrsunfällen zu Fuß oder mit dem Fahrrad besonders häufig (Kinder) oder besonders schwerwiegende Verletzungen bis hin zur Todesfolge (ältere Verkehrsteilnehmer) erleiden. Bei den älteren Menschen kommt das erhöhte Mortalitätsrisiko bei gleicher Unfallschwere als zusätzlicher negativer Einflussfaktor bei den Unfallfolgen hinzu. Mehr als jeder zweite Unfall in Ortschaften ereignet sich an einem Knotenpunkt.

Ziel dieses Forschungsvorhabens war es, einen effizienten Ansatz zur Erhöhung der Verkehrssicherheit zu liefern; durch Sammlung, Dokumentation und Auswertung von Unfalldaten sowie Sicherheitsbetrachtungen und Verhaltensbeobachtungen an ausgewählten Fallbeispielen. Auch um Handlungsempfehlungen für die Regelwerksanwendung bzw. -erstellung formulieren zu können.

Einige bereits bekannte Einflussfaktoren, die zu einer Gefährdung gerade auch von „schwächeren Verkehrsteilnehmern“ führen, konnten auf Basis umfangreicher Unfallanalysen und Verkehrsbeobachtungen sicher verifiziert werden. Zudem konnten neue Erkenntnisse, insbesondere zum Verhalten älterer Pkw-Fahrer, gewonnen werden.

Im Wesentlichen ergeben sich aus den Untersuchungen die folgenden Forderungen, die nachfolgend weiter erläutert werden:

1. Die Verpflichtung zur Einrichtung einer signaltechnisch gesicherten Führung von Linksabbiegern sollte bei bestimmten Rahmenbedingungen, wie z.B. eingeschränkte Sichtverhältnisse oder hohe Verkehrsmengen, verbindlich eingeführt werden. Eine entsprechende, einheitliche sprachliche Regelung sollte in den einschlägigen Entwurfsregelwerken Einzug finden.

2. Die Einhaltung der Sichtbeziehungen ist im Rahmen der Planung von Straßenverkehrsanlagen verbindlich zu gewährleisten und bereits in der (Vor)Entwurfsplanung, z. B. durch die Verpflichtung Sichtdreiecke in Planunterlagen einzuzeichnen, zu berücksichtigen.

3. Bei Neu- und Umbaumaßnahmen von Verkehrsanlagen sind die Gestaltungshinweise aktueller Entwurfsregelwerke verpflichtend einzuhalten. Verkehrsanlagen sind dabei in ihrer Gesamtheit, unter Berücksichtigung der sicherheitsrelevanten Belange aller Verkehrsteilnehmer, zu betrachten. Zudem sollte das Sicherheitsaudit von Straßen in allen Entwurfsphasen verbindlich eingeführt werden.

4. Das Bestandsaudit sollte anlassbezogen (Unfallhäufungsstelle), als systematischer Bestandteil der örtlichen Unfalluntersuchung verbindlich eingeführt werden. Maßnahmen zur Verbesserung der Verkehrssicherheit, die aus dem Bestandsaudit hervorgehen, sollten verbindlich umzusetzen sein. 


\section{Linksabbiegeschutz}

Neben Einbiegen/Kreuzen-Unfällen sind Abbiege-Unfälle die häufigsten Unfälle an Knotenpunkten im Kfz-Verkehr. In der Detailbetrachtung der dreistelligen Unfalltypen steht der Unfalltyp 211 (Zusammenstoß zwischen Linksabbieger und Geradeausfahrer der Gegenrichtung) an erster Stelle noch vor den dreistelligen Unfalltypen der Einbiegen/Kreuzen-Unfälle, sowohl bei älteren als auch bei jüngeren Kraftfahrern (vgl. Abschnitt 4.3).

Im Rahmen der Untersuchung wurde das Verhalten von insgesamt 12.415 PkwFahrern ausgewertet, darunter 2.338 ältere Pkw-Fahrer. In Bezug auf das Linksabbiegen konnte eine Forschungslücke geschlossen werden. Die Auswertungen von 5.964 Linksabbiegern, darunter 1.033 älterer Fahrer, haben gezeigt, dass ältere Fahrer tatsächlich beim Linksabbiegen relativ gesehen mehr Fehler machen als andere Pkw-Fahrer. Die Verhaltensbeobachtung belegen einerseits, dass die Fehlerqualität an denselben Knotenpunkten bei allen Pkw-Fahrern gleich ist. Ältere Kraftfahrer machen im Vergleich mit jüngeren Fahrern also keine grundlegend anderen Fehler an schwierigen Knotenpunkten. Allerdings ist der relative Anteil der Fehler beim Linksabbiegen besonders erhöht.

Weiterhin belegen die Untersuchungen, dass fehlende Orientierungshilfen im Kreuzungsbereich für Linksabbieger (Leit- und Wartelinien), älteren Fahrern an großen, hochfrequentierten Knotenpunkten besondere Schwierigkeiten bereiten. Auch an Knotenpunkten ohne Lichtsignalanlage unterstützen diese Führungshilfen. Daneben sollten Linksabbieger und Geradeausfahrer einer Richtung auf getrennten Fahrstreifen geführt werden. „Vor-Kopf“ angebrachte Linksabbiegestreifen der beiden Gegenrichtungen bieten zusätzliche Sicherheit.

Aufgrund des analysierten Unfallgeschehens an Knotenpunkten und der beobachteten Konfliktsituationen sollten Linksabbieger an Knotenpunkten mit Lichtsignalanalgen signaltechnisch gesichert geführt werden. Eine entsprechend Verpflichtung dazu, sollte neben der Anzahl der Linksabbiegestreifen bei weiteren zu definierenden Randbedingen, wie z. B. der Verkehrsbelastung, verbindlich eingeführt und über eine einheitliche sprachliche Regelung in den Richtlinien berücksichtigt werden.

Ein Linksabbiegeschutz stellt für Kraftfahrer aller Altersgruppen einen erheblichen Sicherheitsgewinn dar und hilft insbesondere älteren Kraftfahrern, auch an großen, stark frequentierten und/oder unübersichtlichen Knotenpunkten, sicher abzubiegen.

\section{Sichtbeziehungen}

Einbiegen/Kreuzen-Unfälle sind die häufigsten Unfalltypen an Knotenpunkten (vgl. Abschnitt 0). Insbesondere an Knotenpunkten ohne Lichtsignalanlage sind daher zwingend die Anfahrsichtweiten für einbiegende oder kreuzende Kraftfahrer einzuhalten. Die Sichtdreiecke sind zudem von beeinträchtigenden Elementen wie Bäumen, Masten, Werbetafeln oder Wartehäuschen/Wetterschutzstände von Haltestellen des ÖV freizuhalten. Bei geschlossener Randbebauung der einmündenden Straße und starkem Radverkehr der Hauptrichtung wäre die 
Signalisierung der einbiegenden und kreuzenden Kfz zum Schutz der Radfahrer wünschenswert.

Kinder verunglücken am häufigsten bei Einbiegen/Kreuzen-Unfällen als Radfahrer (vgl. Abschnitt 4.3). In der Detailbetrachtung der dreistelligen Unfalltypen sticht hier der Unfalltyp 342 (Radfahrer an Einmündung von rechts) hervor; gefolgt von den beiden Unfalltypen 321 (Radfahrer nach Kreuzungsbereich von rechts) und 301 (Radfahrer an Einmündung von links). Insgesamt nehmen diese drei Unfalltypen, bei denen Kinder als Radfahrer verunglücken, über $20 \%$ der Unfälle mit Beteiligung von Kindern ein (vgl. Abschnitt 4.3, Detailbetrachtung dreistelliger Unfalltypen am Beispiel der Verkehrsunfalldaten aus NRW). Neben der Einhaltung der Sichtbeziehungen bzw. der ggf. erforderlichen signaltechnischen Führung der einbiegenden/kreuzenden $\mathrm{Kfz}$, sollten an Einmündungen die Radverkehrsfurten rot eingefärbt werden, um auf kreuzenden Radfahrer besser aufmerksam zu machen.

Die Einhaltung der Sichtbeziehungen stellt einen Sicherheitsgewinn für alle Verkehrsteilnehmer dar. Für die Sicherheit von Kindern ist sie unverzichtbar. Aufgrund ihrer geringeren Körpergröße und ihres noch nicht voll ausgeprägten Gefahrenbewusstseins befinden sich gerade Kinder an Stellen ohne ausreichende Sichtbeziehungen für den Kfz-Verkehr, z. B. durch parkende Fahrzeuge am Fahrbahnrand eingeschränkt, in höchster Gefahr. Dies belegen die Unfalluntersuchungen und die Verhaltensbeobachtungen und gilt analog z. B. auch für Rollstuhlfahrer. Die Unfallanalyse der Unfälle mit behinderten Menschen zeigt, dass mindestens der Teil der Rollstuhlnutzer an Knotenpunkten verhältnismäßig oft von abbiegenden Kraftfahrern übersehen wird und es dadurch zum Teil zu schweren Unfällen kommt.

Daher ist die Einhaltung der Sichtbeziehung ein entscheidender Faktor zu Erhöhung der Verkehrssicherheit, insbesondere für die „schwächeren Verkehrsteilnehmer" und sollte mit Beginn der (Vor-)Entwurfsplanung, z. B. durch die Verpflichtung Sichtdreiecke in Planunterlagen einzuzeichnen, stärker berücksichtigt werden.

\section{Entwurfsregelwerke / Sicherheitsaudit}

Die Verpflichtung zur Einhaltung der Regelwerke ist bisher selbst beim Neu- und Umbau nicht eindeutig geregelt. Viele der festgestellten Defizite, die zum Teil auch im direkten Bezug zum Unfallgeschehen standen, wären bei Knotenpunkten, die gemäß aktuellen Regelwerken gestaltet wären, nicht aufgetreten. Die Ausgestaltung von Straßenverkehrsanlagen im Zuge von Neu- und Umbaumaßnahmen gemäß den Planungsempfehlungen aktueller Regelwerke sowie die Überprüfung der Verkehrssicherheit durch verbindliche Sicherheitsaudits in allen Phasen der Planung sind daher weitere zentrale Forderungen.

Die bestehenden Regelwerke sind dazu geeignet, „schwächere Verkehrsteilnehmer“ (Kinder, ältere und mobilitätsbehinderte Menschen) zu schützen. Wenn sie konsequent angewendet und im Hinblick auf die Verkehrssicherheit voll ausgeschöpft werden. Sie werden u. a. durch Forschungsergebnisse aktualisiert, sodass eine kontinuierliche Verbesserung stattfindet. 
Insbesondere Kinder sind auf diesen Schutz angewiesen. An kritischen Überquerungsstellen wird innen (wie auch allen anderen Fußgängern), sofern keine gesicherten Überquerungsanlagen vorhanden sind, oftmals trotz ihrer Bevorrechtigung als Fußgänger vor abbiegenden Kfz, dieser Vorrang von PkwFahrern nicht eingeräumt. Bei Radfahrern wurde häufig beobachtet, dass sie Fußgängerverkehrsanlagen nutzten, wenn Radverkehrsanlagen nicht vorhanden waren oder durch die bauliche Gestaltung nicht sicher erschienen. Im Gegenzug nutzten Rollstuhlfahrer oder Personen mit Rollatoren oder Kinderwagen die Radverkehrsanlagen oder wichen auf die Fahrbahn aus, wenn Nullabsenkungen an der Fußgänger-Überquerungsanlage nicht vorhanden waren.

Auch um sicherheitsrelevante Nachteile für bestimmte Gruppen zu vermeiden, ist daher auf eine konsequente Anwendung aktueller Entwurfsregelwerke bei Neu- und Umbaumaßnahmen nach dem Prinzip eines Designs für Alle besonderer Wert zu legen.

\section{Bestandsaudit}

Die festgestellten Konflikte und Unfallsituationen wurden an Knotenpunkten im Bestand aufgenommen. Die Gestaltung der Knotenpunkte (darunter waren auch Unfallhäufungsstellen gemäß den Festlegungen des M Uko $2012^{25}$ ) entsprach häufig nicht den Planungsempfehlungen aktueller Regelwerke und somit nicht dem Stand der Technik und der Verkehrssicherheit.

Für den Bestand sind allerdings so gut wie gar keine gestalterischen Vorgaben und nur teils betriebliche Pflichten einzuhalten. Es fehlt an verbindlichen Regelungen für den Bestand.

Ein Bestandsregelwerk für Straßen wäre daher wünschenswert, ist aber u. a. aufgrund begrenzter Ressourcen nicht einzuhalten. Allerdings sollte den in der Erarbeitung befindlichen Empfehlungen für die Durchführung eines Bestandaudits zukünftig eine maßgebende Rolle zukommen.

Das Bestandsaudit von Straßen sollte anlassbezogen in den in den systematischen Vorgang im Rahmen der örtlichen Unfalluntersuchung verbindlich eingeführt werden. Die Ergebnisse dieser Untersuchung sollten dabei Berücksichtigung finden. Im Weiteren sollten die Maßnahmen zur Verbesserung der Verkehrssicherheit, die aus dem Bestandsaudit hervorgehen, verbindlich umzusetzen sein.

„Schwächere Verkehrsteilnehmer“ benötigen einen besonderen Schutz im Straßenverkehr. Allerdings bedarf es keiner grundlegend neuen alters- oder subgruppenspezifischen Entwurfsregelwerke.

Die bestehenden aktuellen Regelwerke sind bei konsequenter Anwendung dazu geeignet, Kindern und älteren Menschen einen hohen Schutz als Fußgänger und Radfahrer zu geben.

\footnotetext{
${ }^{25}$ FGSV [Hrsg.]: Merkblatt zur Örtlichen Unfalluntersuchung in Unfallkommissionen (M Uko), Ausgabe 2012, S. 15
} 
Eine Verschärfung im Hinblick auf die Regelungen zum signaltechnisch geführten Linksabbieger würde allerdings besonders älteren Kraftfahrern helfen und darüber hinaus zur Erhöhung der Verkehrssicherheit an Knotenpunkten im Allgemeinen beitragen. Analog gilt dies für die Gewährleistung ausreichender Sichtbeziehungen, was einen Sicherheitsgewinn für alle Verkehrsteilnehmer bedeutet, insbesondere aber Kindern hilft.

Für Gruppen mobilitätsbehinderter Menschen ergeben sich teils deckungsgleiche, teils aber auch besondere Anforderungen. Aufgrund ihrer zahlenmäßigen Bedeutung sind besonders die Anforderungen der seh- und der gehbehinderten Menschen zu betrachten. So ist auch aus der Unfallanalyse zu erkennen, dass „rollende“ Fußgänger (Rollstuhl- und Rollatornutzer) aufgrund fehlender Bordsteinabsenkungen im Überquerungsbereich der Fußgänger auf Radverkehrsanlagen oder sogar die Fahrbahn ausweichen.

Vor allem dieser Konflikt über die richtige Ausgestaltung der Absenkung im Überquerungsbereich wird sich aufgrund der deutlichen Zunahme der Anzahl gehbehinderter Menschen zukünftig verschärfen. Bisher bereits in vielen Kommunen umgesetzte Lösungen lassen eine Gestaltung nach dem Prinzip eines Designs für Alle vermissen und führen daher teils zu sicherheitsrelevanten Nachteilen für andere Gruppen. Hier sind gesicherte Erkenntnisse über die Akzeptanz bisheriger Lösungen unter Berücksichtigung der geänderten Rahmenbedingungen bzw. sichere und akzeptierte neue Lösungen vonnöten. ${ }^{26}$

Aufgrund von Fortschreibungen auf Basis neuer Erkenntnisse können Empfehlungen in technischen Regelwerken mit unterschiedlichem Veröffentlichungsdatum voneinander abweichen. Zunächst kann dabei vorausgesetzt werden, dass die Anwendung der Regelwerke aktuelleren Datums Verbesserungen hinsichtlich der Verkehrssicherheit mit sich bringt. Unfallkommissionen und Sicherheitsauditoren ziehen für ihre Arbeit die in den Regelwerken veröffentlichten Planungsempfehlungen heran. Daher ist es essentiell, dass sich diese Gruppen stets auf dem aktuellen Wissensstand der Verkehrssicherheitsforschung befinden. Für Sicherheitsauditoren ist bereits eine wiederkehrende Qualifizierung (alle drei Jahre) erforderlich. Das Sicherheitsaudit besitzt jedoch derzeit in Deutschland mit Ausnahme der Straßen des Trans-europäischen Straßennetzes (TERN) keine Verbindlichkeit für die Verkehrsplanung, es wird ggf. von Straßenbaulastträgern freiwillig durchgeführt. Die Arbeit der Unfallkommissionen ist demgegenüber gesetzlich verbindlich festgeschrieben. Es mangelt jedoch bisher an einer verbindlichen und regelmäßigen Qualifizierung der Mitglieder der Unfallkommissionen im Sinne eines kontinuierlichen Qualitätsmanagements. Zudem besteht derzeit keinerlei Verpflichtung, die von einer Unfallkommission erarbeiteten Maßnahmen umzusetzen.

Daher sollte die Verpflichtung zur Einhaltung aktueller Entwurfsregelwerke beim Neu- und Umbau von Straßenverkehrsanlagen eindeutig geregelt sein. Zudem sollten das Sicherheitsaudit von Straßen sowie ein anlassbezogenes Bestandsaudit im Rahmen der örtlichen Unfalluntersuchung, einschließlich der Umsetzung von Maßnahmen zur Erhöhung der Verkehrssicherheit an Unfallhäufungsstellen, verbindlich eingeführt werden.

\footnotetext{
${ }^{26}$ Dieser Problematik widmet sich ein derzeit laufendes, von der BASt betreutes Forschungsvorhaben (FE77.0500/2010 „Barrierefreie Querungsstellen an Hauptverkehrsstraßen - Ausgestaltung von Bordsteinkante und Bodenindikatoren im Detail“; voraussichtlich bis Juni 2013).
} 
Im Weiteren sollte bei der Umsetzung von Maßnahmen statt einer rein betriebswirtschaftlichen Betrachtung einer volkswirtschaftlichen Betrachtung der Vorzug gegeben werden. Untersuchungen belegen einen besseren Wirkungseffekt bei der Durchführung baulicher Maßnahmen.

Neben den anerkannten und bisher genannten Instrumenten zur Verbesserung der Verkehrssicherheit, könnte ein systematisches Qualitätsmanagement speziell für Knotenpunkte, das die Verkehrsanlage als Ganzes betrachtet und sich auf alle Phasen der Lebensdauer der Verkehrsanlage bezieht, einen weiteren Beitrag zur Erhöhung der Verkehrssicherheit leisten. Dieses Qualitätsmanagement kann in ein allgemeines Qualitätsmanagement für Straßenräume integriert werden.

Eine Definition der Komplexität von Knotenpunkten könnte es ferner ermöglichen, speziell für „schwächere Verkehrsteilnehmer" präventiv zu arbeiten und ggf. helfen, die Entstehung von Unfallschwerpunkten zu vermeiden. In diesem Zusammenhang wird weiterer Forschungsbedarf gesehen, auf dessen Basis ein möglicher Zusammenhang zwischen Komplexität und Sicherheitsrisiken validiert werden kann.

Die Art und die Zusammensetzung städtischer Mobilität wird sich zukünftig, z. B. aufgrund geänderter Rahmenbedingen (Gesetzesänderungen, Verkehrsmengen u. w.), verändern. Beispielsweise in der Form starker Zuwächse beim Fußgänger- und Radverkehr bei derzeit relativ geringen Verkehrsflächen oder durch einen höheren Anteil des Radverkehrs auf der Fahrbahn (u. a. auch durch E-Bikes, Pedelecs). Zudem stellt die zunehmende Anzahl von Rollatornutzern zusätzliche Anforderungen an die Verkehrsinfrastruktur. Neben einer wünschenswerten Überprüfung des bestehenden Verkehrsnetzes auf die Benutzbarkeit durch derartige "Verkehrsmittel“ sollten diese Entwicklungen bei zukünftigen Planung von Verkehrsanlagen berücksichtigt werden, um so auch "schwächeren Verkehrsteilnehmern“ die nötige Sicherheit zukommen lassen zu können. 


\section{Abbildungsverzeichnis}

Abbildung 1: Kognitive Entwicklung über die Lebensspanne [Poschadel \& Sommer, 2007: übersetzt aus: Craik \& Bialystok, 2006]

Abbildung 2: Im Straßenverkehr verunglückte Kinder 2008 in absoluten Zahlen [Quelle:

Statistisches Bundesamt 2009]

Abbildung 3: Verunglückte Kinder 2008 in Prozent in Relation zur jeweiligen Altersgruppe [Quelle: Statistisches Bundesamt 2009]

Abbildung 4: Größe eines Kindes abhängig vom Alter in Relation zu einem Auto [Poschadel 2006]

Abbildung 5: Schematisiertes Blickfeld eines Pkw-Fahrers in einer beidseitig beparkten Straße [Poschadel 2006].

Abbildung 6: Beteiligte Pkw-Fahrer an Unfällen mit Personenschaden nach Alter und Geschlecht, absolut und in Prozent [Quelle: Statistisches Bundesamt 2010].

Abbildung 7: Mobilitätseingeschränkte Menschen - Übersicht [Quelle: (FGSV 2011),

korrigierte Fassung].

Abbildung 8: Anzahl schwerbehinderter Menschen und Bevölkerungsentwicklung in Deutschland im Vergleich [Zahlen: Statistisches Bundesamt und BMAS (Bevölkerung 2011 vorläufig)]

Abbildung 9: Zunahme von Mobilitätseinschränkungen und Medikamenteneinnahme im Alter [Dangschat et. al. 2007].

Abbildung 10: Unfälle mit Beteiligung mobilitätsbehinderter Menschen - Unfalltypen (Auswertung Berlin, 2004 bis 2010).

Abbildung 11: Häufigkeit von Unfällen an Knotenpunkten nach Ortslage und Unfalltyp ( $\mathrm{n}=$ 351.351 Unfälle)

Abbildung 12: Verteilung der Unfalltypen von Unfällen an Knotenpunkten, außerorts, ohne Autobahnen ( $\mathrm{n}=65.825$ Unfälle)

Abbildung 13: Verteilung der Unfalltypen von Unfällen an Knotenpunkten, innerorts $(n=$ 285.526 Unfälle).

Abbildung 14: Unfällen mit Personenschaden und schwerwiegende Unfälle mit Sachschaden an Knotenpunkten innerorts $\left(n_{\text {ges }}=351.351\right.$ ), einschließlich der Unfälle mit Beteiligung von Kindern und Älteren

Abbildung 15: Unfällen mit Personenschaden und schwerwiegende Unfälle mit Sachschaden an Knotenpunkten innerorts ( $\mathrm{n}_{\text {ges }}=351.351$ ), anteilig nach Altersgruppen ....

Abbildung 16: Unfälle mit Personenschaden und schwerwiegende Unfälle mit Sachschaden an Knotenpunkten innerorts $\left(\mathrm{n}_{\text {ges }}=285.526\right)$.

Abbildung 17: Hauptverursacher von Unfällen mit Personenschaden und schwerwiegende Unfälle mit Sachschaden an Knotenpunkten innerorts $\left(n_{\text {ges }}=285.526\right)$. . .

Abbildung 18: Verunglückte Hauptverursacher bei Unfällen mit Personenschaden an Knotenpunkten innerorts $\left(\mathrm{n}_{\text {ges }}=66.172\right)$.

Abbildung 19: Verunglückte weitere Beteiligte (ohne Mitfahrer) bei Unfällen mit Personenschaden an Knotenpunkten innerorts $\left(\mathrm{n}_{\text {ges }}=144.172\right)$

Abbildung 20: Kinder als Hauptverursacher und verunglückte weitere Beteiligte

Abbildung 21: Ältere als Hauptverursacher und verunglückte weitere Beteiligte 
Abbildung 22: Häufigste dreistellige Unfalltypen von Unfällen mit Personenschaden und schwerwiegenden Unfällen mit Sachschaden an Knotenpunkten innerorts am Beispiel der Unfalldaten Nordrhein-Westfalen aus 2004 bis $2008\left(n_{\text {ges }}=174.947\right)$

Abbildung 23: Häufigste dreistellige Unfalltypen von Unfällen mit Personenschaden und schwerwiegenden Unfällen mit Sachschaden mit Beteiligung von Kindern an Knotenpunkten innerorts am Beispiel der Unfalldaten Nordrhein-Westfalen aus 2004 bis $2008\left(n_{\text {ges }}=11.554\right)$

Abbildung 24: Häufigste dreistellige Unfalltypen von Unfällen mit Personenschaden und schwerwiegenden Unfällen mit Sachschaden mit Beteiligung von Älteren an Knotenpunkten innerorts am Beispiel der Unfalldaten Nordrhein-Westfalen aus 2004 bis $2008\left(n_{\text {ges }}=34.482\right)$

Abbildung 25: Relevante Unfalltypen für Unfälle mit Beteiligung von Kindern und Älteren an

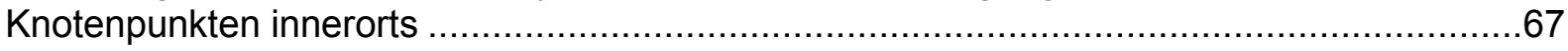

Abbildung 26: Exemplarische Darstellung eines Kamerastandortes .................................73

Abbildung 27: Exemplarische Darstellung der Kamerabilder .........................................74

Abbildung 28: Exemplarische Darstellung der Kamerastandorte und Positionen der Zählposten für die Verhaltensbeobachtungen .74

Abbildung 29: Beteiligte Kinder an Unfällen innerorts ( $\mathrm{n}=400$ Beteiligte) .........................76

Abbildung 30: Beteiligte Ältere an Unfällen innerorts $(n=1.309$ Beteiligte) .......................77

Abbildung 31: Unfälle je Unfalltyp der 15 ausgewählten Knotenpunkte $(n=259) \ldots \ldots \ldots \ldots . . . . .78$

Abbildung 32: Anzahl der Knotenpunkte mit Defiziten je Kategorie ( $n=15$ Knotenpunkte)...81

Abbildung 33: Einbiegende (und wendende) Kraftfahrer missachten Vorrangsrecht überquerender Fußgänger

Abbildung 34: Hilfssignalgeber (gelbes Blinklicht) mit 200 mm Durchmesser an Fußgänger

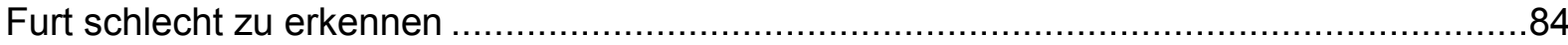

Abbildung 35: Gesamtüberblick Fußgängerverhalten an 15 Knotenpunkten.......................85

Abbildung 36: Einbiegende/kreuzende Fahrzeuge warten auf Radverkehrsfurt bei eingeschränkter Anfahrsicht

Abbildung 37: Radverkehrsfurt aus Sicht der einbiegenden Kraftfahrer nicht zu erkennen ...89

Abbildung 38: Gesamtüberblick Radfahrerverhalten an 15 Knotenpunkten ........................90

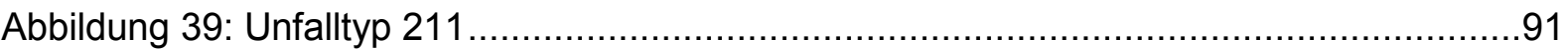

Abbildung 40: Konflikte bei Zugabezeit für Linksabbieger und weit abgesetzter Haltlinie......92

Abbildung 41: Gesamtüberblick Verhalten von Pkw-Fahrern an 15 Knotenpunkten ............93

Abbildung 42: Pkw-Fahrer als Geradeausfahrer an den 15 Knotenpunkten.........................93

Abbildung 43: Pkw-Fahrer als Rechtsabbieger an den 15 Knotenpunkten..........................94

Abbildung 44: Pkw-Fahrer als Linksabbieger an den 15 Knotenpunkten ...........................94

Abbildung 45: Komplexitätsindex zu Unfalldichte (Bild links) und Unfallrate (Bild rechts) für

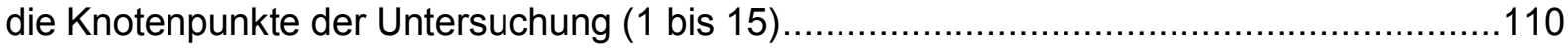

Abbildung 46: Komplexitätsindex zu Unfallkostendichte (Bild links) und Unfallkostenrate (Bild

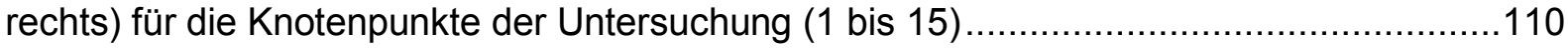

Abbildung 47: Lineare Regressionskurven und Bestimmtheitsmaß für den Zusammenhang zwischen Komplexitätsgrad und Unfalldichte (Bild links) bzw. Unfallrate (Bild rechts) ........111 
Abbildung 48: Lineare Regressionskurven und Bestimmtheitsmaß für den Zusammenhang zwischen Komplexitätsgrad und Unfallkostendichte (Bild links) bzw. Unfallkostenrate (Bild rechts)

Abbildung 49: StVO Zeichen 350 "Fußgänger Überweg".

Abbildung 50: Radverkehrsfurt an einmündender Straße ohne Einfärbung (Bild oben) und mit Einfärbung (Bild unten, Retusche)

Abbildung 51: Sinnbild StVO-Zeichen 138 „Radfahrer kreuzen“ (Bild links) und an einmündender Straße (Bild rechts).

Abbildung 52: Unfalltypen 211, 301, 302 und 321

Abbildung 53: „Vor-Kopf“ gesetzte Linksabbiegestreifen

Abbildung 54: Verflechtungsvorgänge im Kreuzungsbereich am Beispiel des Knotenpunktes

1.

Abbildung 55: Knotenpunkt 1 - Unfalldiagramm, Kamerastandorte und Zählposten.

Abbildung 56: Knotenpunkt 1 - Konflikte bei Zugabezeit für Linksabbieger.

Abbildung 57: Knotenpunkt 2 - Unfalldiagramm, Kamerastandorte und Zählposten.

Abbildung 58: Knotenpunkt 2 - Markierung im Kreuzungsbereich (von links Zufahrt 1, von rechts Zufahrt 3).

Abbildung 59: Knotenpunkt 2 - Aufstellung wartepflichtiger Linksabbieger und Wender im Kreuzungsbereich

Abbildung 60: Knotenpunkt 2 - Sichtbehinderung für Linksabbieger (und Wender) durch Mittelstreifen und Fahrzeuge des Gegenverkehrs

Abbildung 61: Knotenpunkt 2 - Radfahrer auf Gehweg

Abbildung 62: Knotenpunkt 2 - Hilfssignalgeber (gelbes Blicklicht) an Fußgängerfurt........154

Abbildung 63: Knotenpunkt 3 - Unfalldiagramm, Kamerastandorte und Zählposten...........155

Abbildung 64: Kontenpunkt 3 - Blick auf Kreuzungsbereich aus südlicher Richtung ..........156

Abbildung 65: Kontenpunkt 3 - Blick auf Kreuzungsbereich aus nördlicher Richtung .........156

Abbildung 66: Knotenpunkt 3 - Sicht der Linkseinbieger aus Arm 1 auf Gegenverkehr......157

Abbildung 67: Knotenpunkt 4 - Unfalldiagramm, Kamerastandorte und Zählposten ...........158

Abbildung 68: Knotenpunkt 4 - Blick auf Furt 1 in Richtung Zufahrt 4 ...........................159

Abbildung 69: Knotenpunkt 5 - Unfalldiagramm, Kamerastandorte und Zählposten...........160

Abbildung 70: Kontenpunkt 5 - Hauptwegebeziehungen zwischen Schulgebäuden und

Stadtbahn-Haltestelle

Abbildung 71: Knotenpunkt 5 - Aufstellflächen auf Dreiecksinsel am „Freien Rechtsabbieger“ aus Arm 3 in Arm 4 in Blickrichtung Südost (im Bild links ist der rot eingefärbte Bereich für den Radverkehr hervorgehoben).

Abbildung 72: Knotenpunkt 5 - Fußgänger und Radfahrer überqueren Furt an Arm 3 .......162

Abbildung 73: Knotenpunkt 5 - Zufahrt aus Arm 3 in Blickrichtung der Furt .....................163

Abbildung 74: Knotenpunkt 5 - Kinder laufen bei „Rot“ über die Furt an Arm 2 in Richtung der Stadtbahn-Haltestelle.

Abbildung 75: Knotenpunkt 5 - Situation am freien Rechtsabbiegestreifen (Radfahrer von links wartet, Radfahrer von rechts fährt)

Abbildung 76: Knotenpunkt 5 - Sicht der Kfz-Führer aus Arm 2 auf Dreiecksinsel und freien

Rechtsabbiegestreifen. 
Abbildung 77: Knotenpunkt 6 - Situation vor Änderung (Bild links) und nach Änderung (Bild rechts)

Abbildung 78: Knotenpunkt 6 - Unfalldiagramm, Kamerastandorte und Zählposten (Bereiche mit Änderungen sind gelb hinterlegt. Die Unfälle 65, 66, 67, 69, 73 fanden nach bzw. im Jahr der Umsetzung der Maßnahmen statt. Nummerisch nicht aufgeführte Unfälle beziehen sich

auf andere Bereiche des Knotenpunktes).

Abbildung 79: Knotenpunkt 6 - Unfälle vor verkehrstechnischer Änderung (Bild links) und nach oder im Jahr der verkehrstechnischen Änderung (Bild rechts).....

Abbildung 80: Knotenpunkt 6 - Signalfolge für Linksabbieger von Süden

Abbildung 81: Knotenpunkt 7 - Unfalldiagramm, Kamerastandorte und Zählposten (Bereiche mit Änderungen sind gelb hinterlegt. Die Unfälle 17 bis 19 fanden nach bzw. im Jahr der Umsetzung der Maßnahmen statt).....

Abbildung 82: Knotenpunkt 7 - Situation vor Änderung (Bild links) und nach Änderung (Bild rechts)

Abbildung 83: Knotenpunkt 7 - Unfälle vor verkehrstechnischer Änderung (Bild links, 16 Unfälle zwischen 2007 und 2009) und nach oder im Jahr der verkehrstechnischen Änderung (Bild rechts, 3 Unfälle im Jahr 2010).

Abbildung 84: Knotenpunkt 7 - Räumendes Kfz bei bereits grünem Signal der Fußgängerfurt

Abbildung 85: Knotenpunkt 7 - Räumweg für Geradeausfahrer aus Arm 3 zur Fußgängerfurt

Abbildung 86: Knotenpunkt 7 - Wartende Fußgänger du Radfahrer an der Furt an Arm 5 (Blickrichtung Ost)

Abbildung 87: Knotenpunkt 8 - Unfalldiagramm, Kamerastandorte und Zählposten (Bereiche mit Änderungen sind gelb hinterlegt. Die Unfälle 10 bis 12 fanden nach bzw. im Jahr der Umsetzung der Maßnahmen statt)

Abbildung 88: Knotenpunkt 8 - Situation vor Änderung (Bild links) und nach Änderung (Bild rechts)

Abbildung 89: Knotenpunkt 8 - Unfälle vor verkehrstechnischer Änderung (Bild links, 9 Unfälle zwischen 2006 und 2007) und nach oder im Jahr der verkehrstechnischen Änderung (Bild rechts, 3 Unfälle zwischen Nov 2008 und 2010) ..............................................174

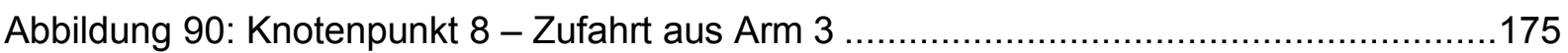

Abbildung 91: Knotenpunkt 8 - Zufahrt aus Arm 1

Abbildung 92: Knotenpunkt 8 - Kind mit Fahrrad und Mutter mit Kinderwagen

Schwierigkeiten bei der Überwindung eines Hochbordes

Abbildung 93: Knotenpunkt 8 - Mutter mit Kinderwagen hat Schwierigkeiten bei der „Überrollung“ kleiner Unebenheiten.

Abbildung 94: Knotenpunkt 9 - Unfalldiagramm, Kamerastandorte und Zählposten (Bereiche mit Änderungen sind gelb hinterlegt. Die Unfälle 17 bis 26 fanden nach bzw. im Jahr der Umsetzung der Maßnahmen statt).....

Abbildung 95: Knotenpunkt 9 - Unfälle vor verkehrstechnischer Änderung (Bild links, 16 Unfälle zwischen 2006 und Nov 2007) und nach verkehrstechnischer Änderung (Bild rechts, 10 Unfälle zwischen Nov 2007 und 2010) 178

Abbildung 96: Knotenpunkt 9 - Blick der Rechtsabbieger auf Fg-LSA auf Mittelstreifen.....179

Abbildung 97: Knotenpunkt 10 - Unfalldiagramm, Kamerastandorte und Zählposten.........180

Abbildung 98: Knoten 10 - Kreuzungsbereich. 
Abbildung 99: Knotenpunkt 10 - Links abbiegende und ausscherende Fahrzeuge (Bild links)

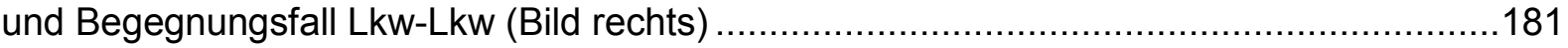

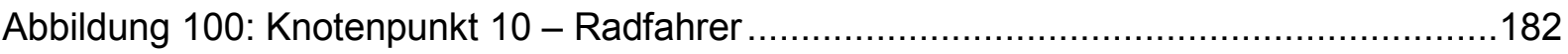

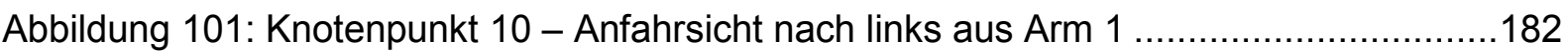

Abbildung 102: Knotenpunkt 10 - Einfahrendes Kfz aus Arm 1..................................183

Abbildung 103: Knotenpunkt 10 - Jugendliche Radfahrerin fährt auf Gehweg (Bild links) und

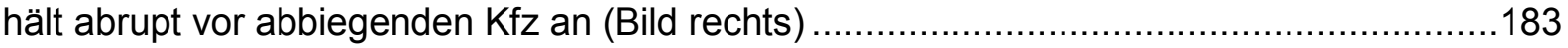

Abbildung 104: Knotenpunkt 11 - Planskizze, Kamerastandorte und Zählposten ...............184

Abbildung 105: Knotenpunkt 11 - Sicht auf Kreuzungsbereich aus Arm 2 .......................186

Abbildung 106: Knotenpunkt 11 - Sicht aus Arm 2................................................... 186

Abbildung 107: Knotenpunkt 11 - Anfahrsicht aus Arm 1 nach links ..................................187

Abbildung 108: Knotenpunkt 11 - Kraftfahrer aus Arm 1 weit vorgefahren.........................187

Abbildung 109: Knotenpunkt 12 - Unfalldiagramm, Kamerastandorte und Zählposten.......188

Abbildung 110: Knotenpunkt 12 - Anfahrt aus Richtung Ost [Kartengrundlage:

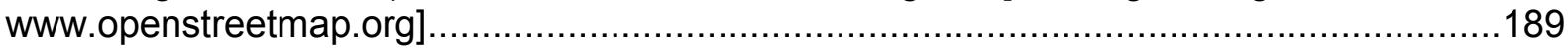

Abbildung 111: Knotenpunkt 12 - Blick auf Knotenpunkt von Osten ................................190

Abbildung 112: Knotenpunkt 12 - Fußgänger an Überquerungsanlage.............................190

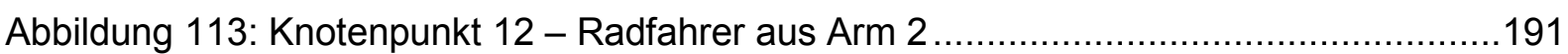

Abbildung 114: Knotenpunkt 12 - Radfahrer auf Mittelinsel .........................................191

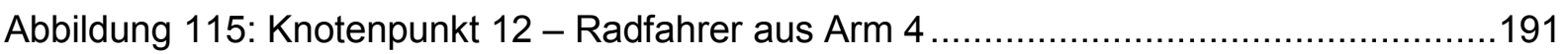

Abbildung 116: Knotenpunkt 13 - Unfalldiagramm (Plangrundlage vor verkehrstechnischer Änderung. Alle Unfälle fanden im Zeitraum vor der Änderung statt.) ................................192

Abbildung 117: Knotenpunkt 13 - Kamerapositionen und Zählposten (Plangrundlage nach

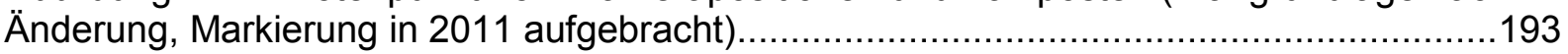

Abbildung 118: Knotenpunkt 13 - Sicht auf Überquerungsanlage aus Arm 1 .....................193

Abbildung 119: Knotenpunkt 13 - Pkw mit abrupten Bremsvorgängen vor FGÜ ...............194

Abbildung 120: Knotenpunkt 13 - Blick auf Überquerungsanlage (FGÜ) ...........................194

Abbildung 121: Knotenpunkt 13 - Neue Markierung und Verkehrsregelung der Zufahrt aus

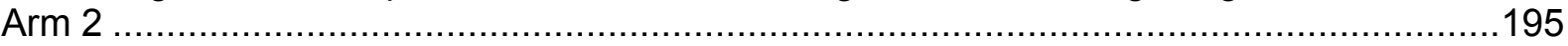

Abbildung 122: Knotenpunkt 14 - Unfalldiagramm, Kamerastandorte und Zählposten.......196

Abbildung 123: Knotenpunkt 14 - Anfahrsicht auf Überquerung 2a..................................197

Abbildung 124: Knotenpunkt 14 - Schlechte Erkennbarkeit / keine Schutzfunktion der Auftstellfläche (Bild links) und zugeparkte Auftstellfläche an der Überquerungsstelle 2a (Bild rechts)

Abbildung 125: Knotenpunkt 14 - Überquerender Fußgänger bei gleichzeitig rechtseinbiegenden und wendenden Fahrzeugen

Abbildung 126: Knotenpunkt 14 - Nebeneinanderaufstellung wartepflichtiger Fahrzeuge im

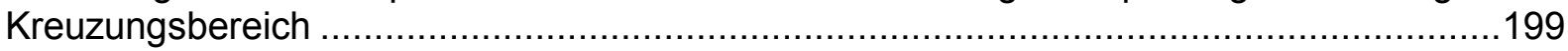

Abbildung 127: Knotenpunkt 15 - Unfalldiagramm, Kamerastandorte und Zählposten.......200 Abbildung 128: Knotenpunkt 15 - Sicht aus Arm 2 ....................................................201

Abbildung 129: Knotenpunkt 15 - Rollatorennutzer an Überquerung (Bild links) und Nullabsenkung auf Breite des Radweges (Bild rechts) 
Abbildung 130: Taktiler Leitstreifen im Fahrbahnbereich über eine lange Furt (Kassel) [Foto: Wiesenhütter, KVC]

Abbildung 131: Überquerungsstelle mit differenzierter Bordhöhe, Sperrfeld und

Auffindestreifen (hier mit Sonderbord) [Foto: Boenke] 235

Abbildung 132: Überquerungsstelle mit differenzierter Bordhöhe - Skizze [FGSV 2011]....236 Abbildung 133: Schallgeber für ein akustisches Freigabesignal in Höhe der Signalgeber und in Furtmitte abstrahlend. 


\section{Tabellenverzeichnis}

Tabelle 1: Aufteilung der Merkzeichen der amtlich registrierten schwerbehinderten Menschen in Deutschland zum 31.12.2011 [Quelle: (Bundesministerium für Arbeit und Soziales (BMAS)

- Referat V2, 2012)]. 40

Tabelle 2: Mobilitätskenngrößen von Personen ab 60 Jahren mit und ohne gesundheitlicher Einschränkungen (MiD 2008)

Tabelle 3: Städte mit Einrichtungen für Menschen mit Behinderungen und polizeiliche Unfalldatenerfassung mit dem Merkmal „Behinderung“.

Tabelle 4: Unfälle im Zusammenhang mit der nicht bestimmungsgemäßen Nutzung von Verkehrsflächen durch Rollstuhlnutzer

Tabelle 5: Übersicht der Bundesländer und der Zeiträume zu den vorliegenden elektronischen Unfalldaten

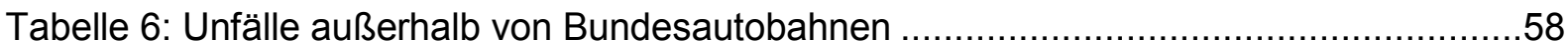

Tabelle 7: Unfälle an Knotenpunkten außerhalb von Bundesautobahnen ..........................58

Tabelle 8: Unfälle an Knotenpunkten innerhalb geschlossener Ortschaften $(n=285.526) \ldots 61$

Tabelle 9: Übersicht der 15 Knotenpunkte für die Detailuntersuchungen............................70

Tabelle 10: Anzahl der Unfälle und Auswertungszeiträume ............................................78

Tabelle 11: Prozentuale Verteilung der Unfallkategorien je Unfalltyp für die Unfälle an den 15

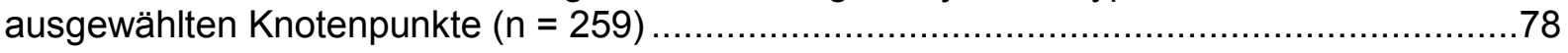

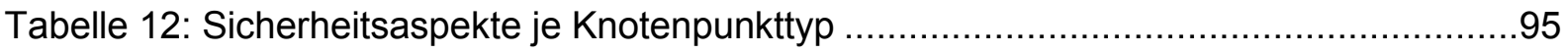

Tabelle 13: Prototypische Merkmale/Situationen für Fußgänger .....................................97

Tabelle 14:Prototypische Merkmale/Situationen für Radfahrer .....................................98

Tabelle 15: Prototypische Merkmale/Situationen für Menschen mit besonderen

Mobilitätseinschränkungen oder Mobilitätsbehinderungen............................................99

Tabelle 16: Prototypische Merkmale/Situationen für Kraftfahrer .......................................100

Tabelle 17: Unfälle an den 15 untersuchten Knotenpunkten vor der Umsetzung baulicher oder verkehrstechnischer Maßnahmen zur Berechnung der Unfallkenngrößen $(n=238) . .108$

Tabelle 18: Unfallkenngrößen und Komplexitätsstufen (K-Index) an den Knotenpunkten der

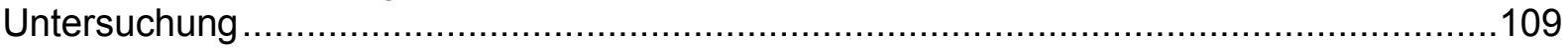

Tabelle 19: Knotenpunkt 11 - Unfallliste „Vorgang“.......................................................185

Tabelle 20: Knotenpunkt 11 - Unfallliste "Beteiligte" .....................................................185

Tabelle 21: Auswertung des Verhaltens von Fußgängern an Kreuzungen mit Lichtsignalanlage (Knotenpunkte 1 bis 9 )

Tabelle 22: Auswertung des Verhaltens von Fußgängern an Kreuzungen mit vorfahrtregelnden Verkehrszeichen (Knotenpunkte 10 bis 13).

Tabelle 23: Auswertung des Verhaltens von Fußgängern an Einmündungen mit vorfahrtregelnden Verkehrszeichen (Knotenpunkte 14 und 15).

Tabelle 24: Auswertung des Verhaltens von Radfahrern an Kreuzungen mit Lichtsignalanlage (Knotenpunkte 1 bis 9 )

Tabelle 25: Auswertung des Verhaltens von Radfahrern an Kreuzungen mit vorfahrtregelnden Verkehrszeichen (Knotenpunkt 10 bis 13) 
Tabelle 26: Auswertung des Verhaltens von Radfahrern an Einmündungen mit vorfahrtregelnden Verkehrszeichen (Knotenpunkte 14 und 15)

Tabelle 27: Auswertung des Verhaltens älterer Pkw-Fahrer an Kreuzungen mit Lichtsignalanlage (Knotenpunkte 1 bis 9)

Tabelle 28: Auswertung des Verhaltens älterer Pkw-Fahrer an Kreuzungen vorfahrtregelnden Verkehrszeichen (Knotenpunkte 10 bis 13)

Tabelle 29: Auswertung des Verhaltens älterer Pkw-Fahrer Einmündungen mit vorfahrtregelnden Verkehrszeichen (Knotenpunkt 14 und 15) ....

Tabelle 30: Prototypische Merkmale/Situationen für Fußgänger 227

Tabelle 31:Prototypische Merkmale/Situationen für Radfahrer 228

Tabelle 32: Prototypische Merkmale/Situationen für Menschen mit besonderen Mobilitätseinschränkungen oder Mobilitätsbehinderungen

Tabelle 33: Prototypische Merkmale/Situationen Kraftfahrer 230

Tabelle 34: Regressionskurven „Komplexitätsindex zu Unfalldichte/Unfallrate“ 239

Tabelle 35: Regressionskurven „Komplexitätsindex zu Unfallkostendichte/Unfallkostenrate“ 


\section{Literatur- und Quellenverzeichnis}

Ampofo-Boateng, K. \& Thomson, J.-A. (1991). Children's perception of safety and danger on the road. British Journal of Psychology, Vol. 82 (4), S. 487-505.

Ball, K., Owsley, C., Sloane, M.E., Roenker, D.L., Bruni, J.R. (1993): Visual attention problems as a predictor of vehicle crashes in older drivers. Investigative Ophthalmology\& Vision Science, 34, 3110-3123.

Ball, D. M., Berch, D. B., Helmers, K. F., Jobe, J. B., Leveck, M. D., Marsiske, M., Morris, J. N., Rebok, G.W., Smith, K., Tennstedt, S. L., Unverzagt, F. W. \& Willis, S. L. (2002). Effects of cognitive training interventions with older adults. A randomized controlled trail. Journal of the American medical Association, November 13, Vol. 288, No. 18, S. 2271 - 2281.

Ball, K. K., Roenker, D. L., Wadley, V. G., Edwards, J. D., Roth, D. L., McGwin, G. J., et al. (2006): Can high-risk older drivers be identified through performance-based measures in a Department of Motor Vehicles setting? Journal of the American Geriatric Society, 54(1), 77-84.

Band, G. \& Kok, A. (2000): Ageeffects on response monitoring in a mental-rotation task. In: Biological Psychology, 51, S. $201-221$.

Basner, B. \& Marées, H. de (1993). Fahrrad- und Straßenverkehrstüchtigkeit von Grundschülern. Serie: Gesundheitsschutz in Schule und Beruf Bd. 1. Gemeindeunfallversicherungsverband Westfalen-Lippe [Hrsg.]. Münster.

Becker, S. \& Albrecht, M. (2003): Verkehrsmedizinische Aspekte im Alter. Zeitschrift für Gerontopsychologie\& -psychiatrie, Vol. 16, No. 3, S. 101-115.

Berufsgenossenschaft für Gesundheitsdienst und Wohlfahrtspflege - BGW (2009): Sicher mobil. Flyer zur Präventionskampagne unter Schirmherrschaft des Bundesministeriums für Arbeit und Soziales. Hamburg. Online verfügbar unter http://www.bgwonline.de/internet/generator/Inhalt/Onlinelnhalt/Statische_20Seiten/Navigation_20links/ Kundenzentrum/Mobilit_C3_A4tsmanagement/Mobilit_C3_A4t_20von_20Menschen_2 Omit_20Behinderungen/sicher-mobil/Kampagnenflyer-sicher-mobil, property=download.pdf, zuletzt geprüft am 21.02.2010.

Böhringer, Dietmar (2007): "Gesicherte Nullabsenkungen": Für blinde Menschen gefährlich gerade noch brauchbar - oder eine gute Lösung? Leonberg.

Böhringer, Dietmar (2010): Es gibt keine Behinderung oder Gefährdung durch „Getrennte Querungsstellen“! Beobachtungen der Fußgängerströme an einer "Getrennten Querungsstelle mit differenzierter Bordhöhe“. Leonberg.

Boenke, Dirk (2010): Sicherung der Mobilität älterer Menschen im Straßenverkehr. Eine Analyse des Status quo bei Verkehrsplanungsprozessen und Empfehlungen für eine zukunftsfähige Verkehrsplanung. Saarbrücken: Südwestdeutscher Verlag für Hochschulschriften. 
Bongert, O. \& Henke, T. (1997). Motorische Radfahrkompetenz von Kindern und Jugendlichen. Serie: Gesundheitsschutz in Schule und Beruf Bd. 10. Gemeinde Unfallversicherungsverband Westfalen-Lippe [Hrsg.]. Münster.

Braver, T. S., Barch, D. M., Keys, B. A., Carter, C. S., Cohen, J. D., Kaye, J. A., Janowsky, J. S., Taylor, S. F., Yesavage, J. A., Mumenthaler, M. S., Jagust, W. J. \& Reed, B. R. (2001). Context processing in older adults: evidence for a theory relating cognitive control to neurobiology in healthy aging. Journal of Experimental Psychology, General, 130, 746-63.

Brust, Ernst (2011): Rollatoren. Das geht nicht! Online-Artikel aus Öko-Test Heft August 2011. Online verfügbar unter http://www.oekotest.de/cgi/index.cgi?artnr=98196; bernr=06; seite=00;co=, $\quad$ zuletzt geprüft am 02.05.2012.

Bundesarbeitsgemeinschaft SELBSTHILFE von Menschen mit Behinderung und chronischer Erkrankung und ihren Angehörigen e. V (2006). In: Selbsthilfe (1).

Bundesministerium für Arbeit und Soziales (BMAS) (2011): 'einfach machen' - Unser Weg in eine inklusive Gesellschaft. Nationaler Aktionsplan der Bundesregierung zur Umsetzung des Übereinkommens der Vereinten Nationen über die Rechte von Menschen mit

Bundesministerium für Verkehr, Bau und Stadtentwicklung - BMVBS [Hrsg.]: „Bürgerfreundliche und behindertengerechte Gestaltung des Straßenraums“. Zweite, vollständig neu bearbeitete Auflage. FMS Media-Service Verlagsgesellschaft $\mathrm{mbH}$. Bad Homburg 2000. ISBN 3-926181-35-4.

Bundesministerium für Verkehr, Bau und Stadtentwicklung - BMVBS [Hrsg.]: Allgemeine Verwaltungsvorschrift zur Straßenverkehrs-Ordnung (VwV-StVO), Vom 22. Oktober 1998, In der Fassung vom 17. Juli 2009

Bundesministerium für Verkehr, Bau und Stadtentwicklung - BMVBS [Hrsg.]: Straßenverkehrs-Ordnung (StVO), Vom 16. November 1970 (Bundesgesetzblatt, Teil I, S. 1565), zuletzt geändert mit Verordnung vom 1. Dezember 2010 (Bundesgesetzblatt, Teil I, S. 1737).

Bundesministerium für Verkehr [Hrsg.]: Hochrechnungsfaktoren für manuelle und automatische Kurzzeitzählungen im Innerortsbereich, aus Forschung Straßenbau und Straßenverkehrstechnik, Heft 732, Ausgabe 1996

Bundesministerium für Familie, Senioren, Frauen und Jugend [Hrsg.] (2002). Vierter Bericht zur Lage der älteren Generation. Bonn.

Bundesministerium für Arbeit und Soziales (BMAS) - Referat V2 (2012): Übersicht über Ausweismerkzeichen 2011. Bonn, 10.07.2012. Email und Tabelle an Studiengesellschaft für unterirdische Verkehrsanlagen - STUVA e. V. 
Cattell, R. B. (1963). Theory of fluid and crystallized intelligence: A critical experiment. Journal of Educational Psychology, Bd. 54(1), S. 1 - 22

Churchill, J. D., Galvez, R., Colcombe, S., Swain, R. A., Kramer, A. F. \&Greenough, W. T. (2002). Exercise, experience and the aging brain. Neurobiology of Aging, Vol. 23, S. $941-955$.

Craik, F.I.M. \& Bialystok, E. (2006). Cognition through the lifespan: mechanisms of change. TRENDS in Cognitive Sciences, Vol.10, No.3, S.131 - 138.

Dangschat, Jens; Blum, Martin; (2007): Mobilität und Verkehr im demografischen Wandel. Wien (Mobilität mit Zukunft, 1).

David, S.-S., Chapman, A.-J., Foot, H.-C. \& Sheehy, N.-P. (1986). Peripheral vision and the aetiology of child pedestrian accidents. British Journal of Psychology, Vol. 77 (1), S. 117-131.

David, S.-S., Foot, H.-C. \& Chapman, A.-J. (1990). Children's sensitivity to traffic hazard in peripheral vision. Applied CognitivePsychology, Vol. 4 (6), S. 471-484.

DBSV - Deutscher Blinden und Sehbehindertenverband e. V (2011): Zahlen und Fakten. Online verfügbar unter http://www.dbsv.org/infothek/zahlen-und-fakten/, zuletzt geprüft am 22.09.2011.

Deutscher Bundestag (15.06.1990): Gesetz über die Statistik der Straßenverkehrsunfälle. Straßenverkehrsunfallstatistikgesetz - StVUnfStatG, vom 15.06.1990 (BGBI. I S. 1078), das zuletzt durch Artikel 298 der Verordnung vom 31.10.2006 (BGBI. I S. 2407) geändert worden ist.

Deutscher Bundestag (2002): Gesetz zur Gleichstellung behinderter Menschen (Behindertengleichstellungsgesetz) vom 27. April 2002, das zuletzt durch Artikel 12 des Gesetzes vom 19. Dezember 2007 geändert worden ist. BGG. Fundstelle: BGBI. I S. 1467, 1468; BGBI. I S. 3024.

Destatis, Unfälle von Senioren im Straßenverkehr 2006, Wiesbaden 2007

DIN - Deutsches Institut für Normung e. V. (2009): Norm DIN 32975, Dezember 2009: Gestaltung visueller Informationen im öffentlichen Raum zur barrierefreien Nutzung

DIN - Deutsches Institut für Normung e. V. (2011): Norm DIN 32984, Oktober 2011: Bodenindikatoren im öffentlichen Raum.

DVR „Innerorts - Raum für Alle“ Hintergrundinformationen zur Schwerpunktaktion „Verkehrssicherheit innerorts“, Bonn 2008

Eickler, Marion (2012): Mit der Bewegung kehrt die Lebensqualität zurück. In: Kölner StadtAnzeiger, 30.06.2012.

Falkenstein, M., Koshlykova, N.A., Kiroj, V.N., Hoormann, J., Hohnsbein, J. (1995): Late ERP components in visual and auditory Go/Nogo tasks. Electroencephalography and Clinical Neurophysiology, 96, 36-43.

Falkenstein, M., Hoormann, J., Hohnsbein, J., (1999a): ERP components in Go/Nogo tasks and their relation to inhibition. ActaPsychologi-ca, 101, 267-291. 
Falkenstein, M., Hohnsbein, J., Hoomann, J. (1999b): Objektivierung altersabhängiger Änderungen von Beanspruchung und Ermüdung bei psychomentalen Belastungen am Bildschirmar-beitsplatz. Bremerhaven: Wirtschaftsverlag NW, (Schriftenreihe der Bundesanstalt für Arbeits-schutz und Arbeitsmedizin, Forschung- Fb 866

Falkenstein, M., Hoormann, J., Hohnsbein, J. (2002): Inhibition-related ERP components: var-iation with age and time-on-task. Journal of Psychophysiology, 16, 167-175.

Falkenstein, M. \&Poschadel, S. (2008): Altersgerechtes Autofahren. In: Wirtschaftspsychologie Bd. 3, 2008, S. 62-71

Falkenstein, M. \& Sommer, S. (2008): Altersbegleitende Veränderungen kognitiver Prozesse mit Bedeutung für das Autofahren. In: In: Schlag, B. [Hrsg.]. Leistungsfähigkeit und Mobilität im Alter. Köln: TÜV Media GmbH.

FGSV - Forschungsgesellschaft für Straßen- und Verkehrswesen e. V. (2010): Richtlinien für Lichtsignalanlagen - Lichtzeichenanlagen für den Straßenverkehr (RiLSA). Ausgabe 2010. Köln (FGSV, 321).

FGSV - Forschungsgesellschaft für Straßen- und Verkehrswesen e. V. (2011): Hinweise für barrierefreie Verkehrsanlagen (H BVA). Ausgabe 2011. Köln (FGSV, 212).

FGSV - Forschungsgesellschaft für Straßen- und Verkehrswesen e. V. (2012): Merkblatt zur Örtlichen Unfalluntersuchung in Unfallkommissionen (M Uko). Ausgabe 2012. Köln (FGSV, 316/1)

Gabler Wirtschaftslexikon, Onlineausgabe. Wiesbaden, Springer Gabler I Springer $\begin{array}{lll}\text { Fachmedien } & \text { Wiesbaden } & \mathrm{GmbH},\end{array}$ (http://wirtschaftslexikon.gabler.de/Definition/komplexitaet.html, letzter Zugriff: 18.08.2012)

Gehring, W. J., Goss, G., Goss, M. G. H., Meyer, D. E. \&Donchin, E. (1993). A neural system for error detection and compensation. Psychological Science, 4, 385-390.

GNP Arbeitskreis Fahreignung (2007): Der motorisierte Krankenfahrstuhl im Straßenverkehr. Informationen zu den Eignungsvoraussetzungen und zur Vorsorgepflicht. Fulda.

Grossmann, Helmut; König, Volker; Ruhe, Carsten (2008): Hinweise - Barrierefreiheit im öffentlichen Verkehrsraum für seh- und hörgeschädigte Menschen. Bremerhaven: Wirtschaftsverlag NW, Verlag für neue Wissenschaft GmbH (direkt - Verbesserung der Verkehrsverhältnisse in den Gemeinden, 64).

Hamburger Verkehrsverbund $\mathrm{GmbH}$ (2011): Taktile Bodenindikatoren. Untersuchungen zur Eignung unterschiedlicher taktiler Bodenindikatoren für eine Verwendung in U- und SBahn-Haltestellen des Hamburger Verkehrsverbundes. Bearbeitet durch Büro KramerAlbrecht Ingenieurgesellschaft $\mathrm{mbH}$ \& Co. KG im Auftrag des HVV. Unter Mitarbeit von Simon Henze und Jens Usadel.

Hasher L, Stoltzfus, E. R, Zacks, R. T. \&Rypma, B. (1991). Age and inhibition. Journal of Experimental Psychology: Learning, Memory and Cognition, 17, 163-9. 
Hautzinger, Heinz (1993): Dunkelziffer bei Unfällen mit Personenschaden. Berichte der Bundesanstalt für Straßenwesen. Bremerhaven: Wirtschaftsverlag NW, Verlag für neue Wissenschaft GmbH (Mensch und Sicherheit, M13).

Hessische Straßen- und Verkehrsverwaltung - HSVV (Wiesbaden) (2010): Unbehinderte Mobilität. Erfahrungen und Untersuchungen. Wiesbaden (HSVV-Heft, 55).

in cognition. PsycholRev 103(3):403-28.

Infas Institut für angewandte Sozialwissenschaften $\mathrm{GmbH}$; Deutsches Zentrum für Luft- und Raumfahrt e. V. (2010): Mobilität in Deutschland 2008. Ergebnisbericht: Struktur Aufkommen - Emissionen - Trends. Forschungsvorhaben FE-Nr. 70.801/2006 im Auftrag des Bundesministeriums für Verkehr, Bau und Stadtentwicklung. Bonn/Berlin.

Januschke-Stämpfli, F. (1978): Zur Psychologie des kindlichen Hören. Dissertation. Zentralstelle der Studentenschaft Zürich.

Kaiser, H.J. \& Oswald, W.D. (2000) Autofahren im Alter - eine Literaturanalyse. Zeitschrift für Gerontopsychologie\& -psychiatrie, Bd. 13(3/4), S. 131 - 170.

Kesting, T. \& Gerlach, J.: (2006) Sicherheitsdefizite von Planungen an innerörtlichen Hauptverkehrsstraßen und Erschließungsstraßen, in: Straßenverkehrstechnik 11/2006, S. $641-651$

König, Roland (2007): Rettungswegkonzepte (Beispiele) für mobilitätseingeschränkte Personen in Sonderbauten. XI. Baurecht \& Brandschutz-Symposium. ZILLER-A.S.S. Sachverständigen $\mathrm{GmbH}$. Frankfurt am Main, 07.01.2007.

Kray, J. \& Lindenberger, U. (2000): Adult age differences in task switching. Psychology and Aging, 15(1), S. 126-147.

Langescheid, Tanja (2005): Überprüfung der Methoden zur Erfassung und Analyse von Schulwegunfällen. Diplomarbeit. Bergische Universität Wuppertal, Wuppertal. Lehrund Forschungsgebiet Straßenverkehrsplanung und Straßenverkehrstechnik.

Li, K.Z., Lindenberger U. (2002): Relations between aging sensory/sensorimotor and cognitive functions. Neuroscience and Biobehavioral Review, 26, 777-83.

Limbourg, M. (1997). Gefahrenkognition und Präventionsverständnis von 3- bis 15jährigen Kindern. In: Sicher Leben [Hrsg.]. Bericht über die 2. Tagung „Kindersicherheit: Was wirkt?" in Essen, 27. und 28. September 1996 in Essen, Wien, S. 313 - 326

Limbourg, M. (1997b). Kind und Verkehr - alles verkehrt? Kindspezifische Mechanismen und Verhaltensmuster als Auslöser für Unfälle im Verkehr. Bericht über die 3. Saarländische Ökopädiatrie-Tagung „Wohin geht die Fahrt? 1997 in Saabrücken

Luna, B., Thulborn, K. R., Munoz, D. P., Merriam, E. P., Garver, K. E, Minshew, N. J., Keshavan, M. S., Genovese, C. R., Eddy, W. F. \& Sweeney, John A. (2001). Maturation of Widely Distributed Brain Function Subserves Cognitive Development. Neurolmage, Vol. 13, S. 786-793.

Määttä, S., Pääkkönen, A., Saavalainen, P. \&Partanen, J. (2005). Selective attention eventrelated potential effects from auditory novel stimuli in children and adults. Clinical Neurophysiology, Vol. 116, S. 129-141. 
Madden, D. J., Gottlob, L. R. \& Allen, P. A. (1999). Adult age differences in visual search accuracy: Attentional guidance and target detectability. Psychological Aging, 14, 68394.

Mager, R., Falkenstein, M., Störmer, R., Brand, S., Müller-Spahn, F. \& Bullinger, A. H. (2005): Auditory distraction in young and middle-aged adults: a behavioural and eventrelated potential study. Journal of Neural Transmission, 112, 1165-1176.

McPhee, L. C., Scialfa, C. T., Dennis, W. M., Ho, G \&Caird, J. K. (2004). Age differences in visual search for traffic signs during a simulated conversation. Human Factors, 46, 674685.

MEYRA-ORTOPEDIA Vertriebsgesellschaft mbH (2005): Leitfaden zur Versorgung mit Elektrofahrzeugen. 2. Auflage. Kalletal.

Milling, P.: Systemtheoretische Grundlagen zur Planung der Unternehmenspolitik. Duncker \& Humblot, Berlin, 1981

Neumann, Peter (2006): Arbeitsmarktsituation behinderter Menschen. In: Leibniz-Institut für Länderkunde [Hrsg.]: Arbeit und Lebensstandard. S. 96 - 97. Nationalatlas Deutschland, Band 7. München.

Oswald, W. D., Hagen, B., Rupprecht, R. \& Gunzelmann, T. (2002): Bedingungen der Erhaltung und Förderung von Selbstständigkeit im höheren Lebensalter (SIMA). Teil XVII. Zusammenfassende Darstellung der langfristigen Trainingseffekte. Zeitschrift für Gerontopsychologie \& -psychiatrie, 15(1), S. $13-31$

Perchet, C. \& Garcia-Larrea, L. (2005). Learning to react: anticipatory mechanisms in children and adults during a visuospatial attention task. Clinical Neurophysiology, Vol. 116, S. 1906-1917.

Persson, J., Sylvester, C. Y., Nelson, J. K., Welsh, K. M., Jonides, J., \& Reuter-Lorenz, P. A. (2004): Selection requirements during verb generation: differential recruitment in older and younger adults. Neuroimage, 23, 1382-1390.

Pfafferott, I. (1994). Straßengestaltung von Kindern. In: Flade, A. [Hrsg.]. Mobilitätsverhalten: Veränderungsmöglichkeiten aus Umweltpsychologischer Sicht. Unter Mitarbeit von Klaus-Peter Kalwitzki. Beltz Psychologie-Verl.-Union. Weinheim. S. 291-300.

Pfeffer, K. \& Barnecutt, P. (1996). Children's auditory perception of movement of traffic sounds. Child Care, Health and Development, Vol. 22 (2), S. 129-137.

Poschadel, S. (2006). Prototypische Kinderunfälle im innerstädtischen Straßenverkehr : Von Unfallanalysen über Präventionsmöglichkeiten zur Entwicklung eines Unfallmodells. Dissertation. Ruhr-Universität Bochum, Fakultät für Psychologie

Poschadel, S. \& Sommer, S. (2007): Anforderungen an die Gestaltung von Fahrtrainings für ältere Kraftfahrer - Machbarkeitsstudie. (Schriftenreihe der Eugen-Otto-Butz-Stiftung, Forschungsergebnisse für die Praxis, Bd. 1). TÜV Media: Köln.

Pro Retina Deutschland e. V. (2008): Altersabhängige Makuladegeneration. (AMD) - Was ist das? Aachen (Informationsservice Pro Retina, 13). 
Rogers, R. D. \& Monsell, S. (1995): Costs of a predictible switch between simple cognitive tasks. Journal of Experimental Psychology: General, 124, S. 207-231.

Rubin GS, Ng ES, Bandeen-Roche K, Keyl PM, Freeman EE, West SK. (2007): A prospective, population-based study of the role of visual impairment in motor vehicle crashes among older drivers: the SEE study. Investigative ophthalmology \& visual science, 48 , 1483-91.

Salthouse TA (1996) The processing-speed theory of adult age differences

Schlag, B. (1990): Empirische Untersuchungen zur Leistungsfähigkeit älterer Kraftfahrer. Zeitschrift für Gerontologie, 23, 260-266.

Schlag, B. [Hrsg.].(2008): Altersbegleitende Leistungsveränderungen in ihrer Bedeutung für Verkehrsteilnahme und Fahrverhalten. In: Schlag, B. [Hrsg.]. Leistungsfähigkeit und Mobilität im Alter. Köln: TÜV Media GmbH.

Schuch, S. \& Koch, I (2003): The role of response selection for inhibition of task sets in task shifting. Journal of Experimental Psychology: Human Perception and Performance, 29 (1), S. 92-105.

Schüller, H. (2001): Geschwindigkeiten und Unfälle auf Stadtstraßen. In: Zeitschrift für Verkehrssicherheit (ZVS), Heft4/2011, S. 181 ff. Köln: TÜV Media Verlag (Herausgeber seit 2012: Kirschbaum Verlag $\mathrm{GmbH}$, Bonn)

Schweizerische Beratungsstelle für Unfallverhütung (o.A.) Autofahren im Alter. Bern. (http://www.bfu.ch/PDFLib/1037_42.pdf; 20.02.2010).

Simoes, A. (2003). The cognitive training needs of older drivers. Recherche Transports Sécurité, No. 79, S. 145 - 155.

Span, M. M., Ridderinkhof, K. R. \& van der Molen, M. W. (2004). Age-related changes in the efficiency of cognitive processing across the life span. Acta Psychologica, Bd. 117, S. 155-183.

Spieler, D. H., Balota, D. A. \& Faust, M. E. (1996): Stroop performance in healthy younger and older adults and individuals with dementia of the Alzheimer's type. Journal of Experimental Psychology. Human Perception and Performance, 22, S. 461-479.

Statistisches Bundesamt [Hrsg.] (2007): „Statistik der schwerbehinderten Menschen Kurzbericht“. Ausgabe 2007. Wiesbaden 2009.

Statistisches Bundesamt [Hrsg.] (2009): Sozialleistungen. Schwerbehinderte Menschen 2007. Wiesbaden (Fachserie 13, Reihe 5.1).

Statistisches Bundesamt [HRSG.] (2009). Kinderunfälle im Straßenverkehr 2008. Wiesbaden.

Statistisches Bundesamt [Hrsg.] (2009): Bevölkerung Deutschlands bis 2060. 12. koordinierte Bevölkerungsvorausberechnung. Begleitmaterial zur Pressekonferenz am 18. November 2009 in Berlin. Wiesbaden.

Statistisches Bundesamt [Hrsg.]: „Bevölkerung: Bundesländer, Stichtag, Altersjahre“. Online abgerufen

unter:

https://www- 
genesis.destatis.de/genesis/online;jsessionid=D872717BADDFAD0856290CAEBBA3A ED9.tcggen1 ?operation=ergebnistabelleUmfang\&levelindex $=3$ \&levelid $=126952398704$ 3\&downloadname=12411-0011 (7.3.2010)

Statistisches Bundesamt [Hrsg.] (2010). Verkehr. Unfälle von Senioren im Straßenverkehr. Wiesbaden.

Statistisches Bundesamt (2012): Bevölkerung und Erwerbstätigkeit. Bevölkerungsfortschreibung. Wiesbaden (Fachserie 1 Reihe 1.3).

Step by Step. Gruppenspezifisches Verhaltens- und Simulationsmodell auf der Grundlage von Telematikerhebungen (2009). Wien. Online verfügbar unter http://www2.ffg.at/verkehr/projektpdf.php?id=714, zuletzt geprüft am 24.07.2012.

Stiftung Warentest: "Mobil auf vier Rädern"; in: test, Ausgabe: 9/2005, Seite: 90 - 95

Thomson, J. A. (1997). Developing safe route planning strategies in young child pedestrians. Journal of Applied Developmental Psychology, Bd. 18 (2), S. 271-281.

Treisman, A. \&Gelade, G. (1980). A feature integration theory of attention. CognitivePsychology, 12, 97-136.

TÜV Kraftfahrt GmbH, Institut für Verkehrssicherheit, Herberg, K.-W. (1998). Untersuchung der Entwicklung der sicherheitsrelevanten Leistungsfähigkeit mit dem Lebensalter. (www.butz-stiftung.de/22.htm; 07.12.05).

VdK Medien- und Anzeigenverwaltung GmbH (2011): Wheel Map. o. O. Online verfügbar unter http://www.vdktv.de/index.php?id=22\&rid=328, zuletzt geprüft am 05.10.2011.

Verband Deutscher Verkehrsunternehmen e. V. - VDV (2012): Barrierefreier ÖPNV in Deutschland. Gesamtbearbeitung durch Studiengesellschaft für unterirdische Verkehrsanlagen - STUVA e. V. 2. vollständig neubearbeitete Auflage. Meerbusch/Düsseldorf: Alba Verlag.

Verhaeghen, P. \& Cerella, J. (2002). Aging, executive control, and attention: a review of meta-analyses. Neuroscience Biobehavior Review, 26, 849- 857.

Wild-Wall, N. \& Falkenstein, M. (2007). Effects of ageing and fatigue on task preparation. Clinical Neurophysiology, 118, 558-69

Wilhelm, Matthias (2009): Wenn Mobilität zur Gefahr wird. Bericht zum Unfallgeschehen von Menschen mit Behinderungen. 3. Auflage (Stand 3/2009). Berufsgenossenschaft für Gesundheitsdienst und Wohlfahrtspflege - BGW. Hamburg (BGW Forschung, SPMobi16).

Yordanova J, Kolev V, Hohnsbein J \&Falkenstein M (2004) Sensorimotor slowing with ageing is mediated by a functional dysregulation of motor-generation processes: evidence from high-resolution eventrelated potentials. Brain 127(2):351-62.

Zimmermann, Tanja (2008): Durchführungshilfen für eine systematische Anpassung des Verkehrsraums an die Bedürfnisse mobilitätseingeschränkter Verkehrsteilnehmer. Auswertung von Unfalldaten mit mobilitätsbehinderten Verkehrsteilnehmern zur Vorbereitung einer Dissertation an der TU Berlin. Unveröffentlicht. Berlin. 


\section{Gesetze und Verordnungen}

Gesetz zur Gleichstellung behinderter Menschen (Behindertengleichstellungsgesetz - BGG) i. d. F. v. 27.04.2002, zuletzt geändert am 19.12.2007. Online abgerufen unter http://www.gesetze-im-internet.de/bundesrecht/bgg/gesamt.pdf (21.02.2010).

Pressegesetz für das Land Nordrhein-Westfalen (Landespressegesetz NRW) i. d. F. v. 24.05.1966, zuletzt geändert am 29.04.2003. Online abgerufen unter http://88.198.44.111/index.php?option=com_content\&task=view\&id=29\&Itemid=26 (22.02.2010).

Sozialgesetzbuch (SGB) Neuntes Buch (IX) - Rehabilitation und Teilhabe behinderter Menschen i. d. F. v. 19.6.2001 zuletzt geändert am 30.7.2009.

Straßenverkehrs-Zulassungs-Ordnung (StVZO) i. d. F. v. 28.09.1988, zuletzt geändert 21.04.2009. Online abgerufen unter http://www.gesetze-iminternet.de/bundesrecht/stvzo/gesamt.pdf (22.02.2010). 


\section{Anlagenverzeichnis}

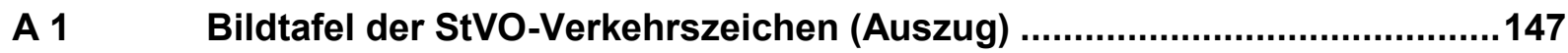

A 2 Sicherheitsanalysen und Verhaltensbeobachtungen im Realverkehr an Kreuzungen mit Lichtsignalanlage ...........................................................148

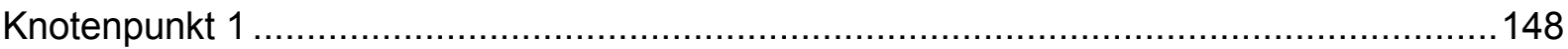

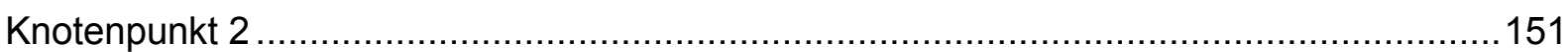

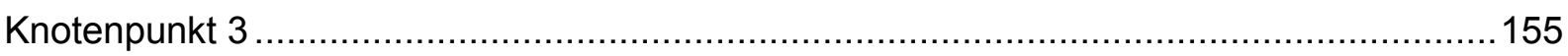

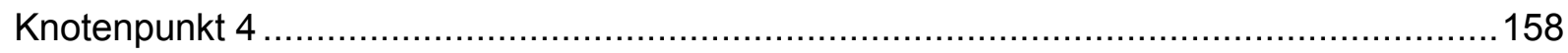

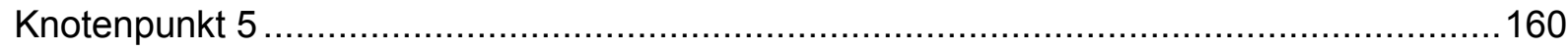

A 3 Sicherheitsanalysen und Verhaltensbeobachtungen im Realverkehr an Kreuzungen mit Lichtsignalanlage und verkehrstechnischer und/oder baulicher Optimierung ............................................................................166

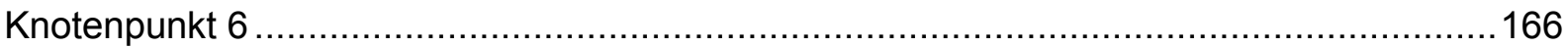

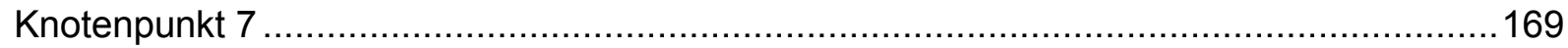

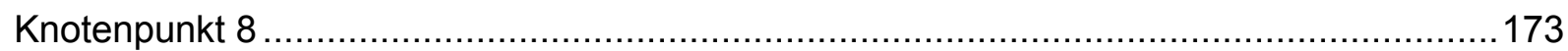

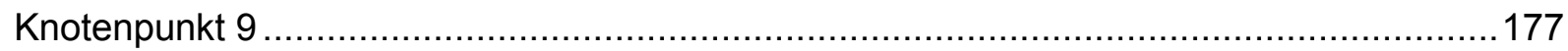

A 4 Sicherheitsanalysen und Verhaltensbeobachtungen im Realverkehr an Kreuzungen mit vorfahrtregelnden Verkehrszeichen ...............................180

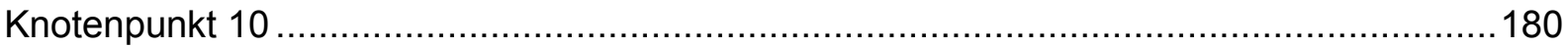

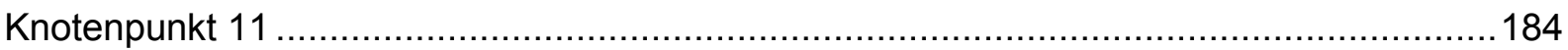

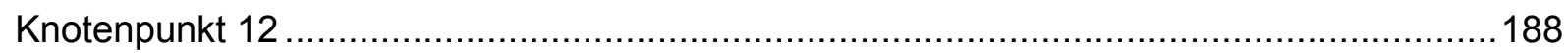

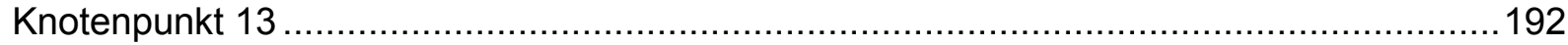

A 5 Sicherheitsanalysen und Verhaltensbeobachtungen im Realverkehr an Einmündungen mit vorfahrtregelnden Verkehrszeichen...........................196

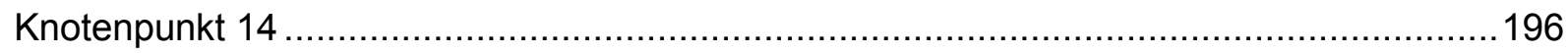

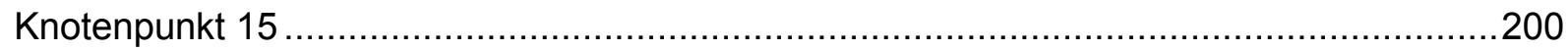

A 6 Detaillierte Auswertung der Verhaltensbeobachtungen nach Gruppe und

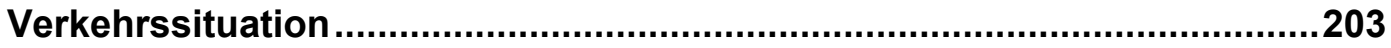

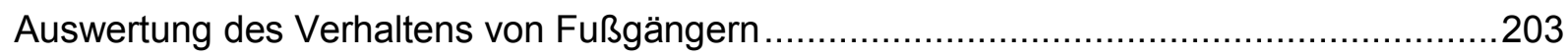

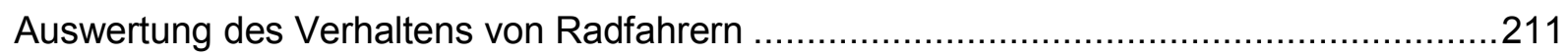

Auswertung des Verhaltens älterer Menschen als Pkw-Fahrer (im Vergleich zu anderen PkwFahrern)

A 7 Prototypische Merkmale/Situationen nach Art der Verkehrsbeteiligung und mögliche Maßnahmen zur Beseitigung der Defizite 
A 8 Empfohlenen Maßnahmen zur Gestaltung von Knotenpunkten insbesondere aus Sicht von Menschen mit Mobilitätseinschränkungen/behinderungen . 232

A 9 Regressionskurven zu Komplexitätsgraden und Unfallkenngrößen... 239 


\section{A 1 Bildtafel der StVO-Verkehrszeichen (Auszug)}

\begin{tabular}{|l|l|l|l|}
\hline Zeichen & Abbildung & Zeichen Nr. & Abbildung \\
\hline Z 133 (Fußgänger) & Z 134 (Fußgängerüberweg) \\
\hline Z 136 (Kinder) & & & \\
\hline
\end{tabular}

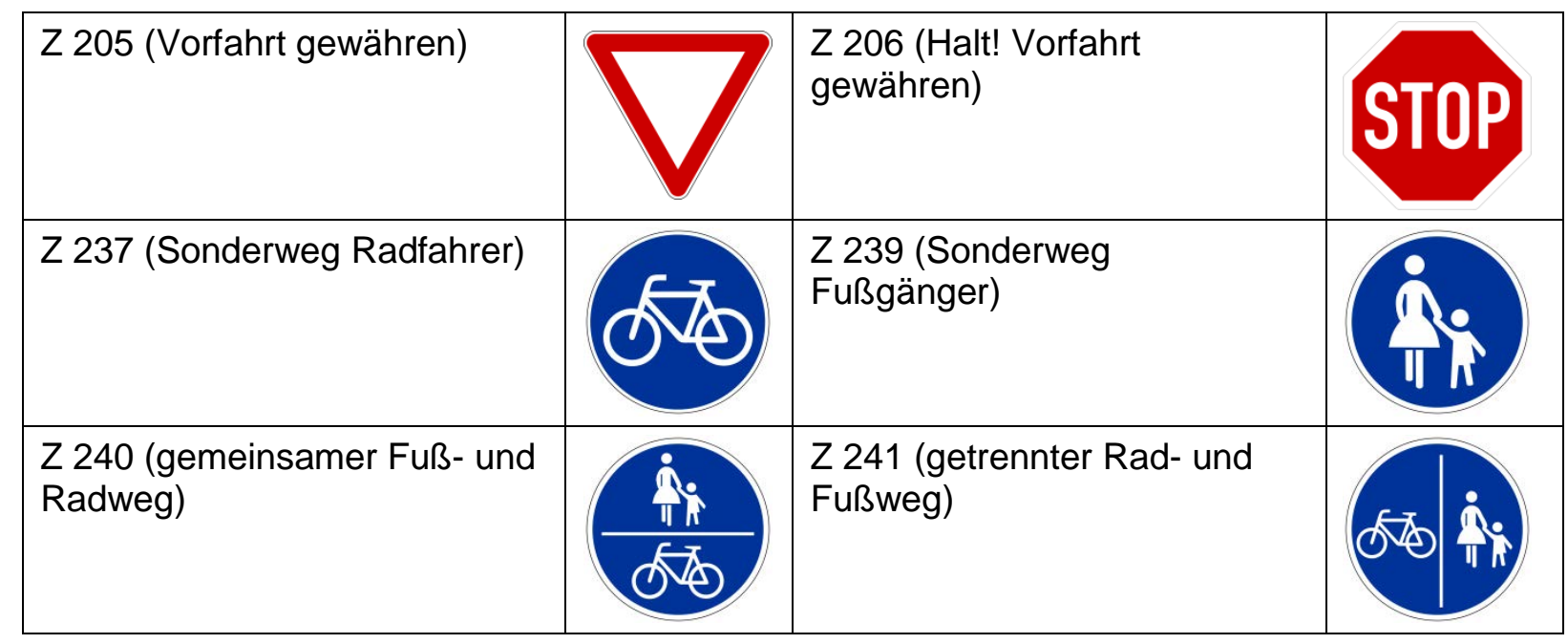

\begin{tabular}{|l|l|l|}
\hline Z 301 (Vorfahrt) & 306 (Vorfahrtstraße) \\
\hline Z 350 (Fußgängerüberweg) & & \\
\hline
\end{tabular}

\begin{tabular}{|l|l|}
\hline Z 720 (Grünpfeil) & \\
\hline
\end{tabular}




\section{A 2 Sicherheitsanalysen und Verhaltensbeobachtungen im Realverkehr an Kreuzungen mit Lichtsignalanlage}

\section{Knotenpunkt 1}

Abbildung 55zeigt zunächst das Unfalldiagramm des Knotenpunktes 1 auf Grundlage der Unfälle mit Personenschaden und schwerem Sachschaden der Jahre 2006 bis 2010, zu denen detaillierte Informationen in Form von anonymisierten Verkehrsunfallanzeigen vorlagen. Unfälle der Kategorie 4 kamen in diesem Zeitraum nicht vor, alle Unfälle sind Unfälle mit Personenschaden. Zudem sind die Kamerapositionen mit den Aufnahmerichtungen und die Positionen der beiden Zählposten für die Verhaltensbeobachtungen dargestellt. Die Verhaltensbeobachtungen wurden im April 2011 durchgeführt.

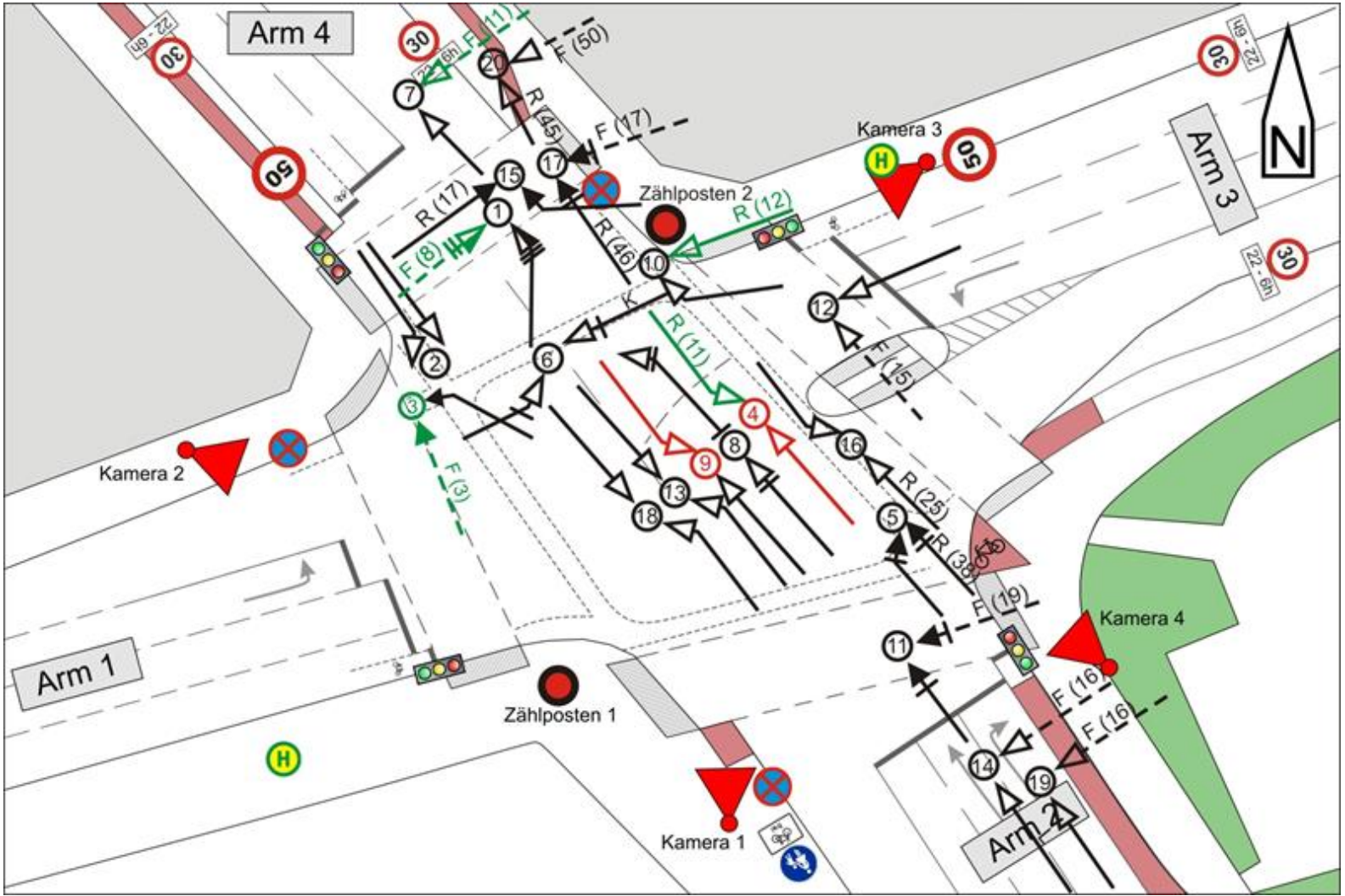

Abbildung 55: Knotenpunkt 1 - Unfalldiagramm, Kamerastandorte und Zählposten

Die wesentlichen Konfliktsituationen an dieser Kreuzung konnten im Kreuzungsbereich festgestellt werden. Hervorgerufen wurden diese Konfliktsituationen vornehmlich durch die im Folgenden beschriebenen Faktoren:

- Die Signalsteuerung des Knotenpunktes ist grundlegend eine 2-Phasensteuerung mit einer nicht gesicherten Führung der Linksabbieger aus Arm 1 und Arm 3 und einer zeitweilig gesicherten Führung der Linksabbieger aus Arm 2 und Arm 4 über eine Zugabezeit (einfeldiger Richtungssignalgeber, „grüner Pfeil“)

- Linksabbieger und Geradeausfahrer aus Arm 2 und Arm 4 nutzen einen gemeinsamen Fahrstreifen (linker Fahrstreifen). Der rechts danebenliegende 
Fahrstreifen wird nur von Geradausfahrern (Arm 2) bzw. von Geradeausfahrern und Rechtsabbiegern (Arm 4) genutzt.

- Nach dem Kreuzungsbereich erfolgt eine Fahrstreifensubtraktion für den Geradeausverkehr von zwei auf einen Fahrstreifen. Ein vorzeitiger Hinweis darauf ist nicht gegeben.

- Im Kreuzungsbereich sind keine Wartelinien für Linksabbieger markiert. Eine äußere Leitlinie ist lediglich für Linksabbieger aus der Zufahrt 3 angebracht.

- Die Haltlinien der Zufahrten sind mit rd. 12 m (Arm 1), rd. 13 m (Arm 2), rd. 10 m (Arm 3) und rd. $15 \mathrm{~m}$ (Arm 4) z. T. weit vom Beginn des Kreuzungsbereiches abgesetzt.

Das Zusammenwirken dieser Umstände, in Verbindung mit hohen Kfz-Verkehrsmengen (DTV-Hochrechnung Knotenpunkt: rd. $43.000 \mathrm{Kfz} / 24 \mathrm{~h}$ ) führte dazu, dass im Kreuzungsbereich unübersichtliche, zum Teil verkehrsgefährdende Situationen entstanden.

Aufgrund fehlender Leit- und Wartelinien stellten sich wartepflichtige Linksabbieger oft falsch auf. Sie fuhren weit in den Kreuzungsbereich ein bzw. gerieten in die Fahrbereiche des Gegenverkehrs.

Geradeausfahrer, die den linken Fahrstreifen nutzten, scherten zum Teil sehr plötzlich und für nachfolgende Fahrer unvermittelt nach rechts aus, wenn vor innen Linksabbieger anhielten, um den Gegenverkehr passieren zu lassen.

Die nötigen Verflechtungsvorgänge im Kreuzungsbereich, bedingt durch die Fahrstreifensubtraktion, konnten allerdings aufgrund hoher Verkehrsmengen der Geradeausfahrer zumeist nicht während der Freigabezeit vollzogen werden. Geradeausfahrer, die den gemeinsamen Fahrstreifen für den linksabbiegenden und geradeausfahrenden Verkehr nutzten, mussten hinter den haltenden Linksabbiegern warten. Ausreichende Zeitlücken ergaben sich für sie erst dann, wenn der Geradeausverkehr bereits rot signalisiert bekam. Dies führte insbesondere in Verbindung mit der Zugabezeit für die Linksabbieger zu konfliktreichen Situationen.

Abbildung 56 veranschaulicht exemplarisch diese Situation. Die Hauptsignalgruppe zeigt rot für die zufahrenden Verkehrsströme der Arme 2 und 4. Den Linksabbiegern wird über den einfeldigen Richtungssignalgeber (grüner Pfeil) die Zugabezeit zum Räumen des Kreuzungsbereiches und damit auch eine sichere Führung (ohne Gegenverkehr) angezeigt ${ }^{27}$. Geradeausfahrer des Gegenverkehrs, die den linken Fahrstreifen nutzen und für die während der Freigabezeit keine ausreichenden Zeitlücken zum Einfädeln in den benachbarten rechten Fahrstreifen entstanden, räumen jetzt ebenfalls den Kreuzungsbereich. Da sie bereits in den Kreuzungsbereich eingefahren sind und hinter der Haltlinie stehen, können sie das rote Signal der Hauptgruppe nicht mehr sehen.

${ }^{27}$ Vgl. StVO, §37, Abs. 2, 1.: „Ein grüner Pfeil links hinter der Kreuzung zeigt an, dass der Gegenverkehr durch Rotlicht angehalten ist und dass Linksabbieger die Kreuzung in Richtung des grünen Pfeils ungehindert befahren und räumen können." (Straßenverkehrsordnung, 1.12.2010) 


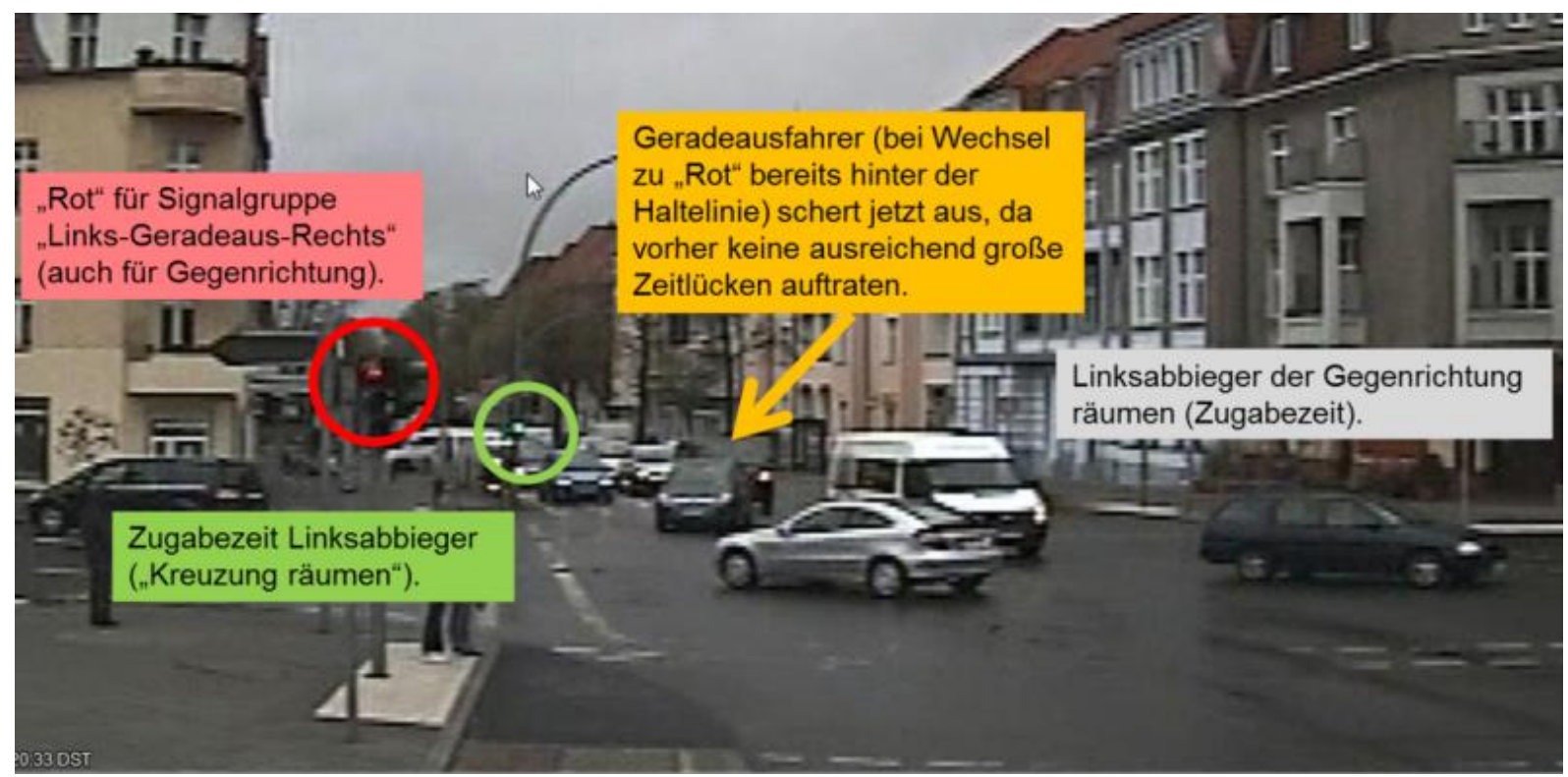

\section{Abbildung 56: Knotenpunkt 1 - Konflikte bei Zugabezeit für Linksabbieger}

Im Weiteren zeigten die Verhaltensbeobachtungen an dieser Kreuzung, dass jüngere wie ältere Kfz-Führer dieselben Fehler beim Linksabbiegen machten. Der relative Anteil der älteren Kfz-Führer war nur unwesentlich höher.

Mit Blick auf die im Kreuzungsbereich, in Verlängerung der Radwege bzw. Schutzstreifen der Zufahrten, markierten Radverkehrsfurten zeigten die Verhaltensbeobachtungen, dass diese kaum von Radfahrern angenommen wurden. Radfahrer aller Altersklassennutzen nutzten eher die Fußgängerfurten.

Rollstuhlfahrer und Personen mit Rollatoren oder Kinderwagen nutzten, dort wo vorhanden, die Nullabsenkungen bzw. Rampen der Radwege und nicht die Flachborde an den Fußgängerfurten.

Spezifische Konfliktsituationen von Kindern und/oder älteren Menschen konnten an diesem Knotenpunkt nicht beobachtet werden. Die Verhaltensbeobachtungen haben vielmehr gezeigt, dass Verkehrsteilnehmer aller Altersgruppen mit den gegebenen Bedingungen Schwierigkeiten haben.

Durch die fehlende Führung der Verkehrsteilnehmer insgesamt und die signaltechnisch nicht bzw. nur zeitweise gesicherte Führung der Linksabbieger, ist von allen Verkehrsteilnehmern ein hohes Maß an Aufmerksamkeit gefordert. Aus verkehrspsychologischer Sicht müsste diese „Reizüberflutung“ durch eine entsprechend übersichtliche Neugestaltung der Kreuzung und die sichere Führung der Linksabbieger reduziert werden.

Für diesen Knotenpunkt ist daher eine sichere Führung der Linksabbieger zu empfehlen. Im Weiteren können die Trennung von Linksabbiegern und Geradeausfahrern durch eigene Linksabbiegestreifen und zusätzliche Leit- und Wartelinien für Linksabbieger im Kreuzungsbereich helfen, die Situation zu ordnen und für alle Verkehrsteilnehmer, auch Radfahrer, übersichtlicher zu gestalten. 


\section{Knotenpunkt 2}

Abbildung 57 zeigt das Unfalldiagramm auf Basis von Unfällen mit Personenschaden und schwerem Sachschaden der Jahre 2007 bis 2010, zu denen detaillierte Informationen in Form von anonymisierten Verkehrsunfallanzeigen vorlagen. Zudem sind die Kamerapositionen mit den Aufnahmerichtungen und die Positionen der beiden Zählposten für die Verhaltensbeobachtungen in dieser Abbildung dargestellt. Die Verhaltensbeobachtungen wurden im Oktober 2010 durchgeführt.

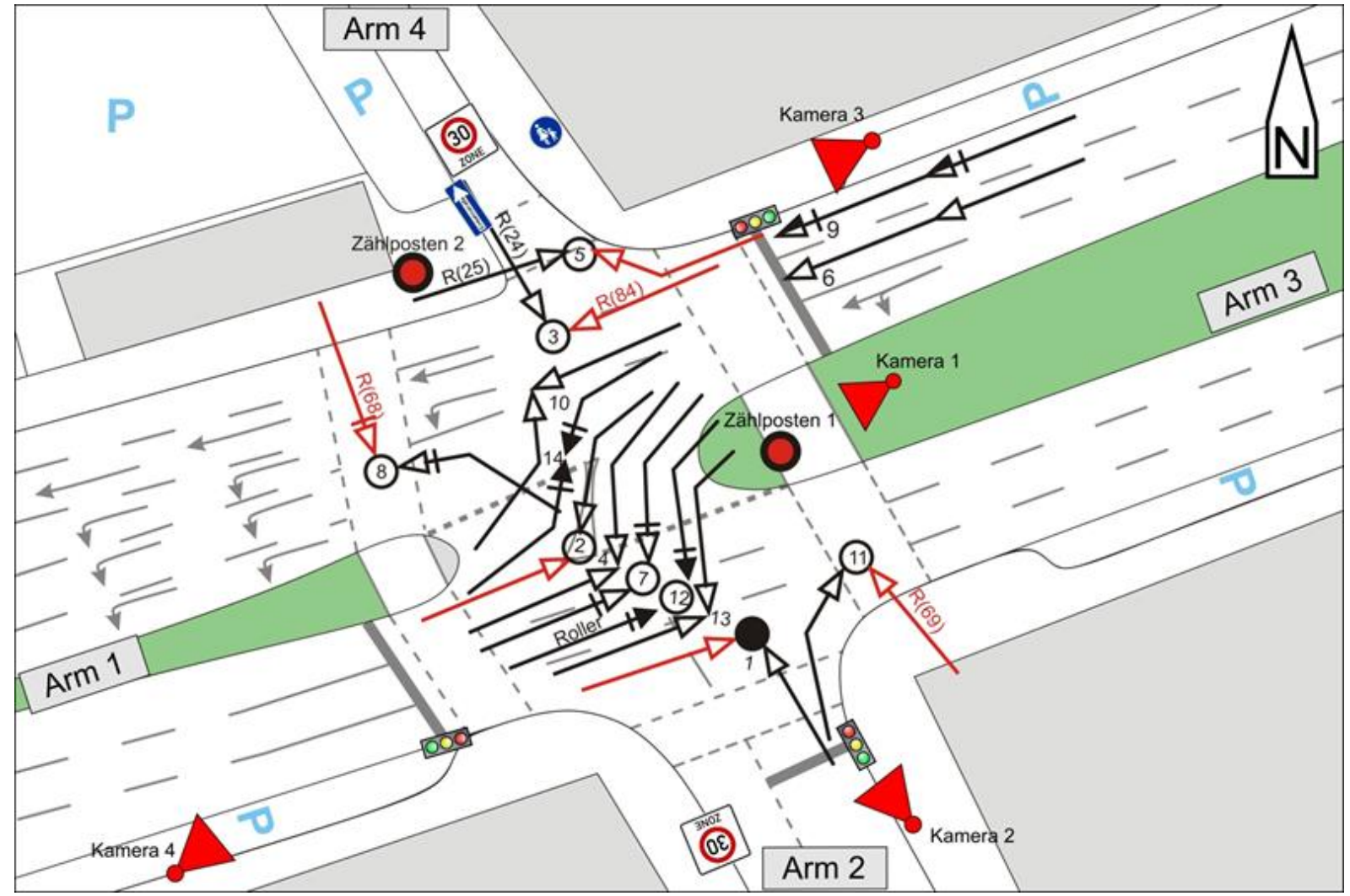

Abbildung 57: Knotenpunkt 2 - Unfalldiagramm, Kamerastandorte und Zählposten

Die wesentlichen beobachteten Konfliktsituationen im Kfz-Verkehr an dieser Kreuzung, die auch dem Unfallbild entsprechen, resultierten aus der nicht gesicherten Führung der Linksabbieger im Zusammenwirken mit einer offensichtlich nicht zweckmäßigen und zu Missverständnissen führenden Markierung im Kreuzungsbereich und Sichtbehinderungen. Zudem müssen die Linksabbieger beider Zufahrten der Hauptrichtung (Arm 1 - 3) jeweils drei Fahrstreifen der Gegenrichtung einsehen und kreuzen ${ }^{28}$.

Die Markierung im Kreuzungsbereich (Abbildung 58) soll den linksabbiegenden (und wendenden) Pkw-Fahrern eine Führung vor der markierten durchgezogenen Linie anzeigen.

${ }^{28}$ Verkehrsbelastungen je Richtungsfahrbahn aus Zufahrt 1 und 3 von etwa 12.000 bis 15.000 $\mathrm{Kfz} / 24 \mathrm{~h}$. 


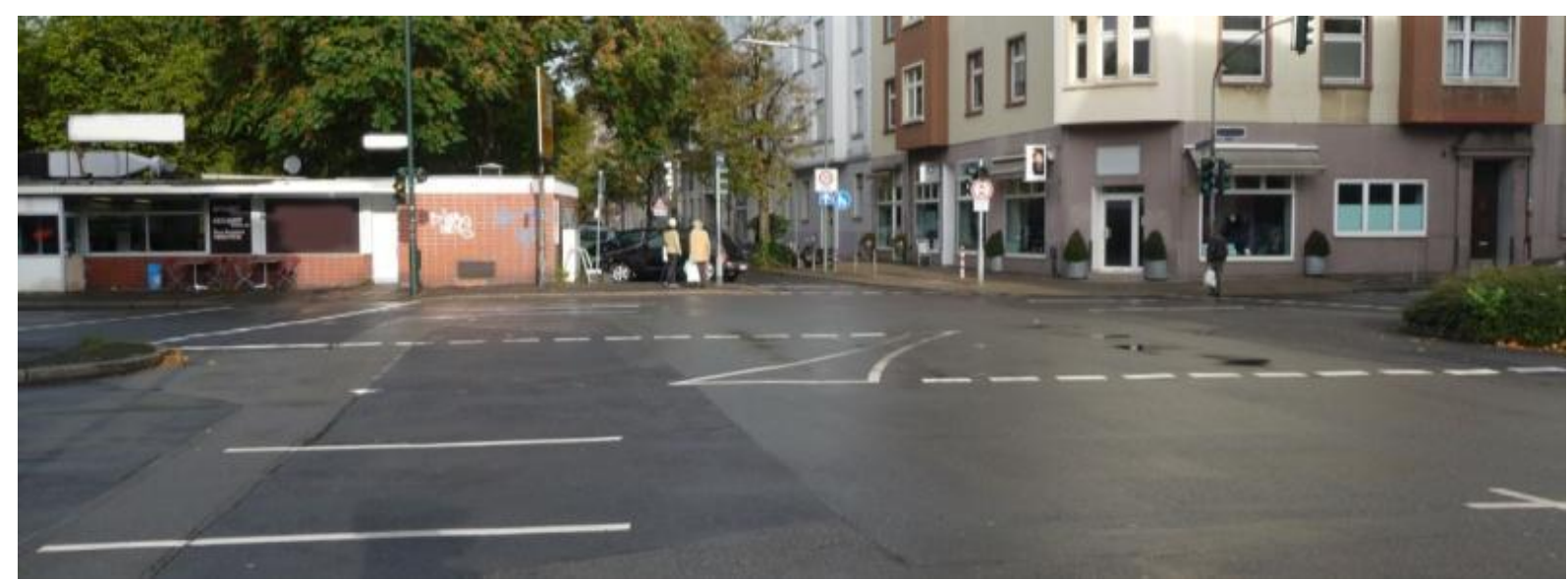

Abbildung 58: Knotenpunkt 2 - Markierung im Kreuzungsbereich (von links Zufahrt 1, von rechts Zufahrt 3)

Die Verhaltensbeobachtungen allerdings zeigten, dass jüngere wie ältere Fahrer auch hinter dieser Leitlinie abbogen bzw. sich im Kreuzungsbereich nebeneinander aufstellten (Abbildung 59). Dies kann auch dem Umstand geschuldet sein, dass Linksabbieger und Geradeausfahrer der Zufahrten 1 und 3 beide die jeweils linken Fahrstreifen nutzen können und die Linksabbieger hier den Weg für die nachfolgenden Fahrzeuge freimachen wollen. Dies führte zu unübersichtlichen Situationen, insbesondere dann, wenn Linksabbieger des Gegenverkehrs sich ebenfalls im Kreuzungsbereich aufstellen (Abbildung 59, Bild links).
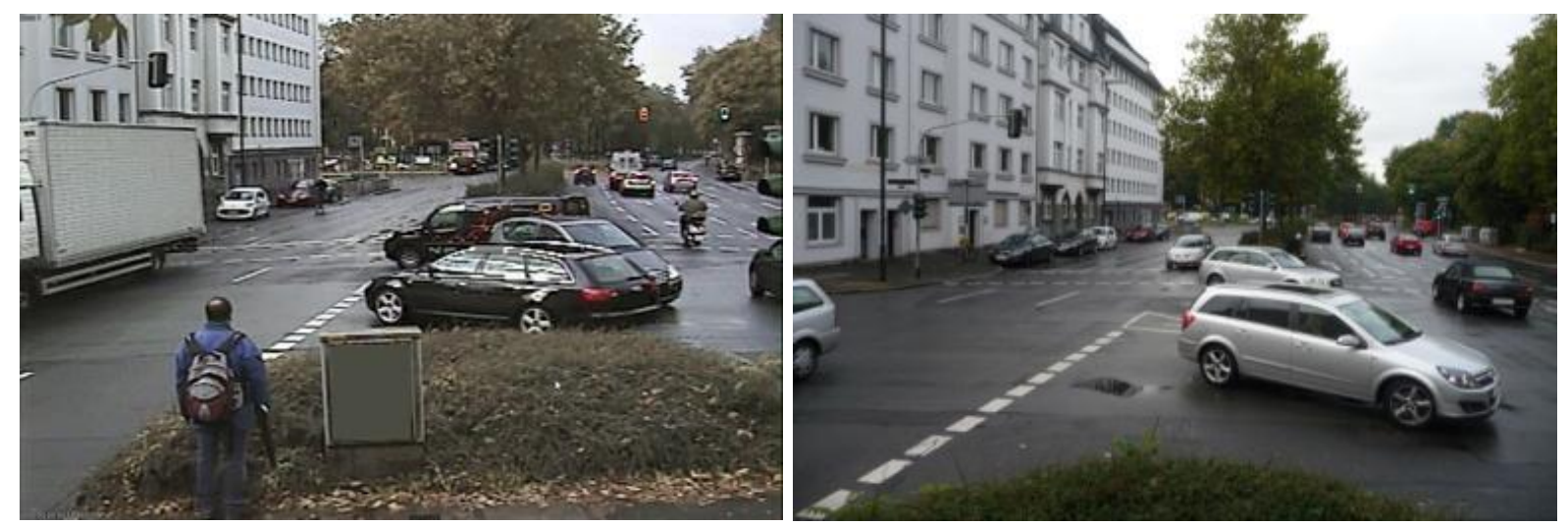

Abbildung 59: Knotenpunkt 2 - Aufstellung wartepflichtiger Linksabbieger und Wender im Kreuzungsbereich

Die Sichtbeziehungen der linksabbiegenden und wendenden Fahrer auf den Gegenverkehr sind durch die Bepflanzung des Mittelstreifens eingeschränkt. Weiter verschlechtern sich die Sichtbeziehungen, wenn wartepflichtige Linksabbieger und Wender der Gegenrichtung ebenfalls im Kreuzungsbereich halten (Abbildung 60). Hinzu kommt, dass die Straße im Verlauf vom Ost nach West gesehen einer leichten Rechtskurve folgt, wodurch die Sicht der Linksabbieger aus Arm 3 auf den Gegenverkehr zusätzlich beeinträchtigt ist. 


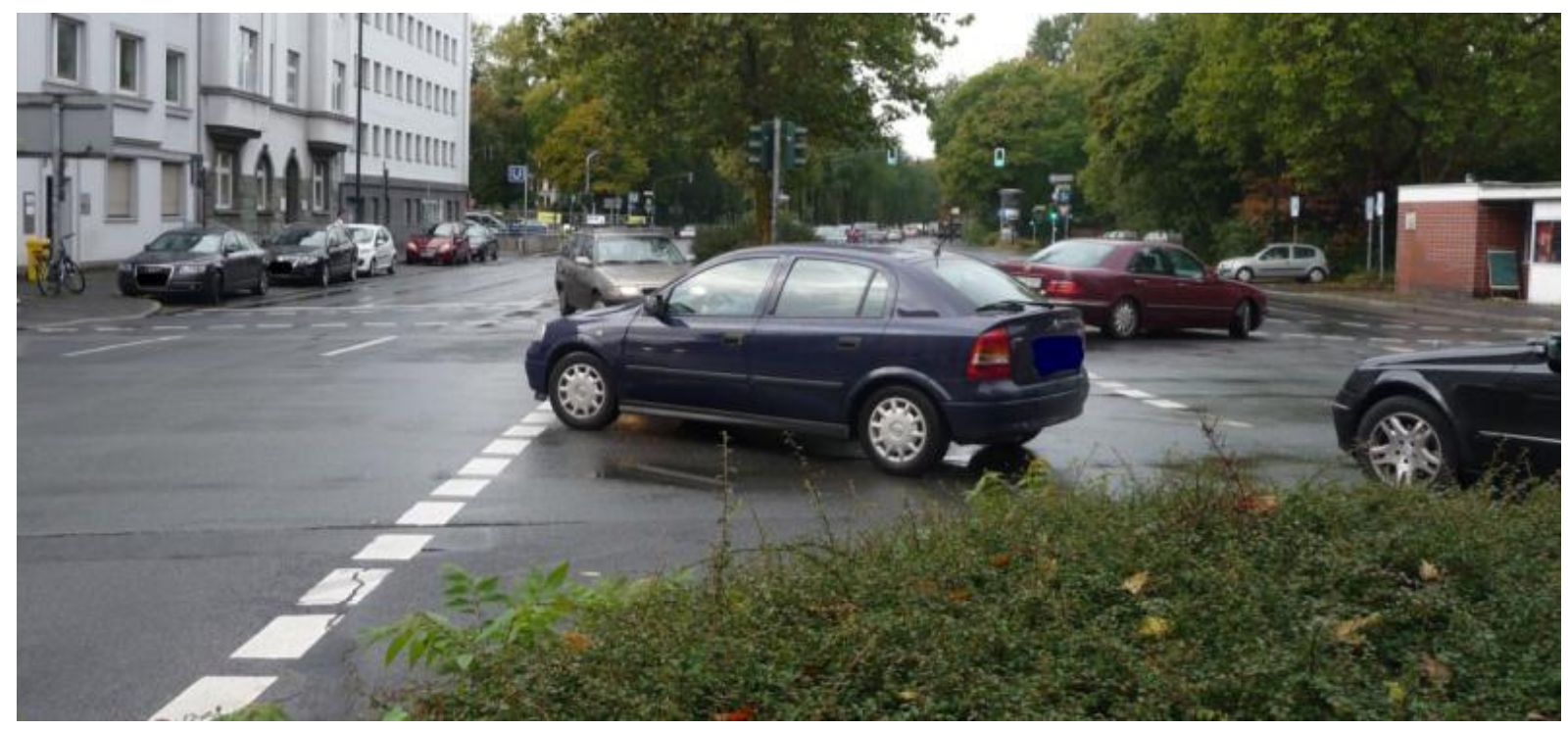

Abbildung 60: Knotenpunkt 2 - Sichtbehinderung für Linksabbieger (und Wender) durch Mittelstreifen und Fahrzeuge des Gegenverkehrs

Die Verhaltensbeobachtungen zeigten zudem eine Gefährdung von Fußgängern, insbesondere im Bereich der nördlichen Furt an Arm 1 durch wendende Fahrzeuge (vom südlichen Teil des Arms 1 in den nördlichen Teil), die sich während ihrer Freigabezeit aufgrund der verkehrlichen Belastung des Geradeausverkehrs von Arm 3 nach 2 nicht einordnen konnten und die Fußgängerfurt zu einem Zeitpunkt kreuzten, in dem die Fußgänger bereits grün signalisiert bekamen. Oft wurde den Fußgängern das Vorrangrecht auch nicht von linkseinbiegenden Kfz aus Arm 2 eingeräumt.

Der Radverkehr wird im Mischverkehr auf der Fahrbahn geführt. Allerdings nutzten die Radfahrer zumeist die Gehwege (Abbildung 61), auch „entgegen der vorgeschriebenen Fahrtrichtung“.

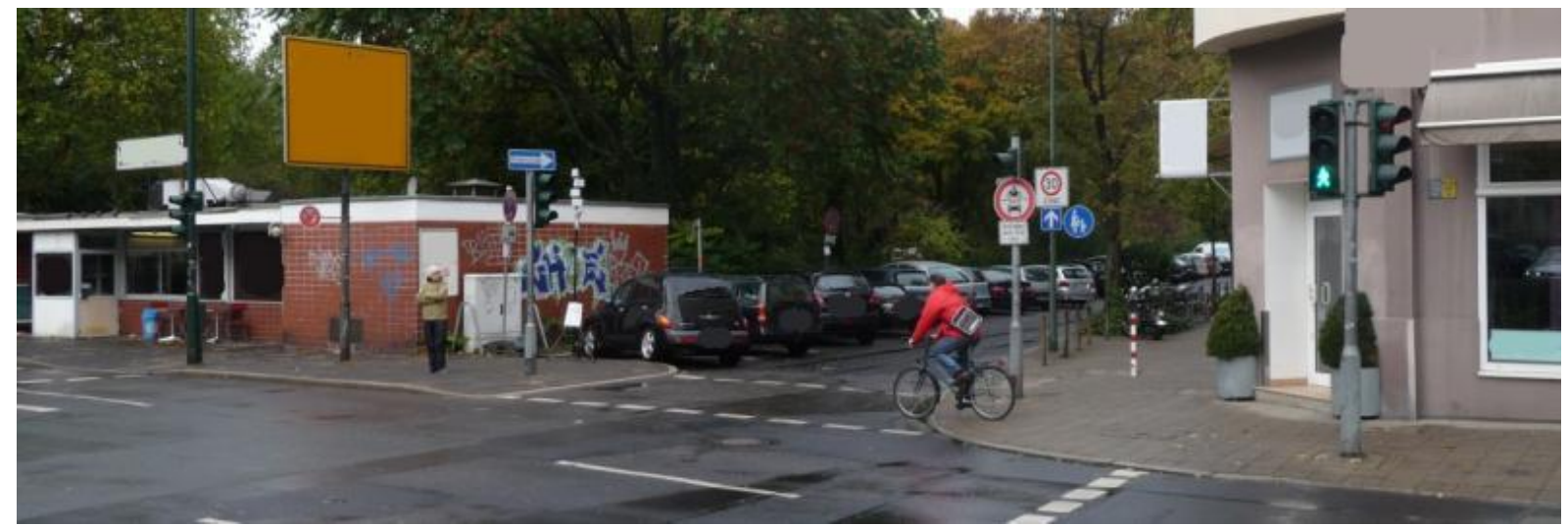

\section{Abbildung 61: Knotenpunkt 2 - Radfahrer auf Gehweg}

Die Verhaltensbeobachtungen zeigten, dass insbesondere ältere Fahrer unsicher in den Kreuzungsbereich beim Linksabbiegen und Wenden einfuhren und die angedachten Haltepositionen nicht fanden. Das falsche Aufstellen im Kreuzungsbereich konnte bei Fahrern aller Altersgruppen beobachteten werden.

Insgesamt müssen an dieser Kreuzung zu viele Reize unter Zeitdruck und bei zum Teil eingeschränkter Sicht verarbeitet werden. 
Zur Erhöhung der Verkehrssicherheit, vornehmlich im Bereich des Kfz-Verkehrs, empfiehlt sich an diesem Knotenpunkt eine signaltechnisch gesicherte Führung der Linksabbieger, auch um Ausschervorgänge des nachfolgenden Geradeausverkehrs nach rechts, um die wartenden Linksabbieger zu passieren, vorzubeugen oder die getrennte Signalisierung der beiden Hauptströme aus Zufahrt 1 und Zufahrt 3.

Im Weiteren können größere Durchmesser der Hilfssignalgeber (gelbes Blicklicht an Fußgängerfurt, vgl. Abbildung 62), z. B. 300 statt 200 mm, zur Sicherheit der gegenüber Linkseinbiegern und Wendern bevorrechtigten Fußgänger beitragen.

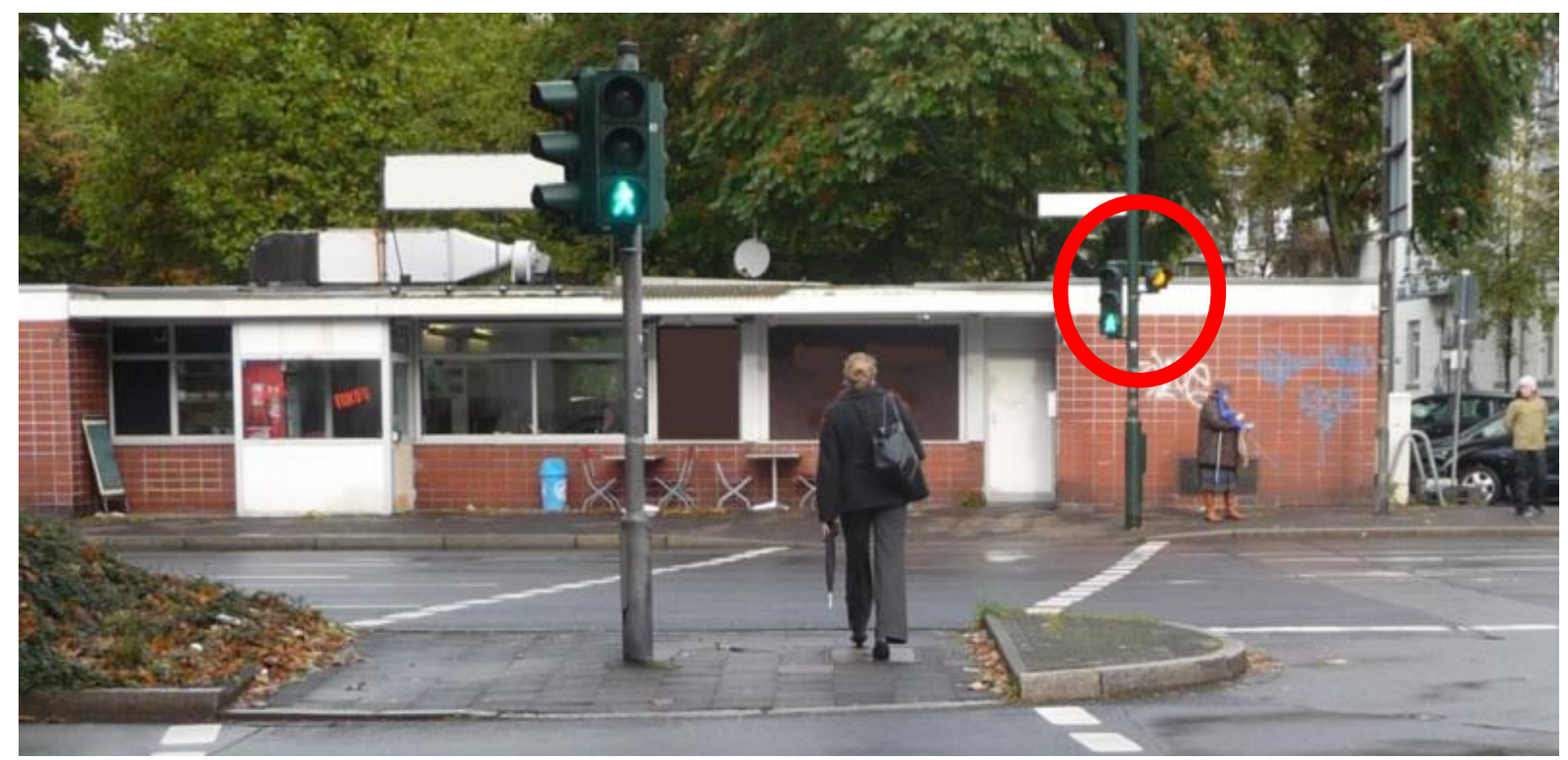

Abbildung 62: Knotenpunkt 2 - Hilfssignalgeber (gelbes Blicklicht) an Fußgängerfurt 


\section{Knotenpunkt 3}

Abbildung 63 zeigt das Unfalldiagramm für Unfälle der Kategorien 1 bis 4 der Jahre 2006 bis 2010, zu denen detaillierte Informationen in Form von anonymisierten Verkehrsunfallanzeigen vorlagen. Zudem sind die Kamerapositionen mit den Aufnahmerichtungen und die Positionen der beiden Zählposten für die Verhaltensbeobachtungen in dieser Abbildung dargestellt. Die Verhaltensbeobachtungen wurden im April 2011 durchgeführt.

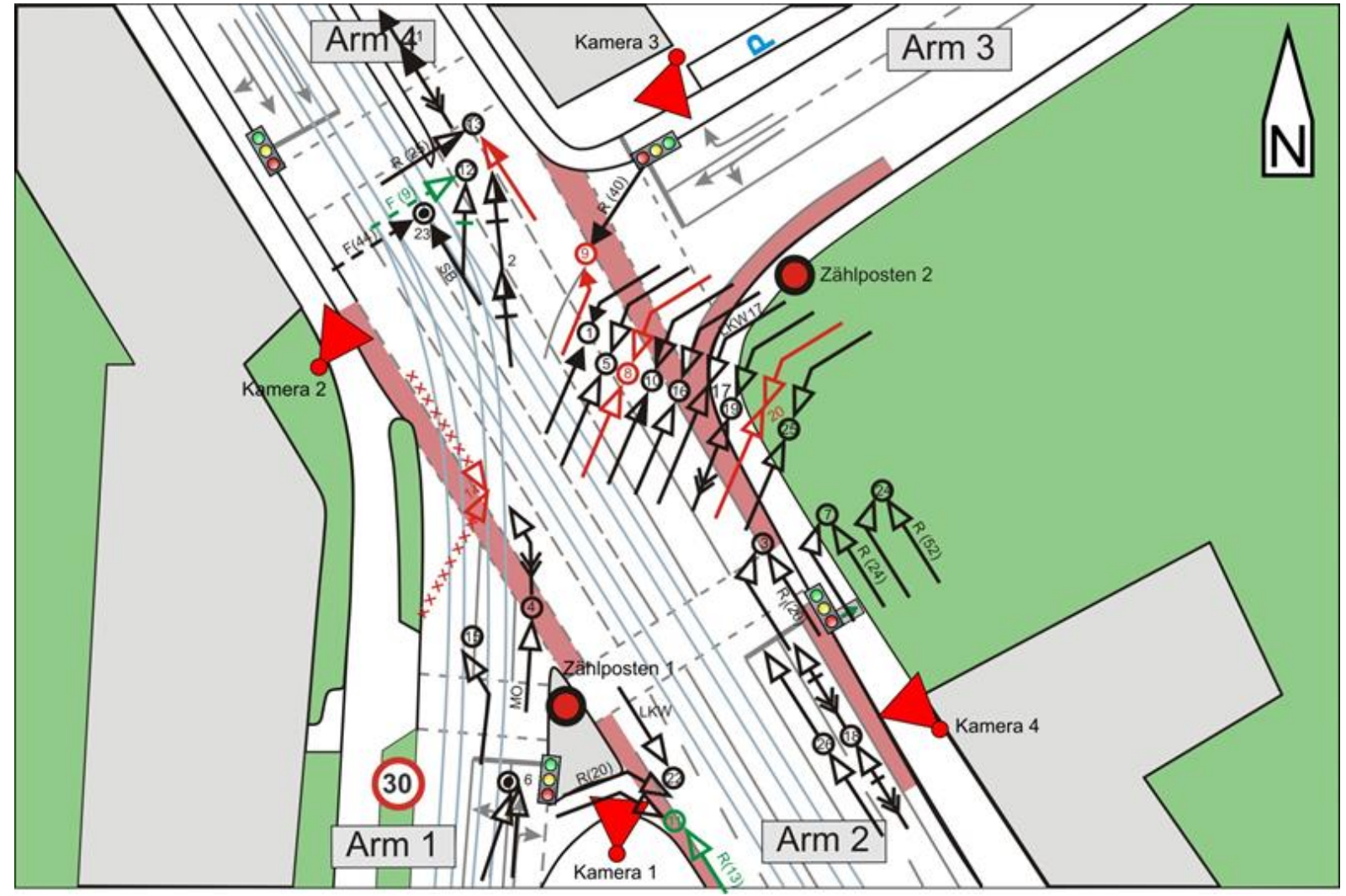

Abbildung 63: Knotenpunkt 3 - Unfalldiagramm, Kamerastandorte und Zählposten

Die an dieser Kreuzung beobachteten Konfliktsituationen entsprechen dem Unfallbild.

Die zufahrenden Verkehrsströme aus Arm 1 und Arm 3 (Nebenstraße) sind grundlegend in einer gemeinsamen Phase signalisiert ${ }^{29}$, die Linkseinbieger werden nicht gesichert geführt. Wartelinien für Linkseinbieger sind nicht aufgebracht und die markierten äußeren Leitlinien sind kaum noch zu erkennen (Abbildung 64 und Abbildung 65). Insgesamt befindet sich die aufgebrachte Markierung in einen schlechten Zustand und ist nicht mehr als Einheit wahrzunehmen (Figur-Grund-Problem). Insbesondere bei älteren Kraftfahrern konnten Unsicherheiten beim Einfahren in den Kreuzungsbereich festgestellt werden.

\footnotetext{
${ }^{29}$ Abweichungen im Signalprogramm ergeben sich durch einfahrende Straßenbahnen.
} 


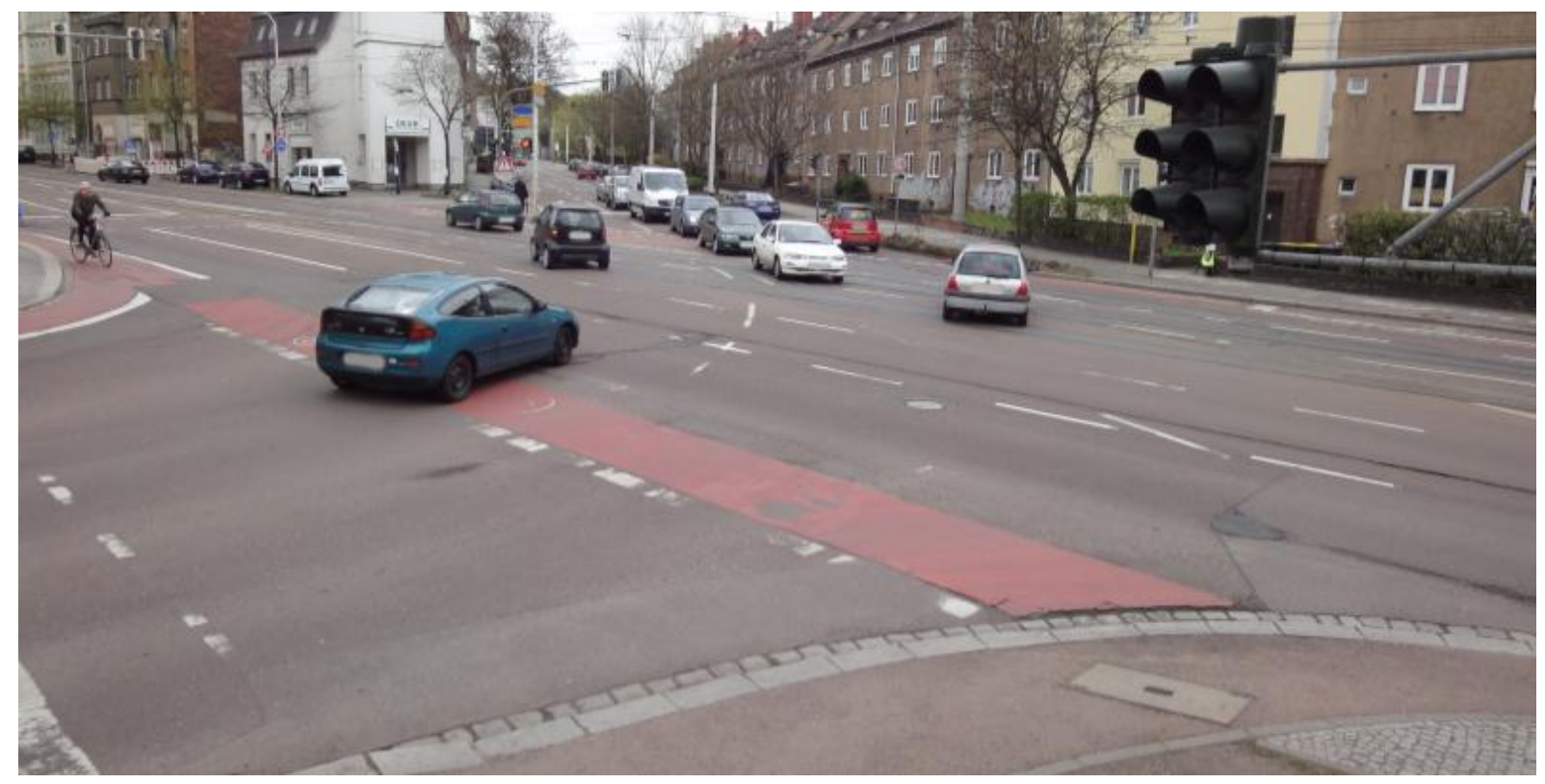

Abbildung 64: Kontenpunkt 3 - Blick auf Kreuzungsbereich aus südlicher Richtung

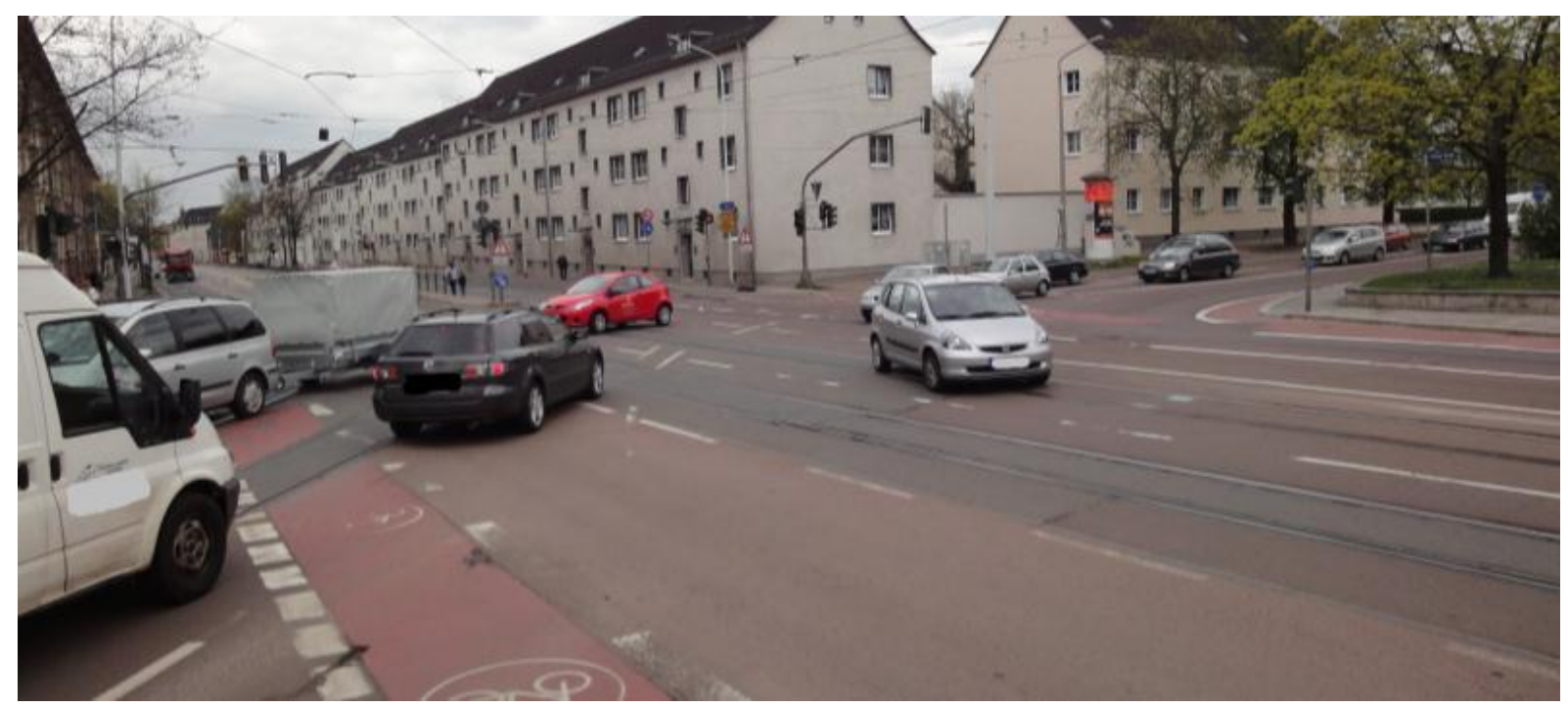

Abbildung 65: Kontenpunkt 3 - Blick auf Kreuzungsbereich aus nördlicher Richtung

Die Schwierigkeit für Linkseinbieger aus Arm 1 wird zudem erhöht, da die untergeordnete Straße in einem nicht rechten Winkel an die Hauptstraße (Achse 2-4) anschließt. Die Sichtbeziehungen zum entgegenkommenden Geradeausverkehr werden durch gleichzeitig einfahrende Linkseinbieger des Gegenverkehrs beeinträchtigt (Abbildung 66). 


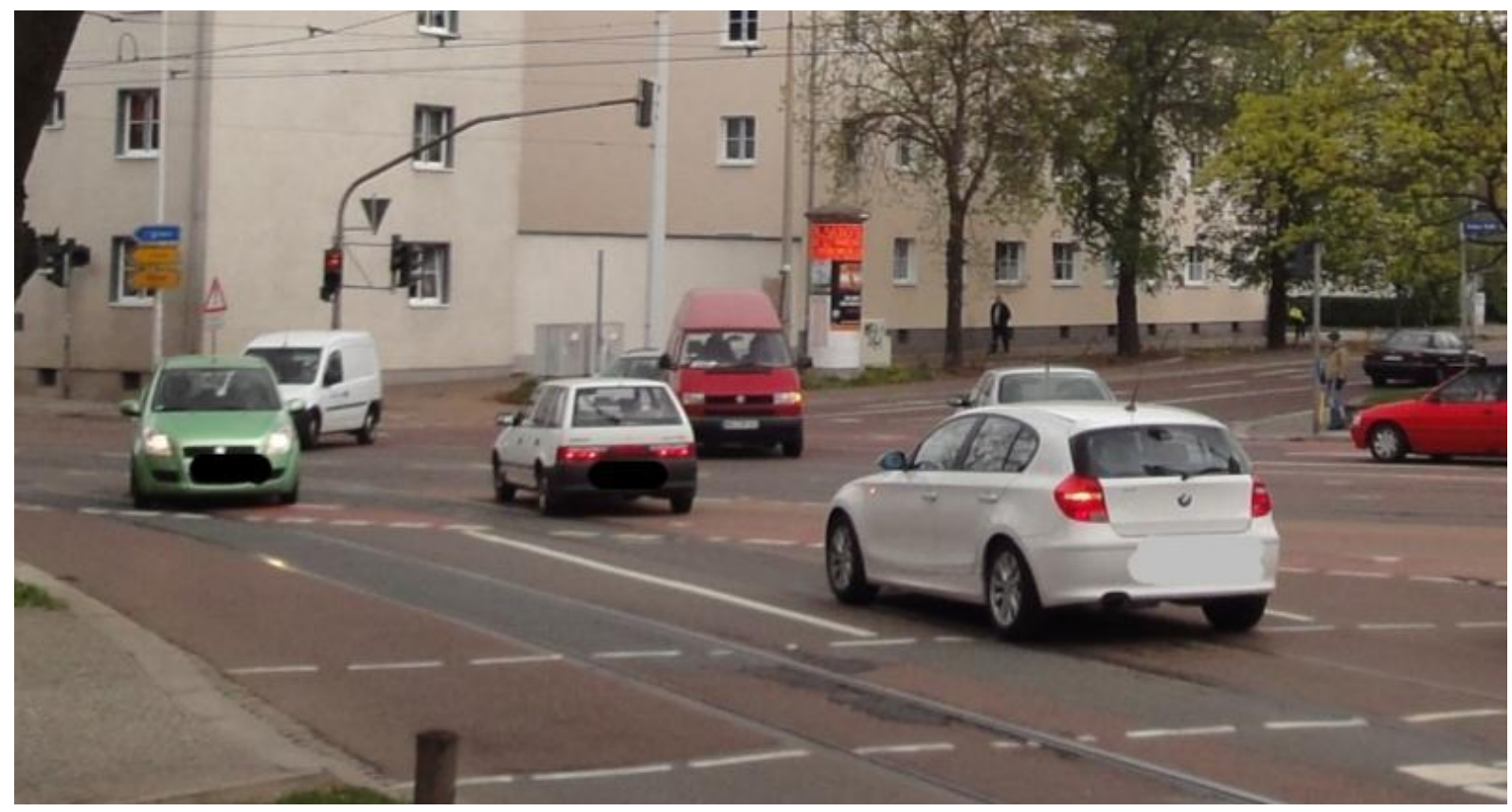

Abbildung 66: Knotenpunkt 3 - Sicht der Linkseinbieger aus Arm 1 auf Gegenverkehr

Im Weiteren konnten an diesem Knotenpunkt Rotlichtverstöße der Kraftfahrer aus Arm 1 und 3 beobachtet werden. Es bleibt zu vermuten, dass ortskundige Kraftfahrer längere Wartezeiten, die sich durch einfahrende Straßenbahnen ergeben können, vermeiden wollen. Durch Rotlichtverstöße von linkseinbiegenden Kraftfahrern aus Arm 1 wurden jedoch Konfliktsituationen mit Fußgängern im Bereich der nordöstlichen Furt an Arm 4, allerdings nur in wenigen Fällen, hervorgerufen.

Zum Teil konnten auch Konfliktsituationen an der Fußgängerfurt von Arm 3 im Zusammenhang mit dem an der Zufahrt 2 angebrachten Grünpfeil (StVO Zeichen 720) ${ }^{30}$ beobachtet werden. Dieses Verkehrszeichen erlaubt Kraftfahrern aus Arm 2 auch bei rotem Signal vorzufahren und rechts abzubiegen. Konfliktsituationen mit Fußgängern entstanden vornehmlich dann, wenn die rechtsabbiegenden Kraftfahrer gegen Ende der Rotzeit an die Haltlinie gelangten, ihr Abbiegemanöver begannen und während ihrer Fahrt in Richtung Furt das Signal von ROT auf GRÜN, auch für die an der Furt 3 wartenden Fußgänger, umschlug. Die Haltlinie der Zufahrt 2 ist mit rd. 24 Metern weit vom Beginn der Furt an Arm 3 abgesetzt. Fußgänger, die direkt bei GRÜN losgehen, rechnen unter Umständen nicht damit, dass unmittelbar Fahrzeuge von rechts kommen können. Kraftfahrer, die den Wechsel von ROT auf GRÜN nicht bemerken konnten, erwarten gegebenenfalls auch nicht, dass sie mit überquerenden Fußgängern rechnen müssen.

Eine gesicherte Führung der Linksabbieger aus Arm 1 und Arm 3 bzw. die getrennte Signalisierung dieser beiden Verkehrsströme, ist an diesem Knotenpunkt zu empfehlen. Zudem sollten die Markierungen insgesamt erneuert werden.

\footnotetext{
${ }^{30}$ Zusatzschild rechts neben dem roten Signal der LSA mit grünem Pfeil auf schwarzen Grund
} 


\section{Knotenpunkt 4}

Abbildung 67 zeigt das Unfalldiagramm für Unfälle der Kategorien 1 bis 4 der Jahre 2006 bis 2009, zu denen Informationen in Form von anonymisierten Verkehrsunfallanzeigen vorlagen. Zudem sind die Kamerapositionen mit den Aufnahmerichtungen und die Positionen der beiden Zählposten für die Verhaltensbeobachtungen in dieser Abbildung dargestellt. Die Verhaltensbeobachtungen wurden im Mai 2011 durchgeführt.

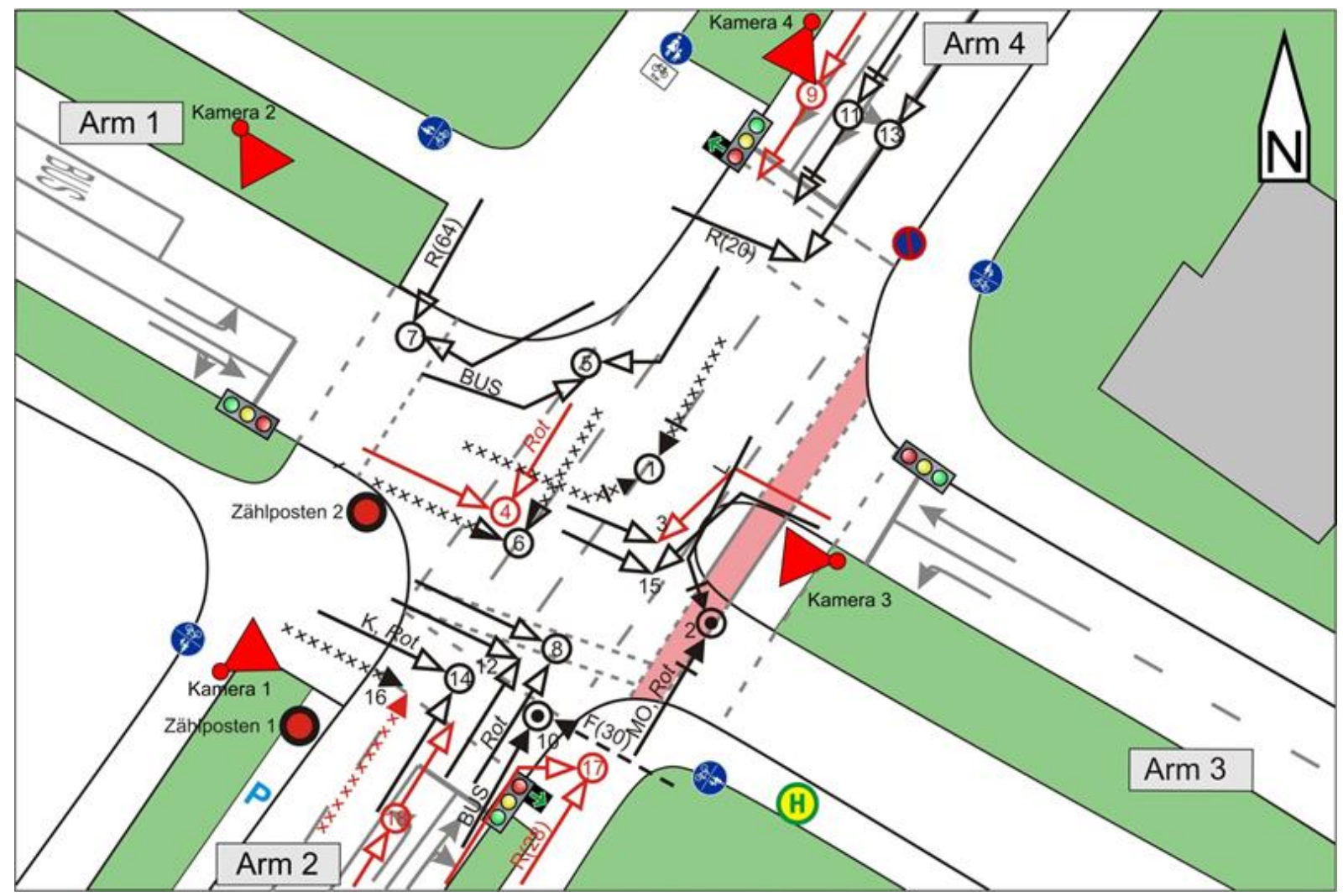

Abbildung 67: Knotenpunkt 4 - Unfalldiagramm, Kamerastandorte und Zählposten

Die bei der Unfallanalyse festgestellten Hauptkonflikte im Kreuzungsbereich und an der Furt des Knotenpunktarms 2 konnten im Rahmen der Verhaltensbeobachtungen nicht festgestellt werden.

Das am häufigsten beobachtete Fehlverhalten von Kraftfahren (älteren wie jüngeren gleichermaßen) wurde im Zusammenhang mit dem Verkehrszeichen 720 (Grünpfeil) beobachtet, da die rechtsabbiegenden Verkehrsteilnehmer häufig nicht vorschriftsmäßig bei rotem Signal anhielten, sondern langsam weiterfuhren.

Konflikte konnten diesbezüglich vor allem zwischen Rechtsabbiegern aus der Zufahrt 4 und Fußgängern und Radfahrern an der Furt des Arms 1 beobachtet werden. Hierzu kam es insbesondere, wenn Rechtsabbieger gegen Ende der Rotzeit die Haltlinie der Zufahrt 4 passierten und bis zu ihrem Erreichen der Furt 2 zwischenzeitlich das Signal für zufahrende KfZ der Zufahrt 4 und für Fußgänger der Furt 2 auf Grün wechselte.

Als Defizite sind in diesem Zusammenhang die mit rd. 20 Metern weit abgesetzte Haltinie der Zufahrt zu nennen. Im Weiteren wird der parallele Gehweg (Zusatzzeichen „Radfahrer frei“) von einer Baumreihe flankiert, die die Sicht für Rechtsabbieger aus der Zufahrt 4 auf diesen Bereich einschränkt (Abbildung 68). 


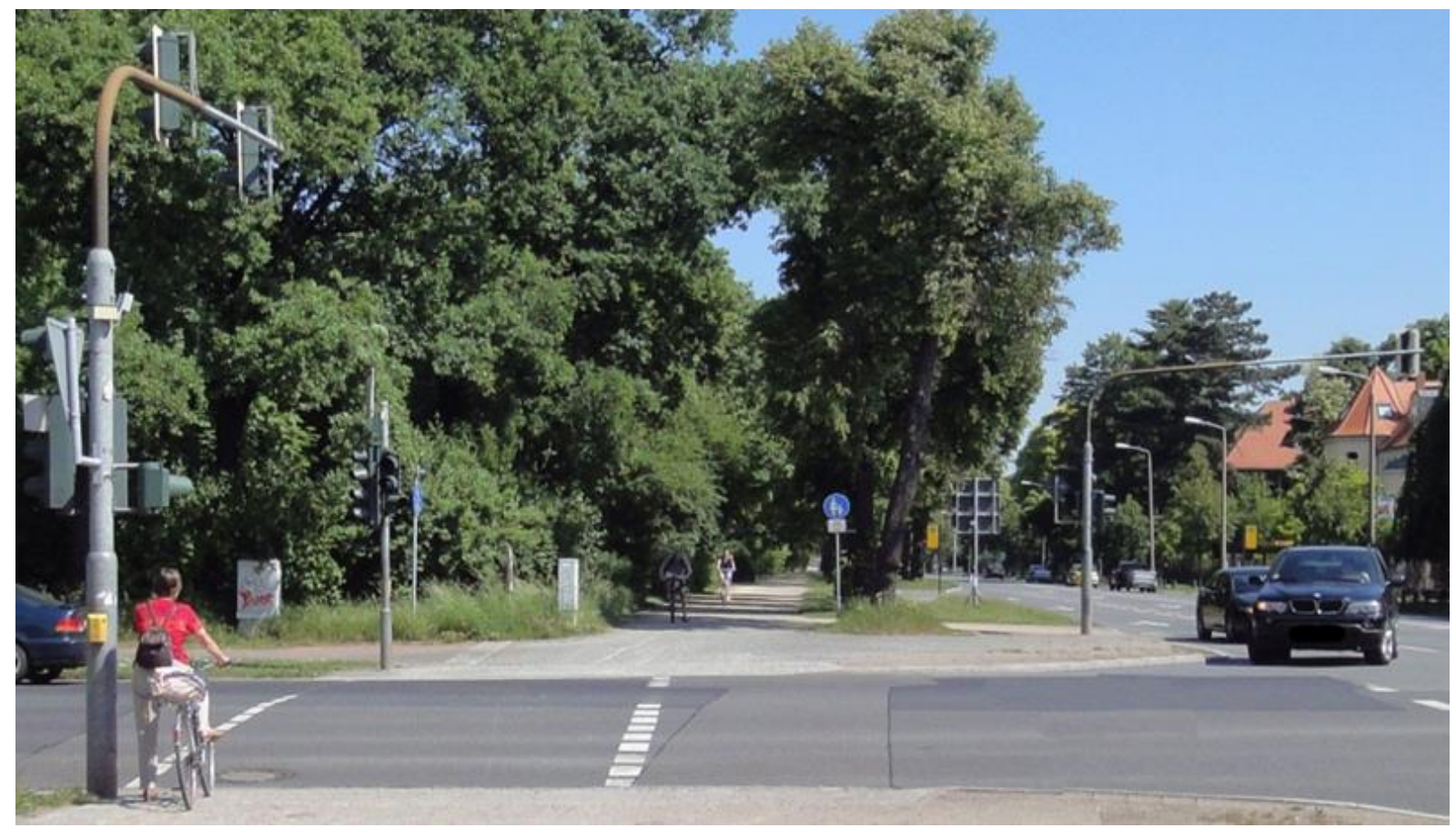

Abbildung 68: Knotenpunkt 4 - Blick auf Furt 1 in Richtung Zufahrt 4

Zwar lässt sich für diese Kreuzung keine „Häufung von Unfällen, bei denen der Grünpfeil ein unfallbegünstigender Faktor war" feststellen (nur Unfall 7 steht in diesem Zusammenhang), dennoch ist der Grünpfeil hier vor dem Hintergrund der Regelungen der VwV-StVo zu überdenken:

„Es (Z 720) darf nicht verwendet werden, wenn der freigegebene Fahrradverkehr auf dem zu kreuzenden Radweg für beide Richtungen zugelassen ist oder der Fahrradverkehr trotz Verbotes in der Gegenrichtung in erheblichem Umfang stattfindet und durch geeignete Maßnahmen nicht ausreichend eingeschränkt werden kann." (Allgemeine Verwaltungsvorschrift zur Straßenverkehrsordnung, zu §37 StVO, Abs. XI Grünpfeil),

Dies trifft für den betrachteten Knotenpunkt bedingt zu. Radfahrer nutzen vornehmlich die Fußgängerfurten zur Überquerung der Straße und fuhren zum Teil auch „entgegen der Fahrtrichtung" auf den gemeinsamen Fuß- und Radwegen (StVO-Zeichen 240). Konflikte zwischen Radfahrern und Fußgängern konnten bei Gehwegbreiten zwischen 3 und 4 Metern allerdings nicht beobachtet werden.

Weitere Konflikte, die sich aus der nicht gesicherten Führung der Linksabbieger der Hauptachse (Arm 2-4) ergeben könnten, wurden nicht beobachtet. In wenigen Fällen konnten Konflikte beobachtet werden, die aus der gemeinsamen Führung von Geradeausfahrern und Linksabbiegern auf einem Fahrstreifen aus der Zufahrt 4 resultieren. Nachfolgende Kraftfahrer wichen zum Teil unvermittelt in die Bereiche des parallel geführten Geradeausverkehrs des rechten Fahrstreifens aus, um wartepflichtige Linksabbieger zu passieren. 


\section{Knotenpunkt 5}

Abbildung 69 zeigt die Kamerapositionen einschließlich der Aufnahmerichtungen für die Verhaltensbeobachtungen, die im März 2011 durchgeführt wurden. Zudem ist das Unfalldiagramm für Unfälle der Kategorien 1 bis 4 der Jahre 2008 bis 2010, zu denen Informationen in Form von anonymisierten Verkehrsunfallanzeigen vorlagen, dargestellt. Der Knotenpunkt war zudem in den Jahren 2003, 2005 und 2006 als Unfallhäufungsstelle gemeldet. Hier ereigneten sich vornehmlich Einbiegen/Kreuzen-Unfälle unter Missachtung des Rotsignals von Kraftfahrern aus Südost (vgl. Abbildung 69 Stelle [1]), ÜberschreitenUnfälle an der Furt an Arm 3 (vgl. Abbildung 69 Stelle [2]), oft auch unter Missachtung des Rotsignals durch die Fußgänger und Unfälle im Bereich des freien Rechtsabbiegestreifens aus der Zufahrt 4 (vgl. Abbildung 69 Stelle [3]), auch mit Beteiligung überquerender Radfahrer.

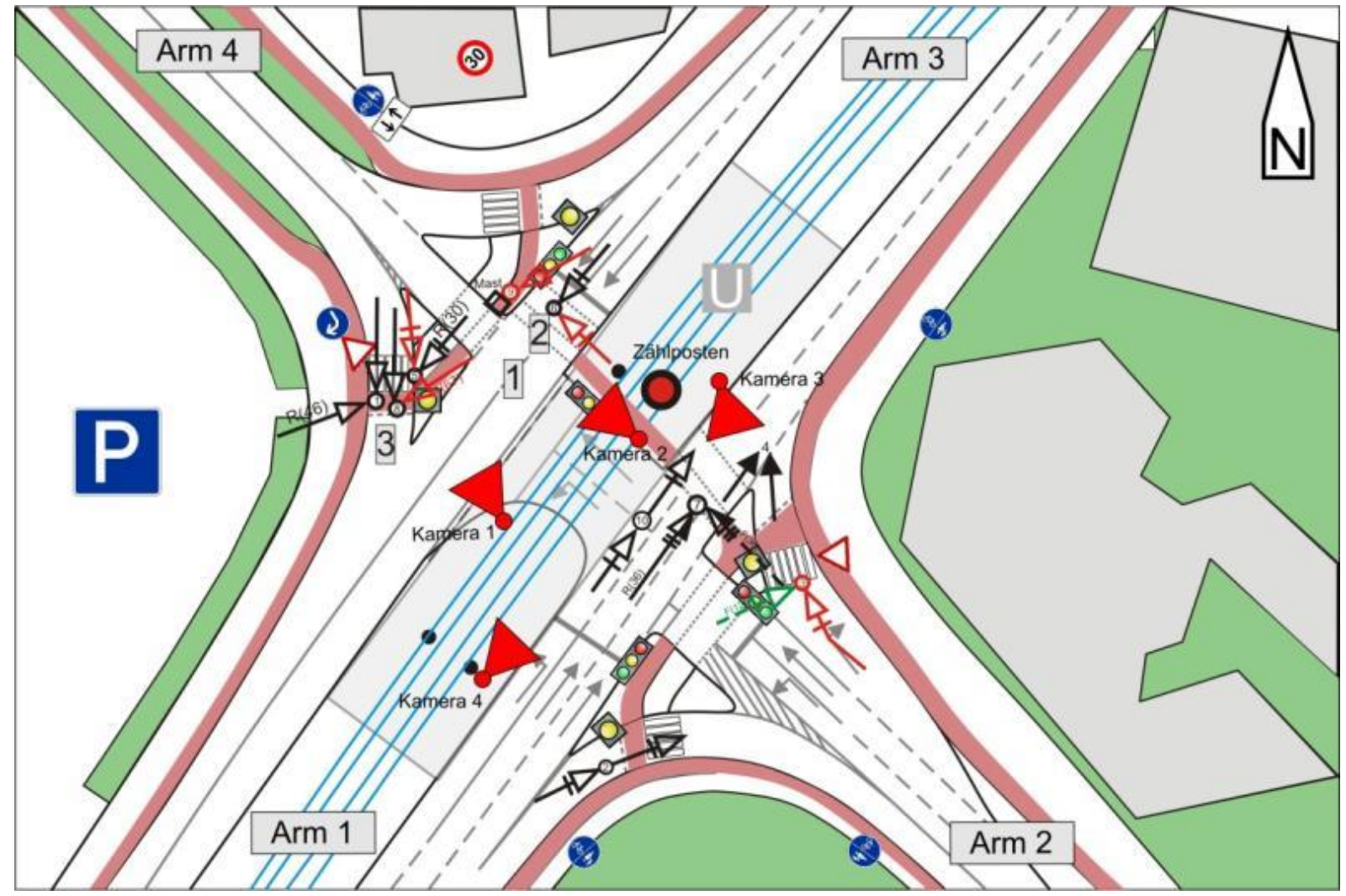

Abbildung 69: Knotenpunkt 5 - Unfalldiagramm, Kamerastandorte und Zählposten

Insgesamt konnten an diesem Knotenpunkt nur wenige Konfliktsituationen beobachtet werden. Diese entsprachen allerdings dem Unfallbild und können in den Zusammenhang zu einem hohen Fuß- und Radverkehrsaufkommen gesehen werden. Zwischen den beiden baulich getrennten Richtungsfahrbahnen der Achse 2-3 befinden sich Gleisanlagen für eine Stadtbahnlinie. Der Zugang zu einer Haltestelle (Brückenlage) befindet sich in der Mitte des Kreuzungsbereiches auf der Höhe des eingezeichneten Zählpostens. Zudem befinden sich Schulen (Gymnasium und Berufskolleg) in nordwestlicher Lage (Arm 4) des Knotenpunktes. Die Hauptwege-Beziehung ist in der Abbildung 70 dargestellt. 


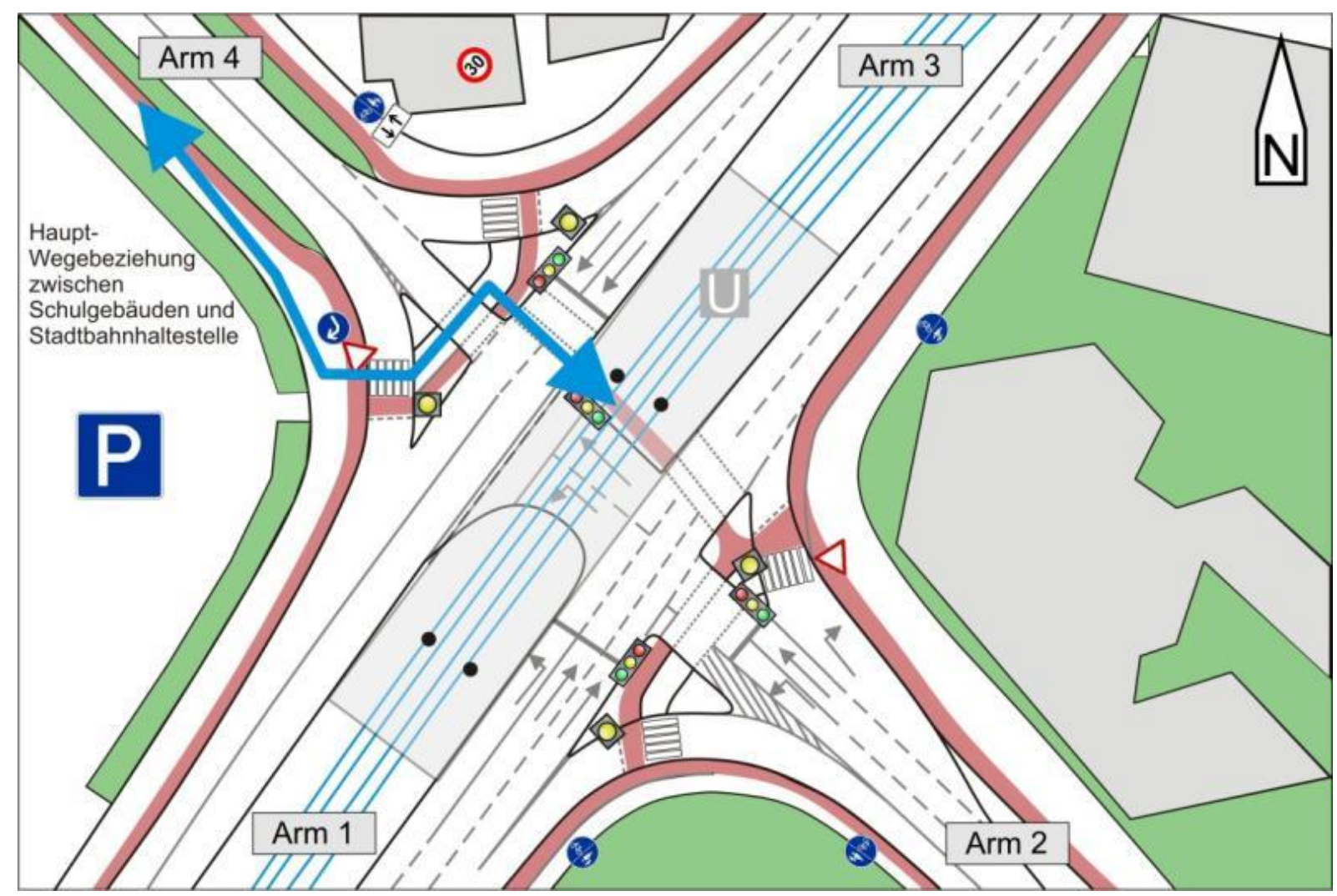

Abbildung 70: Kontenpunkt 5 - Hauptwegebeziehungen zwischen Schulgebäuden und Stadtbahn-Haltestelle

Auf den Dreiecksinseln überlagen sich Rad- und Fußwegebeziehungen (Abbildung 71). Insbesondere bei hohem Fußgängeraufkommen, z. B. nach Schulschluss, kommt es zu unübersichtlichen Situationen, in denen sich Radfahrer und Fußgänger vermischen (Abbildung 72). Gemessen an den pulkweise zu Schulschluss auftretenden Fußgängern, bieten die Aufstellflächen zudem zu wenig Platz. Teilweise konnten sich nicht alle Schüler auf den Dreiecksinseln aufstellen und standen auf der Fahrbahn neben der Dreiecksinsel.
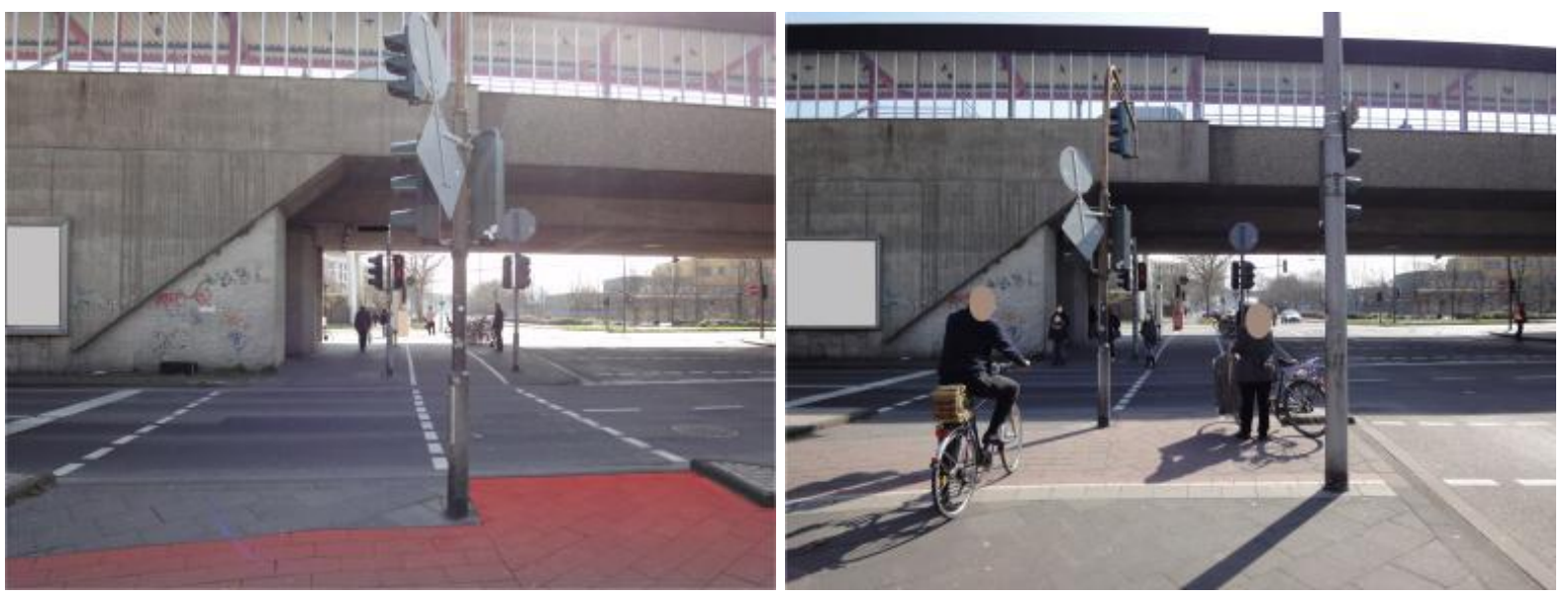

Abbildung 71: Knotenpunkt 5 - Aufstellflächen auf Dreiecksinsel am „Freien Rechtsabbieger“ aus Arm 3 in Arm 4 in Blickrichtung Südost (im Bild links ist der rot eingefärbte Bereich für den Radverkehr hervorgehoben) 


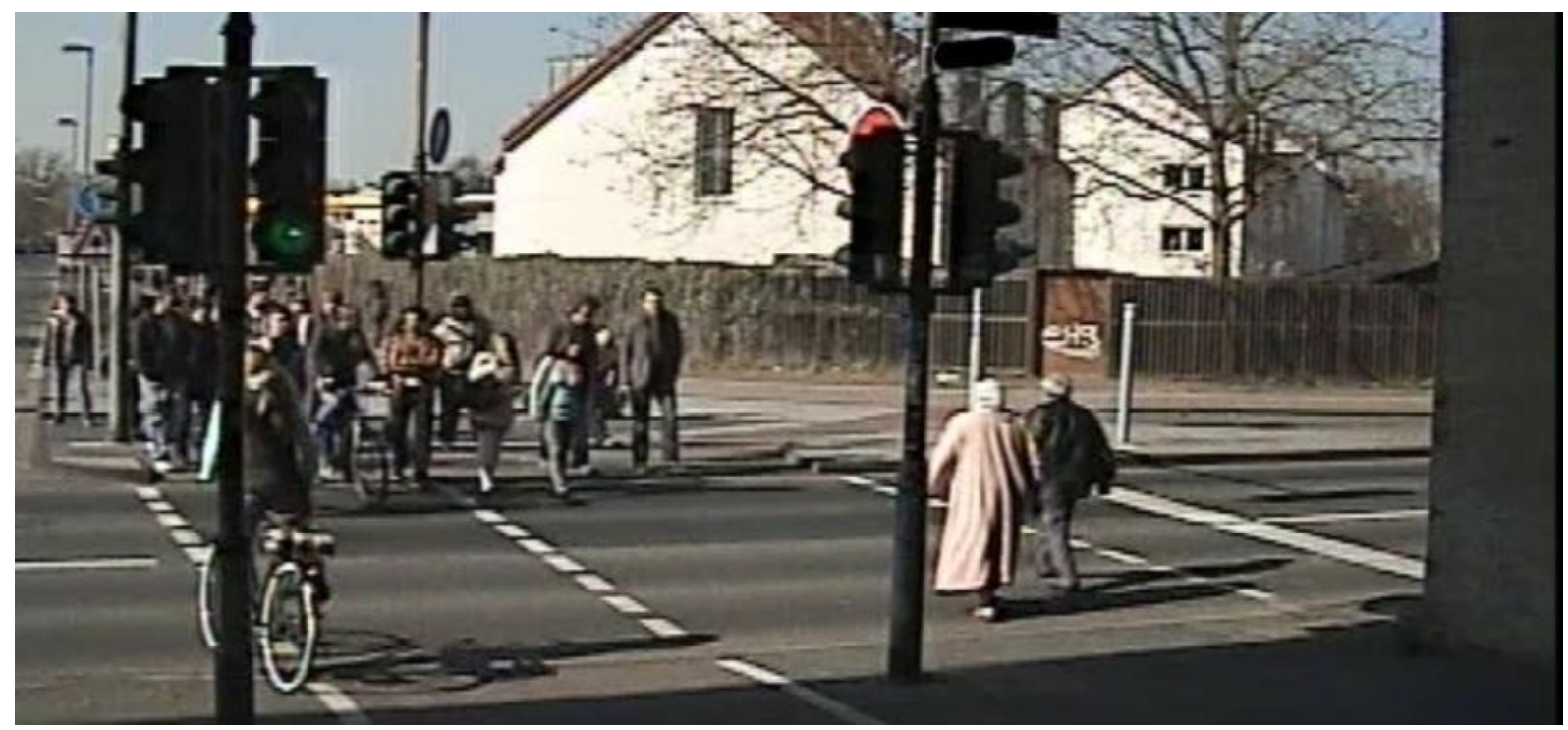

Abbildung 72: Knotenpunkt 5 - Fußgänger und Radfahrer überqueren Furt an Arm 3

Verglichen mit anderen Beobachtungsstandorten konnten an diesem Knotenpunkt viele Rotlichtverstöße von Kindern (Schülern) als Fußgänger beobachtet werden (Abbildung 74). Bei den Überquerungen der Kinder als Fußgänger wurden 194 Verstöße beobachtet (annähernd 17\% aller Querungen), davon über die Hälfte (117) Rotlichtverstöße.

Über die Gründe kann nur spekuliert werden. Zum einen sind die Aufstellflächen teilweise zu klein, zum anderen kann auch das „Peer-Group“-Verhalten eine Rolle spielen, weil es „cool“ sein könnte, sich nicht an Regeln zu halten. Andererseits ist die Straße an dieser Stelle nach links auch sehr weit einsehbar und der Kfz-Verkehr kommt nur aus einer Richtung (vgl. Abbildung 73, aus Sicht des zufahrenden Kfz-Verkehrs). Verschiedentlich wurde beobachtet, wie die Kinder rennen, um noch eine U-Bahn zu erreichen und dabei das Rotsignal missachtet haben (Abbildung 74).

Rotlichtverstöße konnten auch bei allen anderen Fußgängern und Radfahrern an dieser Furt beobachtet werden. Es kann nur spekuliert werden, dass die weit einsehbare Straße und das pünktliche Erreichen der U-Bahn dieses Verhalten begünstigen. 


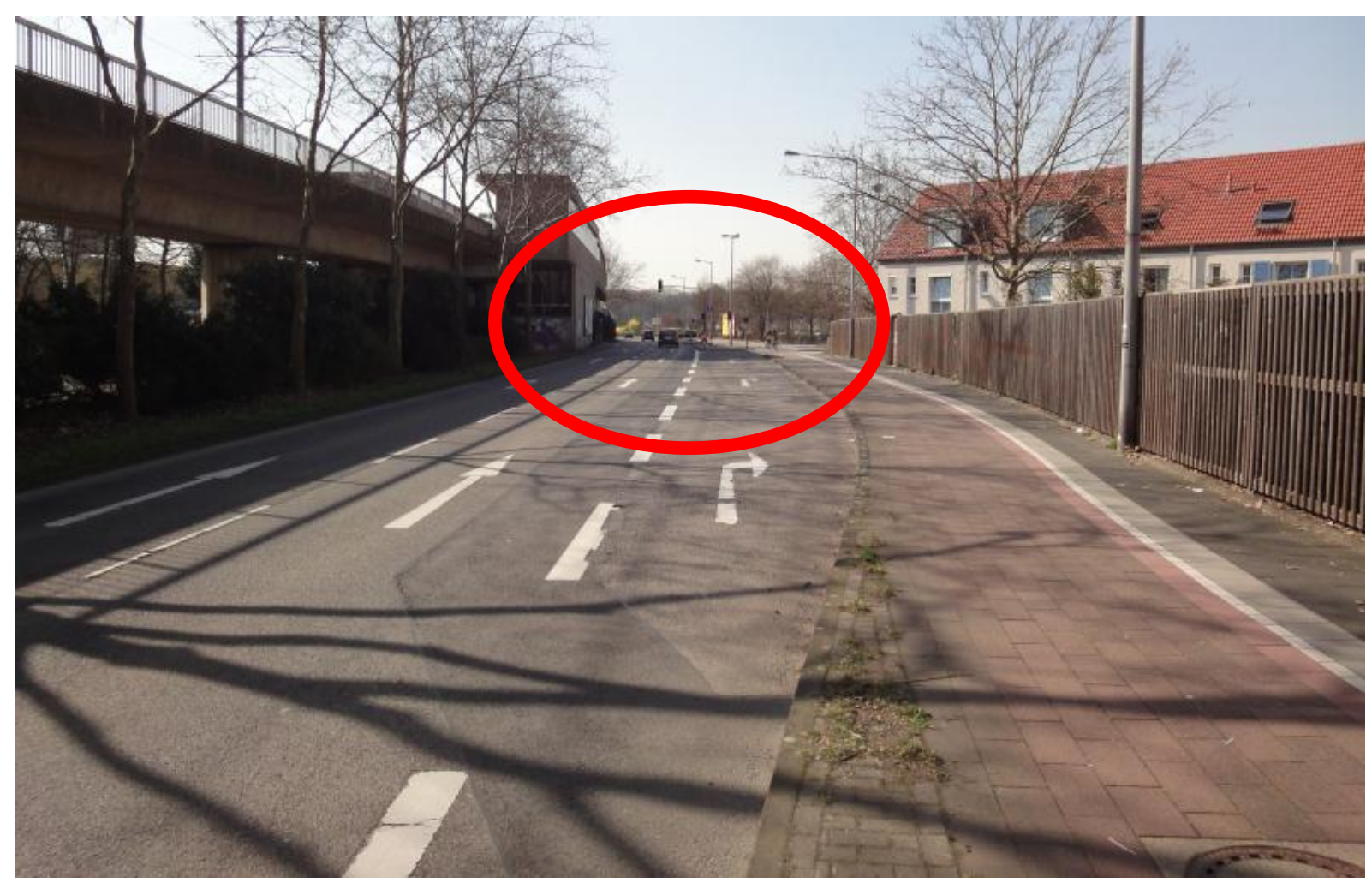

Abbildung 73: Knotenpunkt 5 - Zufahrt aus Arm 3 in Blickrichtung der Furt

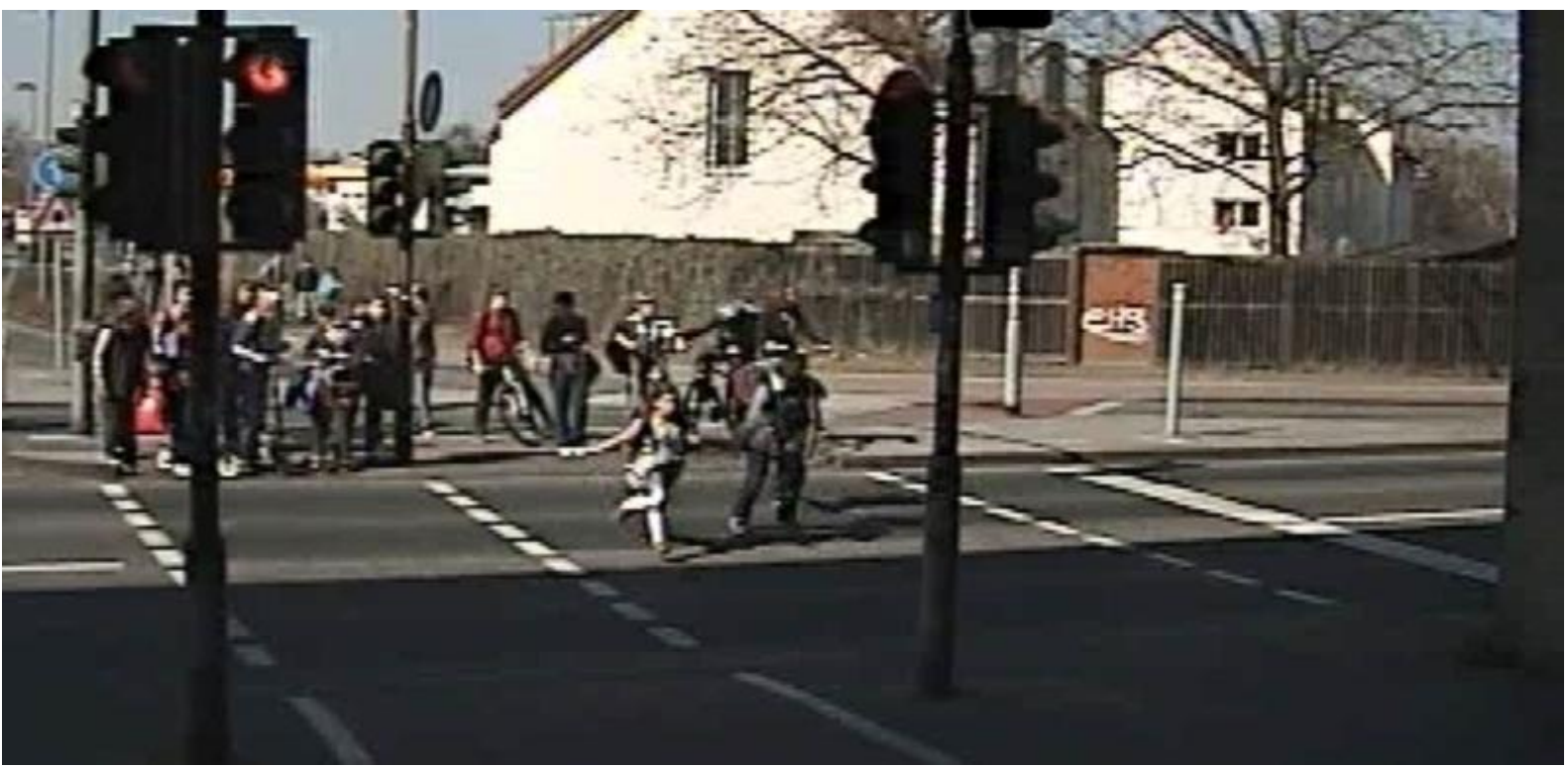

Abbildung 74: Knotenpunkt 5 - Kinder laufen bei „Rot“ über die Furt an Arm 2 in Richtung der Stadtbahn-Haltestelle

An den freien Rechtsabbiegestreifen konnten nur wenige Konfliktsituationen beobachtet werden. Allerdings lässt das Verhalten der Verkehrsteilnehmer darauf schließen, dass sich diese im Hinblick auf die Vorfahrtregelung unsicher sind (Abbildung 75). Unfälle mit von rechts kommenden Radfahrern könnten zudem durch die Einschränkung der Sichtbeziehungen aufgrund der Begrünung zum Parkplatz hin begünstigt werden (Abbildung 76). 


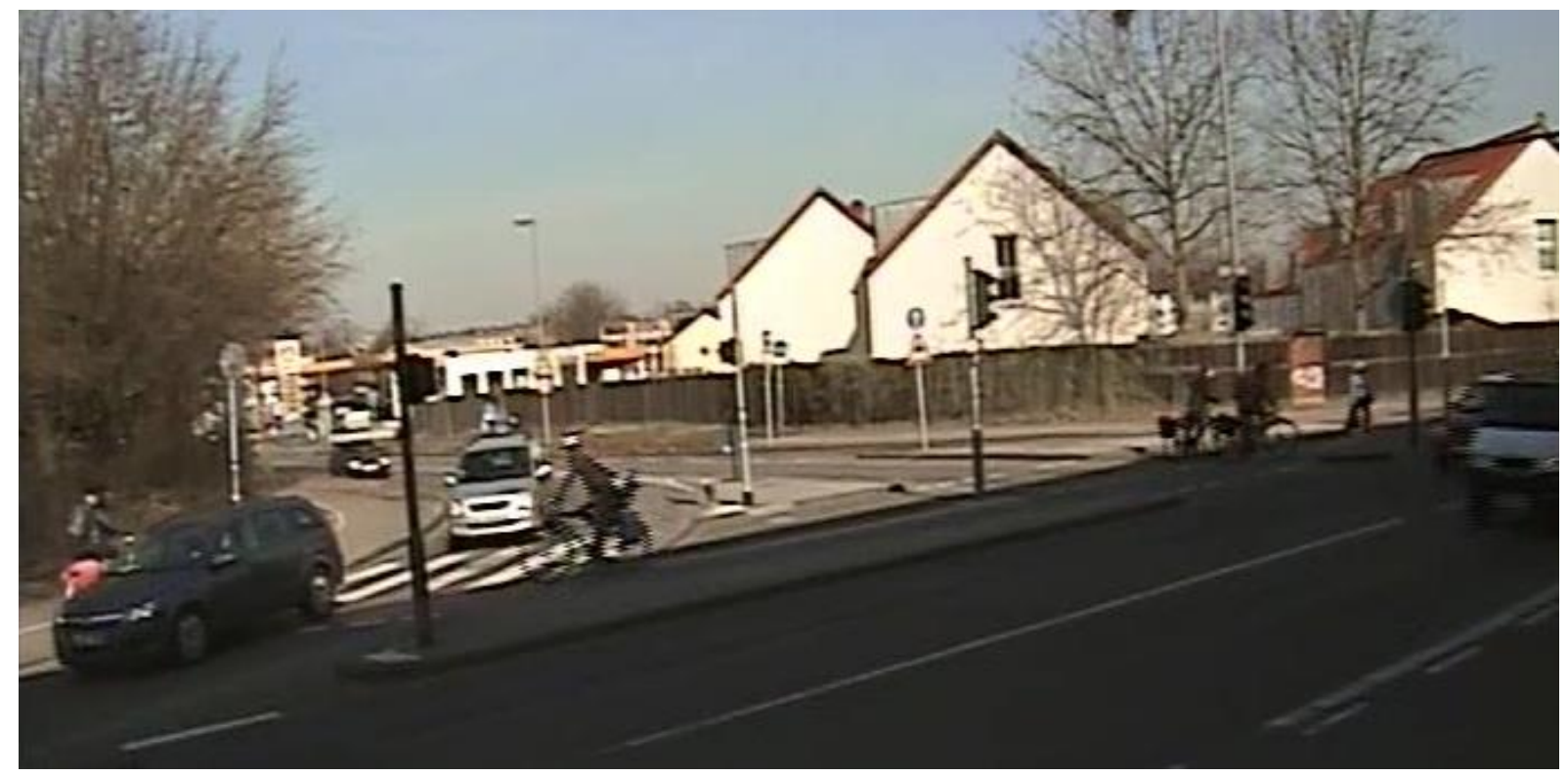

Abbildung 75: Knotenpunkt 5 - Situation am freien Rechtsabbiegestreifen (Radfahrer von links wartet, Radfahrer von rechts fährt)

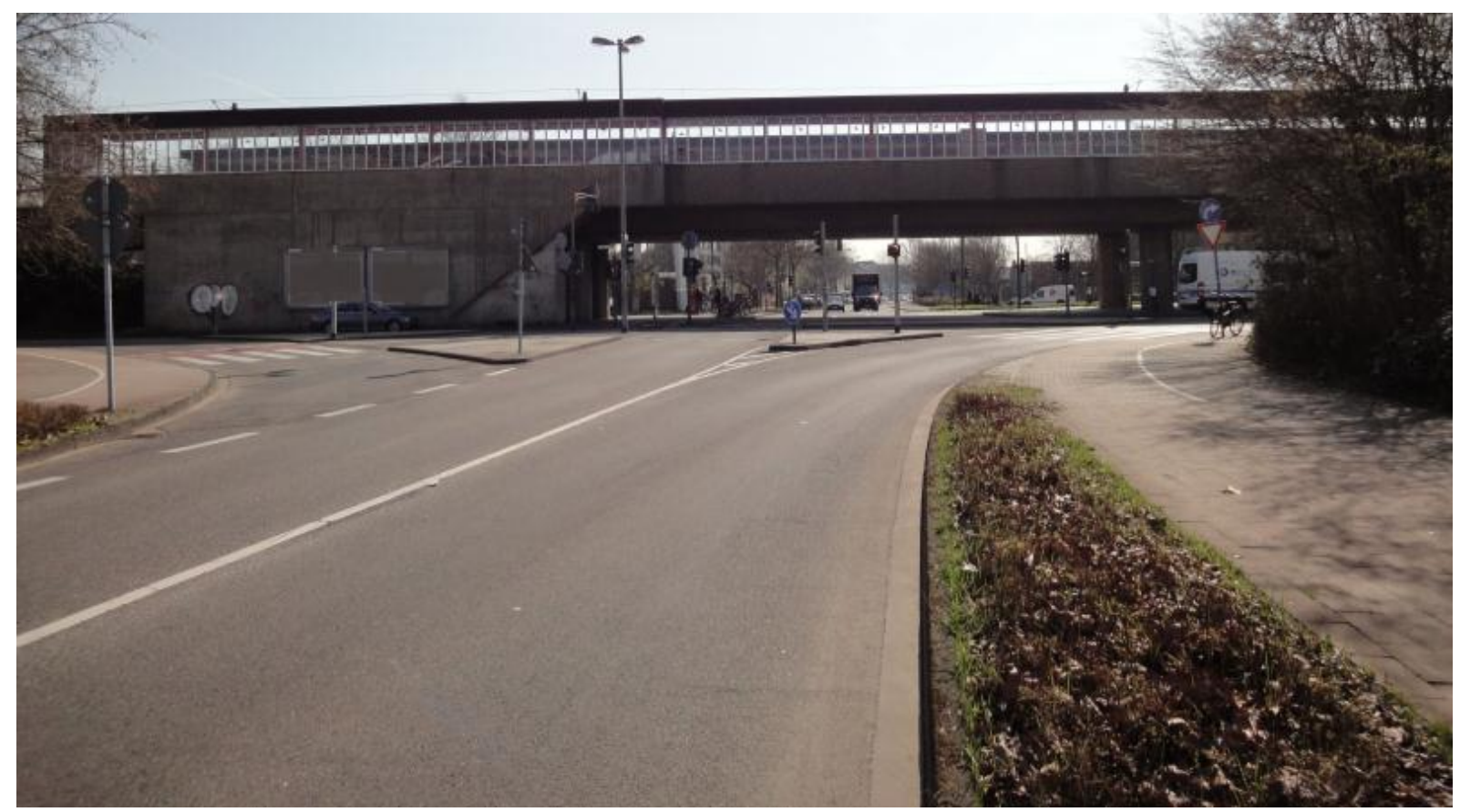

Abbildung 76: Knotenpunkt 5 - Sicht der Kfz-Führer aus Arm 2 auf Dreiecksinsel und freien Rechtsabbiegestreifen

Überwiegend verhielten sich die Kfz-Führer an den freien Rechtsabbiegestreifen korrekt. Selbst in unübersichtlichen Situationen zu Schulschluss mit vielen Fußgängern und Radfahrern. Bei der Auswertung der Videoaufnahmen entstand der Eindruck, dass viele Pkw-Fahrer mit diesen Situationen vertraut waren.

Gemessen an der großen Zahl von Überquerungen von Kindern gab es kaum verkehrsgefährdende Konflikte.

Die Radverkehrsanlagen wurden gut angenommen und meist vorschriftsmäßig genutzt. 


\section{A 3 Sicherheitsanalysen und Verhaltensbeobachtungen im Realverkehr an Kreuzungen mit Lichtsignalanlage und verkehrstechnischer und/oder baulicher Optimierung}

\section{Knotenpunkt 6}

Die Verhaltensbeobachtungen an diesem Knotenpunkt fanden im Mai 2011 statt. Die vorgenommen Änderungen erfolgten im Jahr 2010, vornehmlich zur besseren Führung des Radverkehrs (Abbildung 77).
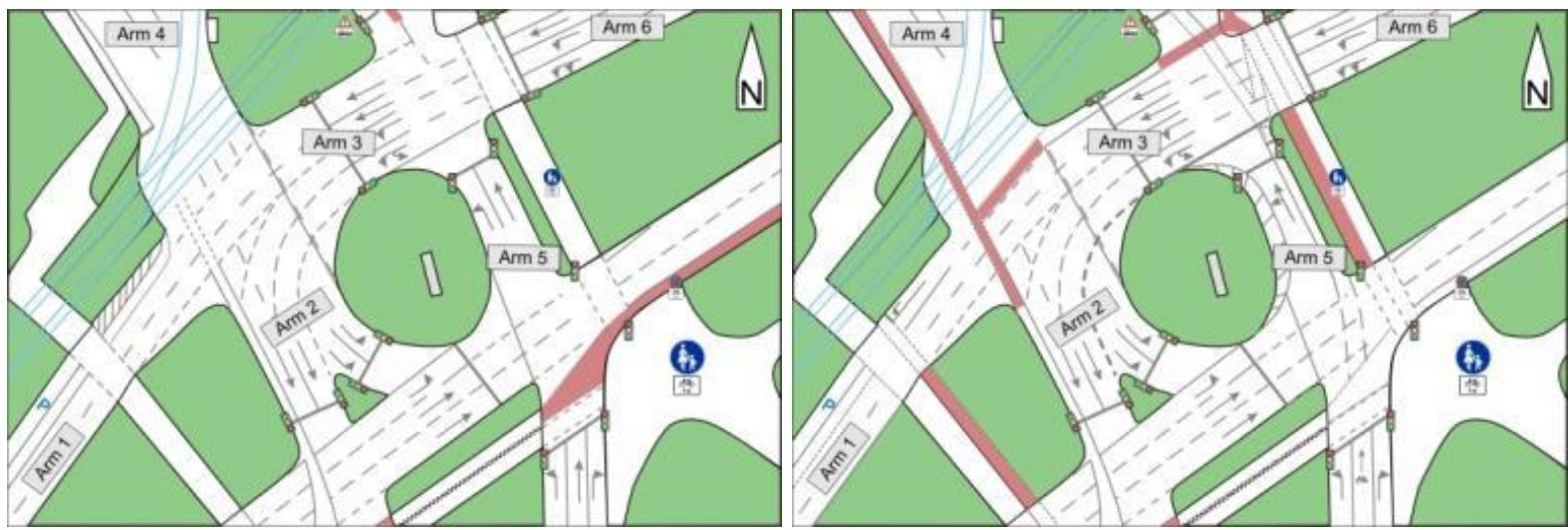

Abbildung 77: Knotenpunkt 6 - Situation vor Änderung (Bild links) und nach Änderung (Bild rechts)

Das Unfalldiagramm auf Grundlage der Unfälle aus 2006 bis 2010 zeigt Abbildung 78. Dargestellt sind hier nur Unfälle, die sich auf den Unfallschwerpunkt des nordwestlichen Teilknotenpunkts beziehen. Diesem Bereich galt auch das vorwiegende Interesse der Verhaltensbeobachtungen. 


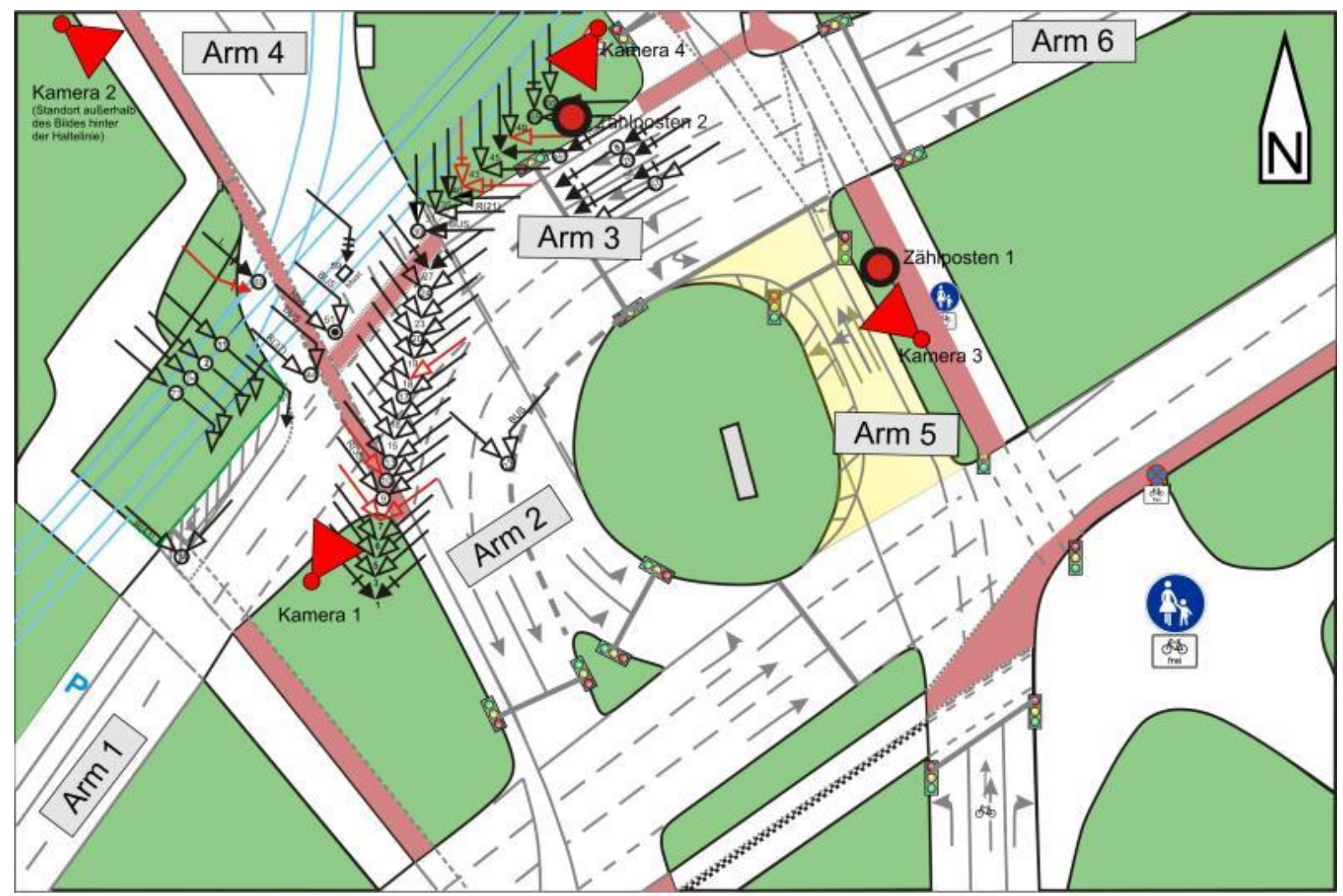

Abbildung 78: Knotenpunkt 6 - Unfalldiagramm, Kamerastandorte und Zählposten (Bereiche mit Änderungen sind gelb hinterlegt. Die Unfälle 65, 66, 67, 69, 73 fanden nach bzw. im Jahr der Umsetzung der Maßnahmen statt. Nummerisch nicht aufgeführte Unfälle beziehen sich auf andere Bereiche des Knotenpunktes)

Insgesamt 37 Unfälle fanden an diesem Teilknotenpunkt zwischen 2006 und 2009 statt, also vor dem Jahr der Umsetzung der Maßnahmen. In 2010 fanden hier 5 Unfälle statt (Abbildung 79).
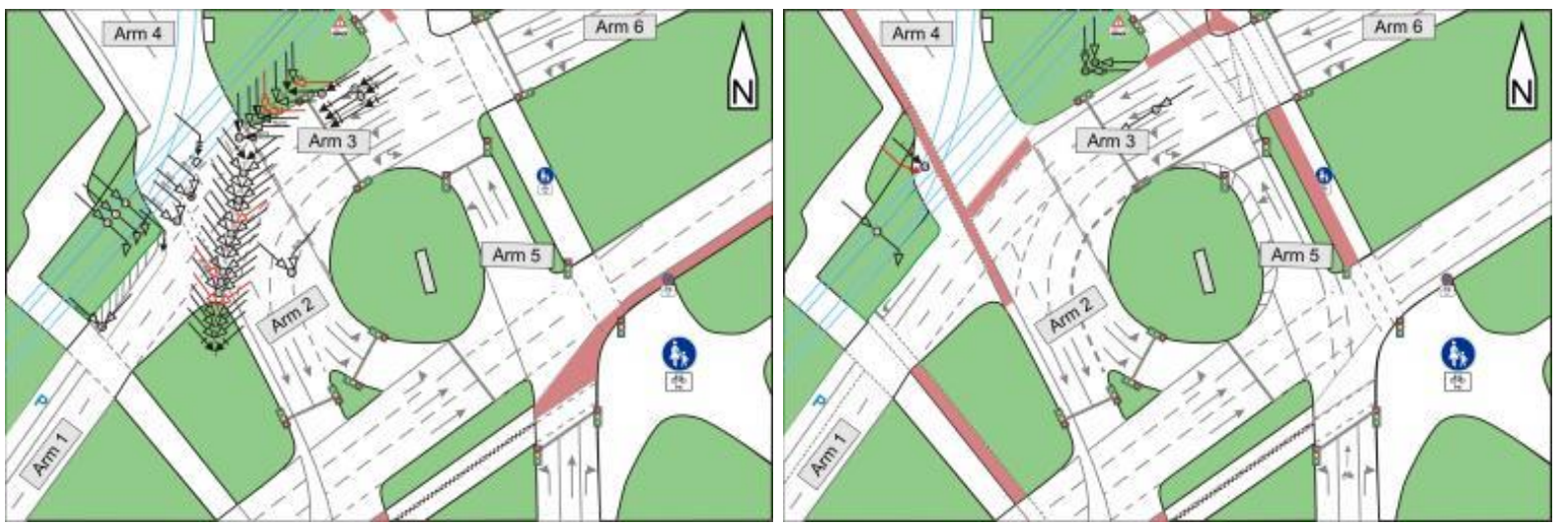

Abbildung 79: Knotenpunkt 6 - Unfälle vor verkehrstechnischer Änderung (Bild links) und nach oder im Jahr der verkehrstechnischen Änderung (Bild rechts)

Bei den Einbiegen/Kreuzen-Unfällen am nordwestlichen Teilknotenpunkt handelte es sich vorwiegend um Umfälle, bei denen die Verkehrsteilnehmer das rote Signal an der Haltlinie des Arms 3 missachteten und in den Kreuzungsbereich einfuhren, wo sie mit Verkehrsteilnehmern aus der Zufahrt 4 kollidierten. 
Rotlichtverstöße konnten zwar beobachtet werden, führten allerdings zu keinen Konfliktsituationen. Die Zwischenzeit dieser beiden Ströme betrug 11 Sekunden (3 räumt / 4 fährt ein), die Haltlinie der Zufahrt 4 (im Plan nicht eingezeichnet) liegt etwa 45 Meter von der Mittelachse des rechten Fahrstreifens der Zufahrt 3 entfernt.

Die genauen Ursachen, die zu den Rotlichtverstößen an der Zufahrt 3 führen, sind nicht bekannt. Das Signalprogramm während der Verhaltensbeobachtungen beinhaltete allerdings einen begünstigten Faktor. "Linksabbieger" aus Süden passieren an diesem Knotenpunkt drei Lichtsignalanlagen. Für sie ergibt sich die "Signalfolge“ zu GRÜN an der ersten LSA, GRÜN an der zweiten LSA und ROT an der dritten LSA (Abbildung 80). Dies kann vermutlich dazu führen, dass das rote Signal an der Haltlinie 3 nicht beachtet wird.

Rotlichtverstöße aus der Zufahrt 6 konnten nicht festgestellt werden. Zudem ist zur Ahndung von Rotlichtverstößen der Zufahrt 6 ist eine stationäre Rotlichtüberwachung auf Höhe der Haltlinie der Zufahrt 3 aufgestellt.

Neben den Rotlichtverstößen an der Zufahrt 3 konnten zudem noch Rotlichtverstöße an der Zufahrt 5 beobachtet werden (vornehmlich Geradeausfahrer).

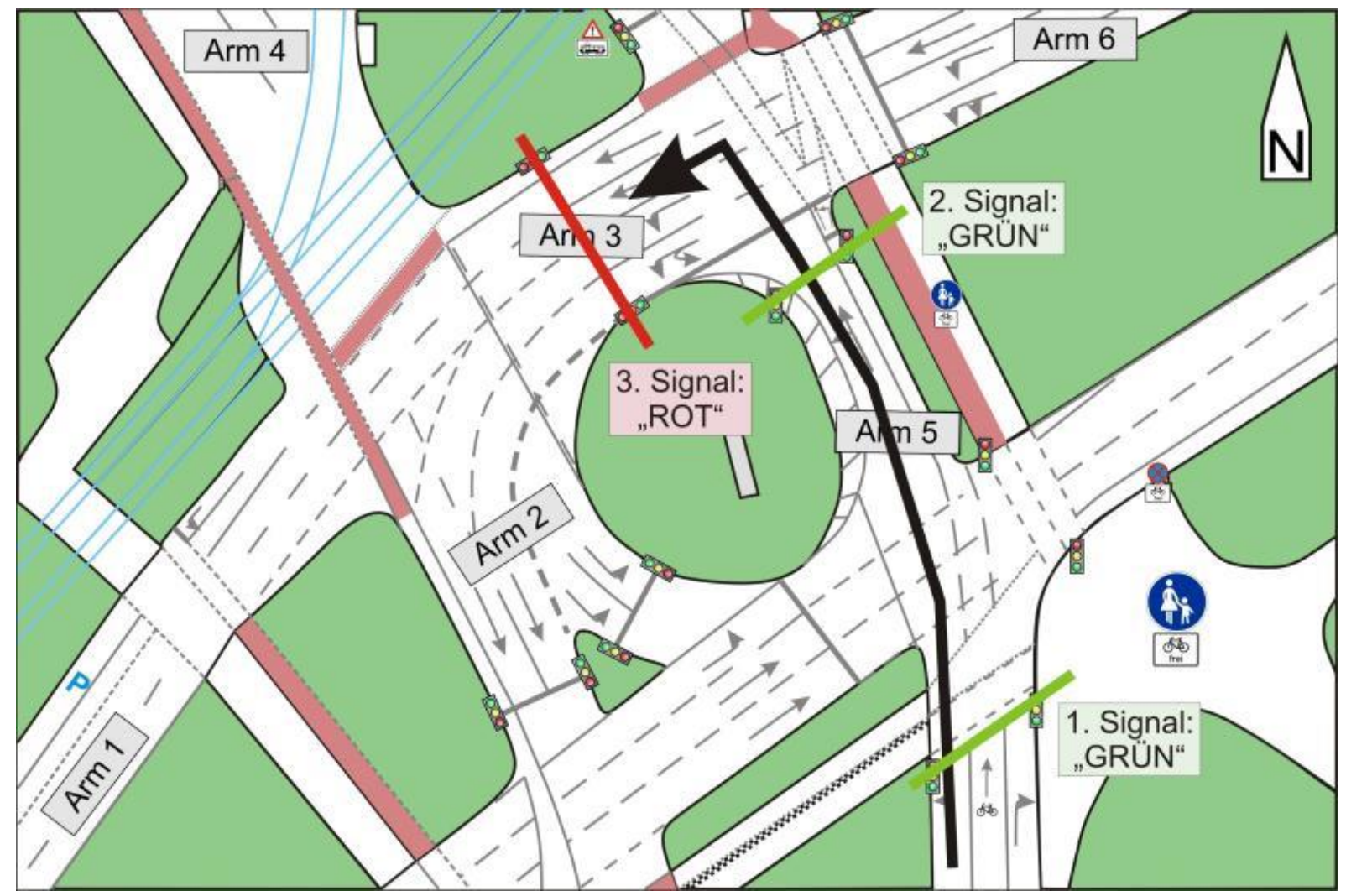

Abbildung 80: Knotenpunkt 6 - Signalfolge für Linksabbieger von Süden

Im Hinblick auf den Radverkehr lässt sich festhalten, dass die Radverkehrsanlagen gut angenommen werden und den Radverkehr sicher über diesen großen Knotenpunkt führen. Auffällig war, dass Radfahrer, die eigens für sie installierten Signalgeber für den Radverkehr, häufig nicht beachtet haben bzw. häufig bei ROT, insbesondere entlang des nördlichen Radweges (parallel zu den Armen 6-3) fuhren, wenn keine Kfz aus Arm 5 kamen. 


\section{Knotenpunkt 7}

Der Knotenpunkt 7 wurde im Wesentlichen wegen Überschreiten-Unfällen mit Beteiligung von Kindern im Rahmen der Bestimmung der 291 Knotenpunkte ausgewählt. Die Abbildung 81 zeigt das Unfalldiagramm auf Basis von Unfällen der Kategorie 1 bis 4 der Jahre 2007 bis 2010. Die Verhaltensbeobachtungen wurden im März 2011 durchgeführt. Östlich des Knotenpunktes befindet sich eine Grundschule (Arm 1) und westlich liegt eine Straßenbahnhaltestelle (Arm 4).

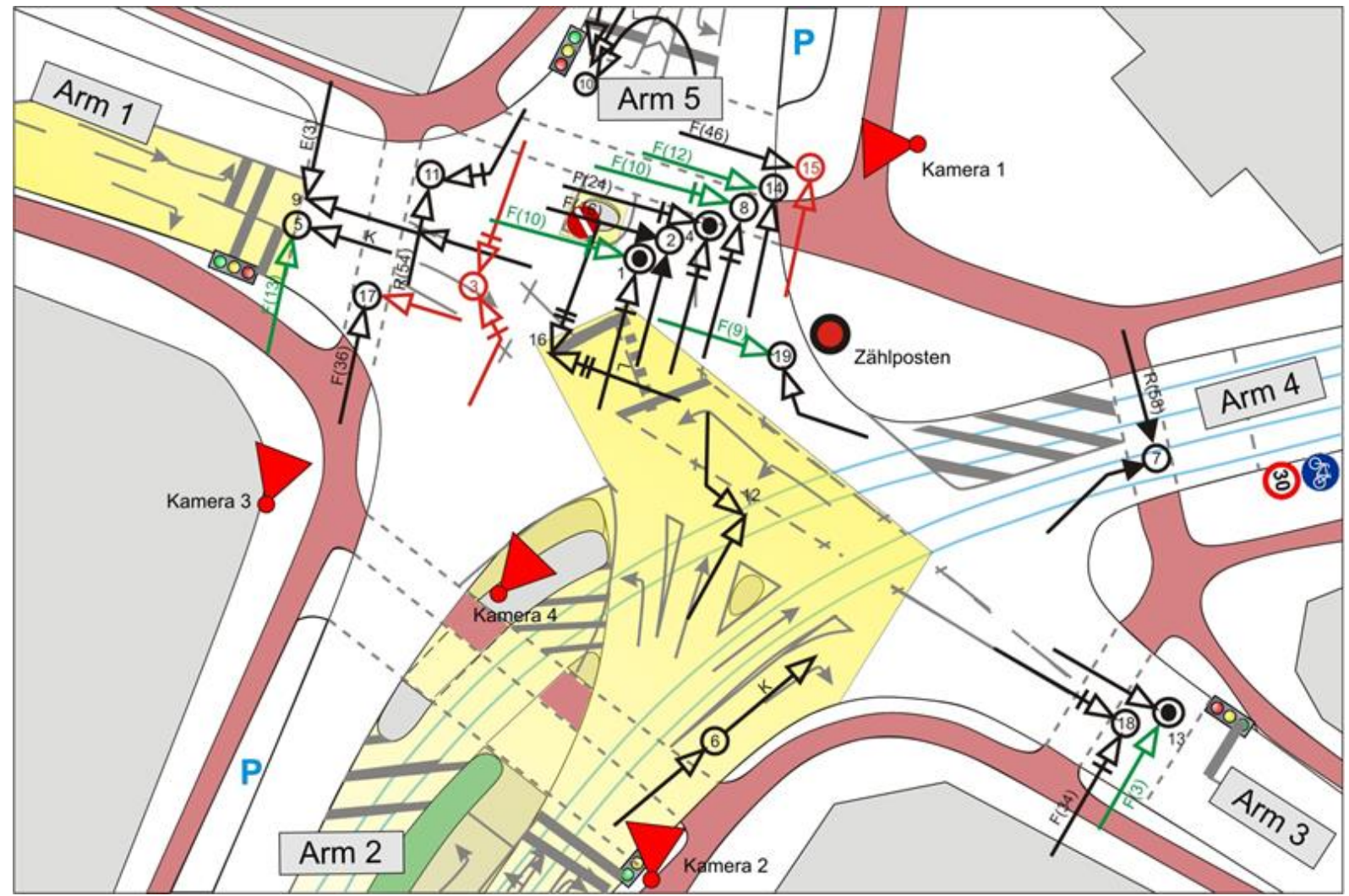

Abbildung 81: Knotenpunkt 7 - Unfalldiagramm, Kamerastandorte und Zählposten (Bereiche mit Änderungen sind gelb hinterlegt. Die Unfälle 17 bis 19 fanden nach bzw. im Jahr der Umsetzung der Maßnahmen statt)

Der Unfallschwerpunkt mit Überschreiten-Unfällen, auch unter Beteiligung von Kindern, ergab sich an der Furt des Arms 5. Dabei wurden die zum Teil bei ROT überquerenden Fußgänger von geradeausfahrenden Kfz aus Arm 2 erfasst.

Zu dieser Situation (vgl. Abbildung 82, Bild links) wurden die linksabbiegenden Kfz der Achse 2-5 bedingt verträglich geführt. Dabei befuhren Geradeausfahrer und Linksabbieger aus Arm 2 zunächst einen gemeinsamen Fahrstreifen, der sich erst im Kreuzungsbereich verzweigte. Die aus Süden kommenden Geradeausfahrer konnten hinter den wartenden Linksabbiegern, die sich auch bis zur Haltlinie zurückstauten, nicht abfließen. Zudem verdeckten wartende Linksabbieger im Kreuzungsbereich, aus Arm 2 und Arm 5, die Sicht für Fußgänger an der Furt 5 auf hinter den Linksabbiegern aus Arm 2 wartende Geradausfahrer. Diese Geradeausfahrer konnten die Kreuzung erst spät räumen, wenn die Linksabbieger den Kreuzungsbereich zum größten Teil verlassen hatten. Zu diesem Zeitpunkt war den zufahrenden Verkehrsteilnehmern aus der Zufahrt 5 bereits rot signalisiert. Wartende 
Fußgänger, die zum Teil die Bahn noch erreichen wollten, sahen dies, überquerten bei ROT und rechneten nicht mehr mit Fahrzeugen, die aus ihrer Sicht von rechts kamen.

Unter anderem aus diesem Grund wurden in 2010 Umbaumaßnahmen durchgeführt. Dabei wurde die Mittelinsel im Arm 2 so zurückgebaut, dass Linksabbieger und Geradeausfahrer getrennt werden konnten (vgl. Abbildung 82, Bild rechts). Im Weitern wurden den Linksabbieger aus Arm 5 eine eigene Signalzeit eingeräumt, so dass eine Sichtbehinderung durch die im Kreuzungsbereich wartenden Linksabbieger beseitigt wurde. Geradeausfahrer aus Arm 5 und Linksabbieger und Geradeausfahrer aus Arm 2 blieben in einer Signalphase. Zudem wurde das Linksabbiegen aus Arm 1 unterbunden.

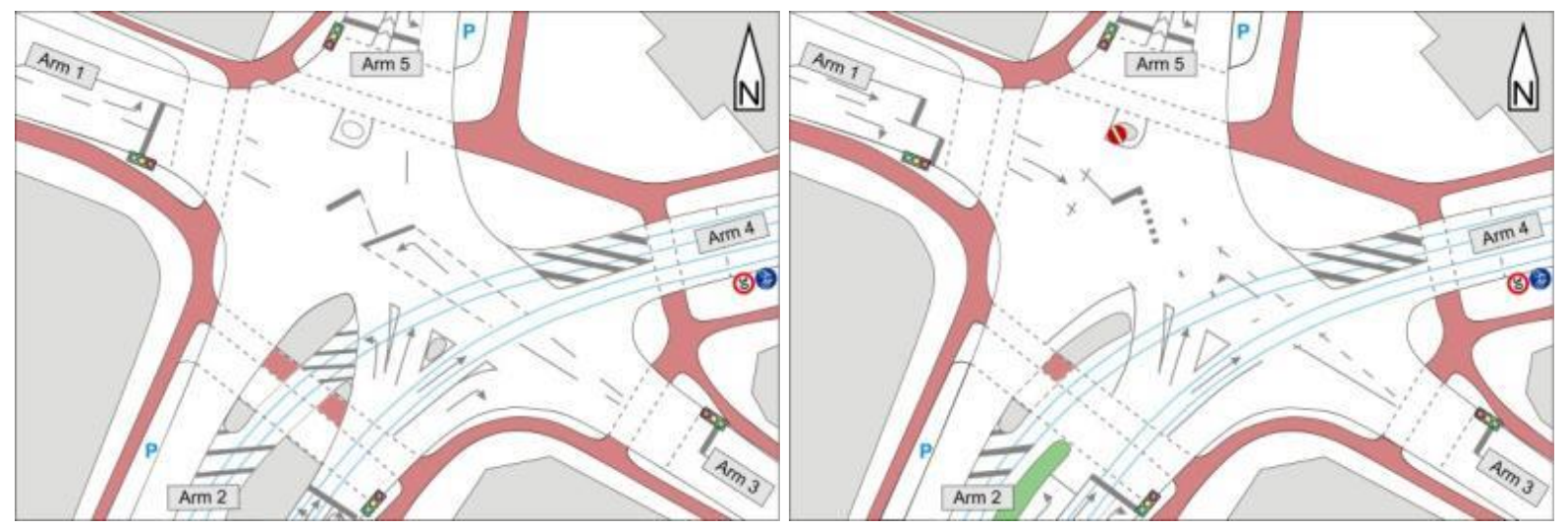

Abbildung 82: Knotenpunkt 7 - Situation vor Änderung (Bild links) und nach Änderung (Bild rechts)

Die Verhaltensbeobachtungen zeigten, dass die Situation für überquerenden Fußgänger an Arm 5 absolut konfliktfrei verlief, auch wenn einige „Rotläufer“, die die Bahn erreichen wollten, beobachtet werden konnten. Auch die Betrachtung der Unfälle, unter Berücksichtigung des kurzen Betrachtungszeitraumes für den Zustand nach der Umsetzung der Maßnahmen, lassen den Schluss zu, dass diese Unfallsituation entschärft werden konnte (Abbildung 83).
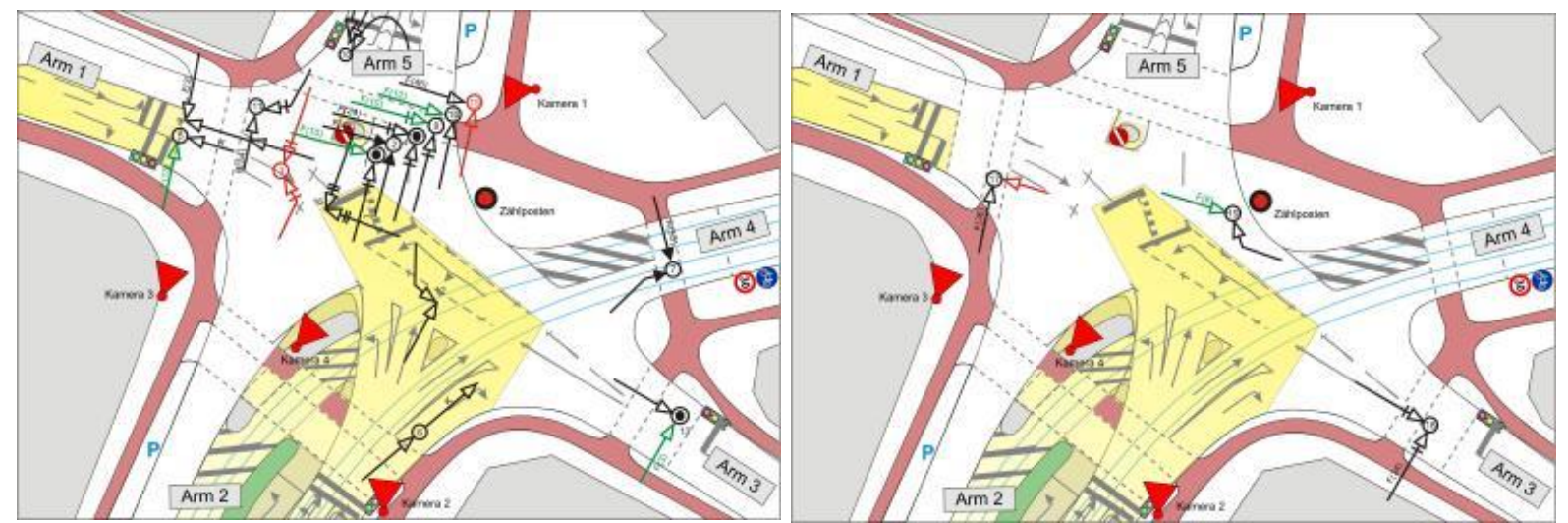

Abbildung 83: Knotenpunkt 7 - Unfälle vor verkehrstechnischer Änderung (Bild links, 16 Unfälle zwischen 2007 und 2009) und nach oder im Jahr der verkehrstechnischen Änderung (Bild rechts, 3 Unfälle im Jahr 2010)

Konflikte zwischen Fußgängern und Kfz konnten an diesem Knotenpunkt allerdings an der Furt des Arms 1 beobachtet werden. Zum Teil befuhren noch kreuzende $\mathrm{Kfz}$ die Furt zu einem Zeitpunkt, da den Fußgängern bereits GRÜN signalisiert wurde (Abbildung 84). 


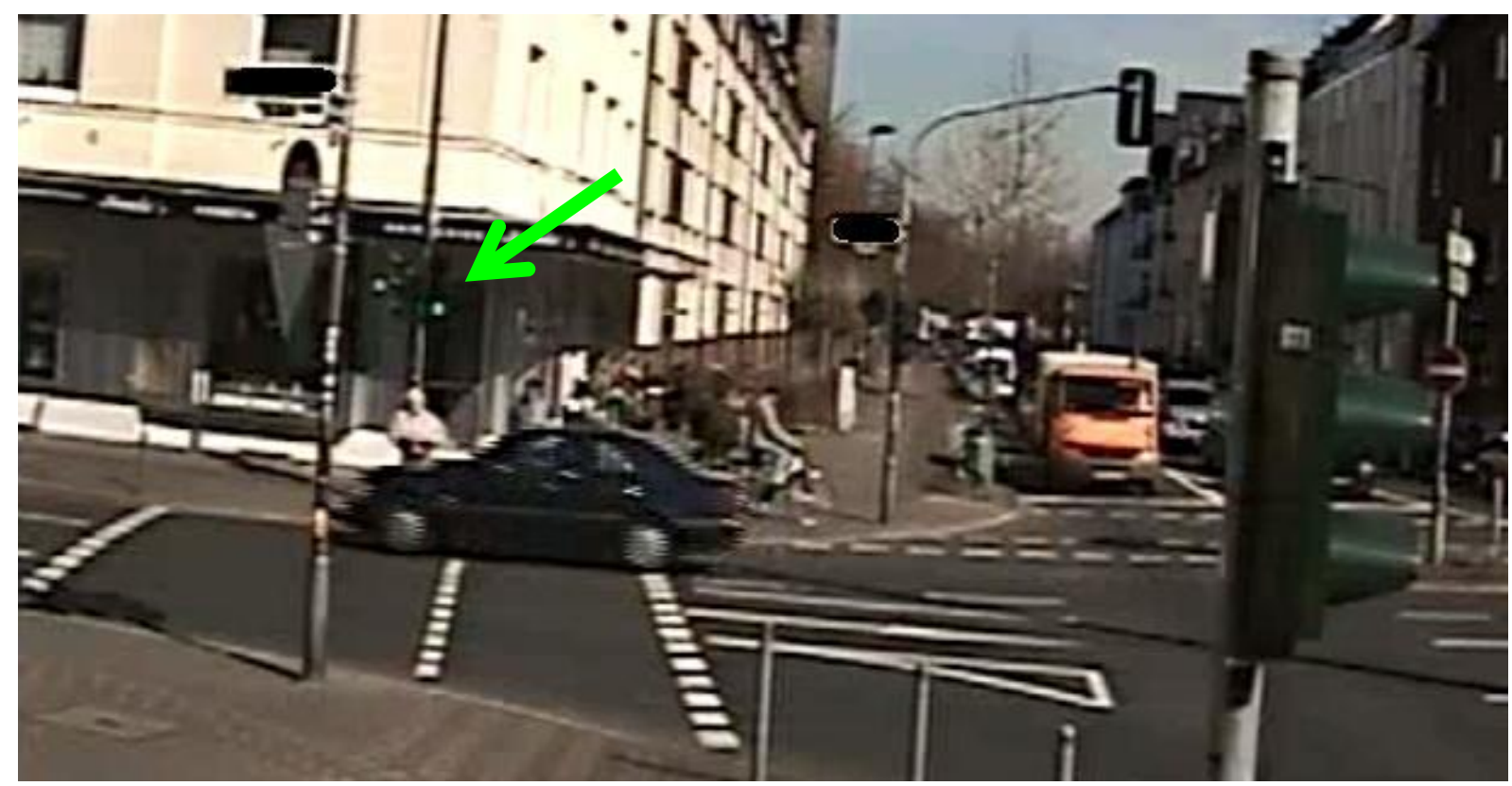

Abbildung 84: Knotenpunkt 7 - Räumendes Kfz bei bereits grünem Signal der Fußgängerfurt

Es ist nicht bekannt, ob es sich bei diesen kreuzenden Kfz aus Arm 3 um Rotlichtverstöße handelte (wenn, dann kurz nach dem Wechsel von GELB auf ROT). Allerdings war die Zwischenzeit ( $\mathrm{Kfz}$ aus 3 räumt und Fußgänger an 1 geht los) am Tag der Verhaltensbeobachtung (werktags, 10 bis 15 Uhr) hier mit 9 Sekunden knapp bemessen. Bei Grundräumwegen von etwa 55 bis 60 Metern (Abbildung 85) ergeben sich gemäß den RiLSA 2010 rechnerisch ${ }^{31}$ 9,05 Sekunden (bei 54,5 Meter Grundräumweg) bzw. 9,6 Sekunden (bei 60 Metern Grundräumweg). Zum Schutz der Fußgänger sollte die Zwischenzeit somit mindestens 10 Sekunden betragen.

\footnotetext{
${ }^{31}$ Zur Berechnung der Zwischenzeit angesetzt: Überfahrzeit $=3 \mathrm{~s}$, Räumgeschwindigkeit $=10 \mathrm{~m} / \mathrm{s}$, Grundräumweg $=54,5$ bzw. $60 \mathrm{~m}$, Fahrzeuglänge $6 \mathrm{~m}$, Einfahrweg $=0 \mathrm{~m}$, Einfahrzeit $=0 \mathrm{~s}$
} 


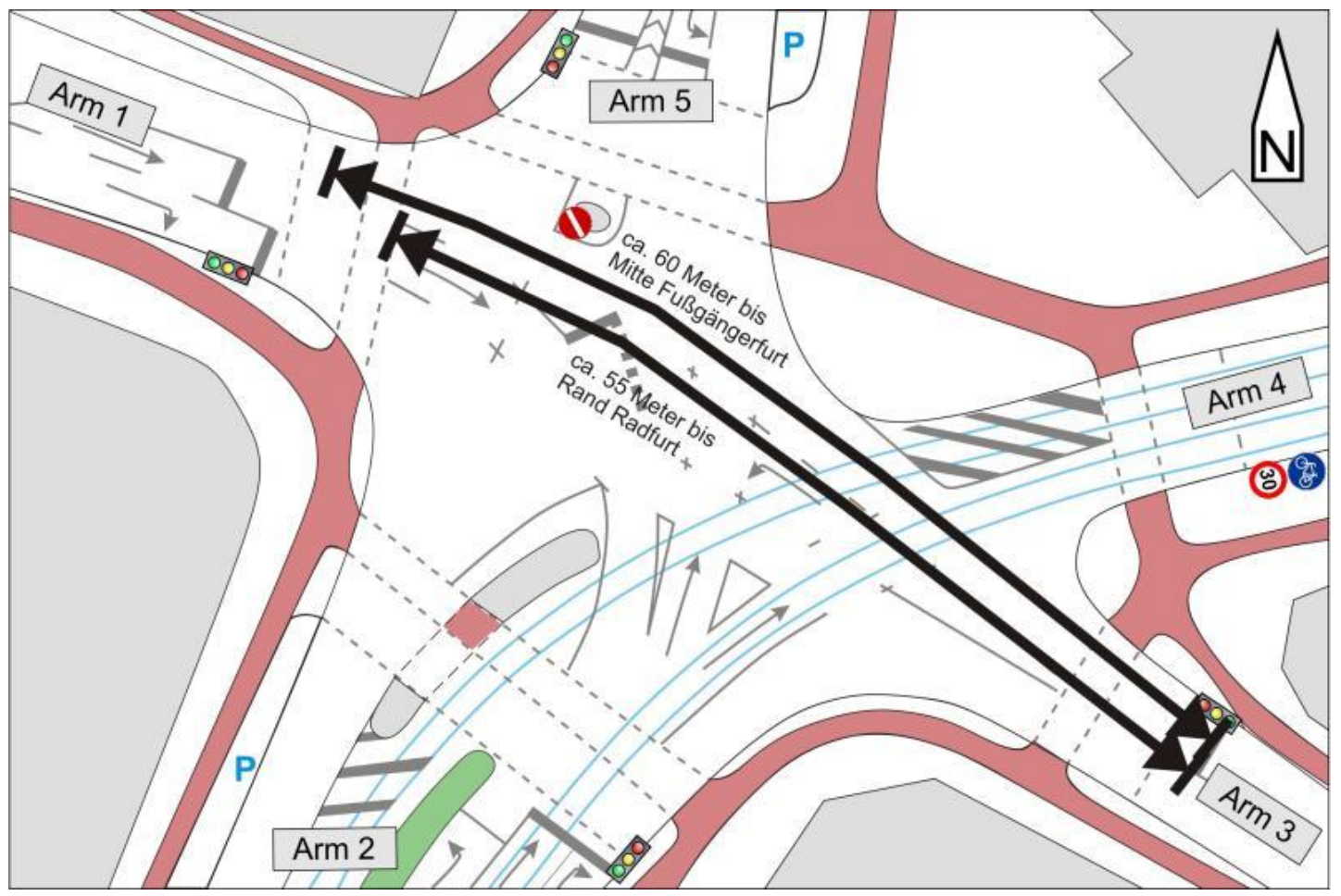

Abbildung 85: Knotenpunkt 7 - Räumweg für Geradeausfahrer aus Arm 3 zur Fußgängerfurt

Nicht optimal ist an diesem Knotenpunkt mit hoher Passantendichte (Geschäftsbesatz, Wochenmarkt, Straßenbahnhaltestelle, Grundschule) die Wegeführung für den Radverkehr, welche sich, insbesondere an den an den Furten, mit denen des Fußverkehrs überlagern (Abbildung 86). Konflikte konnten allerdings nicht beobachtet werden, da vor allem die Radfahrer sich sehr umschauend und defensiv verhielten.

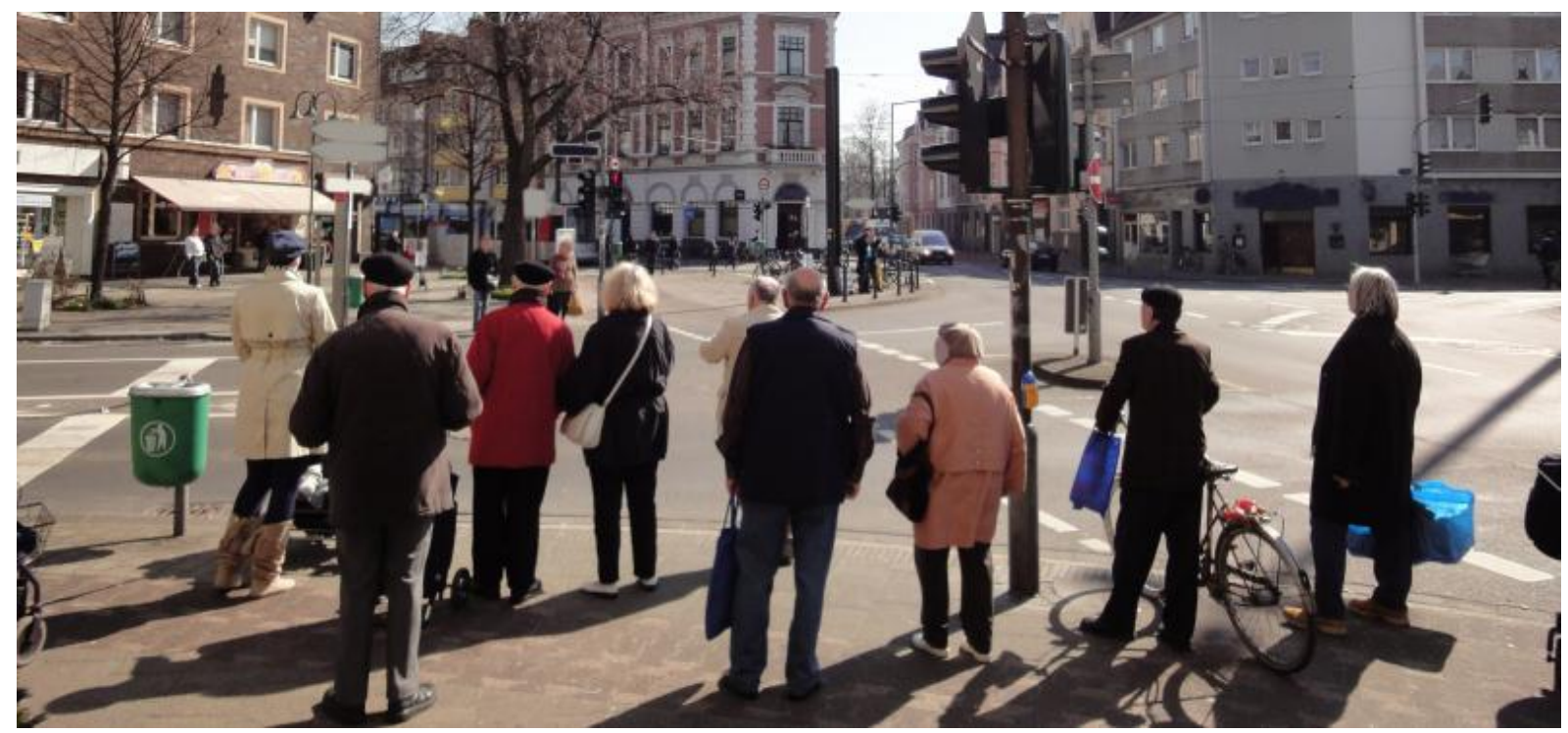

Abbildung 86: Knotenpunkt 7 - Wartende Fußgänger du Radfahrer an der Furt an Arm 5 (Blickrichtung Ost)

Insgesamt konnten an dieser sehr komplexen Kreuzung (vgl. Kap. 9) nur wenige Konflikte beobachtet werden. Die Maßnahmen, die für diesen Knotenpunkt entwickelt und umgesetzt wurden, können als sehr gut erachtet werden. 


\section{Knotenpunkt 8}

Die Verhaltensbeobachtungen an dieser Kreuzung wurden im Mai 2011 durchgeführt. Das Unfalldiagramm auf Basis von Unfällen der Kategorie 1 bis 4 aus dem Zeitraum 2006 bis 2009 zeigt Abbildung 87. Zu sehen ist, dass an diesem Knotenpunkt vornehmlich Unfälle des Typs 211 stattfanden, von Verkehrsteilnehmern der Achse 1-3. Die Signalsteuerung an diesem Knotenpunkt ist eine 2-Phasensteuerung, so dass die Linksabbieger nicht gesichert geführt werden.

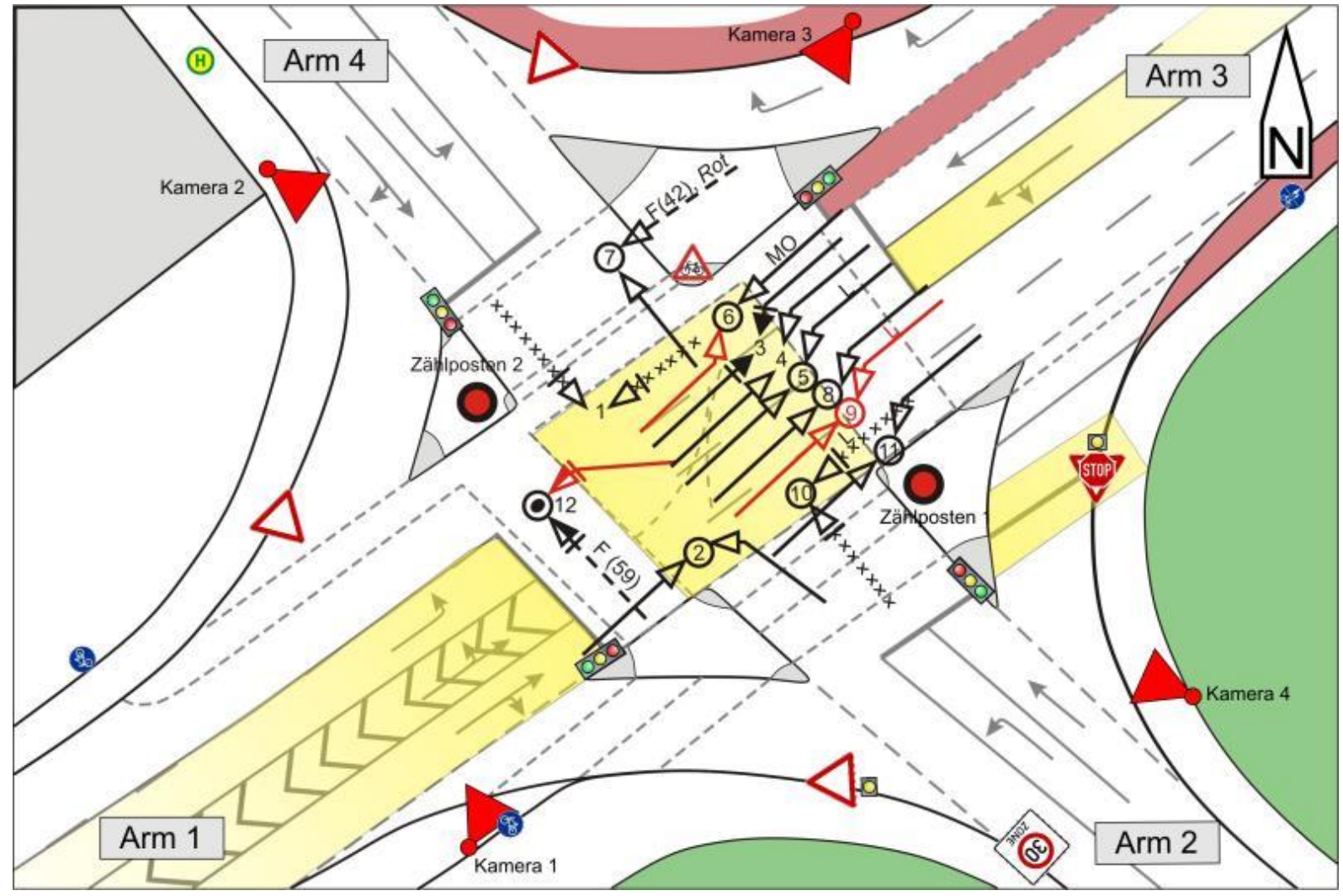

Abbildung 87: Knotenpunkt 8 - Unfalldiagramm, Kamerastandorte und Zählposten (Bereiche mit Änderungen sind gelb hinterlegt. Die Unfälle 10 bis 12 fanden nach bzw. im Jahr der Umsetzung der Maßnahmen statt)

Die Maßnahmen an diesem Knotenpunkt zur Entschärfung der Unfallsituation wurden im Sommer 2008 durchgeführt. Hierbei handelte es sich um eine Änderung der Verkehrsführung der Zufahrten 1 und 3 mit Hilfe einer geänderten Markierung. Im Zustand vor der Änderung wurden Geradausfahrer zweistreifig geführt, die jeweiligen Linksabbieger nutzen mit den Geradeausfahrern den gemeinsamen linken Fahrstreifen (Abbildung 88, Bild links).

Durch die vorgenommen Maßnahmen wurden die Geradeausfahrer von den Linksabbiegern getrennt (Abbildung 88, Bild rechts). Beide Verkehrsströme erhielten nur noch einen Fahrstreifen, der Linksabbiegestreifen aus Arm 1 wurde zur Straßenmitte hin versetzt. Zudem wurden äußere Leitlinien für Linksabbieger im Kreuzungsbereich markiert. Die 2Phasen-Signalsteurung wurde beibehalten, die Linksabbieger werden auch nach der Änderung somit signaltechnisch nicht gesichert geführt. Am „freien Rechtsabbieger“ der Zufahrt 2 wurde das Zeichen „Vorfahrt gewähren“ durch ein „Stopp-Schild“ ersetzt. 


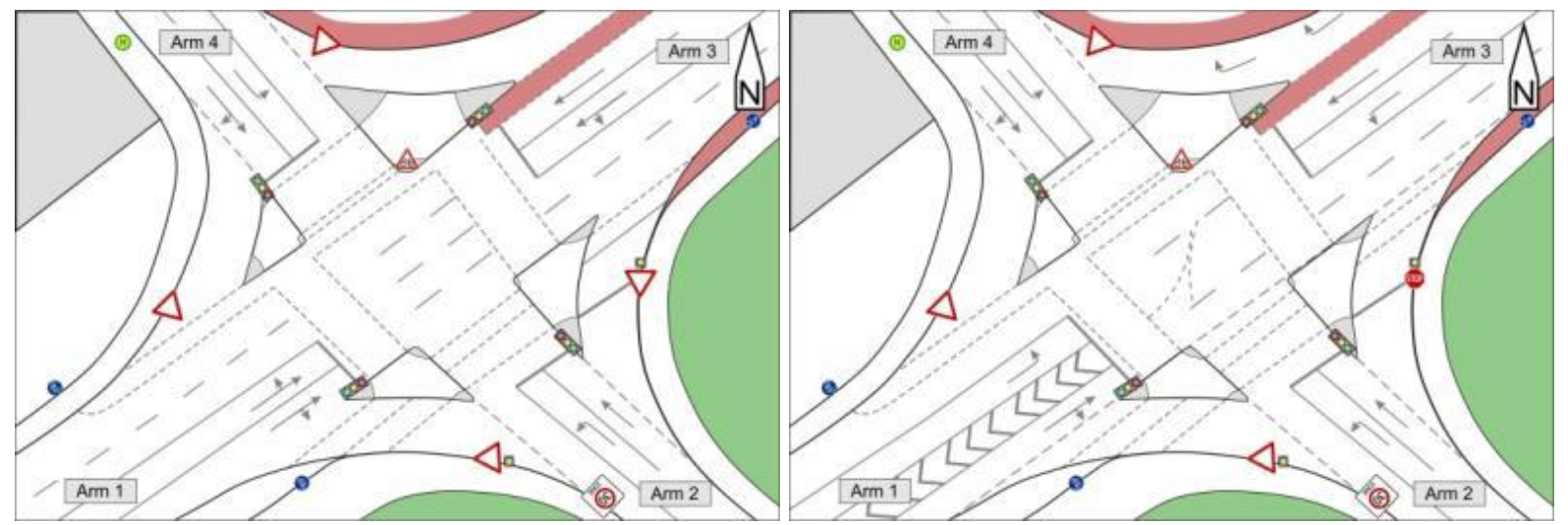

Abbildung 88: Knotenpunkt 8 - Situation vor Änderung (Bild links) und nach Änderung (Bild rechts)

Die Unfälle des Typs 211 konnten mit Hilfe der vorgenommen Maßnahmen erheblich reduziert werden (Abbildung 89). Nach Umsetzung der Maßnahme kam es nur noch zu einem Unfall des Typs 212 in zwei Jahren. Allgemein ist die durchschnittliche Anzahl der Unfälle pro Jahr an diesem Knotenpunkt ebenfalls zurückgegangen.

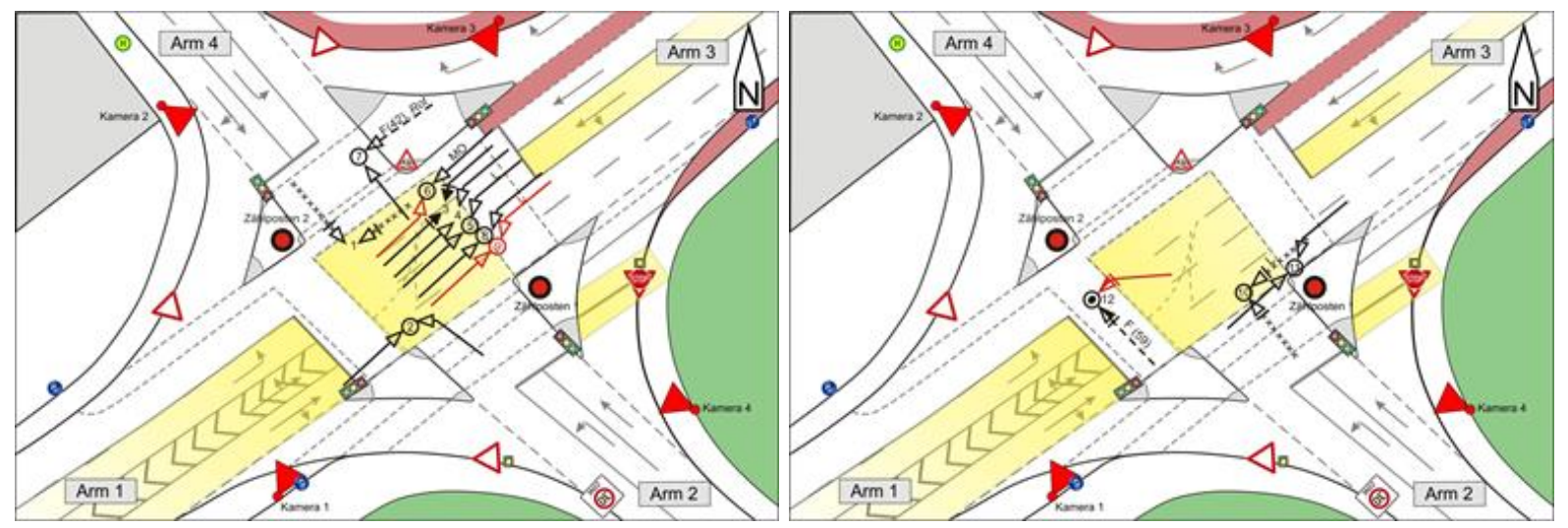

Abbildung 89: Knotenpunkt 8 - Unfälle vor verkehrstechnischer Änderung (Bild links, 9 Unfälle zwischen 2006 und 2007) und nach oder im Jahr der verkehrstechnischen Änderung (Bild rechts, 3 Unfälle zwischen Nov 2008 und 2010)

Auch während der Vor-Ort-Beobachtungen, bei allerdings geringem Verkehrsaufkommen, konnten bei den Linksabbiegern keine Schwierigkeiten festgestellt werden. Die Trennung von Geradeausverkehr und Linksabbieger und die zusätzliche äußere Leitlinie für Linksabbieger bewirkten ein geordnetes und sicheres Linksabbiegen, auch bei der signaltechnisch nicht gesicherten Führung.

Durch den Versatz des Linksabbiegestreifens der Zufahrt 1 zur Straßenmitte hin und „Vor Kopf" des Linksabbiegestreifens der Gegenrichtung wird eine ausreichend große Fläche zur Aufstellung erzeugt, die sich gegenüberstehenden Linksabbieger nehmen sich nicht gegenseitig die Sicht und sie müssen anstatt der vorher zwei Fahrstreifen des Gegenverkehrs nur noch einen Fahrstreifen überqueren und einsehen (Abbildung 90 und Abbildung 91). 


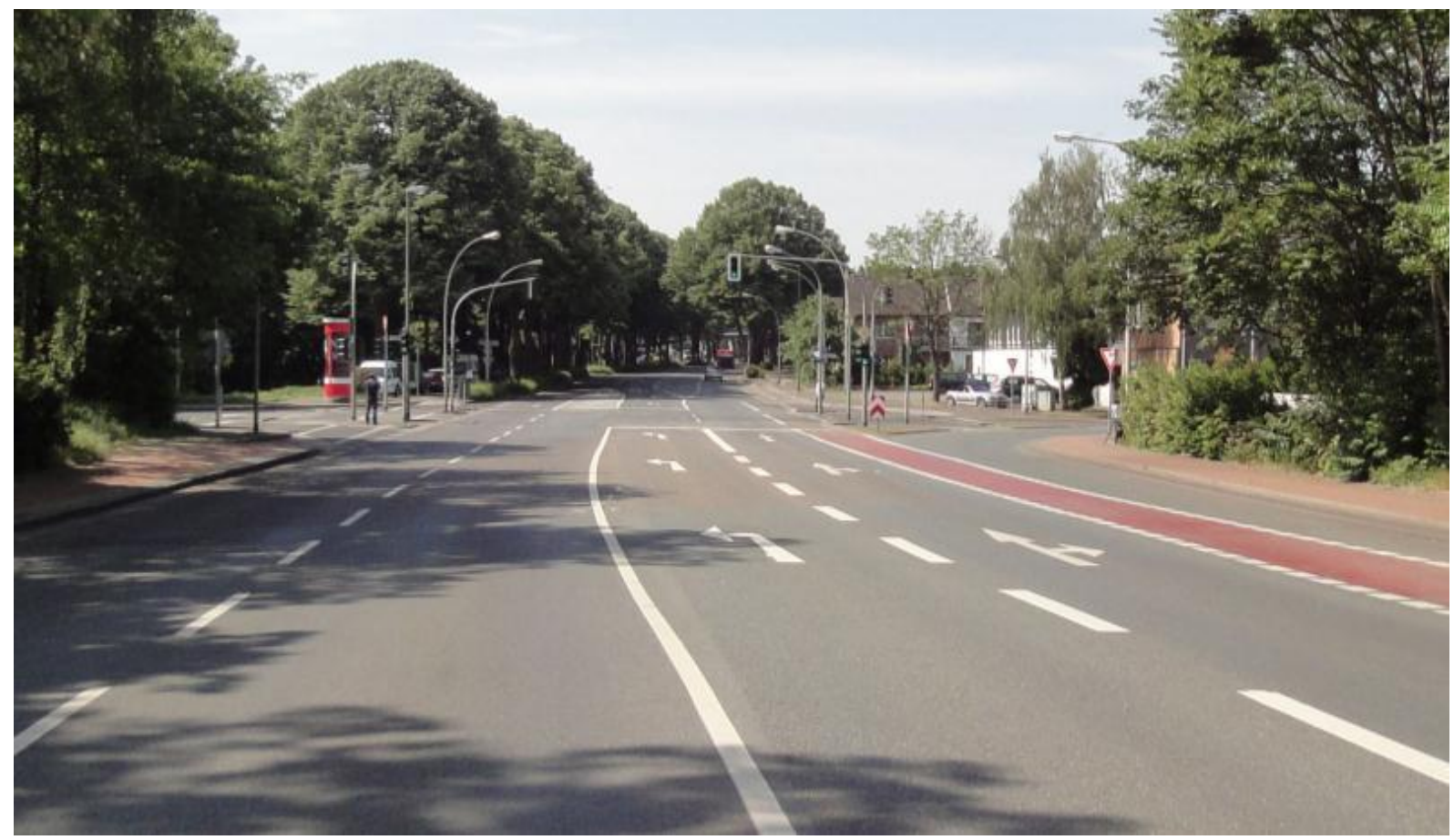

Abbildung 90: Knotenpunkt 8 - Zufahrt aus Arm 3

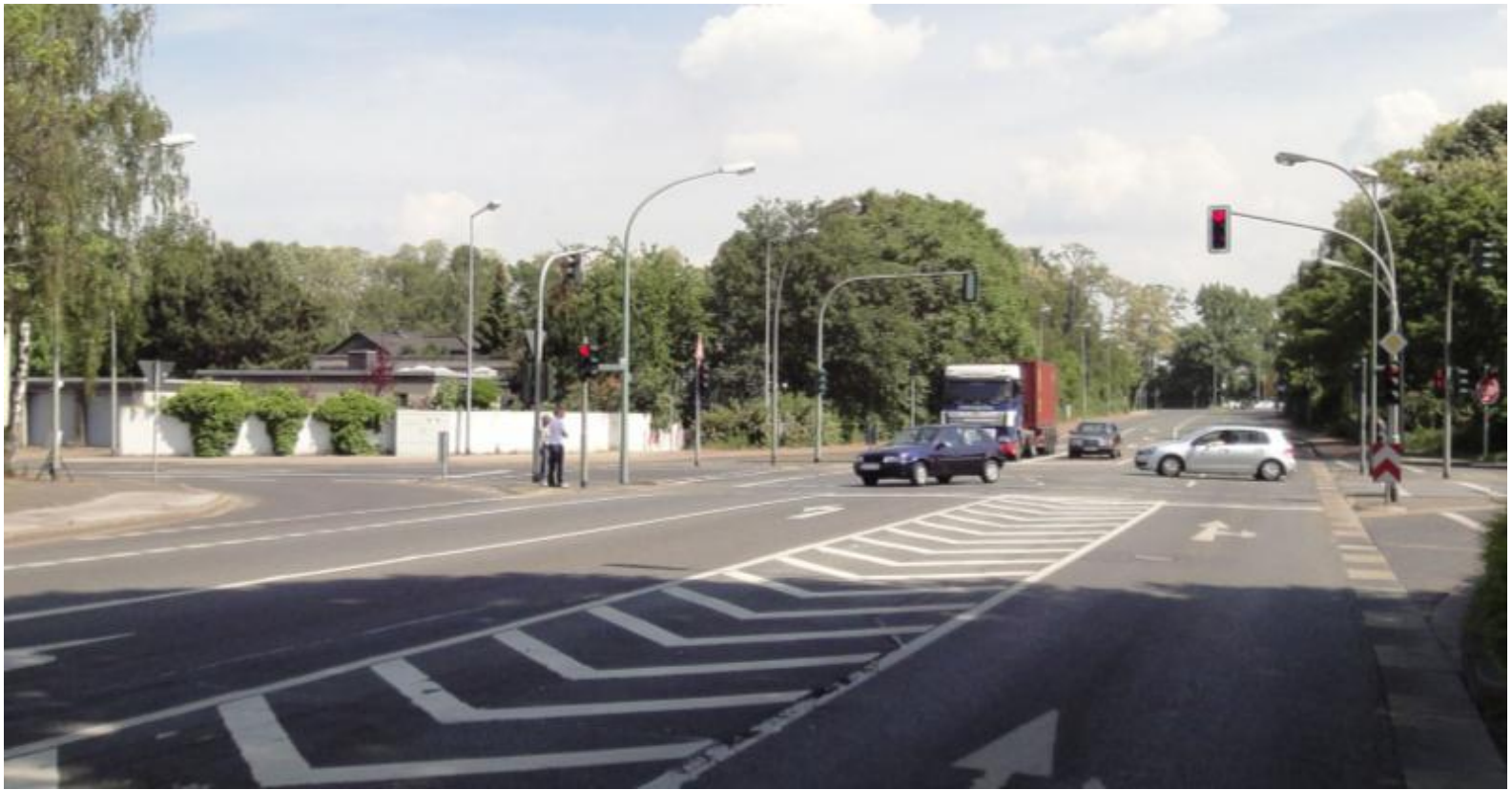

Abbildung 91: Knotenpunkt 8 - Zufahrt aus Arm 1

Die Kreuzung zeigte sich auch insgesamt aus verkehrs- und umweltpsychologischer Sicht am Beobachtungstag unauffällig. Die umgesetzten Maßnahmen werden daher auch als gut geeignet angesehen, Konflikte, die vornehmlich den Unfalltyp 211 entsprechen, zu entschärfen.

Wenige Defizite ergaben sich aus Sicht der Fußgänger, insbesondere aus Sicht mobilitätseingeschränkter Personen, da nicht alle Borde im Bereich der Überquerungen abgesenkt waren. Abbildung 92 zeigt exemplarisch, dass z. B. ein Kind, das ein Fahrrad schiebt, erhebliche Schwierigkeiten dabei hat, ein Hochbord zu überwinden. 


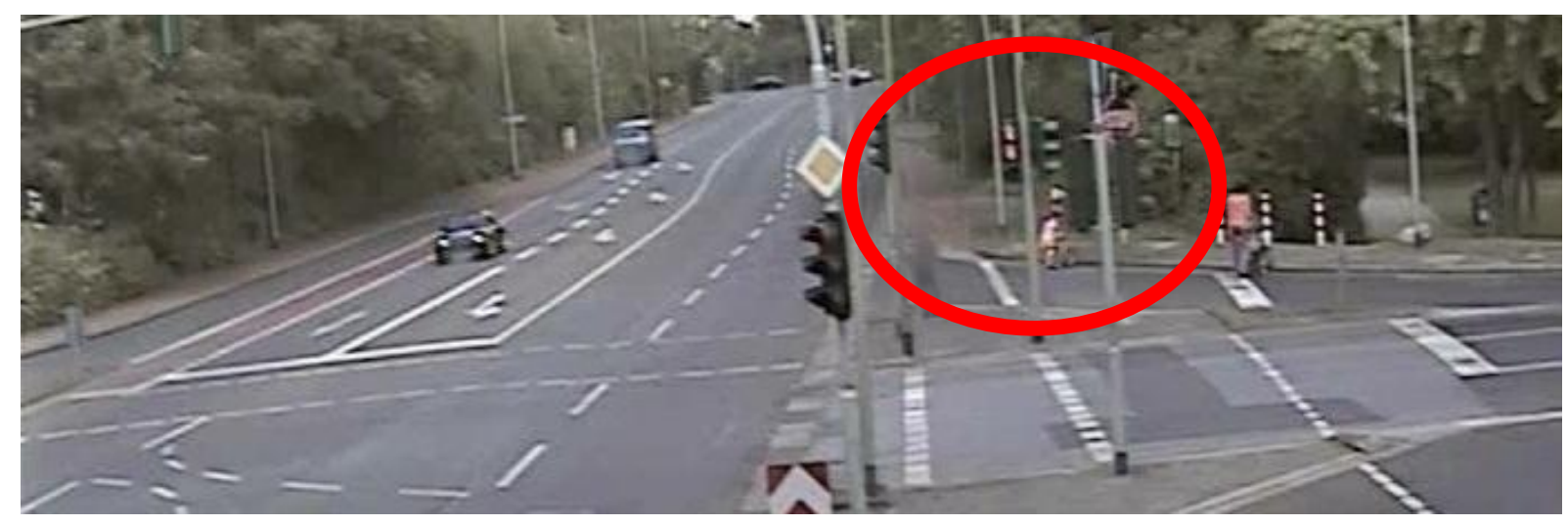

Abbildung 92: Knotenpunkt 8 - Kind mit Fahrrad und Mutter mit Kinderwagen Schwierigkeiten bei der Überwindung eines Hochbordes

Auch an diesem Knotenpunkt konnte beobachtet werden, dass selbst kleinere Unebenheiten (ca. 1-2 cm Höhenunterschied) Schwierigkeiten bei der Überwindung bedeuten, z. B. für Mütter mit Kinderwagen, insbesondere bei kleinen Rollendurchmessern (vgl. Abbildung 93).

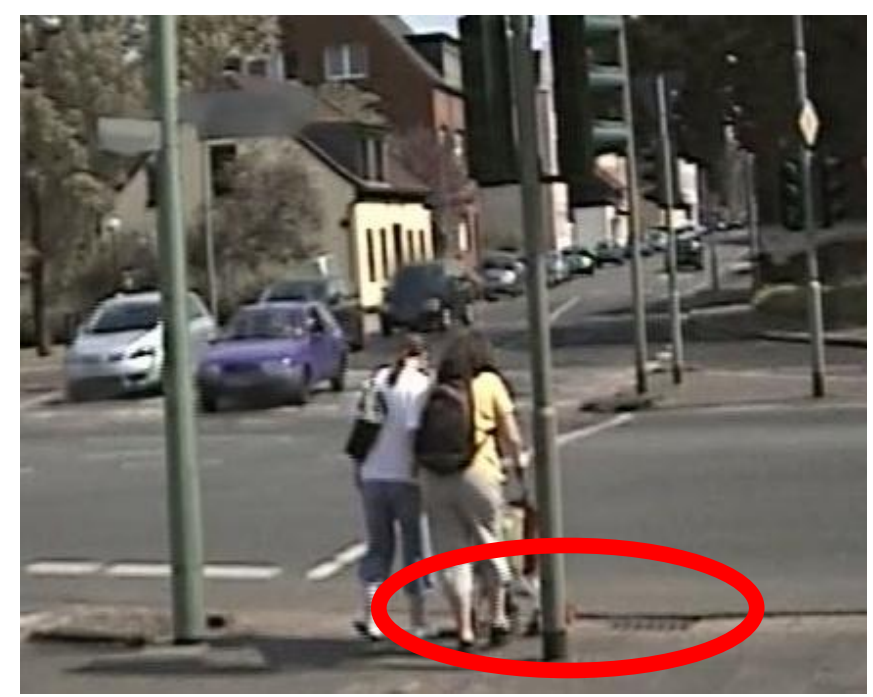

Abbildung 93: Knotenpunkt 8 - Mutter mit Kinderwagen hat Schwierigkeiten bei der „Überrollung“ kleiner Unebenheiten 


\section{Knotenpunkt 9}

Die Verhaltensbeobachtungen an diesem Knotenpunkt wurden im April 2011 durchgeführt. Abbildung 94 zeigt das Unfalldiagramm auf Basis von Unfällen der Kategorie 1 bis 4 aus dem Zeitraum 2006 bis 2010. Auch hier ist der Unfalltyp 211 häufig vertreten.

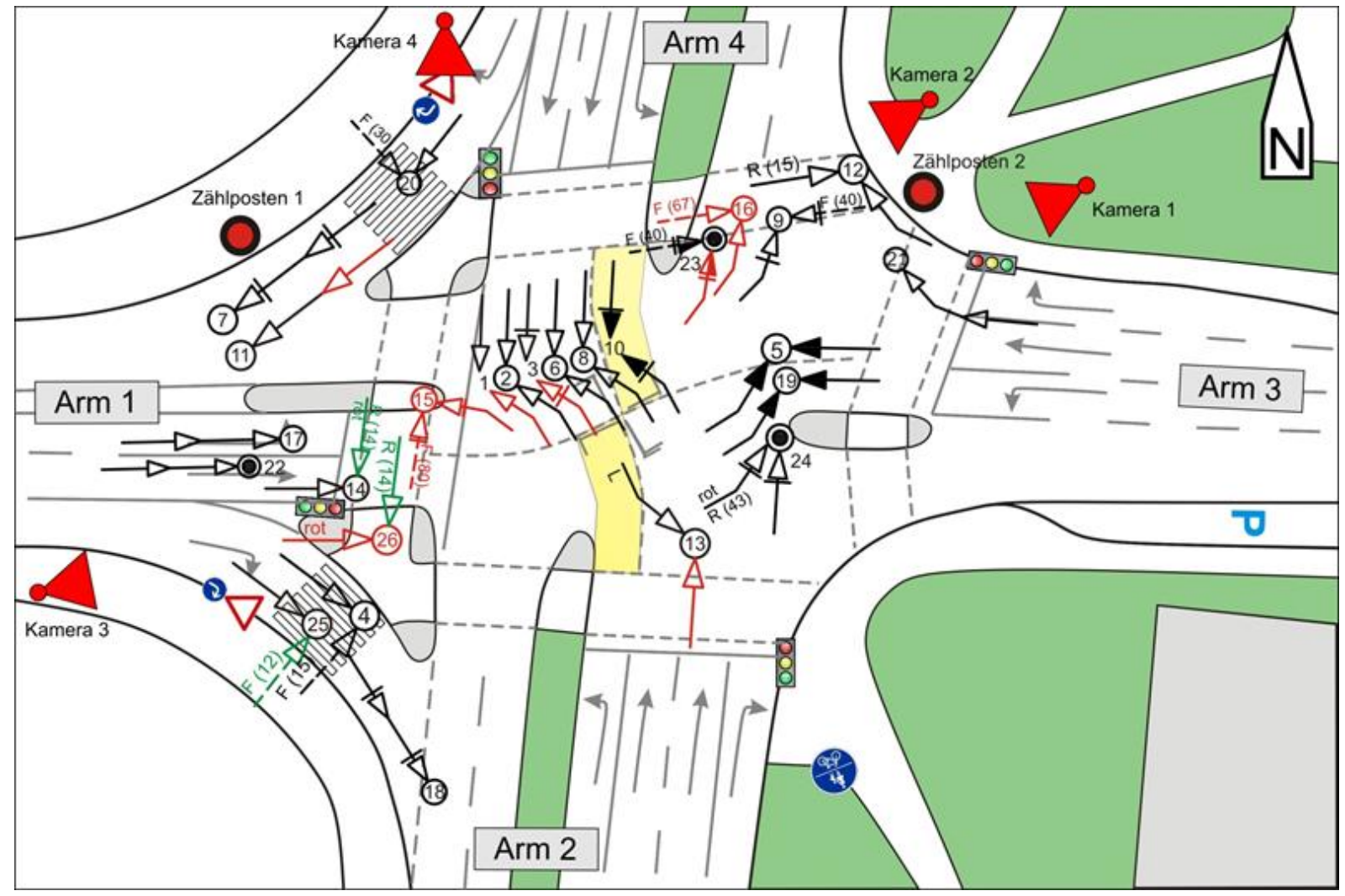

Abbildung 94: Knotenpunkt 9 - Unfalldiagramm, Kamerastandorte und Zählposten (Bereiche mit Änderungen sind gelb hinterlegt. Die Unfälle 17 bis 26 fanden nach bzw. im Jahr der Umsetzung der Maßnahmen statt)

Als Maßnahme zur Erhöhung der Verkehrssicherheit wurden nach diesen Unfällen des Typs 211 an dieser Kreuzung die Linksabbieger der Hauptachse 2-4 signaltechnisch gesichert geführt. Die Unfallentwicklung belegt die Wirksamkeit dieser Maßnahme. Unfälle im Typ 211 sind, zumindest für die gesichert geführten Linksabbieger der Hauptachse, nach Änderung nicht mehr vorgekommen (Abbildung 95). 


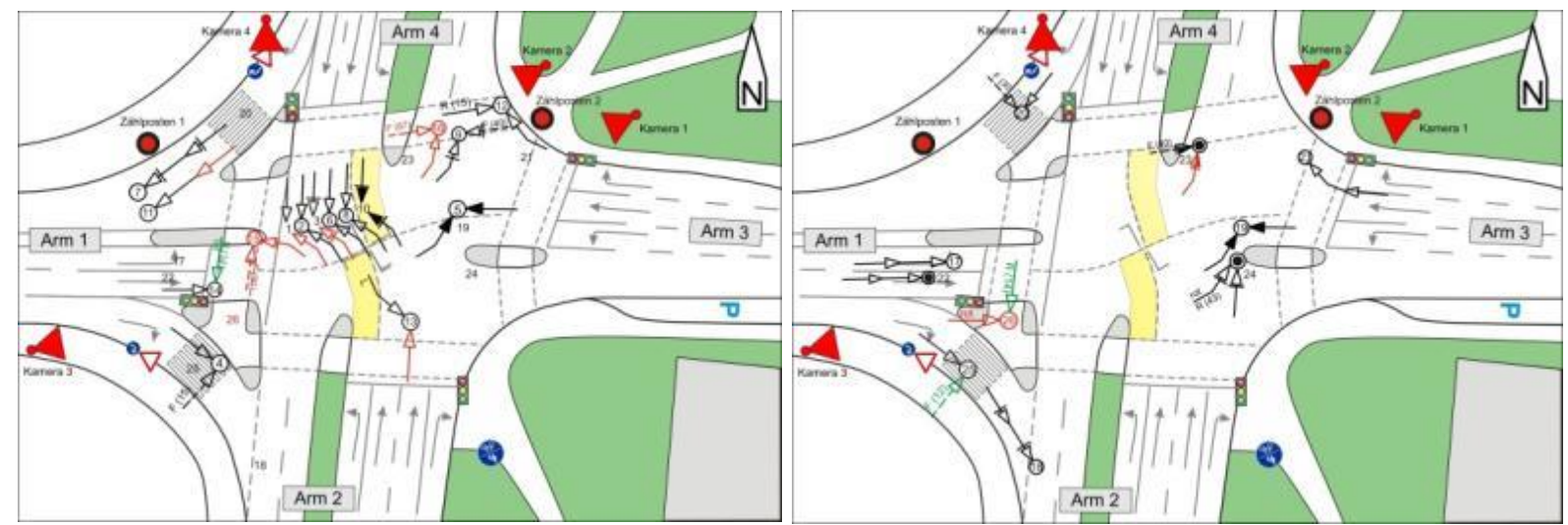

Abbildung 95: Knotenpunkt 9 - Unfälle vor verkehrstechnischer Änderung (Bild links, 16 Unfälle zwischen 2006 und Nov 2007) und nach verkehrstechnischer Änderung (Bild rechts, 10 Unfälle zwischen Nov 2007 und 2010)

Linksabbieger der Nebenachse werden zwar nach wie vor signaltechnisch nicht gesichert geführt, allerdings nutzen sie einen eigenen Fahrstreifen und finden zur Orientierung äußere Leitlinien und Wartelinien im Kreuzungsbereich vor. Die Verhaltensbeobachtungen unterstreichen die Wirksamkeit dieser Führungsform von Linksabbiegern an einem stärker belasteten Knotenpunkt (DTV-Hochrechnung: rd. $30.000 \mathrm{Kfz} / 24 \mathrm{~h}$. Beobachtungszeiträume: $1.823 \mathrm{bzw}$. $1.693 \mathrm{Kfz} / \mathrm{h}$ ). Konflikte, unsicheres Vorfahren oder Warten in Bereichen des Gegenverkehrs der Linksabbieger konnten nicht festgestellt werden.

Mit Bezug auf die Sicherheit auf Fußgänger kann folgender Sachverhalt als Defizit eingestuft werden, wobei diesbezüglich nur wenige Konflikte an der östlichen Furt des Arms 4 beobachtet werden konnten. Um den Fußgängern möglichst die Überquerung in einem Zug zu ermöglichen, sind die hintereinander liegenden Furten hier progressiv signalisiert. Dabei wird das Fußgängersignal auf dem Fahrbahnteiler früher von GRÜN auf ROT geschaltet als das Signal am gegenüberliegenden Fahrbahnrand. Rechtsabbieger aus Arm 3 könnten dieses Signal fehldeuten und ihren vermeintlichen Vorrang gegenüber den Fußgängern durchsetzen wollen ${ }^{32}$. Das Signal der Fußgänger LSA ist mit einer seitlichen Blende versehen, den Blick auf das Signal für Rechtsabbieger aus Arm 3 zeigt Abbildung 96.

\footnotetext{
${ }^{32}$ Vgl. auch FGSV (Hrsg.): Richtlinien für Lichtsignalanlagen RiLSA, Ausgabe 2010, S. 17 f.
} 


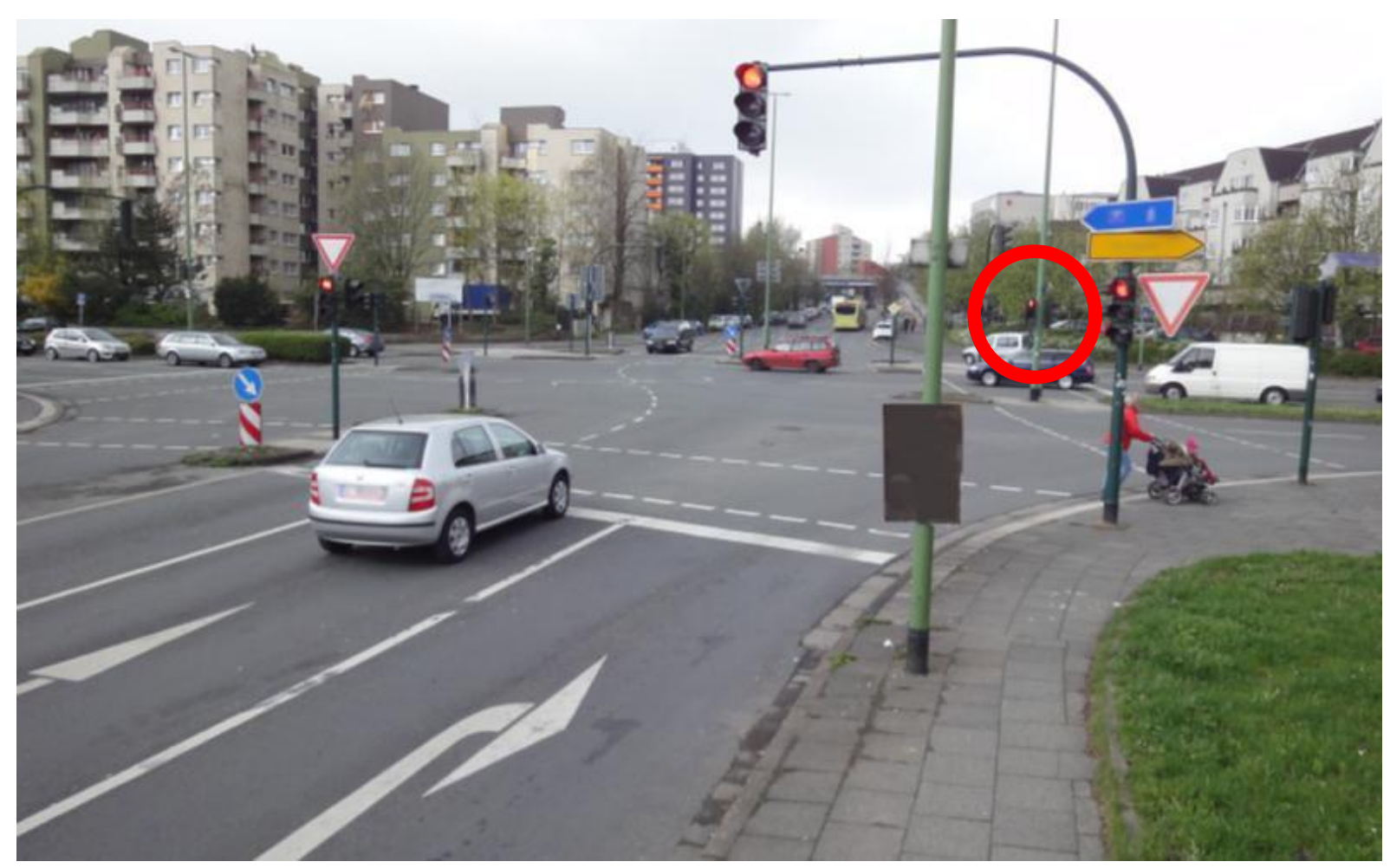

Abbildung 96: Knotenpunkt 9 - Blick der Rechtsabbieger auf Fg-LSA auf Mittelstreifen 


\section{A 4 Sicherheitsanalysen und Verhaltensbeobachtungen im Realverkehr an Kreuzungen mit vorfahrtregelnden Verkehrszeichen}

\section{Knotenpunkt 10}

Die Kamerastandorte und der Zählposten für die Verhaltensbeobachtungen sowie das Unfalldiagramm auf Grundlage von Unfällen mit Personenschaden und schwerem Sachsachschaden aus dem Zeitraum 2006 bis 2009 ist in der Abbildung 97 dargestellt. Die Verhaltensbeobachtungen wurden im Mai 2011 durchgeführt.

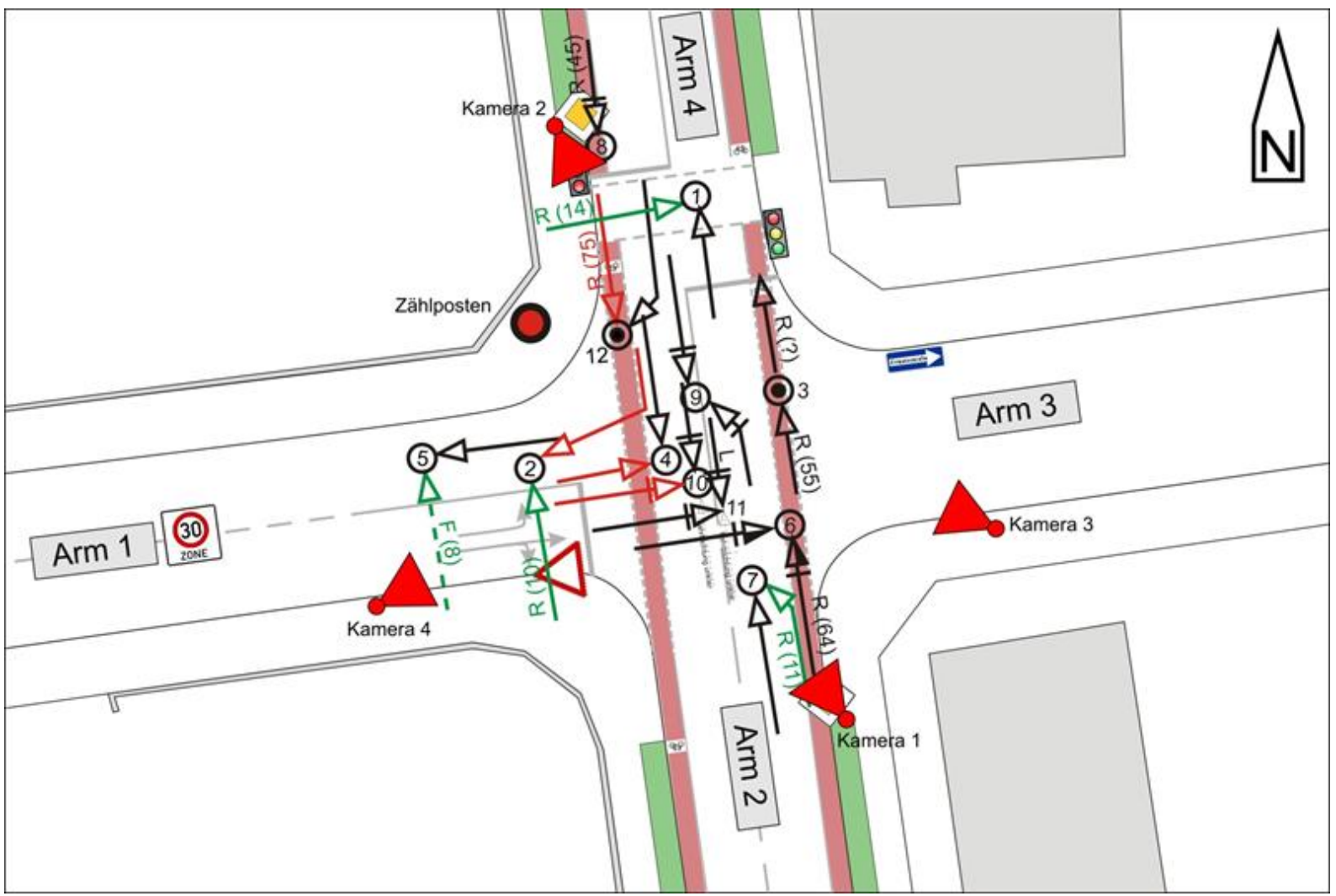

Abbildung 97: Knotenpunkt 10 - Unfalldiagramm, Kamerastandorte und Zählposten

Im Hinblick auf die Unfälle fällt unter anderem auf, dass an 7 der insgesamt 12 Unfälle Radfahrer beteiligt waren. Pkw-Pkw-Unfälle sind ausschließlich Einbiegen-Kreuzen-Unfälle (Unfall 4, 10 und 11). Die wesentlichen Sicherheitsdefizite und beobachteten Konfliktsituationen können zu diesem Unfallgeschehen teilweise in Beziehung gesetzt werden.

Die vorhandenen Radverkehrsanlagen entsprechen in ihrer markierungstechnischen Ausführung Radfahrstreifen (Abbildung 98). Sie sind mit einer Breite von 1 Meter ohne Markierung allerdings schmal bemessen und entsprechen damit auch nicht den 
Breitenmaßen für Radfahrstreifen gemäß den Empfehlungen für Radverkehrsanlagen ${ }^{33}$, sondern dem Mindestmaß für Schutzstreifen.

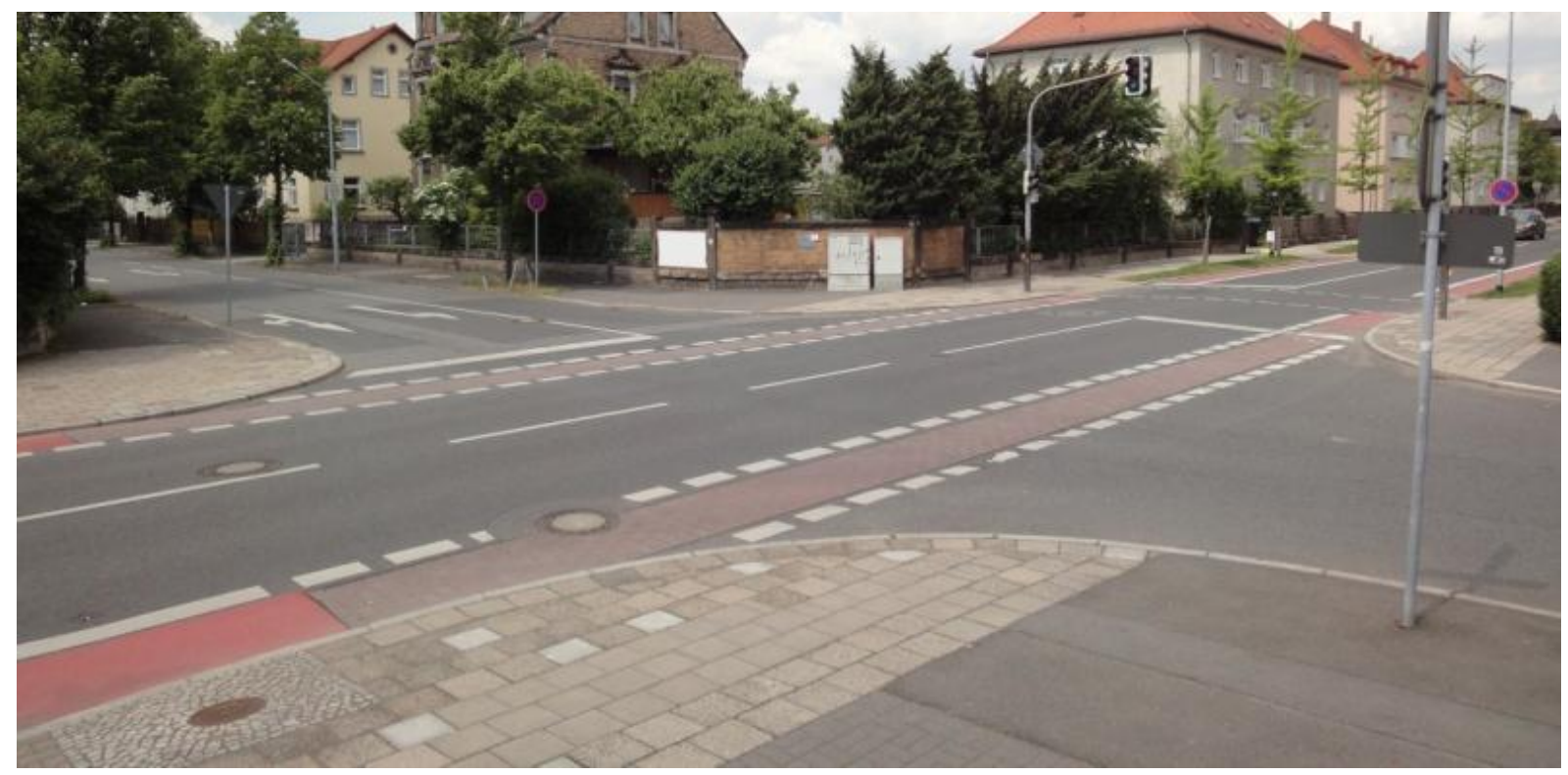

\section{Abbildung 98: Knoten 10 - Kreuzungsbereich}

Radfahrern wird hier eine Sicherheit signalisiert, die sie nicht haben. Eine Gefährdung für Radfahrer können insbesondere dann eintreten, wenn auf den ansonsten nicht allzu hoch belasteten Zufahrten (DTV-Hochrechnung Knotenpunkt: rd. 13.000 Kfz/24h), Kraftfahrzeuge zeitweilig im Pulk zufahren. Im Fall von Linksabbiegern werden die Radfahrstreifen von nachfolgenden Geradeausfahrern als Ausweichfläche genutzt (Abbildung 99, Bild links). Auch im Begegnungsfall Lkw-Lkw, bei einer Fahrstreifenbreite von jeweils 3,20 Metern, wird der den Radfahrern zur Verfügung gestellte Raum eingeschränkt (Abbildung 99, Bild rechts).
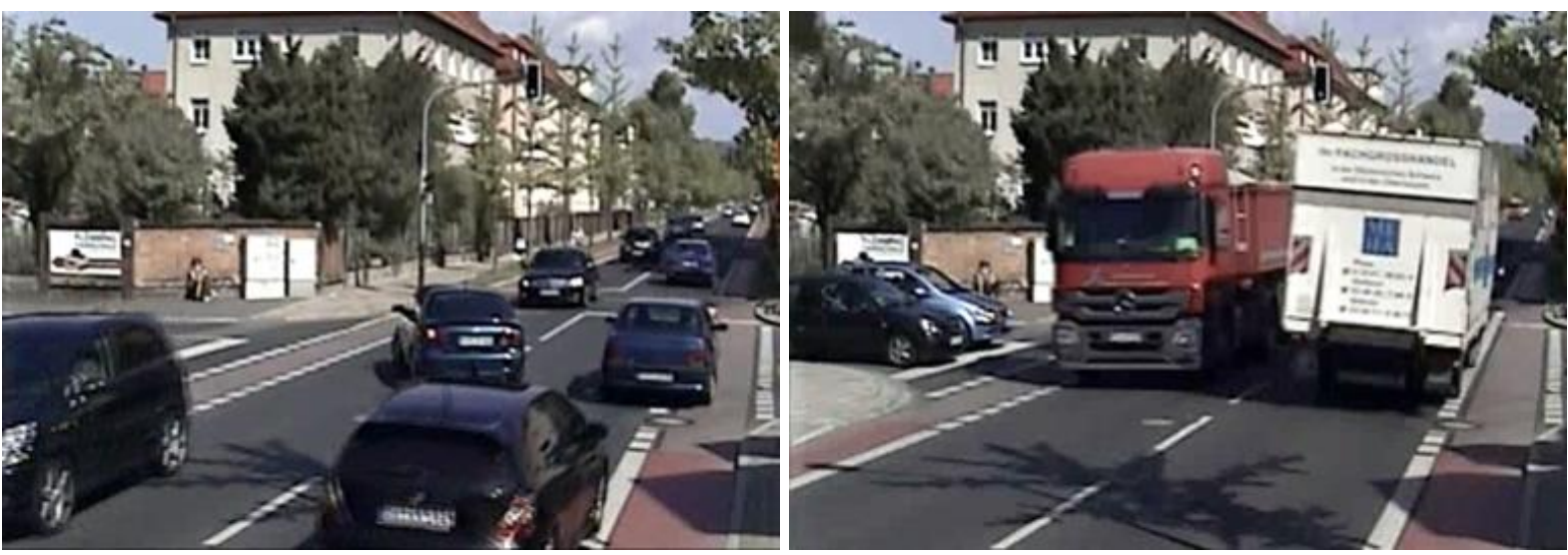

Abbildung 99: Knotenpunkt 10 - Links abbiegende und ausscherende Fahrzeuge (Bild links) und Begegnungsfall Lkw-Lkw (Bild rechts)

Es kann diesen Umständen geschuldet sein, dass einige Radfahrer, aus allen Altersklassen, zu beobachten waren, die anstatt auf der Straße, auf dem Gehweg fuhren (Abbildung 100).

${ }^{33}$ Vgl. Empfehlungen für Radverkehrsanlagen, FGSV (Hrsg.): Breite für Schutzstreifen: 1,50 m (Regelmaß) bzw. 1,25 m (Mindestmaß). Breite für Radfahrstreifen: 1,85 m (Regelmaß). S. 16, Ausgabe 2010 
Auffällig allerdings war, dass nahezu alle abbiegenden Radfahrer aus den Zufahrten 2 und 4 zur Querung der Straße die Fußgänger LSA (Anforderungssignalanlage) nutzten.

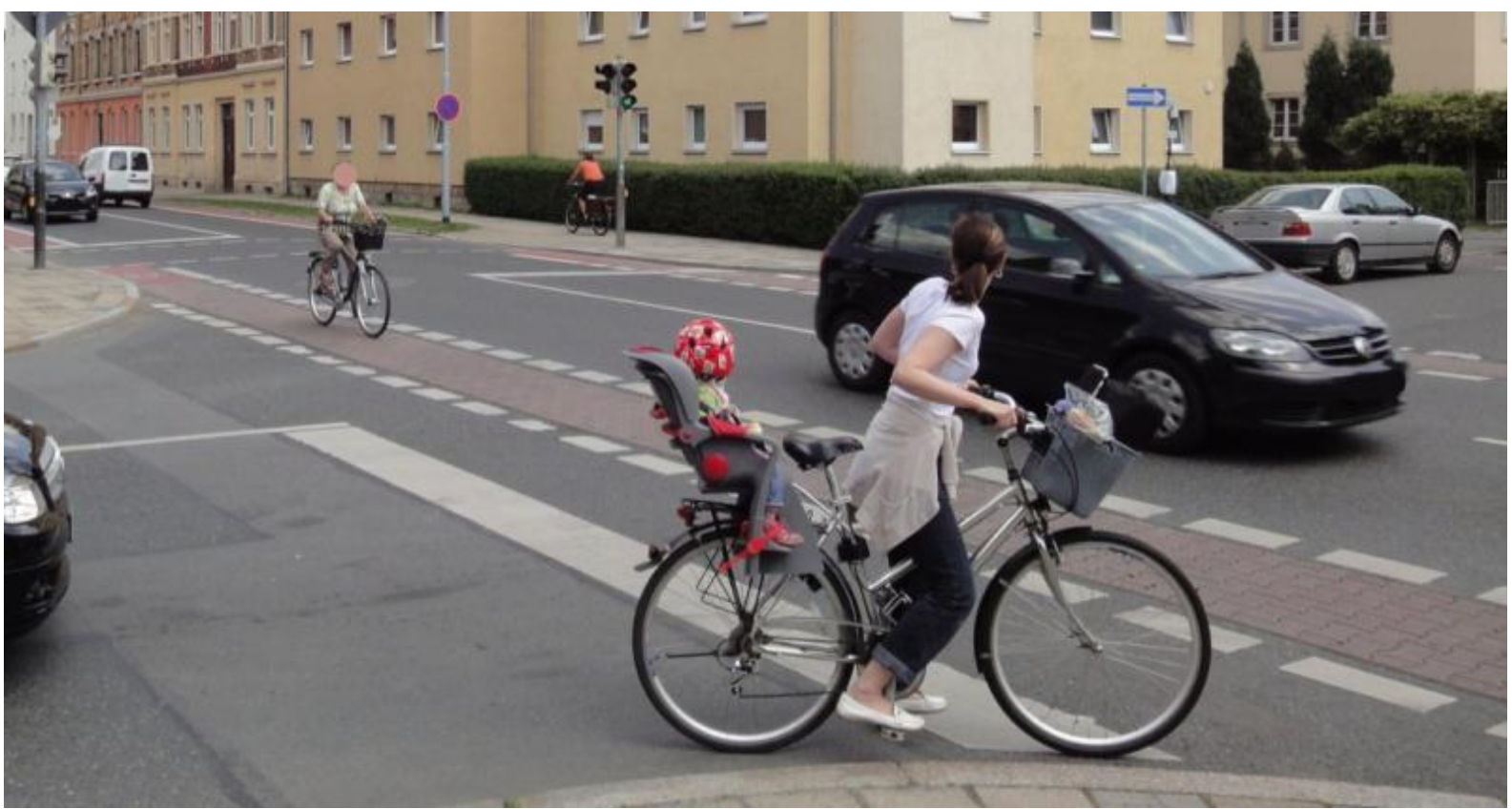

\section{Abbildung 100: Knotenpunkt 10 - Radfahrer}

Die Mindestsichtfelder für einbiegende oder kreuzende Kfz aus Arm 1 sind an diesem Knotenpunkt planmäßig eingehalten. Die weite Sicht nach links ist für zufahrende $\mathrm{Kfz}$ aus Arm 1 allerdings durch eine Baumreihe eingeschränkt (Abbildung 101). Sowohl Pkw-Fahrer als auch Busfahrer, die aus diesem Arm einbiegen oder kreuzen wollten, fuhren oft bis auf den Radfahrstreifen vor (Abbildung 102).

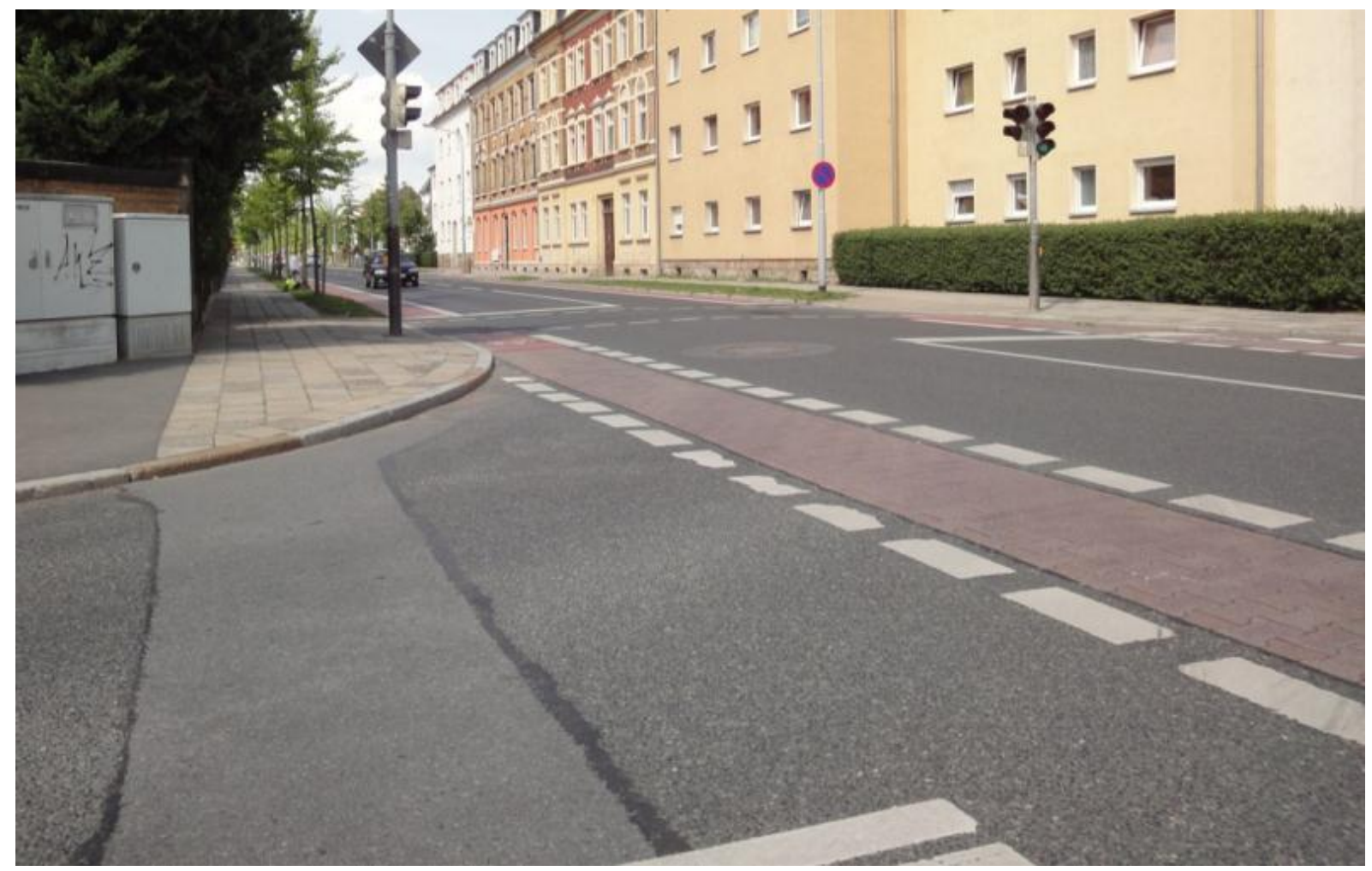

Abbildung 101: Knotenpunkt 10 - Anfahrsicht nach links aus Arm 1 


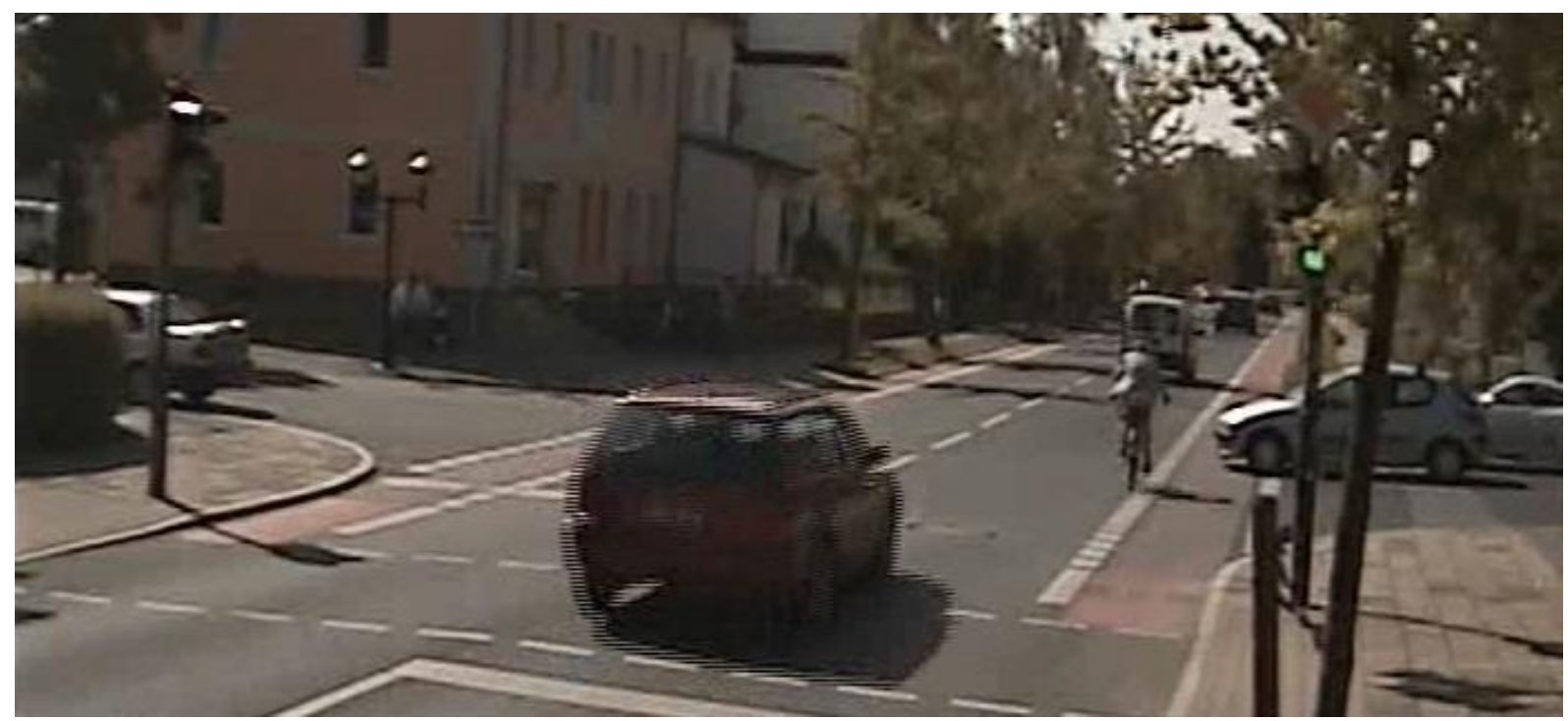

Abbildung 102: Knotenpunkt 10 - Einfahrendes Kfz aus Arm 1

Zum Teil sehr unübersichtliche Zustände für alle Verkehrsteilnehmer im Kreuzungsbereich kamen dann auf, wenn Fußgänger an der Überquerung von Arm 4 die Freigabezeit anforderten. Geradausfahrende Kraftfahrzeuge aus Arm 2 fuhren in den Kreuzungsbereich ein und hielten an der Haltlinie vor der Furt. Gleichzeitig nutzen Kraftfahrer aus Arm 1 die Situation (von links ist kein Verkehr zu erwarten), um links einzubiegen oder zu kreuzen. Hierdurch wurde der Kreuzungsbereich zeitweise vollständig blockiert. Eine Vollsignalisierung oder eine Haltlinie vor dem Kreuzungsbereich kann dazu beitragen, diese unübersichtlichen Situationen erst gar nicht aufkommen zu lassen.

Bei der Art und Anzahl der Verstöße unterschieden sich ältere Verkehrsteilnehmer nicht von Jüngeren. Insgesamt konnten an diesem Knotenpunkt nur wenige Konfliktsituationen beobachtet werden, die aber durchaus dem Unfallbild entsprachen. Unter anderem an der Überquerung von Arm 1 wenn Radfahrer - in diesem Fall eine Jugendliche - im Bereich des Gehweges die Straße überquerten, womit ein abbiegender Kraftfahrer aus Arm 2 nicht rechnete (Abbildung 103).
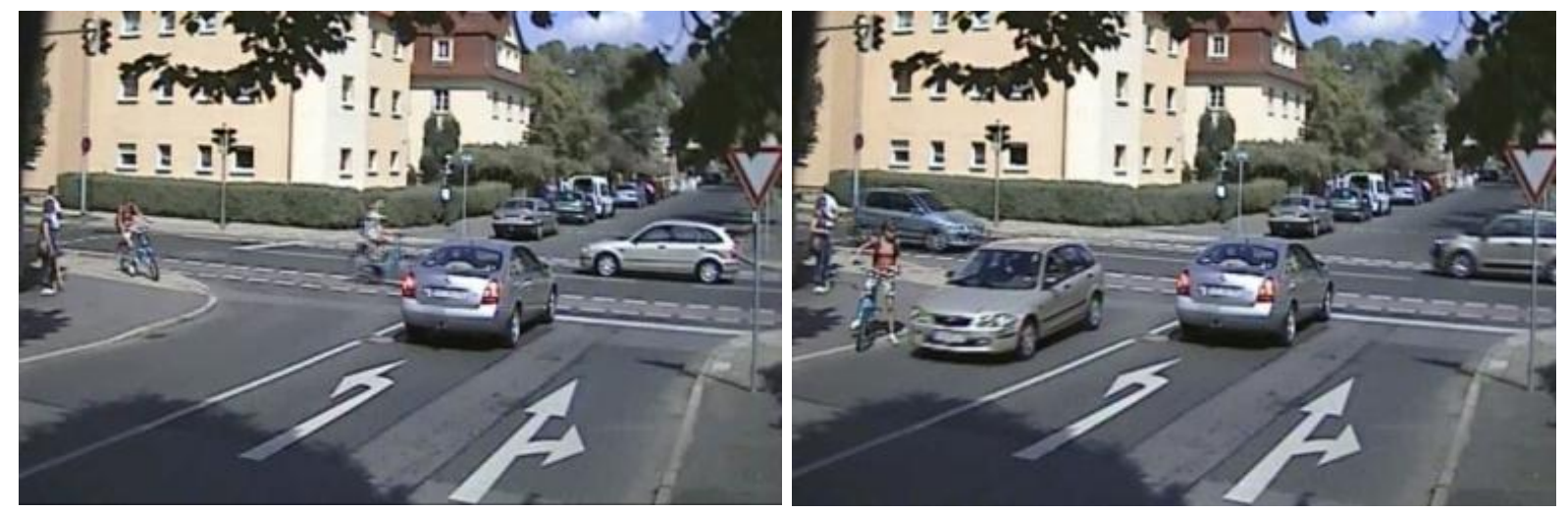

Abbildung 103: Knotenpunkt 10 - Jugendliche Radfahrerin fährt auf Gehweg (Bild links) und hält abrupt vor abbiegenden Kfz an (Bild rechts) 


\section{Knotenpunkt 11}

Die Abbildung 104 zeigt die Planskizze des Knotenpunktes 11, einschließlich der Standorte der Kameras und der Zählposten. Die Verhaltensbeobachtungen wurden im Oktober 2010 durchgeführt. Anonymisierte Verkehrsunfallanzeigen lagen zu diesem Knotenpunkt nicht vor. Alternativ sind in Tabelle 19 und Tabelle 20 die Unfalllisten für Unfälle mit Personenschaden und schwerem Sachschaden aus dem Zeitraum 2004 bis 2009 aufgeführt. Diese legen dar, dass an diesem Knotenpunkt vornehmlich Abbiege-Unfälle vom Typ 211 und EinbiegenKreuzen-Unfälle stattgefunden haben.

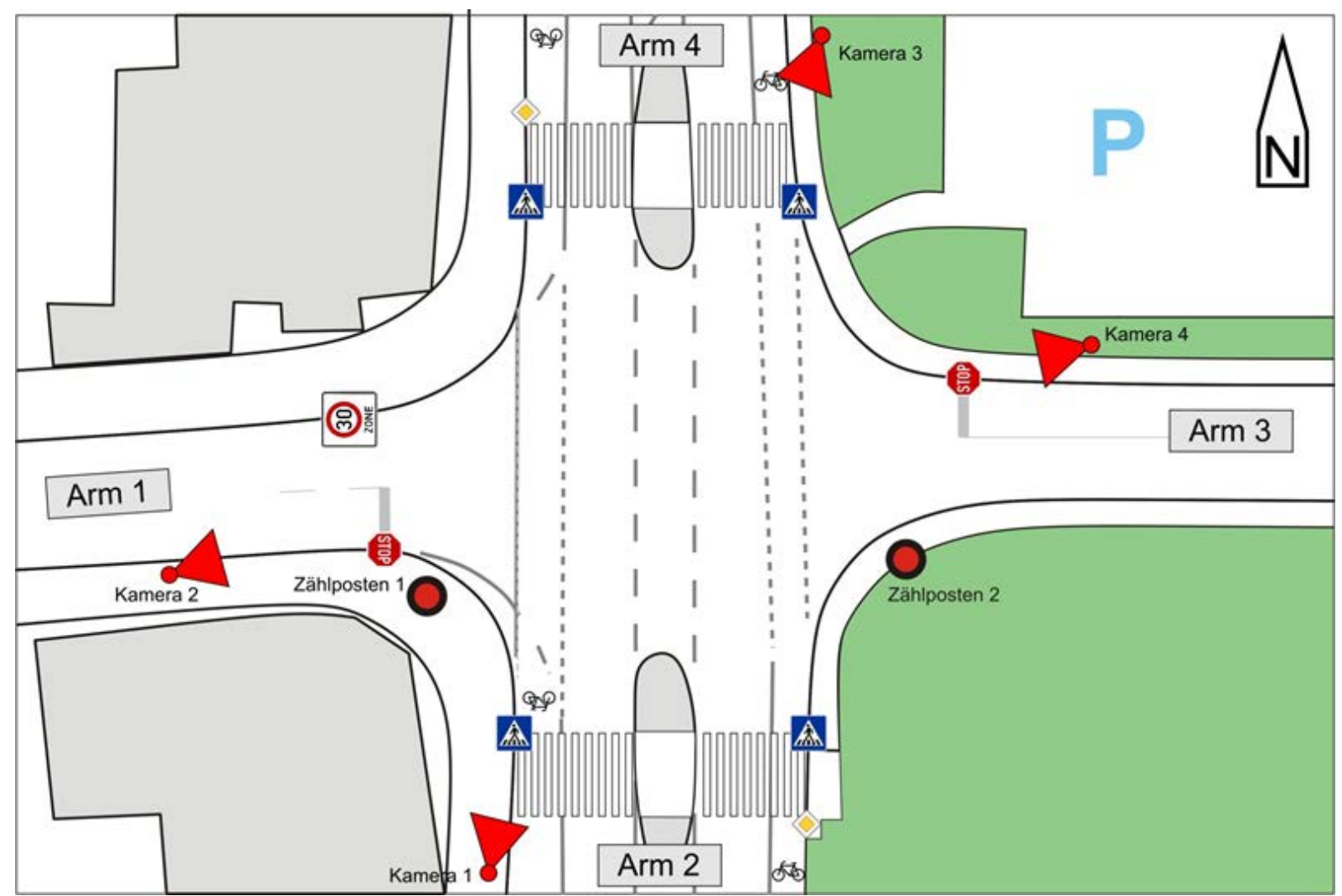

Abbildung 104: Knotenpunkt 11 - Planskizze, Kamerastandorte und Zählposten 
Tabelle 19: Knotenpunkt 11 - Unfallliste „Vorgang“

\begin{tabular}{|c|c|c|c|c|c|c|c|c|c|c|}
\hline 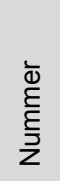 & 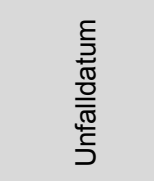 & $\begin{array}{l}0 \\
\frac{\pi}{\pi} \\
\frac{1}{0} \\
\frac{1}{0} \\
0 \\
3\end{array}$ & $\begin{array}{l}\stackrel{+}{N} \\
\stackrel{N}{N} \\
\stackrel{5}{J}\end{array}$ & 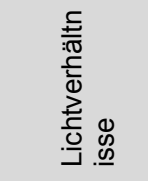 & 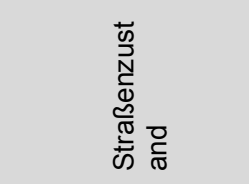 & 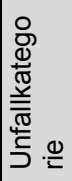 & 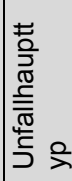 & 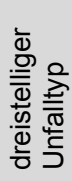 & 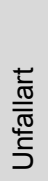 & 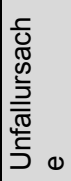 \\
\hline 1 & 10.04 .2004 & $\mathrm{Sa}$ & 10:57 & Tageslicht & nass (auch feucht) & 3 & 3 & 321 & 5 & 28 \\
\hline 2 & 06.11 .2004 & $\mathrm{Sa}$ & 14:07 & Tageslicht & nass (auch feucht) & 4 & 2 & 211 & 5 & 35 \\
\hline 3 & 10.01 .2005 & Mo & $14: 35$ & Tageslicht & trocken & 3 & 3 & 372 & 5 & 49 \\
\hline 4 & 20.12 .2005 & $\mathrm{Di}$ & 11:54 & Tageslicht & nass (auch feucht) & 2 & 3 & 301 & 5 & 28 \\
\hline 5 & 25.08 .2006 & $\mathrm{Fr}$ & 11:00 & Tageslicht & trocken & 3 & 3 & 303 & 0 & 28 \\
\hline 6 & 11.11 .2006 & Sa & $10: 20$ & Tageslicht & nass (auch feucht) & 4 & 3 & 301 & 5 & 28 \\
\hline 7 & 02.01 .2007 & Di & 11:00 & Tageslicht & nass (auch feucht) & 3 & 2 & 211 & 5 & 35 \\
\hline 8 & 15.01 .2007 & Mo & $15: 27$ & Tageslicht & trocken & 4 & 2 & 211 & 5 & 35 \\
\hline 9 & 05.09 .2007 & $\mathrm{Mi}$ & 10:55 & Tageslicht & trocken & 3 & 2 & 231 & 2 & 14 \\
\hline 10 & 25.01 .2008 & $\mathrm{Fr}$ & $7: 45$ & Dunkelheit & trocken & 3 & 3 & 303 & 5 & 28 \\
\hline 11 & 21.02.2008 & Do & 15:20 & Tageslicht & trocken & 3 & 3 & 301 & 5 & 28 \\
\hline 12 & 09.03 .2008 & So & 16:13 & Tageslicht & trocken & 3 & 2 & 211 & 5 & 35 \\
\hline 13 & 06.05 .2008 & $\mathrm{Di}$ & $16: 20$ & Tageslicht & trocken & 3 & 2 & 211 & 4 & 35 \\
\hline 14 & 13.05 .2008 & Di & 18:45 & Tageslicht & trocken & 3 & 3 & 301 & 5 & 35 \\
\hline 15 & 06.11 .2008 & Do & $17: 45$ & Dunkelheit & trocken & 3 & 3 & 311 & 5 & 28 \\
\hline 16 & 01.01 .2009 & Do & $3: 20$ & Dunkelheit & trocken & 4 & 1 & 121 & 1 & 1 \\
\hline 17 & 23.03.2009 & Mo & $14: 25$ & Tageslicht & nass (auch feucht) & 4 & 3 & 321 & 5 & 28 \\
\hline 18 & 22.06.2009 & Mo & 11:07 & Tageslicht & trocken & 3 & 6 & 602 & 1 & 14 \\
\hline 19 & 04.09 .2009 & $\mathrm{Fr}$ & $13: 44$ & Tageslicht & trocken & 2 & 2 & 211 & 5 & 35 \\
\hline 20 & 28.11 .2009 & $\mathrm{Sa}$ & 20:05 & Dunkelheit & nass (auch feucht) & 2 & 2 & 211 & 5 & 35 \\
\hline 21 & 07.12 .2009 & Mo & 12:10 & Tageslicht & trocken & 4 & 2 & 211 & 5 & 35 \\
\hline
\end{tabular}

Tabelle 20: Knotenpunkt 11 - Unfallliste "Beteiligte"

\begin{tabular}{|c|c|c|c|c|c|c|c|c|c|c|}
\hline 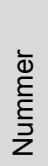 & 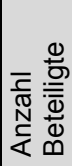 & 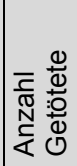 & 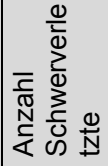 & 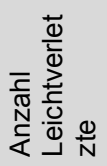 & 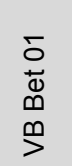 & $\begin{array}{l}\delta \\
0 \\
\overline{0} \\
\infty \\
\bar{\Phi} \\
\frac{\omega}{<}\end{array}$ & 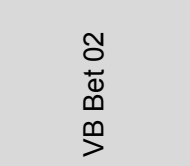 & 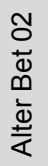 & 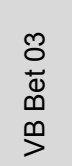 & 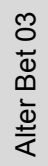 \\
\hline 1 & 2 & 0 & 0 & 1 & Pkw & 51 & Fahrrad & 74 & & \\
\hline 2 & 3 & 0 & 0 & 0 & Pkw & 53 & Pkw & 41 & Lkw & 45 \\
\hline 3 & 2 & 0 & 0 & 1 & Pkw & 45 & Fahrrad & 64 & & \\
\hline 4 & 2 & 0 & 1 & 0 & Pkw & 78 & Krad & 24 & & \\
\hline 5 & 2 & 0 & 0 & 1 & Pkw & 48 & Pkw & 67 & & \\
\hline 6 & 2 & 0 & 0 & 0 & Pkw & 71 & Pkw & 52 & & \\
\hline 7 & 2 & 0 & 0 & 1 & Pkw & 56 & Pkw & 31 & & \\
\hline 8 & 2 & 0 & 0 & 0 & Pkw & 65 & Pkw & 23 & & \\
\hline 9 & 2 & 0 & 0 & 1 & Pkw & 53 & Pkw & 18 & & \\
\hline 10 & 2 & 0 & 0 & 1 & Pkw & $?$ & Moped & 16 & & \\
\hline 11 & 2 & 0 & 0 & 1 & Pkw & 18 & Fahrrad & 72 & & \\
\hline 12 & 2 & 0 & 0 & 1 & Pkw & 20 & Moped & 42 & & \\
\hline 13 & 2 & 0 & 0 & 1 & Pkw & 48 & Moped & 54 & & \\
\hline 14 & 2 & 0 & 0 & 1 & Pkw & 77 & Leichtkraftrad & 63 & & \\
\hline 15 & 2 & 0 & 0 & 1 & Pkw & 46 & Krad & 55 & & \\
\hline 16 & 2 & 0 & 0 & 0 & Pkw & 17 & Pkw & $?$ & & \\
\hline 17 & 2 & 0 & 0 & 0 & Pkw & 41 & Pkw & 25 & & \\
\hline 18 & 2 & 0 & 0 & 1 & Pkw & 41 & Mofa & 15 & & \\
\hline 19 & 2 & 0 & 1 & 0 & Pkw & 39 & Leichtkraftrad & 29 & & \\
\hline 20 & 2 & 0 & 2 & 0 & Pkw & 36 & Pkw & 19 & & \\
\hline 21 & 2 & 0 & 0 & 0 & Pkw & 86 & Pkw & 51 & & \\
\hline
\end{tabular}


Zu den Faktoren, die die Verkehrssicherheit an diesem Knotenpunkt beeinträchtigen können, zählt, dass die Hauptstraße (Arm 2 - 4) einer langen Geraden entspricht und breite Fahrstreifen (4 Meter) aufweist, was hohe Geschwindigkeiten des geradausfahrenden KfzVerkehrs, auch im Bereich der Überquerungsanlage, bewirken kann (vgl. Abbildung 105).

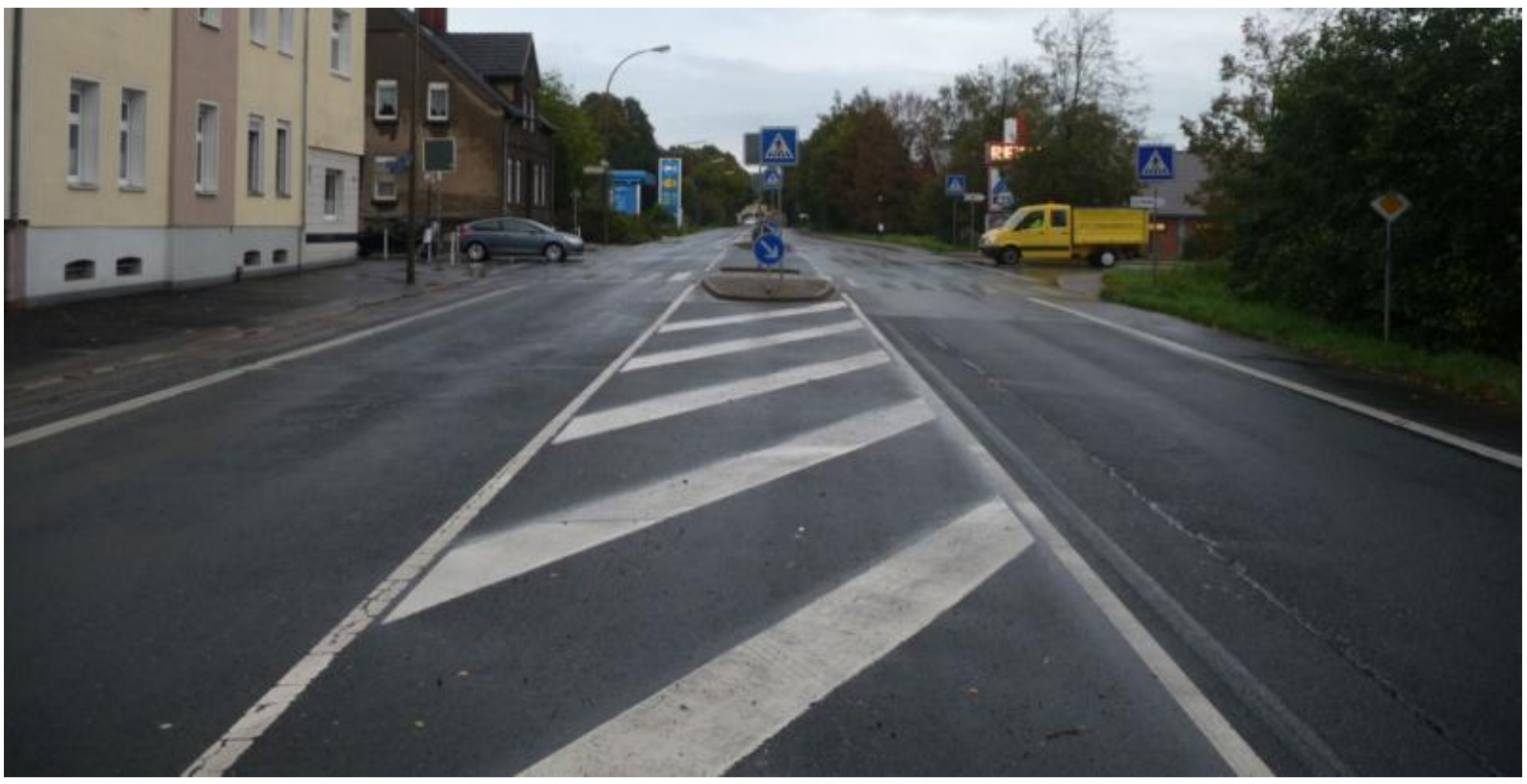

Abbildung 105: Knotenpunkt 11 - Sicht auf Kreuzungsbereich aus Arm 2

Die Radverkehrsfurten an den Armen 1 und 3 sind für einbiegende und kreuzende Kraftfahrer schlecht zu erkennen (vgl. Abbildung 106).

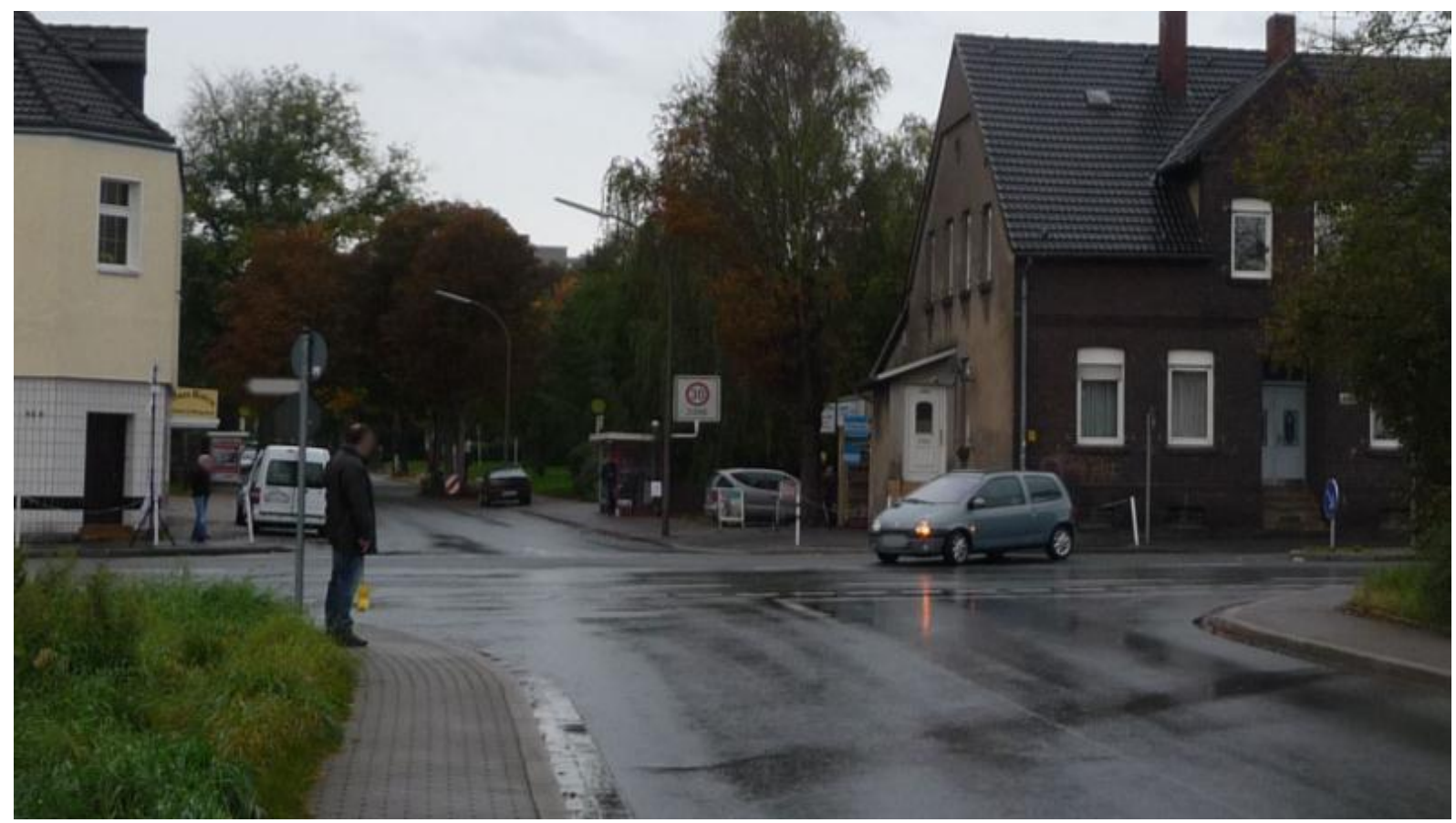

\section{Abbildung 106: Knotenpunkt 11 - Sicht aus Arm 2}

Die Anfahrsicht aus Arm 1 nach links ist durch Bepflanzung und eine Grundstücksmauer eingeschränkt (Abbildung 107). Kraftfahrer fuhren zum Teil bis auf den Radfahrstreifen in den Kreuzungsbereich ein (Abbildung 108). Zudem verbirgt sich, für wartende Kraftfahrer der 
Zufahrt 1 nicht zu erkennen, die Ausfahrt einer Tankstell direkt hinter der Grundstücksmauer aus der unvermittelt Fahrzeuge auf die Hauptstraße einbiegen können.

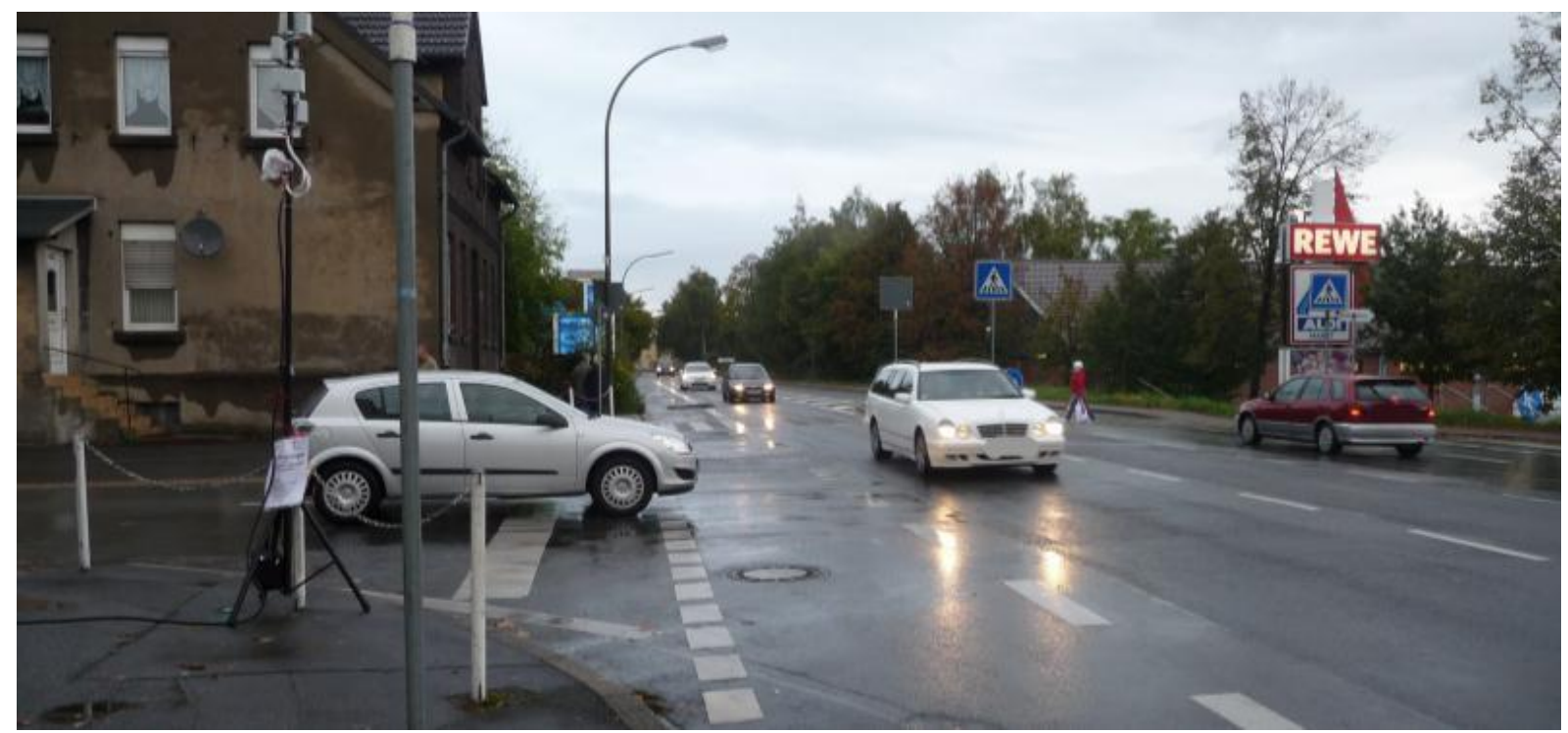

Abbildung 107: Knotenpunkt 11 - Anfahrsicht aus Arm 1 nach links

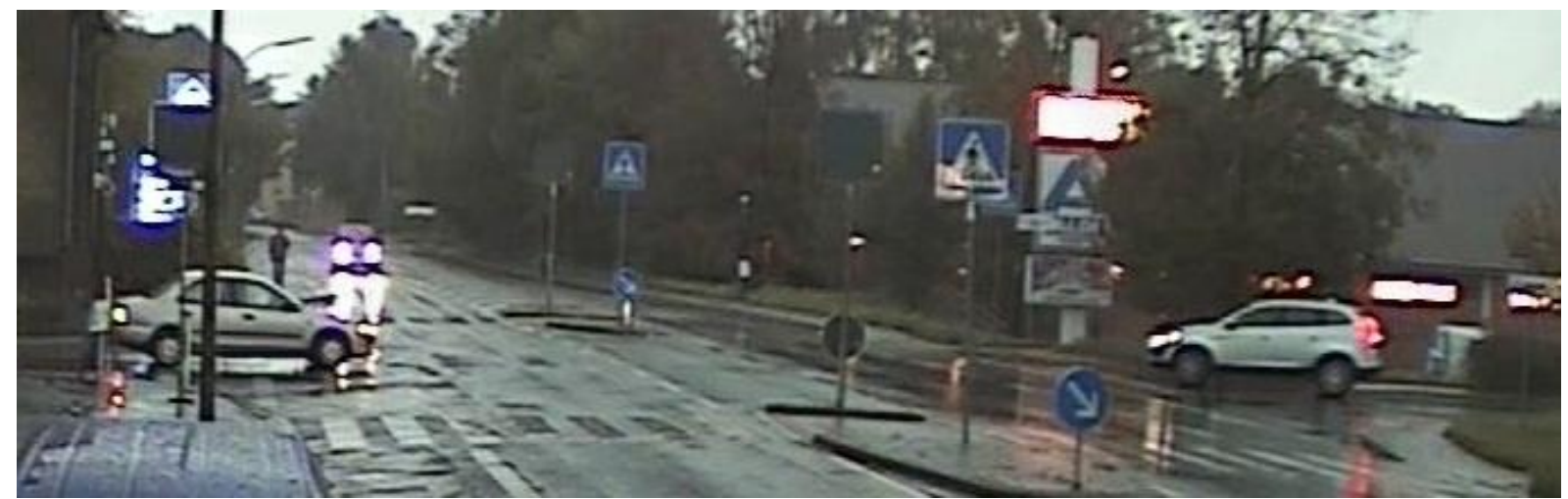

Abbildung 108: Knotenpunkt 11 - Kraftfahrer aus Arm 1 weit vorgefahren

Die Konfliktsituationen, die während der Verhaltensbeobachtung erfasst wurden, entsprechen grundlegend dem Unfallbild (Abbiege- und Einbiegen-Kreuzen-Unfälle). Zudem wurde eine häufige Missachtung des Zeichens 206 „Halt! Vorfahrt gewähren!“ (Stopp-Schild) aus den untergeordneten Straßen festgestellt, dies von jüngeren wie älteren Kraftfahrern gleichermaßen.

Für diesen Knotenpunkt empfiehlt sich aus Gründen der Verkehrssicherheit eine Vollsignalisierung. Die Kreuzung scheint auf den ersten Blick übersichtlich, ist allerdings in Wirklichkeit schwer einsehbar. Zudem führen Sichtbehinderungen und hohe Geschwindigkeiten auf der Hauptstraße (messtechnisch nicht erfasst) dazu, dass viele optische Reize unter Zeitdruck bewältigt werden müssen. 


\section{Knotenpunkt 12}

Abbildung 109 zeigt das Unfalldiagramm auf Basis von Unfällen mit Personenschaden und schwerem Sachschaden für den Zeitraum 2006 bis 2008. Die Kamerastandorte und Zählposten hinsichtlich der Verhaltensbeobachtungen sind ebenfalls aufgeführt. Die Verhaltensbeobachtungen wurden im Mai 2011 durchgeführt.

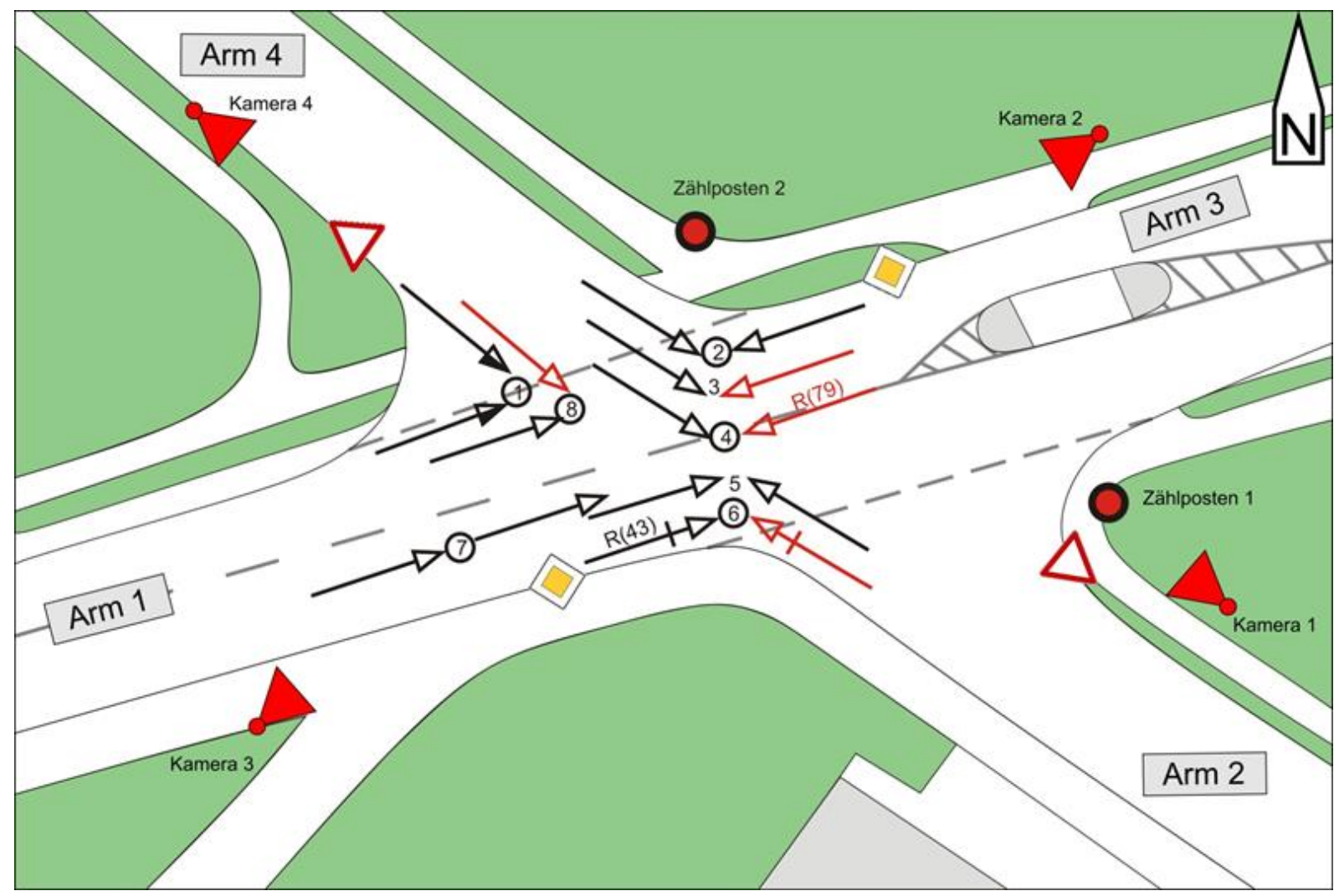

Abbildung 109: Knotenpunkt 12 - Unfalldiagramm, Kamerastandorte und Zählposten

Während der Verhaltensbeobachtungen konnten an diesem Knotenpunkt keine Konfliktsituationen erfasst werden. Insgesamt zeigt sich ein aus verkehrs- und umweltpsychologischer Sicht unauffälliges Bild.

Faktoren, die Einbiegen-Kreuzen-Unfälle begünstigen können, liegen ggf. in der Straßengeometrie der Hauptstraße begründet. Diese folgt von Osten her kommend einer langgezogenen Rechtskurve und führt als Vorfahrtstraße im Vorfeld an einer Eimündung vorbei (Abbildung 110). 


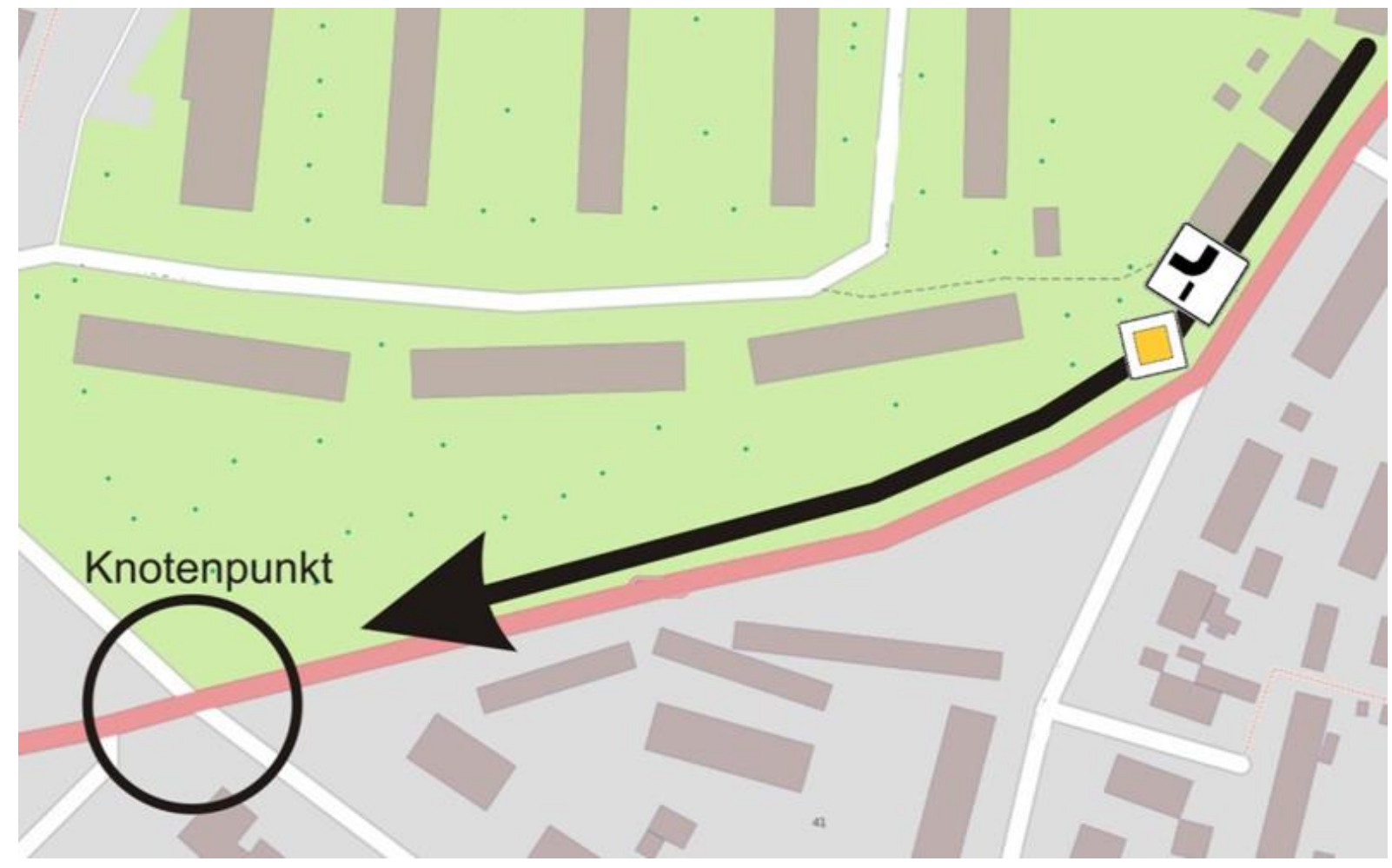

Abbildung 110: Knotenpunkt 12 - Anfahrt aus Richtung Ost [Kartengrundlage: www.openstreetmap.org]

Kraftfahrer, die diesem Verlauf folgen, sind an der im Vorfeld gelegen Einmündung dazu angehalten, rechts zu blinken. Aufgrund des sehr stumpfen Winkles der Kurve, stellt sich der eingeschaltete rechte Blinker im weiteren Verlauf der Straße in einigen Fällen allerdings nicht automatisch zurück, sodass die Fahrzeuge mit eingeschaltetem rechten Blinker an die Kreuzung fahren, allerdings nicht rechts abbiegen, sondern weiter geradeaus fahren.

Passanten wiesen auf diesen Umstand hin. Während der Aufnahmen konnten ebenfalls einige Fälle beobachtet werden. Zudem kann einer Unfallanzeige entnommen werden, dass dies die Ursache für den Unfall war (Unfall 2).

Neben diesem Sonderfall folgt die Straße einer langen Geraden, was ggf. zu hohe Geschwindigkeit bewirken kann (Abbildung 111). 


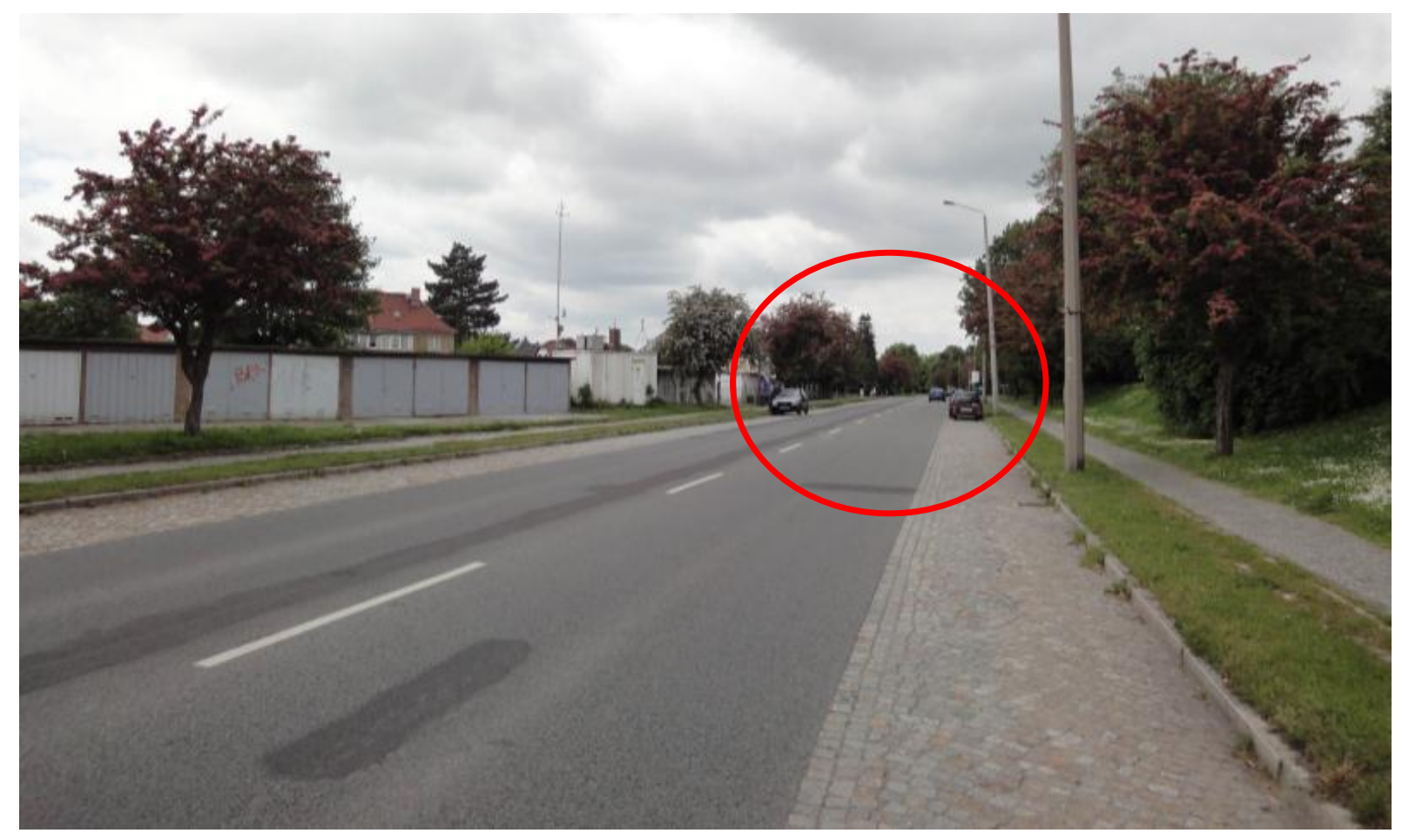

Abbildung 111: Knotenpunkt 12 - Blick auf Knotenpunkt von Osten

Weitere Defizite, die allerdings nicht mit dem Unfallgeschehen direkt in Verbindung stehen, konnten u. a. an der Überquerungsanlage ausgemacht werden. Die angebrachte Bake mit dem Zeichen 222 „Vorgeschriebene Vorbeifahrt - rechts vorbei“ kann die Sicht auf dort wartenden Fußgänger verdecken (Abbildung 112).

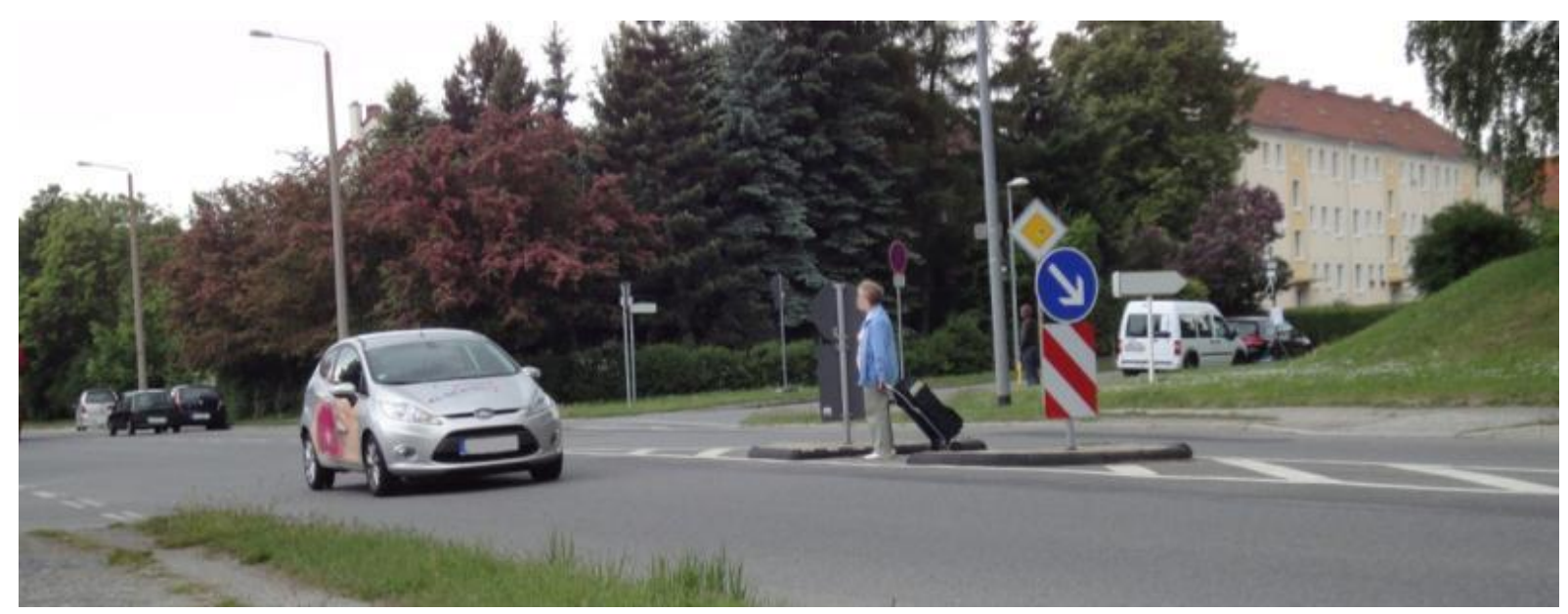

Abbildung 112: Knotenpunkt 12 - Fußgänger an Überquerungsanlage

Radverkehrsanlagen sind an dieser Kreuzung nicht vorhanden. Radfahrer nutzten zumeist den Gehweg bzw. fuhren oder schoben zur Überquerung der Hauptstraße ihr Rad über die Überquerungsanlage (Abbildung 113 und Abbildung 114) und fuhren nur in den seltensten Fällen auf der Straße (Abbildung 115). 


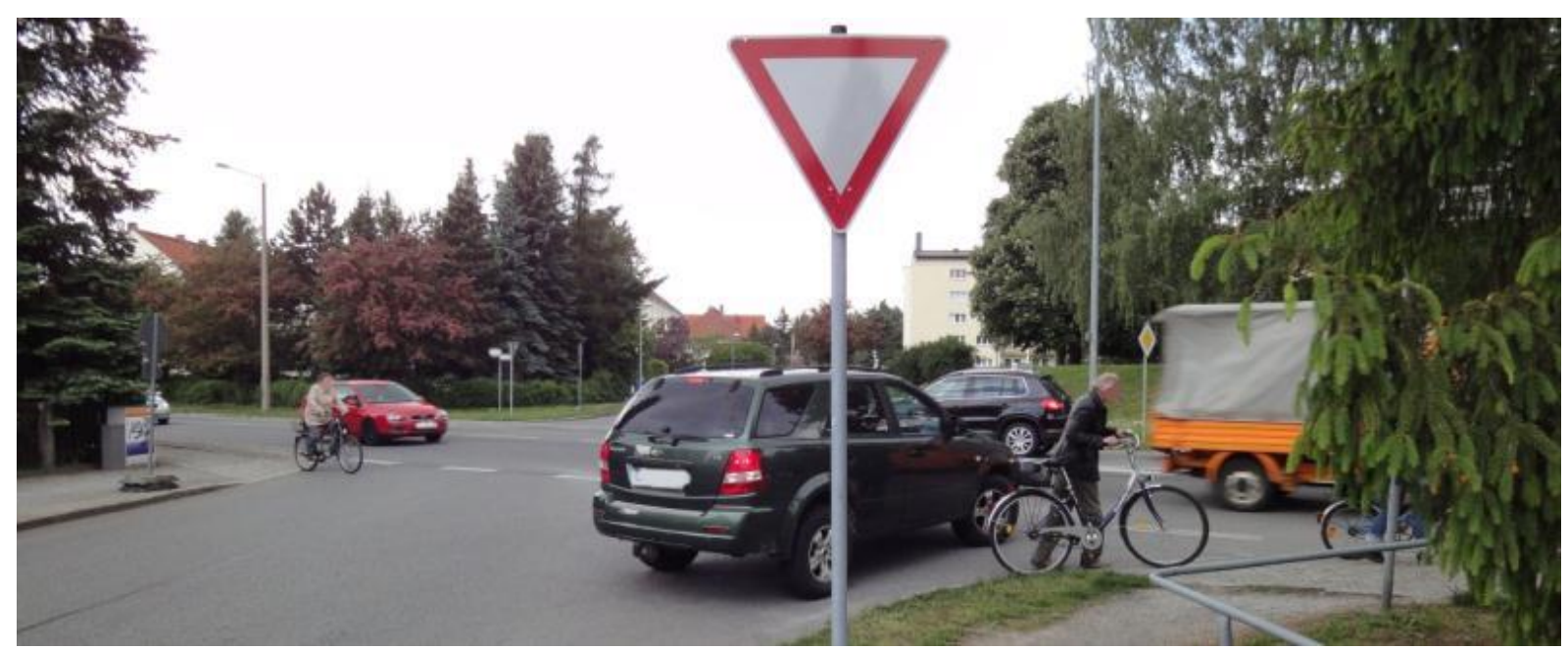

Abbildung 113: Knotenpunkt 12 - Radfahrer aus Arm 2

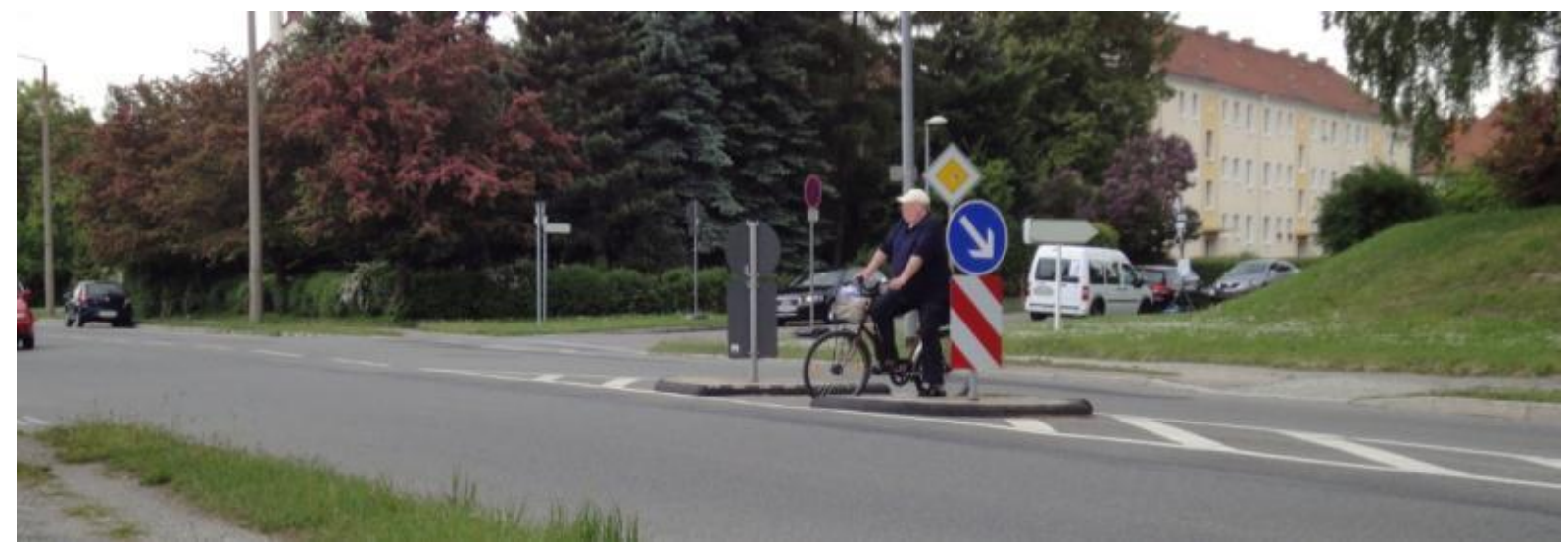

Abbildung 114: Knotenpunkt 12 - Radfahrer auf Mittelinsel

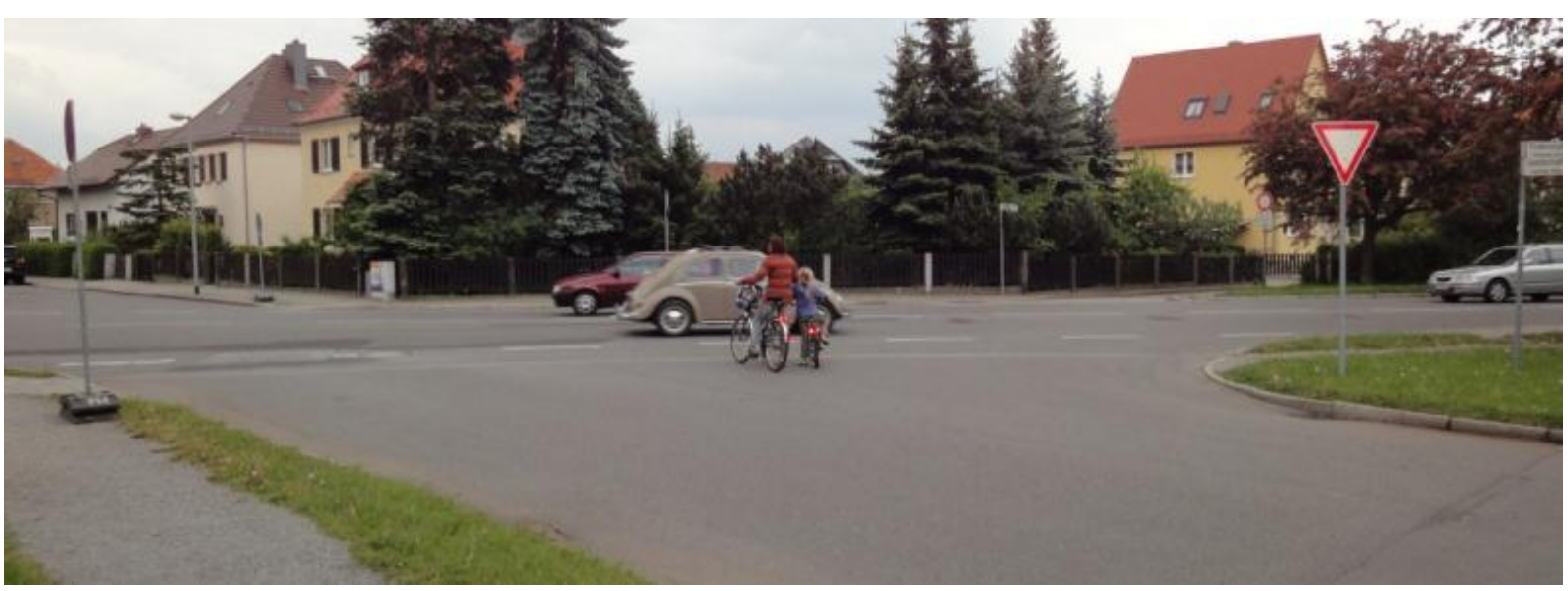

Abbildung 115: Knotenpunkt 12 - Radfahrer aus Arm 4 


\section{Knotenpunkt 13}

Das Unfalldiagramm auf Grundlage von Unfällen mit Personenschaden und schwerem Sachsachschaden aus dem Zeitraum 2004 bis 2010 ist in der Abbildung 116 dargestellt. Kurz vor den Videoaufnahmen, die im März 2011 durchgeführt wurden, wurde in der Zufahrt 2 eine neue Markierung aufgebracht (Abbildung 117). Alle aufgeführten Unfälle fanden zeitlich vor dieser Änderung statt.

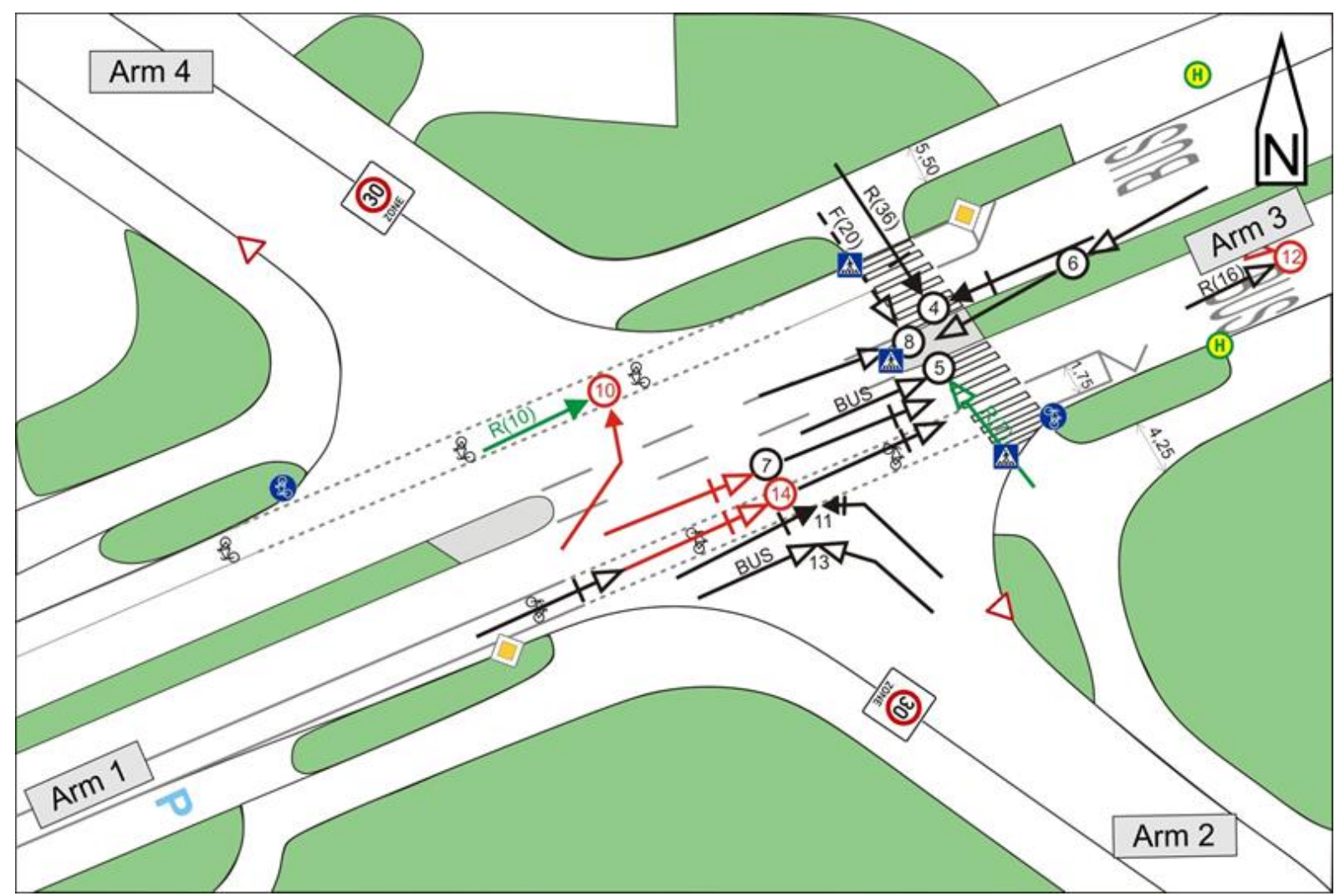

Abbildung 116: Knotenpunkt 13 - Unfalldiagramm (Plangrundlage vor verkehrstechnischer Änderung. Alle Unfälle fanden im Zeitraum vor der Änderung statt.) 


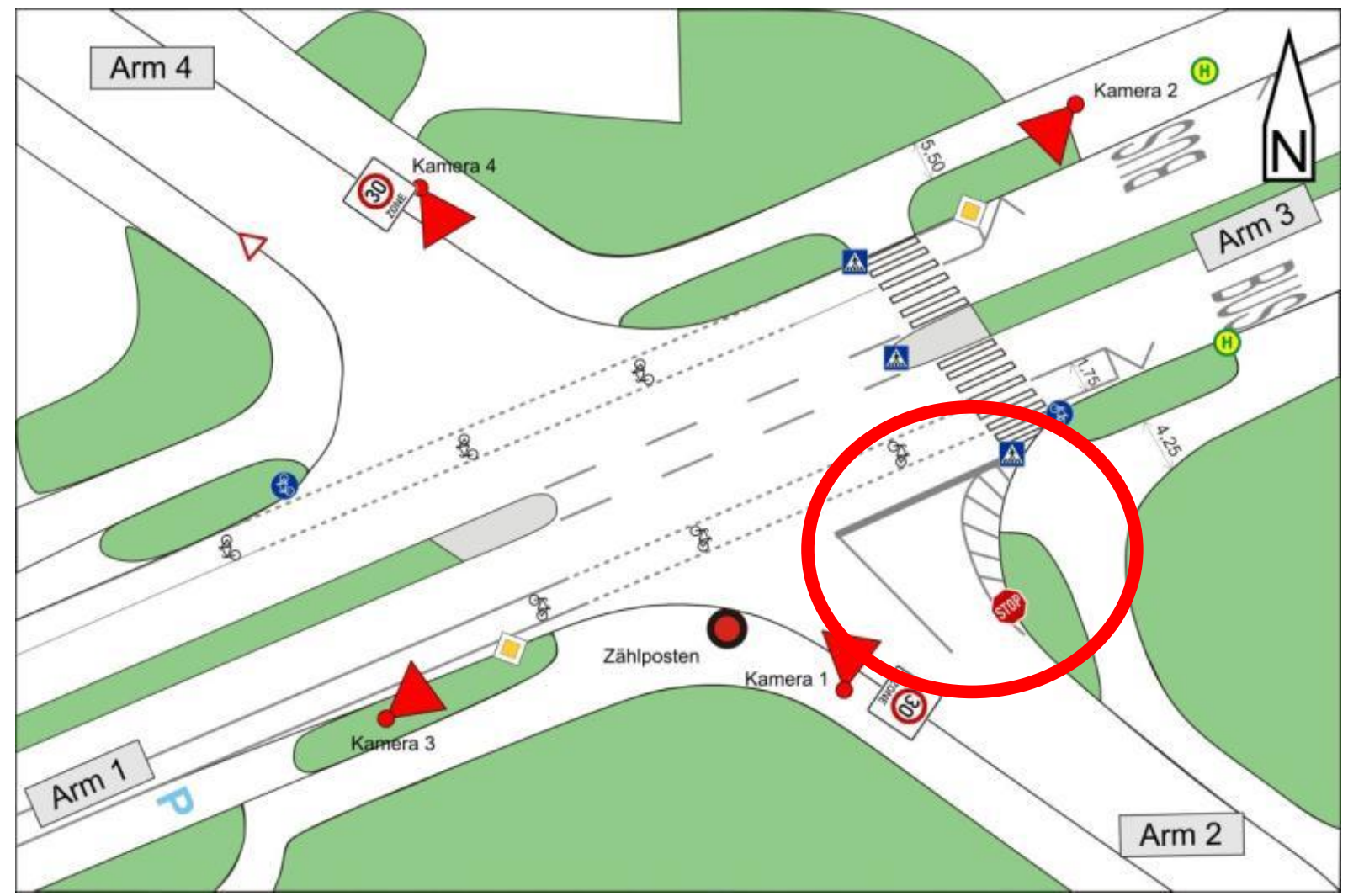

Abbildung 117: Knotenpunkt 13 - Kamerapositionen und Zählposten (Plangrundlage nach Änderung, Markierung in 2011 aufgebracht)

Insgesamt konnten an diesem Knotenpunkt nur wenige Konfliktsituationen beobachtet werden. Allerdings können diese in Beziehung zum Unfallgeschehen am Fußgängerüberweg gesetzt werden, der zur Überquerung einer langen, geraden Hauptstraße dient (Abbildung 118). Aus Arm 1 zufahrende Kfz bremsten zum Teil sehr spät und abrupt vor dem FGÜ ab, um dort wartende Fußgänger passieren zu lassen (Abbildung 119).

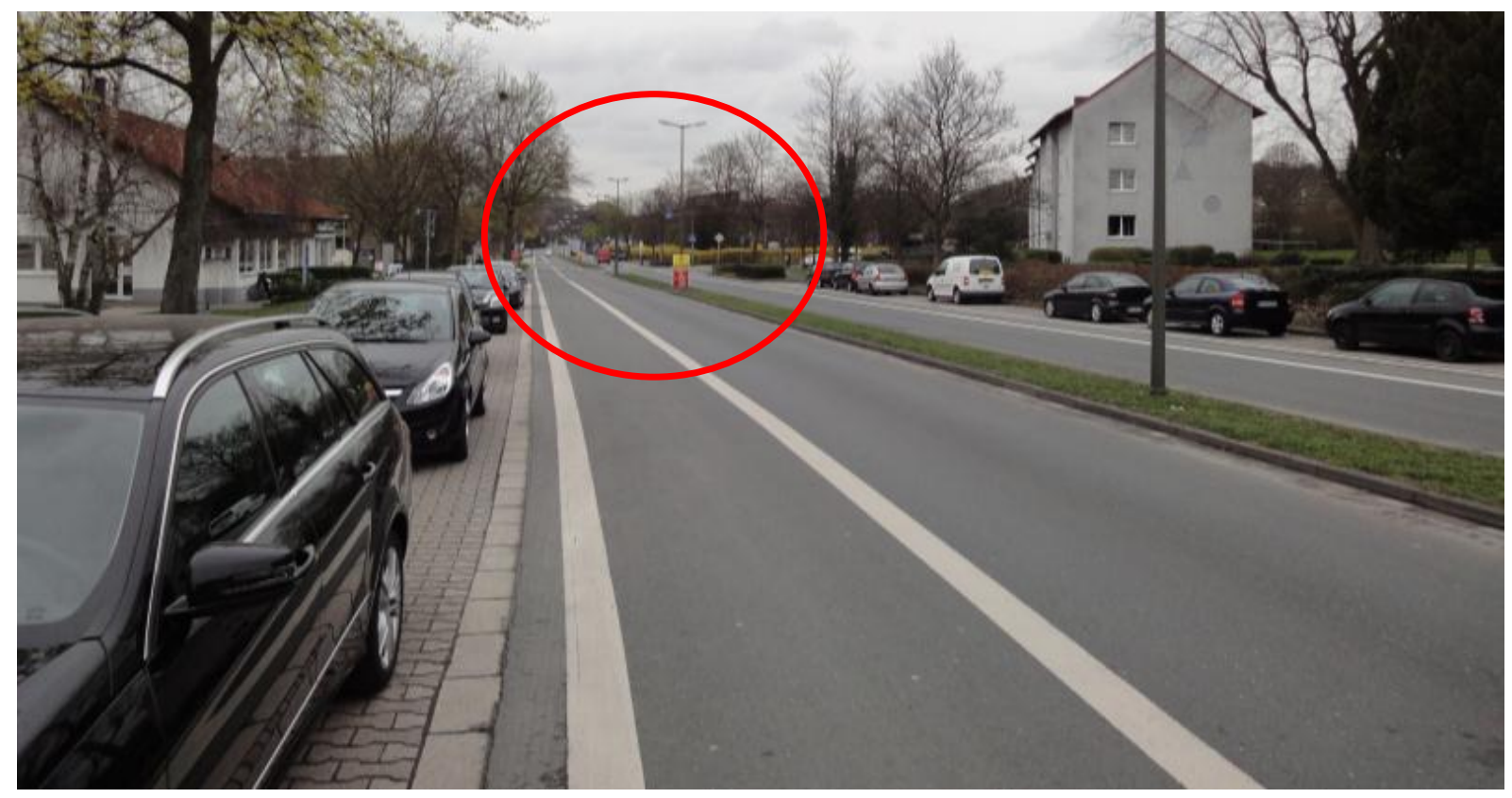

Abbildung 118: Knotenpunkt 13 - Sicht auf Überquerungsanlage aus Arm 1 

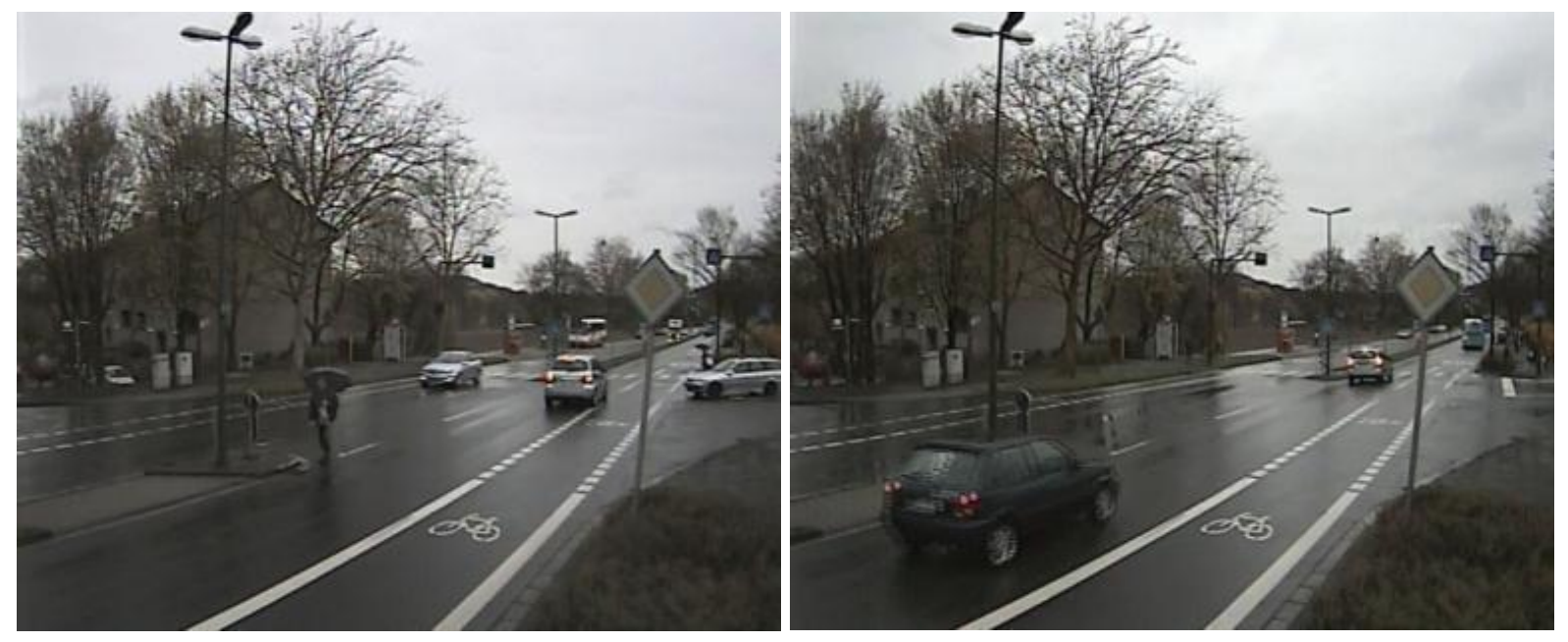

Abbildung 119: Knotenpunkt 13 - Pkw mit abrupten Bremsvorgängen vor FGÜ

Die Vor-Ort-Beobachtungen legen den Schluss nahe, dass die dort wartenden Fußgänger, die aus Sicht der Kraftfahrer vor Bäumen und einer Hecke stehen, insbesondere bei Regen und Dämmerung spät identifiziert werden können. Zur Verbesserung der Sichtbeziehungen, wurden allerdings die direkt an der Überquerung stehenden Hecken (aus Sicht der Fahrer hinter den wartenden Fußgängern) etwa eineinhalb Jahre vor der Vor-Ort-Untersuchung zurückgeschnitten (Abbildung 120).

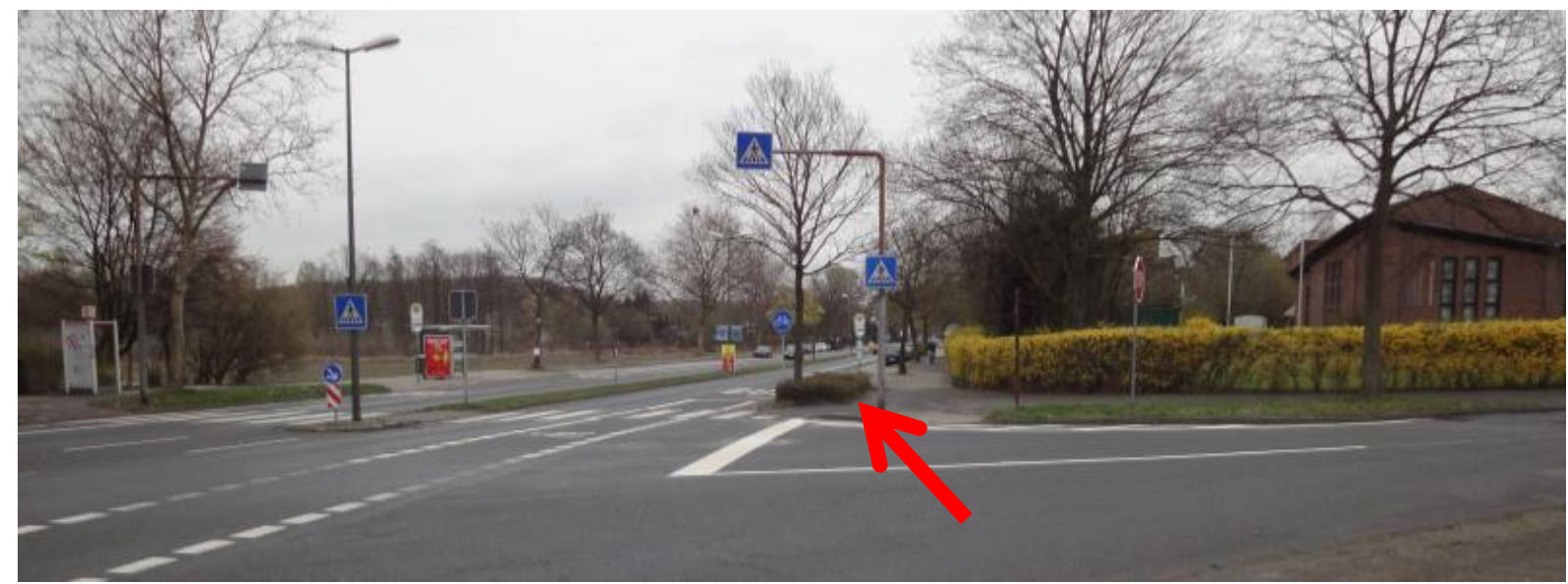

Abbildung 120: Knotenpunkt 13 - Blick auf Überquerungsanlage (FGÜ)

Zur Verbesserung der Verkehrssicherheit wurde zudem die Zufahrtsregelung aus Arm 2 geändert. Das vorher angebrachte Zeichen 205 „Vorfahrt gewähren!“ wurde durch das Zeichen 206 „Halt! Vorfahrt gewähren!“ (Stopp-Schild) ersetzt. Dies in Verbindung mit einer Markierung, die Kraftfahrer in einem nahezu senkrechten Winkel an die übergeordnete Straße heranführt, wodurch die Sichtbeziehungen nach links verbessert werden (Abbildung 121). 

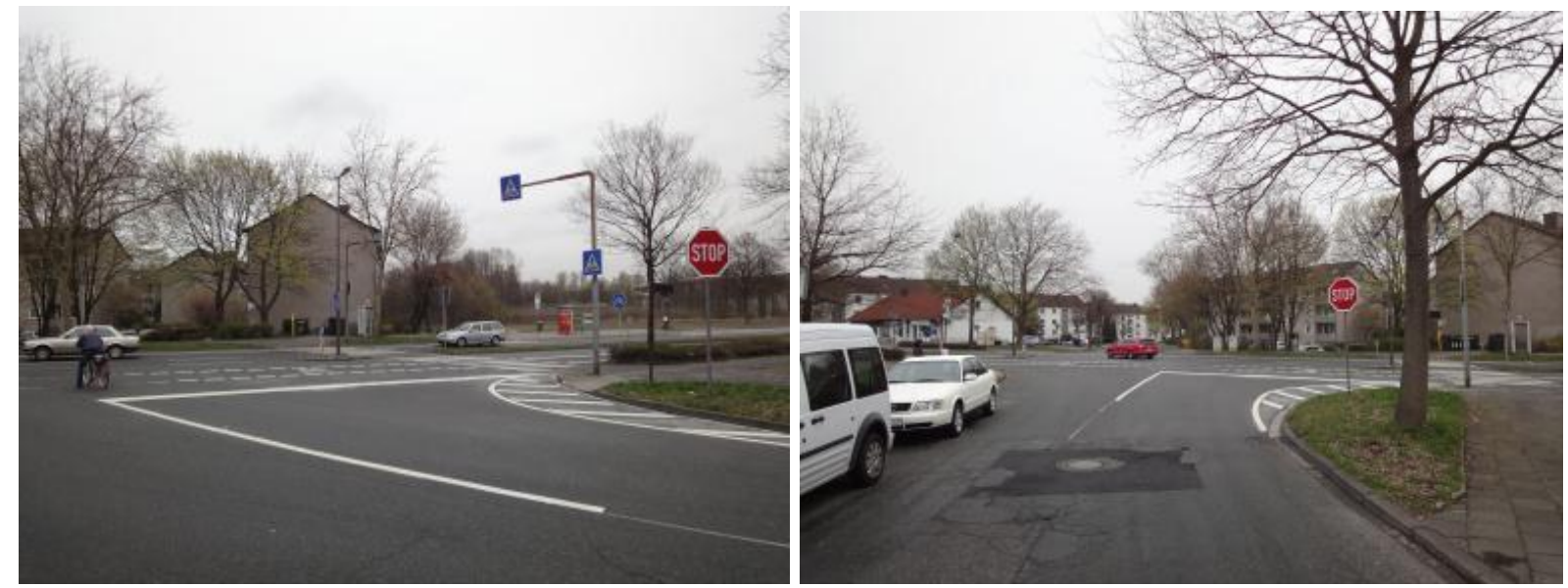

Abbildung 121: Knotenpunkt 13 - Neue Markierung und Verkehrsregelung der Zufahrt aus Arm 2

Allerdings konnte auch an diesem Knotenpunkt festgestellt werden, dass Kraftfahrer aller Altersklassen sich häufig nicht vorschriftsmäßig „am Stopp-Schild verhalten“, sondern ohne inr Fahrzeug vollständig zum Stehen gebracht zu haben in die Hauptstraße einbiegen. 


\section{A 5 Sicherheitsanalysen und Verhaltensbeobachtungen im Realverkehr an Einmündungen mit vorfahrtregelnden Verkehrszeichen}

\section{Knotenpunkt 14}

Abbildung 122 zeigt das Unfalldiagramm für Unfälle der Kategorien 1 bis 4 der Jahre 2006 bis 2009, zu denen detaillierte Informationen in Form von anonymisierten Verkehrsunfallanzeigen vorlagen. Zudem sind die Kamerapositionen und Aufnahmerichtungen für die Verhaltensbeobachtungen in dieser Abbildung dargestellt. Die Verhaltensbeobachtungen wurden im April 2011 durchgeführt.

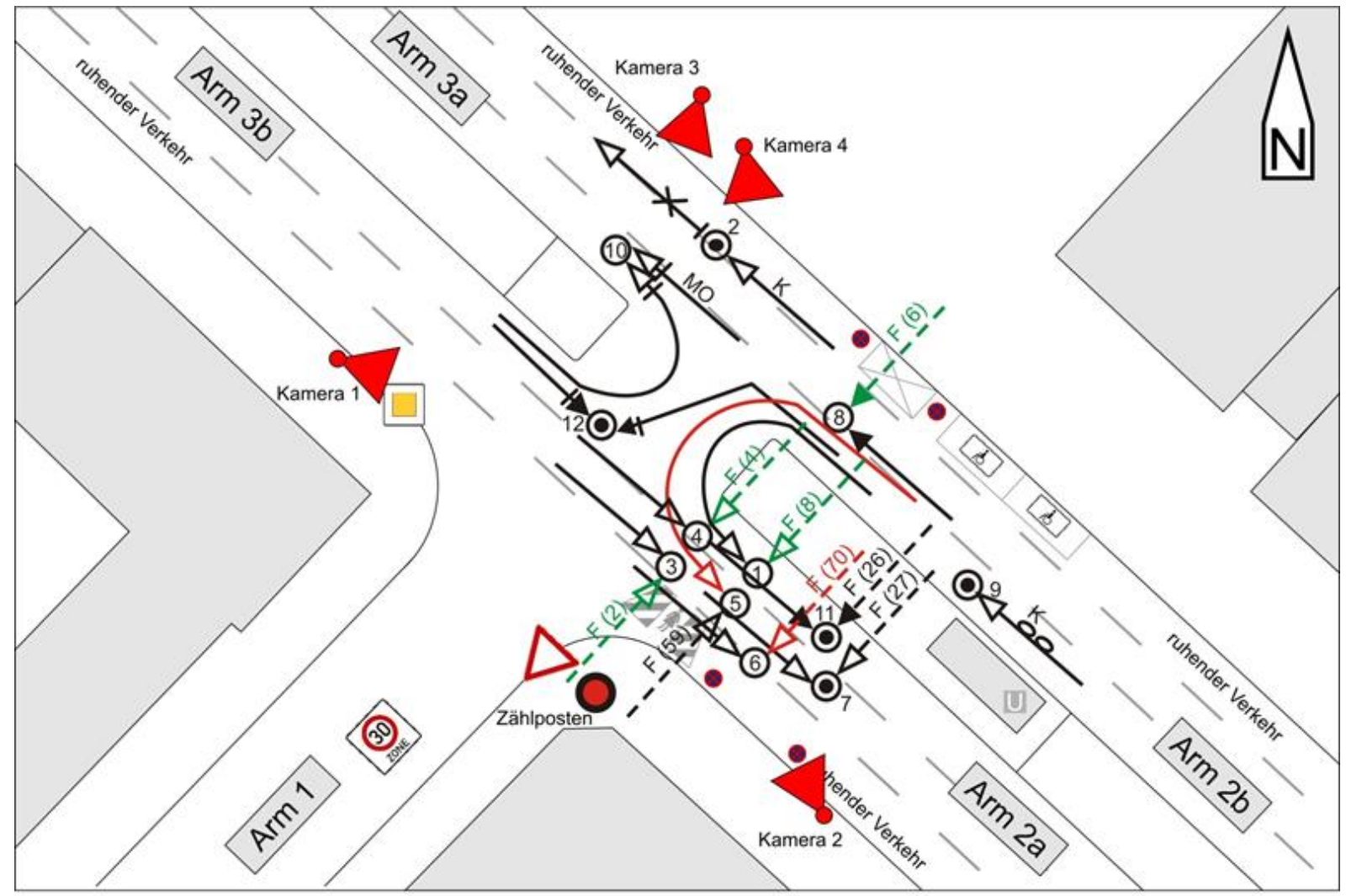

Abbildung 122: Knotenpunkt 14 - Unfalldiagramm, Kamerastandorte und Zählposten

Das Unfalldiagramm zeigt den Unfallschwerpunkt an der Überquerung des Knotenpunktarms 2a. Zwar wurden fünf der dort geschehenen sieben Unfälle mit Beteiligung von Fußgängern, von den Fußgängern selber verursacht, dennoch lassen sich an dieser Überquerung auch deutliche Sicherheitsdefizite ausmachen.

Die Hauptgefahren, insbesondere für Fußgänger, ergeben sich an den Überquerungen 2a und 2b. Hier müssen Fußgänger, die z. B. den Zugang zur U-Bahn-Haltestelle im Bereich des Mittelstreifens erreichen wollen, Richtungsfahrbahnen mit jeweils zwei Fahrsteifen 
überqueren, die in beiden Richtungen eine verkehrliche Belastung von etwa 15.000 $\mathrm{Kfz} / 24 \mathrm{~h}^{34}$ aufweisen.

Die wesentlichen Sicherheitsdefizite lassen sich an der Überquerung 2a ausmachen. Die Überquerungsstelle ist unzureichend ausgewiesen und für geradeausfahrende Kfz-Führer aus Arm 3b schwer zu erkennen (Abbildung 123).

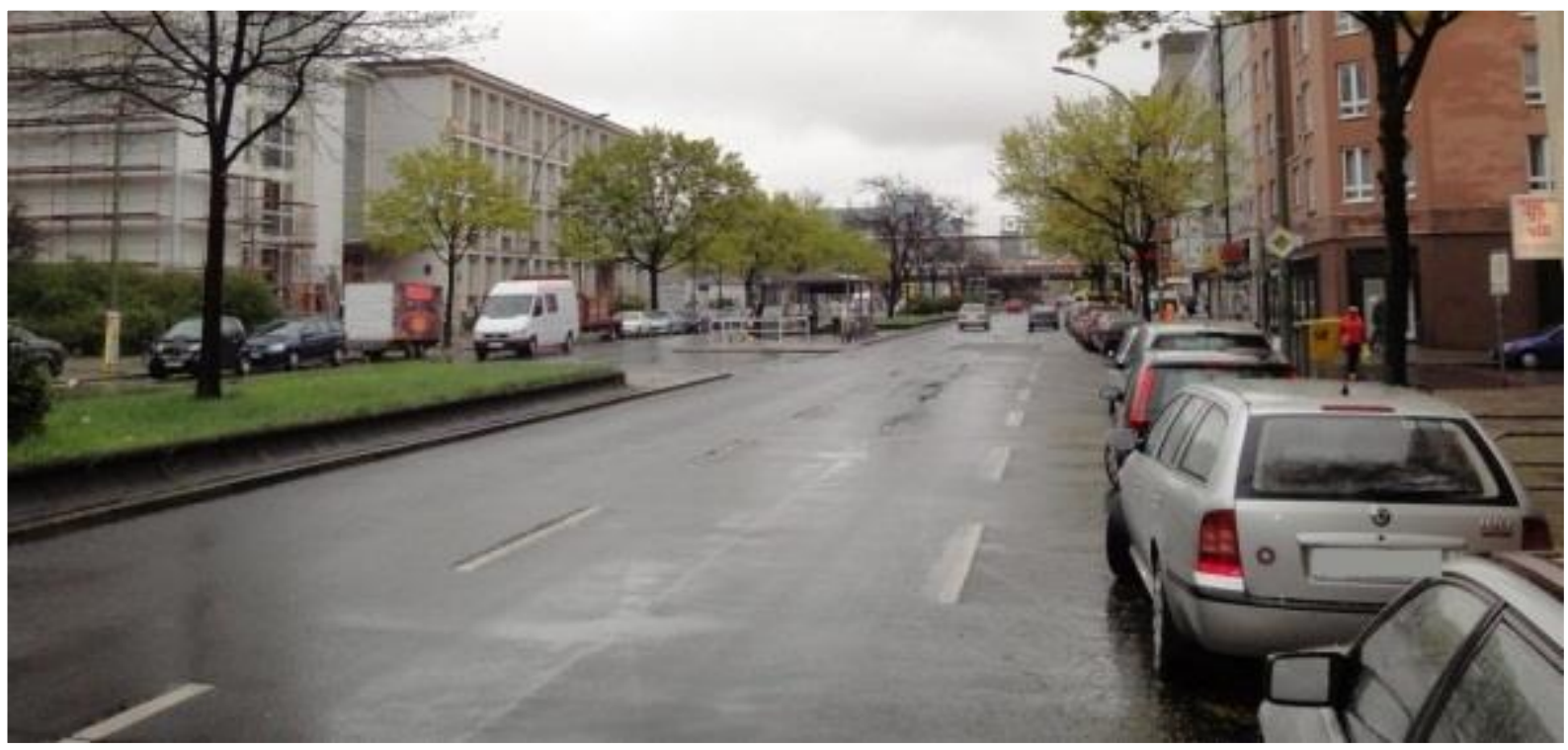

Abbildung 123: Knotenpunkt 14 - Anfahrsicht auf Überquerung 2a

Hinzu kommt, dass die Sichtbeziehung zwischen geradeausfahrendem Kfz-Verkehr und wartepflichtigen Fußgängern durch die am Fahrbahnrand parkenden Fahrzeuge beeinträchtigt ist.

Die Auftstellfläche (Sperrfläche) an dieser Überquerung ist nicht dazu geeignet, Fußgängern den nötigen Schutz zu bieten. Aufgrund der parkenden Fahrzeuge am Fahrbahnrand des Arms 3a, müssen die Fußgänger nah an den befahrenen (mittleren) Fahrstreifen herantreten, um die Straße einsehen zu können. Zudem liegt die Auftstellfläche in der direkten Fahrlinie der aus der untergeordneten Straße rechtseinbiegenden Fahrzeuge (Abbildung 124, links). Auch das Parken ist hier nicht wirksam unterbunden. Die Auftstellfläche war während der Beobachtungszeit nahezu ständig durch kurzzeitig parkende Fahrzeuge blockiert (Abbildung 124, rechts).

\footnotetext{
${ }^{34}$ Hochrechnung auf Basis von zwei 1-Stundenzählung (Zählung 1: 10-11 Uhr, Zählung 2: 13-14 Uhr) an einem mittleren Werktag.
} 

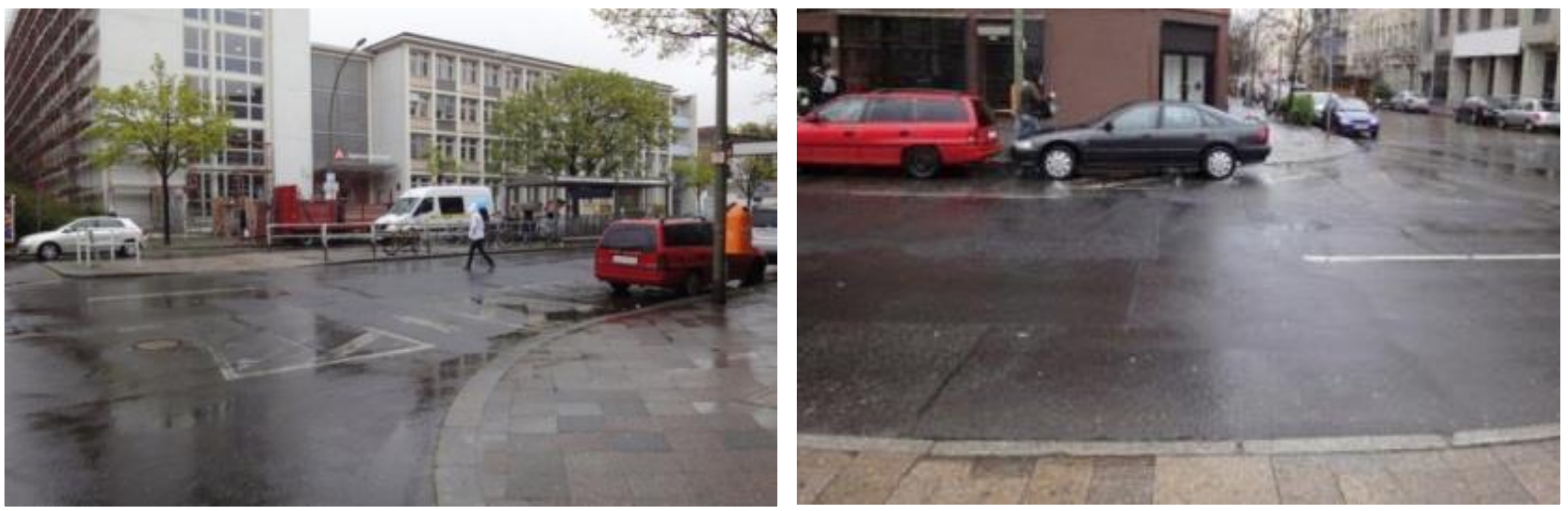

Abbildung 124: Knotenpunkt 14 - Schlechte Erkennbarkeit / keine Schutzfunktion der Auftstellfläche (Bild links) und zugeparkte Auftstellfläche an der Überquerungsstelle 2a (Bild rechts)

Aufgrund der hohen Verkehrsstärke der Hauptrichtung (Arm 2-3) entstehen zum Teil lange Wartezeiten für Fußgänger, aber auch für einbiegende Kfz aus Arm 1 und für abbiegende und wendende $\mathrm{Kfz}$ aus Arm 2b. Die entstehenden kurzen Zeitlücken im Hauptstrom werden oft gleichzeitig von einbiegenden, abbiegenden und wendenden Kfz sowie von Fußgängern, die in dieser Situation bevorrechtigt sind, genutzt (Abbildung 125).

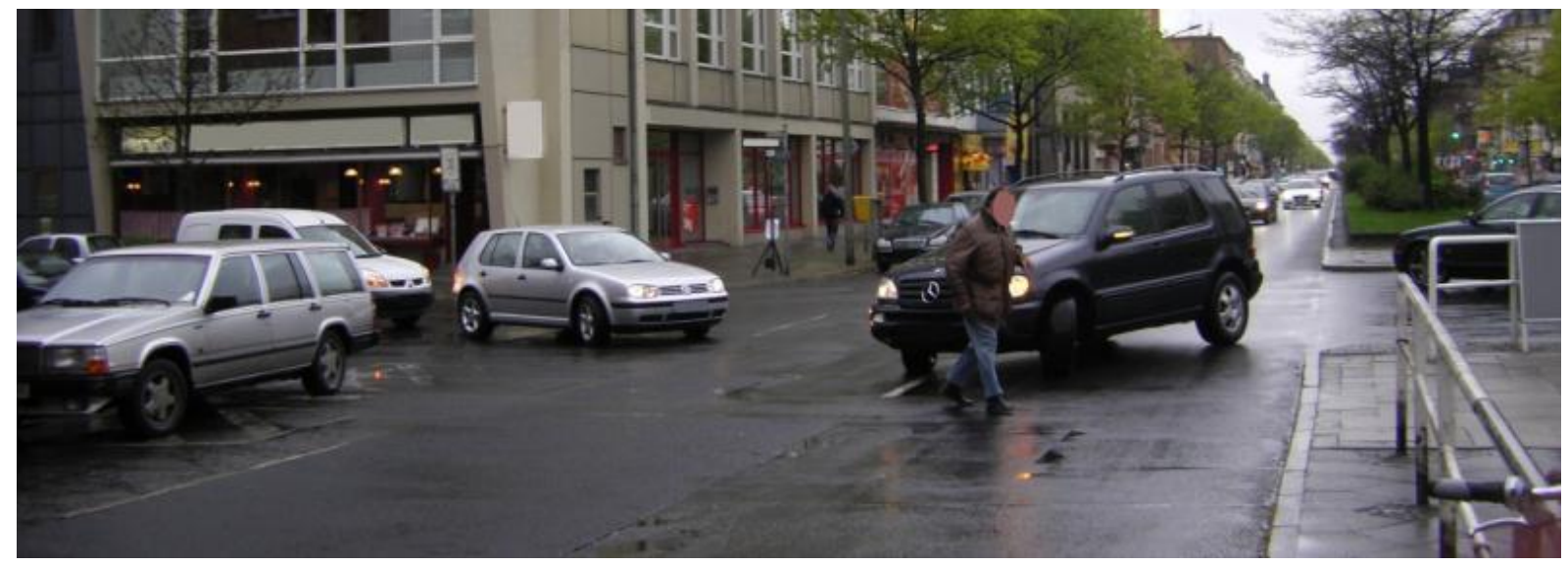

Abbildung 125: Knotenpunkt 14 - Überquerender Fußgänger bei gleichzeitig rechtseinbiegenden und wendenden Fahrzeugen

Die Verhaltensbeobachtungen belegen, dass den Fußgängern in dieser Situation das Vorrangsrecht gegenüber den einbiegenden und wendenden $\mathrm{Kfz}$ bei der Straßenüberquerung oft nicht eingeräumt wird bzw. sich die Kfz-Führer nicht so verhalten, dass eine Gefährdung anderer Verkehrsteilnehmer ausgeschlossen ist. Situationen mit „Beinahe-Konflikten“ zwischen Fußgängern und Kfz konnten häufig beobachtet werden.

Eine weitere Schwierigkeit ergibt sich aus der Gestaltung des Kreuzungsbereiches. Leit- und Wartelinien für Linksabbieger (und Wender) sind nicht angebracht. Die Fläche ermöglicht zudem, dass sich Linksabbieger und Wender beider Fahrtrichtungen nebeneinander aufstellen können (Abbildung 126), was zu unübersichtlichen Situationen führt. Für Fußgänger ist es zum Teil nicht zu erkennen, ob die dort wartenden Fahrzeuge links in Arm 1 abbiegen oder von Arm 2b nach Arm 2a wenden werden. 


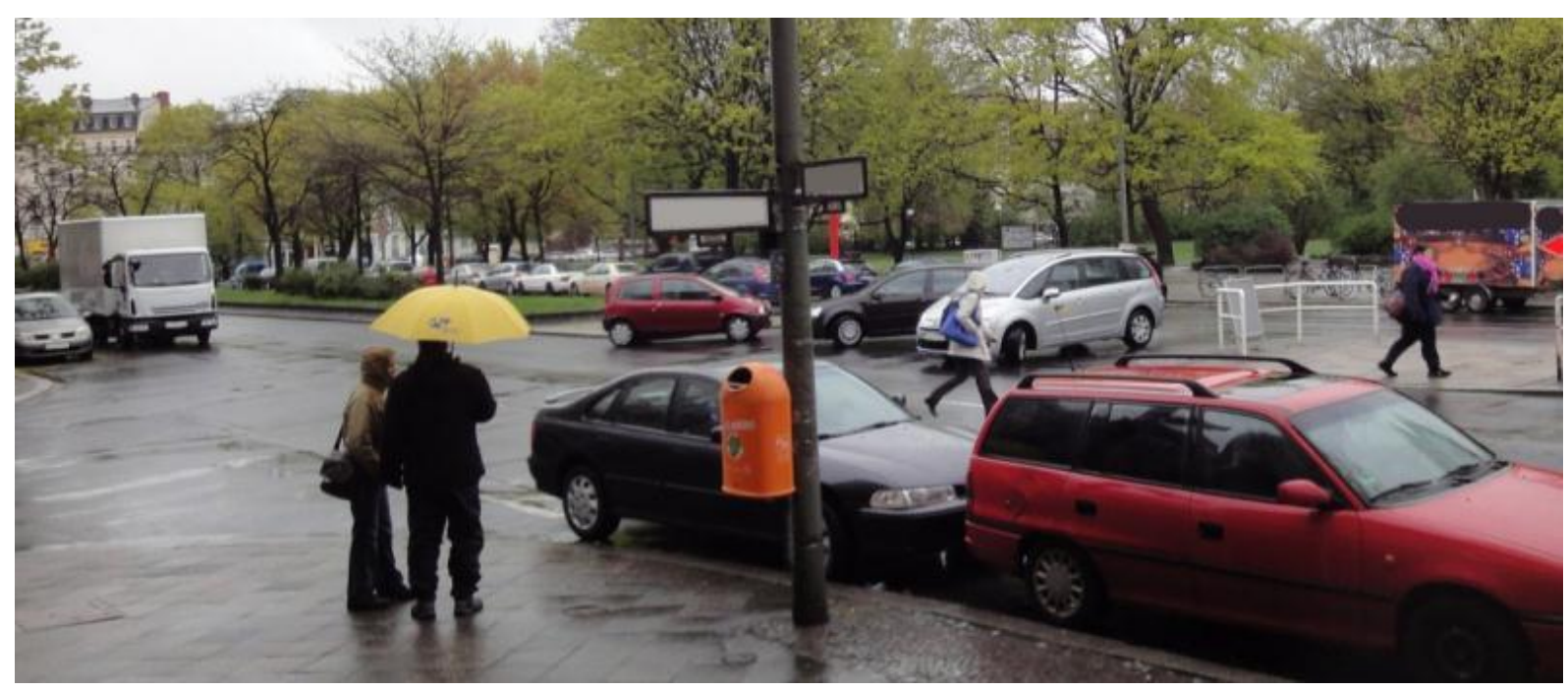

Abbildung 126: Knotenpunkt 14 - Nebeneinanderaufstellung wartepflichtiger Fahrzeuge im Kreuzungsbereich

Im Ergebnis der Verhaltensbeobachtungen bleibt festzuhalten, dass zahlreiche Konfliktsituationen mit einer erheblichen Gefährdung von Kindern beobachtet werden konnten. Die beobachteten Konflikte entsprachen auch Unfallbild.

Aufgrund der Verkehrsmengen im Kfz-Verkehr (je Richtungsfahrbahn der Hauptachse etwa $15.000 \mathrm{Kfz} / 24 \mathrm{~h}$ ) bei gleichzeitig hohem Überquerungsbedarf, ist die Installation einer Lichtsignalanlage und die damit verbundene sichere Führung der Fußgänger die optimale Lösung für diesen Knotenpunkt.

In Ergänzung zur Lichtsignalanlage können vorgezogene Seitenräume im Bereich der Überquerung einen weiteren Schutz bieten bzw. die Sichtbeziehungen zwischen Fußgängern und Kfz-Führern verbessern. 


\section{Knotenpunkt 15}

Der Knotenpunkt 15 wurde im Wesentlichen aufgrund von Unfällen mit Beteiligung von Kindern in die Detailanalyse und Verhaltensbeobachtungen mit aufgenommen. Diese Unfälle wiesen in der makroskopischen Analyse elektronischer Datenbestände aus 2004 bis $2008 \mathrm{u}$. a. die bei Kindern häufig vorkommenden Einbiegen-Kreuzen-Unfälle vom Typ 342 auf (Radfahrer vom Radweg, von rechts kommend). Von 7 Unfällen aus diesem Zeitraum, waren sechs vom Typ 342, davon 3 mit Beteiligung eines Kindes.

Abbildung 127 zeigt das Unfalldiagramm für Unfälle der Kategorien 1 bis 4 der Jahre 2007 bis $2010 \mathrm{zu}$ denen Informationen aus anonymisierten Verkehrsunfallanzeigen vorlagen. Zudem sind die Kamerapositionen und Aufnahmerichtungen für die Verhaltensbeobachtungen in dieser Abbildung dargestellt. Die Verhaltensbeobachtungen wurden im Mai 2011 durchgeführt.

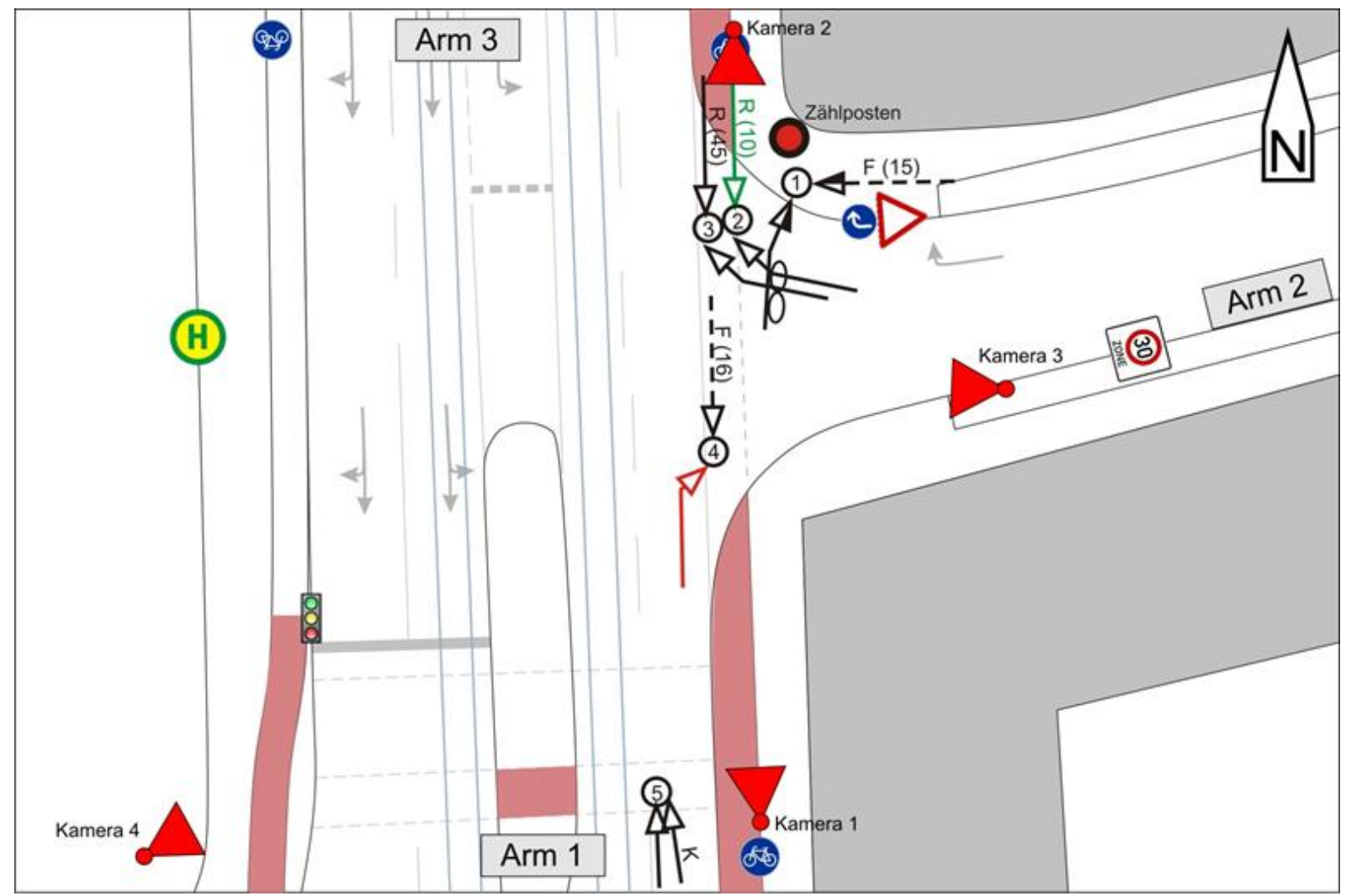

Abbildung 127: Knotenpunkt 15 - Unfalldiagramm, Kamerastandorte und Zählposten

Während der Verhaltensbeobachtungen konnten Radfahrer, die die Einrichtungsradwege zu beiden Seiten der Hauptstraße (Arm 1-3) entgegen der vorgeschriebenen Fahrtrichtung nutzten, beobachtet werden. Allerdings konnten nur wenige Konfliktsituationen an der Überquerung an Arm 2 mit einbiegenden Fahrzeugen festgestellt werden, die allerdings dem Unfallbild, insbesondere Typ 342, entsprachen.

Zu den Defiziten, die an dieser Einmündung den Unfalltyp 342 begünstigen können, zählt die eingeschränkte Sicht der KfZ-Führer aus Arm 2, bedingt durch die geschlossenen Randbebauung zu beiden Seiten der Straße, auf mögliche Radfahrer aus Arm 3. Zudem ist die markierte, aber nicht eingefärbte Radverkehrsfurt im Bereich der Überquerung schlecht zu erkennen (Abbildung 128). 


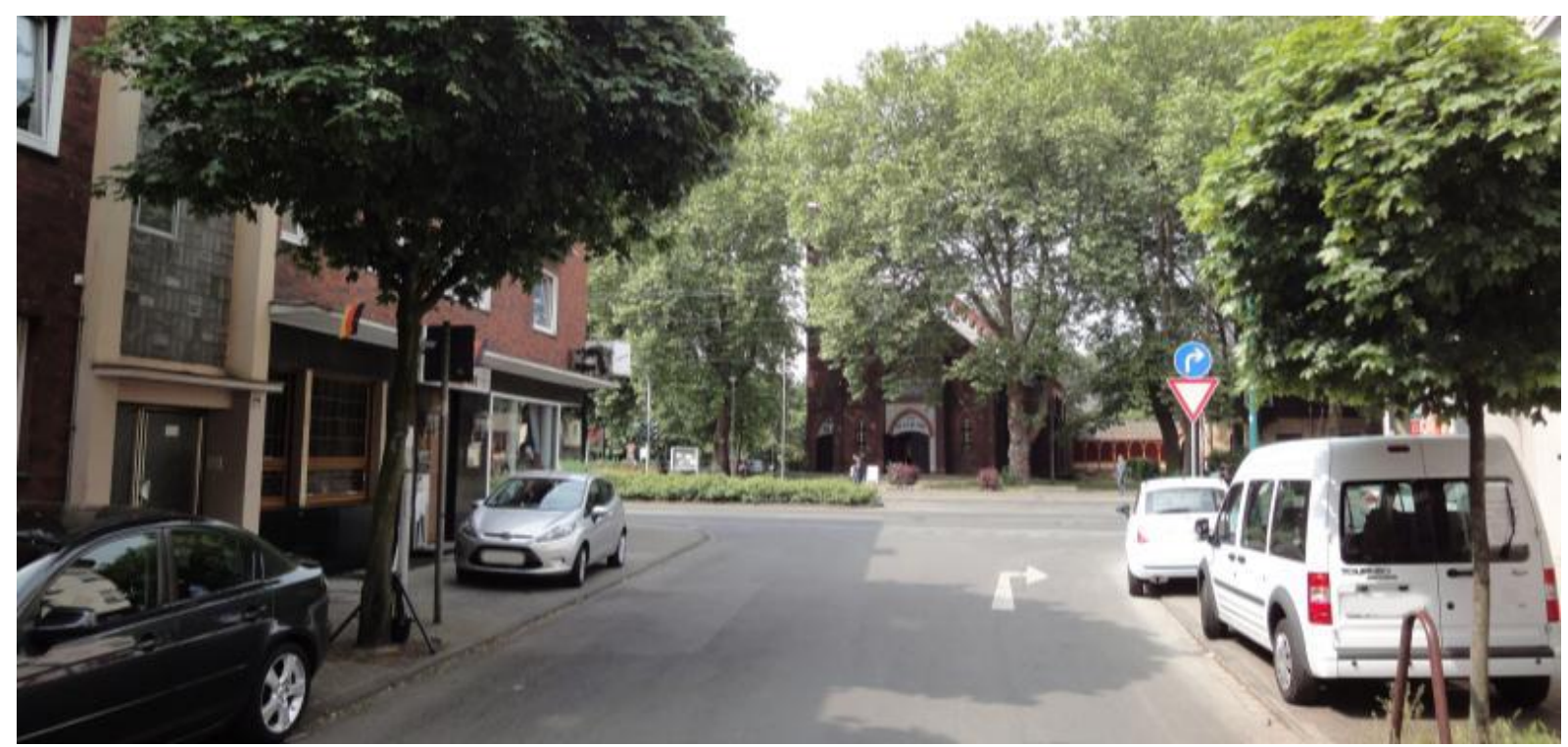

\section{Abbildung 128: Knotenpunkt 15 - Sicht aus Arm 2}

Eine weitere, vom festgestellten Unfallgeschehen unabhängige Auffälligkeit an dieser Einmündung ergibt sich durch den Umstand, dass die Borde im Bereich der Überquerung an Arm 2 nur auf der Breite des Radeweges auf Nullniveau abgesenkt sind. Personen mit Kinderwagen oder Rollatoren nutzten hier die Nullabsenkung und somit den Radweg bzw. die Radverkehrsfurt zur Überquerung (Abbildung 129).
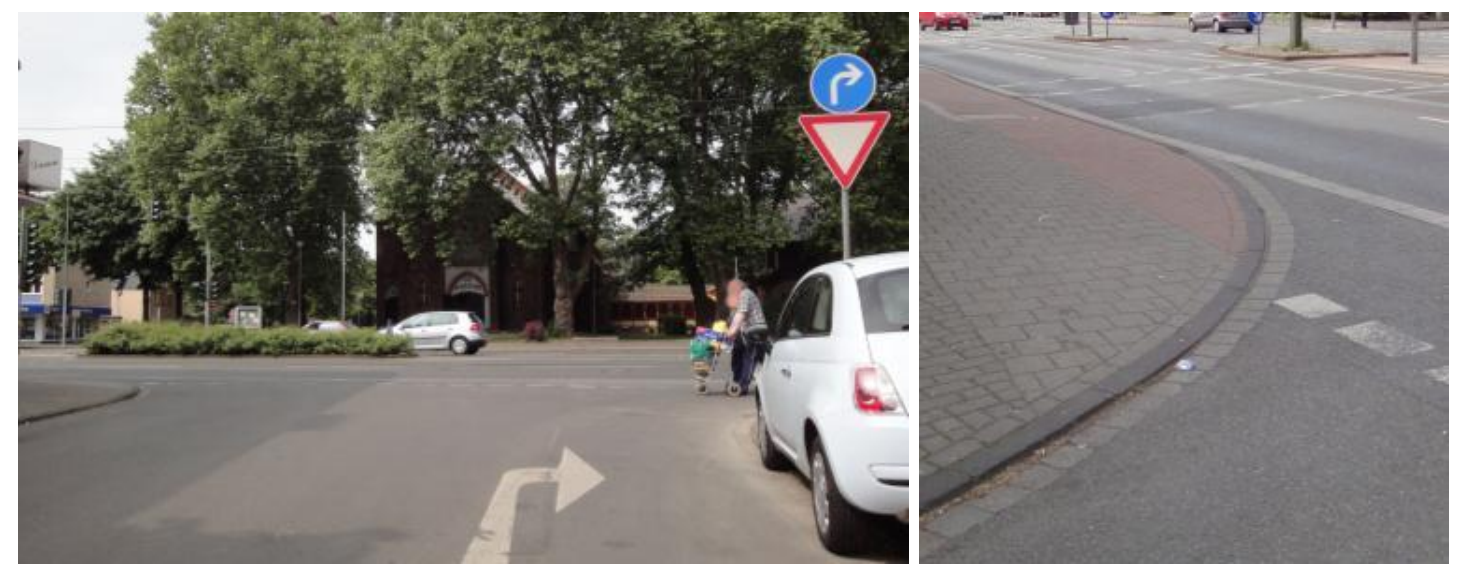

Abbildung 129: Knotenpunkt 15 - Rollatorennutzer an Überquerung (Bild links) und Nullabsenkung auf Breite des Radweges (Bild rechts)

Aufgrund der durchaus vorkommenden Situation, dass Radfahrer die Einrichtungsradwege entgegen der vorgeschriebenen Fahrtrichtung nutzen, empfiehlt sich die Überprüfung der Radwegebeziehungen und die Schaffung ausreichender Überquerungsmöglichkeiten über die Hauptstraße. Im Weiteren kann eine rote Einfärbung der Radverkehrsfurt an der Überquerung des Arms 2, ggf. mit zusätzlichem Hinweis durch das Zeichen 138 der StVO „Radfahrer kreuzen“, die Aufmerksamkeit der einbiegenden KfZ-Führer auf Radfahrer erhöhen und somit zur Verbesserung der Situation beitragen.

Auch die Verlegung des Radweges vom Niveau des Gehweges auf die Straße, in Ausführung als Radfahrstreifen, kann dazu beitragen, dass Radfahrer hier nicht entgegen der vorgeschriebenen Richtung fahren. Zudem würde sich, durch die damit verbundene Verbreiterung des Gehweges und die Trennung von Rad- und Fußverkehr im Bereich der 
Überquerung, die Situation für Fußgänger verbessern. Konflikte zwischen mobilitätseingeschränkten Personen, die die Nullabsenkung nutzen, und Radfahrern, wären weitgehend ausgeschaltet. 


\section{A 6 Detaillierte Auswertung der Verhaltensbeobachtungen nach Gruppe und Verkehrssituation}

Insgesamt wurden etwa 300 Stunden Videomaterial an den 15 beobachteten Knotenpunkten aufgenommen und anschließend im Detail ausgewertet.

Nachfolgend werden die Besonderheiten der einzelnen schwächeren Verkehrsteilnehmer nach Gruppe und Verkehrssituation dargestellt.

Hierbei werden die Videoauswertungen in Form von Balkendiagrammen für jeden Knotenpunkt dargestellt. Das Hauptmerkmal für jede Subgruppe ist die Frage, ob die beobachtete Einzelperson ein StVO-konformes Verhalten gezeigt hat. Hierdurch können die relativen Anteile zwischen konformen und nicht-konformen Verhalten für jede Subgruppe miteinander verglichen werden. In einem weiteren Schritt wurde bei der Auswertung festgehalten, worin das nicht konforme Verhalten besteht. Diese Ergebnisse sind im Wesentlichen in die Darstellung der Sicherheitsanalysen und Verhaltensbeobachtungen (Abschnitt 6.3) eingeflossen, woraus sich u. a. auch spezifischen Gefährdungspotenziale für einzelne Verkehrssituationen ergeben.

Bei der Darstellung der spezifischen Subgruppen wird immer zwischen der Vollzählzeit (in der alle Verkehrsteilnehmer gezählt wurden und das Verhalten aller Verkehrsteilnehmer ausgewertet wurde) und der „übrigen Zeit“ unterschieden. In der Vollzählzeit (in der Regel zwischen 10 und $11 \mathrm{Uhr}$ ) wurde eine Vollzählung aller Verkehrsteilnehmer am beobachteten Knotenpunkt vorgenommen, die eine weitere Grundlage für die Auswertung darstellt. Für die Verhaltensbeobachtung wurden alle Verkehrsteilnehmer ausgewertet, die in dieser Zeit an den Zählposten vorbeifuhren oder vorbeigingen. Für diese Zeit kann deshalb ein genauer Vergleich zwischen der intendierten Untergruppe und den restlichen Verkehrsteilnehmern gezogen werden. Die übrige Zeit (in der Regel von 11 bis 13 Uhr und von 14 bis 15 Uhr) wurde nur das Verhalten der schwächeren Verkehrsteilnehmer ausgewertet. Auswertdaten der Videobeobachtung und Daten der 2 stündlichen Verkehrszählungen wurden für die folgende Auswertung kombiniert.

Bei der Ermittlung der Fehleranzahl sind auch „doppelte“ Fehler möglich: So wird z. B. ein Radfahrer, der die Fußgängerfurt nutzt und gleichzeitig bei ROT losfährt mit 2 Fehlern notiert. In Balkendiagrammen entspricht eine Ziffer jedoch immer einer Person. Es kann allerdings mehr Fehler (in den Bemerkungen) als Personen geben.

\section{Auswertung des Verhaltens von Fußgängern}

Nachfolgend wird das Verhalten als Fußgänger der verschiedenen Zielgruppen an den Knotenpunkten miteinander verglichen. Wichtige Erkenntnisse zur Verbesserung der Gestaltung von Knotenpunkten, die auch auf der Beobachtung des Verkehrsverhaltens der verschiedenen Fußgängergruppe beruhen, wurden in den vorangegangenen Kapiteln beschrieben. Die quantitativen Daten der Fußgängergruppen werden in Balkendiagrammen nebeneinander dargestellt (Tabelle 21 bis Tabelle 23). 
Tabelle 21: Auswertung des Verhaltens von Fußgängern an Kreuzungen mit Lichtsignalanlage (Knotenpunkte 1 bis 9)

\begin{tabular}{|c|c|c|c|c|c|c|c|}
\hline \multirow[t]{2}{*}{$\begin{array}{l}\text { Kno- } \\
\text { ten- } \\
\text { punkt } \\
1\end{array}$} & $\begin{array}{c}250 \\
200 \\
150 \\
100 \\
50 \\
0\end{array}$ & 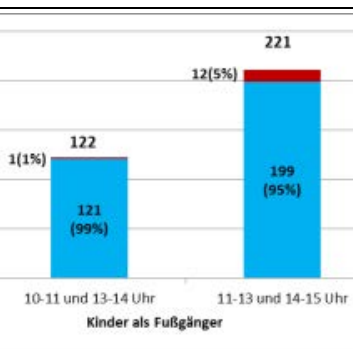 & 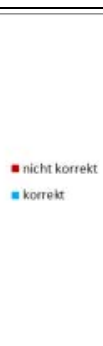 & 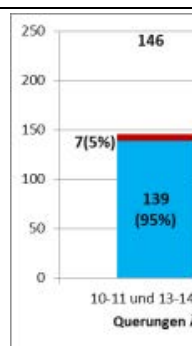 & 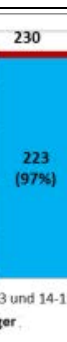 & 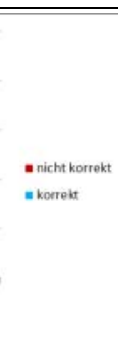 & \\
\hline & $\begin{array}{c}350 \\
300 \\
250 \\
200 \\
150 \\
100 \\
50 \\
50\end{array}$ & 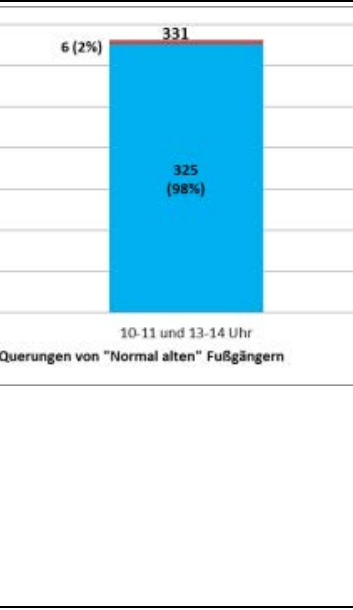 & 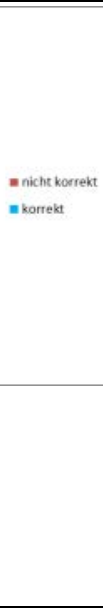 & \multicolumn{4}{|c|}{$\begin{array}{l}\text { Kinder: außer } 5 \text { Rotlichtverstößen keine } \\
\text { schwerwiegenden Fehler, die restlichen } \\
\text { Fehler beziehen sich vor allem auf den } \\
\text { nicht kürzesten Weg bei der } \\
\text { Fahrbahnüberquerung. } \\
\text { Ältere Menschen: außer } 2 \\
\text { Rotlichtverstößen keine schwerwiegenden } \\
\text { Fehler, nicht der kürzeste Weg vor allem } \\
\text { bei der Querung. } \\
\text { Fußgänger (16-64 J.): Außer } 2 \\
\text { Rotlichtverstößen, ebenfalls vor allem } \\
\text { nicht der kürzeste Weg bei der Querung. } \\
\text { Fazit: Alle Gruppen begehen nach Art und } \\
\text { Quantität etwas dieselben (wenigen) } \\
\text { Fehler. }\end{array}$} \\
\hline \multirow[t]{2}{*}{$\begin{array}{l}\text { Kno- } \\
\text { ten- } \\
\text { punkt } \\
2\end{array}$} & \begin{tabular}{|c|}
50 \\
50 \\
40 \\
30 \\
30 \\
20 \\
10 \\
0 \\
0 \\
\end{tabular} & 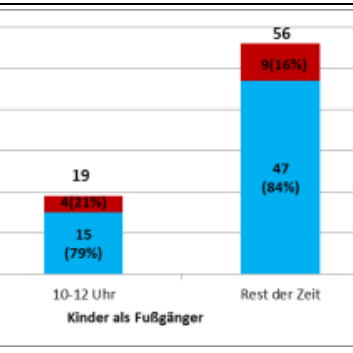 & 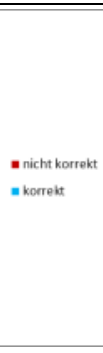 & 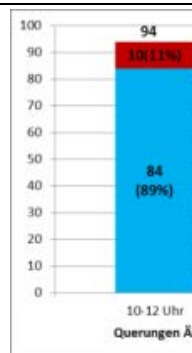 & 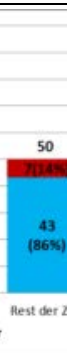 & 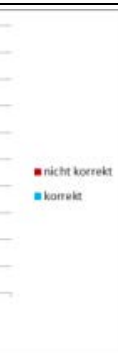 & \\
\hline & $\begin{array}{l}300 \\
250 \\
200 \\
150 \\
100 \\
50\end{array}$ & 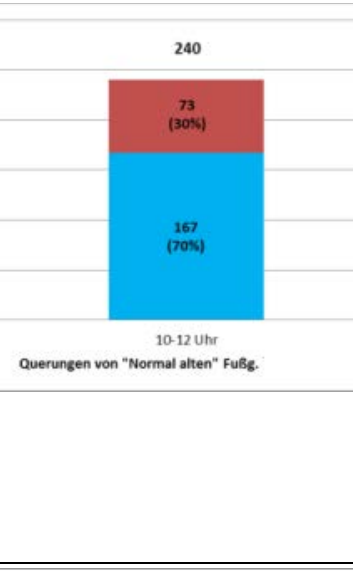 & 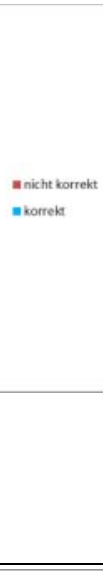 & \multicolumn{4}{|c|}{$\begin{array}{l}\text { Kinder: Alle Fehler von Kindern wurden } \\
\text { von Kindern begangen, die an der Hand } \\
\text { ihrer Begleitpersonen gingen: Teilweise } \\
\text { sogar bei ROT. Darunter ist kein einziger } \\
\text { Verstoß eines Kindes, das allein zu Fuß } \\
\text { unterwegs war. } \\
\text { Ältere Menschen: Es gibt hierbei auch } \\
\text { "nur“ einen Rotverstoß, } 4 \text { Senioren sind } \\
\text { noch bei „Gelb“ (einer Besonderheit bei } \\
\text { Fg-LSA) losgegangen. } \\
\text { Demgegenüber stehen } 22 \text { Rotlicht- und } 17 \\
\text { Gelblichverstöße der } 16-64 \text { Jährigen. } \\
\text { Kinder (sofern sie allein gehen) verhalten } \\
\text { sich am regelkonformsten. }\end{array}$} \\
\hline $\begin{array}{l}\text { Kno- } \\
\text { ten- } \\
\text { punkt } \\
3\end{array}$ & $\begin{array}{l}30 \\
25 \\
20 \\
15 \\
10 .\end{array}$ & 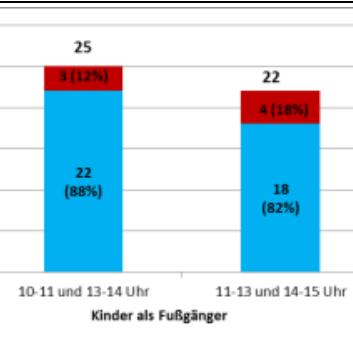 & 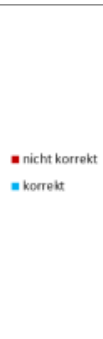 & 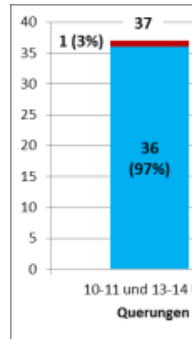 & $\begin{array}{l}3 \text { 3und 14-1. } \\
\text { gerer }\end{array}$ & 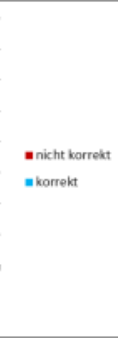 & \\
\hline
\end{tabular}




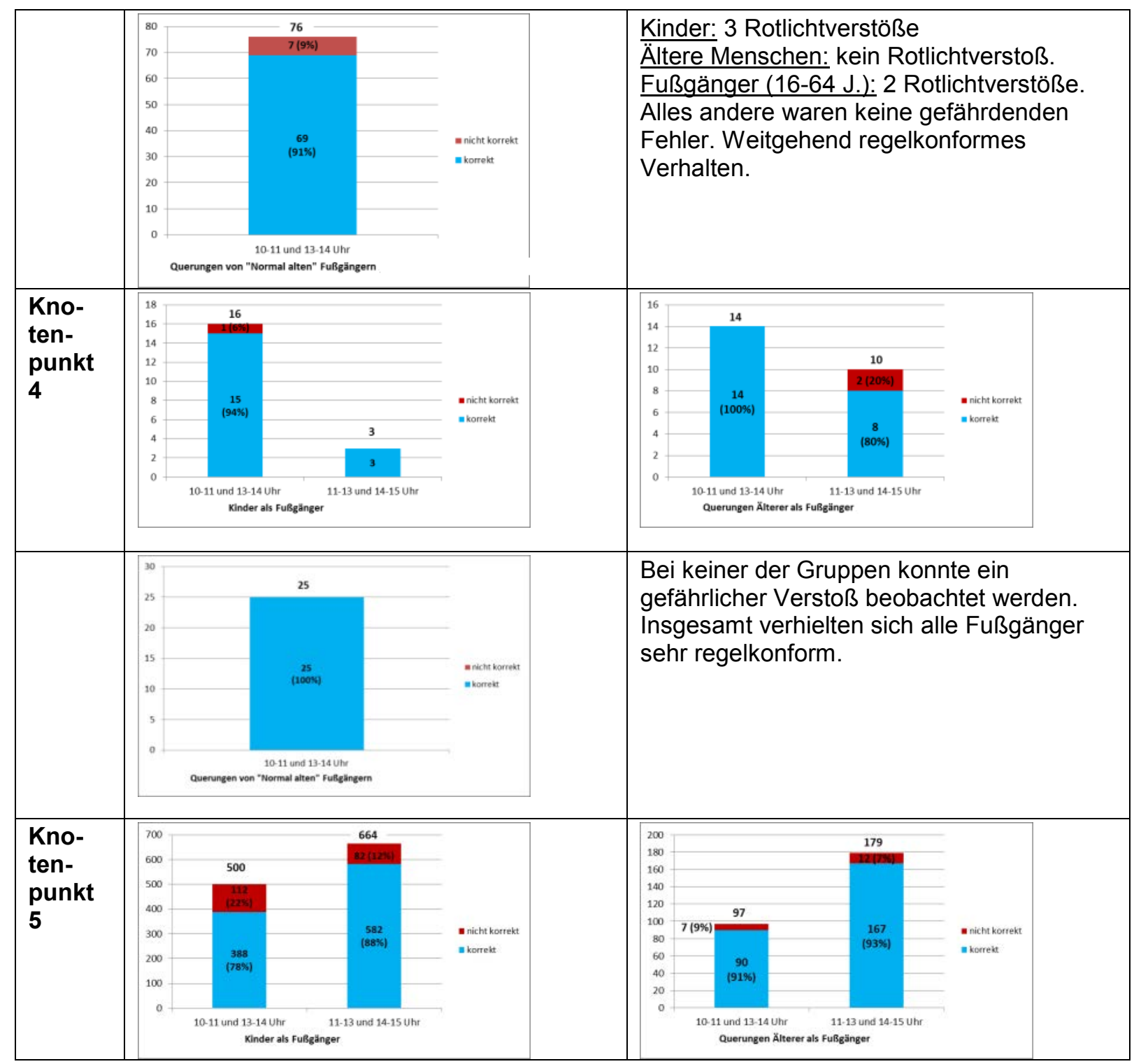




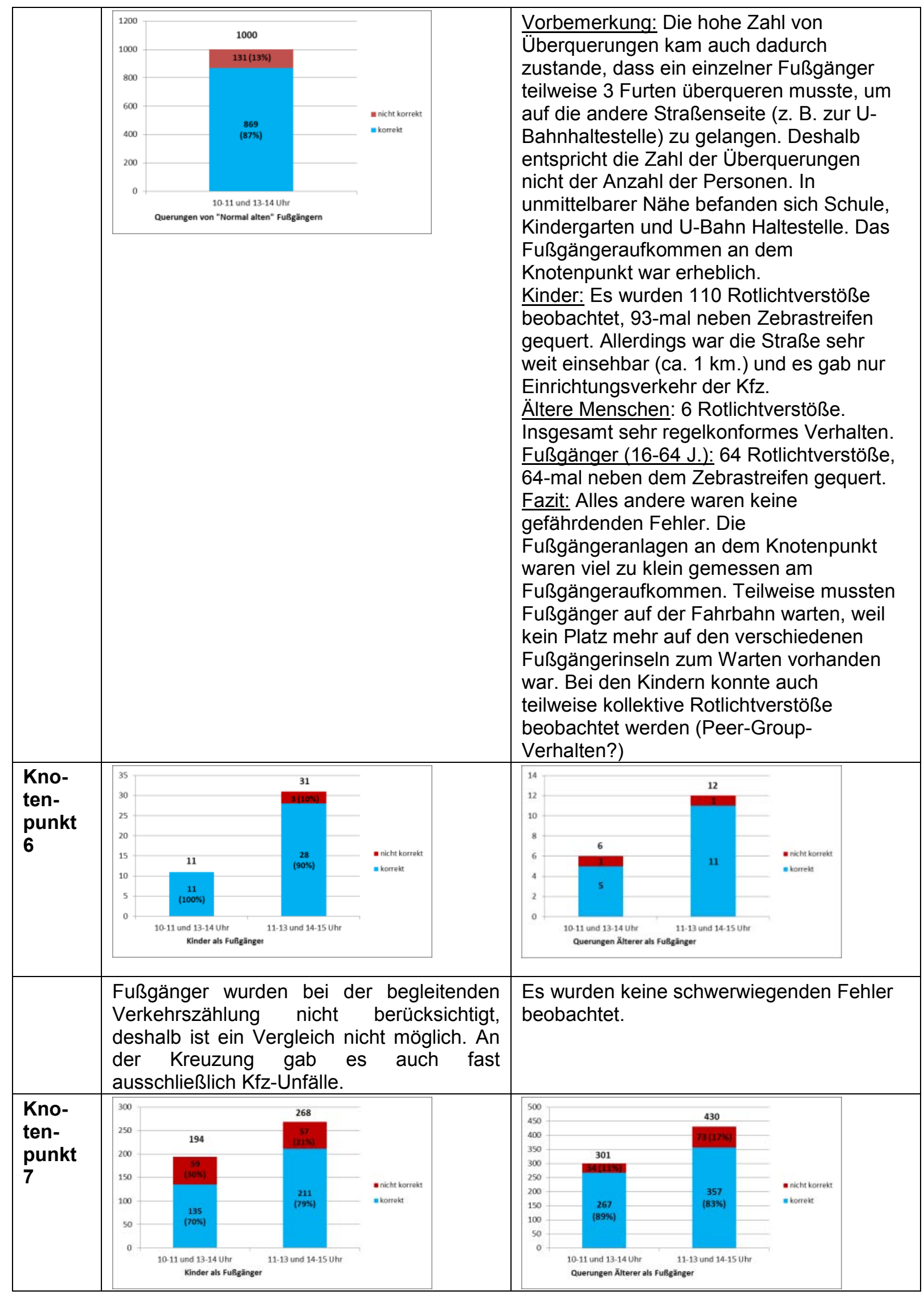




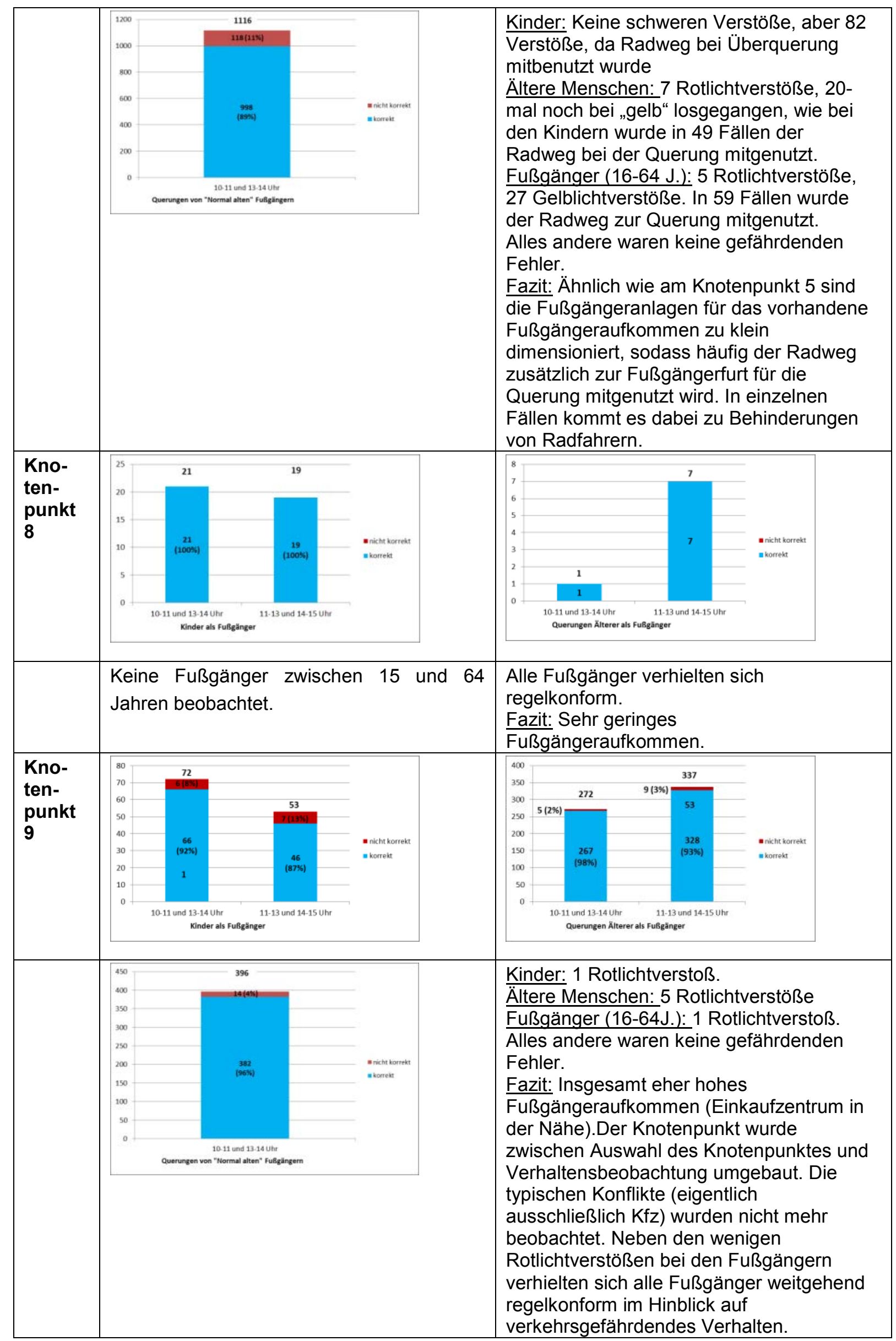


Tabelle 22: Auswertung des Verhaltens von Fußgängern an Kreuzungen mit vorfahrtregelnden Verkehrszeichen (Knotenpunkte 10 bis 13)

\begin{tabular}{|c|c|c|c|c|c|c|c|c|}
\hline \multirow[t]{2}{*}{$\begin{array}{l}\text { Kno- } \\
\text { ten- } \\
\text { punkt } \\
10\end{array}$} & \begin{tabular}{|c|}
60 \\
50 \\
40 \\
30 \\
30 \\
20 \\
10 \\
0 \\
0
\end{tabular} & \begin{tabular}{|c|c|c|}
\multicolumn{1}{|c|}{53} & \\
& \\
\end{tabular} & $\begin{array}{l}\text { Enichthortekt } \\
\text { = korreke }\end{array}$ & $\begin{array}{r}50 \\
50 \\
45 \\
40 \\
35 \\
30 \\
25 \\
20 \\
15 \\
10 \\
5 \\
0 \\
0\end{array}$ & \begin{tabular}{|c|c|}
38 \\
$6(16 \%)$ \\
\\
32 \\
$(84 \%)$ \\
\\
$\begin{array}{r}10-11 \text { und 13-1 } \\
\text { Querunge }\end{array}$ \\
\end{tabular} & $\begin{array}{l}43 \\
(12 \%) \\
38 \\
(88 \%) \\
\\
3 \text { und 14- } \\
\text { ger }\end{array}$ & $\begin{array}{l}\text { =nicht korrekt } \\
\text { =koreteit }\end{array}$ & \\
\hline & \begin{tabular}{|c|c}
190 \\
120 \\
100 \\
80 \\
60 \\
40 \\
20 \\
0
\end{tabular} & \begin{tabular}{|c|c|} 
& 122 \\
& 17 \\
& \\
& \\
$14 \%)$ \\
105 \\
$(86 \%)$ \\
\\
10.11 und 13-14 Uhr \\
\\
\\
Querungen von "Normal alten" Fußgangern \\
\end{tabular} & $\begin{array}{l}\text { Enicht korrekt } \\
\text { "korrekt }\end{array}$ & \multicolumn{5}{|c|}{$\begin{array}{l}\text { Kinder: 3_Rotlichtverstöße. } \\
\text { Ältere Menschen:2 Rotlichtverstöße. } \\
\text { Fußgänger (16-64J.): } 4 \text { Rotlichtverstöße. } \\
\text { Alles andere waren keine gefährdenden } \\
\text { Fehler. } \\
\text { Fazit: Die Fußgänger-LSA war mit einem } \\
\text { Anforderungskontakt ausgestattet, der sehr } \\
\text { schnell die LSA für Fußgänger freischaltete } \\
\text { (etwa } 10 \text { Sek. Anforderungszeit). Trotzdem } \\
\text { kam es zu Rotlichtverstößen der } \\
\text { Fußgänger, die sich nicht erklären lassen. } \\
\text { Der Knotenpunkt rangiert in der Kategorie } \\
\text { „vorfahrtregelnde Zeichen“ da es sich um } \\
\text { eine reine Fußgänger LSA handelte. Der } \\
\text { direkt benachbarte Knotenpunkt wurde } \\
\text { durch Vorfahrtzeichen geregelt. }\end{array}$} \\
\hline \multirow[t]{2}{*}{$\begin{array}{l}\text { Kno- } \\
\text { ten- } \\
\text { punkt } \\
11\end{array}$} & \begin{tabular}{|c|}
14 \\
12 \\
10 \\
8 \\
6 \\
4 \\
2 \\
0 \\
0
\end{tabular} & $\begin{array}{l}\text { 10.12 Uhr } \\
\text { Kinder als Fußgänger }\end{array}$ & $\begin{array}{l}\text { - nicht korrekt } \\
=\text { "korrekt }\end{array}$ & \begin{tabular}{|c|}
45 \\
40 \\
35 \\
30 \\
25 \\
20 \\
15 \\
10 \\
5 \\
0
\end{tabular} & $\begin{array}{c}\text { 10-12 Uhr } \\
\text { Querungen }\end{array}$ & $\begin{array}{l}11 \\
11 \\
\text { (100\%) } \\
\text { lest der } 2 \\
4\end{array}$ & 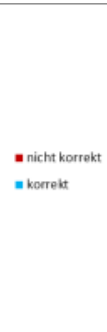 & \\
\hline & $\begin{array}{l}120 \\
100 \\
80 \\
60 \\
40 \\
20 \\
0\end{array}$ & 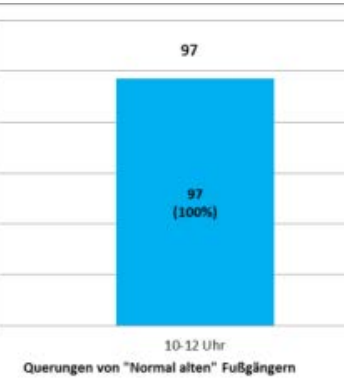 & 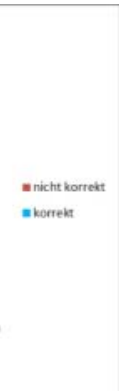 & \multicolumn{5}{|c|}{$\begin{array}{l}\text { Ältere und Erwachsene verhielten sich } \\
\text { absolut korrekt als Fußgänger, ein Kind } \\
\text { ging neben dem Zebrastreifen. Es wurde } \\
\text { kein gefährliches Verhalten beobachtet, } \\
\text { hohe Regelkonformität. }\end{array}$} \\
\hline $\begin{array}{l}\text { Kno- } \\
\text { ten- } \\
\text { punkt } \\
12\end{array}$ & $\begin{array}{l}8 \\
7 \\
6 \\
5 \\
4 \\
3 \\
2 \\
1 \\
0 \\
\end{array}$ & $\begin{array}{l}10: 11 \text { und } 13: 14 \text { Uthr } \\
\text { Kinder ats fubganger }\end{array}$ & $\begin{array}{l}\text { - nichl kortekt } \\
\text { =korrekt }\end{array}$ & $\begin{array}{l}60 \\
50 \\
40 \\
30 \\
20 \\
10\end{array}$ & $\begin{array}{c}\text { 10-11 und 13-1 } \\
\text { Querungen }\end{array}$ & $\begin{array}{c}33 \\
7(21 \%) \\
\\
26 \\
(79 \%)\end{array}$ & $\begin{array}{l}\text { Enicht korreats } \\
\text { =koretelt }\end{array}$ & \\
\hline
\end{tabular}




\begin{tabular}{|c|c|c|c|c|}
\hline & 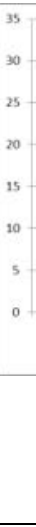 & 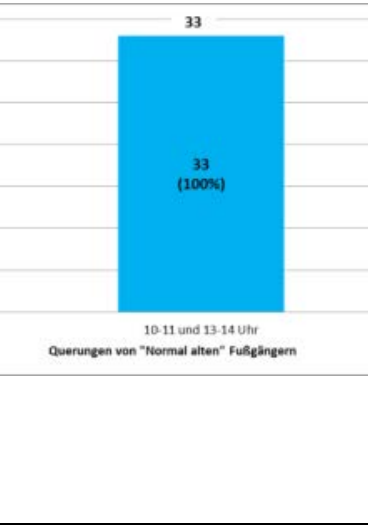 & 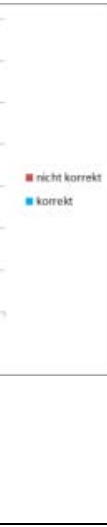 & $\begin{array}{l}\text { Die wenigen Kinder und Fußgänger (16-64 } \\
\text { J.) haben sich absolut korrekt verhalten. } \\
\text { Ältere Menschen gingen teilweise auf der } \\
\text { Straße. } \\
\text { Fazit: Es gab sehr wenig } \\
\text { Fußgängerverkehr. Die Fußgängeranlagen } \\
\text { an dem Knotenpunkt waren ein einem } \\
\text { schlechten Zustand und nicht asphaltiert } \\
\text { (bis auf die eigentliche Fahrbahn). } \\
\text { Dementsprechend gingen Ältere manchmal } \\
\text { auf der Fahrbahn (um nicht zu stolpern). } \\
\text { Wirklich gefährliche Situationen wurden } \\
\text { nicht beobachtet. }\end{array}$ \\
\hline $\begin{array}{l}\text { Kno- } \\
\text { ten- } \\
\text { punkt } \\
13\end{array}$ & $\begin{array}{l}70 \\
60 \\
50 \\
40 \\
30 \\
20 \\
10 \\
10\end{array}$ & 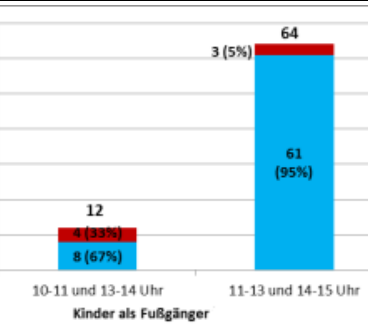 & $\begin{array}{l}\text { - nicht korrokt } \\
=\text { "korereit }\end{array}$ & 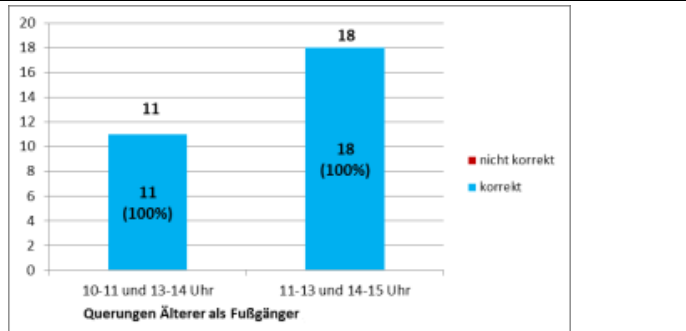 \\
\hline & $\begin{array}{l}30 \\
25 \\
20 \\
15 \\
10 \\
10\end{array}$ & 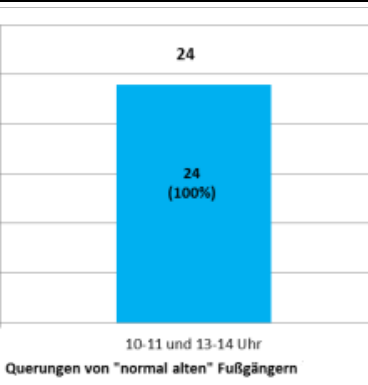 & $\begin{array}{l}\text { = nicht korrekt } \\
\text { =korrekt }\end{array}$ & $\begin{array}{l}\text { Kinder: Alle } 7 \text { Kinder, die sich nicht richtig } \\
\text { verhielten, gingen (teilweise) neben dem } \\
\text { Zebrastreifen über die Straße } \\
\text { Ältere Menschen und Fußgänger (16-64 J.) } \\
\text { verhielten sich alle korrekt. } \\
\text { Fazit: Insgesamt hohe Regelkonformität, } \\
\text { kein gefährliches Verhalten beobachtet. }\end{array}$ \\
\hline
\end{tabular}


Tabelle 23: Auswertung des Verhaltens von Fußgängern an Einmündungen mit vorfahrtregelnden Verkehrszeichen (Knotenpunkte 14 und 15)

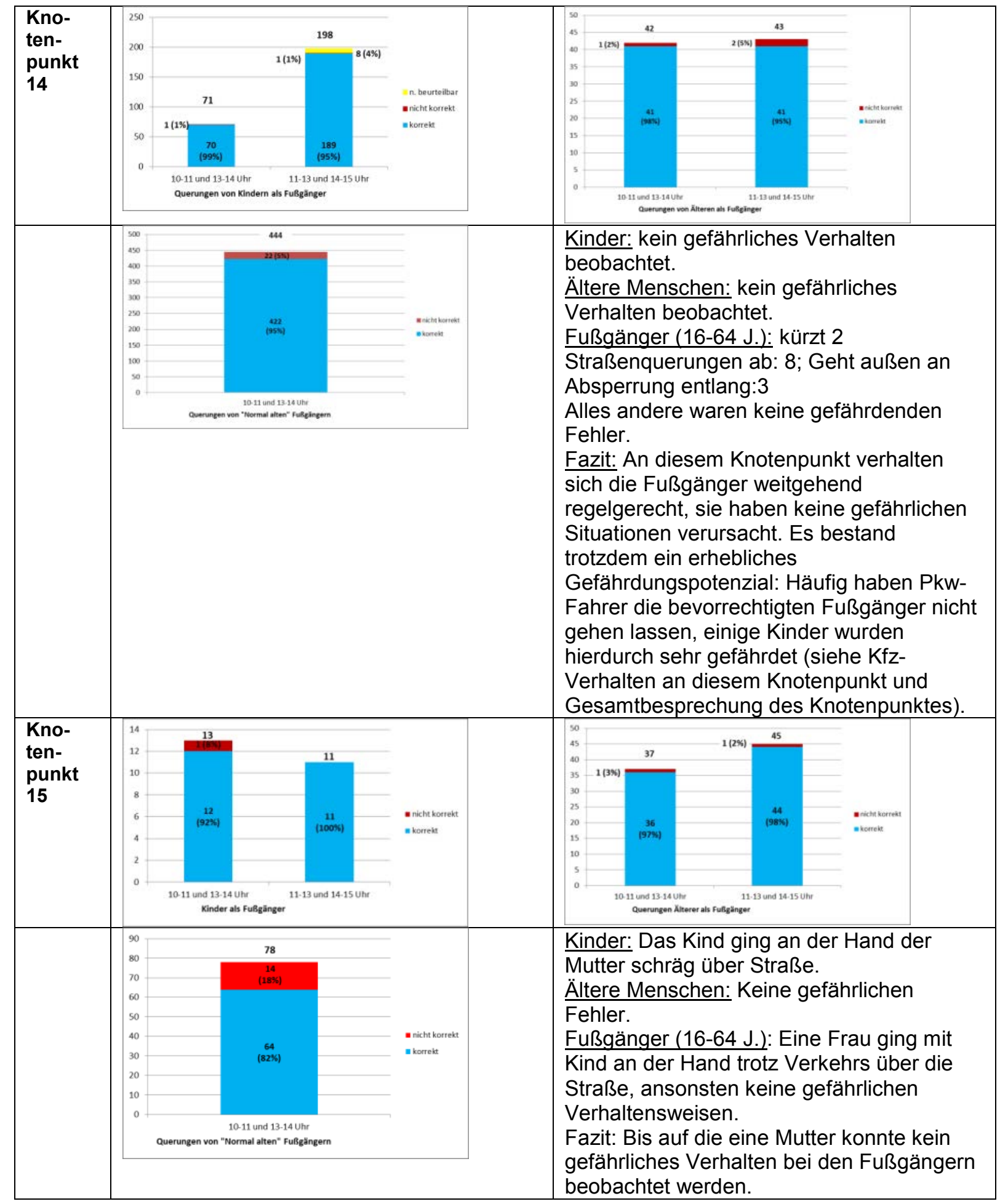




\section{Auswertung des Verhaltens von Radfahrern}

Nachfolgend wird das Verhalten als Radfahrer der verschiedenen Zielgruppen an den Knotenpunkten miteinander verglichen. Wie bereits in den vorangegangenen Kapiteln erwähnt, wurden wichtige Erkenntnisse zur Verbesserung der Gestaltung von Knotenpunkten, die auch auf der Beobachtung des Verkehrsverhaltens der verschiedenen Fußgängergruppe beruhen, schon in den vorangegangenen Kapiteln detailliert besprochen.

Die quantitativen Daten der Radfahrer werden in Balkendiagrammen nebeneinander dargestellt (Tabelle 24 bis Tabelle 26 ).

Tabelle 24: Auswertung des Verhaltens von Radfahrern an Kreuzungen mit Lichtsignalanlage (Knotenpunkte 1 bis 9)

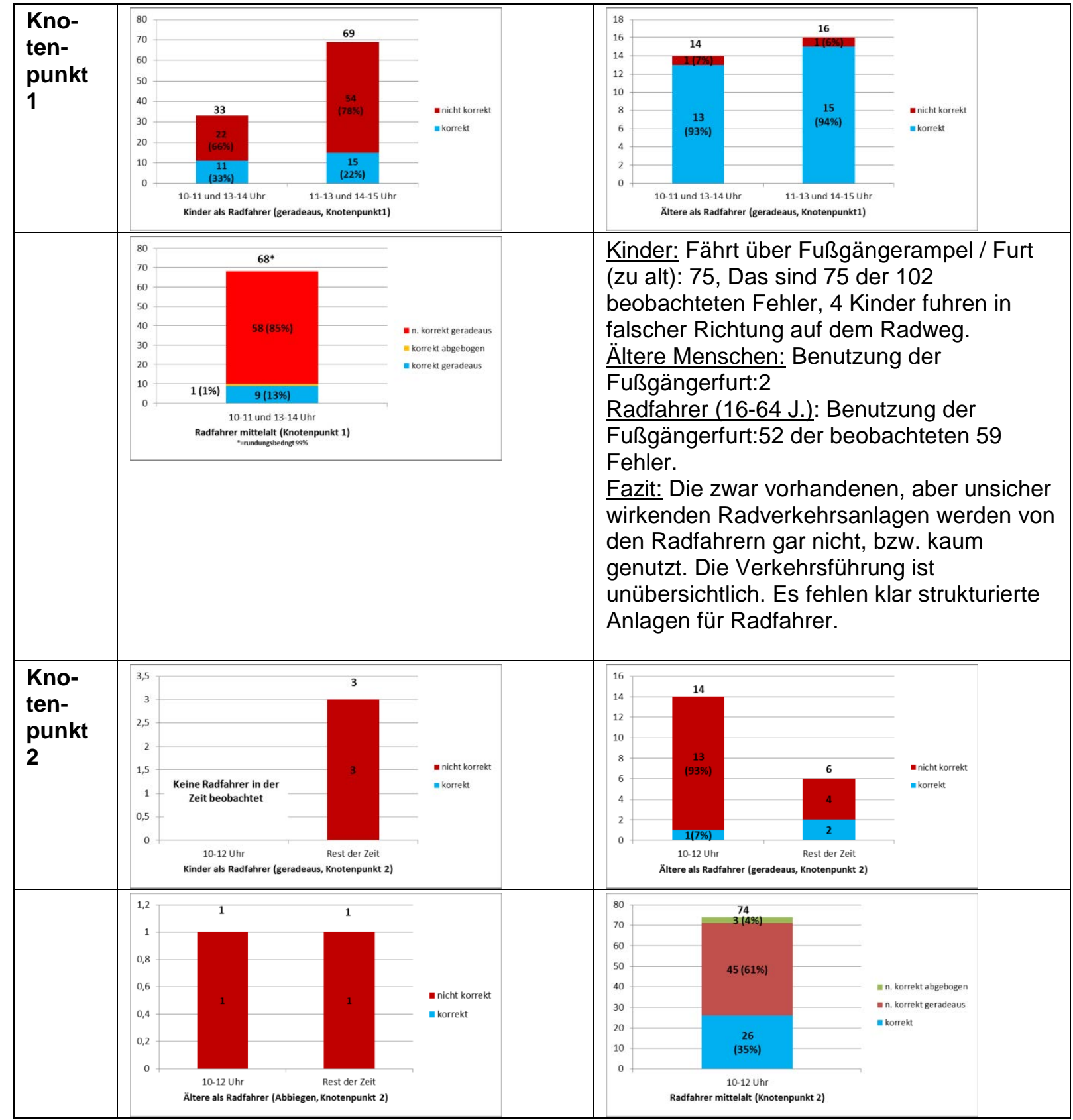




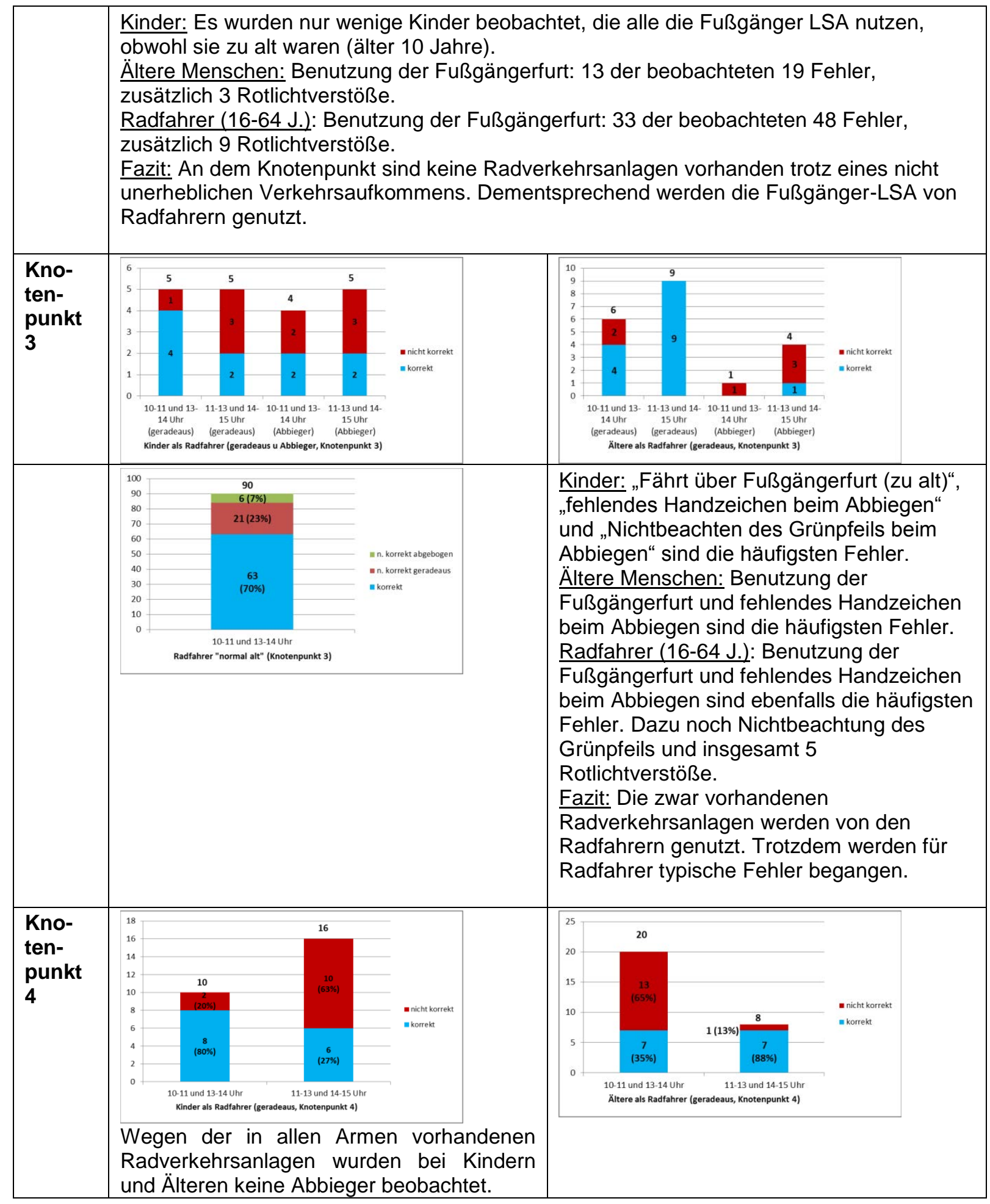




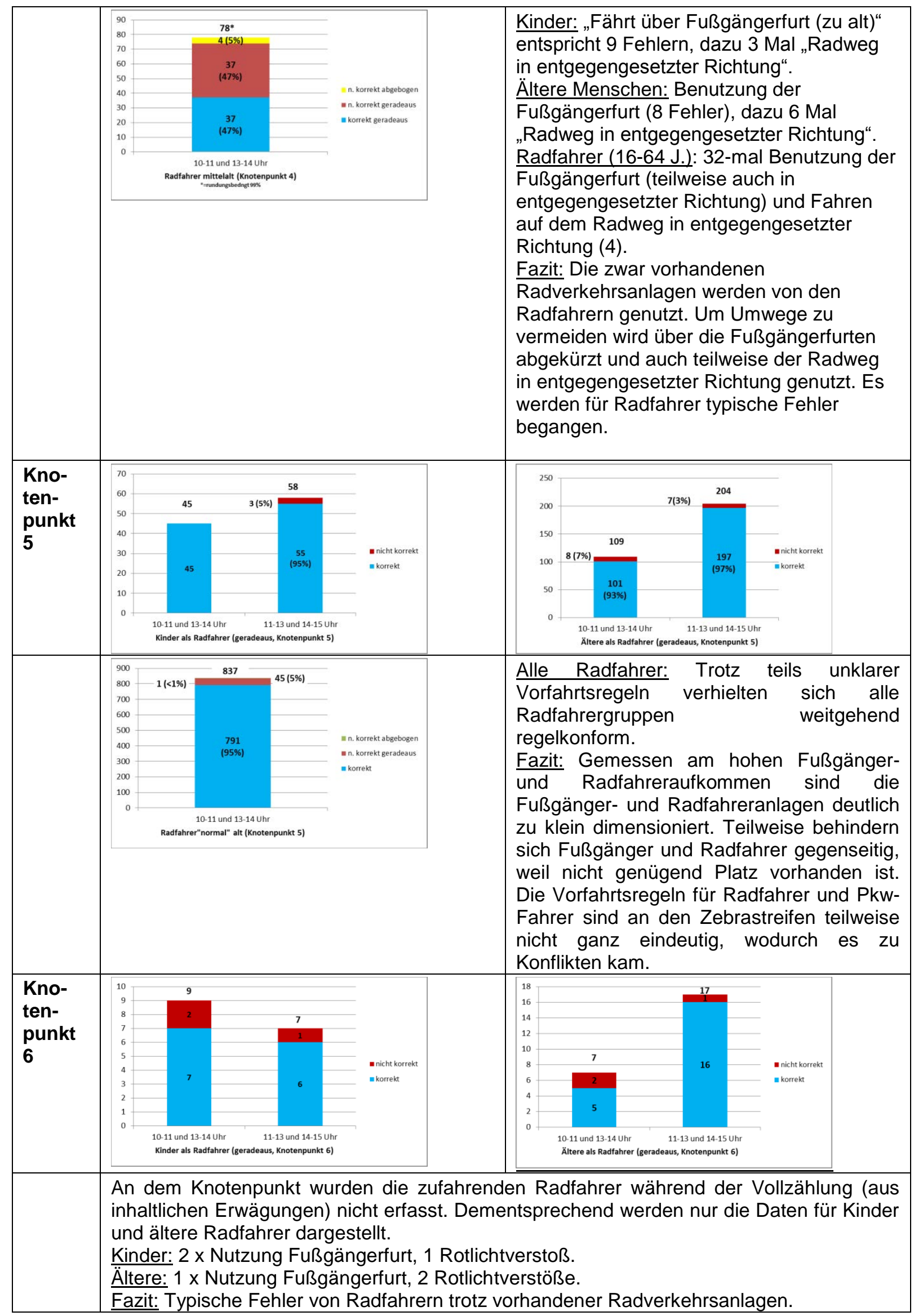




\begin{tabular}{|c|c|c|c|c|c|c|c|}
\hline \multirow[t]{2}{*}{$\begin{array}{l}\text { Kno- } \\
\text { ten- } \\
\text { punkt } \\
7\end{array}$} & $\begin{array}{l}12 . \\
10 . \\
8 \\
6 .\end{array}$ & 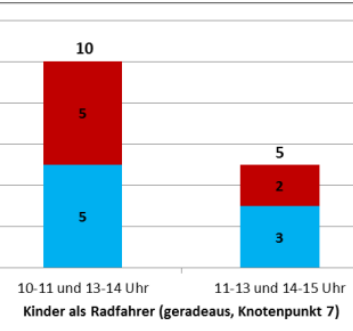 & 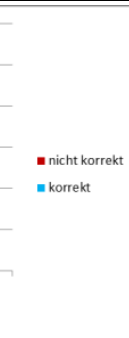 & $\begin{array}{c}60 \\
50 \\
40 \\
30 \\
20 \\
10 \\
0 \\
0\end{array}$ & 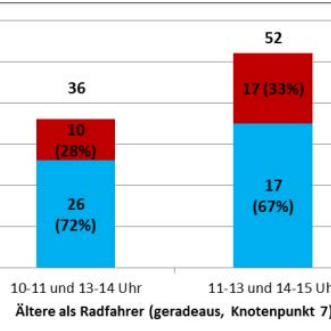 & 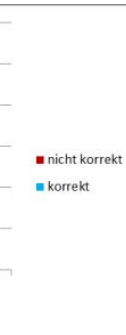 & \\
\hline & $\begin{array}{l}120 \\
100 \\
80 \\
60 \\
40 \\
20 \\
0\end{array}$ & 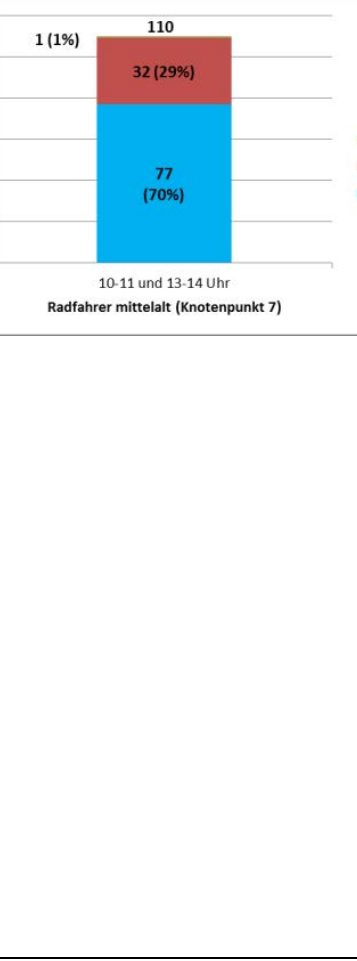 & 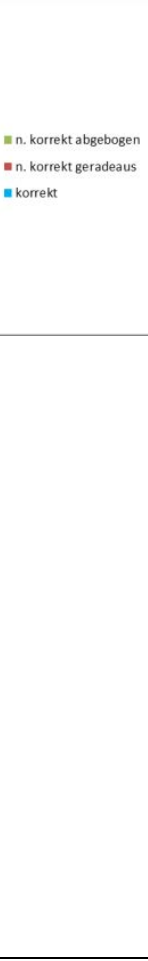 & \multicolumn{4}{|c|}{$\begin{array}{l}\text { Kinder: Alle Fehler beziehen sich auf } \\
\text { Nutzung der Fußgängerfurt trotz } \\
\text { vorhandener Radverkehrsanlagen. } \\
\text { Ältere Menschen: } 25 \text { der } 27 \text { beobachteten } \\
\text { Fehler beziehen sich auf die Benutzung der } \\
\text { Fußgängerfurt statt des Radweges. } \\
\text { Radfahrer (16-64 J.): } 26 \text { der } 32 \\
\text { beobachteten Fehler beziehen sich auf die } \\
\text { Benutzung der Fußgängerfurt statt des } \\
\text { Radweges. Hinzu kommen } 6 \\
\text { Gelblichtverstöße und ein Rotlichtverstoß. } \\
\text { Fazit: Die zwar vorhandenen } \\
\text { Radverkehrsanlagen werden von den } \\
\text { Radfahrern genutzt. Um Umwege zu } \\
\text { vermeiden (die Radwege sind nur in einer } \\
\text { Richtung um den Knotenpunkt herum gut } \\
\text { ausgebaut), wird über die Fußgängerfurten } \\
\text { abgekürzt. Es werden für Radfahrer } \\
\text { typische Fehler begangen. Die } \\
\text { Aufstellflächen für Radfahrer und } \\
\text { Fußgänger sind insgesamt deutlich zu klein } \\
\text { dimensioniert (gemessen am Aufkommen), } \\
\text { sodass sich immer wieder Behinderungen } \\
\text { ergeben. }\end{array}$} \\
\hline \multirow[t]{3}{*}{$\begin{array}{l}\text { Kno- } \\
\text { ten- } \\
\text { punkt } \\
8\end{array}$} & $\begin{array}{r}3,5 \\
3 \\
2,5 \\
2 \\
1,5 \\
1 \\
0,5 \\
0\end{array}$ & 2 & 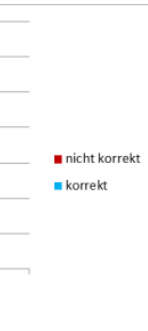 & 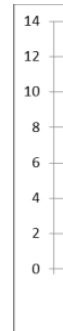 & 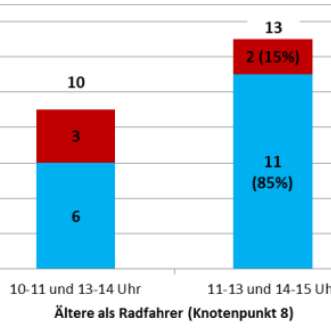 & $\begin{array}{l}\text { mnicht korrekt } \\
\text { ekorenth }\end{array}$ & \\
\hline & $\begin{array}{r}3,5 \\
3 \\
2,5 \\
2 \\
1,5\end{array}$ & 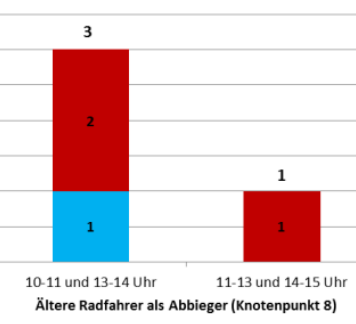 & $\begin{array}{l}\text { mnicht korreekt } \\
\text { =nkorrektt }\end{array}$ & $\begin{array}{c}18 \\
16 \\
12 \\
12 \\
10 \\
8 \\
6 \\
4 \\
2 \\
2 \\
0\end{array}$ & 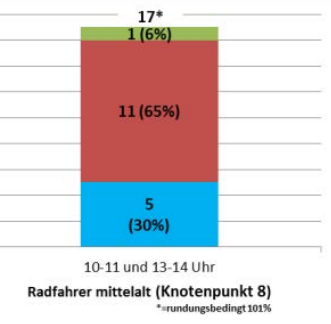 & 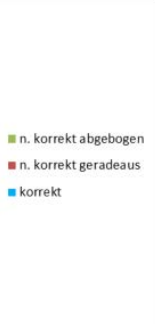 & \\
\hline & \multicolumn{7}{|c|}{$\begin{array}{l}\text { Kinder: Alle Kinder haben sich korrekt verhalten. } \\
\text { Ältere: } 3 \times \text { fehlendes Handzeichen beim Abbiegen, } 3 \times \text { Nutzung Fußgängerfurt, } 2 \times \\
\text { Nutzung des Radweges in falscher Richtung. } \\
\text { Radfahrer }(16-64 \text { J.): } 11 \times \text { Nutzung Fußgängerfurt, } 2 \times \text { Nutzung des Radweges in falscher } \\
\text { Richtung. } \\
\text { Fazit: Typische Fehler von Radfahrern trotz vorhandener Radverkehrsanlagen. }\end{array}$} \\
\hline
\end{tabular}




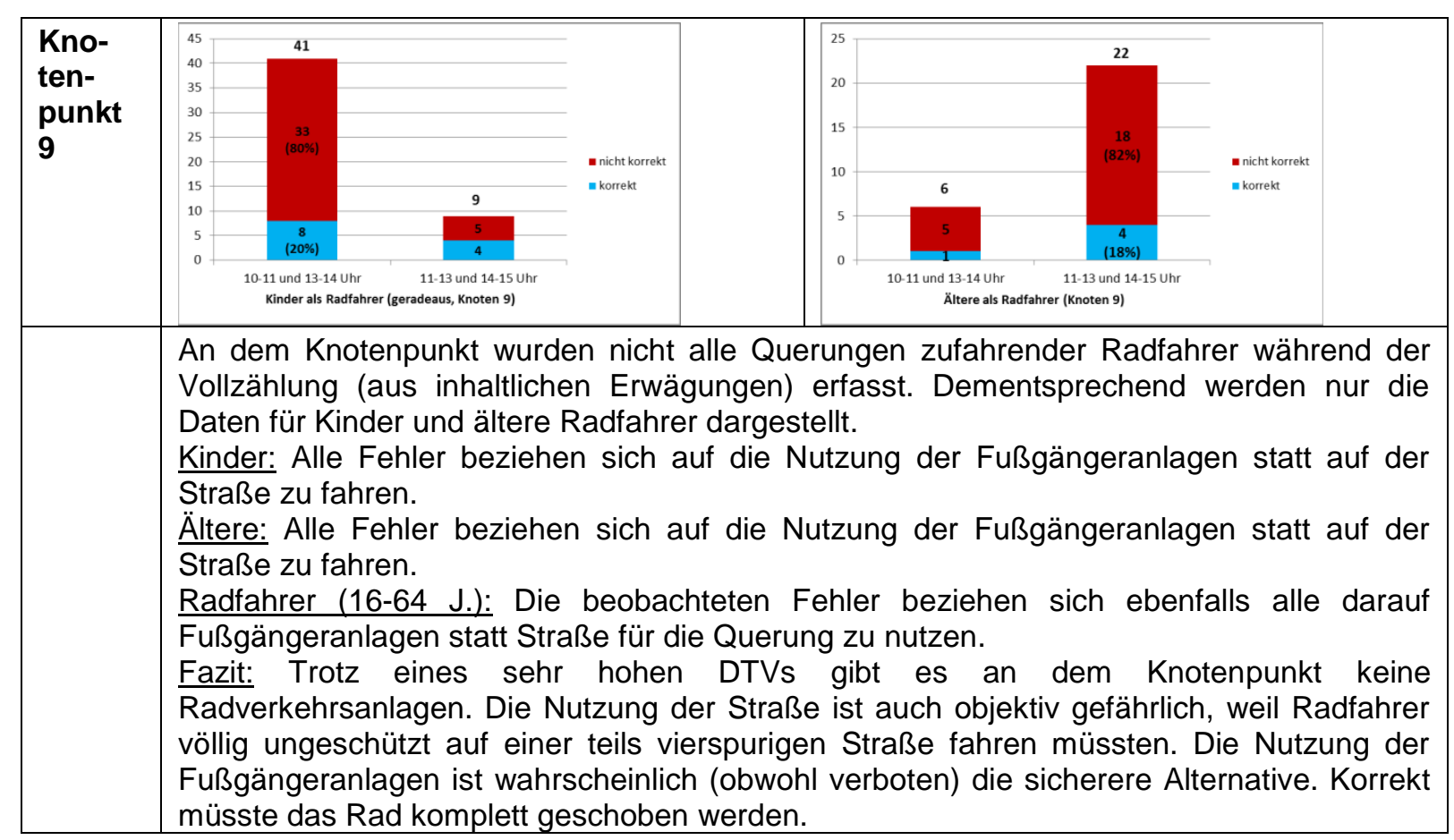


Tabelle 25: Auswertung des Verhaltens von Radfahrern an Kreuzungen mit vorfahrtregelnden Verkehrszeichen (Knotenpunkt 10 bis 13)

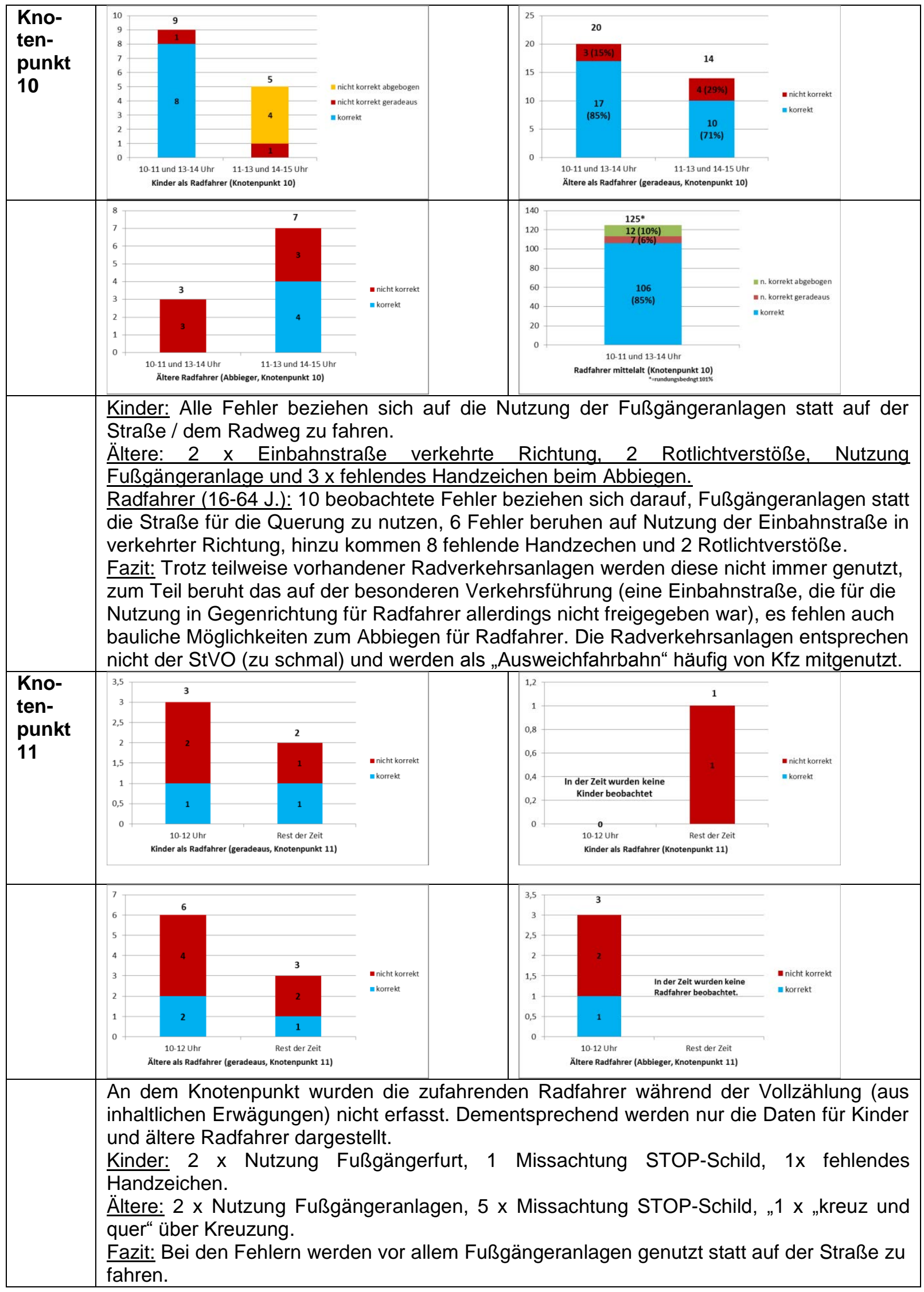




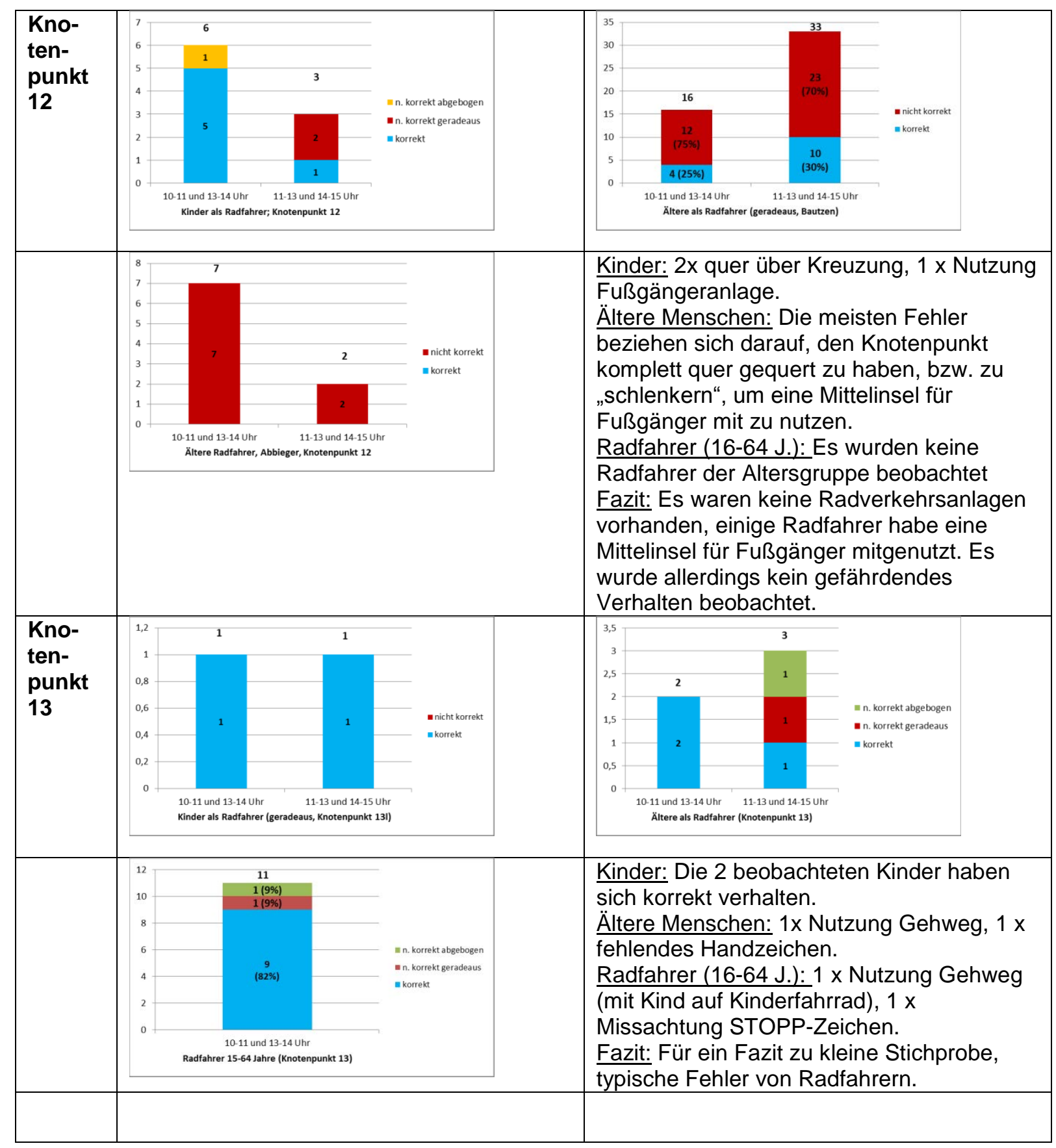


Tabelle 26: Auswertung des Verhaltens von Radfahrern an Einmündungen mit vorfahrtregelnden Verkehrszeichen (Knotenpunkte 14 und 15)

\begin{tabular}{|c|c|c|c|c|}
\hline $\begin{array}{l}\text { Kno- } \\
\text { ten- } \\
\text { punkt } \\
14\end{array}$ & & 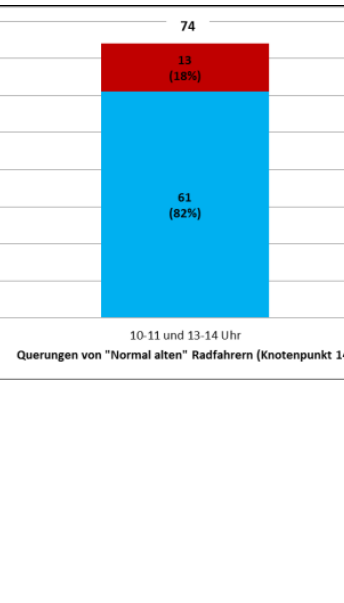 & 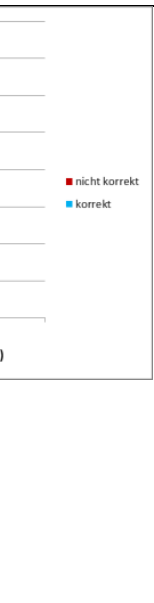 & $\begin{array}{l}\text { Kinder: Kinder als Radfahrer konnten - } \\
\text { wohl auch wegen des sehr schlechten } \\
\text { Wetters - nicht beobachtet werden. } \\
\text { Ältere: Ältere Menschen als Radfahrer } \\
\text { konnten nicht beobachtet werden. } \\
\text { Radfahrer (16-64 J.): Insgesamt wurden } 74 \\
\text { Radfahrer (23 querende und } 51 \text { geradeaus } \\
\text { fahrende) an dem Tag beobachtet. } 10 \\
\text { Fehler beziehen sich auf die Nutzung der } \\
\text { Fußgängeranlagen, bei den Abbiegern fehlt } \\
2 \text { x das Handzeichen. } \\
\text { Fazit: Es sind keine Radverkehrsanlagen } \\
\text { trotz einer sehr großen DTV vorhanden: Es } \\
\text { handelt sich um typische Fehler von } \\
\text { Radfahrern. }\end{array}$ \\
\hline $\begin{array}{l}\text { Kno- } \\
\text { ten- } \\
\text { punkt } \\
15\end{array}$ & $\begin{array}{l}25 \\
20 \\
15 \\
10 \\
10 \\
5\end{array}$ & 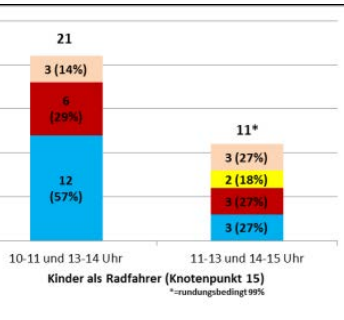 & 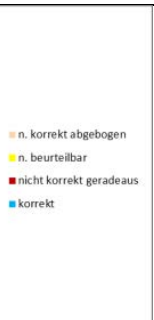 & 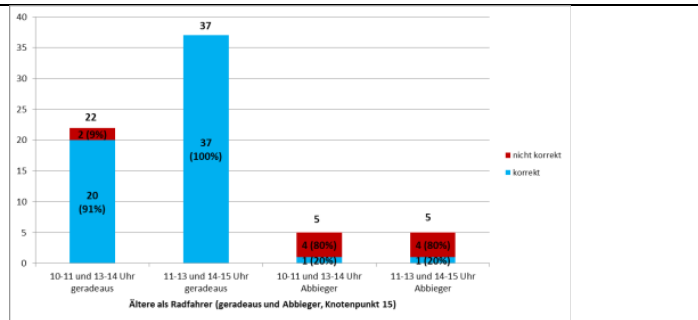 \\
\hline
\end{tabular}




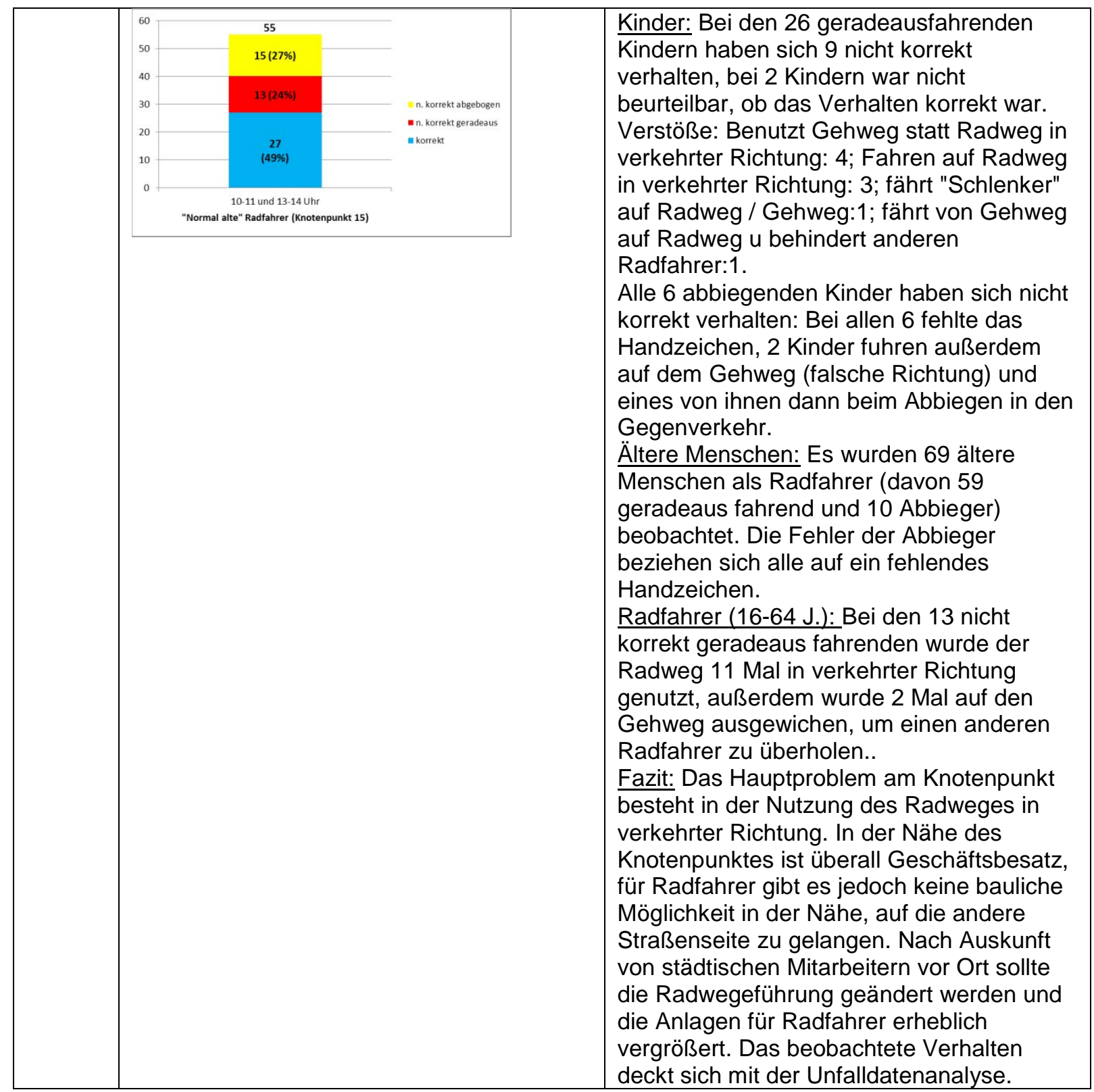




\section{Auswertung des Verhaltens älterer Menschen als Pkw-Fahrer (im Vergleich zu anderen Pkw-Fahrern)}

Die quantitativen Daten der Pkw-Fahrer werden in Balkendiagrammen nebeneinander dargestellt (Tabelle 27 bis Tabelle 29).

Tabelle 27: Auswertung des Verhaltens älterer Pkw-Fahrer an Kreuzungen mit Lichtsignalanlage (Knotenpunkte 1 bis 9)

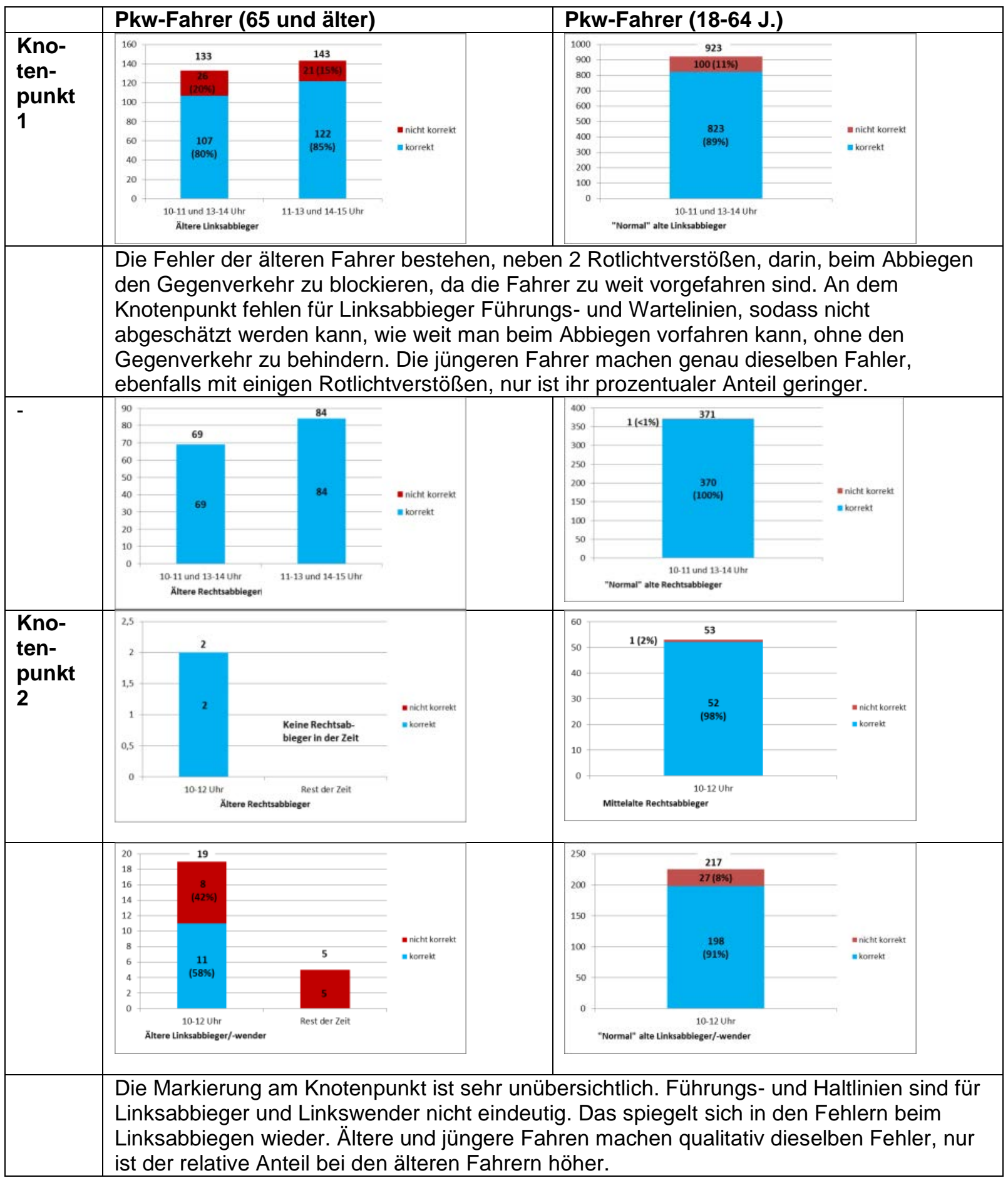




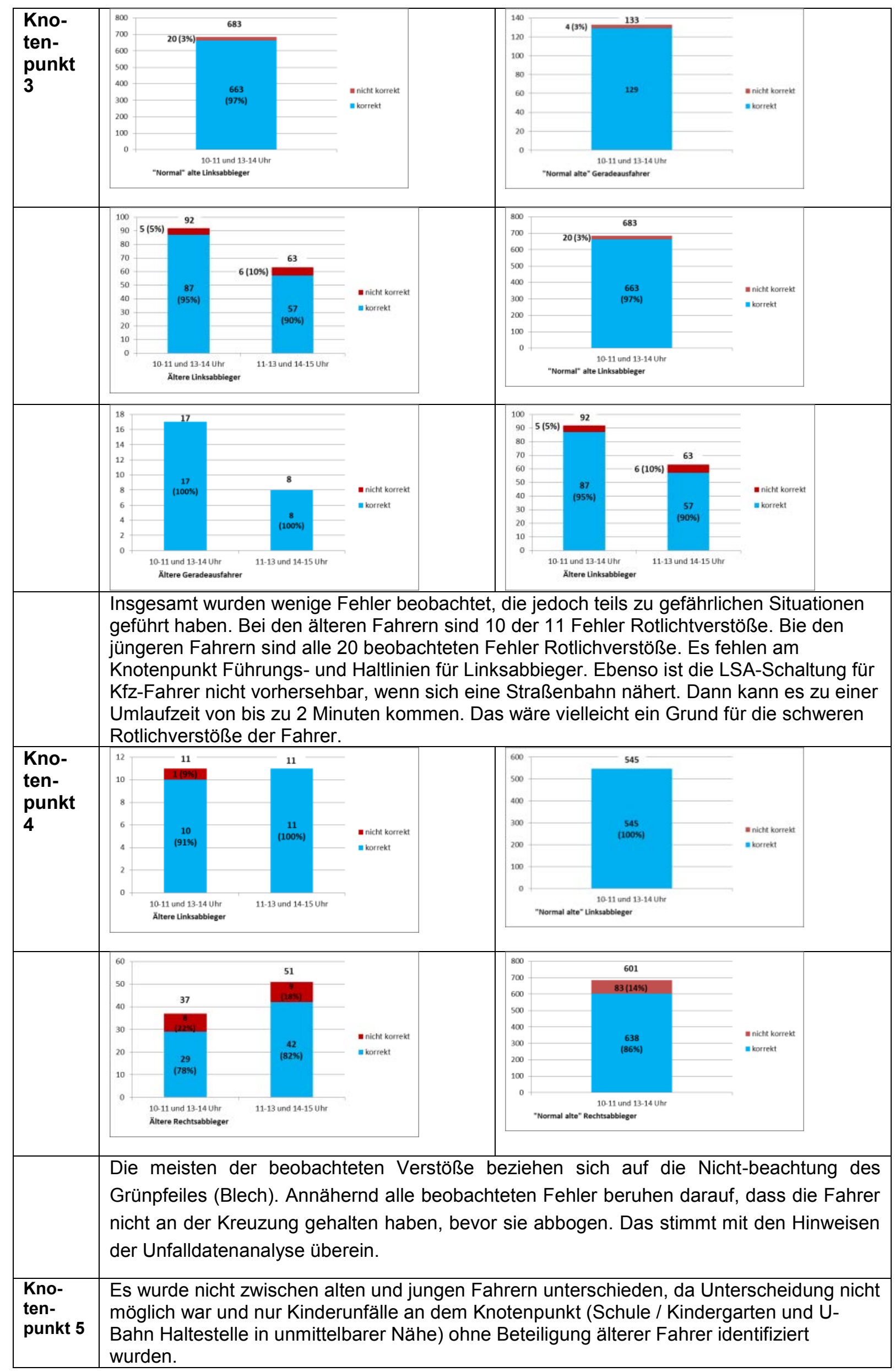




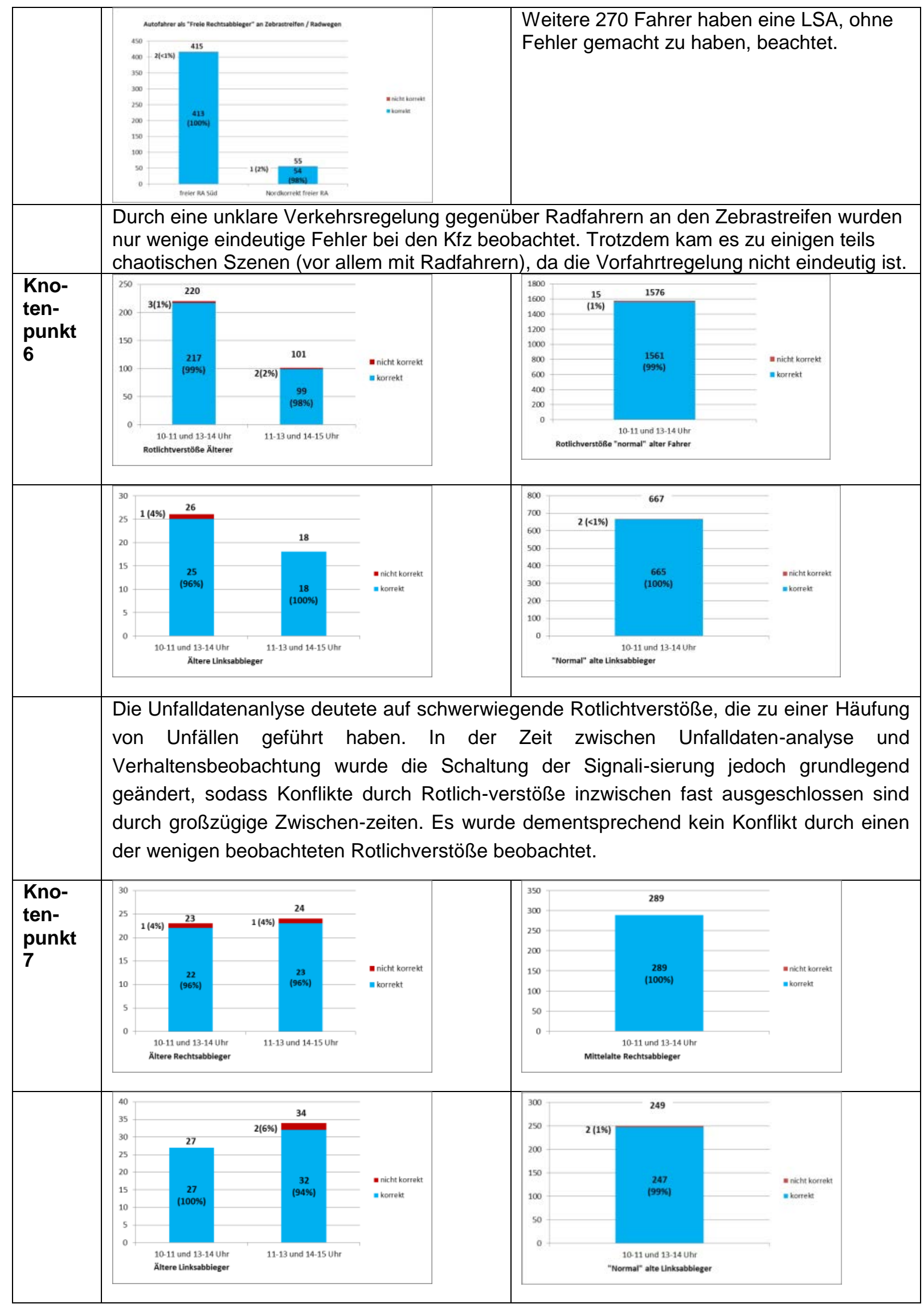




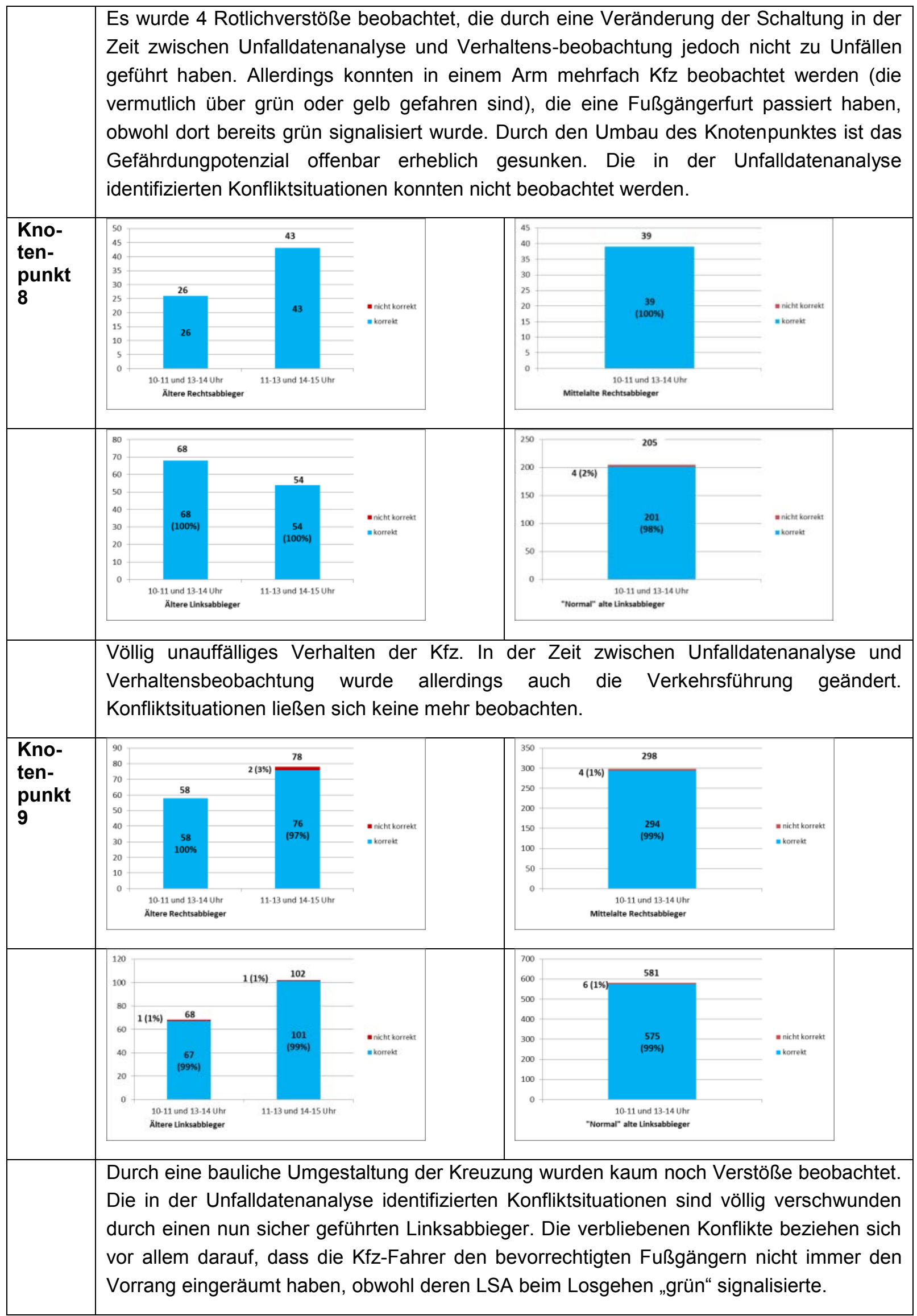


Tabelle 28: Auswertung des Verhaltens älterer Pkw-Fahrer an Kreuzungen vorfahrtregelnden Verkehrszeichen (Knotenpunkte 10 bis 13)

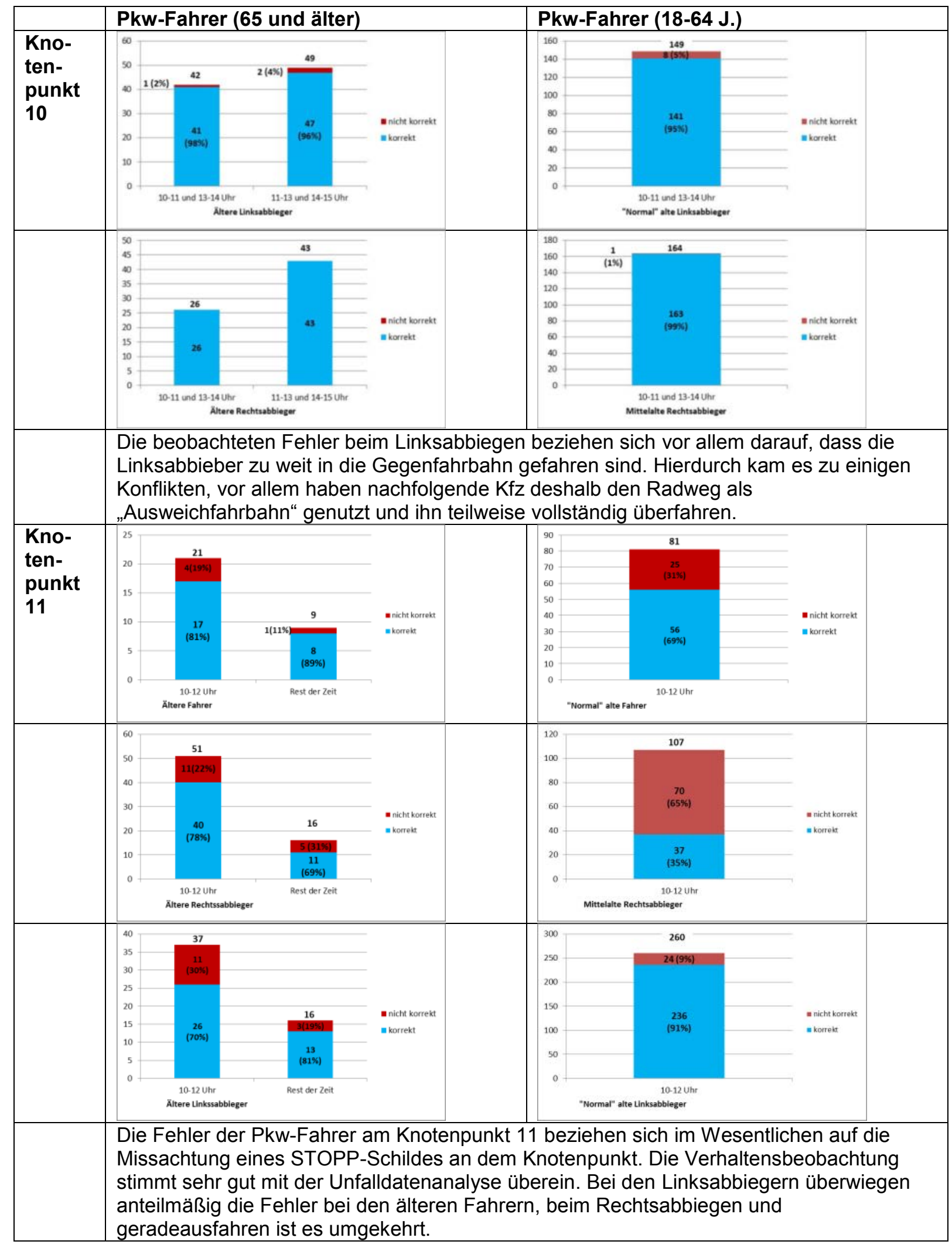




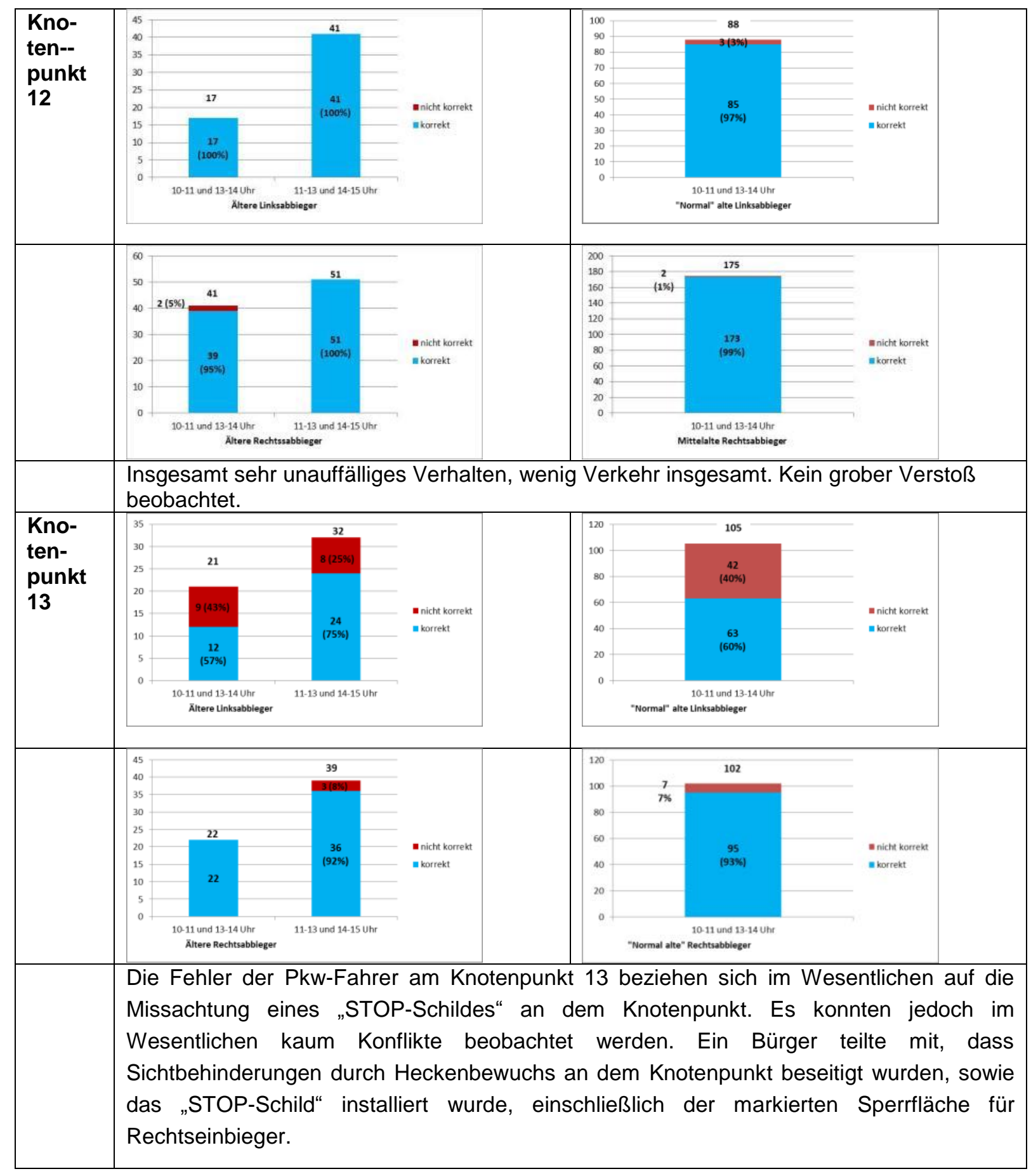


Tabelle 29: Auswertung des Verhaltens älterer Pkw-Fahrer Einmündungen mit vorfahrtregelnden Verkehrszeichen (Knotenpunkt 14 und 15)

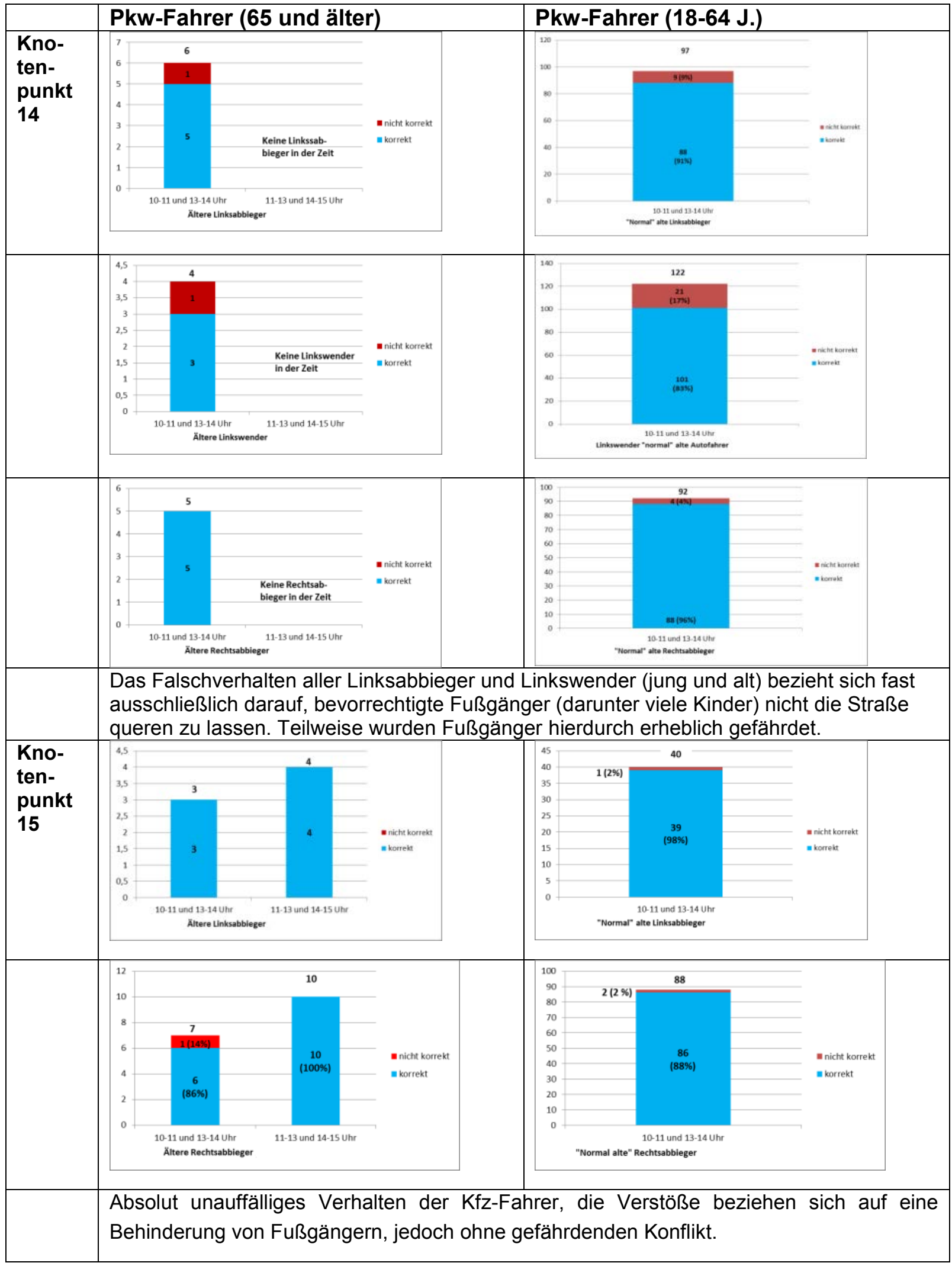




\section{A 7 Prototypische Merkmale/Situationen nach Art der Verkehrsbeteiligung und mögliche Maßnahmen zur Beseitigung der Defizite}

Tabelle 30: Prototypische Merkmale/Situationen für Fußgänger

\begin{tabular}{|c|c|c|c|c|c|}
\hline $\begin{array}{l}\text { Lfd.- } \\
\mathrm{Nr} .\end{array}$ & Merkmale/Situationen & Verhalten/Konflikte & $\begin{array}{l}\text { Knotenpunktart / } \\
\text { Verkehrsregelung }\end{array}$ & Bezug zu Unfällen & $\begin{array}{l}\text { Mögliche Maßnahmen / } \\
\text { Hinweise }\end{array}$ \\
\hline 1 & $\begin{array}{l}\text { Keine } \\
\text { Überquerungsanlage } \\
\text { an mehrstreifiger } \\
\text { Fahrbahn bei hohen } \\
\text { Verkehrsmengen }\end{array}$ & $\begin{array}{l}\text { - Unsicheres } \\
\text { Überquerungsverh } \\
\text { alten } \\
\text { - „Vor- und } \\
\text { Zurückgehen“ } \\
\text { - Bei längerer } \\
\text { Wartezeit werden } \\
\text { auch kurze } \\
\text { Zeitlücken } \\
\text { genutzt, riskante } \\
\text { Überquerungsvers } \\
\text { uche }\end{array}$ & $\begin{array}{l}\text { Einmündungen (VZ) } \\
\text { und Kreuzungen (VZ) }\end{array}$ & Überschreiten-Unfälle & $\begin{array}{l}\text { - Fußgänger-LSA } \\
\text { (Anforderungsanlage) } \\
\text { bzw. Signalisierung des } \\
\text { Knotenpunktes }\end{array}$ \\
\hline 2 & $\begin{array}{l}\text { Sichtbeziehungen } \\
\text { zwischen } \\
\text { Fußgängern und Kfz- } \\
\text { Verkehr durch } \\
\text { ruhenden Verkehr } \\
\text { eingeschränkt }\end{array}$ & $\begin{array}{l}\text { - Unsicheres } \\
\text { Überquerungsverh } \\
\text { alten } \\
\text { - Gefahr } \\
\text { insbesondere für } \\
\text { Kinder und } \\
\text { Rollstuhlfahrer }\end{array}$ & $\begin{array}{l}\text { Einmündungen (VZ) } \\
\text { und Kreuzungen (VZ) }\end{array}$ & Überschreiten-Unfälle & $\begin{array}{l}\text { - Wirksamens (bauliches) } \\
\text { Unterbinden von Parken } \\
\text { im Nahbereich der } \\
\text { Überquerung, z. B. } \\
\text { Poller } \\
\text { - Vorgezogene } \\
\text { Seitenräume } \\
\text { - Überquerungsanlage } \\
\text { (LSA) }\end{array}$ \\
\hline 3 & $\begin{array}{l}\text { Erkennbarkeit der } \\
\text { Überquerungsstelle } \\
\text { ist für den Kfz- } \\
\text { Verkehr nicht } \\
\text { gegeben, wartende } \\
\text { Fußgänger werden } \\
\text { spät gesehen } \\
\text { (Straßenführung, } \\
\text { Parken am } \\
\text { Fahrbahnrand, keine } \\
\text { Beschilderung, } \\
\text { Bepflanzung) }\end{array}$ & $\begin{array}{l}\text { - Hohe } \\
\text { Geschwindigkeite } \\
\text { n im Kfz-Verkehr } \\
\text { - Z. T. abruptes } \\
\text { Abbremsen der } \\
\text { Kfz zu beobachten } \\
\text { - Unsicheres } \\
\text { Überquerungsverh } \\
\text { alten der } \\
\text { Fußgänger }\end{array}$ & $\begin{array}{l}\text { Einmündungen (VZ) } \\
\text { und Kreuzungen (VZ) }\end{array}$ & $\begin{array}{l}\text { Überschreiten-Unfälle } \\
\text { und bedingt Unfälle } \\
\text { im Längsverkehr }\end{array}$ & $\begin{array}{l}\text { Fußgängerüberweg, } \\
\text { einschließlich } \\
\text { hinweisender } \\
\text { Beschilderung / } \\
\text { Beleuchtung (StVO- } \\
\text { Zeichen 134, 350, vgl. } \\
\text { Anlage A 1) } \\
\text { Parken am } \\
\text { Fahrbahnrand in } \\
\text { unmittelbarer Nähe der } \\
\text { Überquerungsstelle } \\
\text { wirksam (baulich) } \\
\text { unterbinden } \\
\text { Vorgezogene } \\
\text { Seitenräume } \\
\text { - Überquerungsanlage } \\
\text { (LSA) }\end{array}$ \\
\hline 4 & $\begin{array}{l}\text { Keine oder nicht } \\
\text { ausreichend } \\
\text { gesicherte } \\
\text { Aufstellfläche für } \\
\text { Fußgänger an } \\
\text { Überquerung }\end{array}$ & $\begin{array}{l}\text { - Ausweichen auf } \\
\text { Fahrbahn oder } \\
\text { Radverkehrsanlag } \\
\text { en } \\
\text { - Unkontrolliertes, } \\
\text { unvorhersehbares } \\
\text { und unsicheres } \\
\text { Überquerungsverh } \\
\text { alten von } \\
\text { Fußgängern }\end{array}$ & $\begin{array}{l}\text { Einmündungen (VZ) } \\
\text { und Kreuzung (VZ } \\
\text { und LSA) }\end{array}$ & Überschreiten-Unfälle & $\begin{array}{l}\text { - Vorgezogene } \\
\text { Seitenräume }\end{array}$ \\
\hline 5 & $\begin{array}{l}\text { Durchmesser } \\
\text { Warnsignal (gelbes } \\
\text { Blinklicht) an } \\
\text { Fußgängerfurten } \\
\text { beträgt } 200 \mathrm{~mm}\end{array}$ & $\begin{array}{l}\text { - Schlechte } \\
\text { Wahrnehmbarkeit } \\
\text { für Kfz-Verkehr }\end{array}$ & $\begin{array}{l}\text { Knotenpunkte mit } \\
\text { LSA }\end{array}$ & $\begin{array}{l}\text { Kein Bezug auf Basis } \\
\text { der Datengrundlage } \\
\text { herstellbar. }\end{array}$ & $\begin{array}{l}\text { - Warnsignal mit } 300 \text { mm } \\
\text { Durchmesser } \\
\text { (Empfehlung RiLSA) }\end{array}$ \\
\hline
\end{tabular}




\begin{tabular}{|c|c|c|c|c|c|}
\hline $\begin{array}{l}\text { Lfd.- } \\
\mathrm{Nr} .\end{array}$ & Merkmale/Situationen & Verhalten/Konflikte & $\begin{array}{l}\text { Knotenpunktart / } \\
\text { Verkehrsregelung }\end{array}$ & Bezug zu Unfällen & $\begin{array}{l}\text { Mögliche Maßnahmen / } \\
\text { Hinweise }\end{array}$ \\
\hline 6 & $\begin{array}{l}\text { Fußgängersignalanla } \\
\text { gen } \\
\text { (Anforderungssignala } \\
\text { nlagen) }\end{array}$ & $\begin{array}{l}\text { - Werden gut } \\
\text { angenommen, } \\
\text { wenn } \\
\text { Anforderungsdaue } \\
\text { r kurz ist, trotz } \\
\text { einzelner } \\
\text { „Rotläufer". }\end{array}$ & Knotenpunkte mit VZ & $\begin{array}{l}\text { Kein Bezug auf Basis } \\
\text { der Datengrundlage } \\
\text { herstellbar. }\end{array}$ & $\begin{array}{l}\text { Kurze } \\
\text { Anforderungsdauer } \\
\text { - Haltlinie für Kfz vor } \\
\text { kreuzender Straße }\end{array}$ \\
\hline
\end{tabular}

Tabelle 31:Prototypische Merkmale/Situationen für Radfahrer

\begin{tabular}{|c|c|c|c|c|c|}
\hline $\begin{array}{l}\text { Lfd.- } \\
\mathrm{Nr} .\end{array}$ & Merkmale/Situationen & Verhalten/Konflikte & Knotenpunktart & Bezug zu Unfällen & $\begin{array}{l}\text { Mögliche Maßnahmen } \\
\text { Hinweise }\end{array}$ \\
\hline 1 & $\begin{array}{lr}\text { Erkennbarkeit der } \\
\text { Radverkehrsfurt an } \\
\text { einmündenden } \\
\text { Straßen ist nicht } \\
\text { gegeben }\end{array}$ & $\begin{array}{l}\text { - Einbiegende und } \\
\text { kreuzende } \\
\text { Kraftfahrer fahren } \\
\text { zügig bis an den } \\
\text { Fahrbahnrand und } \\
\text { warten auf den } \\
\text { Bereichen der } \\
\text { Radverkehrsfurt }\end{array}$ & $\begin{array}{l}\text { Einmündungen (VZ) } \\
\text { und Kreuzungen (VZ) }\end{array}$ & $\begin{array}{l}\text { Einbiegen/Kreuzen- } \\
\text { Unfälle }\end{array}$ & $\begin{array}{l}\text { Einfärben der } \\
\text { Radverkehrsfurten } \\
\text { - Hinweis auf kreuzende } \\
\text { Radfahrer aus beiden } \\
\text { Richtungen für } \\
\text { einbiegende/kreuzende } \\
\text { Kfz (z. B. durch } \\
\text { Beschilderung StVO- } \\
\text { Zeichen } 138 \text { „Radfahrer } \\
\text { kreuzen“ oder } \\
\text { Piktogramme, vgl. } \\
\text { Anlage A 1) }\end{array}$ \\
\hline 2 & $\begin{array}{l}\text { Nicht regelkonform } \\
\text { gestaltete und/oder } \\
\text { dimensionierte } \\
\text { Radverkehrsanlagen } \\
\text { (z. B. Aufstellflächen } \\
\text { für indirekte } \\
\text { Linksabbieger fehlen, } \\
\text { zu geringe Breiten } \\
\text { bei Radfahrstreifen, } \\
\text { überlagernde } \\
\text { Wegebeziehungen) }\end{array}$ & $\begin{array}{l}\text { - Gefährdung von } \\
\text { Radfahrern durch } \\
\text { überholende und } \\
\text { abbiegende Kfz } \\
\text { - Anlagen werden } \\
\text { von Radfahrern } \\
\text { zum Teil nicht } \\
\text { genutzt } \\
\text { - Radfahrer } \\
\text { weichen auf die } \\
\text { (sichereren) } \\
\text { Anlagen für } \\
\text { Fußgänger aus } \\
\text { - Behinderung/Konfl } \\
\text { ikte mit } \\
\text { Fußgängern }\end{array}$ & Alle & $\begin{array}{l}\text { Bedingter Bezug zu } \\
\text { Abbiege- und } \\
\text { Einbiegen/Kreuzen- } \\
\text { Unfällen, vor allem, } \\
\text { wenn Radfahrer auf } \\
\text { Gehwege } \\
\text { ausweichen und } \\
\text { Kraftfahrer hier nicht } \\
\text { mit Radfahrern } \\
\text { rechnen. }\end{array}$ & $\begin{array}{l}\text { Einhaltung der } \\
\text { Empfehlungen von } \\
\text { Entwurfsregelwerken } \\
\text { beim Bau von } \\
\text { Radverkehrsanlagen }\end{array}$ \\
\hline 3 & $\begin{array}{lr}\text { Nicht ausreichende } \\
\text { und/oder } & \text { sich } \\
\text { überlagernde } & \\
\text { Aufstellflächen } & \text { für } \\
\text { Radfahrer } & \text { und } \\
\text { Fußgänger } & \end{array}$ & $\begin{array}{l}\text { - Gegenseitige } \\
\text { Behinderung. } \\
\text { Allerdings keine } \\
\text { Konflikte } \\
\text { beobachtet, da die } \\
\text { Radfahrer, } \\
\text { insbesondere bei } \\
\text { hoher } \\
\text { Passantendichte } \\
\text { sich zumeist } \\
\text { defensiv verhalten }\end{array}$ & Alle & $\begin{array}{l}\text { Kein Bezug auf Basis } \\
\text { der Datengrundlage } \\
\text { herstellbar. }\end{array}$ & $\begin{array}{l}\text { Ausbildung } \\
\text { ausreichender } \\
\text { Aufstellflächen } \\
\text { - Aufstellflächen für } \\
\text { Radfahrer (indirektes } \\
\text { Linksabbiegen) }\end{array}$ \\
\hline 4 & $\begin{array}{l}\text { Radwegenetz } \\
\text { (Straßenzug) ohne } \\
\text { ausreichende } \\
\text { Überquerungsmöglic } \\
\text { hkeiten für Radfahrer }\end{array}$ & $\begin{array}{l}\text { - Radfahrer auf } \\
\text { Einrichtungsradwe } \\
\text { gen entgegen der } \\
\text { vorgeschriebenen } \\
\text { Fahrtrichtung } \\
\text { - Radfahrer nutzen } \\
\text { Gehwege (in } \\
\text { beiden } \\
\text { Fahrtrichtungen) }\end{array}$ & Alle & $\begin{array}{l}\text { Einbiegen/Kreuzen- } \\
\text { Unfälle } \\
\text { (insbesondere Typ } \\
342 \text { ) }\end{array}$ & $\begin{array}{l}\text { - Schaffung } \\
\text { ausreichender und } \\
\text { geeigneter } \\
\text { Überquerungsmöglichke } \\
\text { iten } \\
\text { - Ggf. Zwei- } \\
\text { Richtungsradwege (mit } \\
\text { Hinweis auf kreuzenden } \\
\text { Radfahrer für } \\
\text { einbiegende/kreuzende } \\
\text { Kfz) }\end{array}$ \\
\hline
\end{tabular}


Tabelle 32: Prototypische Merkmale/Situationen für Menschen mit besonderen Mobilitätseinschränkungen oder Mobilitätsbehinderungen

\begin{tabular}{|c|c|c|c|c|c|}
\hline $\begin{array}{l}\text { Lfd.- } \\
\text { Nr. }\end{array}$ & Merkmale/Situationen & Verhalten/Konflikte & Knotenpunktart & Bezug Unfälle & $\begin{array}{l}\text { Mögliche Maßnahmen } \\
\text { Hinweise }\end{array}$ \\
\hline 1 & $\begin{array}{l}\text { Keine } \\
\text { Überquerungsbereich } \\
\text { e mit Nullabsenkung } \\
\text { vorhanden }\end{array}$ & $\begin{array}{l}\text { Menschen mit } \\
\text { Rollatoren und } \\
\text { Rollstühlen } \\
\text { weichen auf } \\
\text { Radverkehrsanlag } \\
\text { en (z. T. auch auf } \\
\text { die Fahrbahn) aus }\end{array}$ & Alle & $\begin{array}{l}\text { Abbiege-Unfälle und } \\
\text { Einbiegen/Kreuzen- } \\
\text { Unfälle }\end{array}$ & $\begin{array}{l}\text { - Überquerungsstellen mit } \\
\text { Nullabsenkung bzw. mit } \\
\text { differenzierten } \\
\text { Bordhöhen ausführen }\end{array}$ \\
\hline 2 & Bord mit $3 \mathrm{~cm}$ Auftritt & $\begin{array}{l}\text { Menschen mit } \\
\text { Rollatoren und } \\
\text { Rollstuhlfahrer } \\
\text { haben auch bei } 3 \\
\text { cm Bordhöhe z. T. } \\
\text { erhebliche } \\
\text { Probleme, dieses } \\
\text { Hindernis zu } \\
\text { überwinden }\end{array}$ & Alle & $\begin{array}{l}\text { Kein Bezug auf Basis } \\
\text { der Datengrundlage } \\
\text { herstellbar. }\end{array}$ & $\begin{array}{l}\text { - Überquerungsstellen mit } \\
\text { Nullabsenkung bzw. mit } \\
\text { differenzierten } \\
\text { Bordhöhen ausführen }\end{array}$ \\
\hline 3 & $\begin{array}{l}\text { Keine oder } \\
\text { unzureichende } \\
\text { Anlagen für blinde } \\
\text { und sehbehinderte } \\
\text { Menschen }\end{array}$ & $\begin{array}{l}\text { Wurde an nahezu } \\
\text { allen } \\
\text { Knotenpunkten } \\
\text { beobachtet, } \\
\text { zumeist keine } \\
\text { Neubauten, } \\
\text { festgestellt. } \\
\text { Konflikte konnten } \\
\text { allerdings nicht } \\
\text { beobachtet } \\
\text { werden (kaum } \\
\text { blinde oder } \\
\text { sehbehinderte } \\
\text { Menschen } \\
\text { beobachtet) }\end{array}$ & Alle & $\begin{array}{l}\text { Kein Bezug auf Basis } \\
\text { der Datengrundlage } \\
\text { herstellbar. }\end{array}$ & $\begin{array}{l}\text { Bodenindikatoren, } \\
\text { akustische und taktile } \\
\text { Signalgeber } \\
\text { Kontrastreiche } \\
\text { Markierungen von } \\
\text { Bordsteinkanten }\end{array}$ \\
\hline
\end{tabular}


Tabelle 33: Prototypische Merkmale/Situationen Kraftfahrer

\begin{tabular}{|c|c|c|c|c|c|}
\hline $\begin{array}{l}\text { Lfd.- } \\
\text { Nr. }\end{array}$ & Merkmale/Situationen & Verhalten/Konflikte & Knotenpunktart & $\begin{array}{l}\text { Bezug zu } \\
\text { Unfällen }\end{array}$ & $\begin{array}{l}\text { Mögliche Maßnahmen / } \\
\text { Hinweise }\end{array}$ \\
\hline 1 & $\begin{array}{l}\text { Nicht gesichert } \\
\text { geführte } \\
\text { Linksabbieger }\end{array}$ & $\begin{array}{l}\text { - Schwierigkeiten } \\
\text { für Ältere und } \\
\text { Jüngere } \\
\text { gleichermaßen }\end{array}$ & $\begin{array}{l}\text { Kreuzung (LSA und } \\
\text { VZ) }\end{array}$ & $\begin{array}{l}\text { Abbiege- } \\
\text { Unfälle (Typ } \\
\text { 211) }\end{array}$ & $\begin{array}{l}\text { - Signaltechnisch } \\
\text { gesichert geführte } \\
\text { Linksabbieger } \\
\text { Trennung von } \\
\text { Linksabbiegern und } \\
\text { Geradeausfahrern } \\
\text { - Entgegengesetzte } \\
\text { Linksabbiegerstreifen } \\
\text { „Vor Kopf setzen“ }\end{array}$ \\
\hline 2 & $\begin{array}{l}\text { Keine } \\
\text { Orientierungshilfen } \\
\text { für Linksabbieger } \\
\text { (Leitlinie, } \\
\text { Wartelinie) im } \\
\text { Kreuzungsbereich }\end{array}$ & $\begin{array}{l}\text { - Ungeordnete } \\
\text { Aufstellpositione } \\
\text { n (auch für nMIV } \\
\text { problematisch) } \\
\text { - Nebeneinandera } \\
\text { ufstellung } \\
\text { (,Doppelabbiege } \\
\text { r“) } \\
\text { - „Unsicheres } \\
\text { - Vorziehen“ } \\
\text { - "Vortaster“ }\end{array}$ & $\begin{array}{l}\text { Kreuzung (VZ und } \\
\text { LSA bei } \\
\text { signaltechnisch nicht } \\
\text { gesichert geführten } \\
\text { Linksabbieger) }\end{array}$ & $\begin{array}{l}\text { Abbiege- } \\
\text { Unfälle }\end{array}$ & $\begin{array}{l}\text { - Leit- und Wartelinien } \\
\text { markieren und } \\
\text { instandhalten }\end{array}$ \\
\hline 3 & $\begin{array}{l}\text { Sichtbehinderung } \\
\text { (für Linksabbieger) } \\
\text { durch Begrünung } \\
\text { auf Mittelstreifen } \\
\text { (oder Verkehr der } \\
\text { Gegenrichtung) }\end{array}$ & $\begin{array}{l}\text { - Schwierigkeiten } \\
\text { für Jüngere wie } \\
\text { Ältere } \\
\text { gleichermaßen }\end{array}$ & $\begin{array}{l}\text { Kreuzung (VZ und } \\
\text { LSA bei } \\
\text { signaltechnisch nicht } \\
\text { gesichert geführten } \\
\text { Linksabbieger) }\end{array}$ & $\begin{array}{l}\text { Abbiege- } \\
\text { Unfälle }\end{array}$ & $\begin{array}{l}\text { Beseitigung der } \\
\text { Sichtbehinderung } \\
\text { Trennung von } \\
\text { Linksabbiegern und } \\
\text { Geradeausfahrern } \\
\text { - Leit- und Wartelinien } \\
\text { markieren und } \\
\text { instandhalten } \\
\text { - Entgegengesetzte } \\
\text { Linksabbiegerstreifen } \\
\text { „vor Kopf setzen“ }\end{array}$ \\
\hline 4 & $\begin{array}{l}\text { Eingeschränkte } \\
\text { Anfahrsicht, z. B. } \\
\text { durch Baumreihe, } \\
\text { Hecken oder } \\
\text { ruhenden Verkehr }\end{array}$ & $\begin{array}{l}\text { Vorziehen in } \\
\text { den } \\
\text { Kreuzungsberei } \\
\text { ch, z. T. auf } \\
\text { Radfahrstreifen } \\
\text { - Unsicheres } \\
\text { Anfahren }\end{array}$ & $\begin{array}{l}\text { Einmündung (VZ) und } \\
\text { Kreuzung (VZ) }\end{array}$ & $\begin{array}{l}\text { Einbiegen/Kreu } \\
\text { zen-Unfälle }\end{array}$ & $\begin{array}{l}\text { Einhaltung der } \\
\text { Sichtdreiecke } \\
\text { Beseitigung der } \\
\text { Sichtbehinderung, z. B. } \\
\text { wirksames (bauliches) } \\
\text { Verhindern von Parken } \\
\text { am Fahrbahnrand im } \\
\text { Nahbereich der } \\
\text { Kreuzung/Einmündung }\end{array}$ \\
\hline
\end{tabular}




\begin{tabular}{|c|c|c|c|c|c|}
\hline $\begin{array}{l}\text { Lfd.- } \\
\text { Nr. }\end{array}$ & Merkmale/Situationen & Verhalten/Konflikte & Knotenpunktart & $\begin{array}{l}\text { Bezug zu } \\
\text { Unfällen }\end{array}$ & $\begin{array}{l}\text { Mögliche Maßnahmen / } \\
\text { Hinweise }\end{array}$ \\
\hline 5 & $\begin{array}{l}\text { Links abbiegender } \\
\text { und geradeaus } \\
\text { fahrender Verkehr } \\
\text { auf einem } \\
\text { Fahrstreifen bei } \\
\text { mehr als einem } \\
\text { Fahrstreifen in der } \\
\text { Zufahrt }\end{array}$ & $\begin{array}{l}\text { - Nachfolgende } \\
\text { Verkehrsteilneh } \\
\text { mer scheren } \\
\text { nach rechts } \\
\text { (auch auf } \\
\text { Radfahrstreifen/ } \\
\text { Schutzstreifen) } \\
\text { aus } \\
\text { - Besondere } \\
\text { Gefährdung bei } \\
\text { Zugabezeit für } \\
\text { Linksabbieger } \\
\text { wenn } \\
\text { Geradeausverke } \\
\text { hr (der } \\
\text { Gegenrichtung) } \\
\text { noch räumen } \\
\text { muss } \\
\text { - Schwierigkeiten } \\
\text { für Ältere und } \\
\text { Jüngere } \\
\text { gleichermaßen } \\
\text { - Benötigt viel } \\
\text { Aufmerksamkeit }\end{array}$ & $\begin{array}{l}\text { Kreuzung (LSA und } \\
\text { VZ) }\end{array}$ & $\begin{array}{l}\text { Unfälle im } \\
\text { Längsverkehr } \\
\text { und bedingt } \\
\text { Abbiege- } \\
\text { Unfälle }\end{array}$ & $\begin{array}{l}\text { Trennung von } \\
\text { Linksabbiegern und } \\
\text { Geradeausfahrern }\end{array}$ \\
\hline 6 & $\begin{array}{l}\text { Fahrstreifenredukti } \\
\text { on hinter } \\
\text { Kreuzungsbereich } \\
\text { bei } 2 \text { oder mehr } \\
\text { Fahrstreifen des } \\
\text { zufahrenden } \\
\text { Geradeausverkehr } \\
\text { s }\end{array}$ & $\begin{array}{l}\text { - Riskante } \\
\text { Verflechtungsvo } \\
\text { rgänge im } \\
\text { Kreuzungsberei } \\
\text { ch } \\
\text { - Plötzliches } \\
\text { Ausscheren der } \\
\text { Geradeausfahre } \\
\text { r nach rechts }\end{array}$ & Kreuzung (LSA) & $\begin{array}{l}\text { Unfälle im } \\
\text { Längsverkehr }\end{array}$ & $\begin{array}{l}\text { - Gleiche Anzahl von } \\
\text { Fahrstreifen für } \\
\text { Geradeausverkehr vor } \\
\text { und hinter der Kreuzung } \\
\text { - Verflechtungsbereich } \\
\text { hinter den Knotenpunkt } \\
\text { legen }\end{array}$ \\
\hline 7 & $\begin{array}{l}\text { Grünpfeil (StVO- } \\
\text { Zeichen 720, vgl. } \\
\text { Anlage A 1), } \\
\text { insbesondere bei } \\
\text { weit abgesetzter } \\
\text { Haltlinie }\end{array}$ & $\begin{array}{l}\text { - Verhalten der } \\
\text { Verkehrsteilneh } \\
\text { mer oft nicht } \\
\text { StVO-konform } \\
\text { - Wird von } \\
\text { Jüngeren und } \\
\text { Älteren } \\
\text { gleichermaßen } \\
\text { missachtet } \\
\text { - Gefährdung von } \\
\text { Radfahrern und } \\
\text { Fußgängern an } \\
\text { „paralleler" Furt }\end{array}$ & Kreuzung (LSA) & $\begin{array}{l}\text { Abbiege- } \\
\text { Unfälle }\end{array}$ & $\begin{array}{l}\text { - Verzicht auf Grünpfeil } \\
\text { (Anordnung gemäß } \\
\text { VWV-StVO) }\end{array}$ \\
\hline 8 & $\begin{array}{l}\text { Lange, gerade } \\
\text { Straßenflucht der } \\
\text { Hauptachse }\end{array}$ & $\begin{array}{l}\text { - Subjektive } \\
\text { Einschätzung: } \\
\text { zu hohe } \\
\text { Geschwindigkeit } \\
\text { en } \\
\text { - Spätes, z. T. } \\
\text { abruptes } \\
\text { Abbremsen an } \\
\text { Fußgänger- } \\
\text { Überquerungsa } \\
\text { nlage }\end{array}$ & Kreuzung (VZ) & $\begin{array}{l}\text { Überschreiten- } \\
\text { Unfälle und } \\
\text { Unfälle im } \\
\text { Längsverkehr }\end{array}$ & $\begin{array}{l}\text { - Ausreichende } \\
\text { Kennzeichnung der } \\
\text { Überquerungsstelle, } \\
\text { z. B. mit } \\
\text { (innenbeleuchtetem) } \\
\text { Richtzeichen } 350 \text { oder } \\
\text { mit Gefahrenzeichen } \\
\text { 133, 134 (vgl. Anhang A } \\
\text { 1) }\end{array}$ \\
\hline
\end{tabular}




\section{A 8 Empfohlenen Maßnahmen zur Gestaltung von Knotenpunkten insbesondere aus Sicht von Menschen mit Mobilitätseinschränkungen/-behinderungen}

\section{Sichtbeziehungen:}

Gute Sichtbeziehungen zwischen Kraftfahrzeugverkehr und überquerenden Fußgängern spielen gerade für mobilitätsbehinderte Menschen eine wichtige Rolle.

Die Unfallanalyse der Unfälle mit behinderten Menschen zeigt, dass mindestens der Teil der Rollstuhlnutzer an Knotenpunkten verhältnismäßig oft von abbiegenden Kraftfahrern übersehen wird (Abschnitt 3.2.5.5) und es dadurch zum Teil zu schweren Unfällen kommt. Gerade Rollstuhlnutzer sind durch Ihre niedrige Sitzposition für Kraftfahrer schwerer zu erkennen (analog der Problematik bei Kindern).

\section{Mittelinseln:}

Überquerungen an nichtsignalisierten Knotenpunkten (aber auch an signalisierten Überquerungsstellen und auf der Strecke) sollten möglichst mit Überquerungshilfen oder Mittelinseln ausgestattet werden.

Aufgrund verringerter Gehgeschwindigkeit oder Problemen bei der (akustischen) Orientierung ist es hilfreich, wenn Verkehrsströme des Kraftfahrzeugverkehrs getrennt werden. So können sich überquerende Fußgänger zunächst auf eine Fahrtrichtung konzentrieren und die Überquerungslängen werden verkürzt. Gleichzeitig wird die Situation auch für Pkw-Fahrer weniger komplex.

Mittelinseln sollten nach denselben Prinzipien wie Zu- und Abgang zur Überquerung gestaltet werden. Das bezieht sich vor allem auf Bordsteinabsenkungen (z. B. differenzierte Bordhöhen) und Ausstattung mit Bodenindikatoren.

An Überquerungsstellen an signalisierten Knotenpunkten spielt die Trennung der Verkehrsströme eine geringere Rolle, da die Überquerung im Schutz der Signalisierung erfolgt. Allerdings kann der Einbau einer Mittelinsel - vor allem bei größeren Überquerungslängen - auch dort zweckmäßig sein, um insbesondere für Menschen mit einer geringen Gehgeschwindigkeit während der Grünphase eine für sie sicher zu bewältigende Überquerungslänge anbieten zu können.

\section{Taktile Bodeninformationen:}

Insbesondere an komplexen, größeren Knotenpunkten ist es angebracht, Bodenindikatorbasierte Leitsysteme für blinde und sehbehinderte Menschen zu verlegen.

Die einer festgelegten Verlegungslogik folgenden Bodenindikator-basierte Leitsysteme können die Orientierung blinder und sehbehinderter Menschen an Knotenpunkten verbessern. Folgende Elemente werden an Überquerungsbereichen verlegt (vgl. Abbildung 132 und zu weiteren Details s. (DIN 2011) und (FGSV 2011):

- Auffindestreifen

Auffindestreifen dienen der besseren Auffindbarkeit von Überquerungsstellen. Sie sind aus Noppenplatten hergestellt, mindestens $60 \mathrm{~cm}$ tief und über die gesamte Breite der Gehbahn verlegt. Kreuzt ein Auffindestreifen einen Radweg, wird der 
Auffindestreifen unterbrochen. An lichtsignalgeregelten Überquerungsstellen führt der Auffindestreifen an den Lichtsignalmast. Ist der Lichtsignalmast auf diese Weise taktil auffindbar, kann ein evtl. Vorhandenes akustisches Ortungssignal in den Nachtstunden abgeschaltet werden, um die Schallemissionen für Anwohner zu reduzieren.

- Richtungsfeld

Das Richtungsfeld unterstützt Langstocknutzer bei der Festlegung der Überquerungsrichtung, besonders im Bogen und bei schiefwinkligen Überquerungsanlagen. Dieses Feld liegt unmittelbar hinter dem Bord mit einer Tiefe von mindestens $60 \mathrm{~cm}$. Die Breite entspricht der Breite der Überquerungsstelle bei Überquerungen in Längsrichtung. Bei seitlich liegenden Überquerungsstellen (z. B. auf der Strecke) ist eine Breite entsprechend der Breite des Auffindestreifens ausreichend, da über den Auffindestreifen direkt zum Richtungsfeld geführt wird. Das Richtungsfeld wird aus Rippenplatten verlegt. Die Rippenstruktur zeigt in Längsrichtung die einzuschlagende Gehrichtung bei der Überquerung an. Das bedeutet, dass bei einer schiefwinkligen Überquerung auch das Richtungsfeld schiefwinklig eingebaut werden muss.

- Sperrfeld

Das Sperrfeld dient der Absicherung eines auf Fahrbahnniveau abgesenkten Bereichs bei der Überquerungsstelle mit differenzierter Bordhöhe (s Abschnitt zu Bordsteinhöhe und Absenkung, S. 119). Das Sperrfeld besteht aus Rippenplatten, die parallel zum Bordstein verlegt werden. Es hat eine Tiefe von mindestens $60 \mathrm{~cm}$ und sichert die Absenkung über die volle Breite ab (inkl. Übergangssteinen).

Die strukturierten Oberflächen von Bodenindikatoren unterstützen blinde und sehbehinderte Menschen in der Orientierung. Allerdings können die Strukturen die Überrollung für Rollstuhlund Rollatornutzer erschweren. Hinweise auf geeignete Strukturen finden sich in der DIN 32984 (DIN 2011) und den Hinweisen für barrierefreie Verkehrsanlagen (FGSV 2011). Allerdings können hinsichtlich von idealen Lösungen für beide Gruppen noch keine abschließenden Aussagen getroffen werden. Einer Lösung in dieser Frage widmet sich derzeit ein laufendes Forschungsvorhaben. ${ }^{35}$ Ein oft praktizierter Kompromiss liegt darin, die Felder mit Bodenindikatoren nur in der Mindesttiefe von $60 \mathrm{~cm}$ auszuführen. Sie könne so i. d. R. von Langstocknutzern ertastet werden (auch podotakti ${ }^{36}$ ) und bilden einen geringeren Widerstand bei der Überrollung als Felder in einer Tiefe von $90 \mathrm{~cm}$.

Bodenindikator-basierte Leitsysteme sollten immer auch visuell kontrastierend ausgeführt werden, damit sie für sehbehinderte Menschen besser nutzbar sind. Diese Gruppe ist zahlenmäßig insbesondere zukünftig von größerer Bedeutung. Der Nutzen der Maßnahme wird somit bedeutend erweitert.

Auch im Bereich einer Furt können taktile Informationen eingebracht werden. Gerade bei langen Furten ohne Mittelinsel ist dies für Langstocknutzer hilfreich, da dort die Gefahr des

\footnotetext{
35 FE-Nr. 70.0500/2010 „Barrierefreie Querungsstellen an Hauptverkehrsstraßen - Ausgestaltung der Bordsteinkante im Detail“; bearbeitet durch die STUVA und Partner; voraussichtlich bis Mitte 2013.

${ }^{36}$ Podotaktil $=$ mit den Füßen.
} 
Abdriftens besteht (Abbildung 130). Bodeninformationen im Fahrbahnbereich dürfen allerdings nicht visuell kontrastierend ausgeführt werden, um eine Verwechslungsgefahr mit Markierungen zu vermeiden.

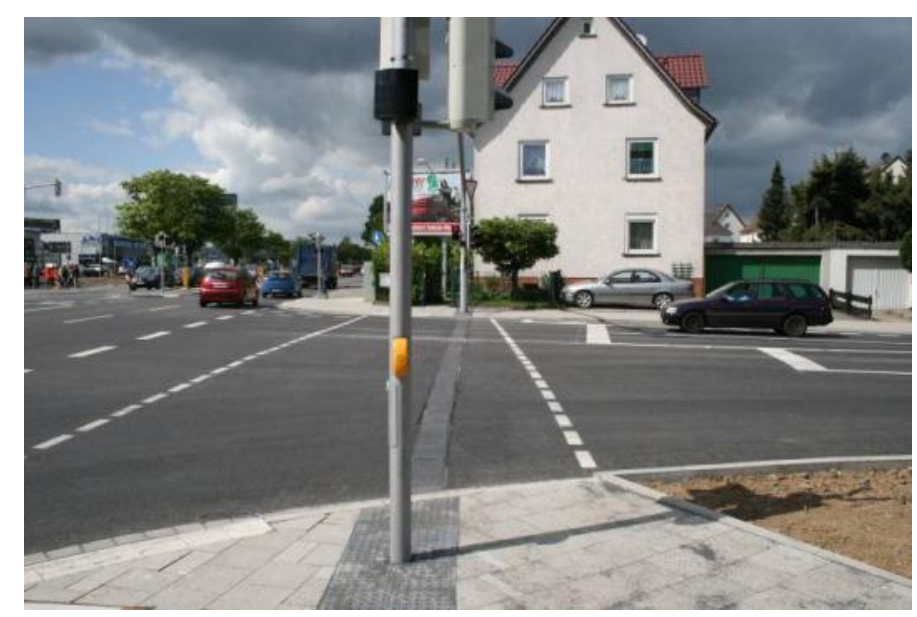

Abbildung 130: Taktiler Leitstreifen im Fahrbahnbereich über eine lange Furt (Kassel) [Foto: Wiesenhütter, KVC]

\section{Bordsteinhöhe und Absenkungen:}

Wenn möglich, sollte ein Teil der Bordsteinkante im Überquerungsbereich auf Fahrbahnniveau abgesenkt werden, während ein anderer Abschnitt auf einer eindeutig ertastbaren Höhe verbleibt (differenzierte Bordhöhe, Abbildung 131). Damit kann den Anforderungen von Rollstuhl- und Rollatornutzern und eben auch von Langstocknutzern entsprochen werden.

Die Absenkung ist gemäß den Vorgaben in den technischen Regelwerken ausreichend abzusichern („Sperrfeld“), damit sich keine Risiken für blinde und sehbehinderte Menschen ergeben, unbemerkt auf die Fahrbahn zu gelangen. Kann eine Überquerungsstelle nicht mit differenzierten Bordhöhen gestaltet werden (z. B. aus Platzgründen, Gründen der Wasserableitung usw.), besteht unter Berücksichtigung einer akzeptierten und verkehrssicheren Lösung für alle Fußgänger derzeit nur die Möglichkeit, die Kompromisslösung mit einer Bordhöhe von $3 \mathrm{~cm}$ auszuführen. 


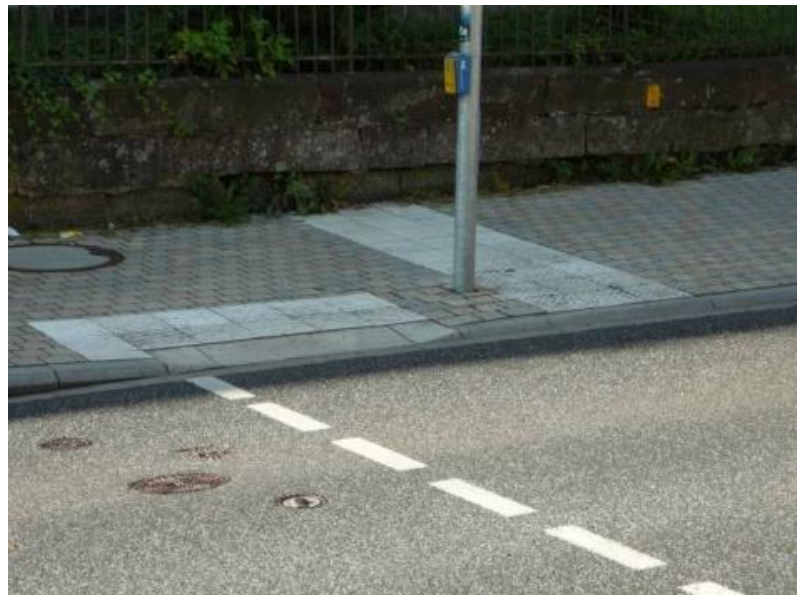

Abbildung 131: Überquerungsstelle mit differenzierter Bordhöhe, Sperrfeld und Auffindestreifen (hier mit Sonderbord) [Foto: Boenke]

Es ist allerdings festzustellen, dass aufgrund der Zunahme der Anzahl von Menschen mit Sehbehinderungen und Gehbehinderungen der Zielkonflikt über die Höhe der Bordsteinkante an Überquerungsstellen immer größer wird. Eine wissenschaftlich fundierte Lösung neben den beiden genannten Möglichkeiten gibt es derzeit allerdings nicht. Die Analyse der Unfälle mit Beteiligung mobilitätsbehinderter Menschen (vgl. Abschnitt 3.2.5) sowie die Verhaltensbeobachtung (Abschnitt 6.5.2) zeigen, dass gerade die rollenden Gruppen eine Überquerung an Bordsteinkanten bevorzugen, die auf Fahrbahnniveau abgesenkt sind. Fehlt diese, werden auch Flächen genutzt, die bestimmungsgemäß nicht dem Fußgängerverkehr gewidmet sind (z. B. Radverkehrsanlagen, sofern sich dort eine Absenkung befindet oder auch Teile der Kfz-Fahrbahnen). Dies führt zu Konflikten mit dem Rad- und Kraftfahrzeugverkehr.

\section{Differenzierte Bordhöhen - Ausführung}

Aus Gründen der Verkehrssicherheit für alle Verkehrsteilnehmer sollten unter Beachtung der derzeit gültigen technischen Regelwerke folgende Gestaltungsgrundsätze beachtet werden (vgl. Abbildung 132):

- Die Absenkung auf Fahrbahnniveau sollte auf $1 \mathrm{~m}$ Breite begrenzt werden, um den Sicherheitsansprüchen blinder und sehbehinderter Menschen zu genügen. Für eine Umsetzung größerer Breiten fehlen derzeit noch gesicherte Evaluationen.

- Die Nullabsenkung sollte durch ein taktil erfassbares „Sperrfeld“ aus Bodenindikatoren abgesichert werden (Tiefe mind. $60 \mathrm{~cm}$, Breite entspricht der Breite des abgesenkten Bereichs).

- Der Bordstein im Überquerungsbereich für blinde und sehbehinderte Menschen sollte angehoben werden (auf $6 \mathrm{~cm}$ ), um eine taktil eindeutig ertastbare Kante (zur Absicherung und zur Ausrichtung) herzustellen. Die $3 \mathrm{~cm}$-Kante ist ein Kompromiss und kann für Langstocknutzer $u$. U. bereits zu gering sein (je nach Ausführung und Randbedingungen). Durch die Anhebung werden Verbesserungen für beide Gruppen erreicht (Absenkung nutzt gehbehinderten Menschen).

- Die Zuführung zur Überquerungsstelle für blinde und sehbehinderte Menschen sollte über einen taktil ertastbaren „Auffindestreifen“ erfolgen. Dieser läuft in einer Tiefe von 
mindestens $60 \mathrm{~cm}$ über die gesamte Gehbahn (bis zur inneren Leitlinie, z. B. Gebäudekante).

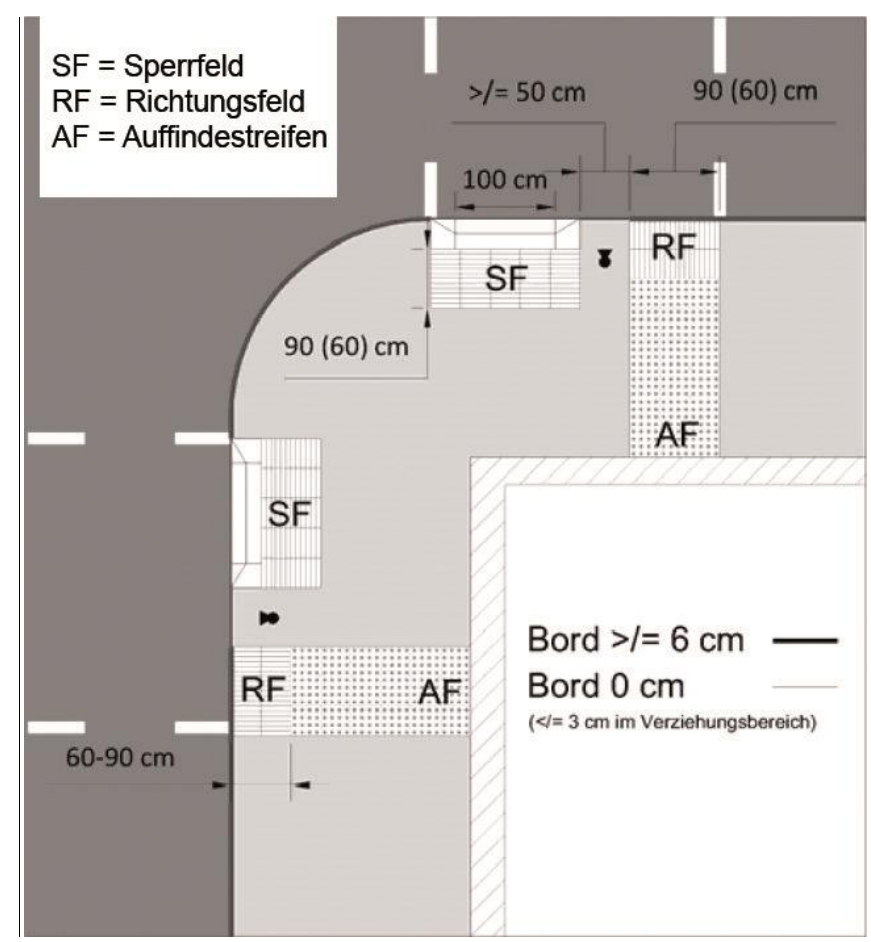

Abbildung 132: Überquerungsstelle mit differenzierter Bordhöhe - Skizze [FGSV 2011]

Die differenzierte Bordhöhe lässt sich allerdings nicht an jedem Knotenpunkt umsetzen. Randbedingungen wie Wasserführung, Platzangebot im Seitenraum usw. können eine Rolle spielen, warum eine Überquerungsanlage mit differenzierter Bordhöhe nicht umzusetzen ist. Daher wird die bisherige Kompromisslösung mit einem Auftritt von $3 \mathrm{~cm}$ (mindestens noch mittelfristig) eine Rolle spielen. Hier gilt es, den bisherigen Kompromiss an die geänderten Anforderungen der Nutzer weiterzuentwickeln, ohne die Verkehrssicherheit zu verringern. Dieser Problemstellung widmet sich derzeit ein Forschungsvorhaben ${ }^{37}$ im Auftrag des BMVBS, betreut von der BASt.

\section{Markierung von Bordsteinkanten mit Auftritt:}

Bordsteinkanten im Bereich von Überquerungsstellen sollten visuell kontrastierend markiert werden (vgl. DIN 2009)..

Niedrige Hindernisse werden insbesondere von sehbehinderten Menschen schlecht erkannt. Dazu zählen auch Bordsteinkanten, vor allem, wenn sie auf ein niedriges Niveau abgesenkt wurden. Dadurch ergeben sich Stolperfallen, die zu Stürzen mit teils schweren Verletzungen führen können.

\section{Akustische und taktile Signalgeber an Lichtsignalanagen:}

37 FE-Nr. 70.0500/2010 „Barrierefreie Querungsstellen an Hauptverkehrsstraßen - Ausgestaltung der Bordsteinkante im Detail“; bearbeitet durch die STUVA und Partner; voraussichtlich bis Mitte 2013. 
Lichtsignalanlagen an Knotenpunkten an Hauptverkehrsstraßen sollten zur Unterstützung einer sicheren Überquerung mit akustischen und taktilen Signalgebern ausgestattet werden; insbesondere, wenn die Überquerungsstellen von blinden oder sehbehinderten Menschen genutzt werden (vgl. RiLSA S. 65) (FGSV 2010).

Blinde und sehbehinderte Menschen benötigen in komplexen Situationen Orientierungshilfen, um sich selbstständig und sicher im Verkehrsraum bewegen zu können. Insbesondere bei Knotenpunkten an Hauptverkehrsstraßen handelt es sich um derartige komplexe Situationen:

- Durch oftmals hohe Verkehrsstärken ist eine Überquerung nur an signalisierten Überquerungsstellen möglich,

- die Verkehrsbelastung sorgt für einen hohen Geräuschpegel, welcher die akustische Orientierung erschweren kann,

- die Überquerungslängen sind häufig wegen mehrstreifiger Fahrbahnen groß. Damit besteht die Gefahr, dass blinde und stark sehbehinderte Menschen von der direkten Gehlinie abweichen und in den Kreuzungsbereich hineinlaufen.

Akustische und taktile (Vibrationsplatten) Signalgeber können an diesen Stellen eine wichtige Hilfestellung zur besseren Orientierung geben. Die akustischen Signalgeber („Akustische Leuchttürme“) sind dabei etwa in Höhe der Signalgeber und jeweils in Furtrichtung abstrahlend $\mathrm{zu}$ montieren, damit das Signal unterbrechungsfrei und in gleichbleibender Qualität (z. B. verdeckt durch Personen) zu hören ist (Abbildung 133). Sie sollten mindestens bis zur Furtmitte strahlen. Die taktilen Signalgeber werden i. d. R. an der Unterseite des Anforderungstasters eingebaut. Sie dienen gleichzeitig als Anforderungstaster für das akustische Freigabesignal. Die Anforderung dient dem Zweck, die Schallemissionen für Anwohner auf ein notwendiges Minimum zu verringern.

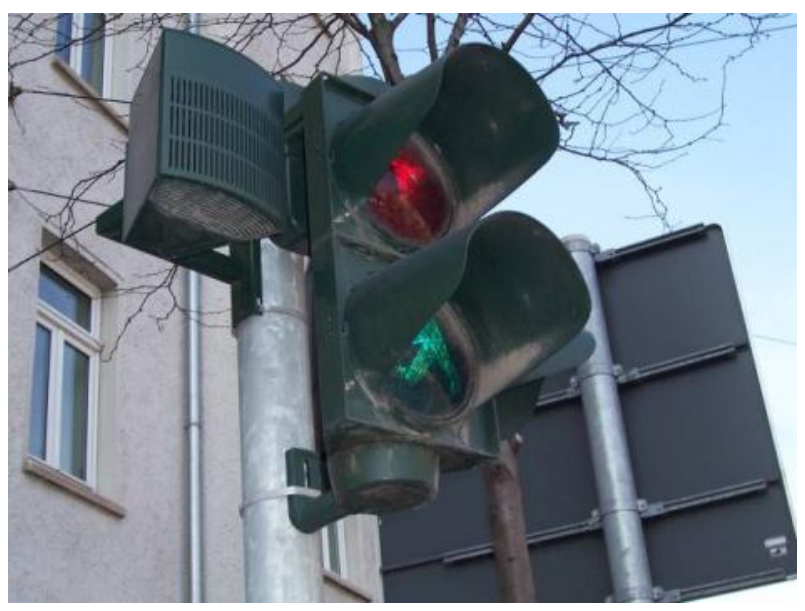

Abbildung 133: Schallgeber für ein akustisches Freigabesignal in Höhe der Signalgeber und in Furtmitte abstrahlend

\section{Räumgeschwindigkeit an signalisierten Fußgängerfurten:}

Für signalisierte Überquerungsstellen sollten für den Fußgängerverkehr maximal der nach den Richtlinien für Lichtsignalanlagen (RiLSA) (FGSV 2010) untere Grenzwert für die Räumgeschwindigkeit eingesetzt werden. 
Viele mobilitätseingeschränkte Menschen sind deutlicher langsamer unterwegs, als der Durchschnitt der Bevölkerung. Dies sollte auch bei den Grünphasen für den Fußgängerverkehr berücksichtigt werden. Dem kann durch die Ansetzung einer geringeren Räumgeschwindigkeit bei der Bemessung berücksichtigt werden. Die RiLSA nennen als unteren Grenzwert $1,0 \mathrm{~m} / \mathrm{s}$.

Für die Mindestfreigabezeit gilt bei Furten, die mit Zusatzeinrichtungen für blinde und sehbehinderte Menschen ausgestattet sind: Rechnerisch muss bei der Grünphase mindestens die gesamte Furtlänge zurückgelegt werden können (vgl. RiLSA S. 28) (FGSV 2010). 


\section{A 9 Regressionskurven zu Komplexitätsgraden und Unfallkenngrößen}

Tabelle 34: Regressionskurven „Komplexitätsindex zu Unfalldichte/Unfallrate“

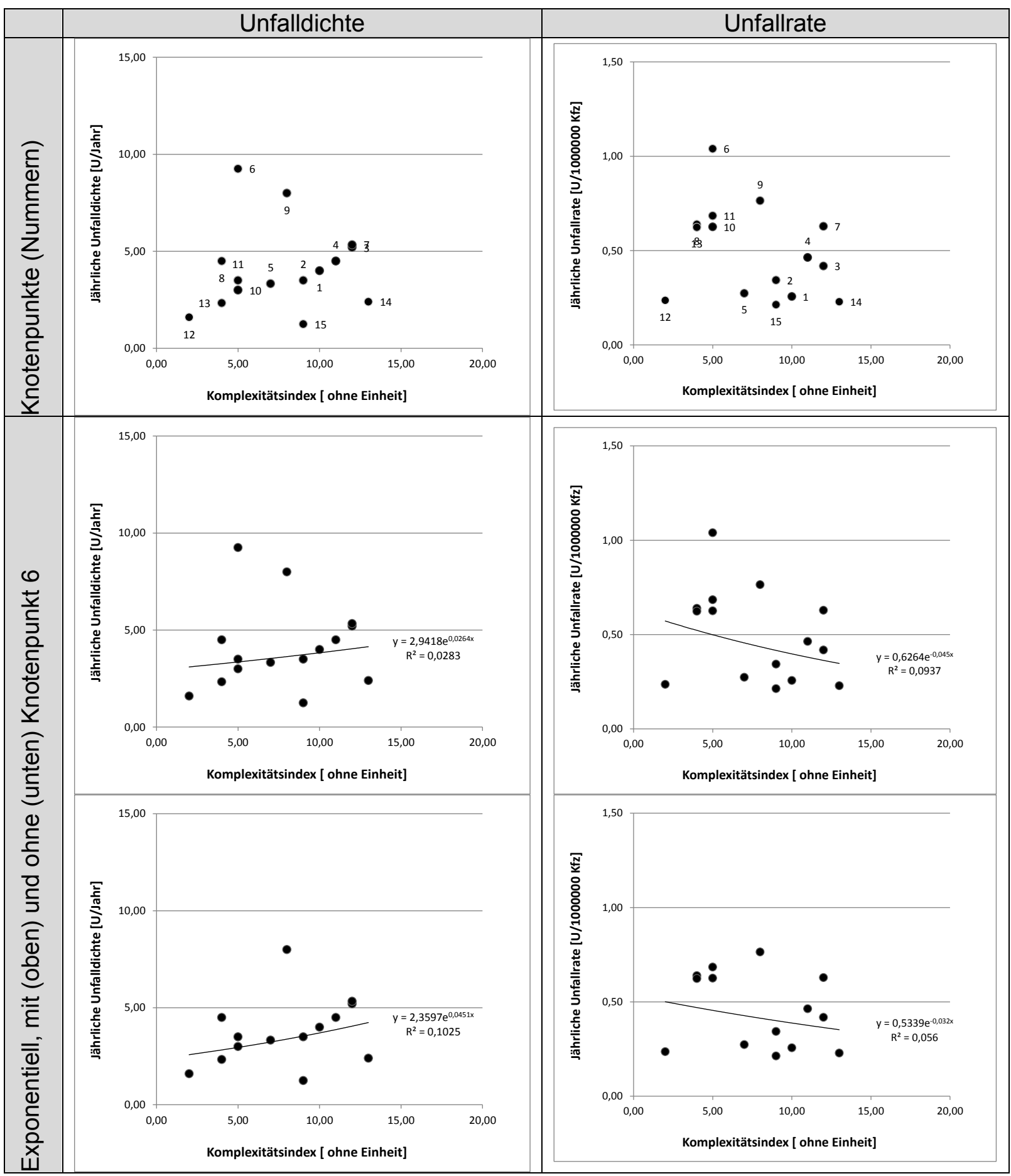




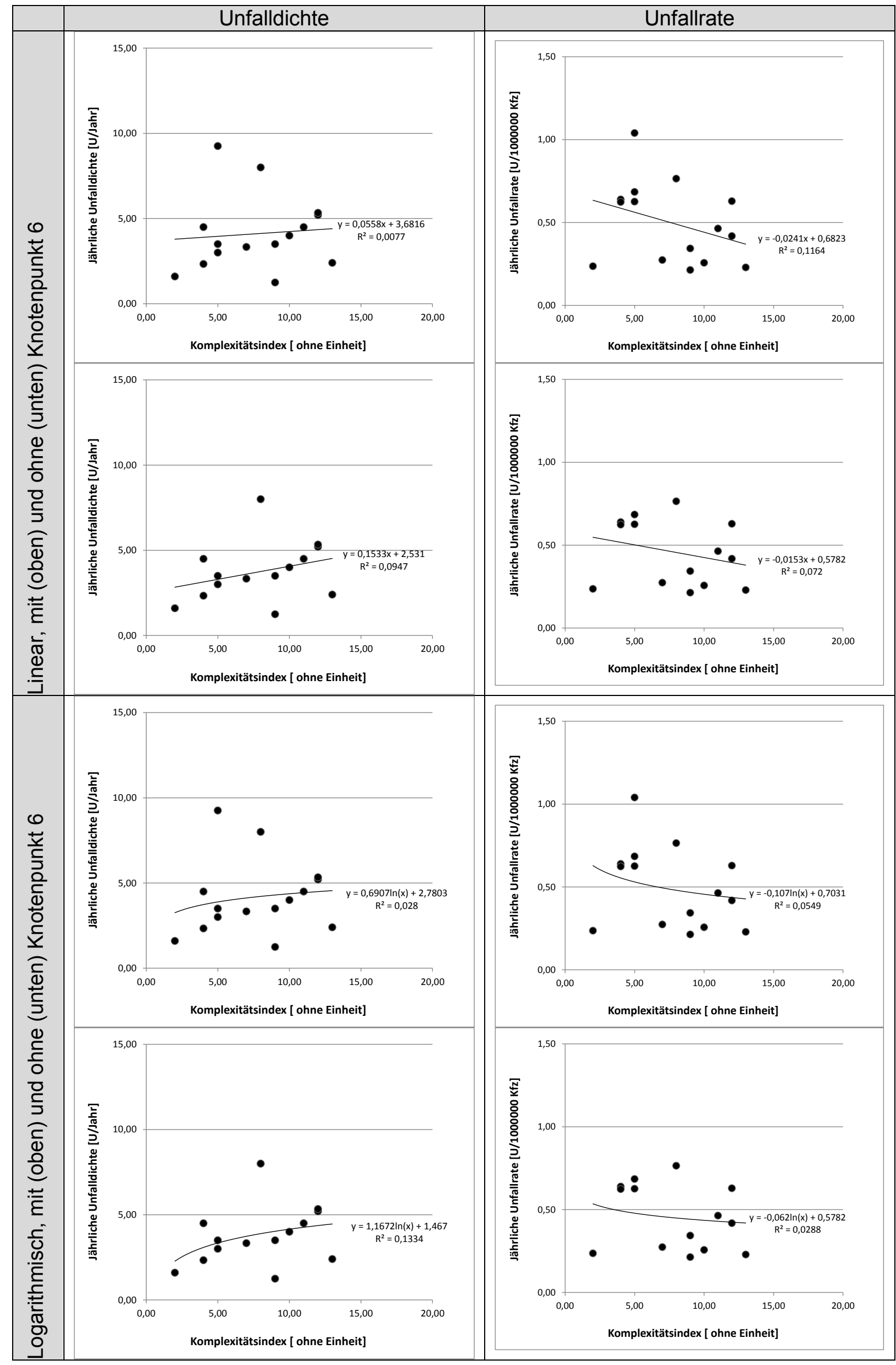




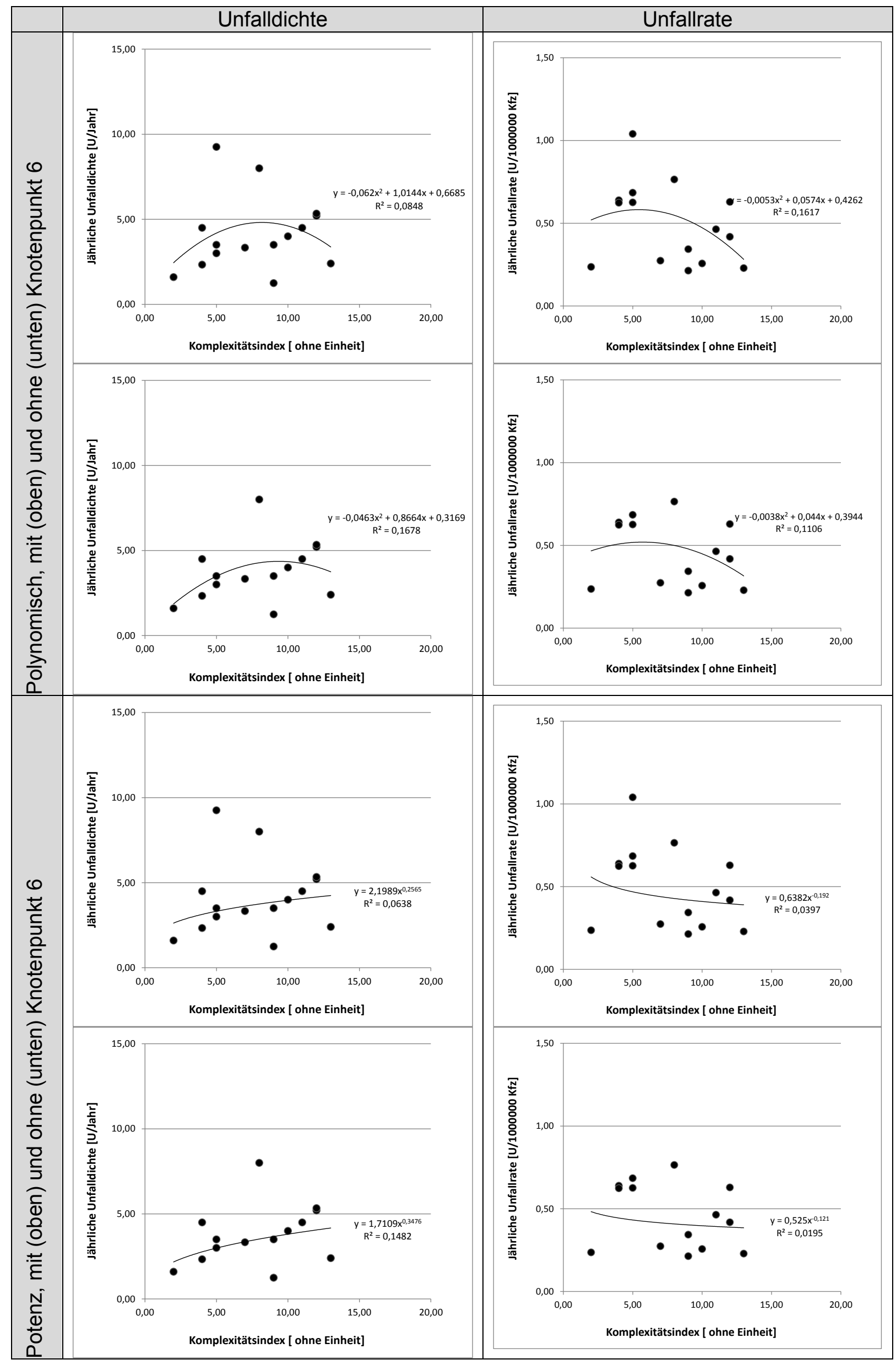


Tabelle 35: Regressionskurven „Komplexitätsindex zu Unfallkostendichte/Unfallkostenrate“

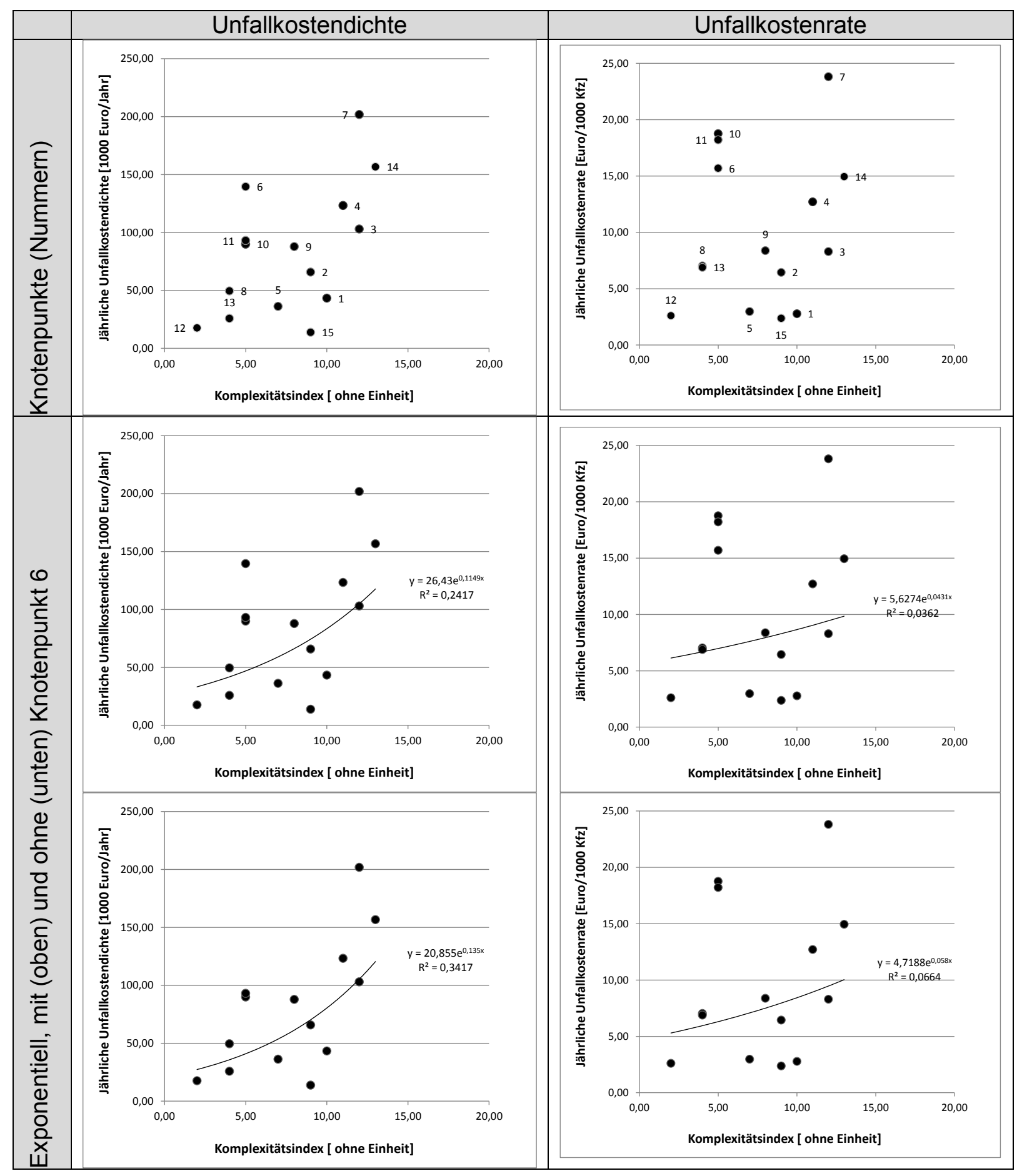




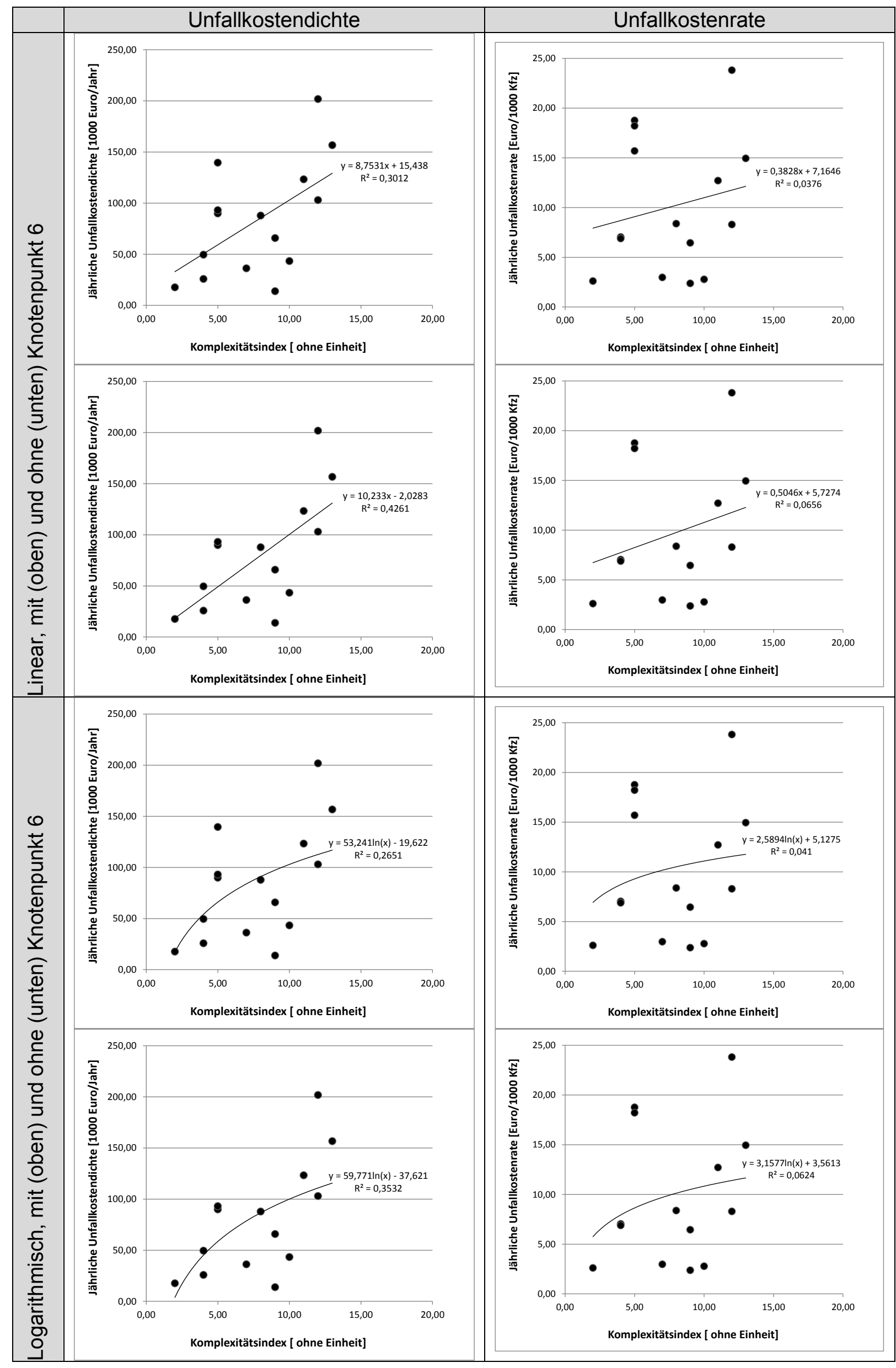




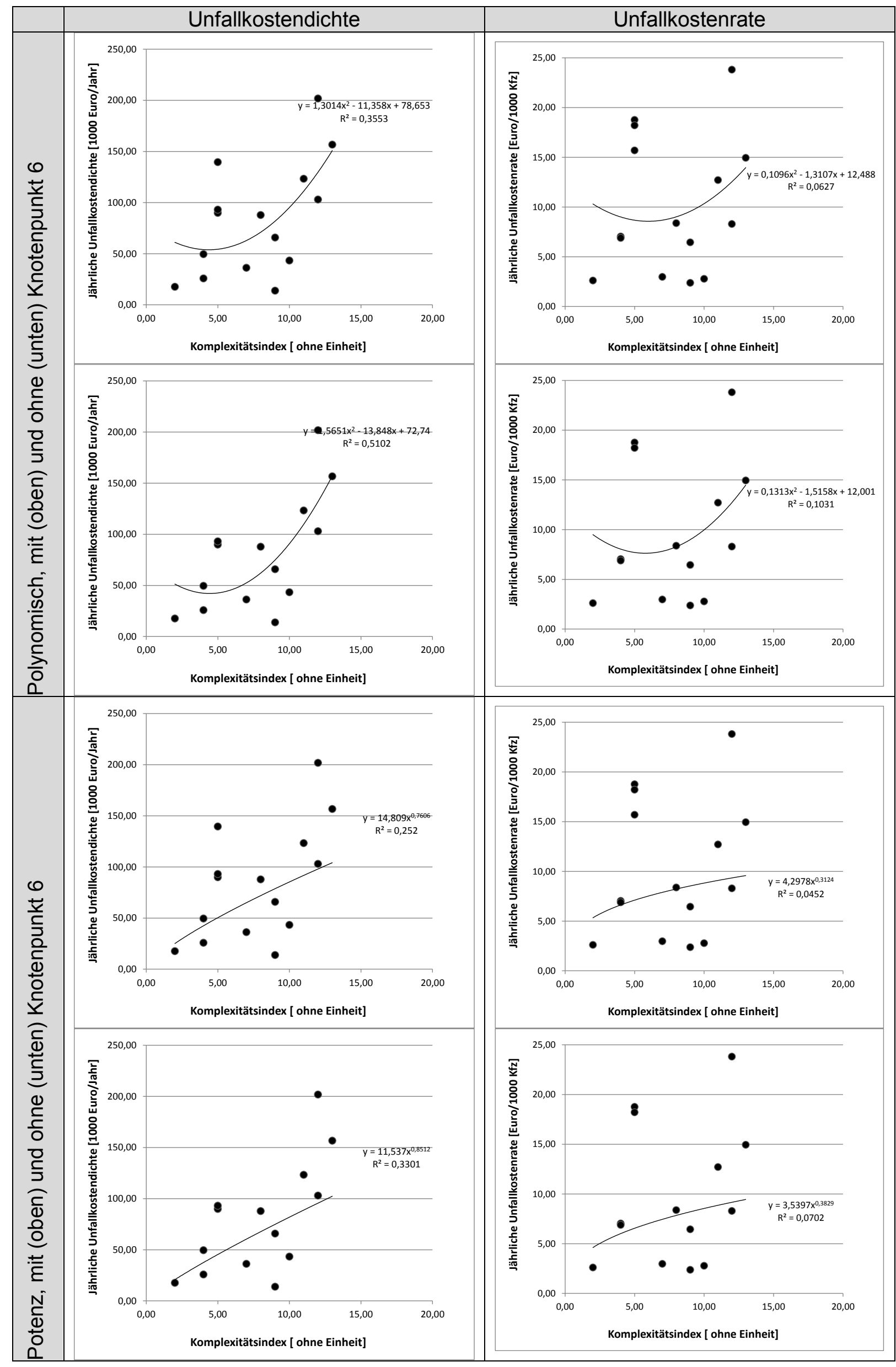




\section{GDV}

DIE DEUTSCHEN VERSICHERER

\section{Gesamtverband der Deutschen Versicherungswirtschaft e.V.}

Wilhelmstraße 43/43G, 10117 Berlin

Postfach 0802 64, 10002 Berlin

Tel.: 030/20 20 - 50 00, Fax: 030/20 20-6000

www.gdv.de, www.udv.de 